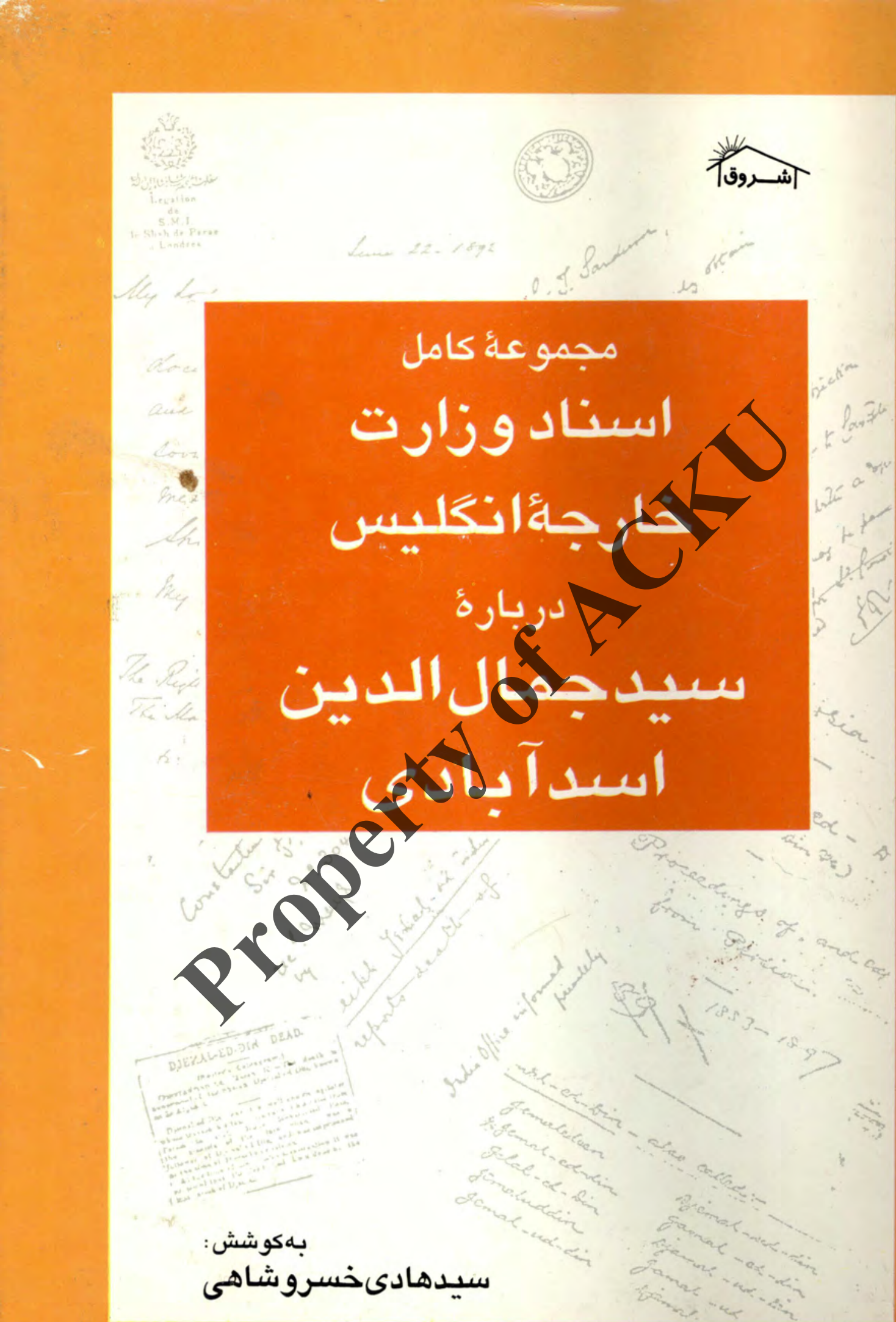




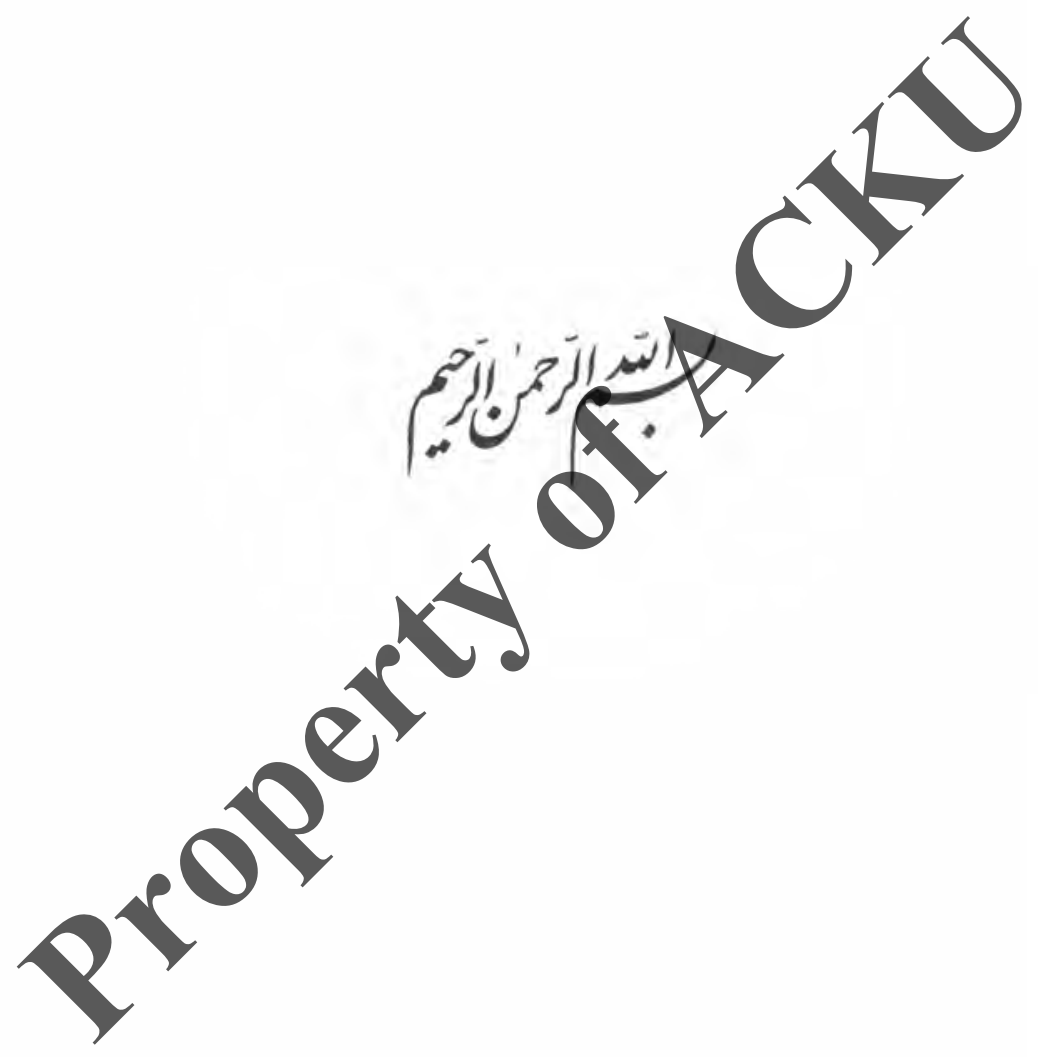




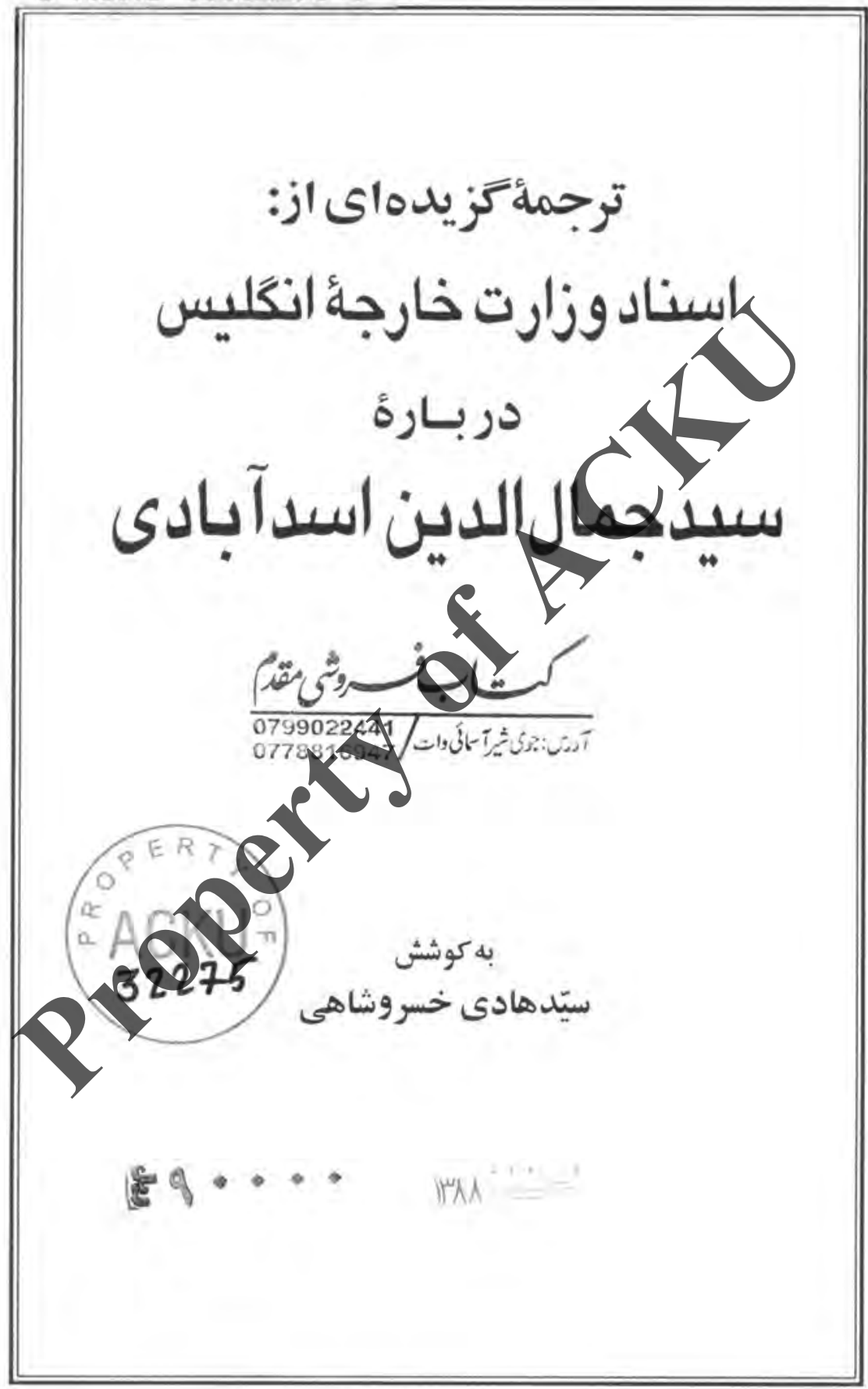




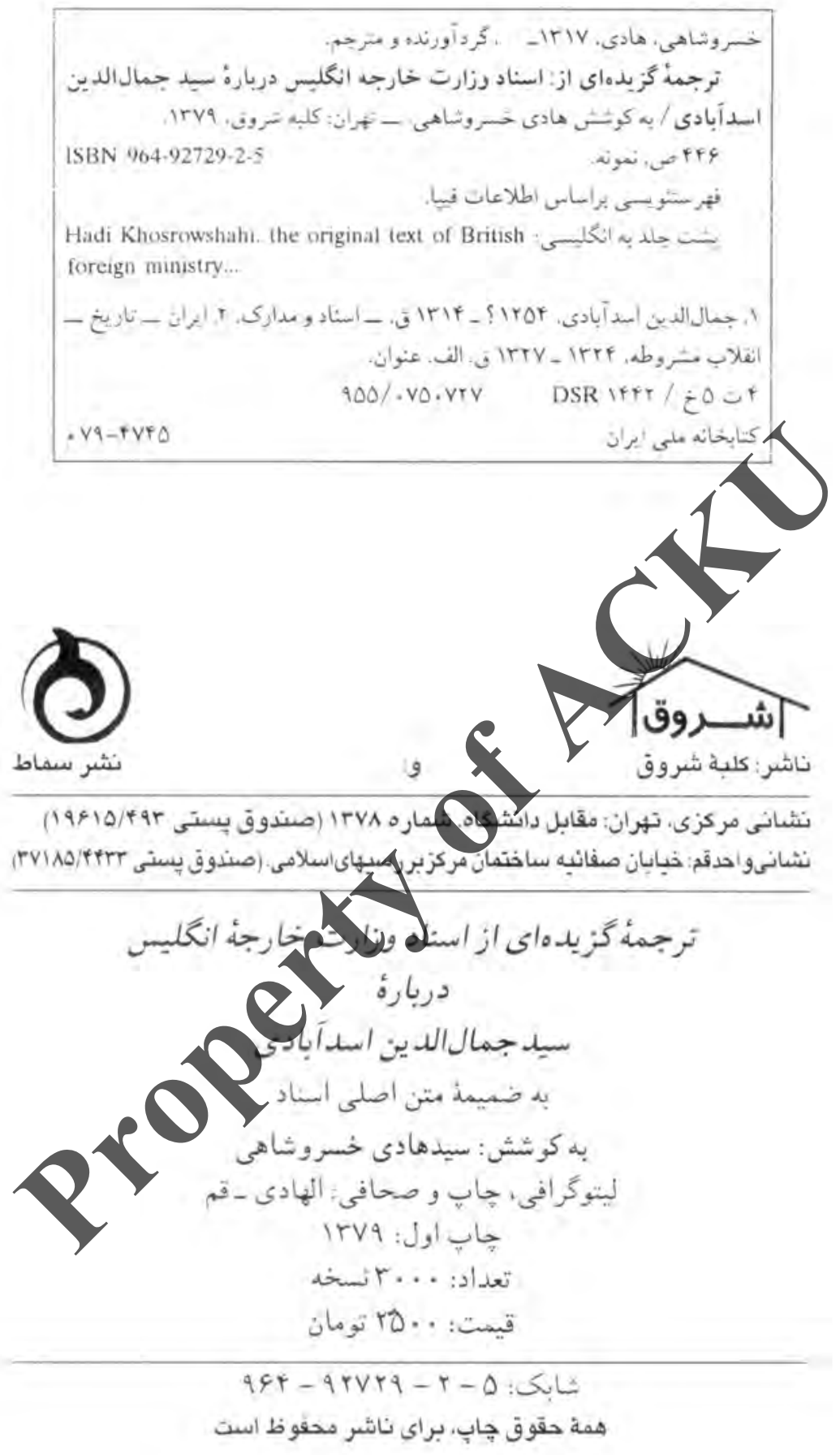




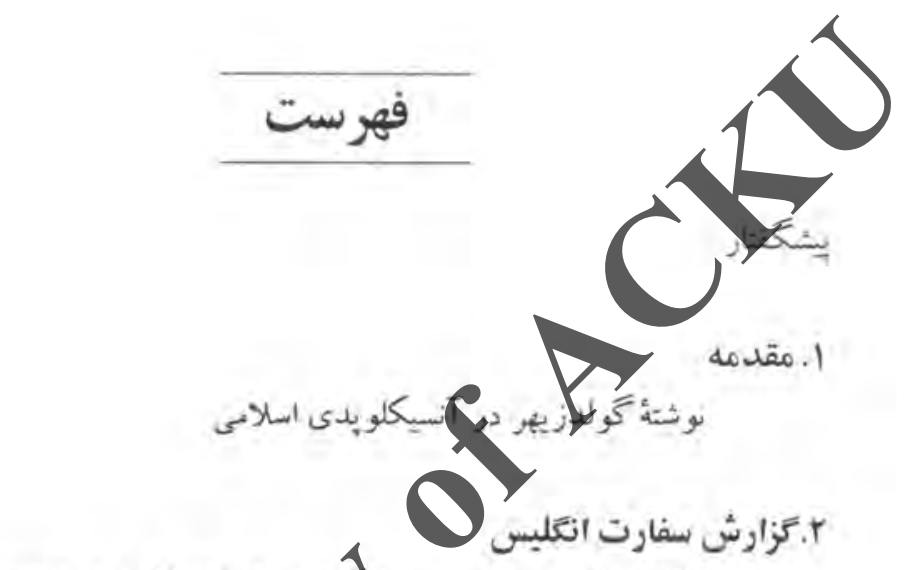

N

11

10

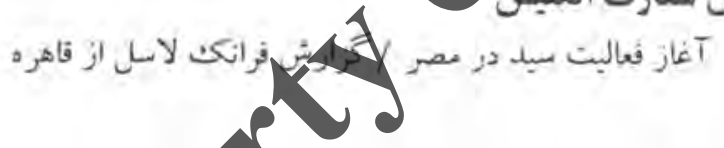

19

r. فعاليت مان سيد در فرانسه

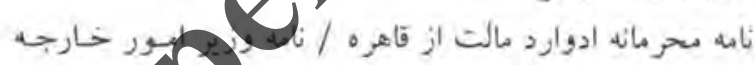

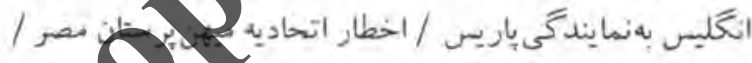

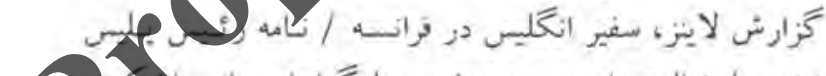

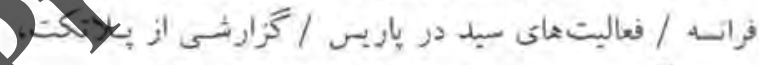

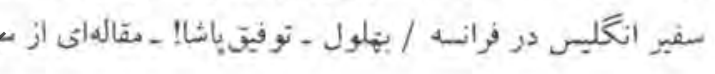

F

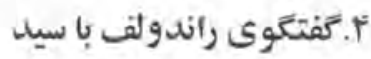

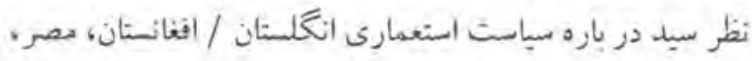

$$
\text { مودان }
$$

FV

ه. هديفار سيد با دوروموندولف

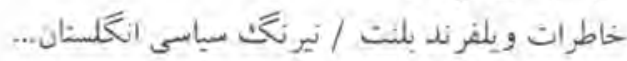


$\Delta r$ 7. اقدلأمات سيل در مسكو و بترزبوركت

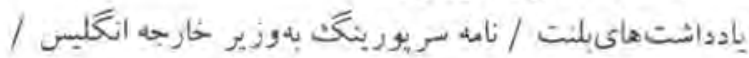

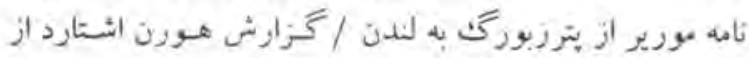

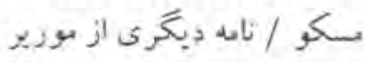

$9 \Delta$ Y. د سنتير و تبعيل سيل از ايران

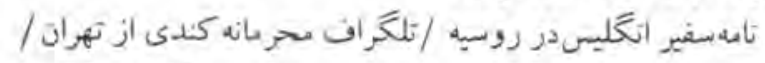

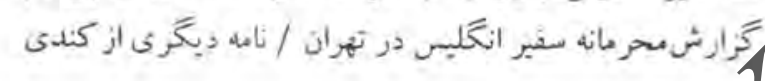

$\mathrm{Vr}$

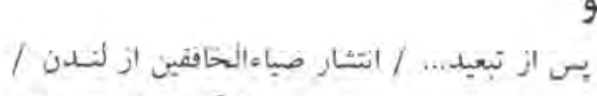

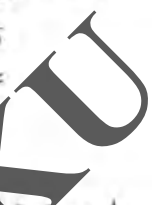

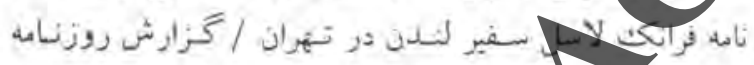

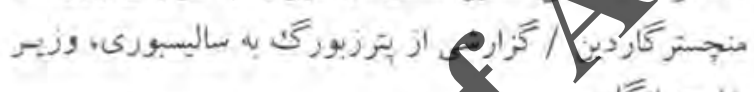

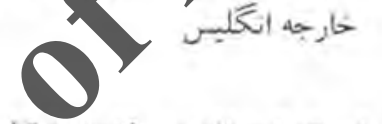

AT

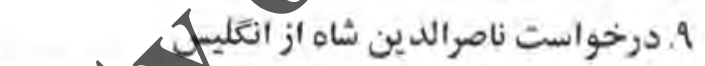

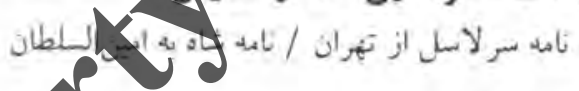

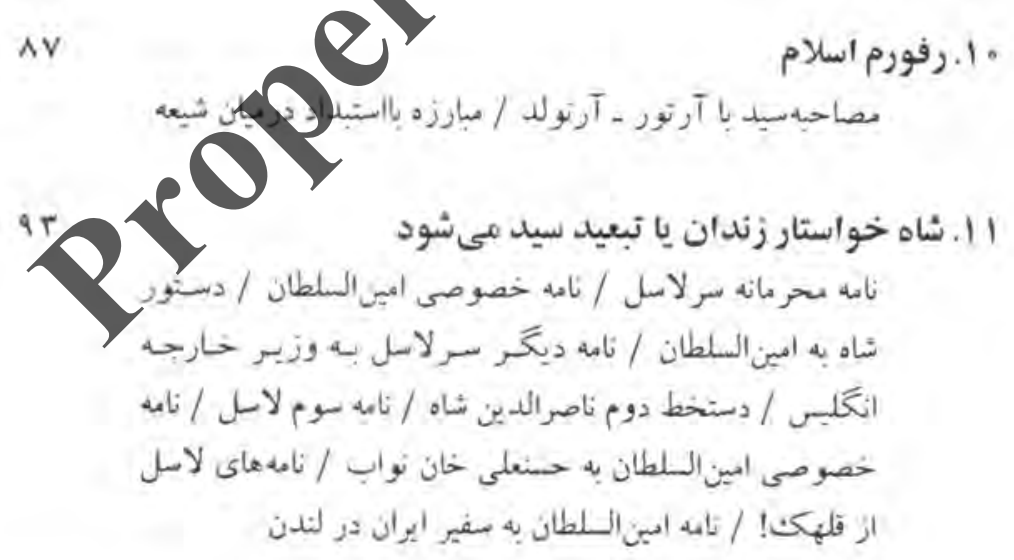

$1 \circ r$

لمهرست اعلام

$1 \cdot 9$

مثن انتليسى اسناد ومّزارشها 


\section{درباره ترجمه تزيده اسناد}

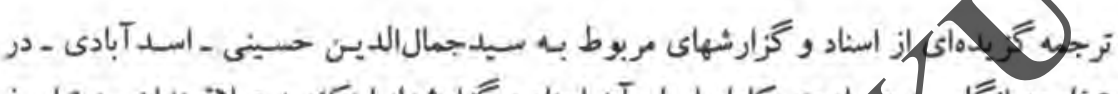

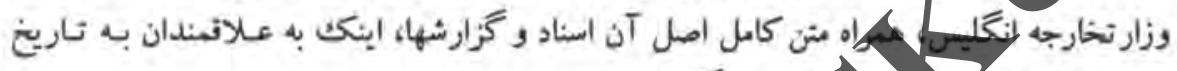

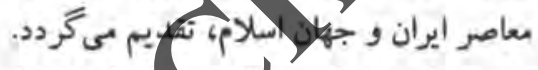

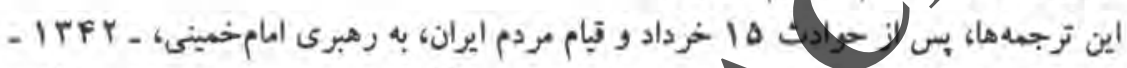

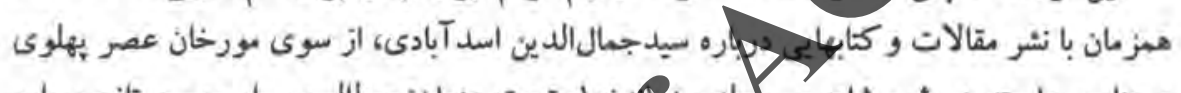

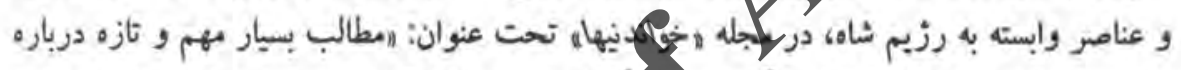

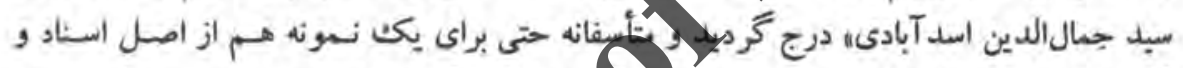

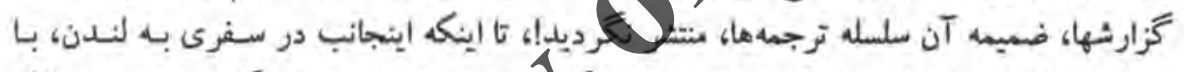

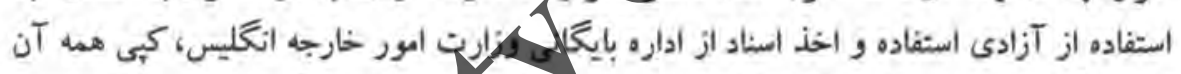

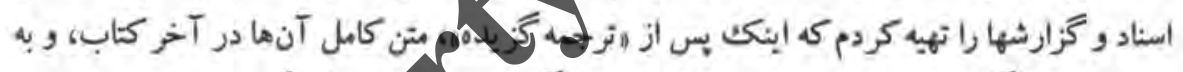

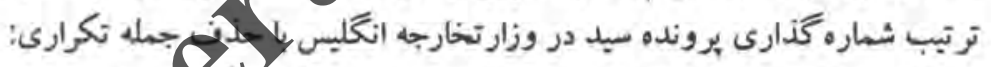

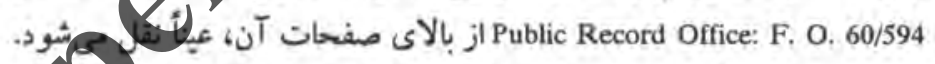

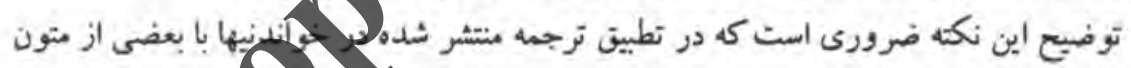

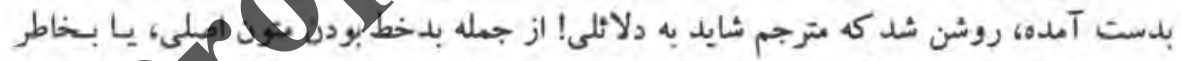

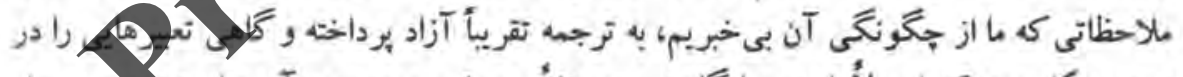

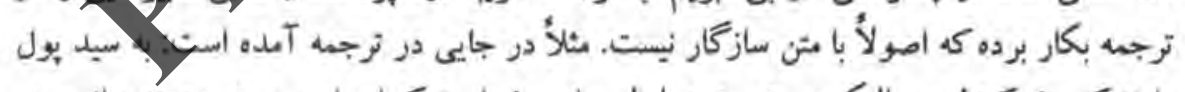

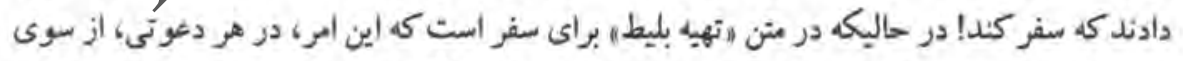

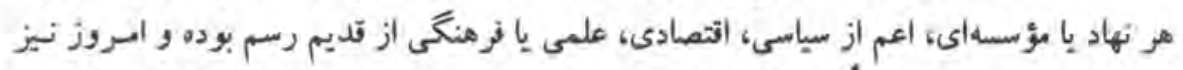

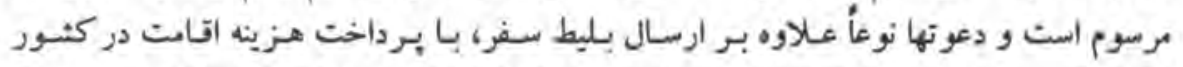

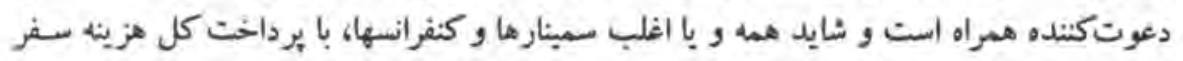

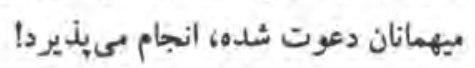

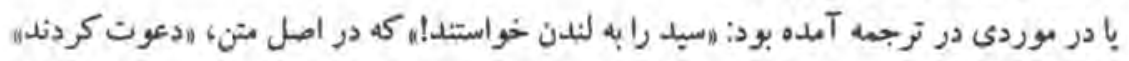

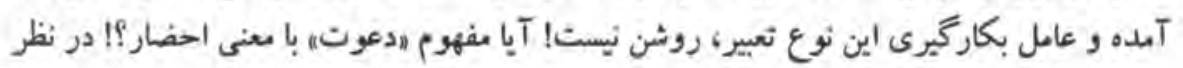




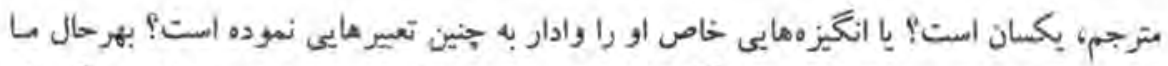

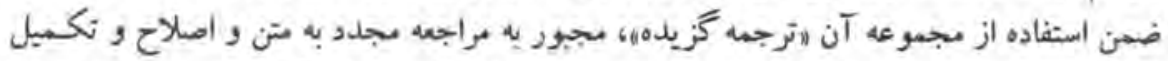

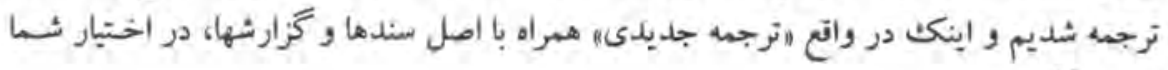
ترار مىكيرد.

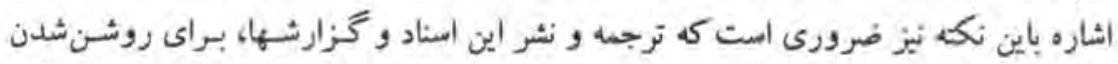

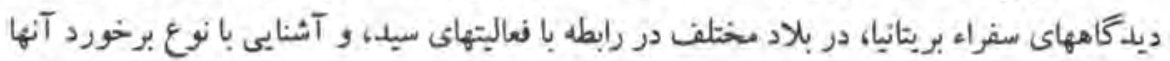

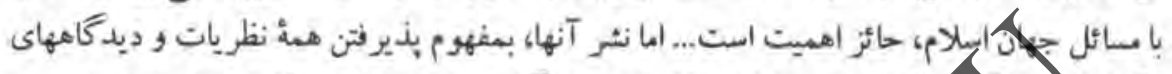

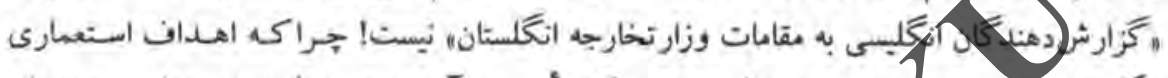

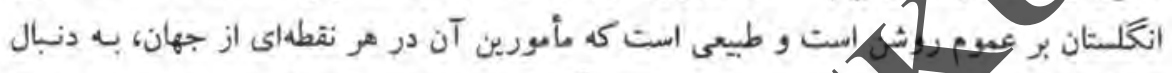

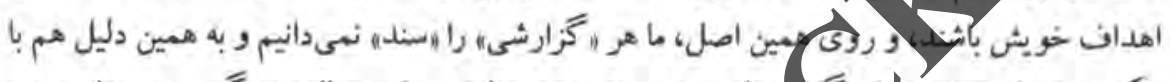

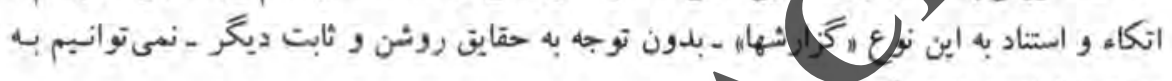
"اداورى نهابى" بنشينيم!

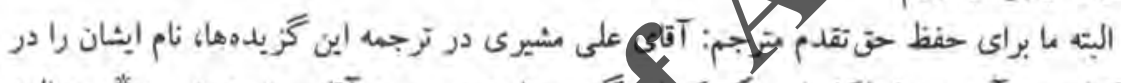

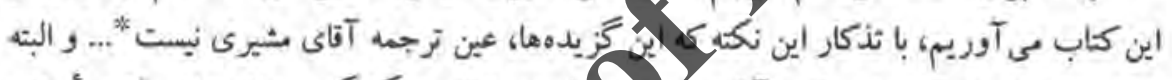

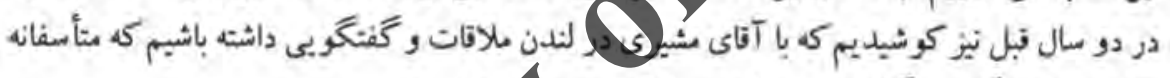

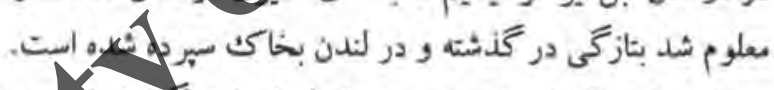

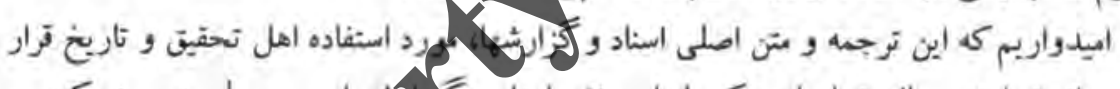

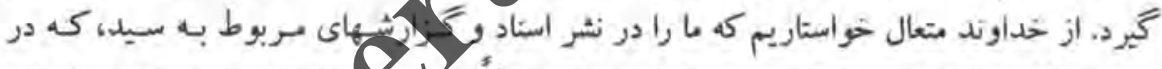

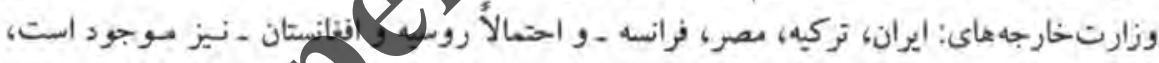

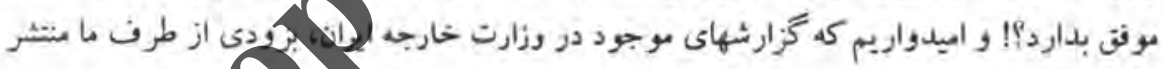
م 1 كردمد....

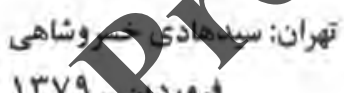
irva- فو

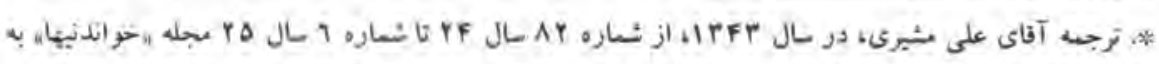

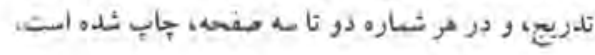




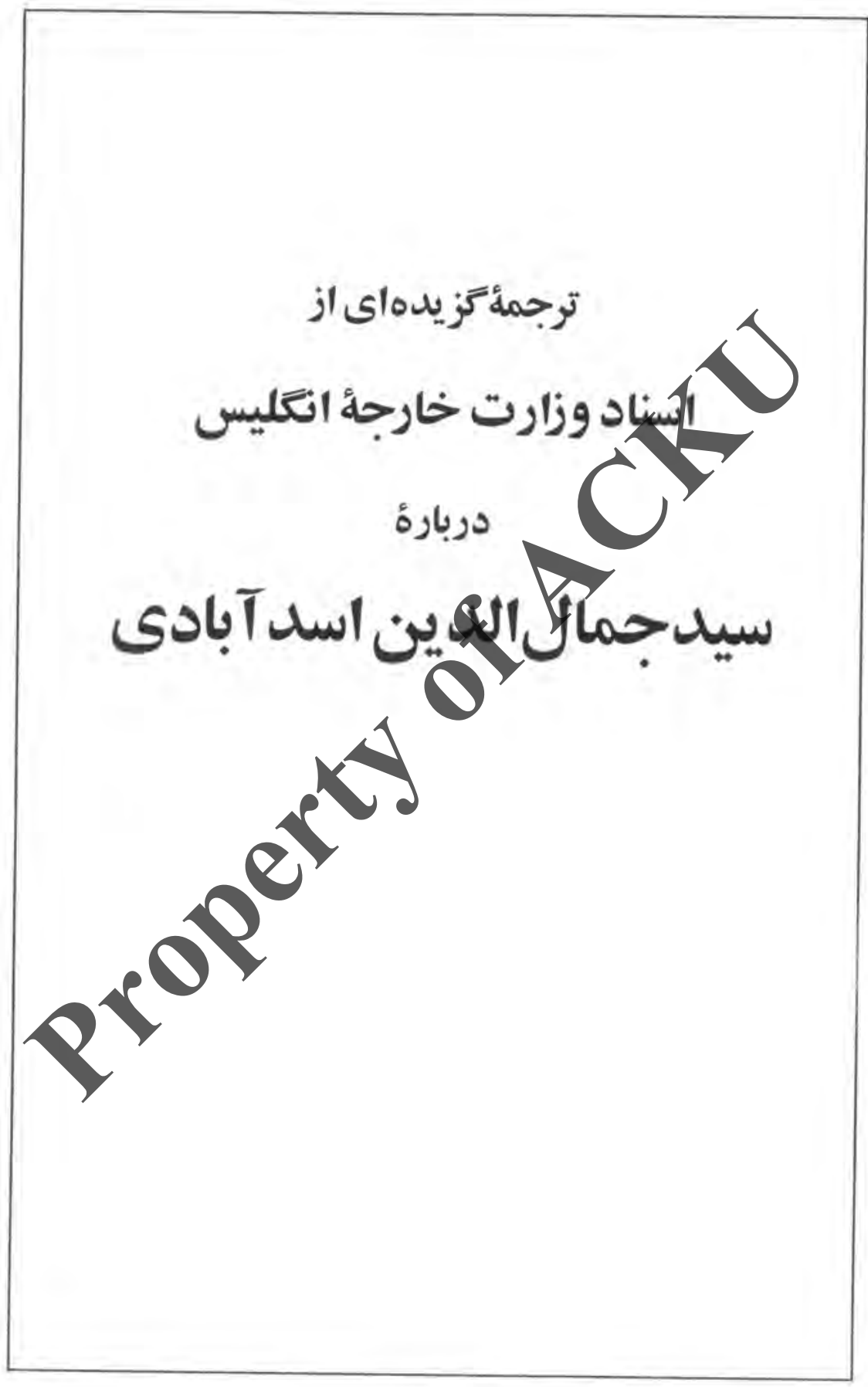




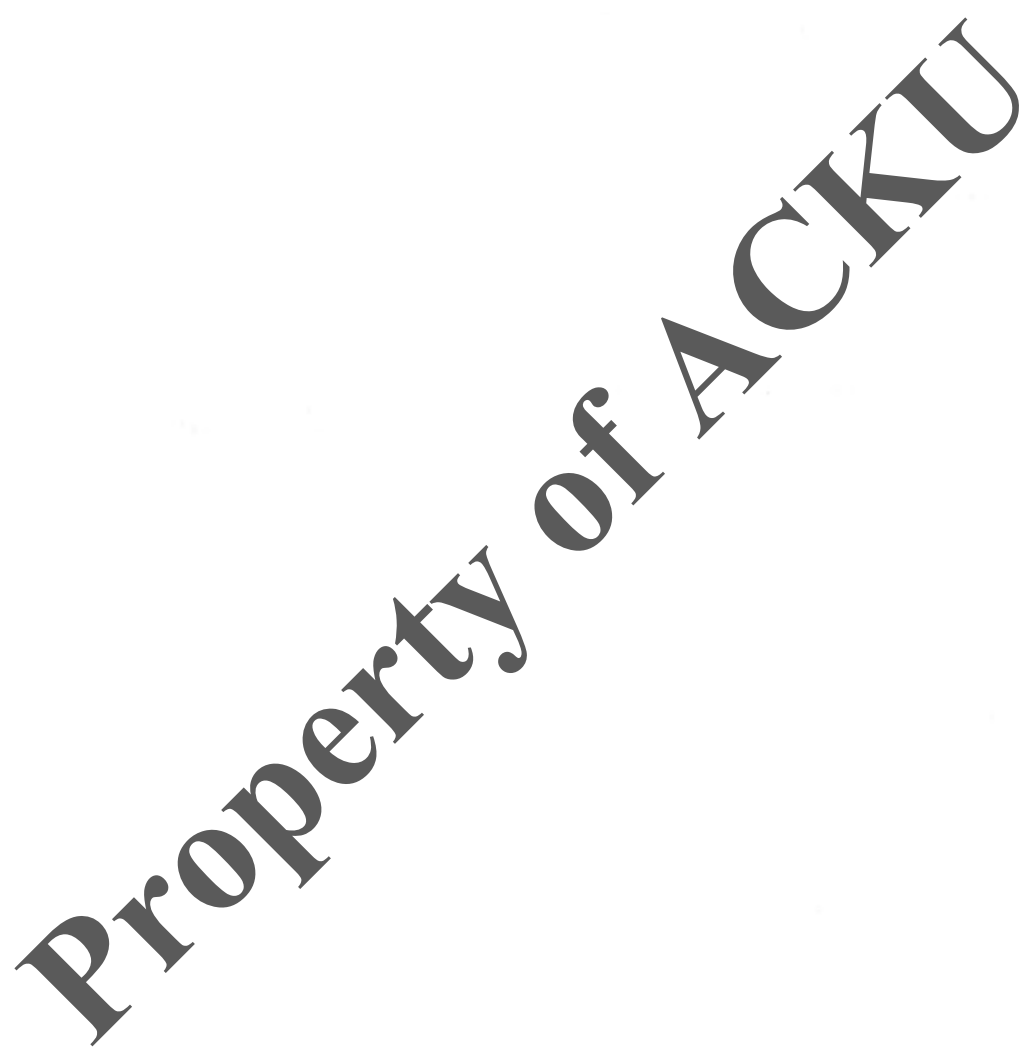




\section{مقدمه}

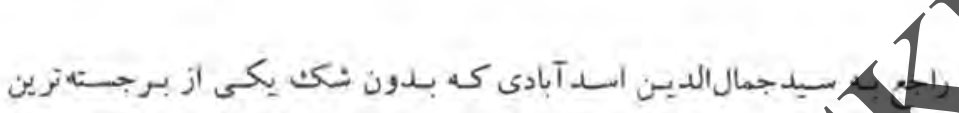

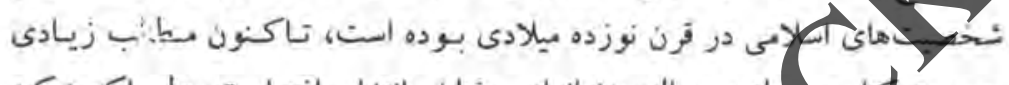

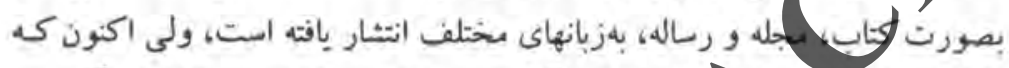

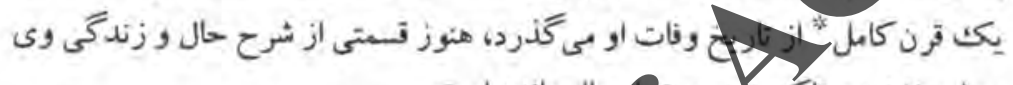

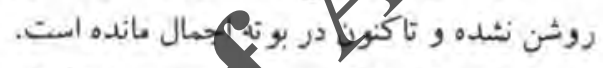

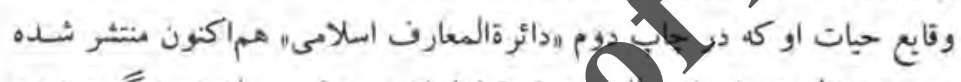

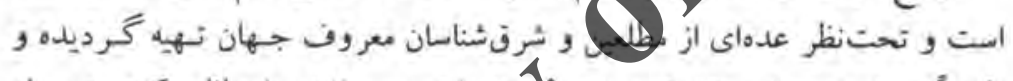

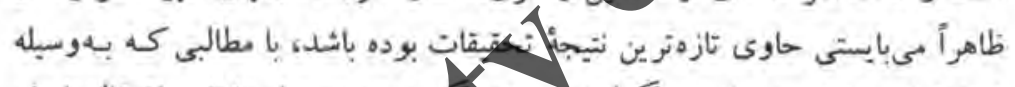

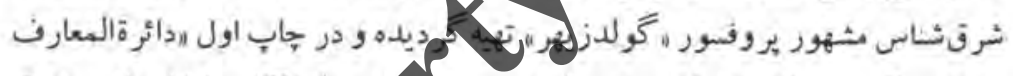

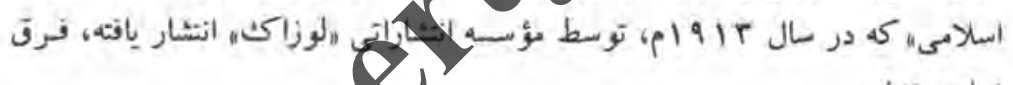
8 زيادى نداردن.

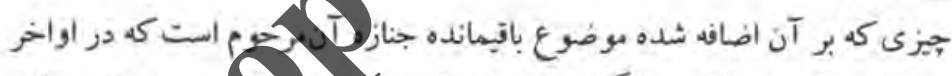

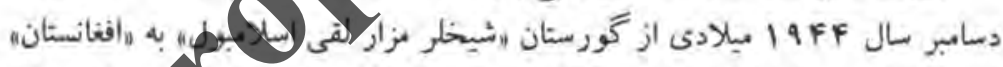

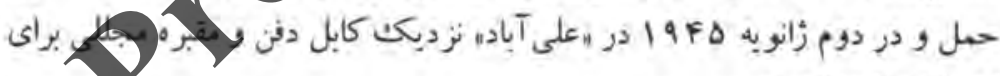
او ساخته شده است.

كَّرجه در سالهاى اخير بر اطلاعاتى كه نسابقاً بهدست آهده بود كمى اضافه شده.

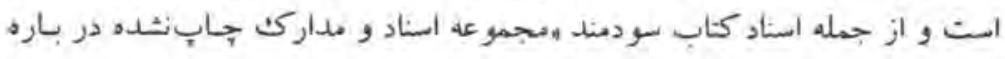

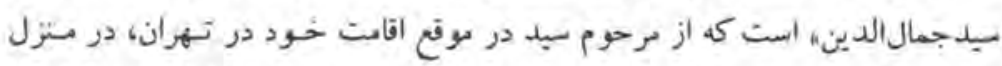

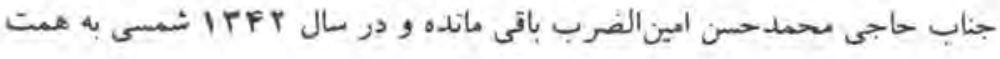

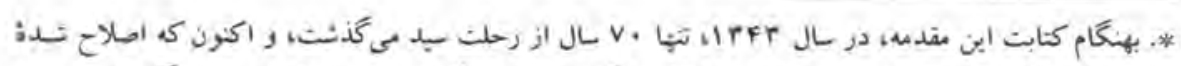

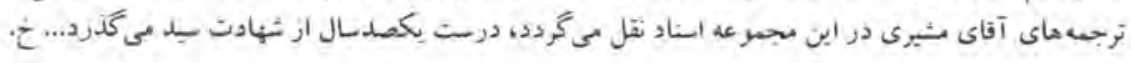


آقايان دكتر اصغر مهذوى و ايرج افنشار تدوين و تنظبم و منتشر خرديده است.

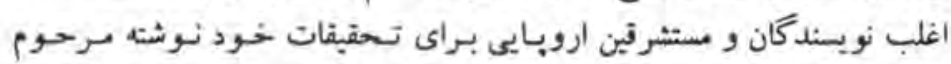

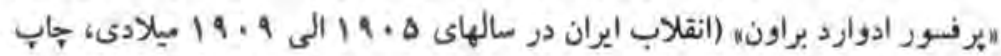

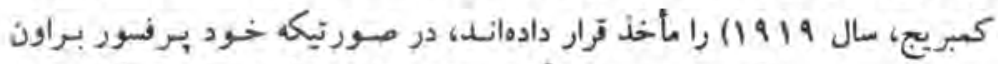

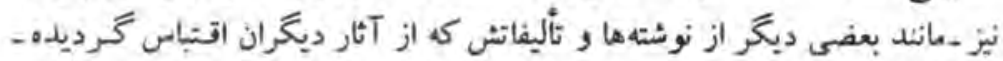

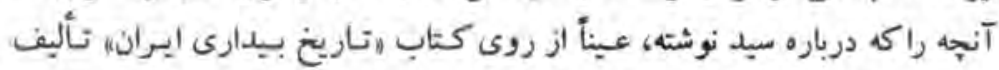

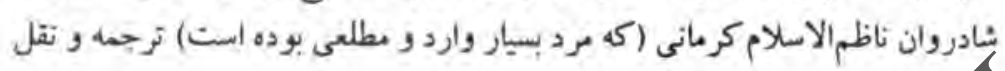

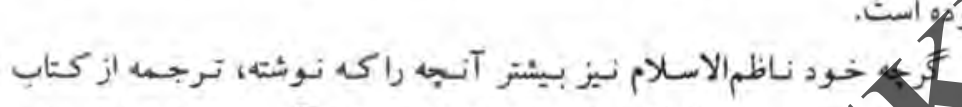

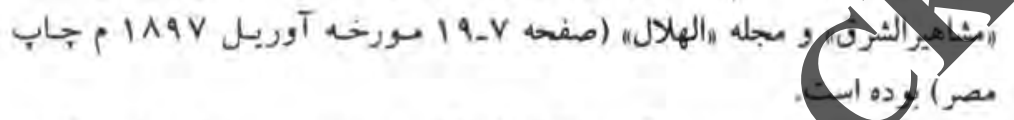

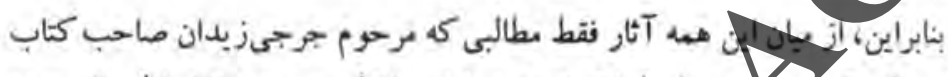

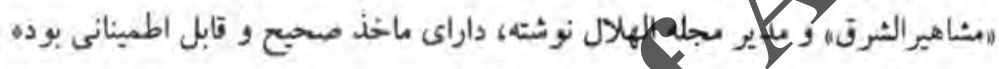

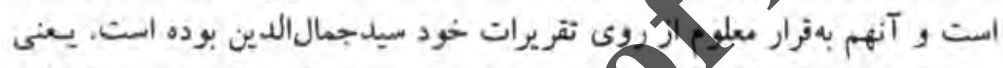

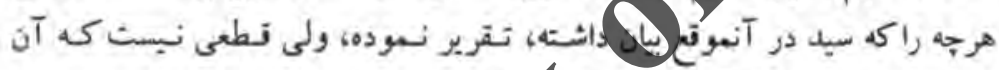

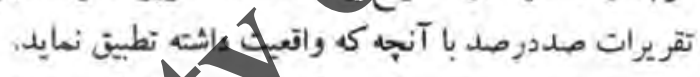

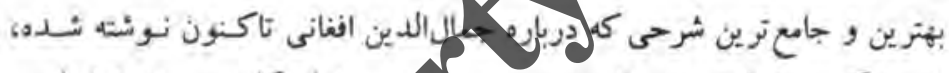

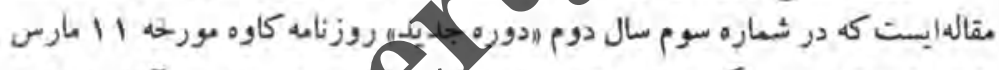

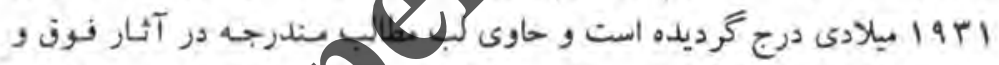

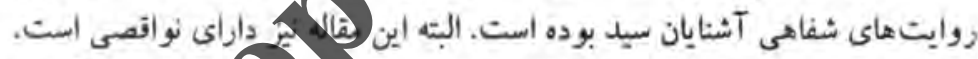

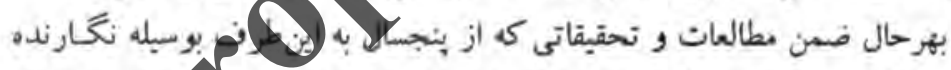

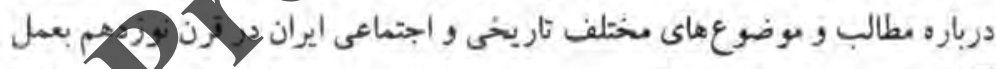

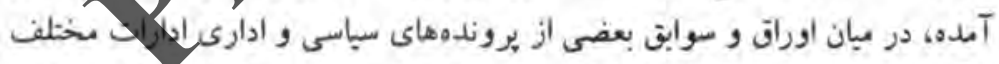

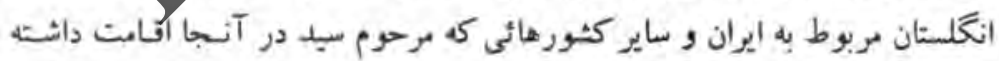

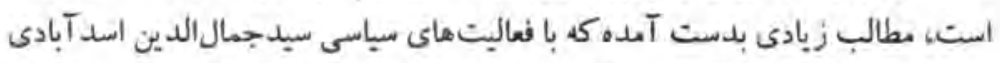

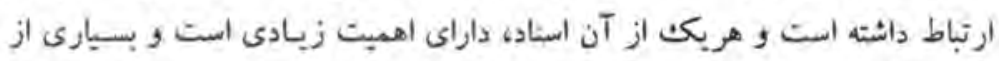

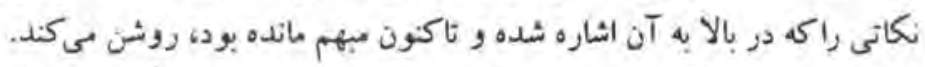

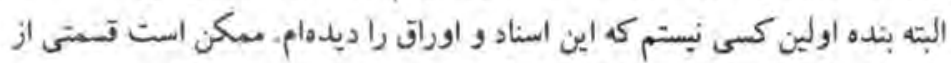

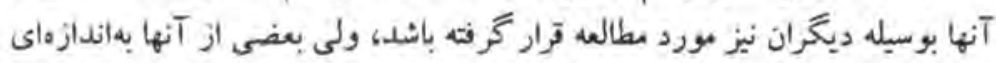

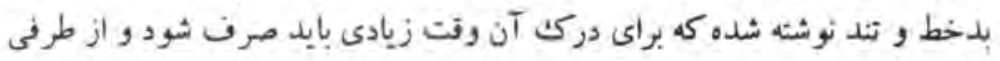




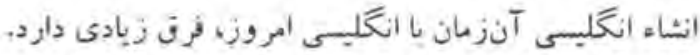

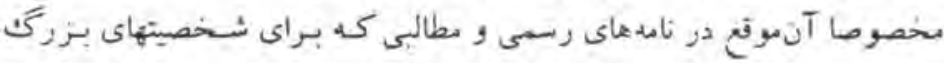

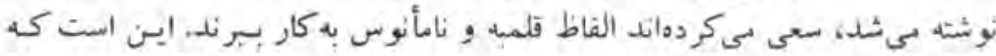
دنبال كردن ائَ فيل تحقيقات نحتاج بي نطالعه طولانى و از حوصله بسيارى خـارج

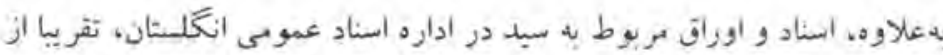

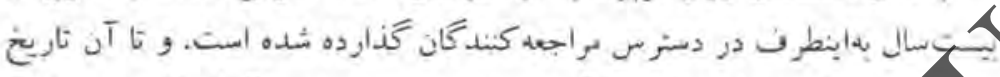

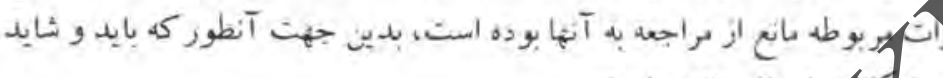

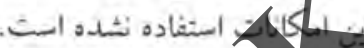

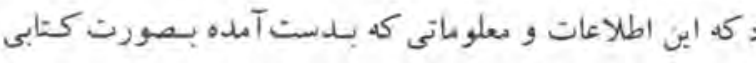

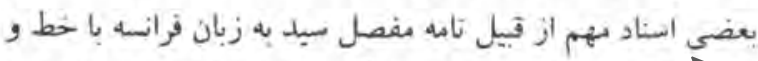

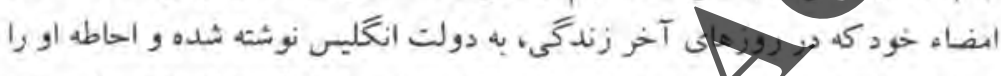

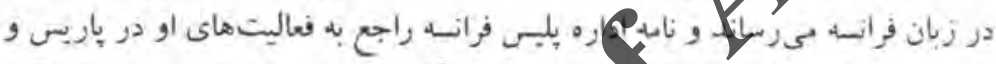

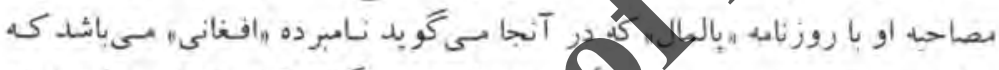

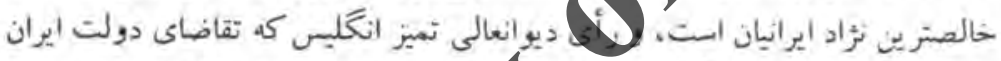

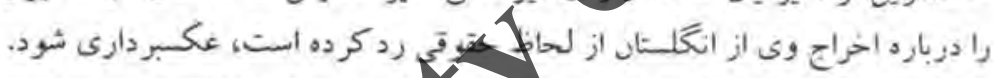

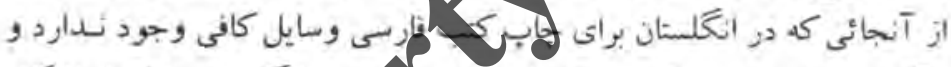

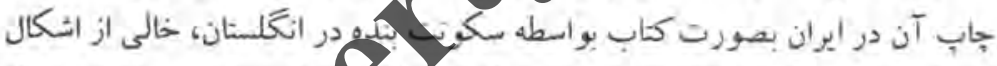

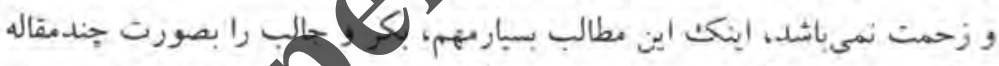

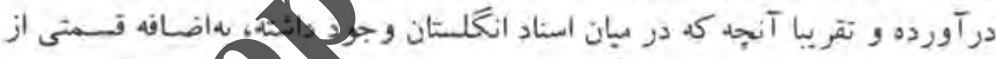

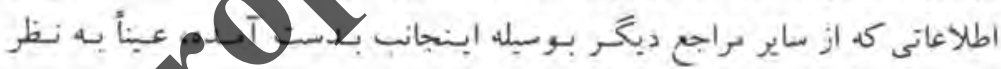

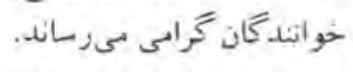

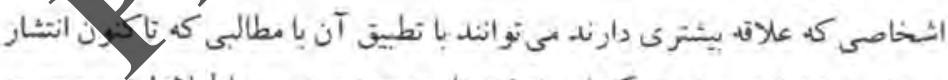

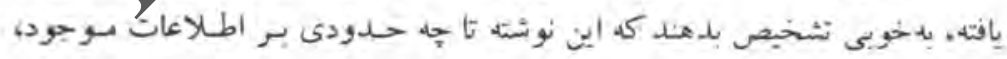

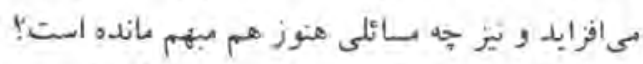

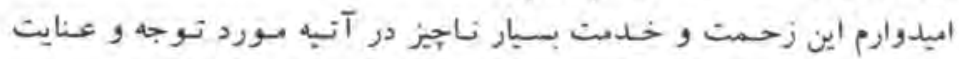

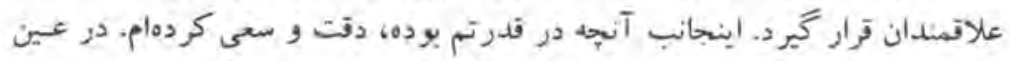

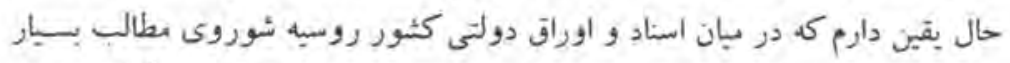

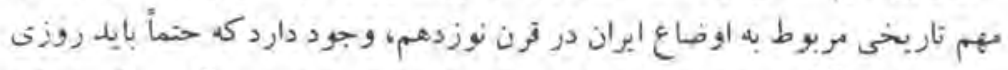

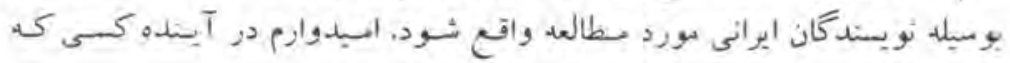




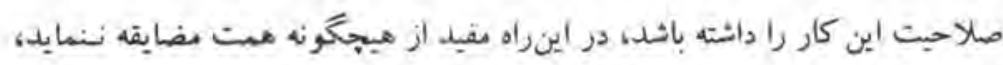

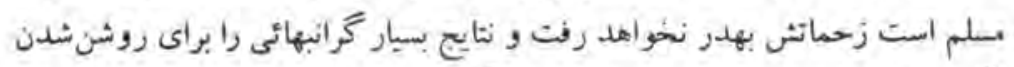

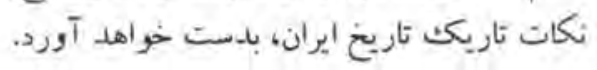

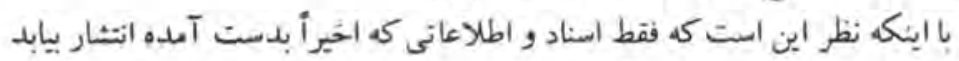

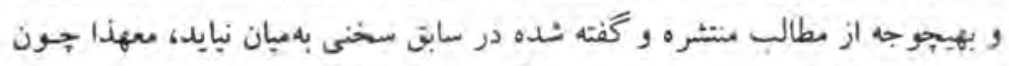

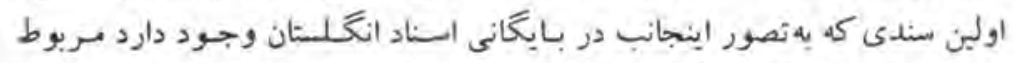

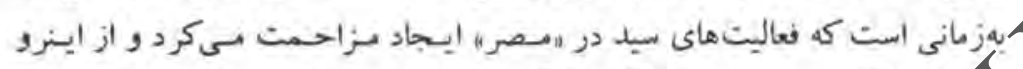

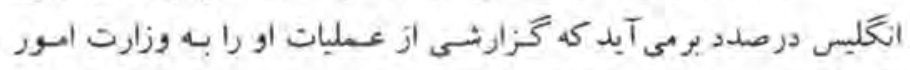

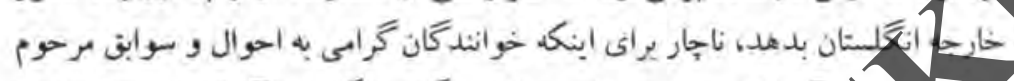

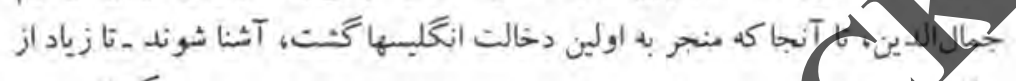

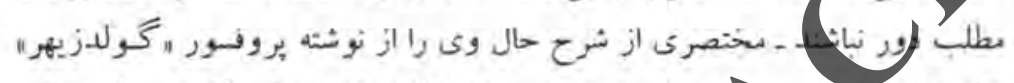

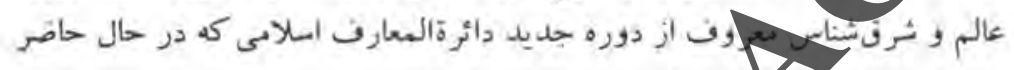

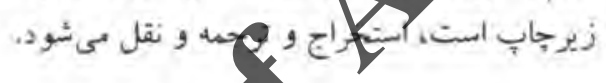

$$
\text { لندن _ مشيرى }
$$




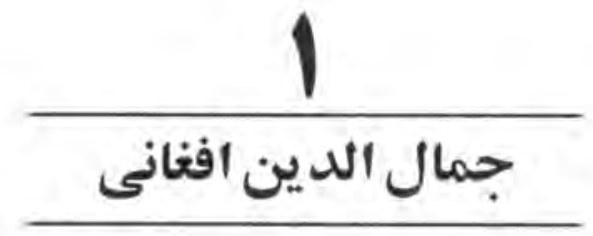

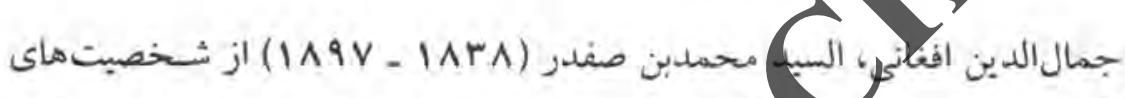

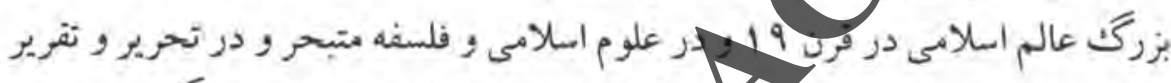

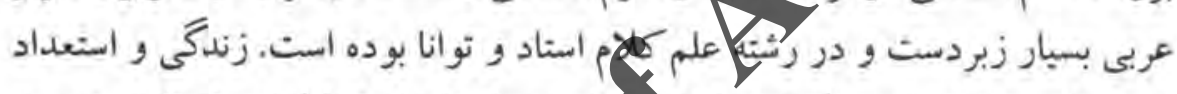

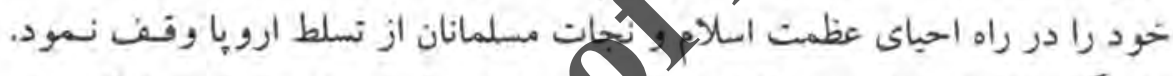

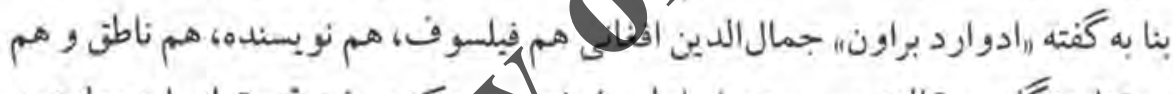

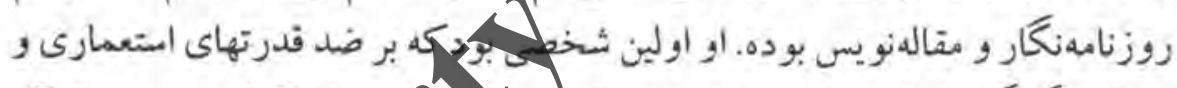

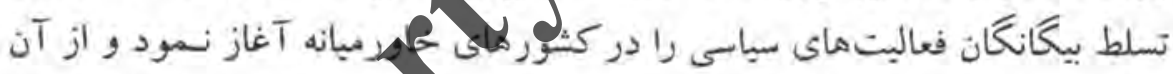

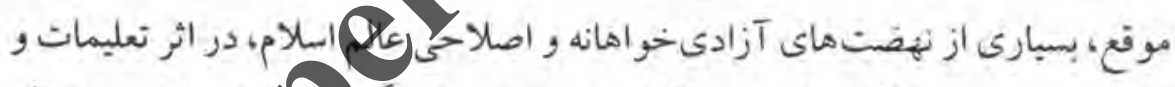

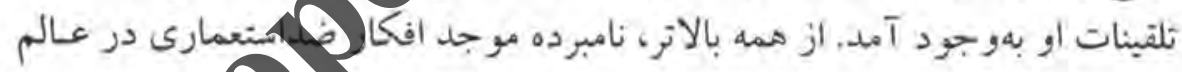

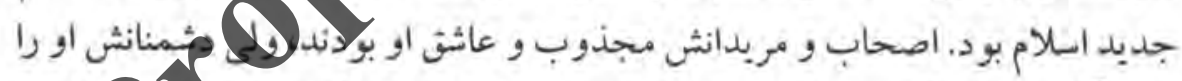

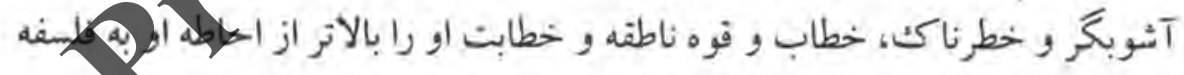
عقليه مي دانستند!

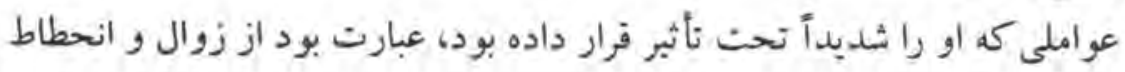

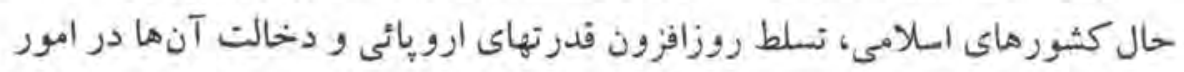

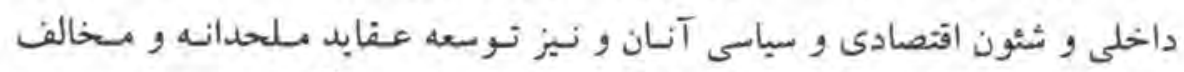

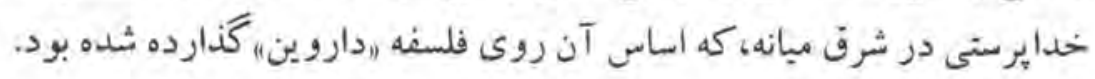

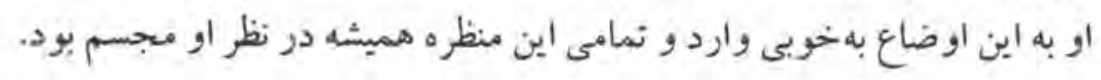




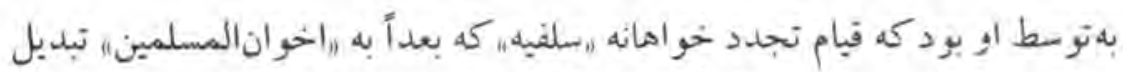

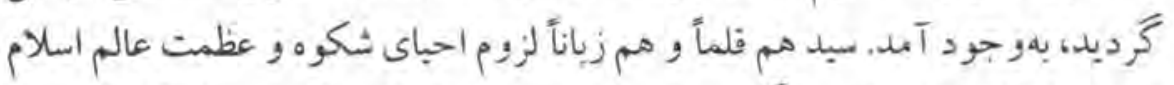

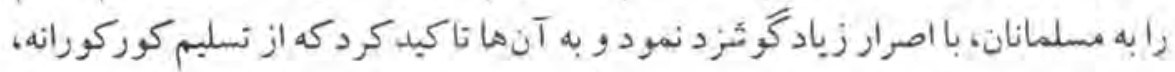

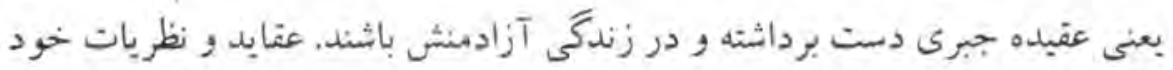

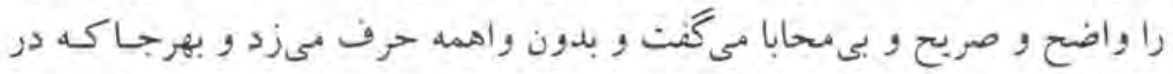

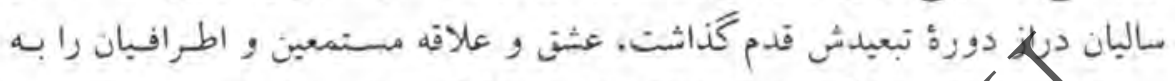

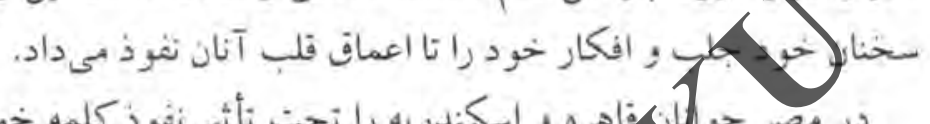

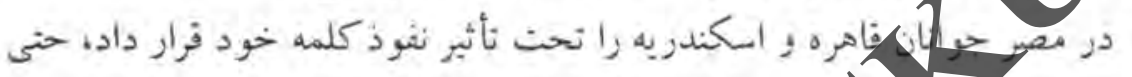

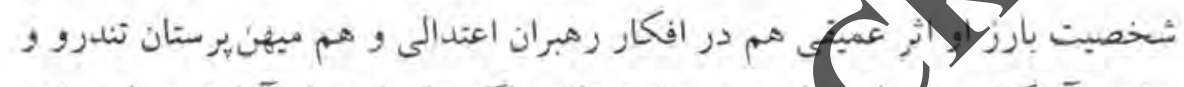

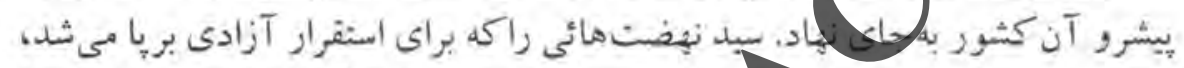

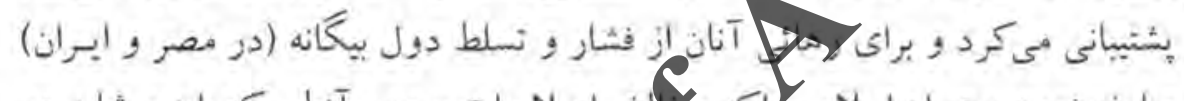

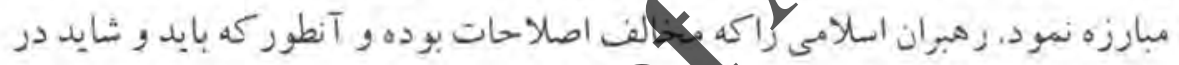

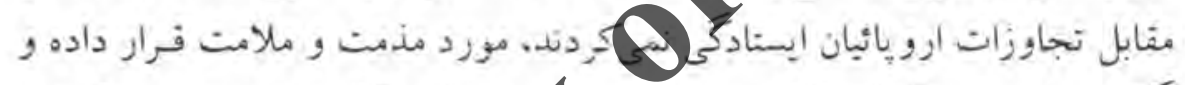

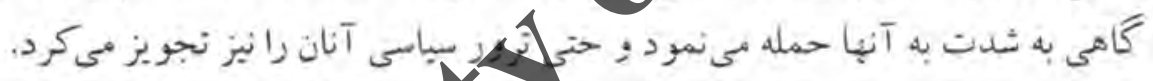

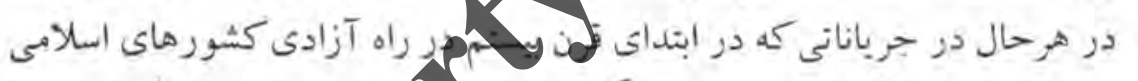

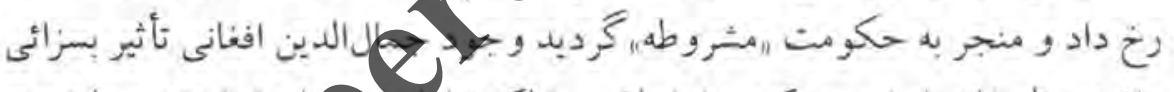

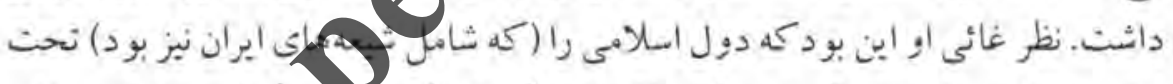

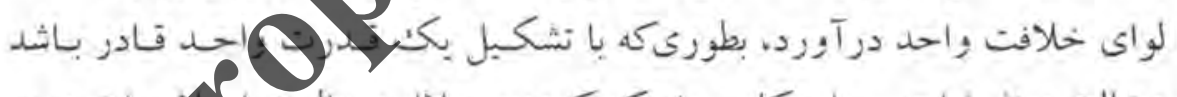

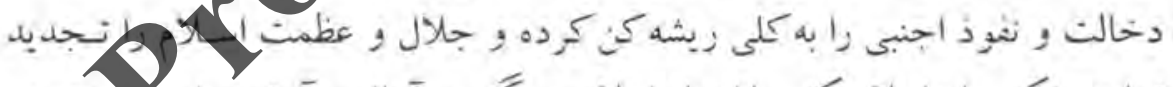

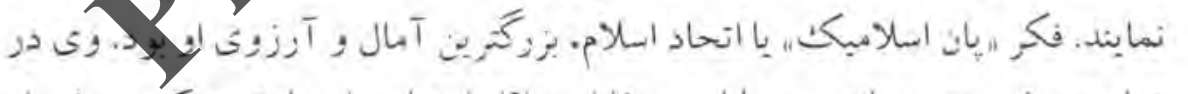

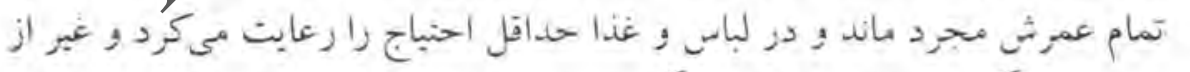

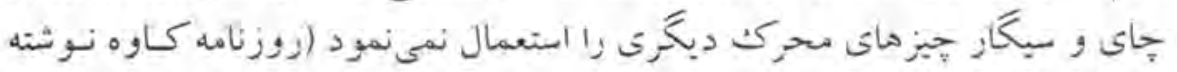

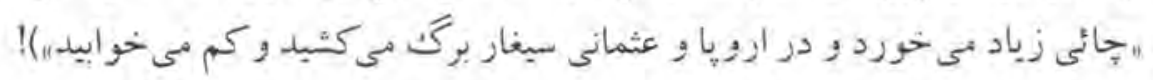

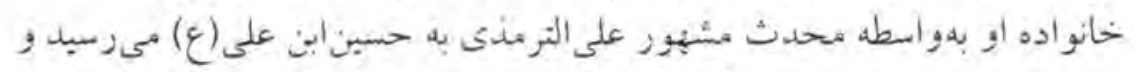

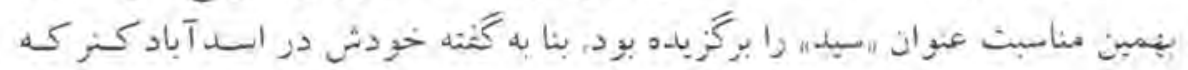




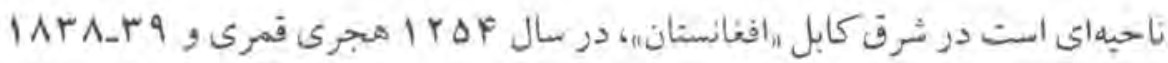

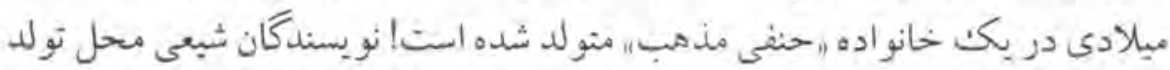

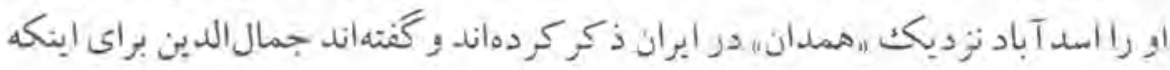

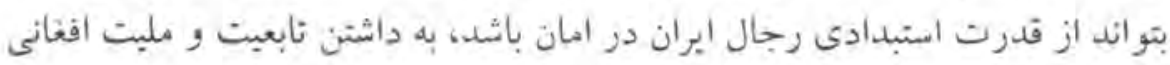
منعلر ور متظاهر بو ده است.

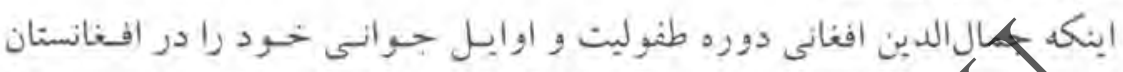

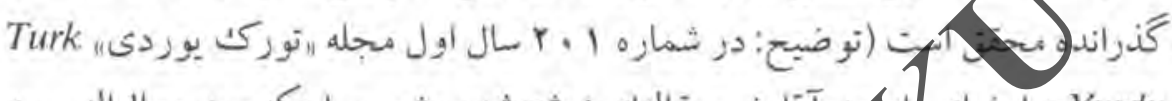
Yurdu

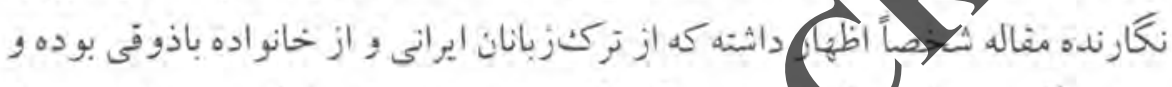

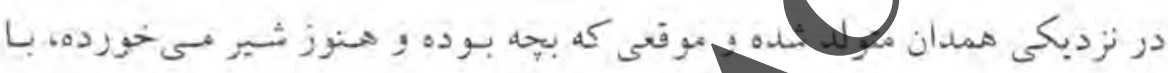

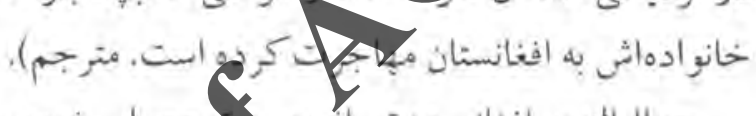

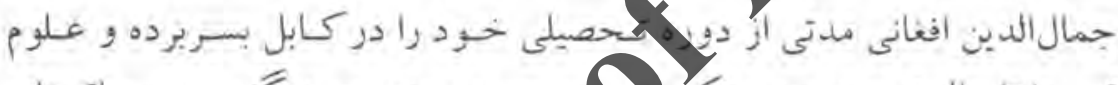

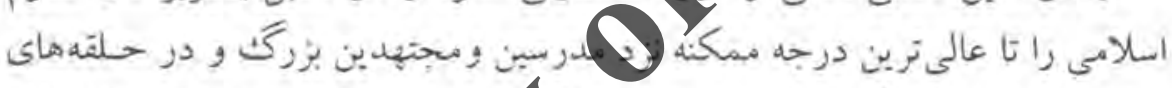

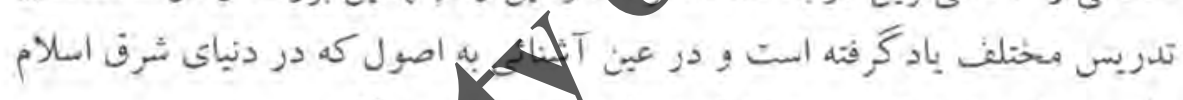

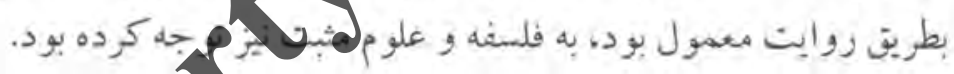

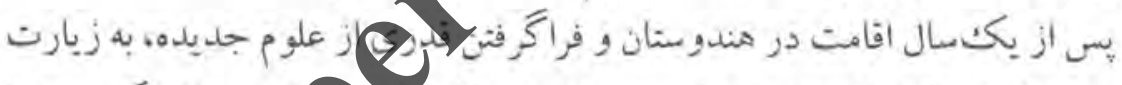

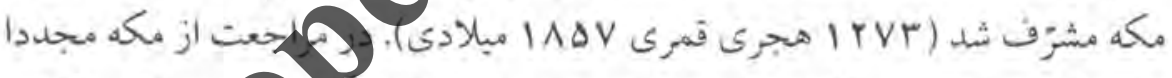

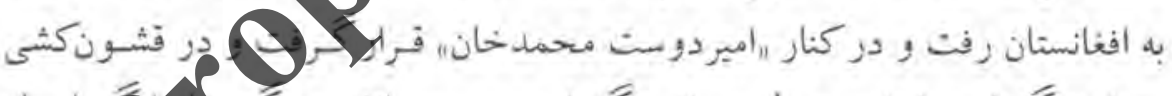

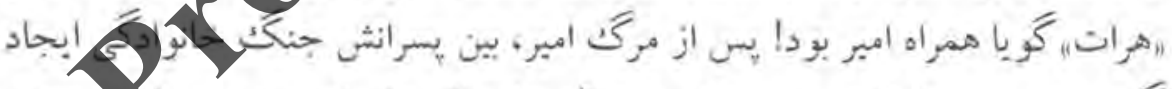

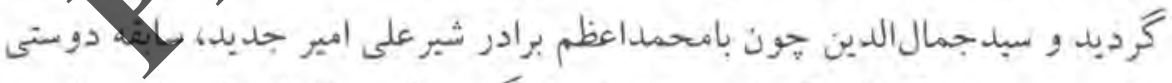

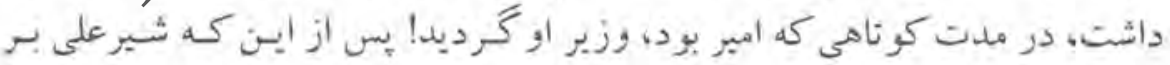

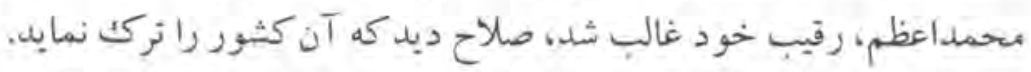

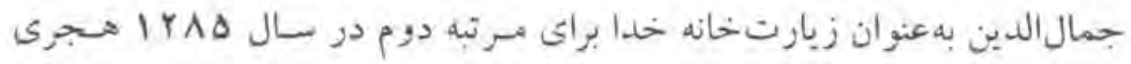

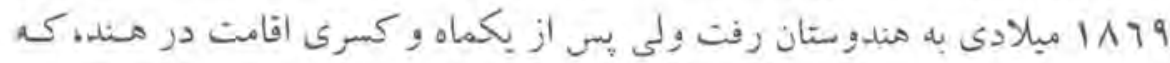

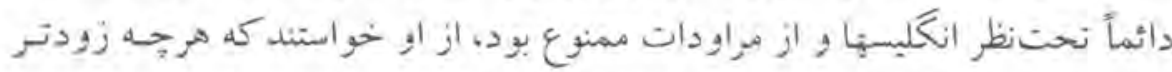




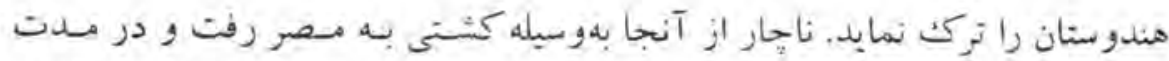

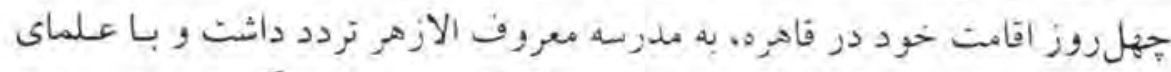

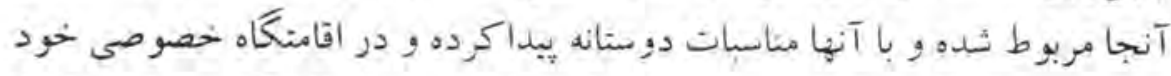

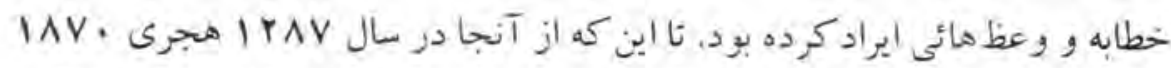

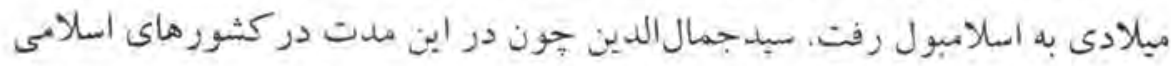

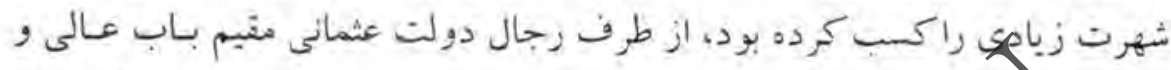

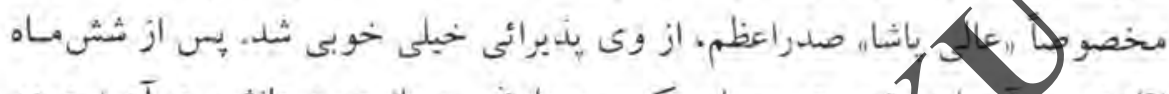

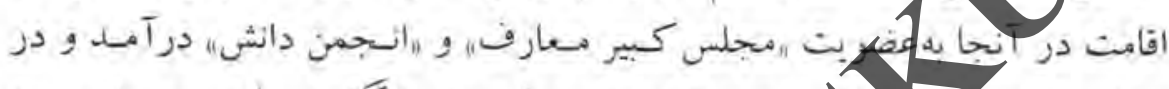

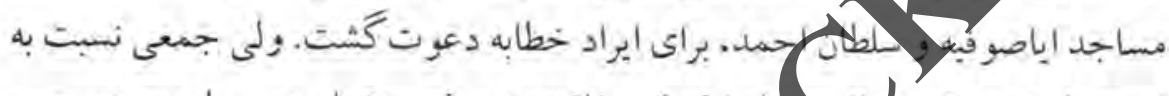

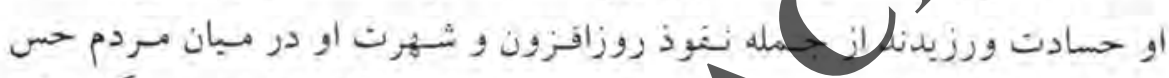

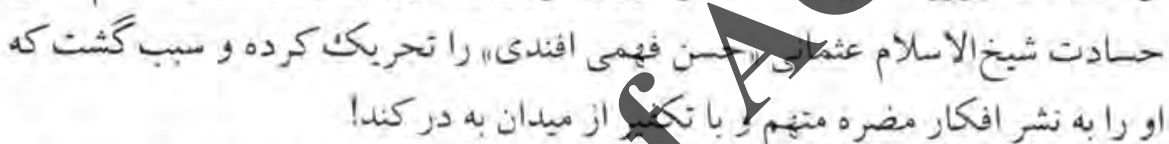

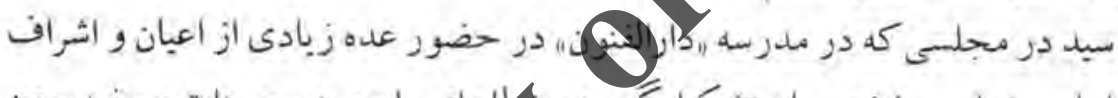

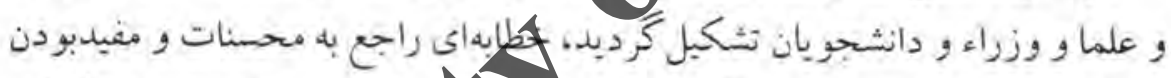

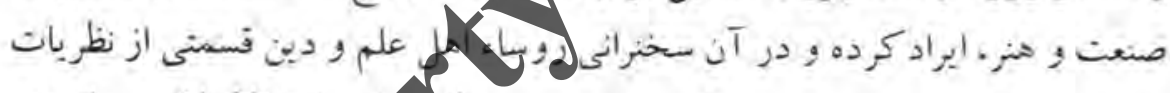

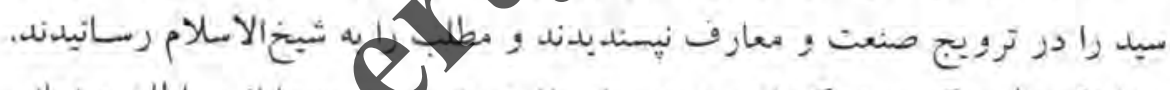

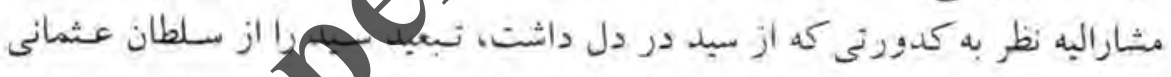

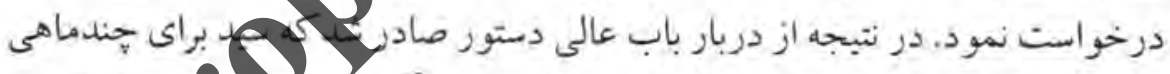

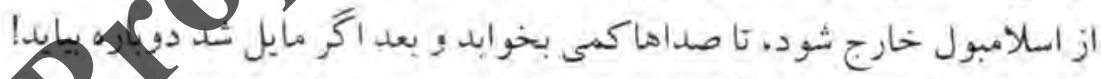

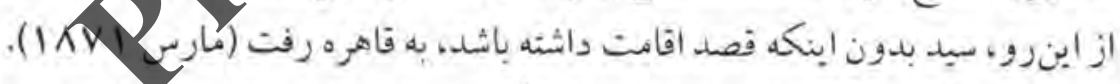

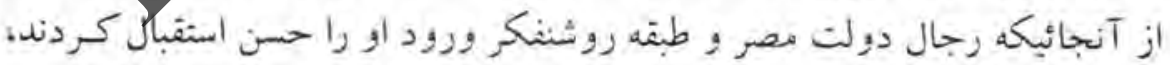

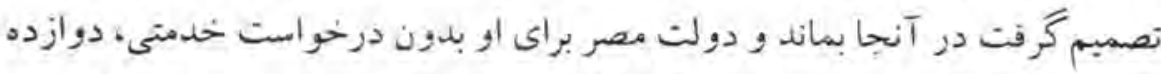

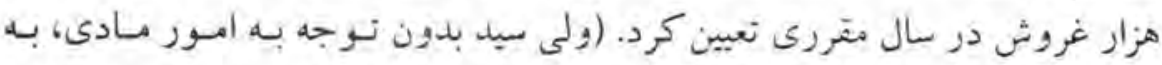

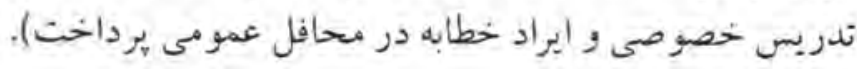




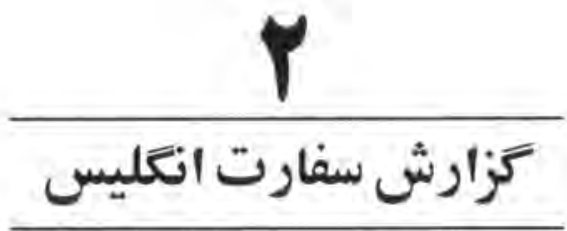

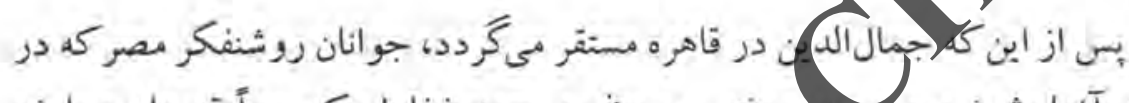

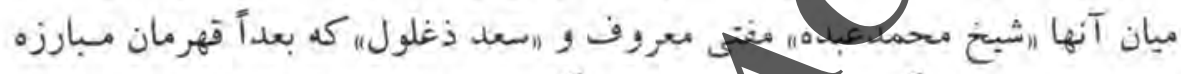

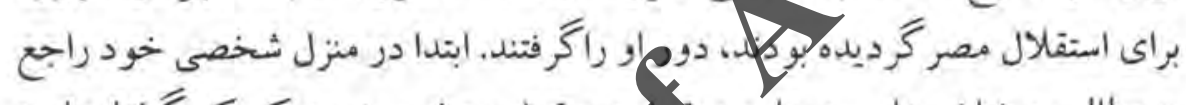

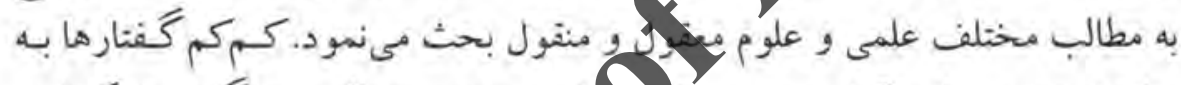

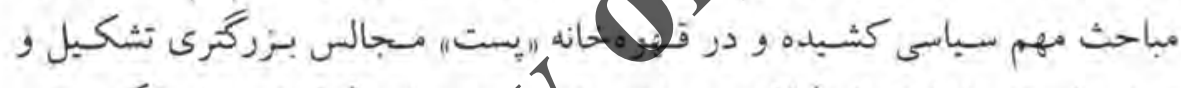

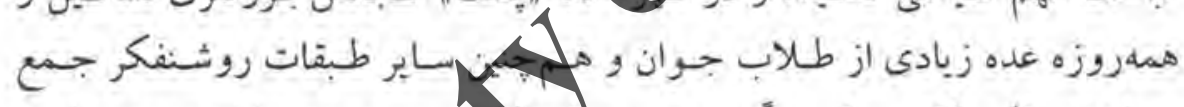

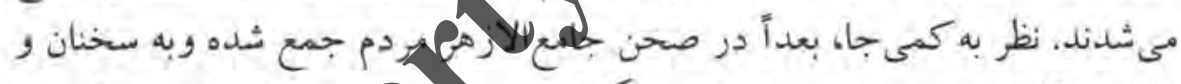

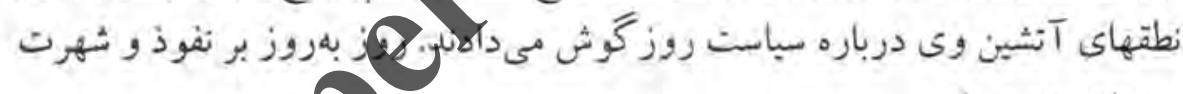

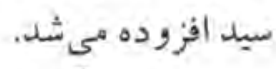

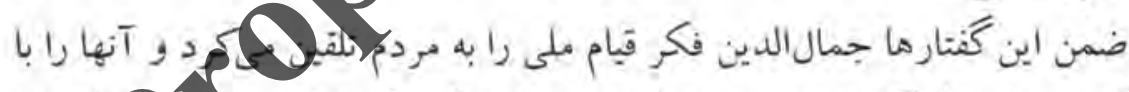

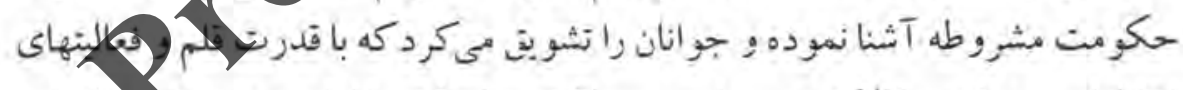

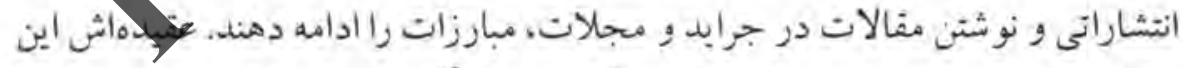

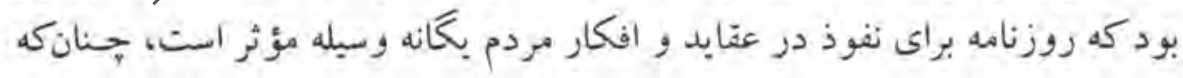

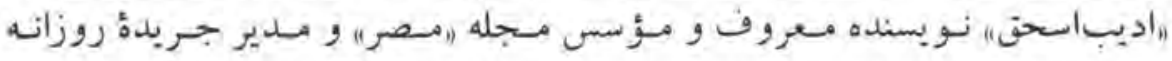

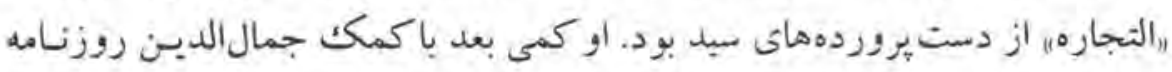

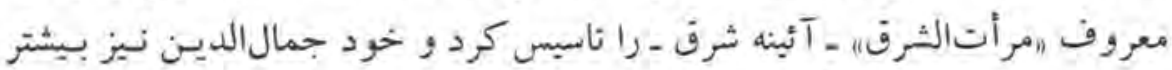

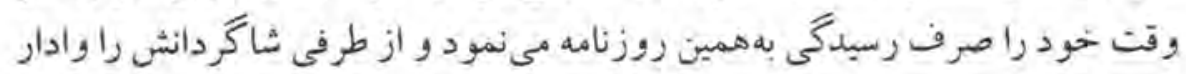


ميكرد كم نوشتن منالات در مضو لات مختلف را دنبال نمايند.

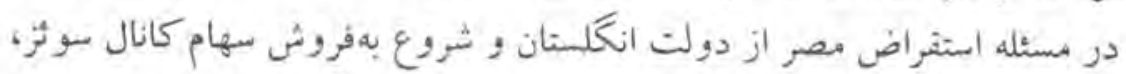

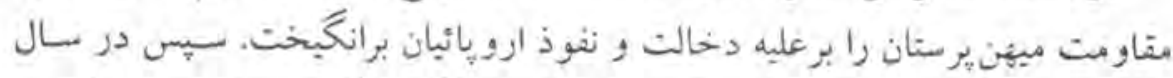

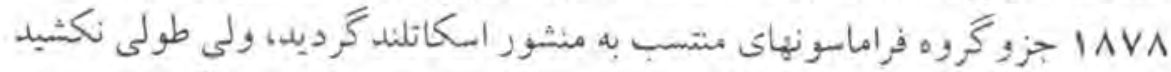

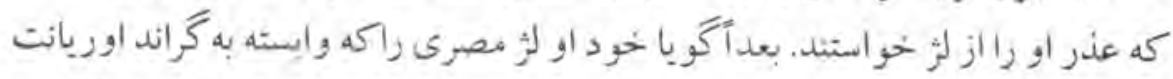

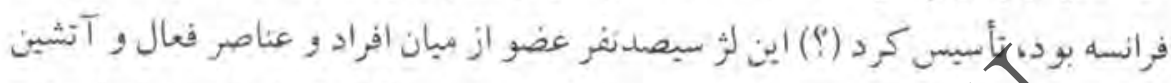

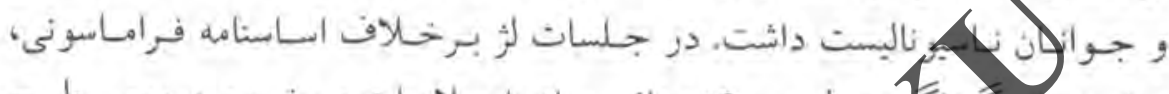

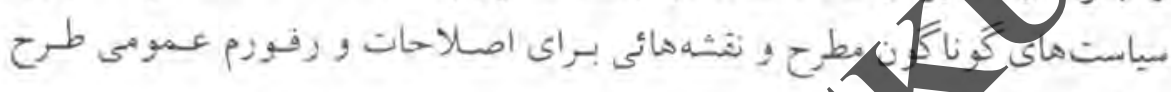

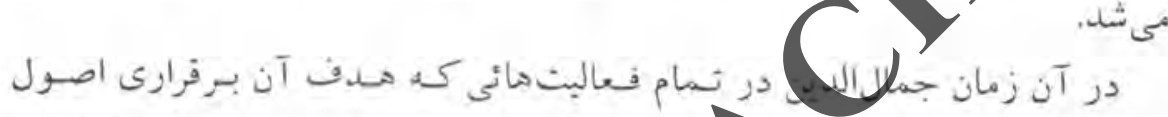

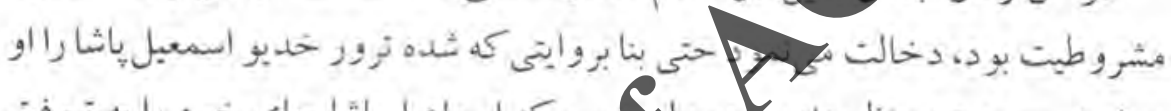

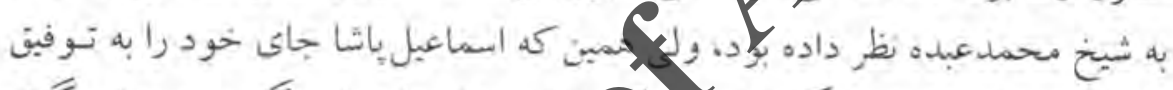

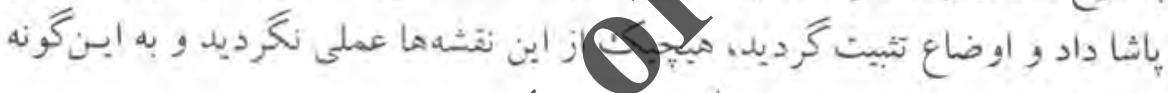
فعاليتها ثقريبا خاتمه داده شُد.'

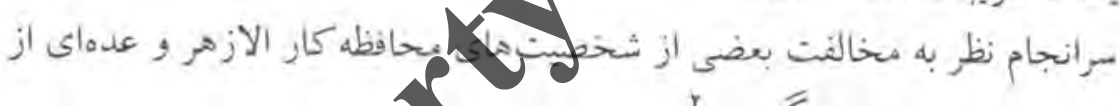
8

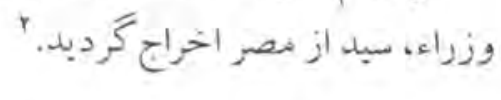

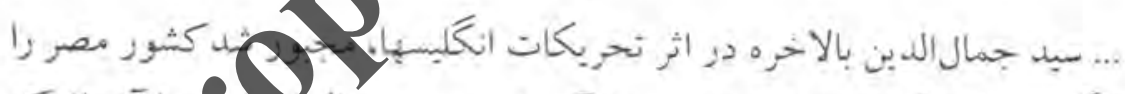

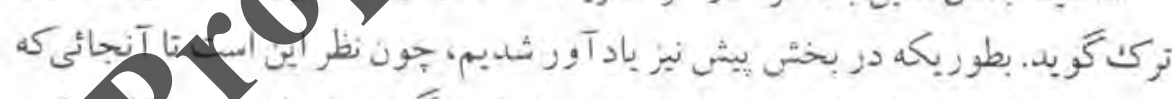

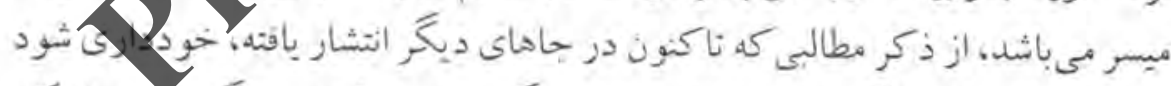

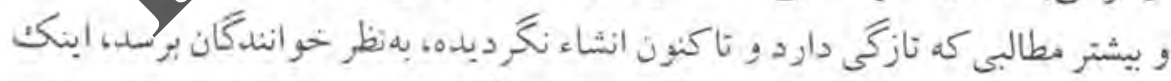

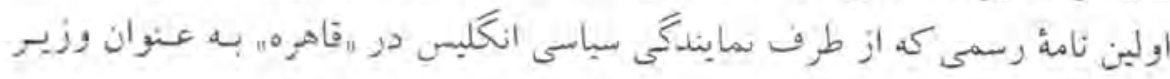

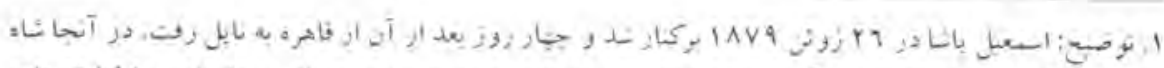

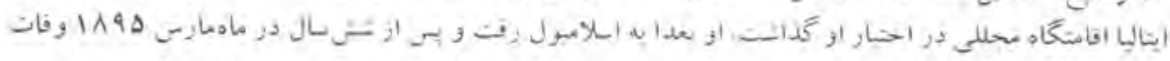

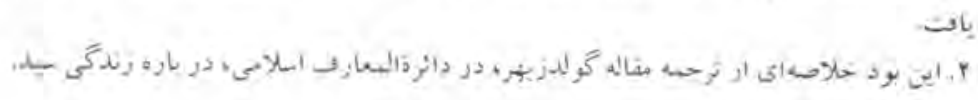




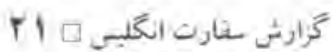

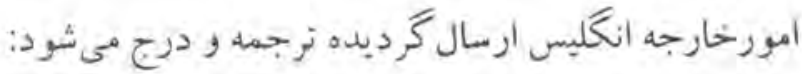

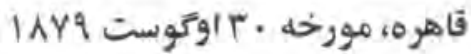

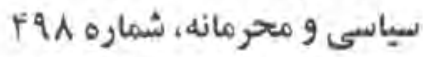

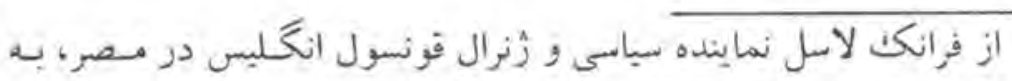

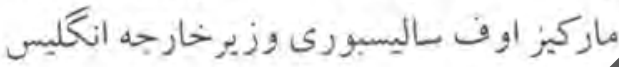

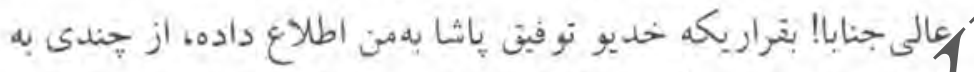

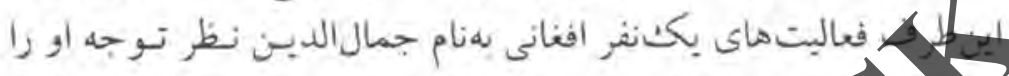

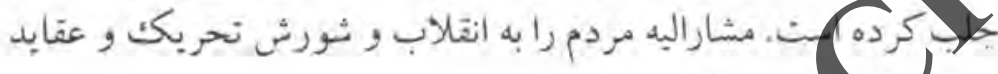
:و

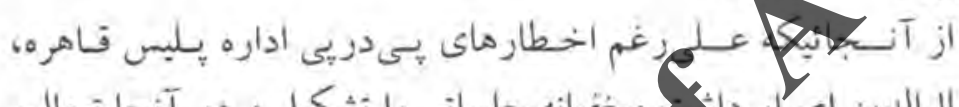

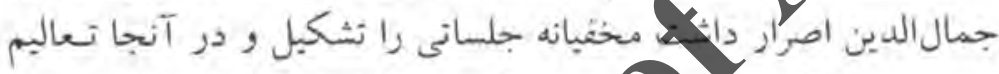

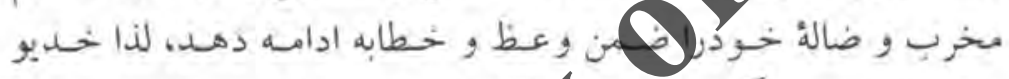

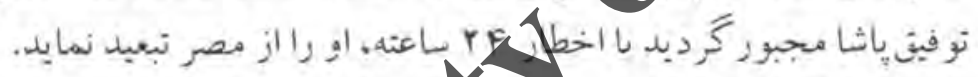

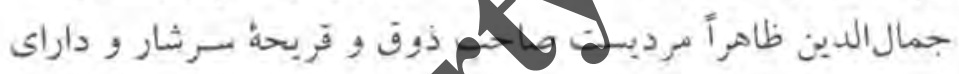

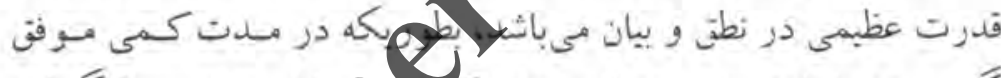

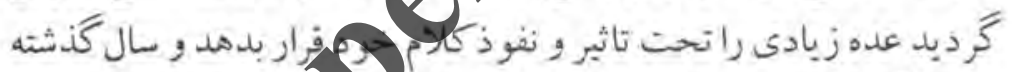

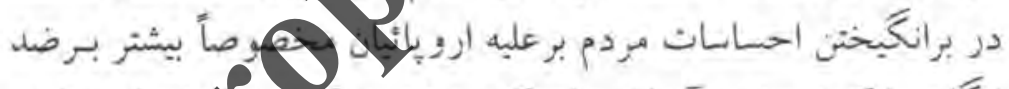

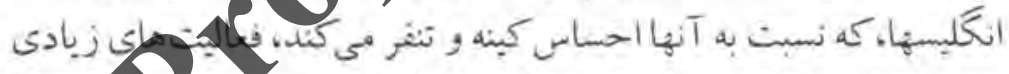

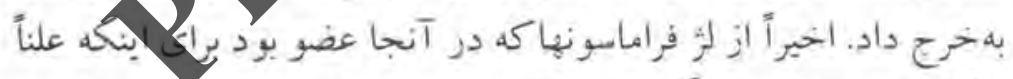

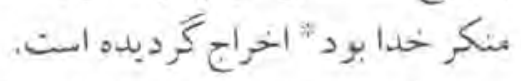

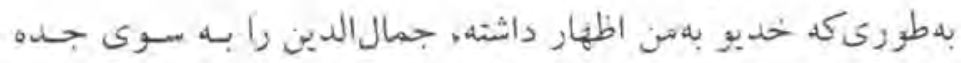

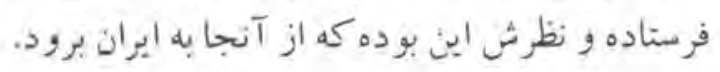
امضاء فرانكت لاسيل 
فسمتي از مندرجات اين نامه با اظهارات يروفسور كولدزيهر و, ساير نسويسندكان

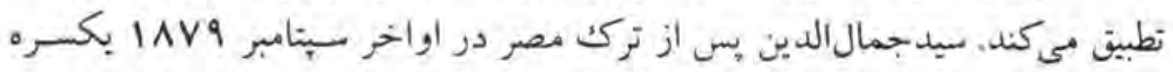

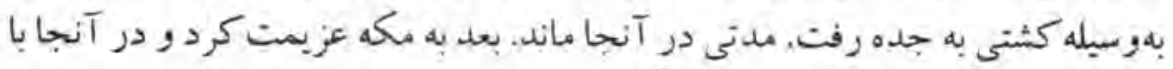

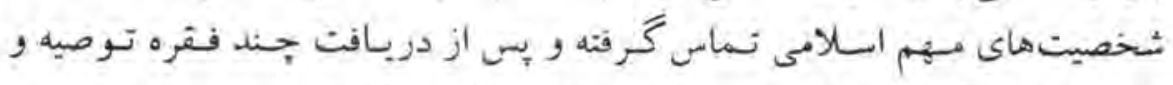

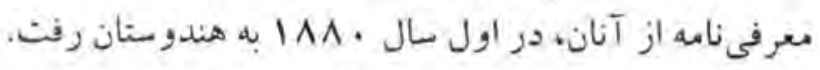

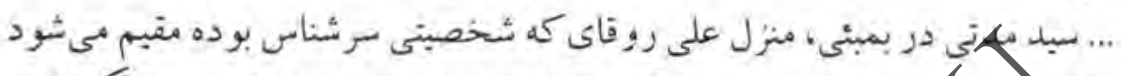

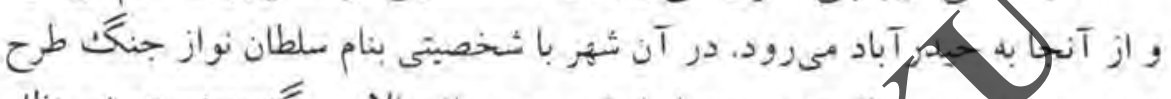

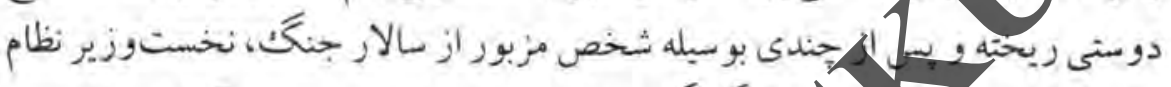

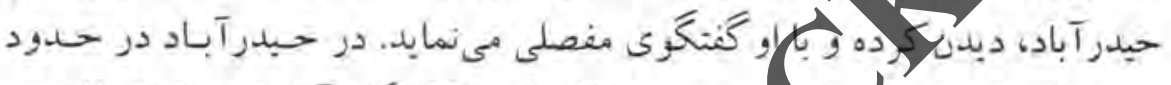

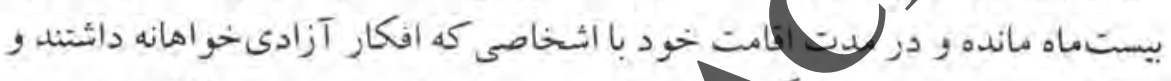

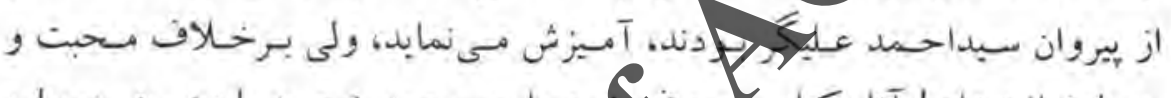

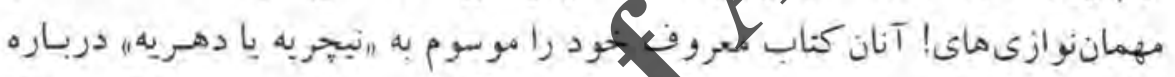

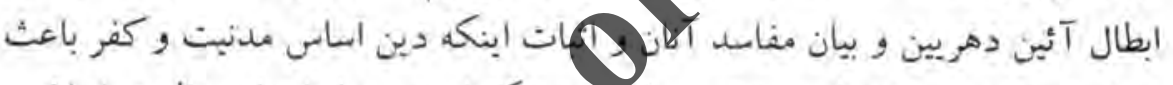

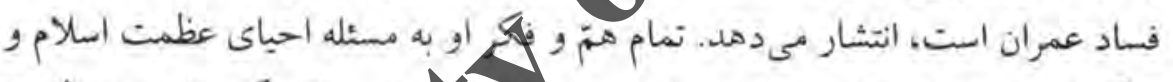

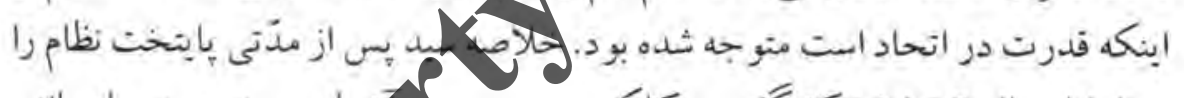

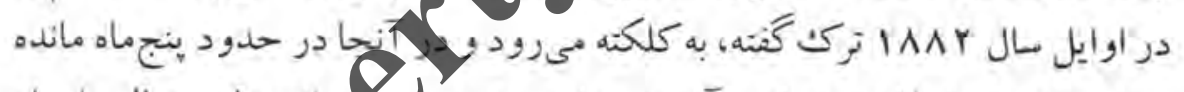

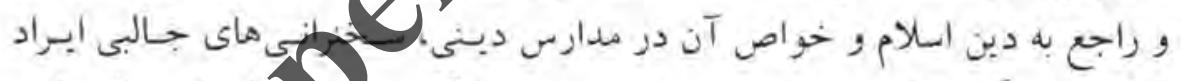

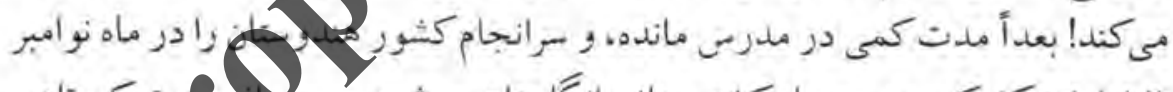

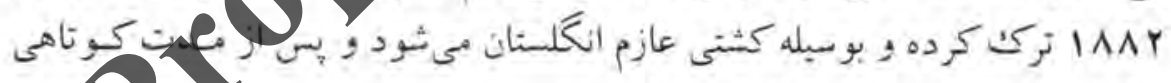

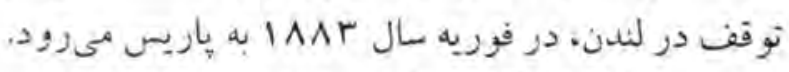

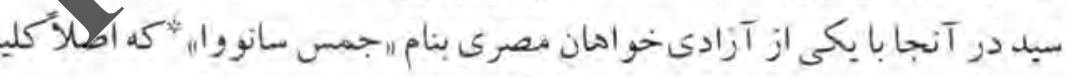

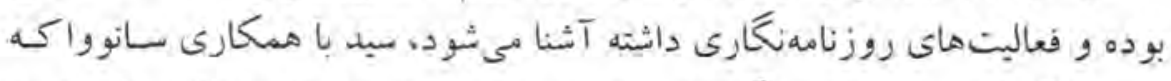

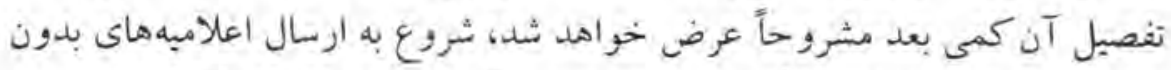

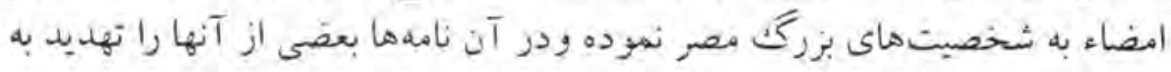

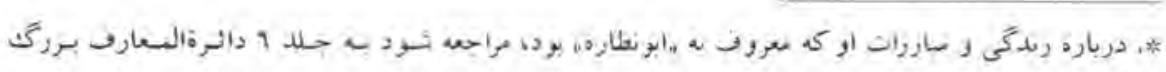




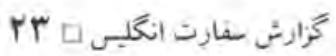

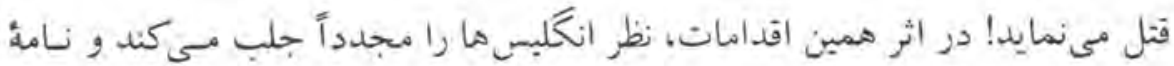

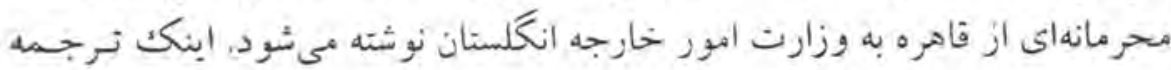

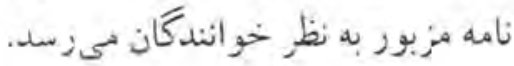

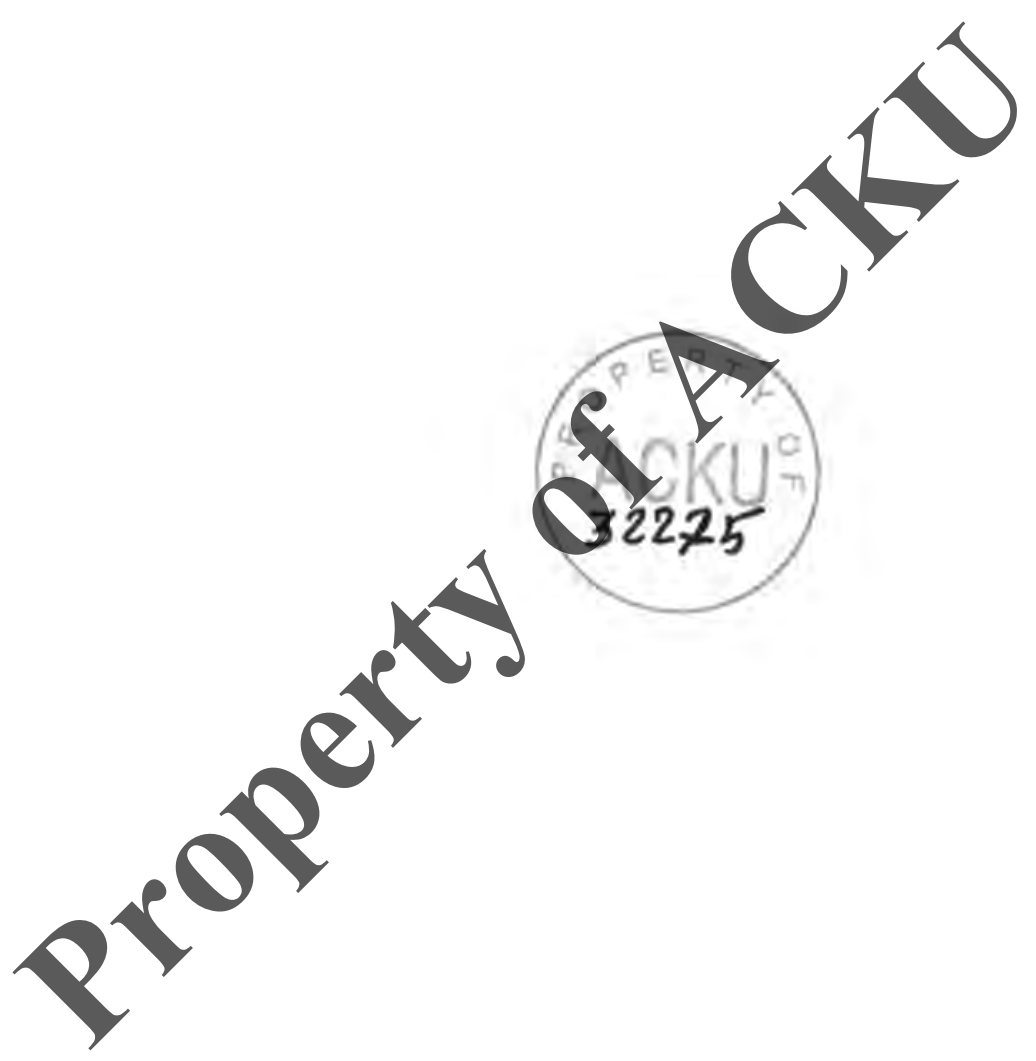




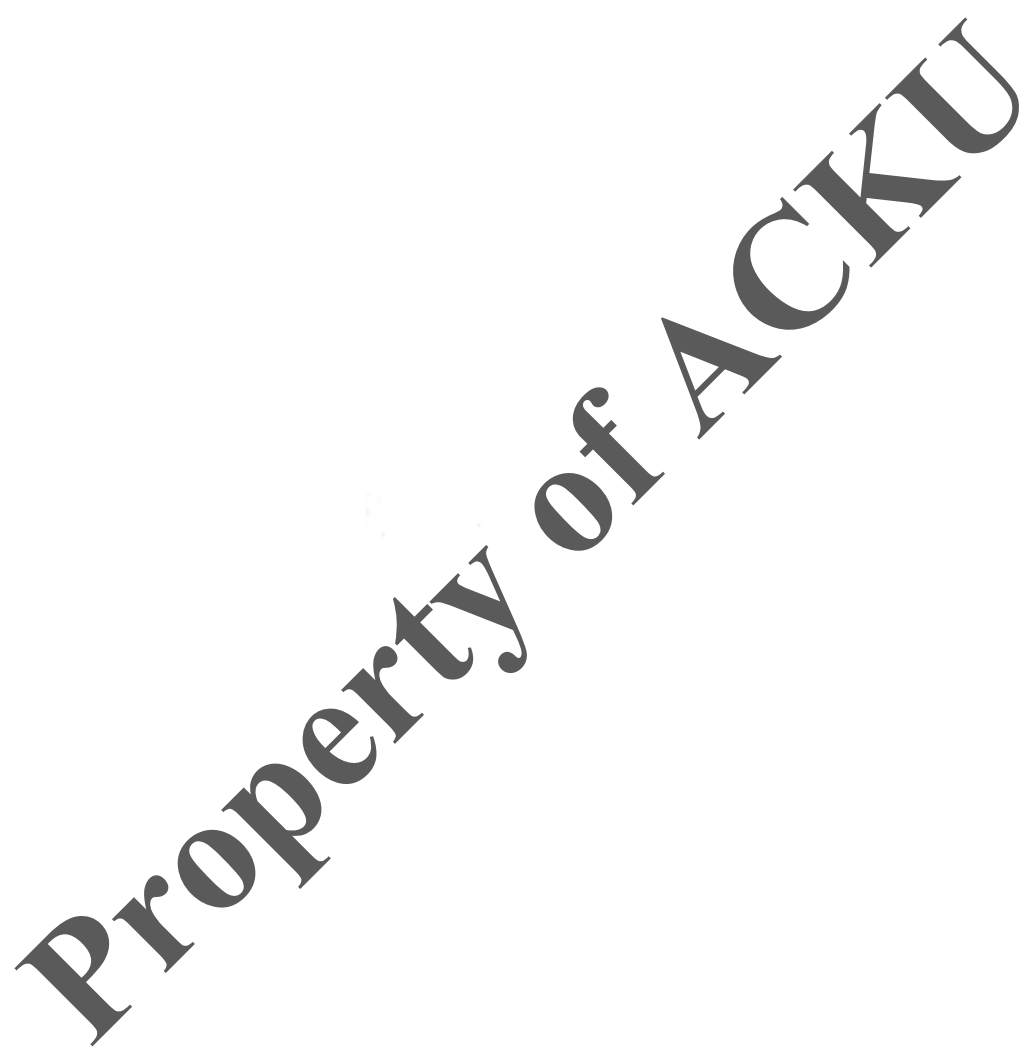




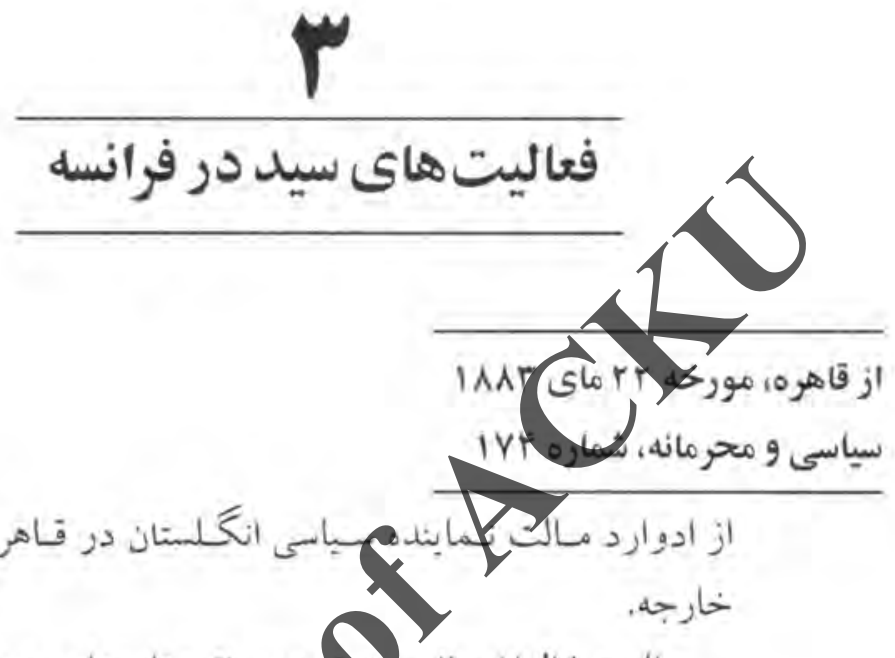

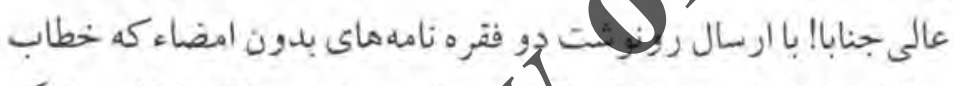

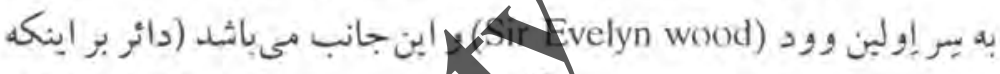

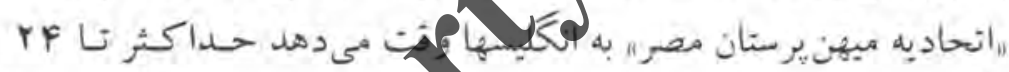

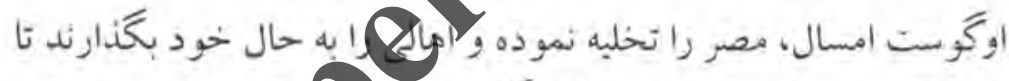

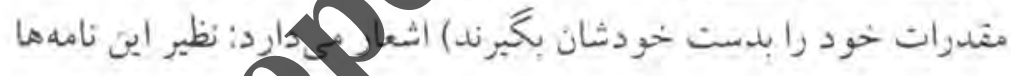

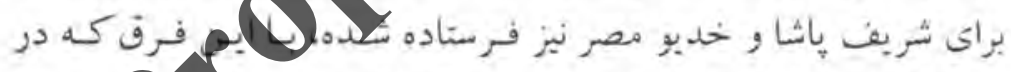

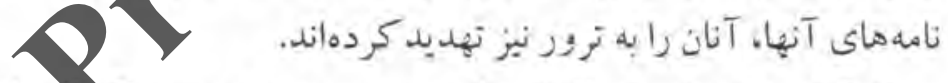

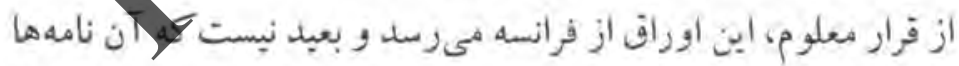

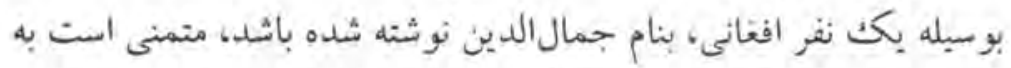

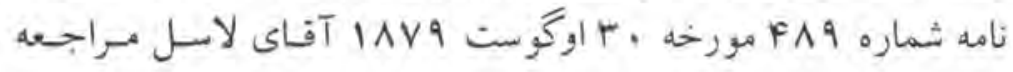
فرمايند.

جمال الدين با شخصى كه خود را جمس سانووا معرفى كرده و ناشر

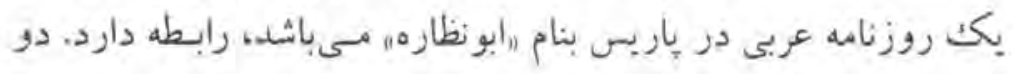




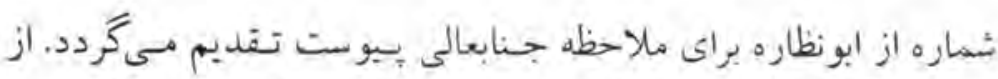

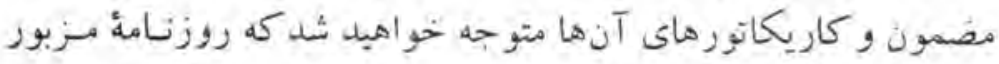

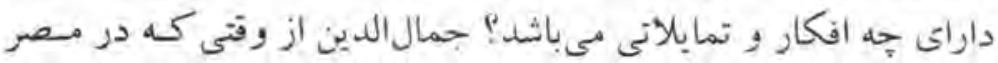

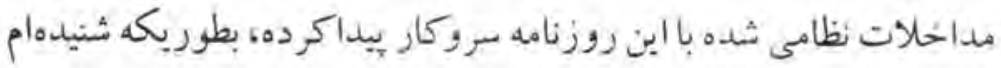

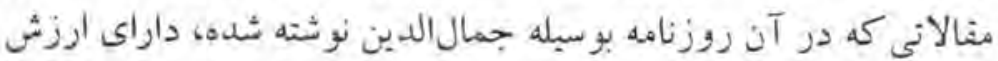

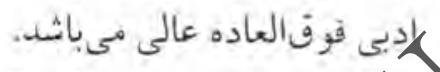

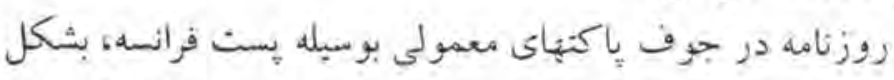

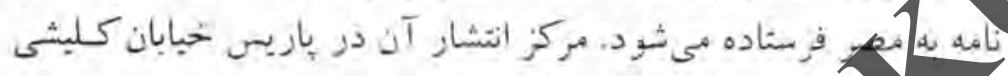
با نامههاى بدون امضاء، از آن جا مسلوم مىشود atpxyat

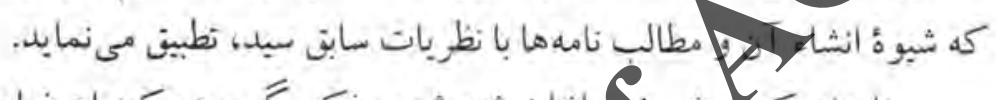

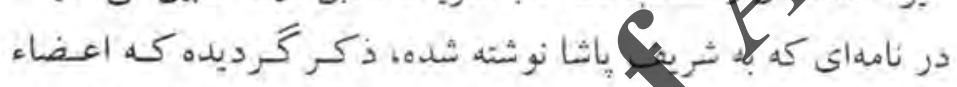

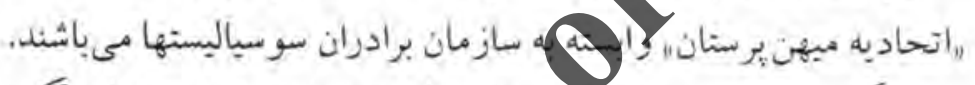

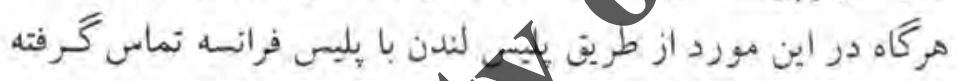

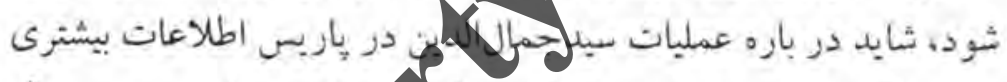

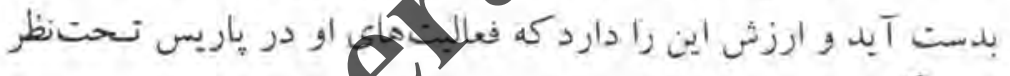
فراز بـيرد. امضاء ادوارد مالت

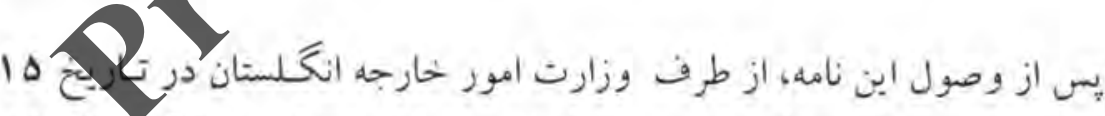

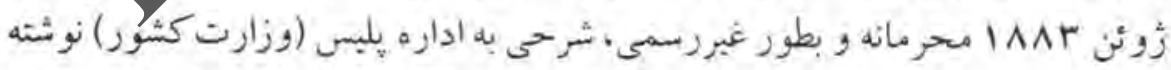

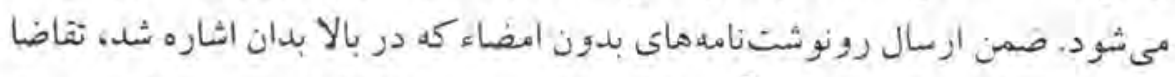

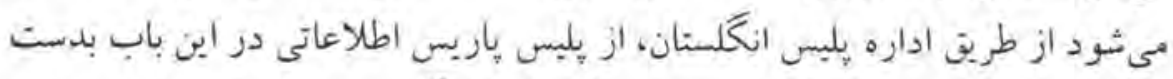

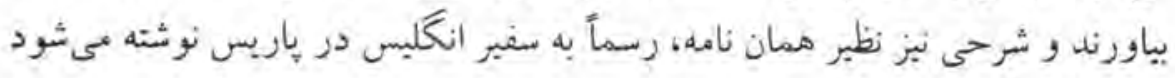
كه ترجمه آن درج مئرد 


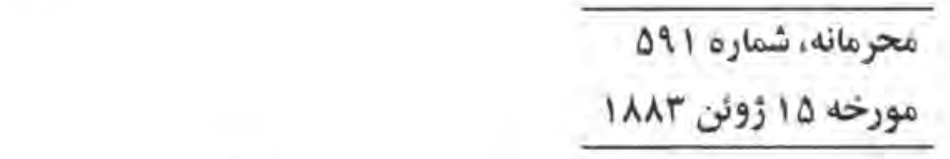

نامه وزارت امور خارجه به سفارت انكلِيس در خياريس

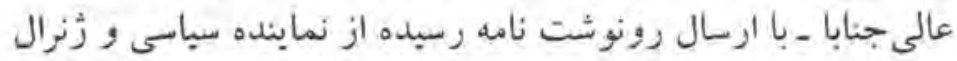

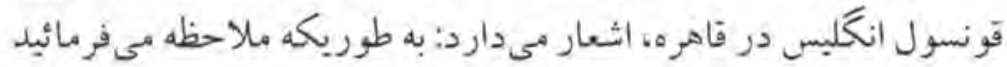

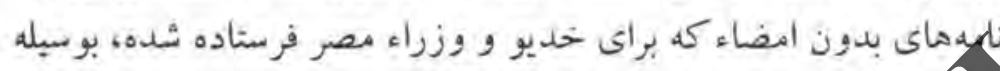

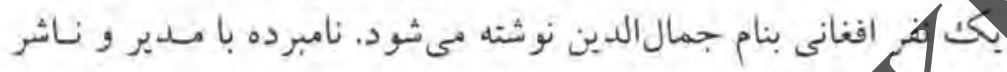

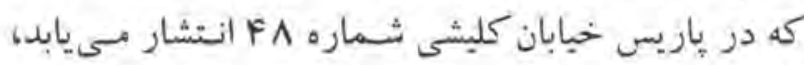

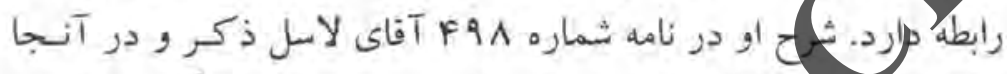

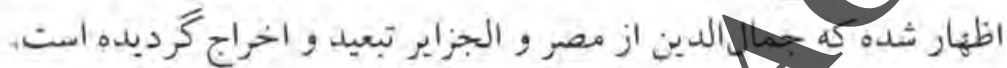

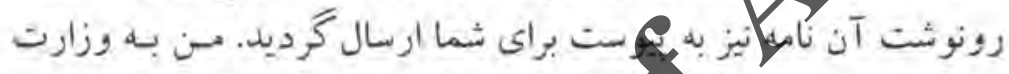

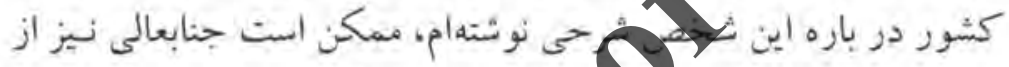

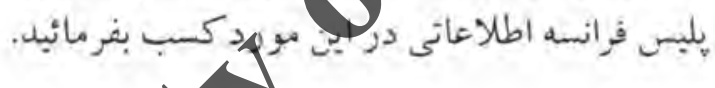
المخاء وزير امورخارجه

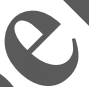
نامله اتحاديه ميهن ير ستان

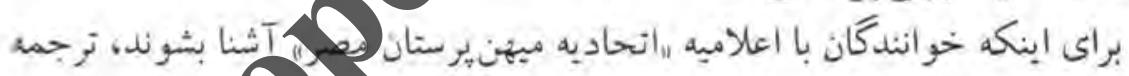

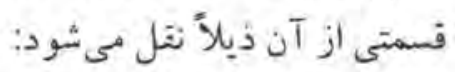

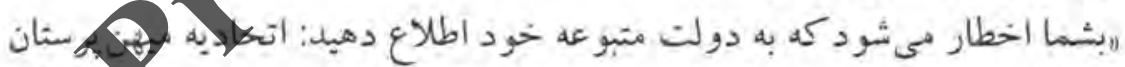

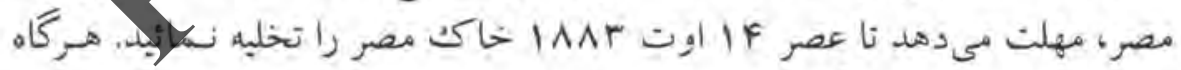

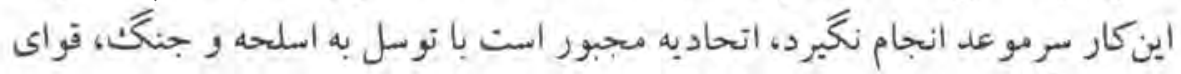

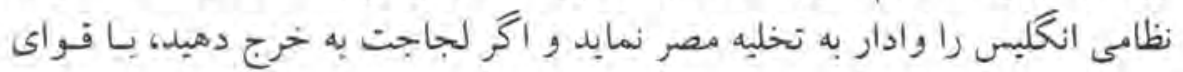

$$
\text { روسبه روبرو خواهيد شد. }
$$

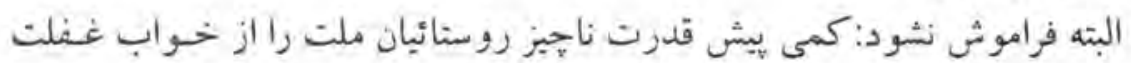

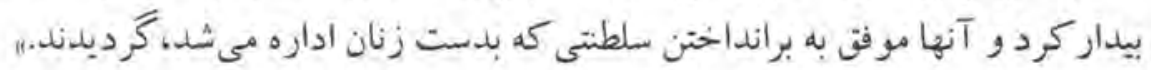

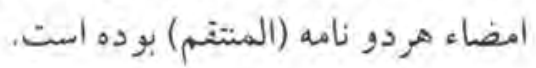


A

نامل سفير انتليس در فرانسه

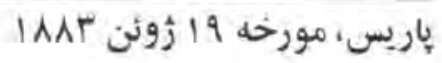

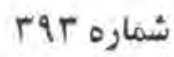

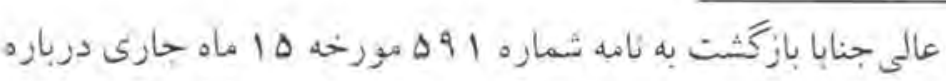

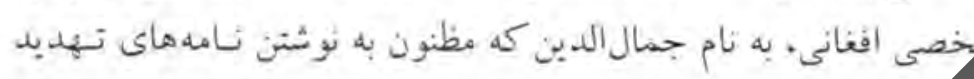

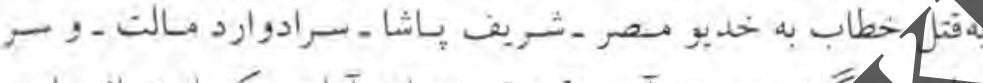

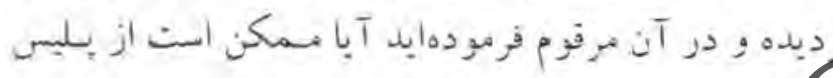

$$
\text { فران }
$$

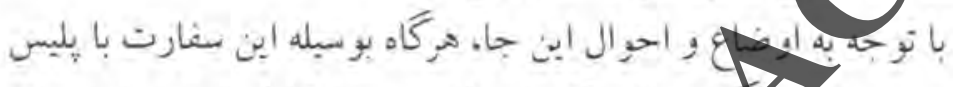

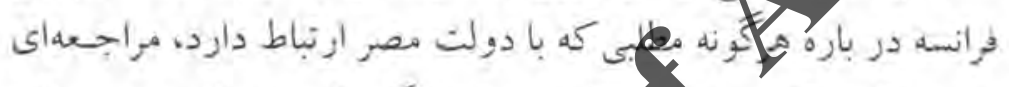

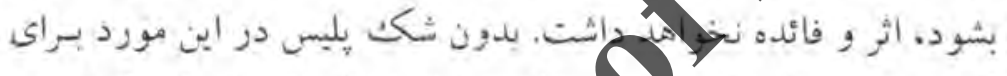

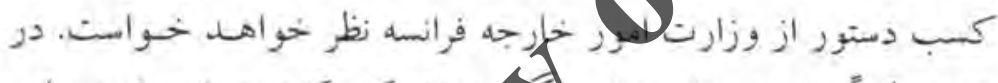

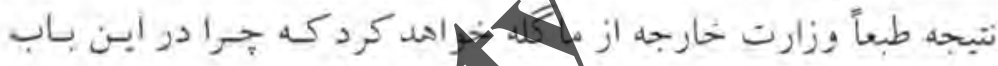

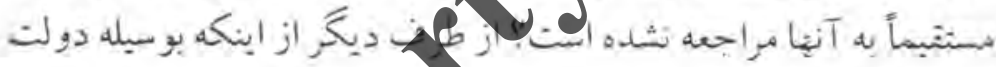

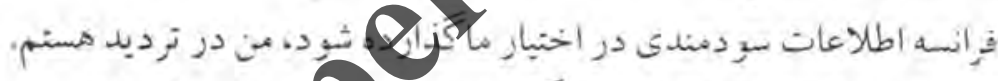

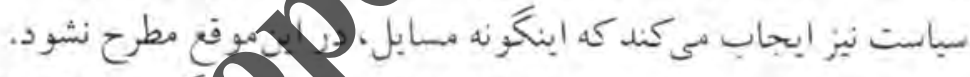

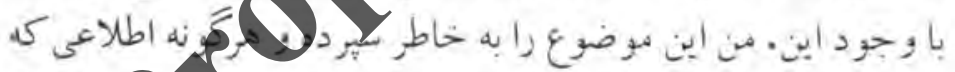

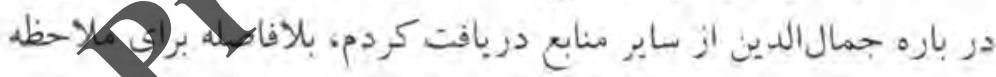
حنابعالى تثديم خواهم نمثزد

امضاء لاينز

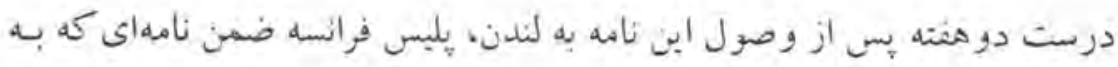

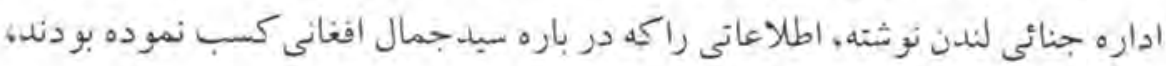

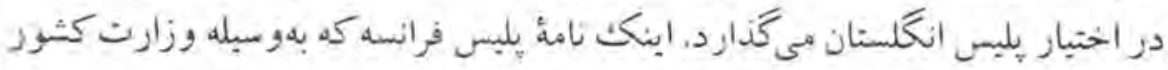


به وزارت اهور خارجه انكُلستان فرستاده شده انست.

\section{نامه رئِيس خليس فرانسه}

\section{باريس ل خؤئي}

از رئيس يليس فرانسه ـ آقاى مديركل ادارة أمور جنائى لندن

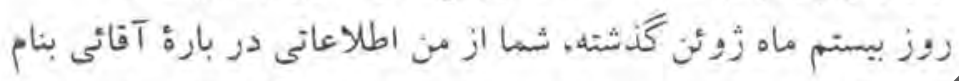

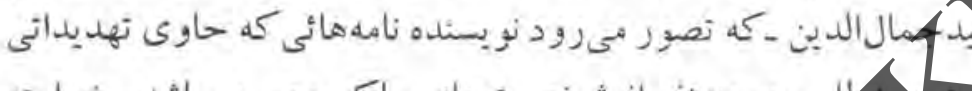

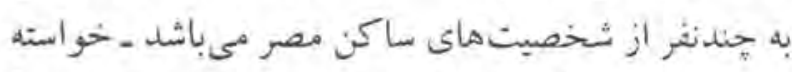

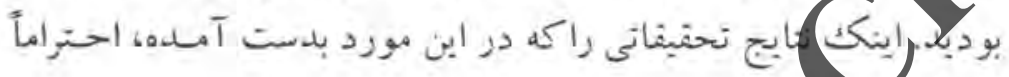

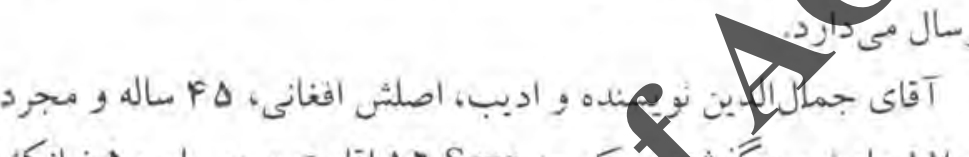

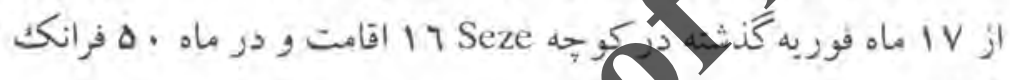
كرايه مي يردازد، 1110

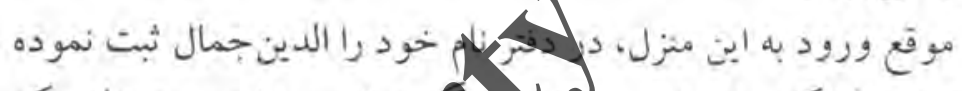

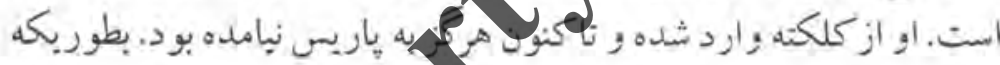

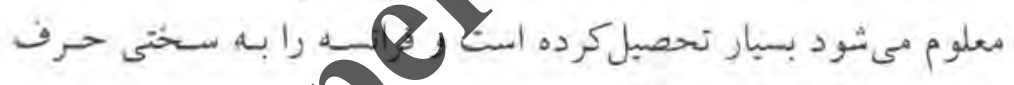
مىزئد و هشت زبان مي مدائد.

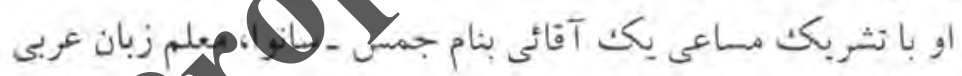

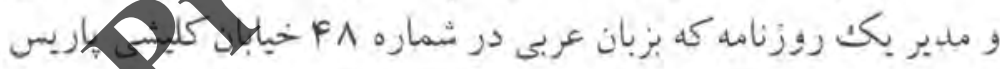

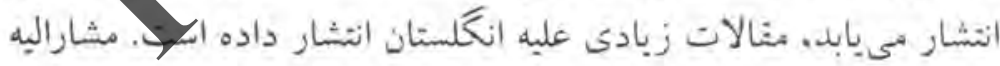

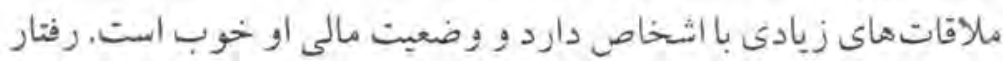

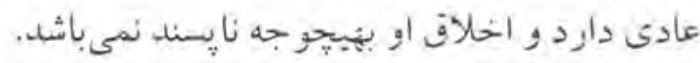

با إحتر أمات فائقه. امضاء ناخواناست 


\section{فعاليتهاى سيد در ياريس}

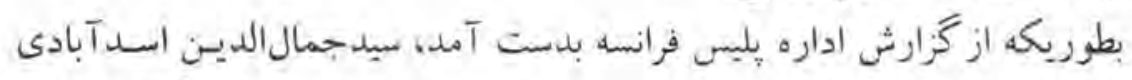

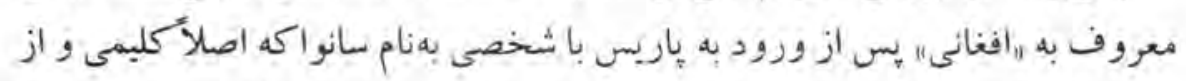

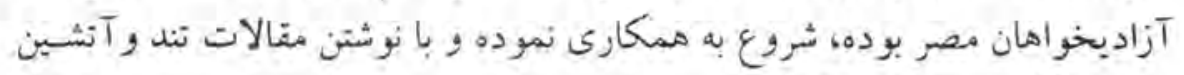

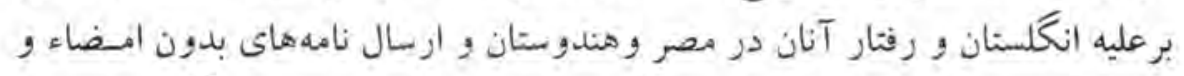

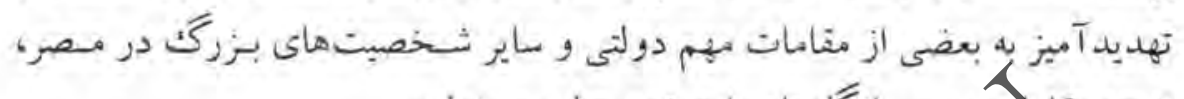

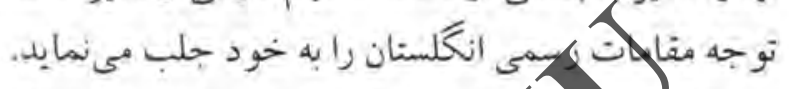

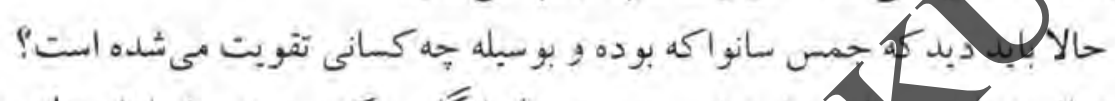

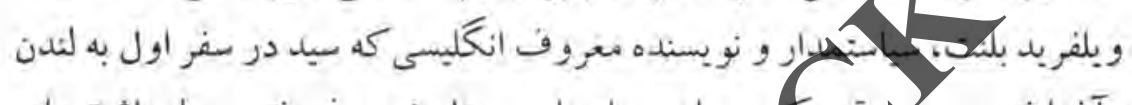

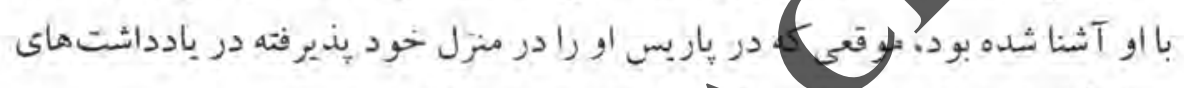

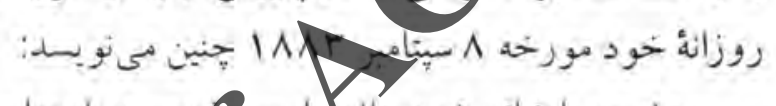

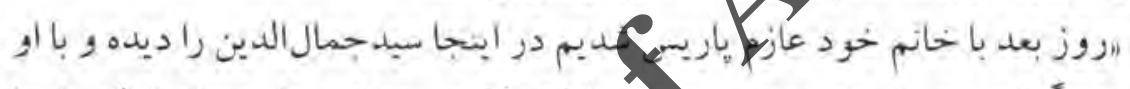

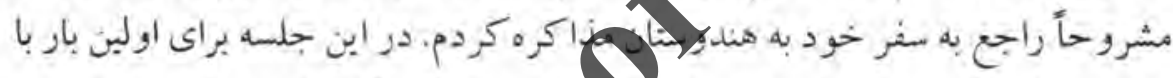

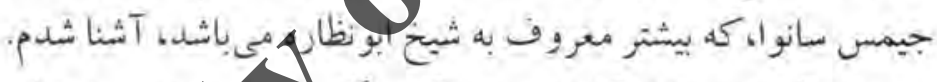

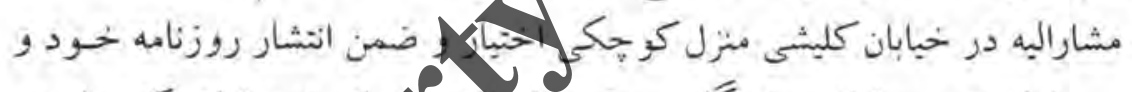

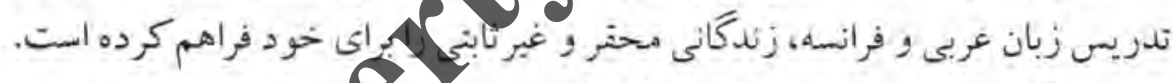

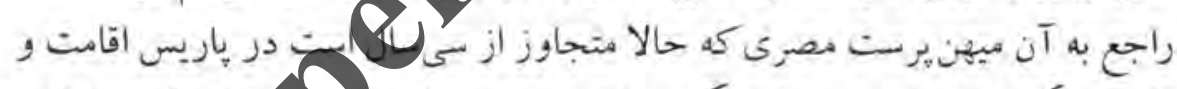

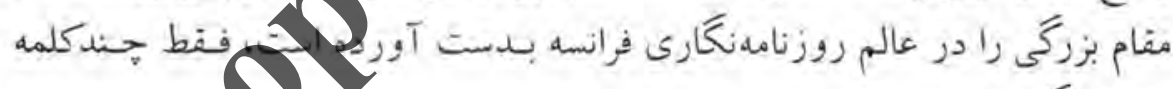
خواهم كُفت:

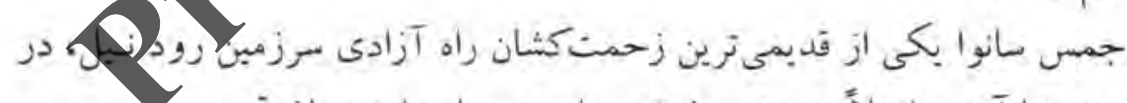

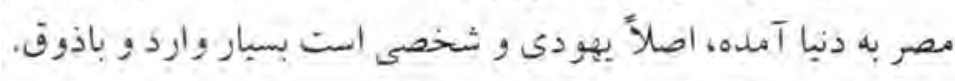

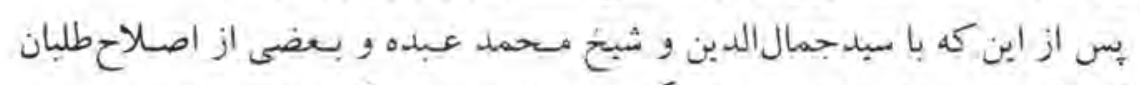

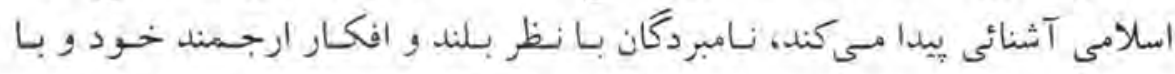

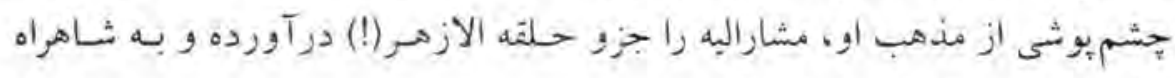
سياست و ادب هدايت مى نمايند. 
جون مرد شوخ وخوش مشربى بوده، او را تشويق به انتشار مطالب فكاهى نموده ور

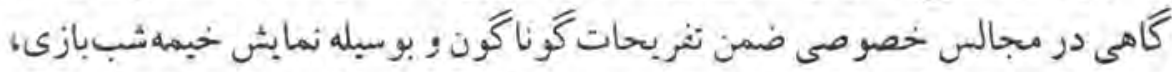

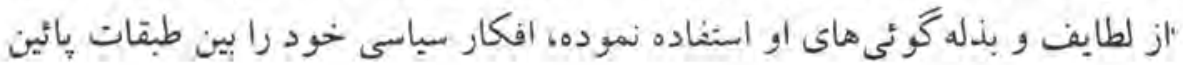

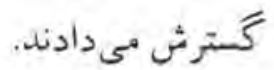

اين تمهيد خبلى زودتر از انتظارى كمه داشتئد نتبجه داد. هدف تمسخر و تنقيد سانوا

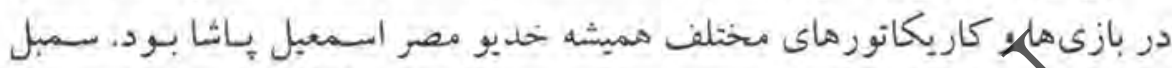

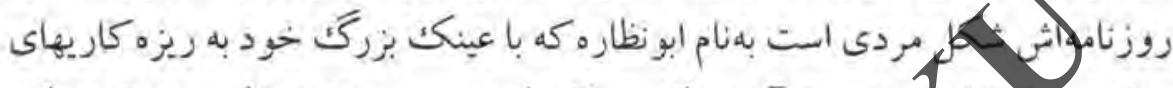

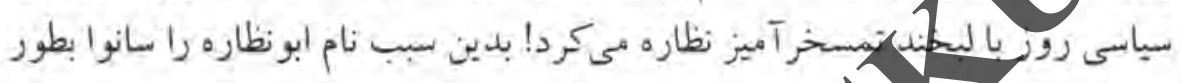

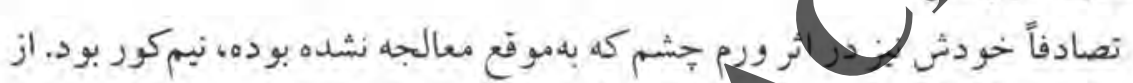

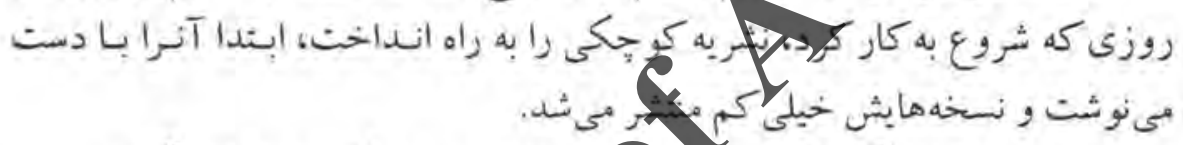

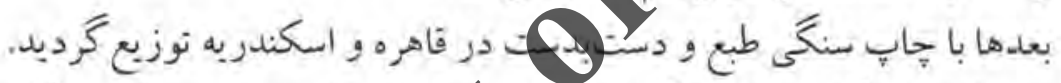

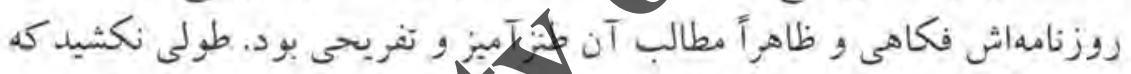

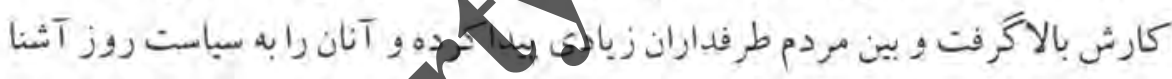

8 نمود.

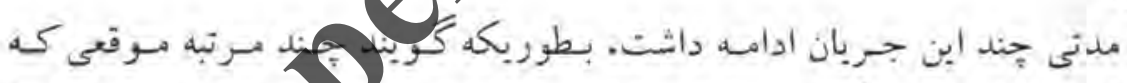

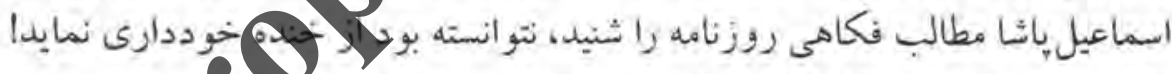

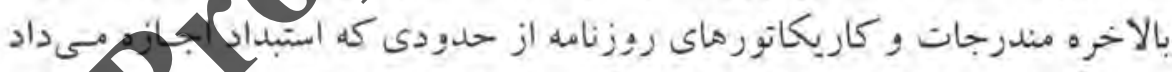

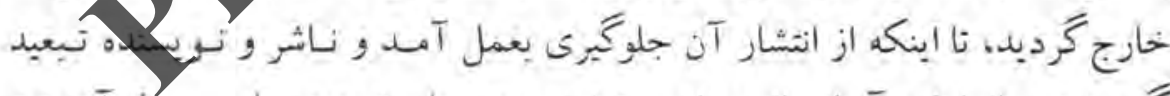

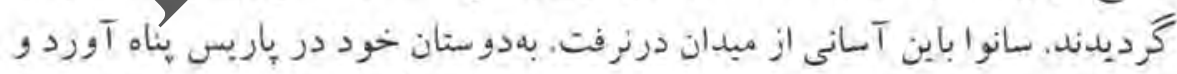

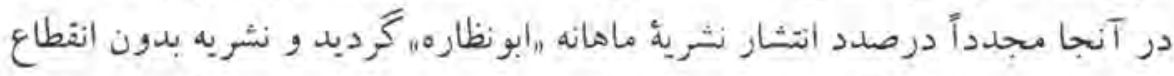

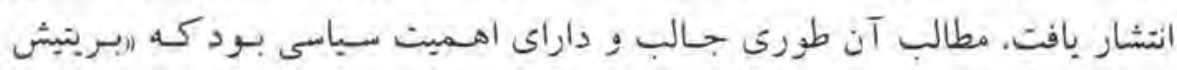

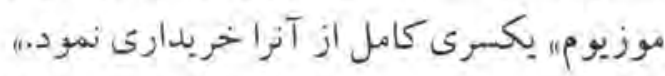

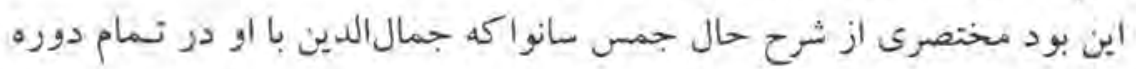




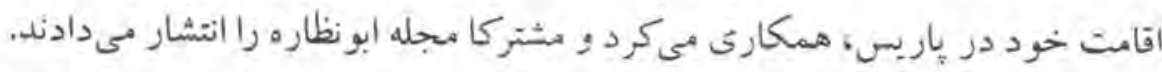

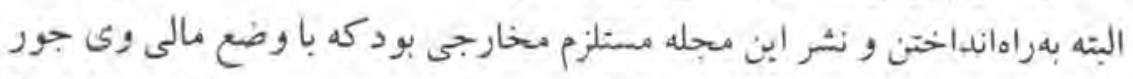

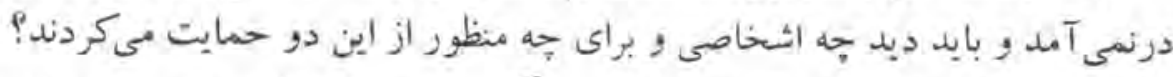

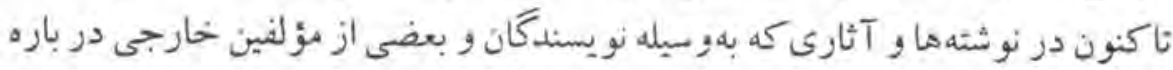

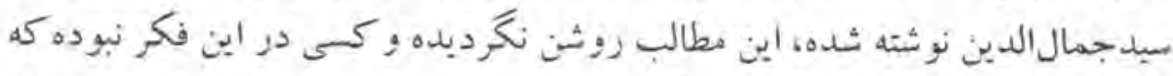

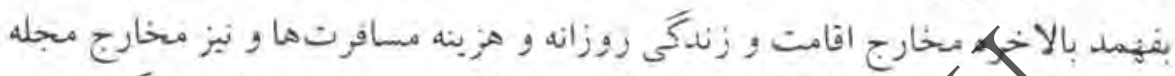

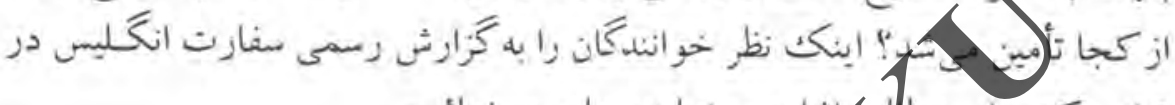

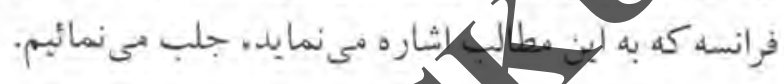

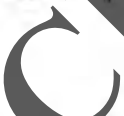

تزارشى از تباريس

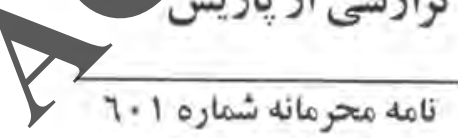

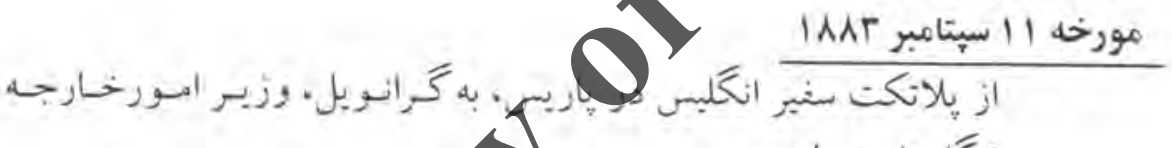

انخملستان در لندن.

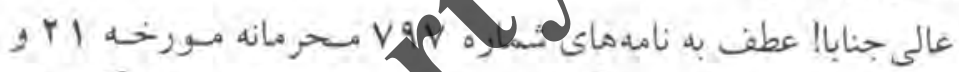
شماره

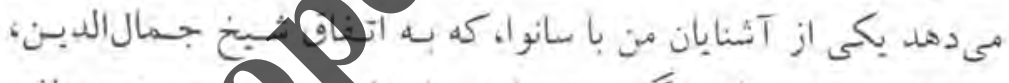

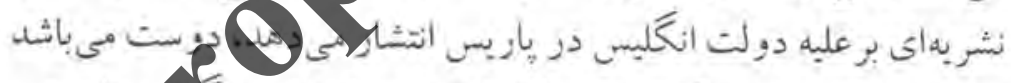

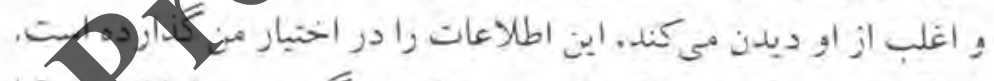

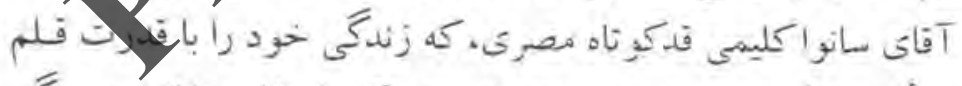

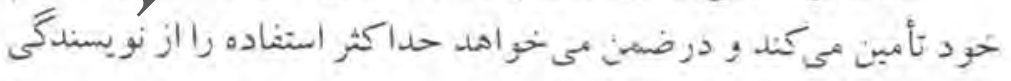

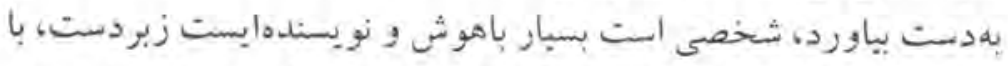

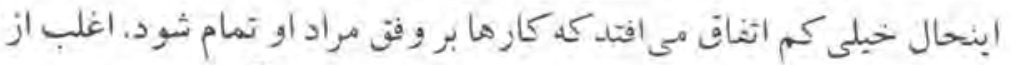

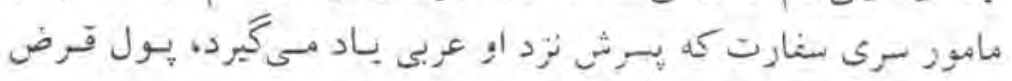
كى كند.

من هتوز موفتي نشدهام ايزمطلب راكه از كمجا اين شخص يول براى 
رأهانداختئ روزنامه خود بلمست ثي آورد، كشف كنم؟ بعيد نيست جهون

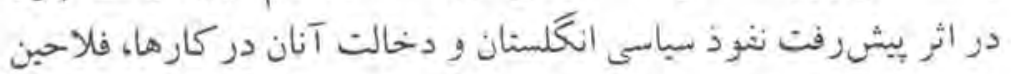

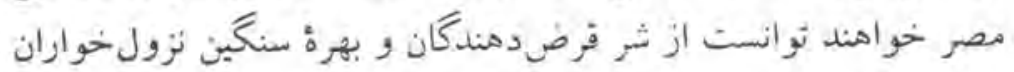

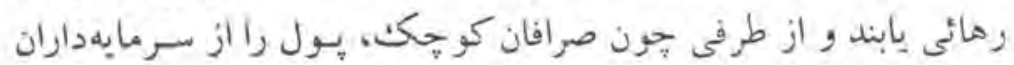

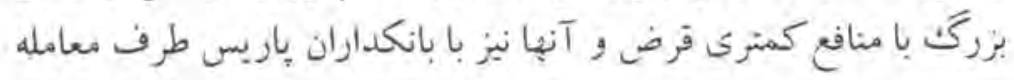

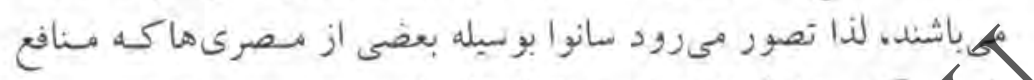

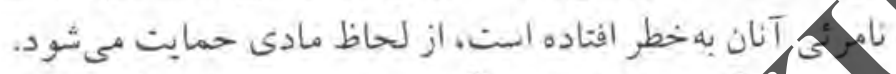

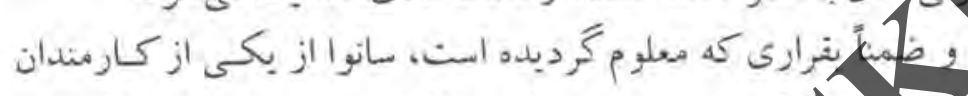

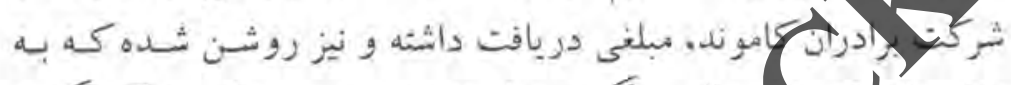

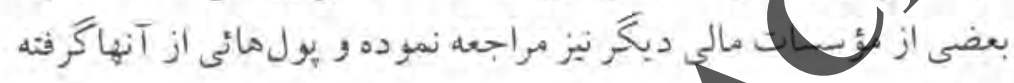

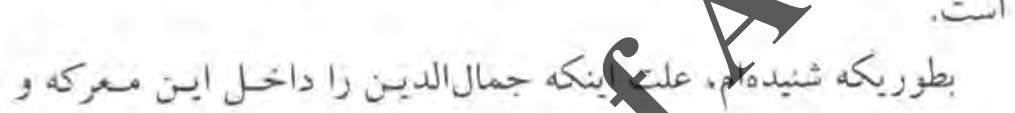

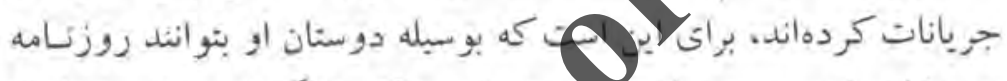

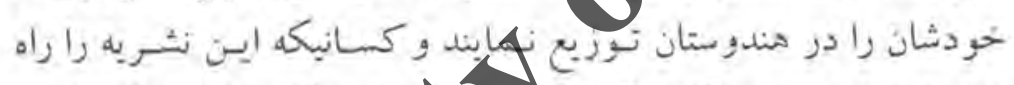

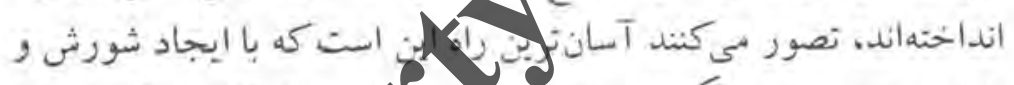

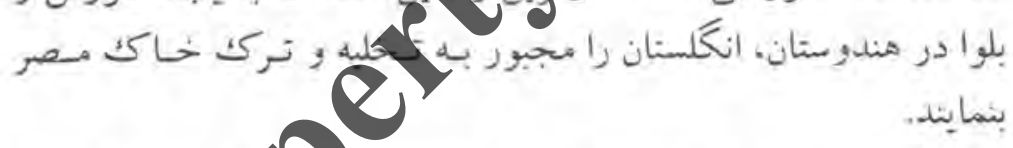

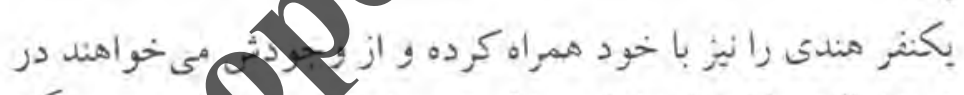

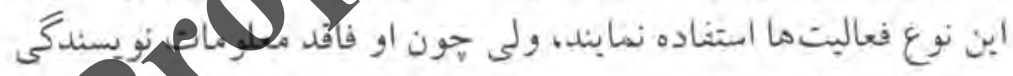

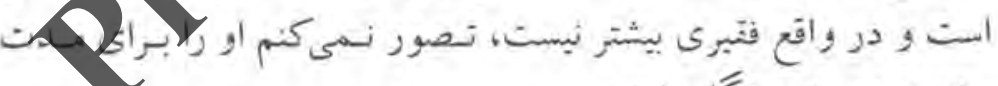
بشترى تزد خود نغاه دارئد.

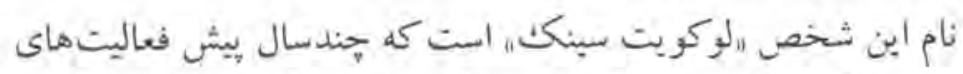

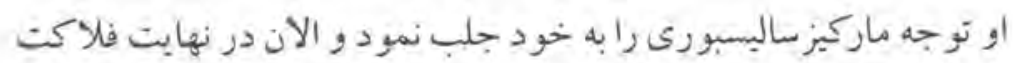

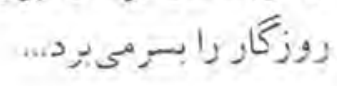

أمخاء - بلإتكت

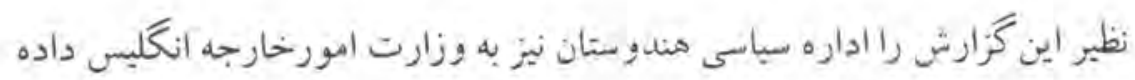




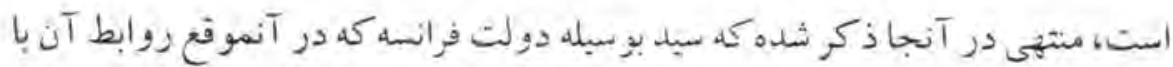

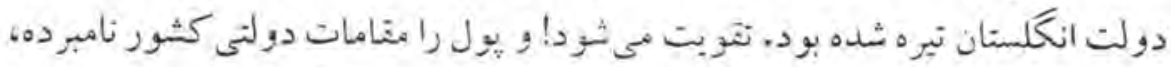

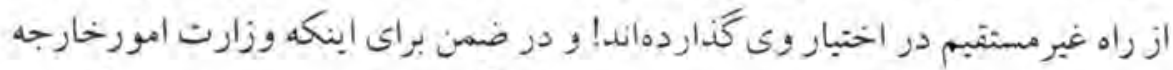

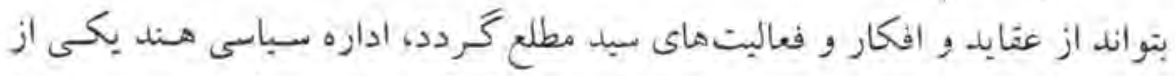

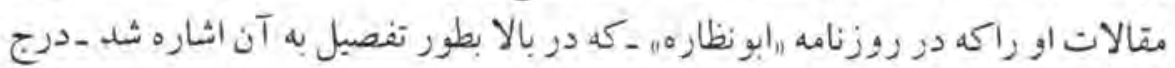

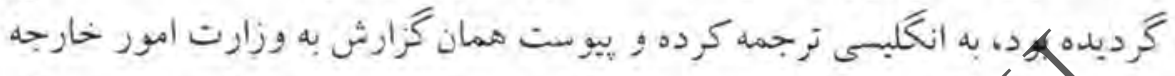

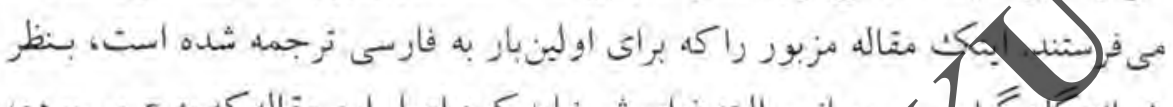

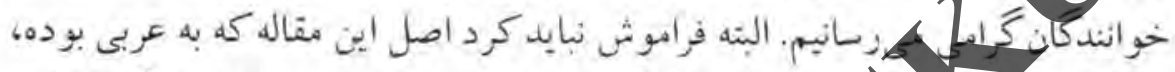

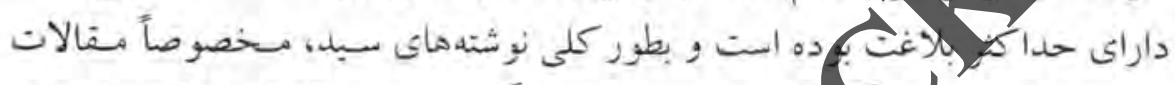

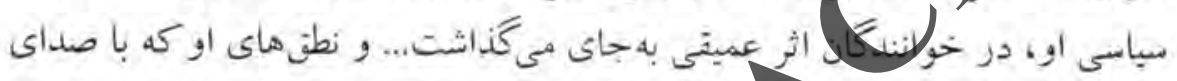

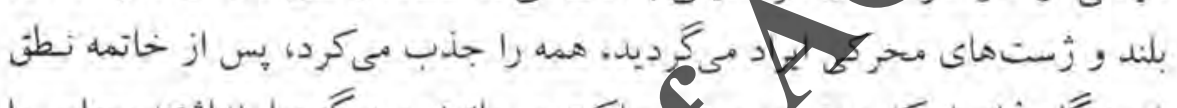

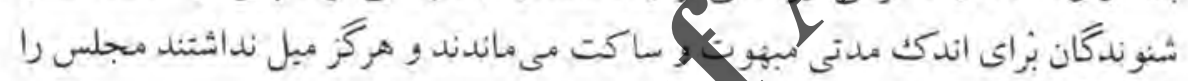
ترك بركو بندا
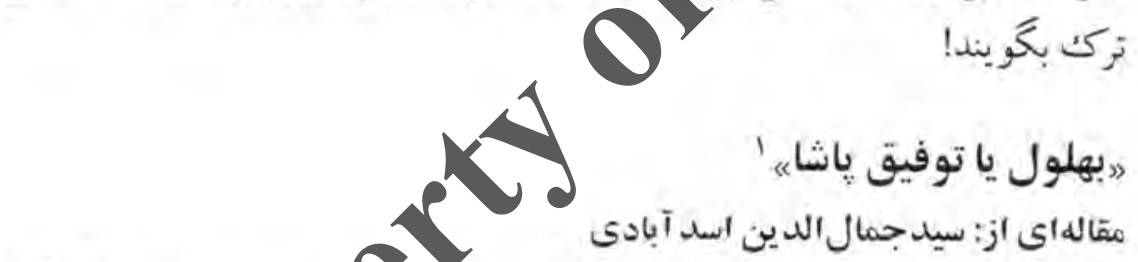

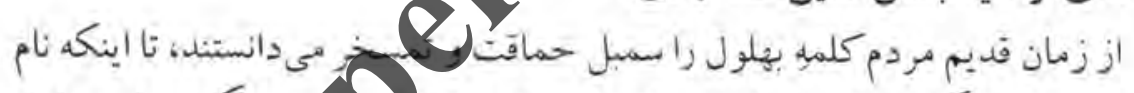

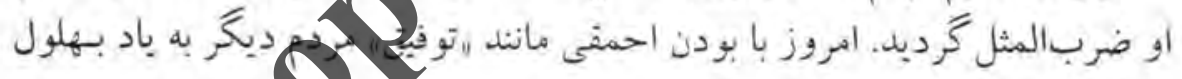

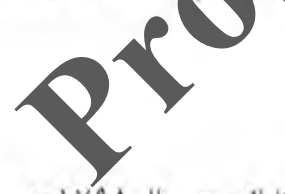

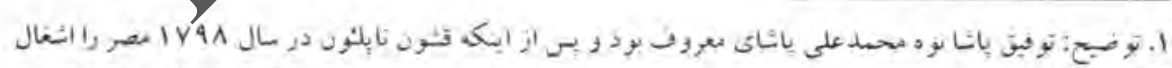

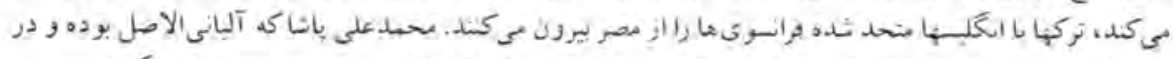

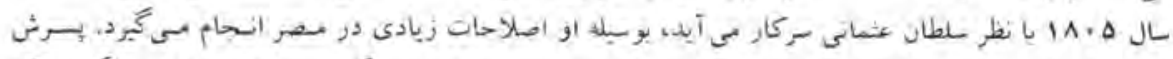

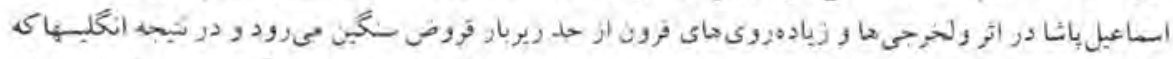

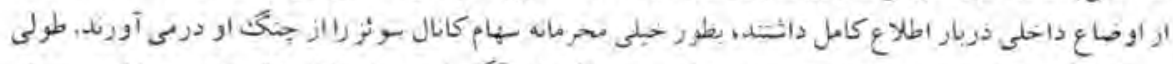

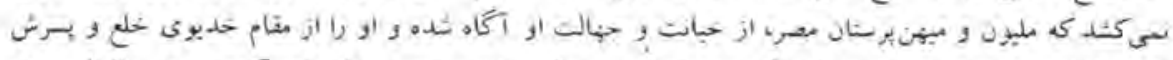

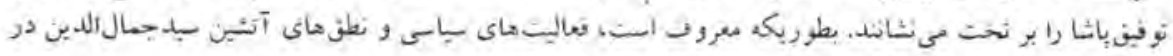

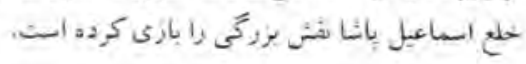




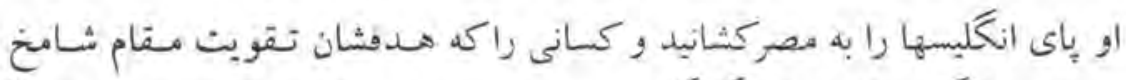

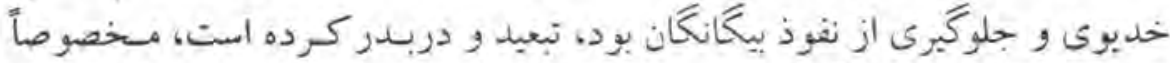

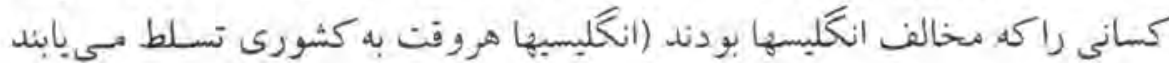

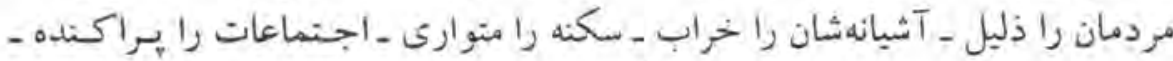

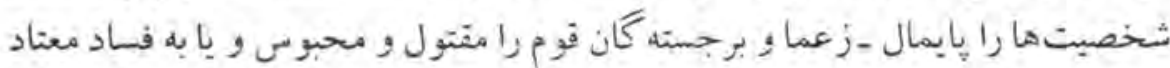

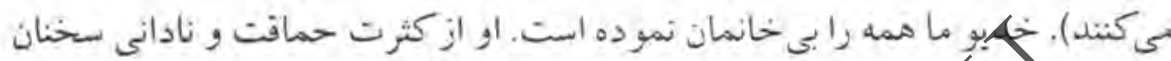

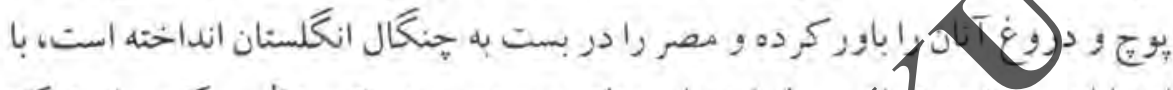

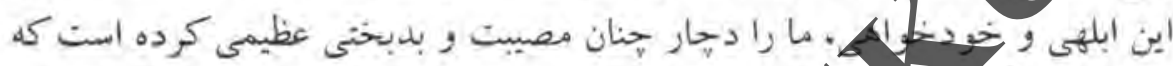

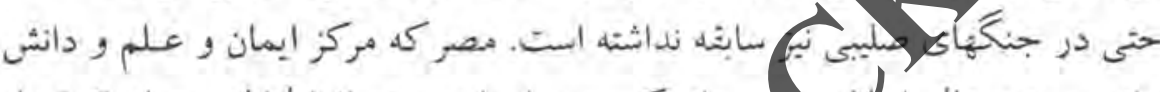

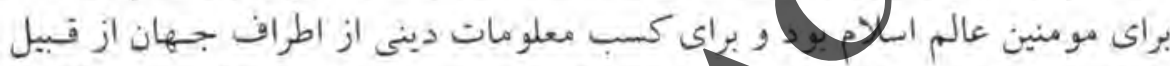

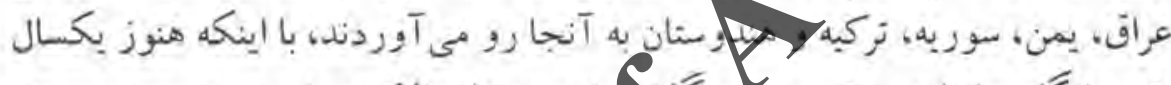

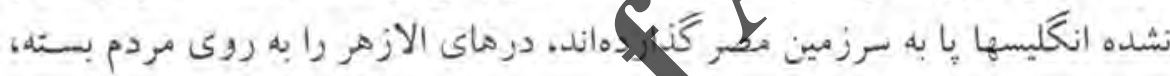

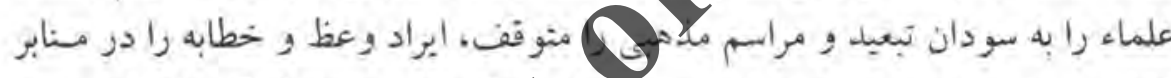

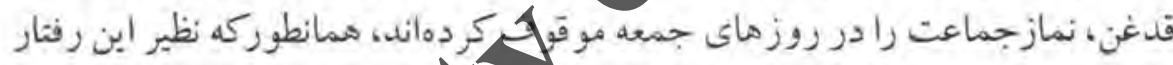

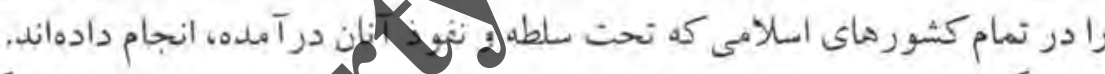

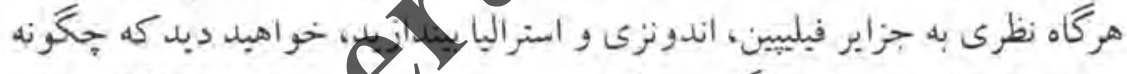

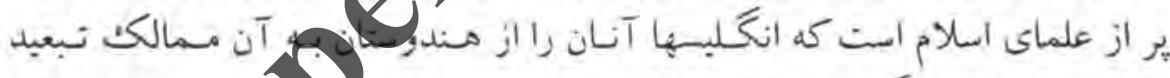

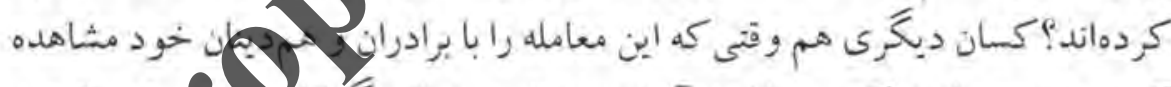

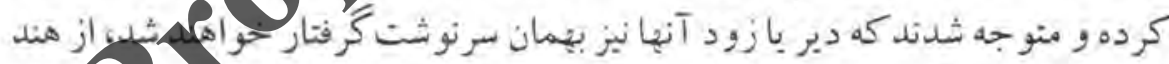

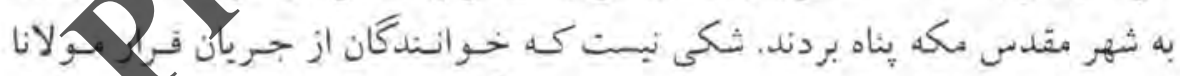

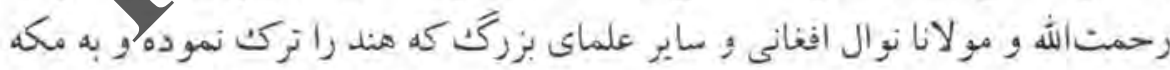

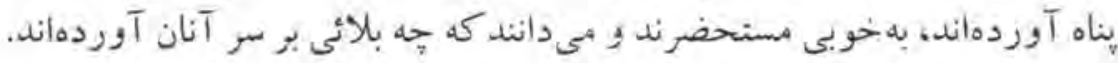

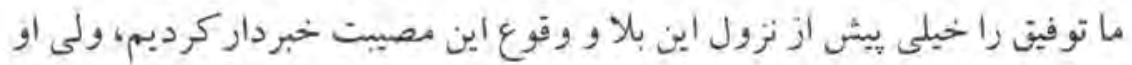

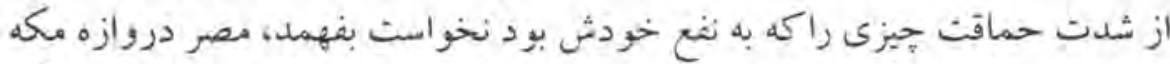

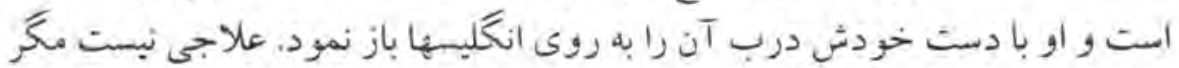

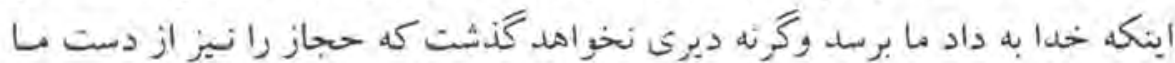




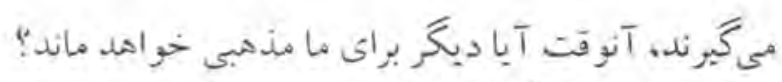

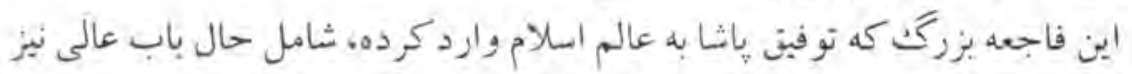

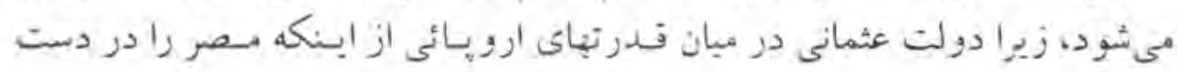

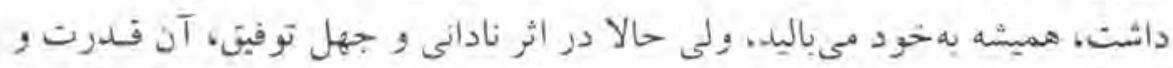

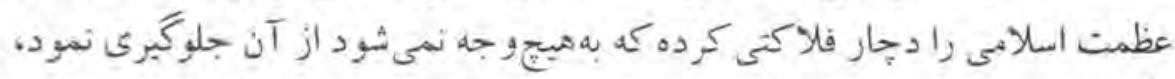

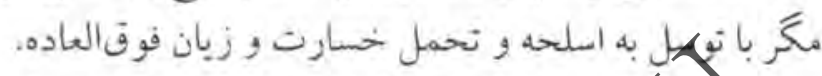

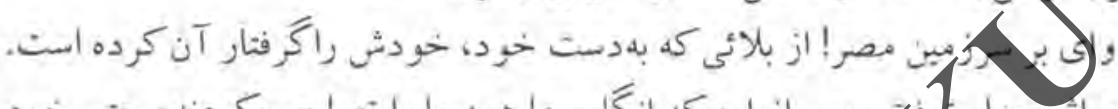

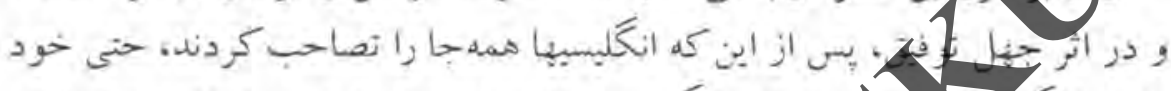

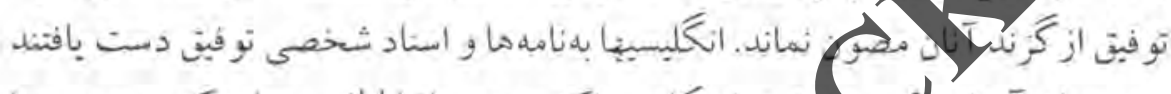

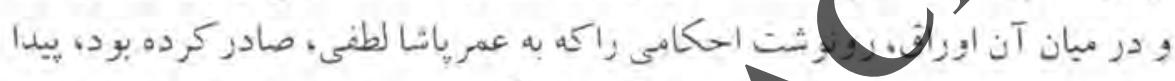

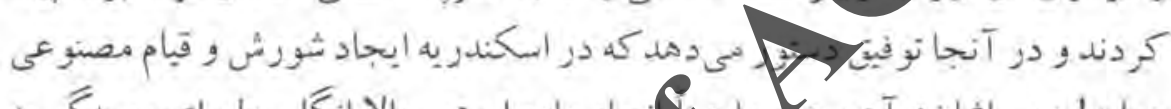

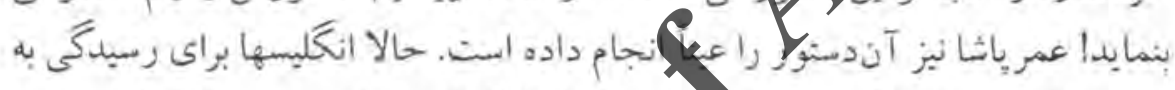

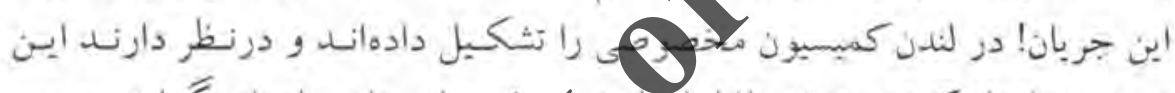

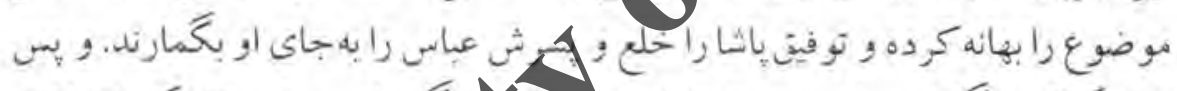

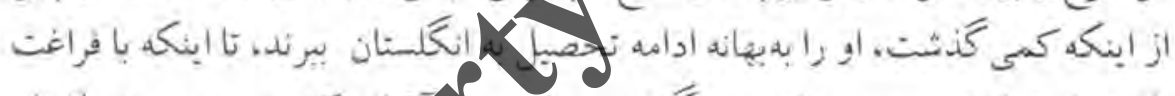

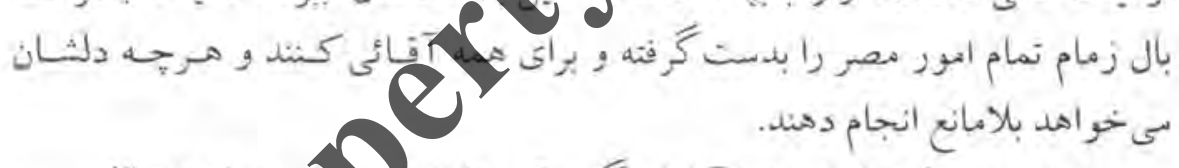

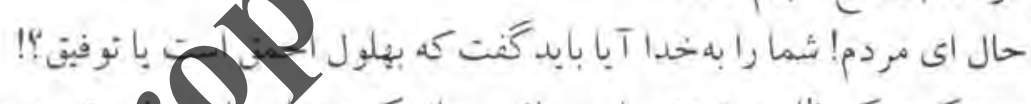

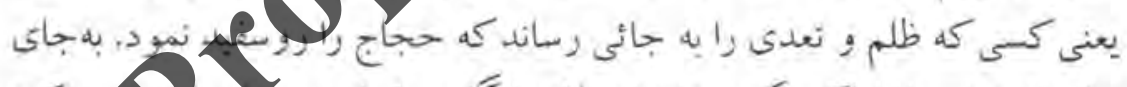

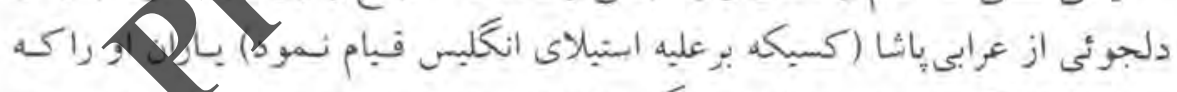

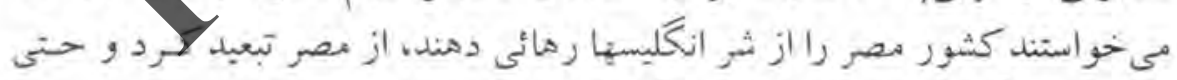

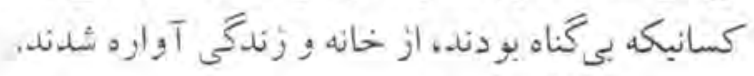

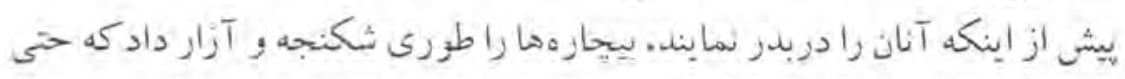

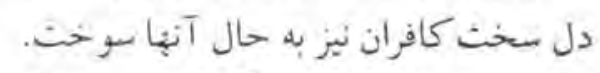

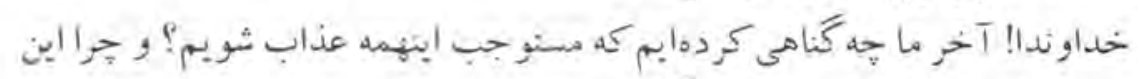

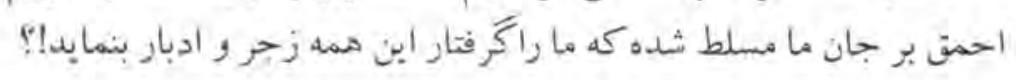




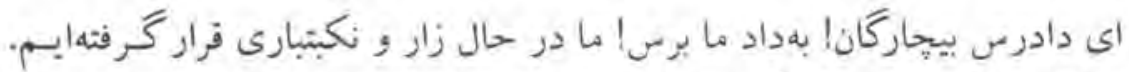

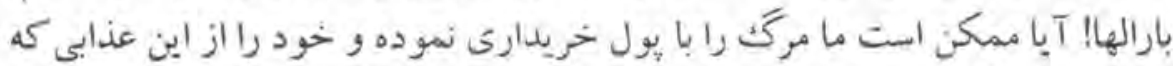

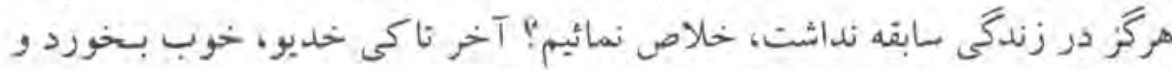
بنوشد و در حال عيش و نوشى در بسنر راحتى و رفاه بسر بيرد، ولى سايرين در حر حال

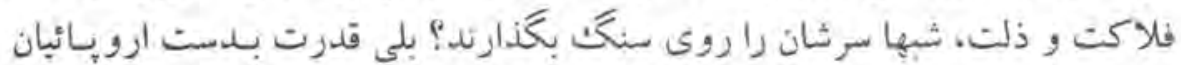

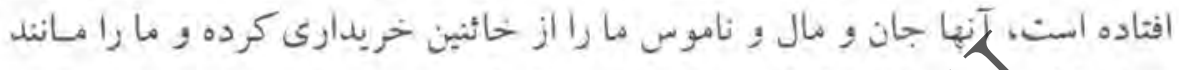

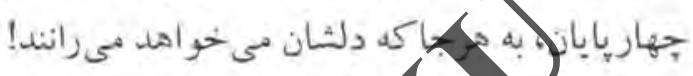

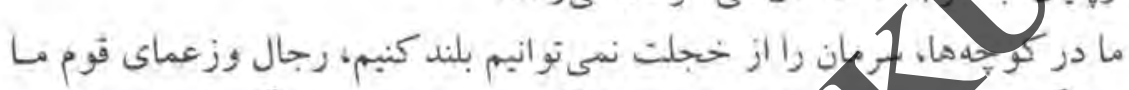

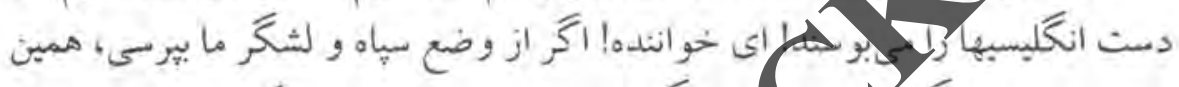

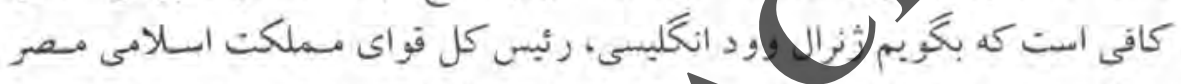

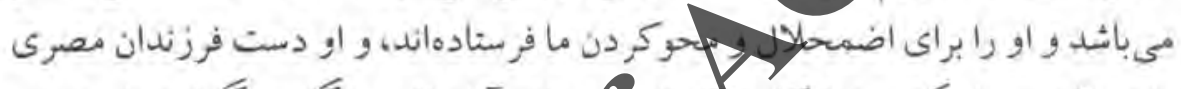

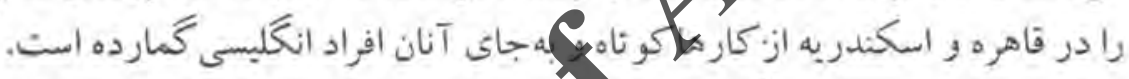

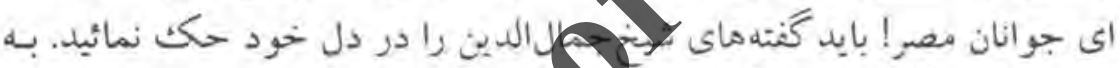

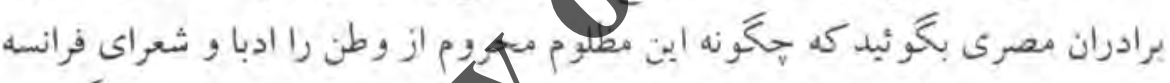

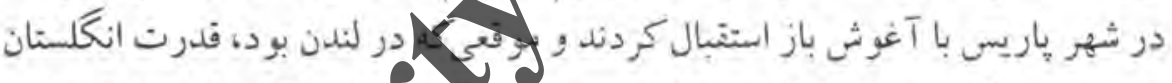

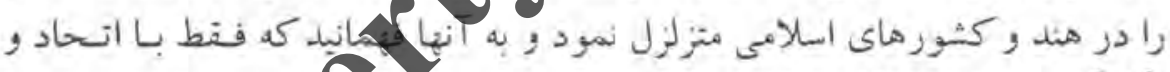

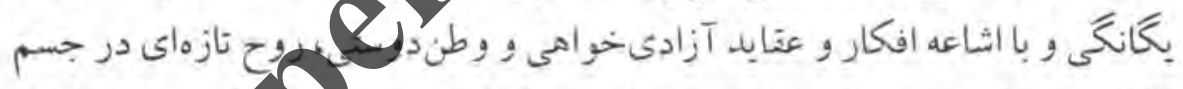

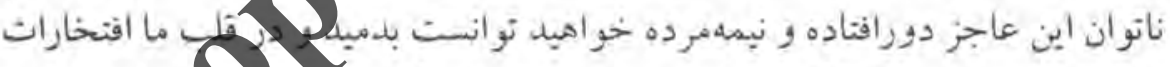

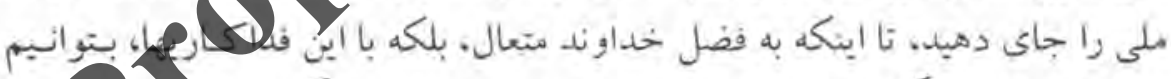

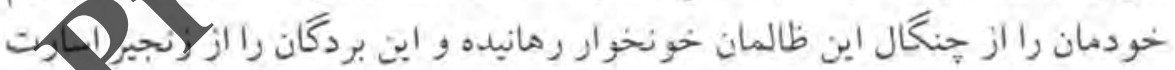
جمال الدين الحسبنى خ الأصى

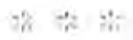

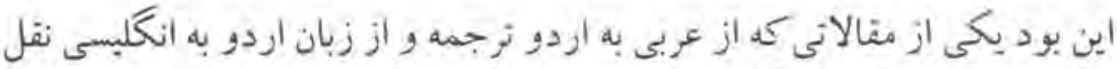

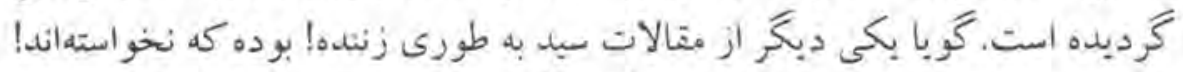

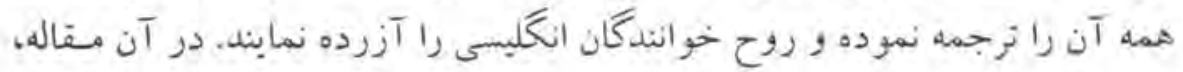


انخليسيها را از فول فيلسوف معروف خودثان بعنى داروبن، ميمون خطاب نموده

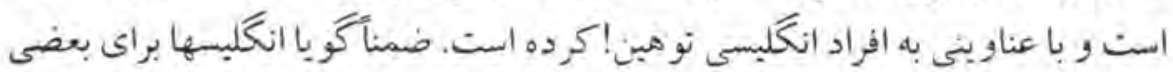

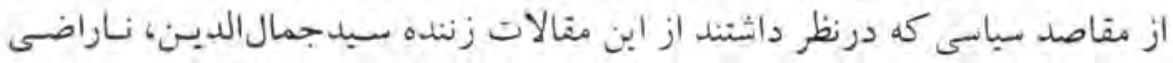

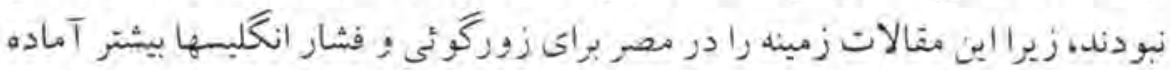

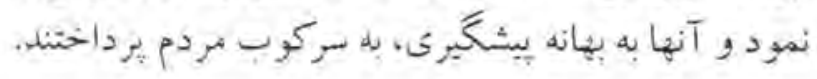

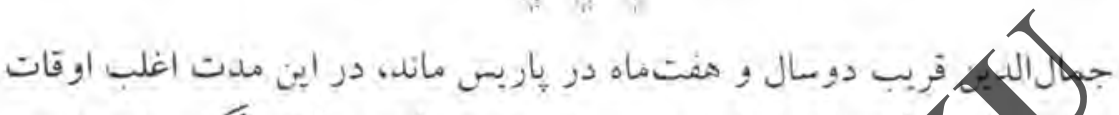

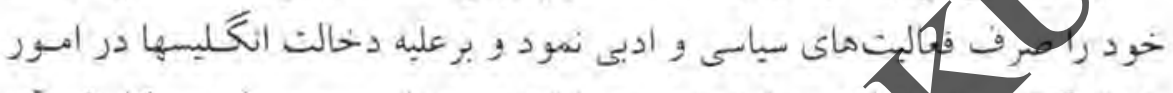

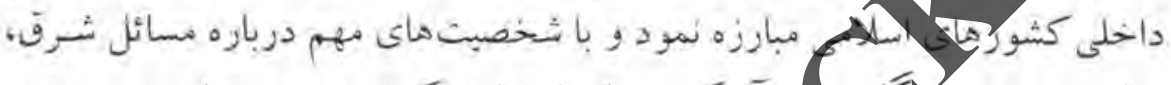

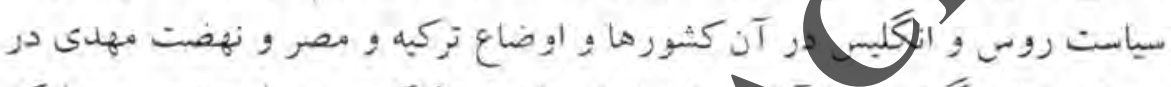

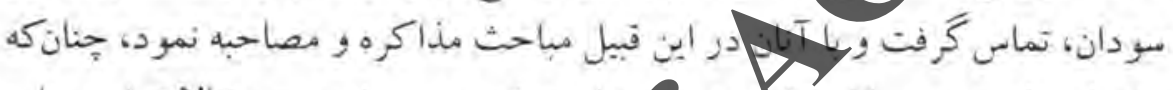

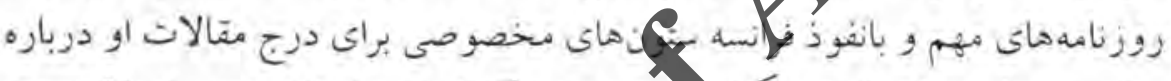

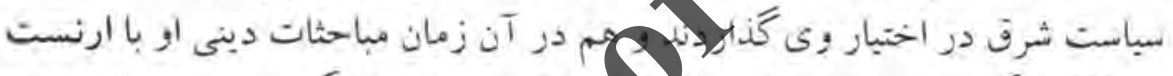

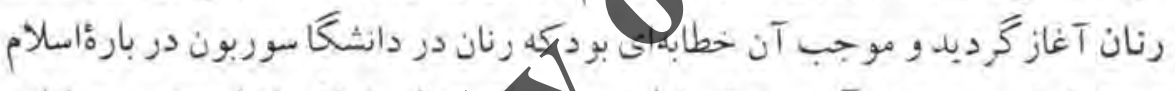

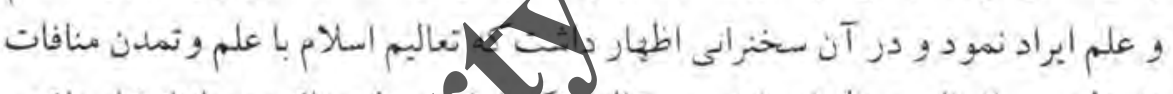

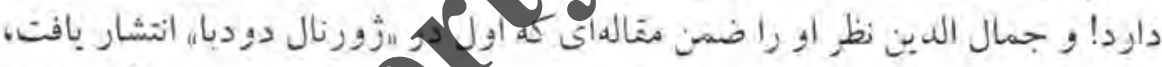

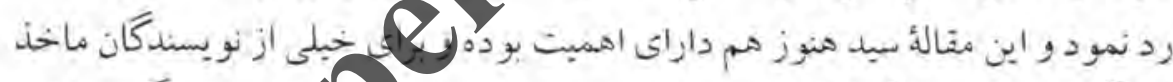

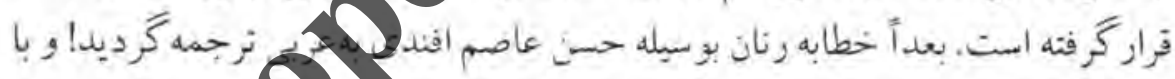

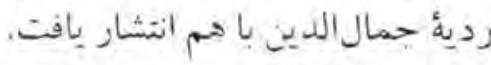

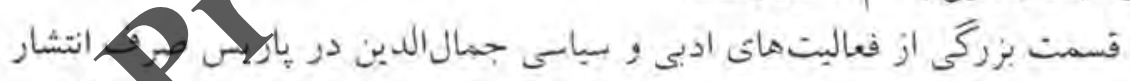

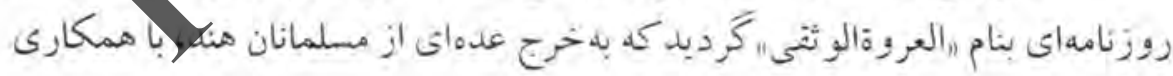

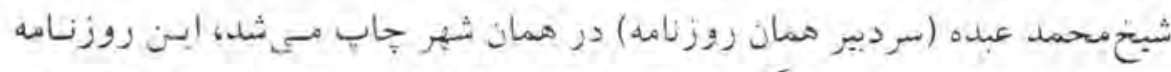

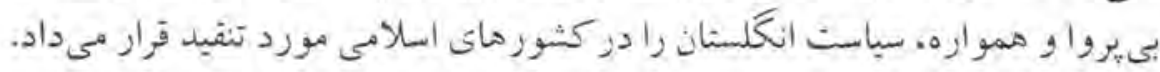

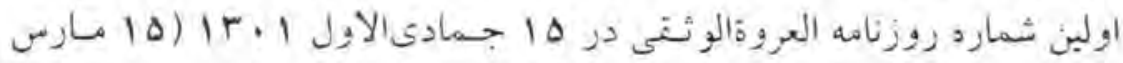
(IANF

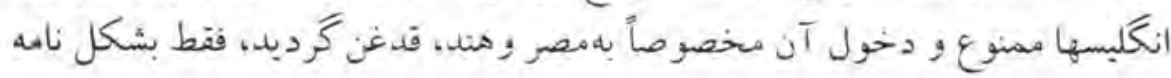




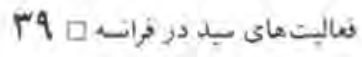

به دست عذه معدودي از اشخاصيكه بهنظر جمال الدين وجود آنان در سباست دوز

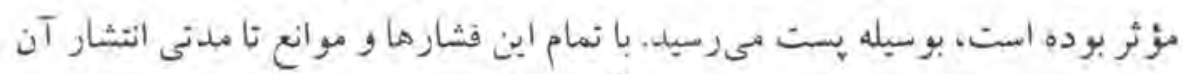

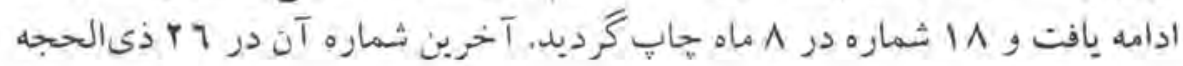

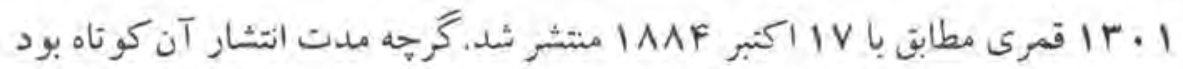

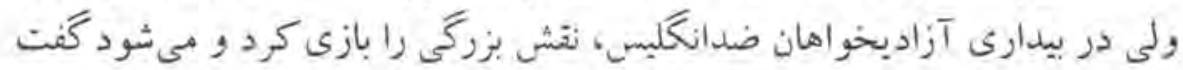

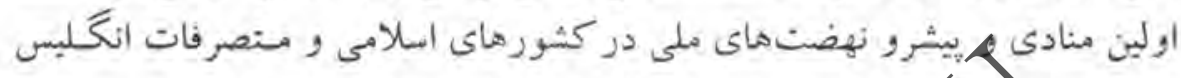

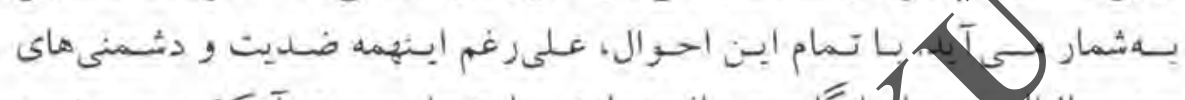

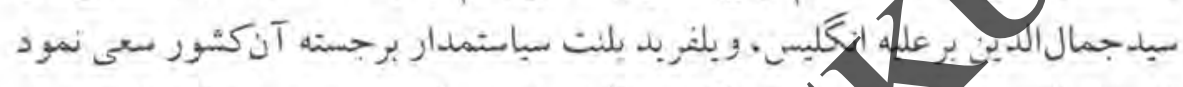

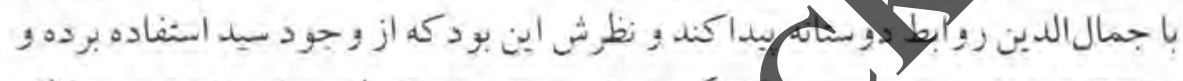

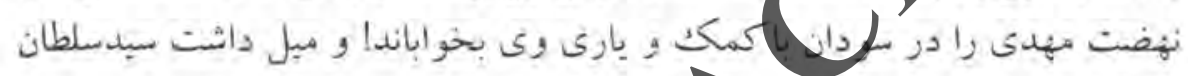

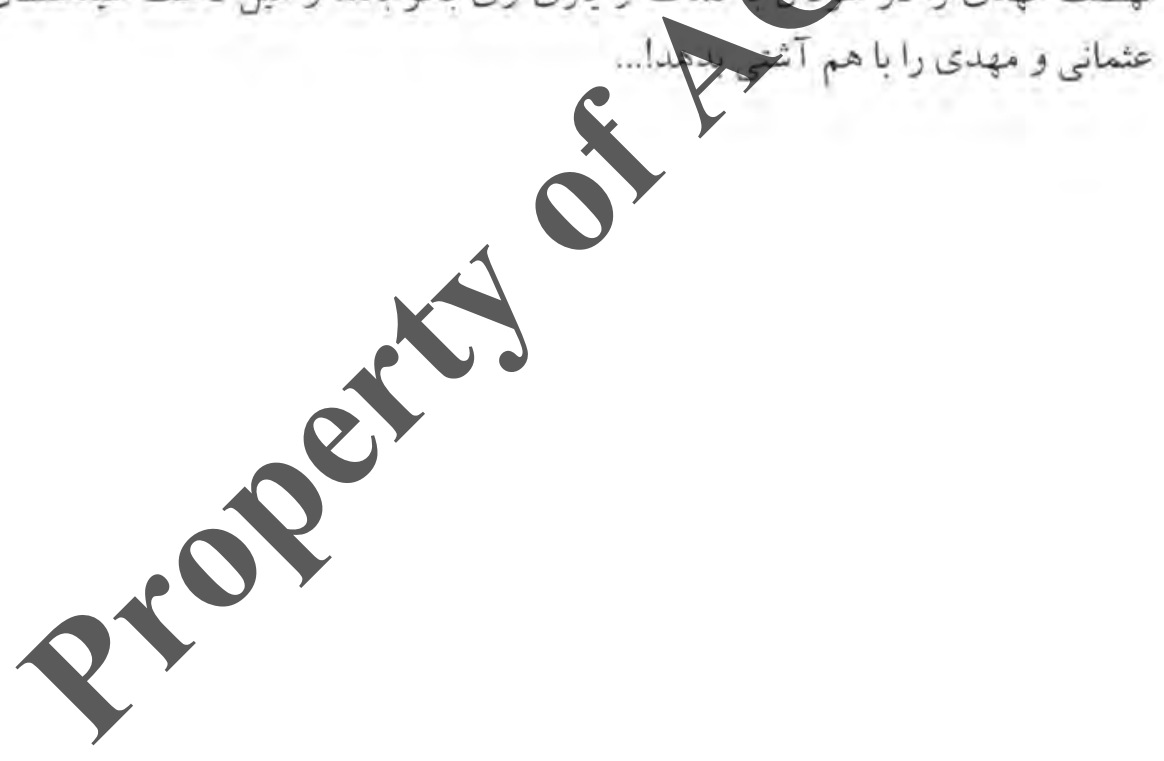




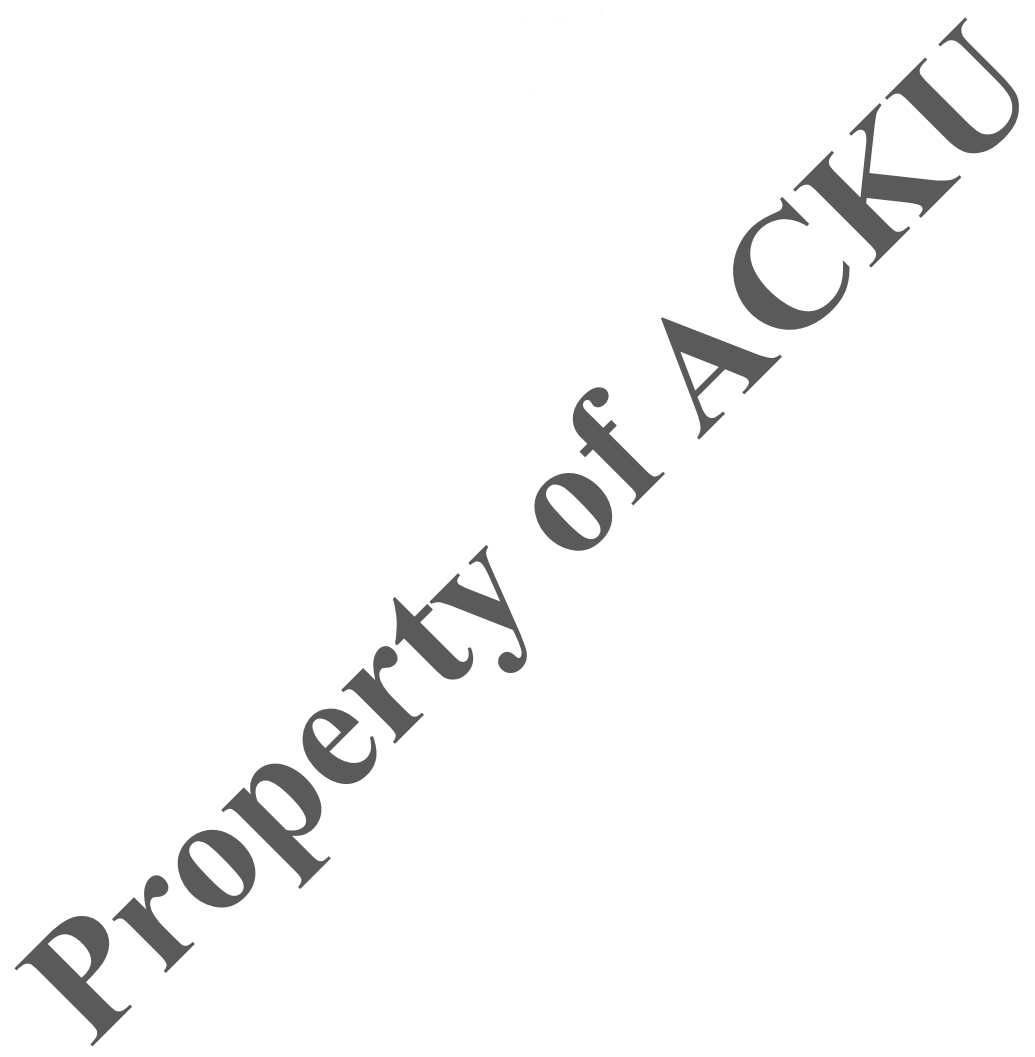




\section{$f$ \\ كفتكوى راندولف با سيد}

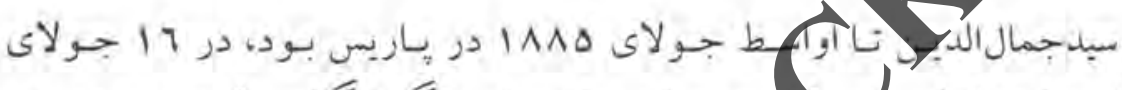

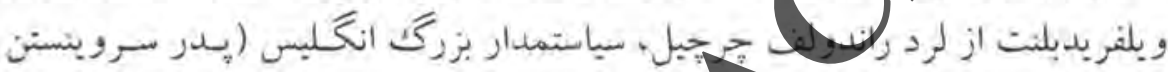

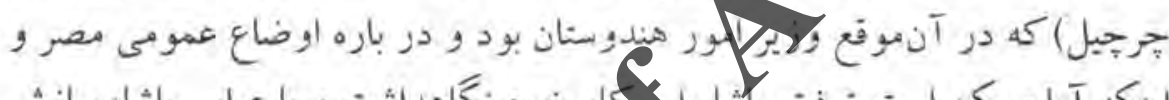

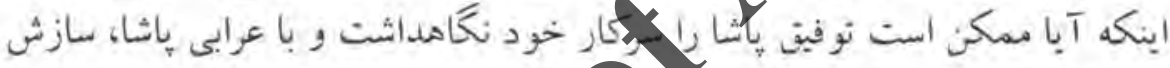

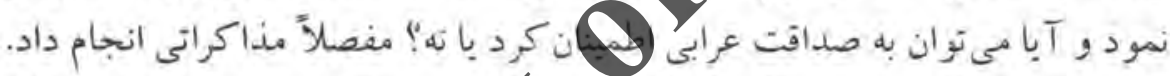

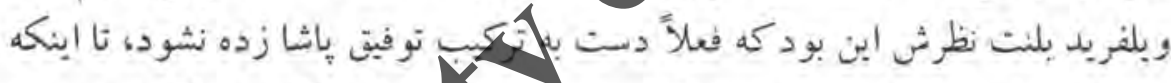

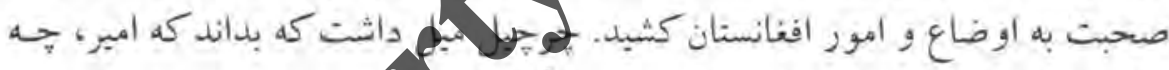

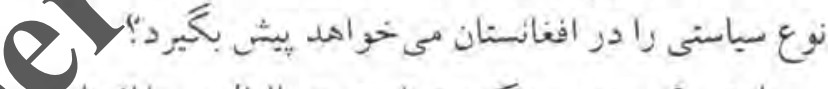

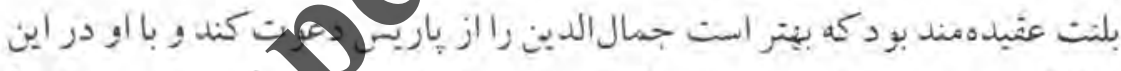

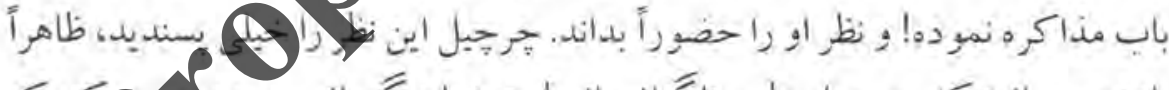

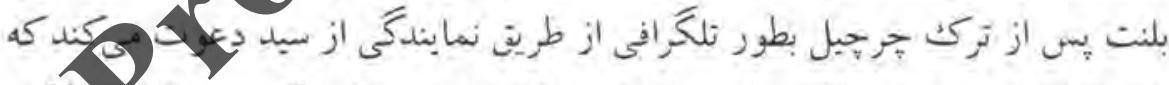

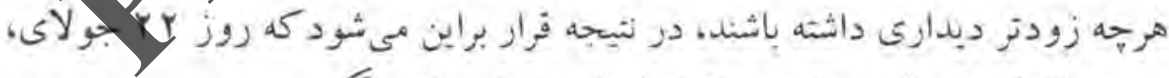

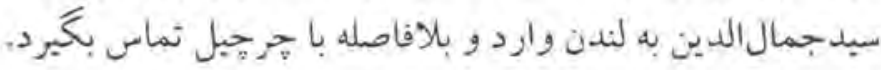

$$
\text { بلنت در يادداشت هايش مى نويسن: }
$$

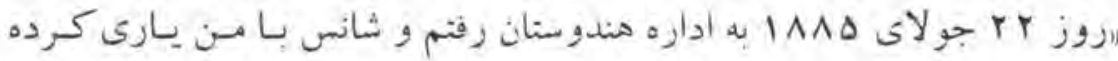

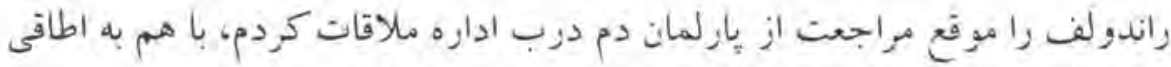

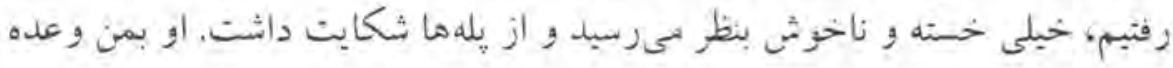


دادكه فودا ساعت دمونيم جمال الدين رادر اطاق كارش بيفيرد.

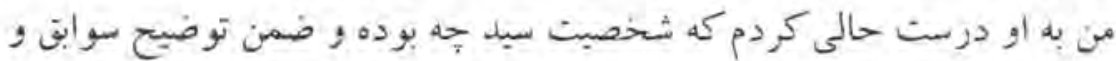

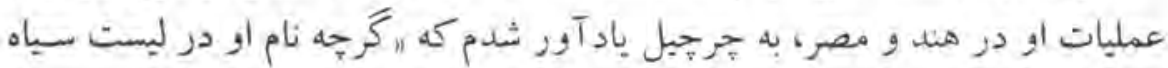

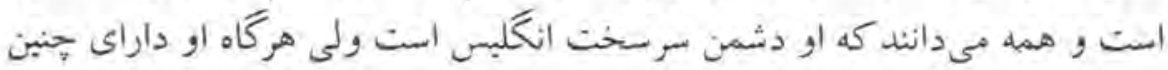

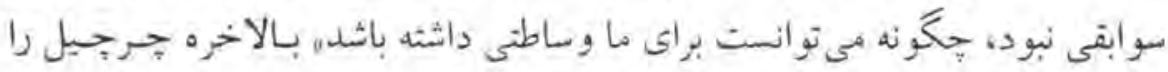

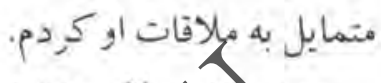

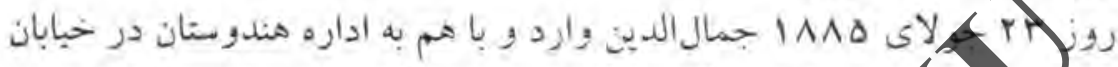

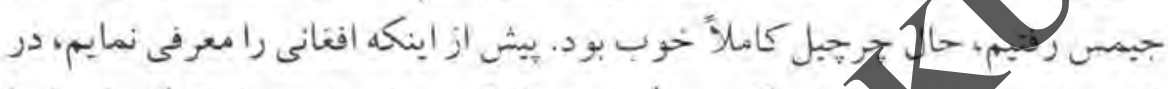

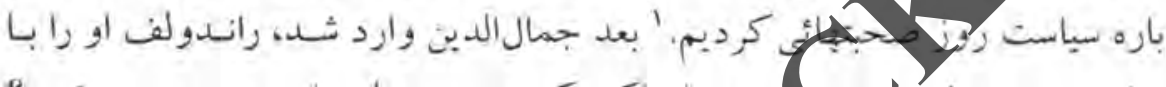

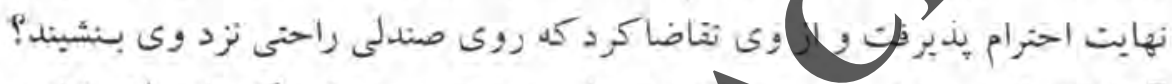

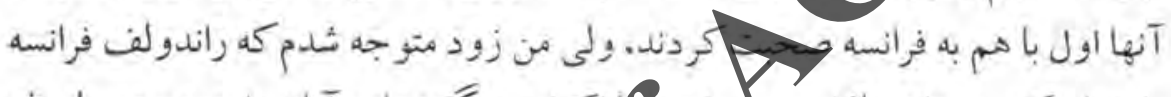

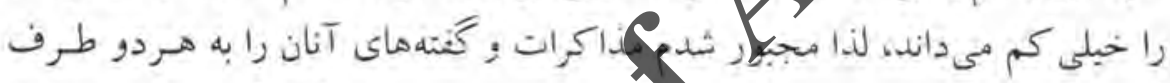

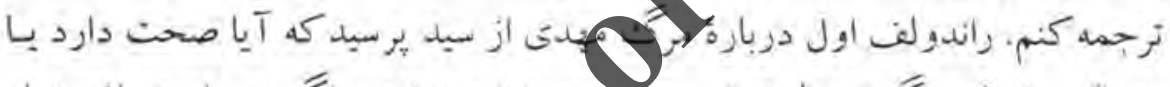

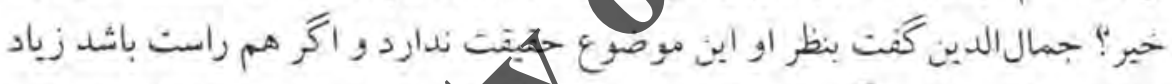

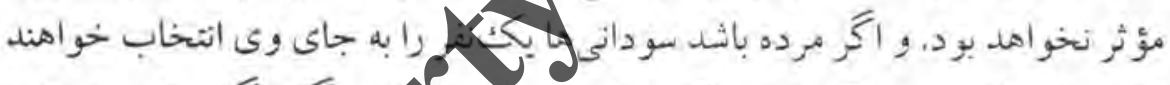

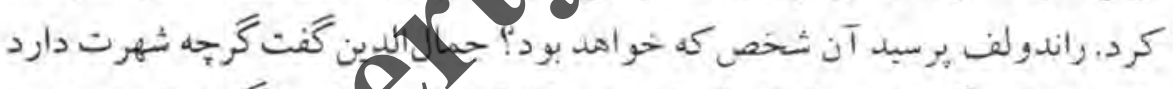

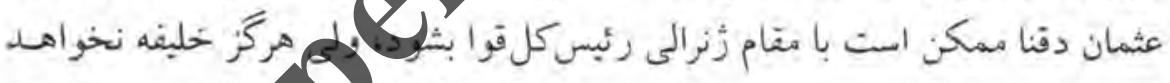

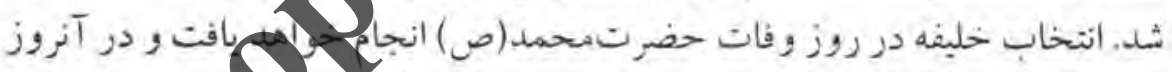

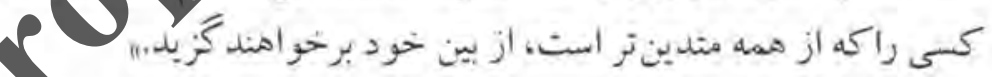

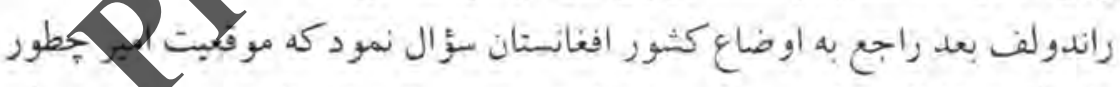

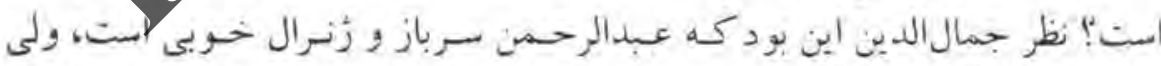

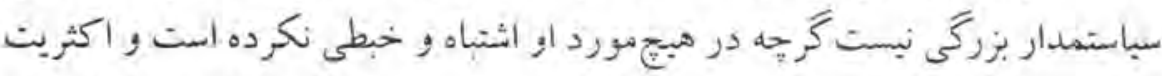

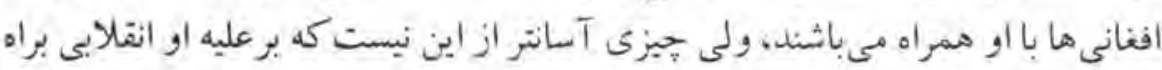

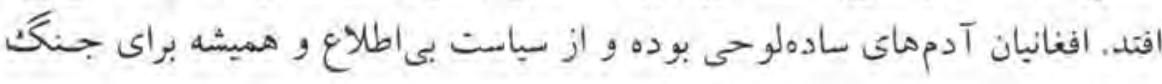




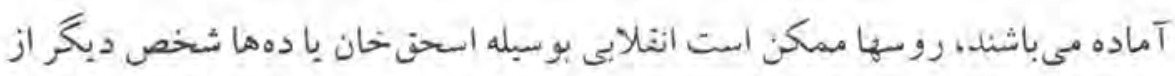

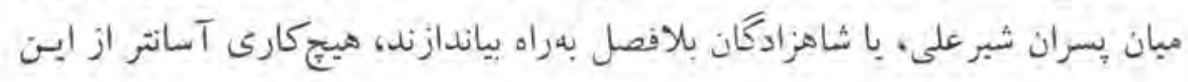

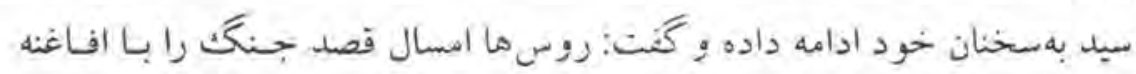

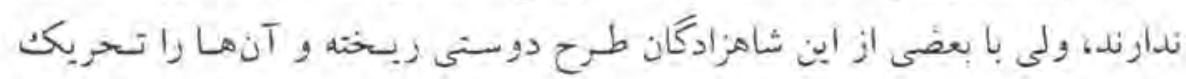

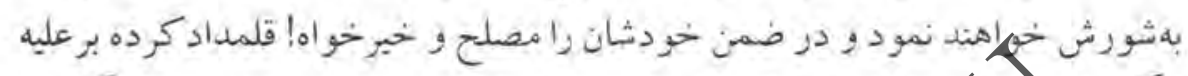

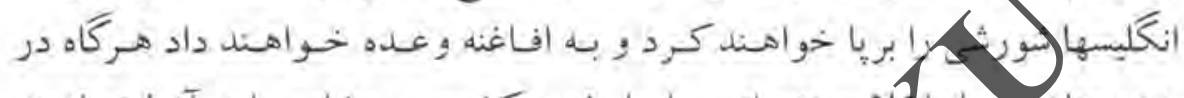

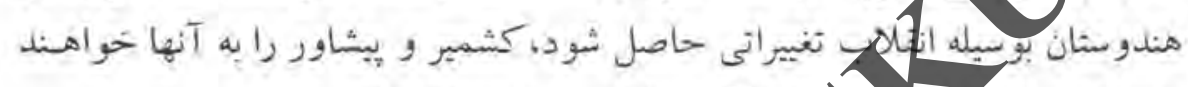

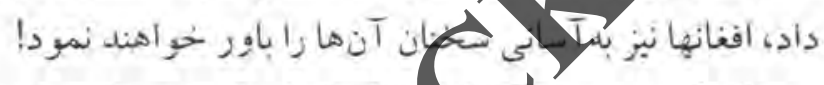

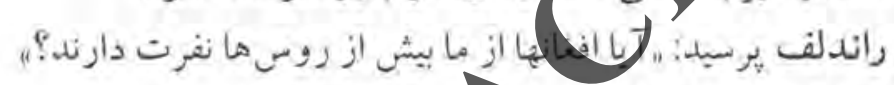

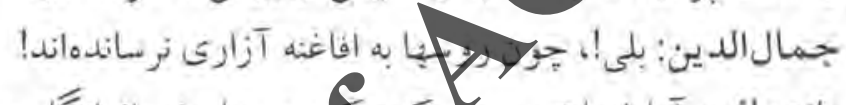

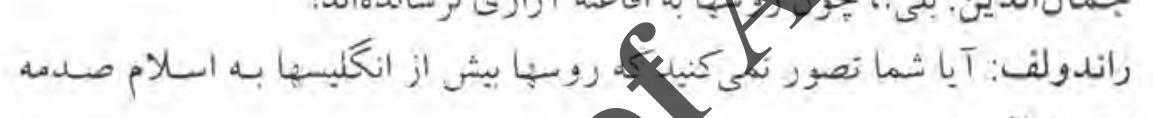
ربانيداندب!

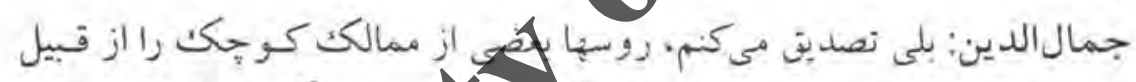

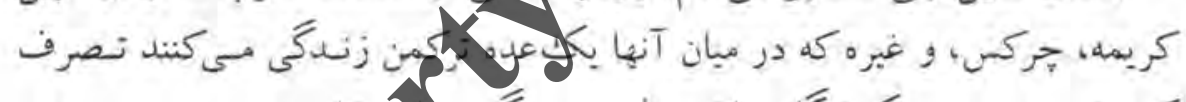

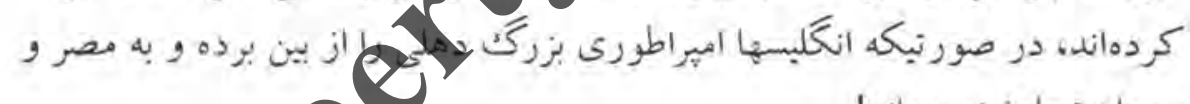
سودان نتجاوز نمودماندا

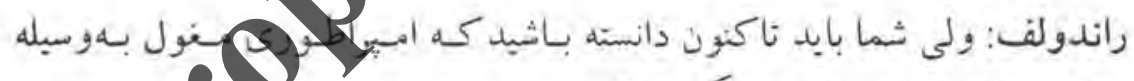
راجه هاى هندى از بين رفت نه انكليسيها!

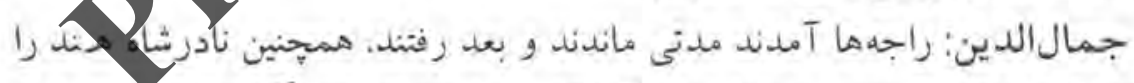

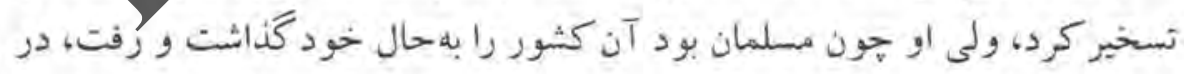

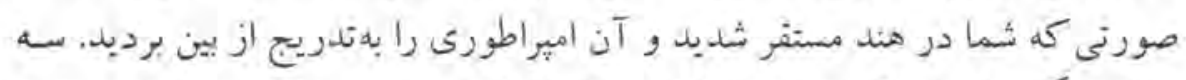

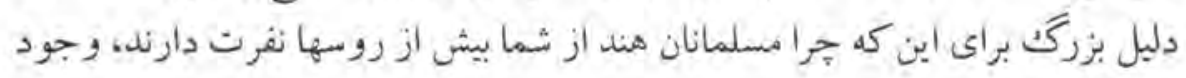
2.

اول: براي اين كه شما اميراطرزى دملى را مـضمحل كر ديد، دوم: بـليل إينكه 


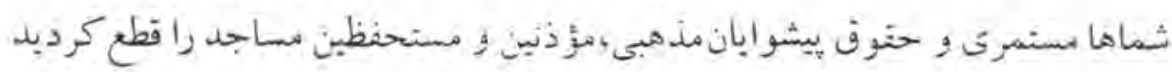

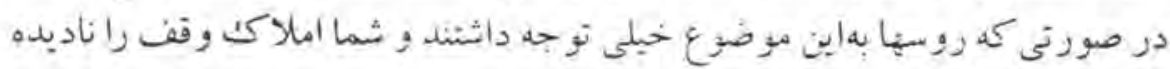

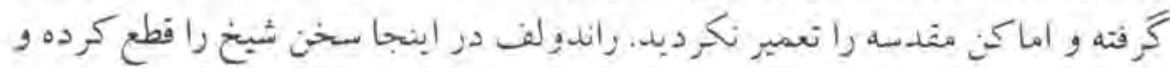

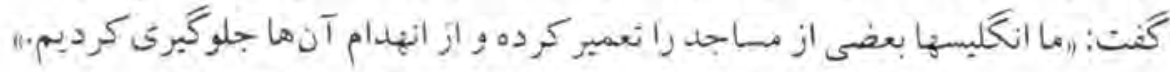

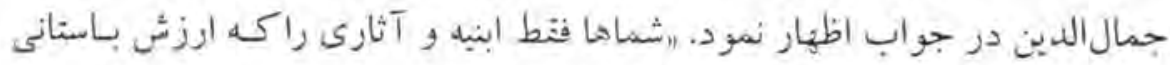

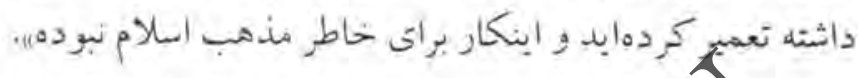

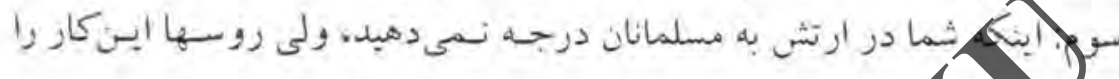

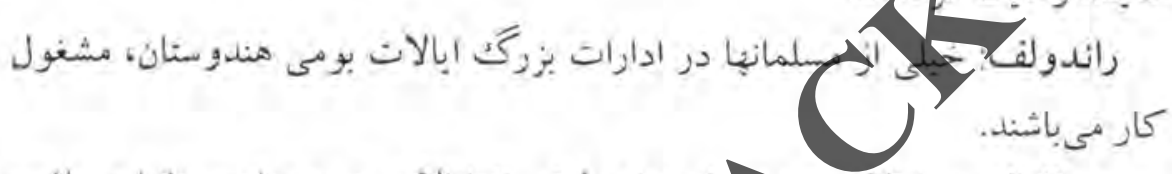

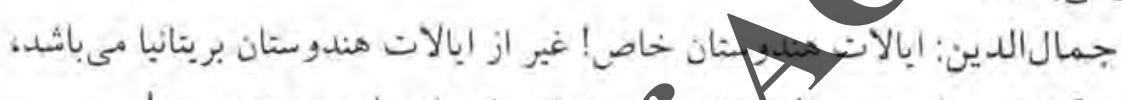

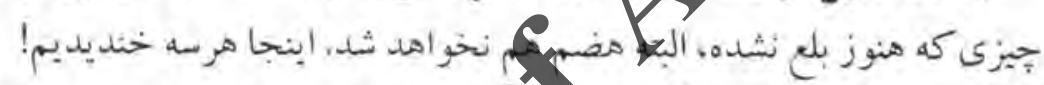

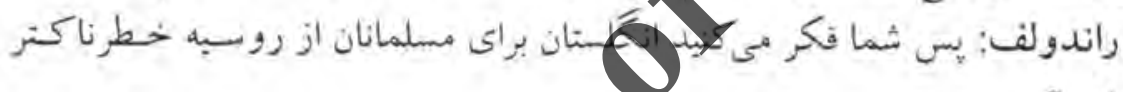

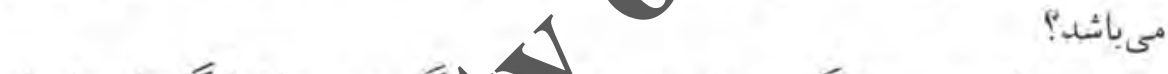

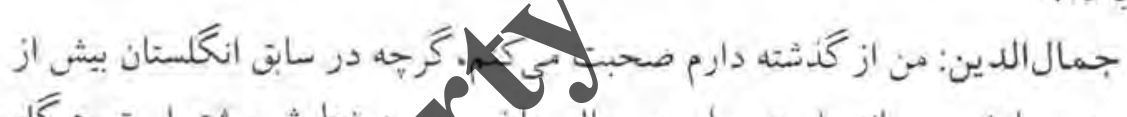

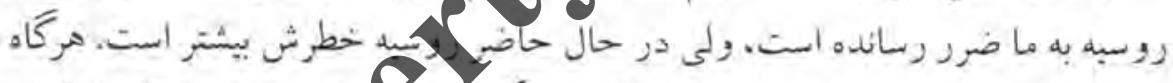

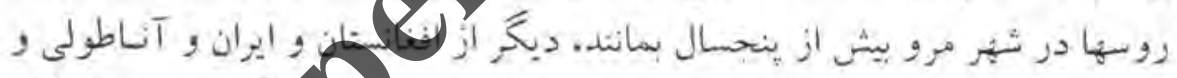

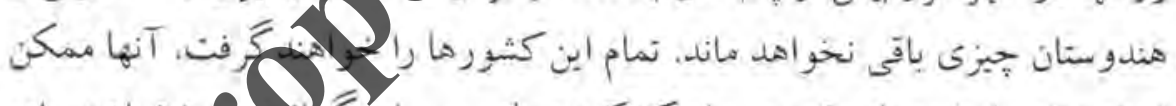

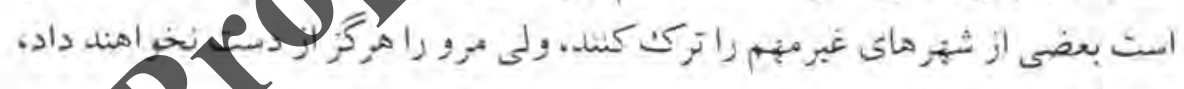
ور آنجا مسنقر خواهند شدي.

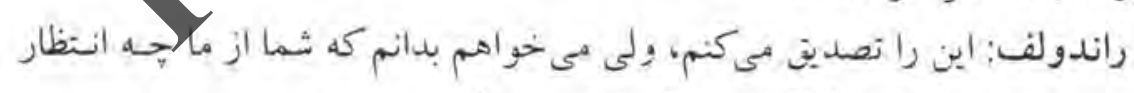

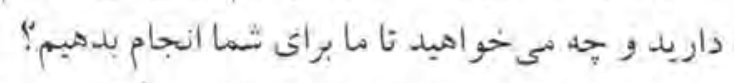

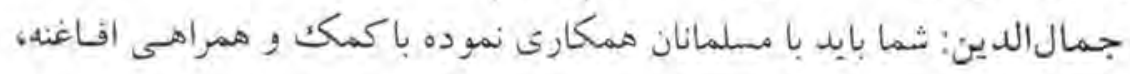

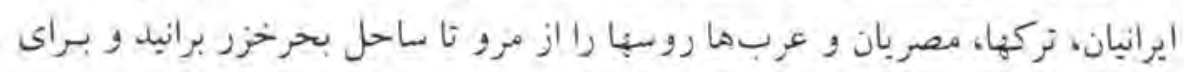

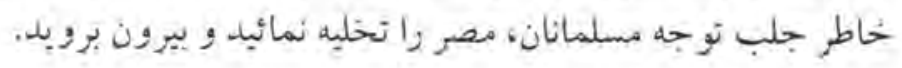




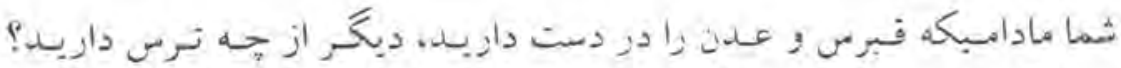

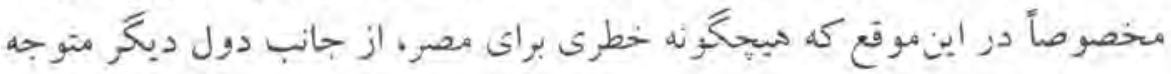
نمى باثد.

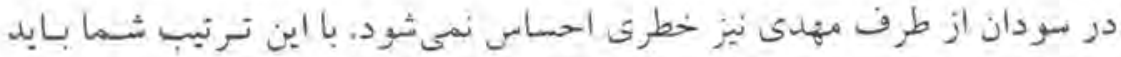

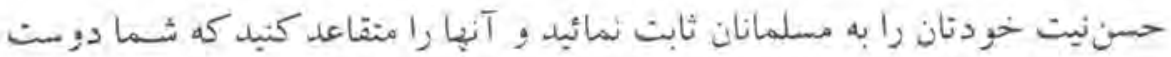

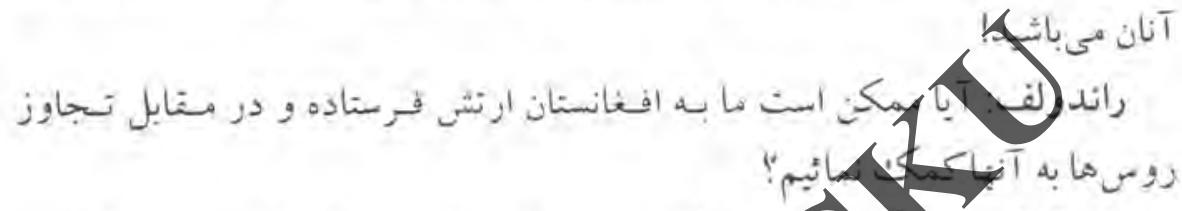

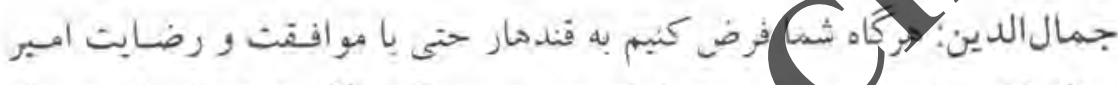

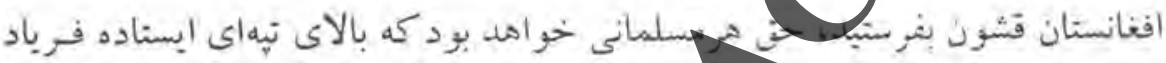

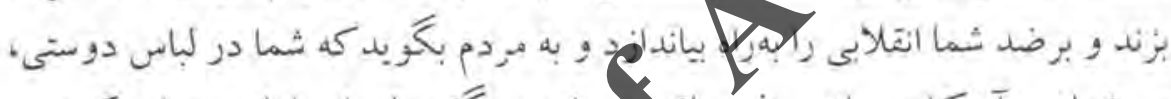

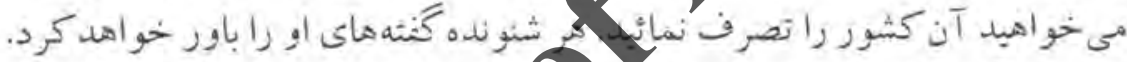

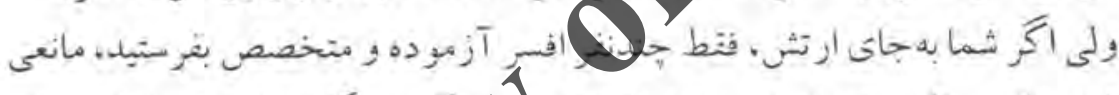

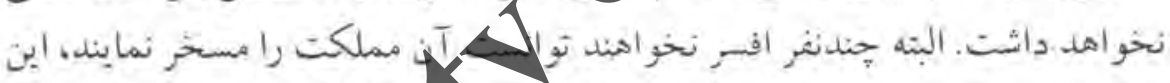

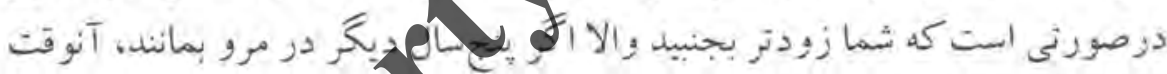
$8>$ دير خواهد شدل

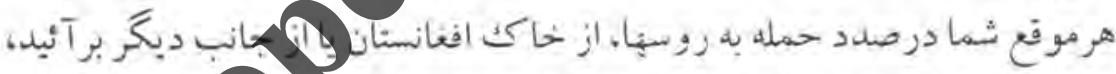

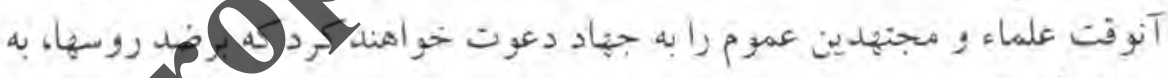

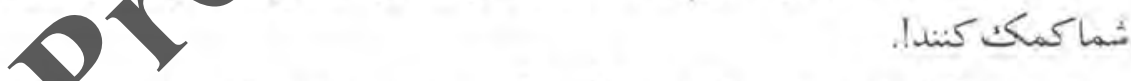

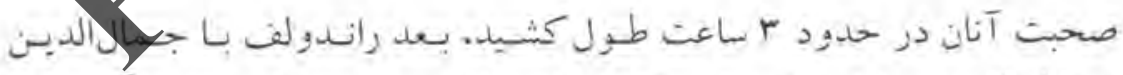

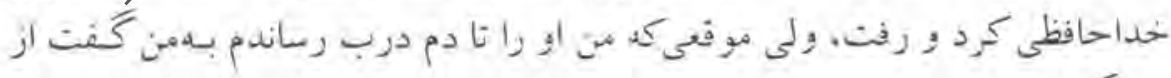

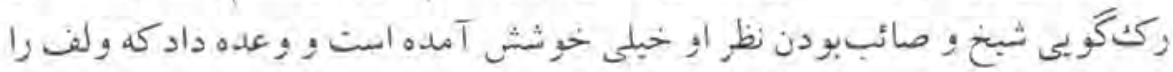

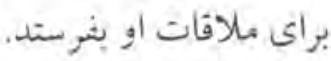




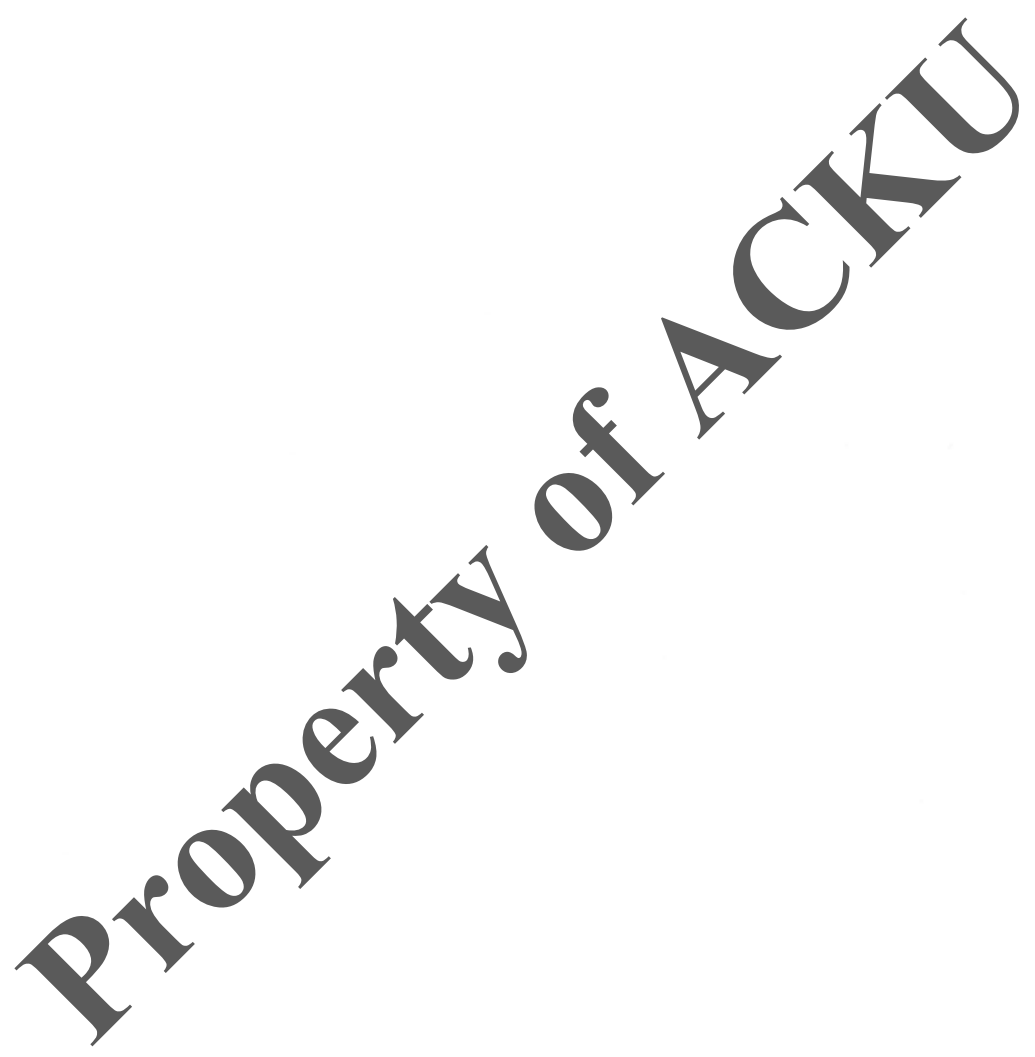




\section{$\Delta$}

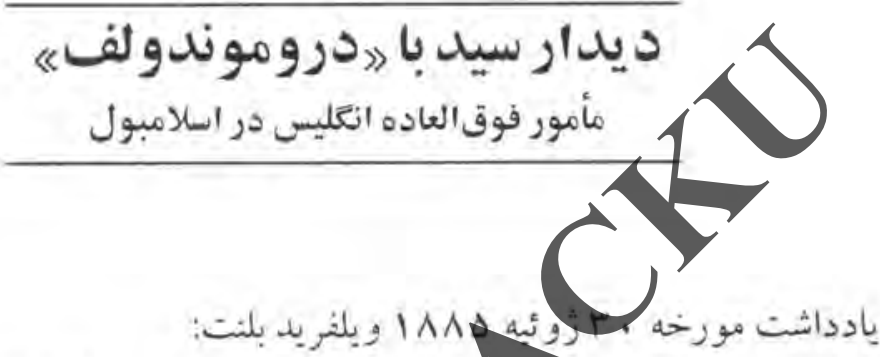

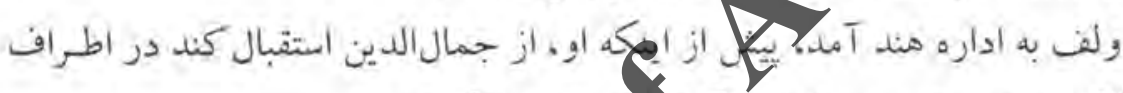

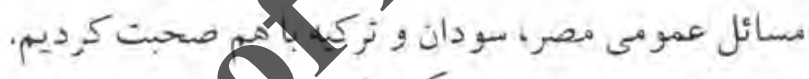

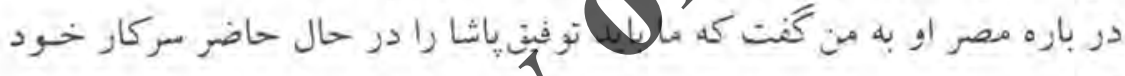

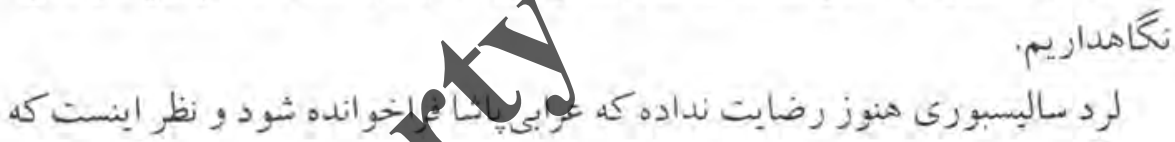

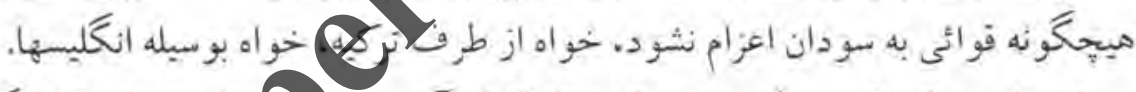

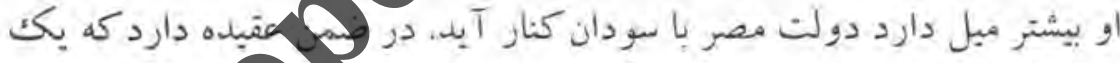

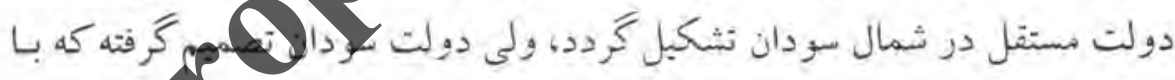
سلطان عثمانى روابط تازهائ برقرار ثمايد.

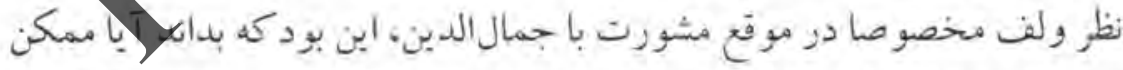

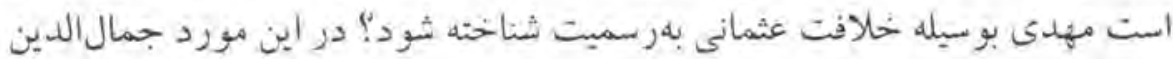

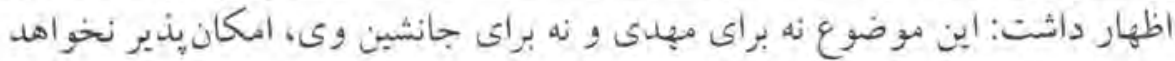

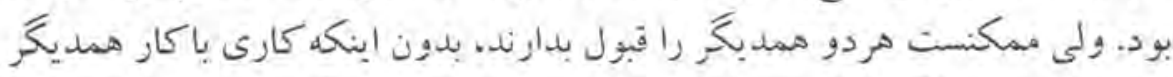

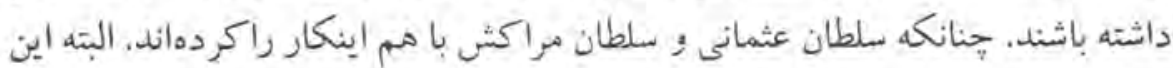

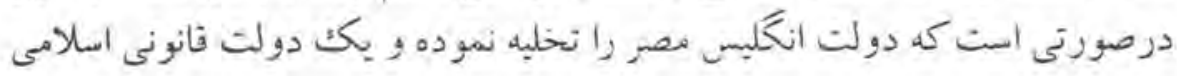




$$
\text { جاى آنرابخيرد. }
$$

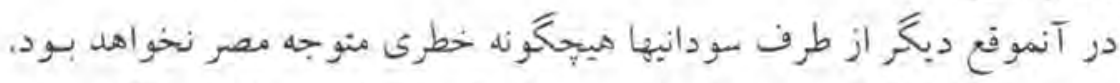

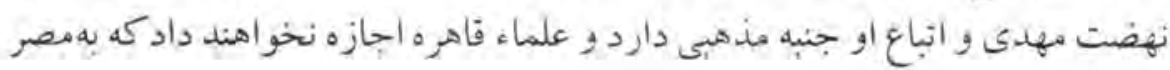
حمله نمايند.

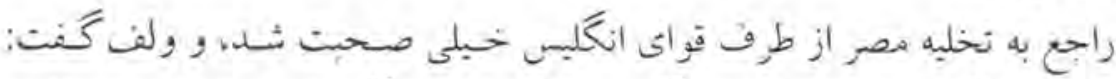

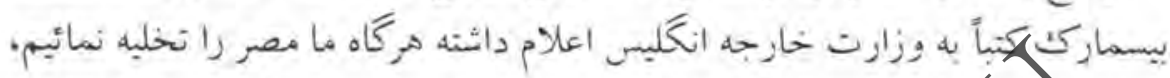

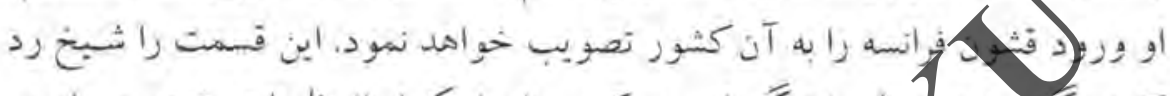

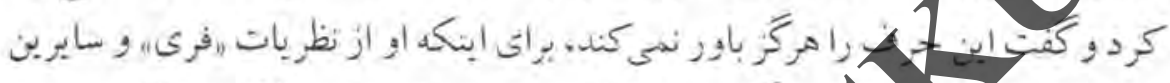

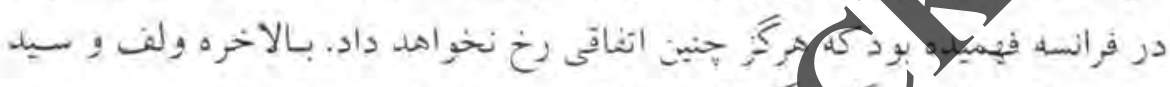

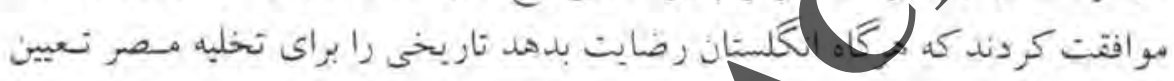

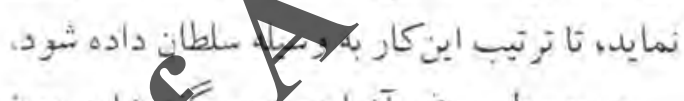

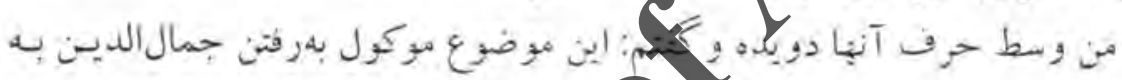

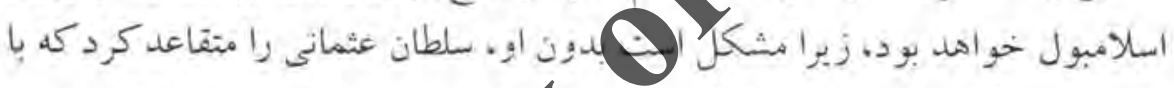

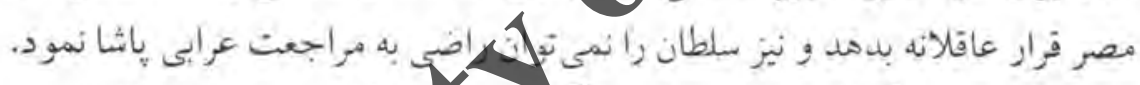

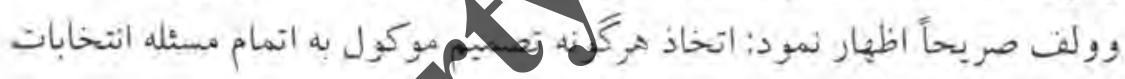

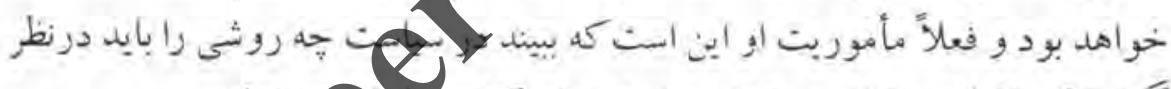

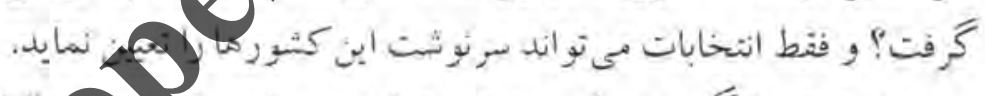

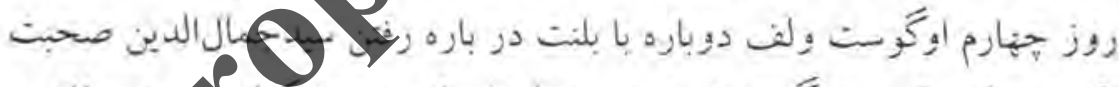

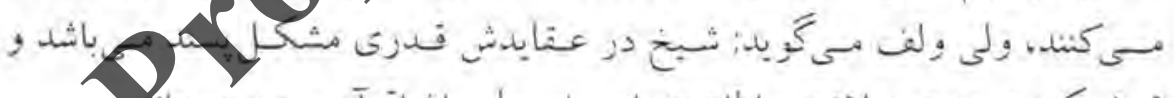

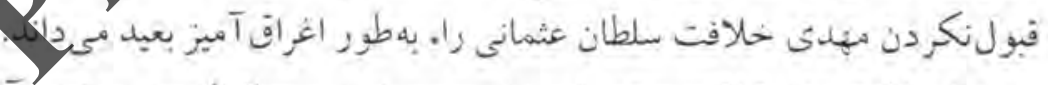

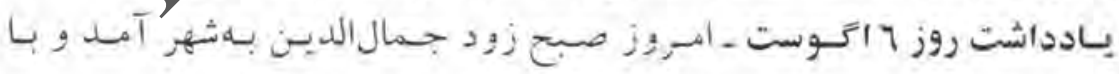

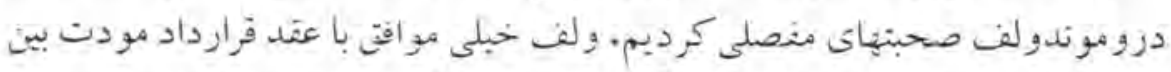

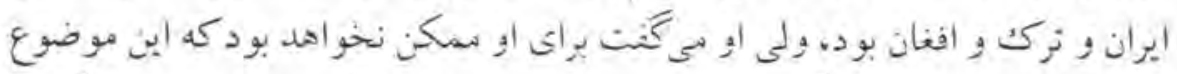

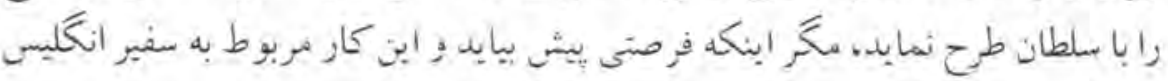
مي باثشا. 


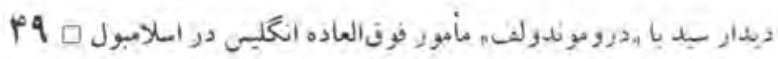

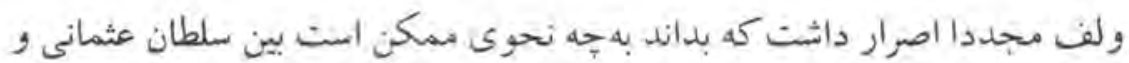

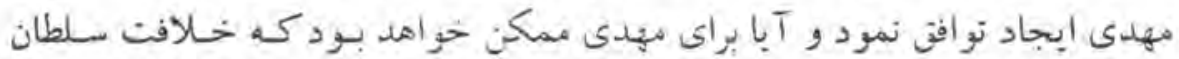
عثماتى را قبول نمايد؟ جمال الدين كَّت: جيزي راكه سلطان به آن واقعاً تو جه دارد اينست كـه تـاريخ

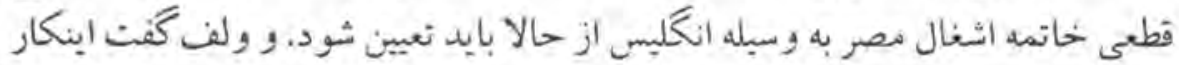

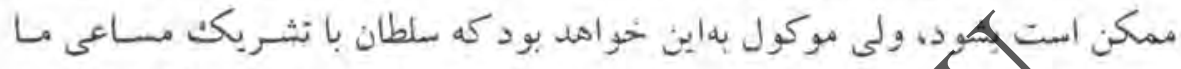

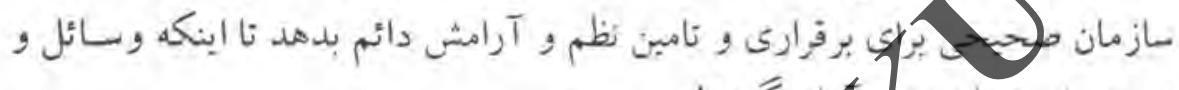

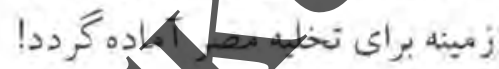

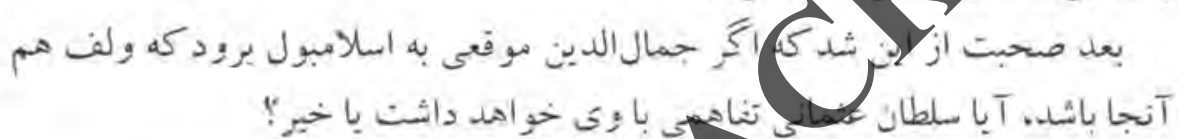

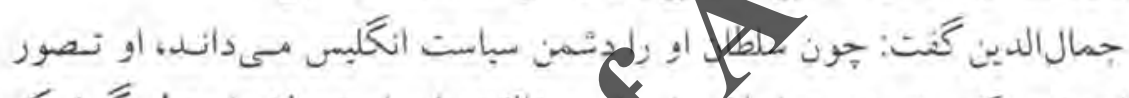

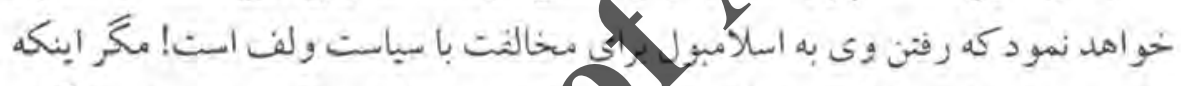

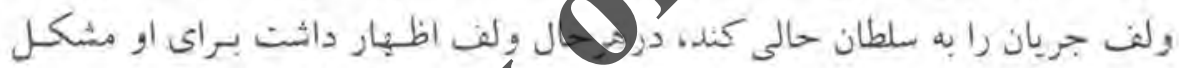

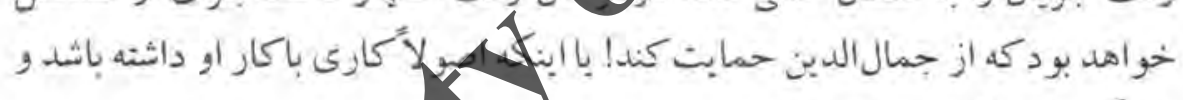

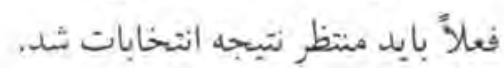

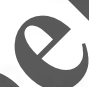

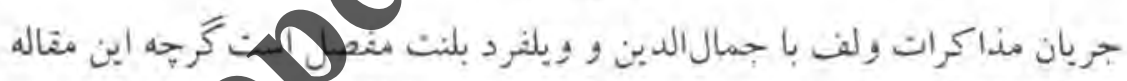

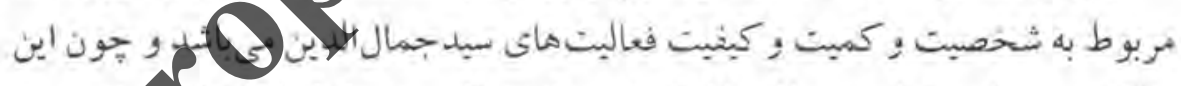

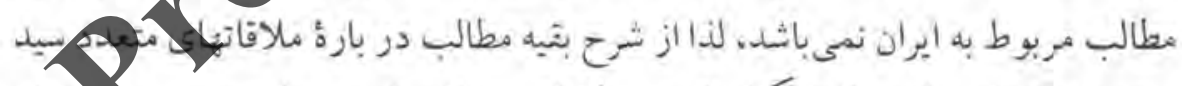

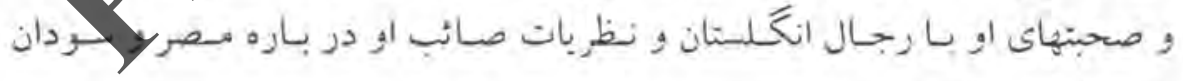
خوددارى مىشود.

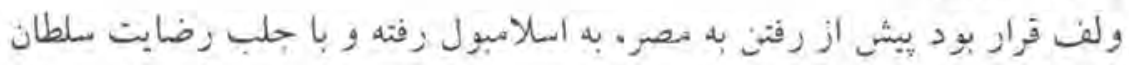

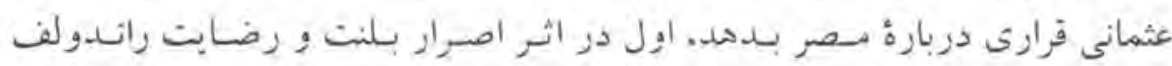

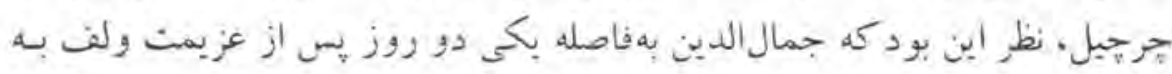

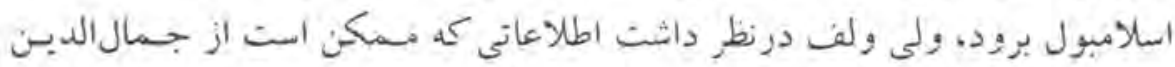


كسب نمايد تا اينكه موقع ورود بن اسلامبول اوضاع سياسى آنجا رادر دست داشته

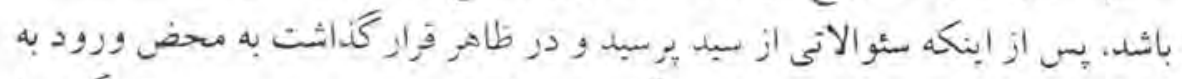

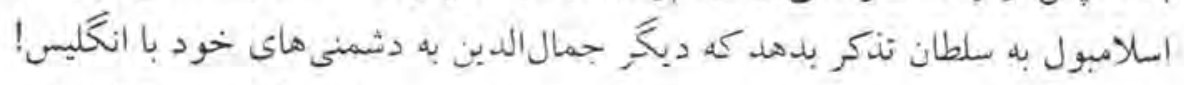

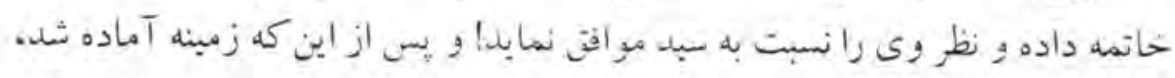

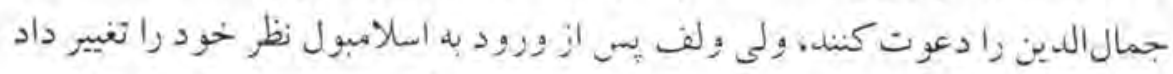

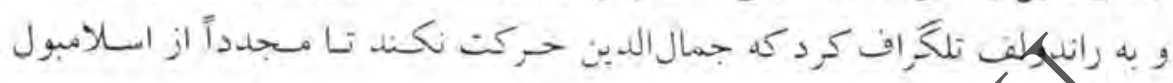

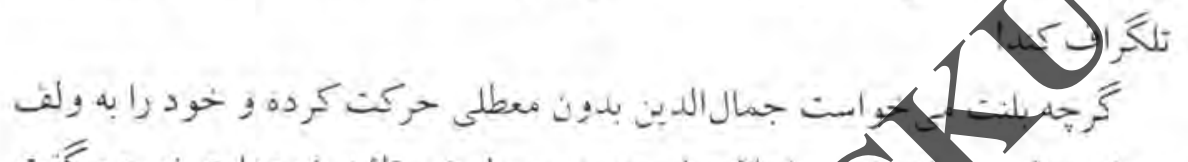

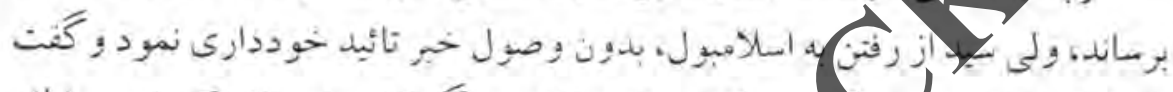

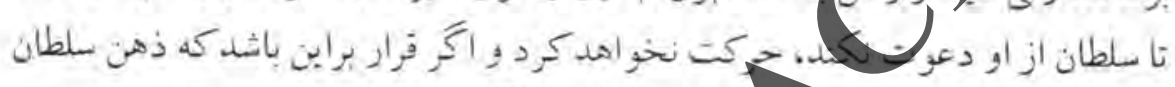

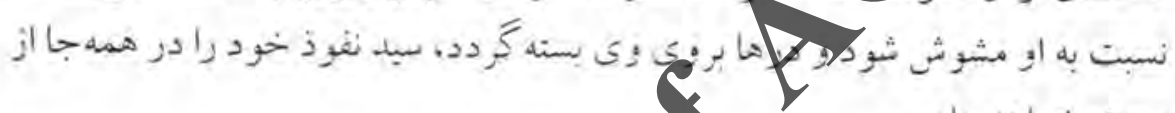
دمبت خواهد داد.

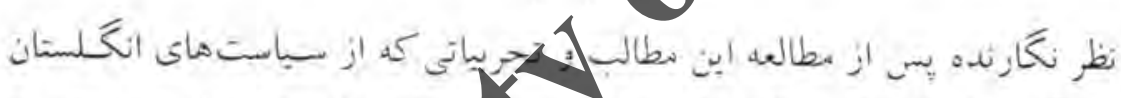

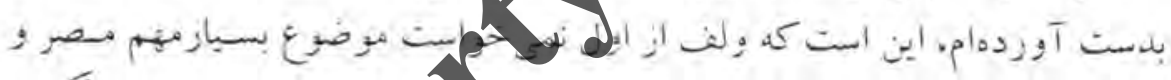

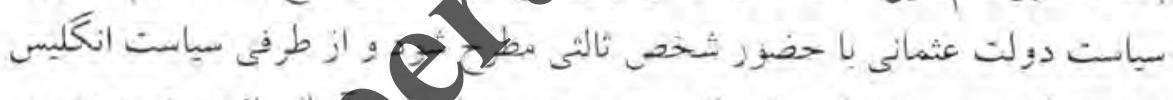

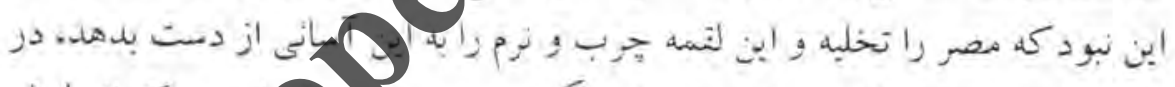

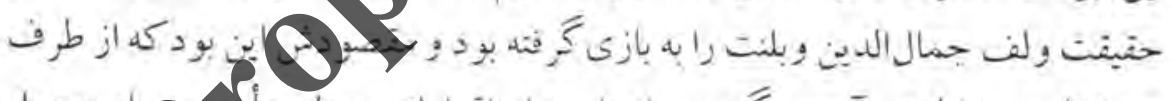

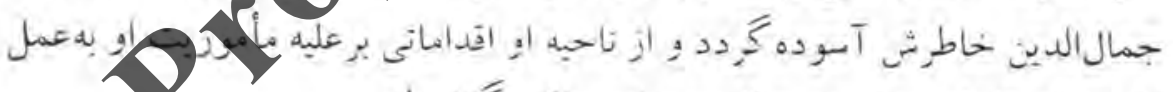

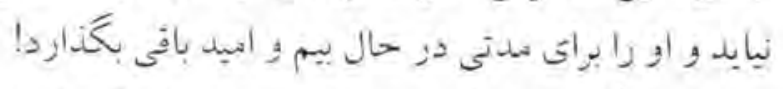

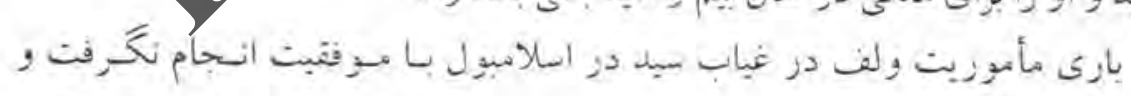

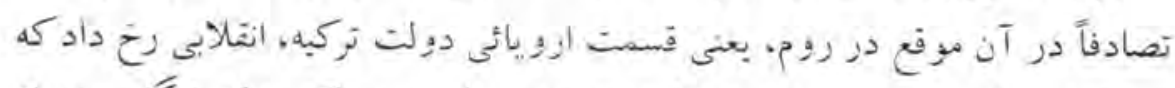

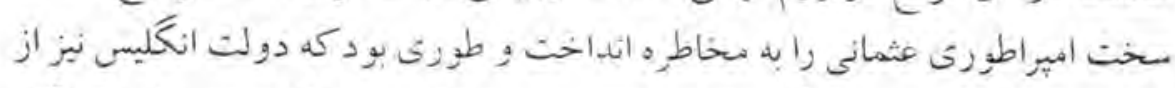

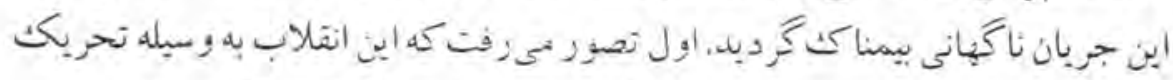

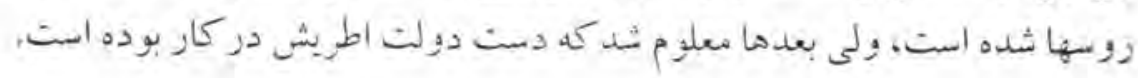




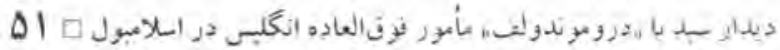

در اين موفع سلطان در اسلامبول به ياد سيد مى افتد و مبل داشت با او راجع بـه

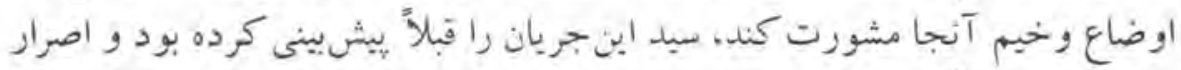

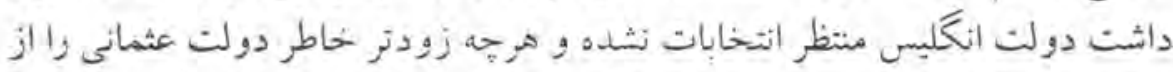

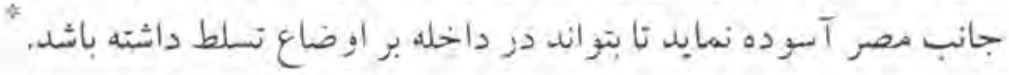

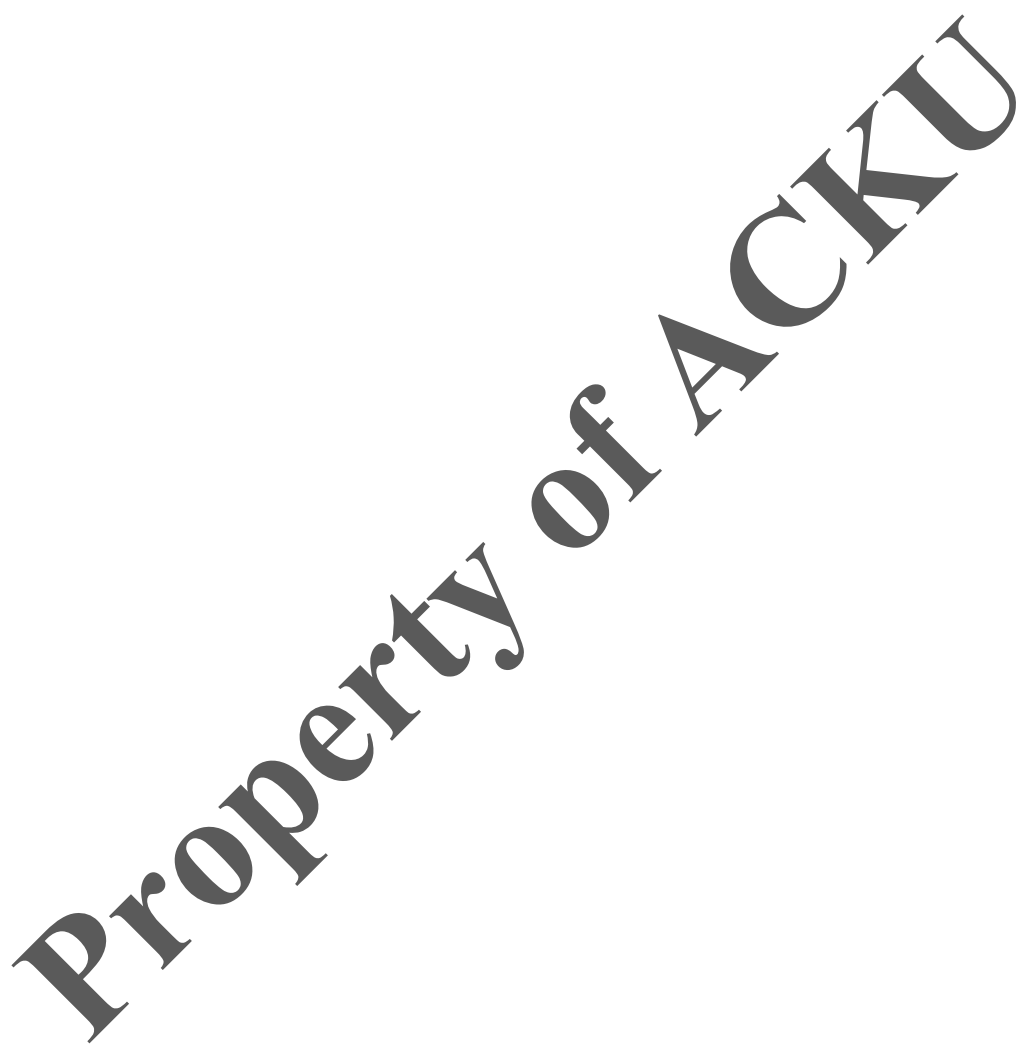

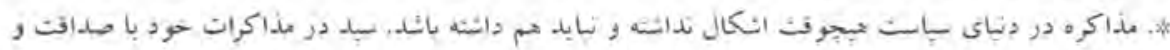

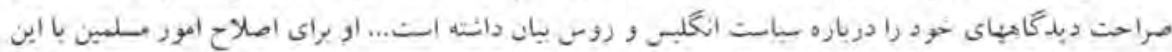

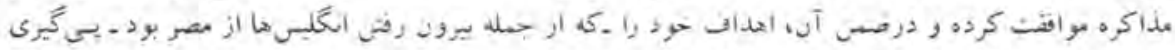

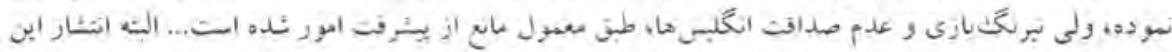

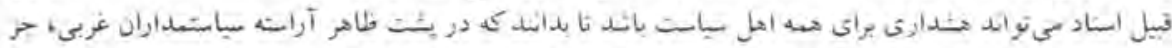

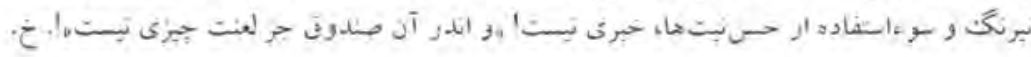




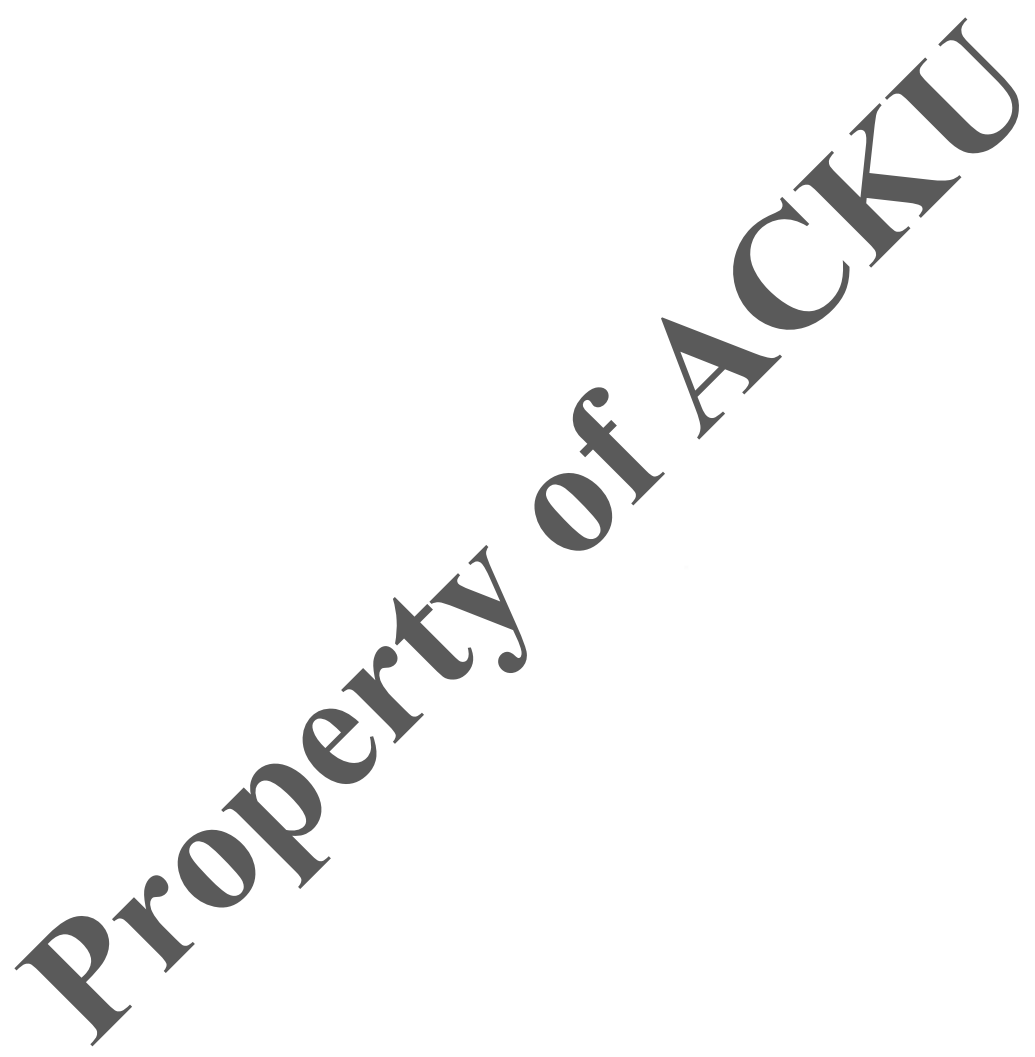




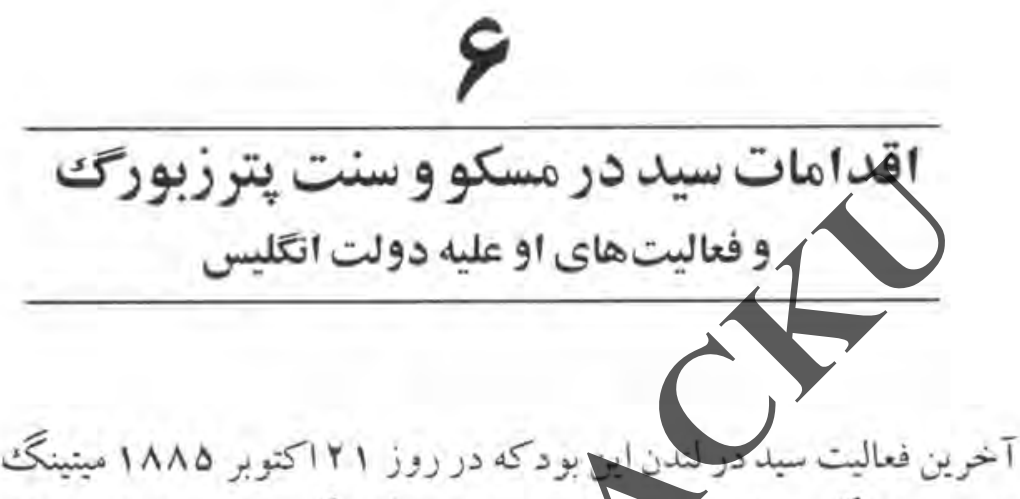

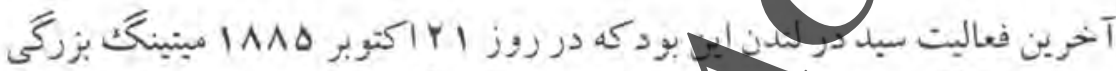

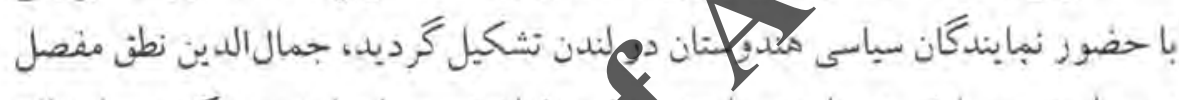

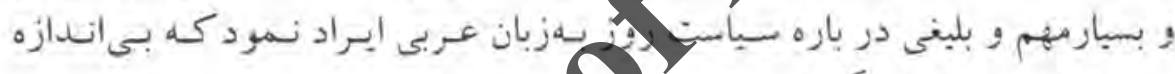

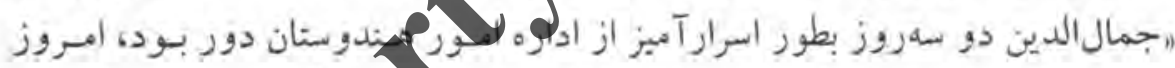

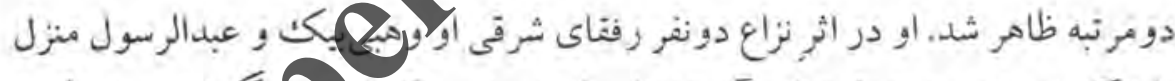

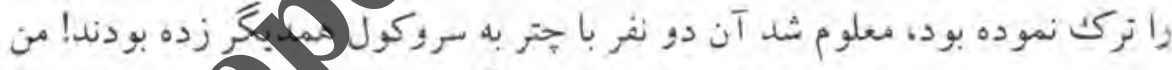

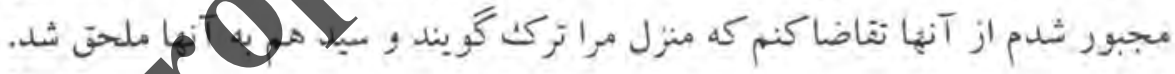

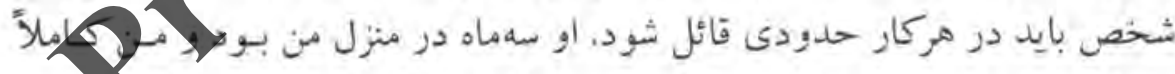

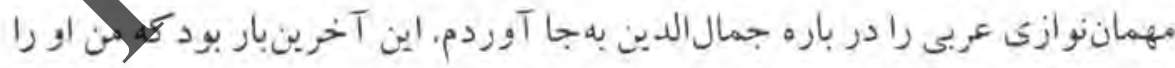

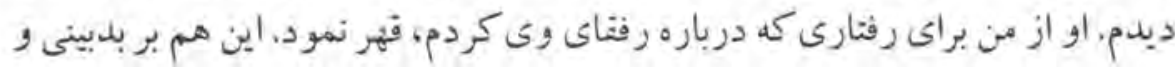

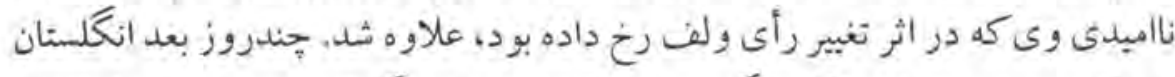

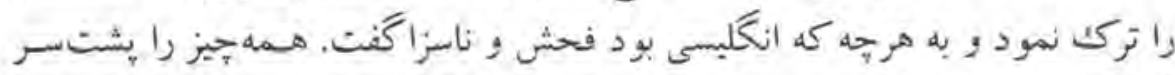

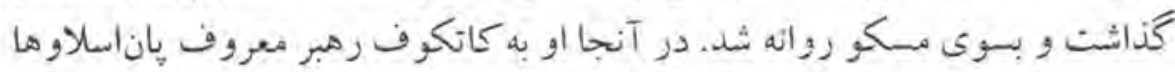

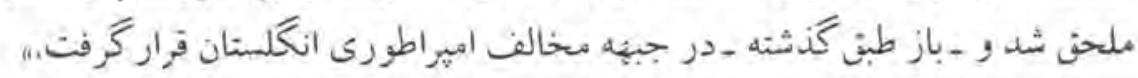




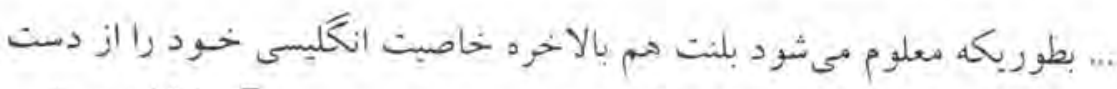

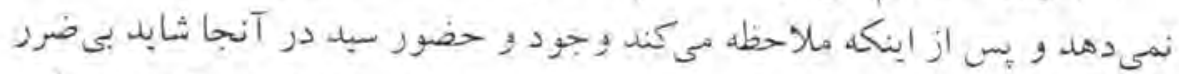

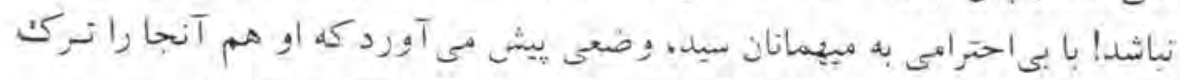

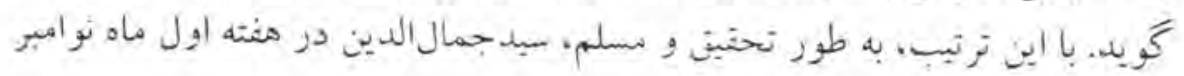

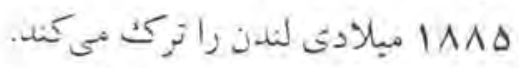

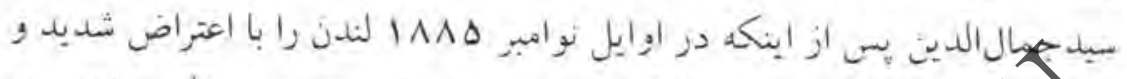

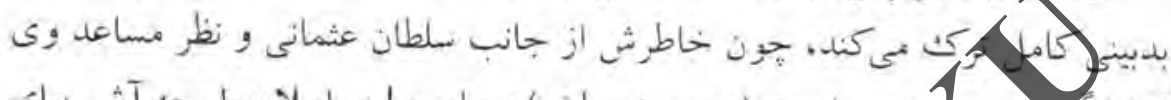

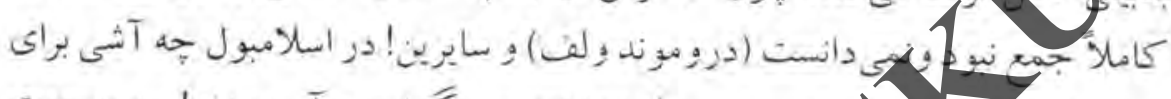

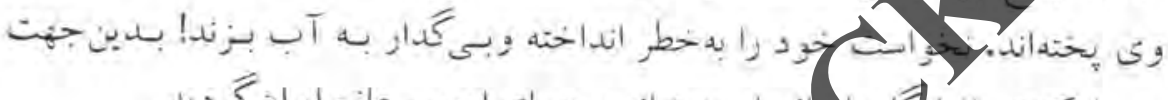

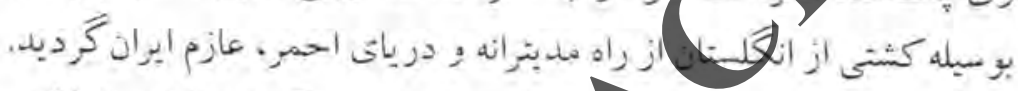

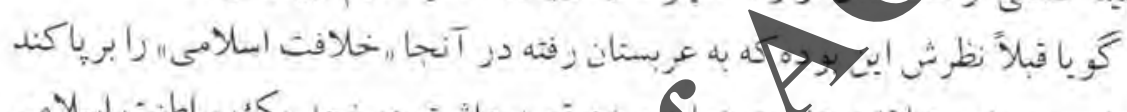

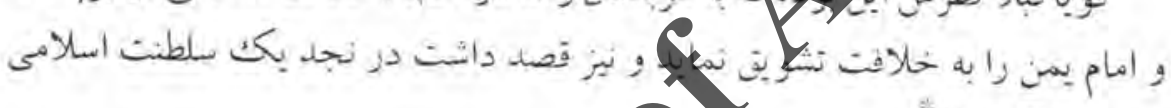

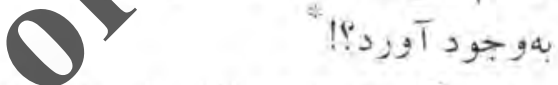

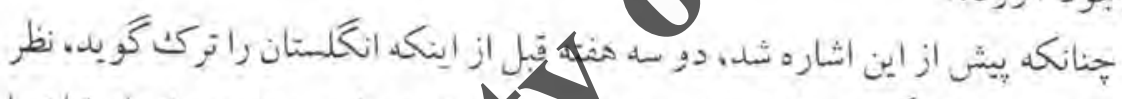

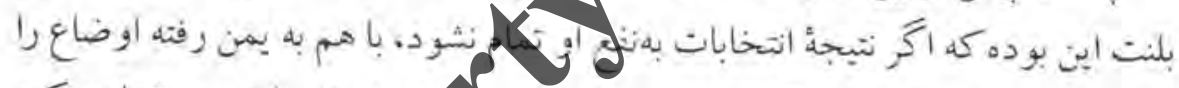

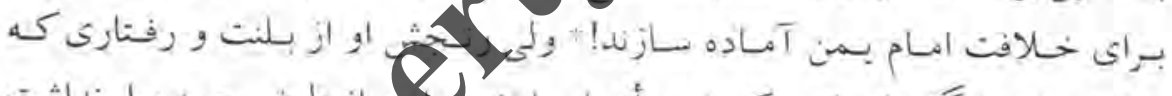

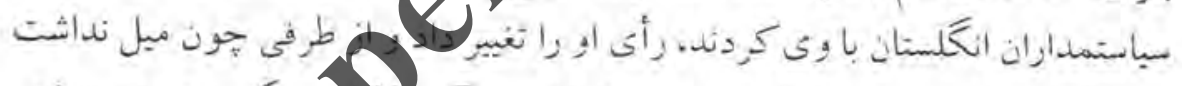

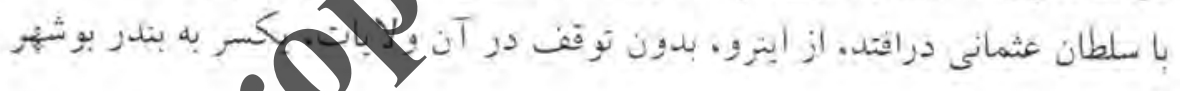
T. Tد

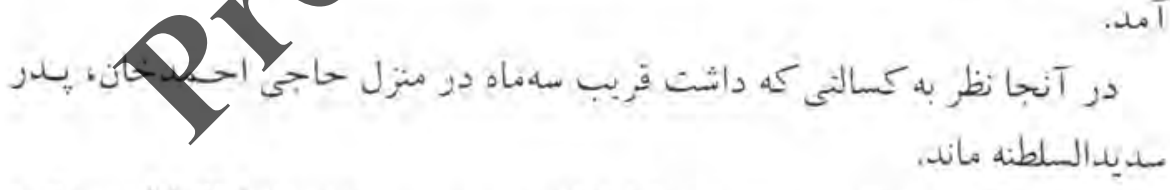

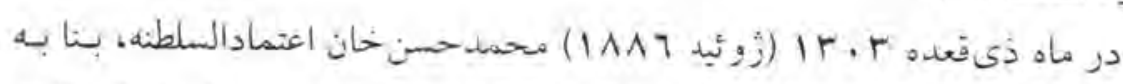

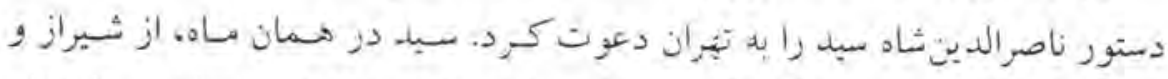

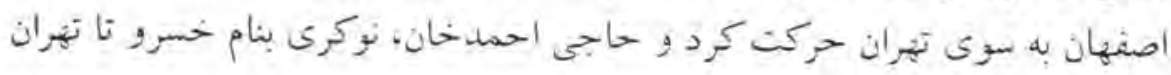




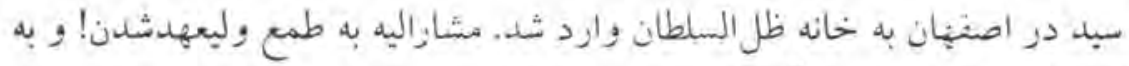

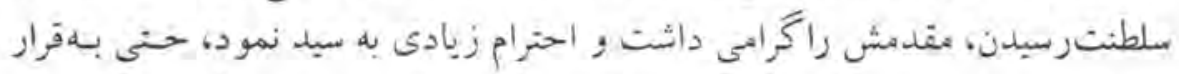

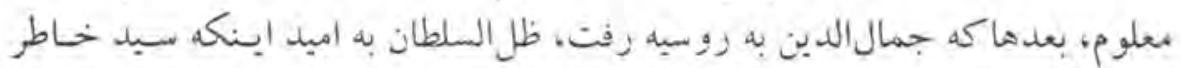

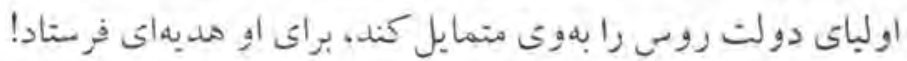

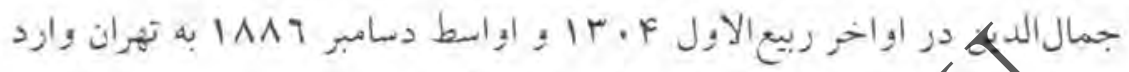

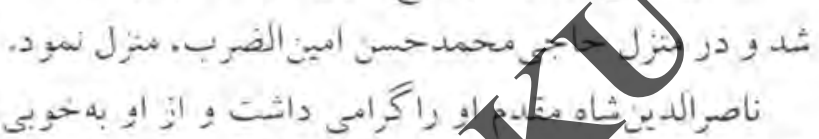

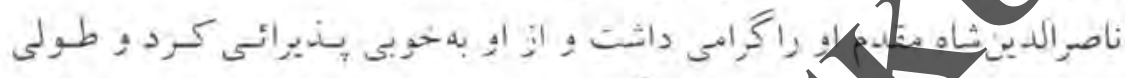

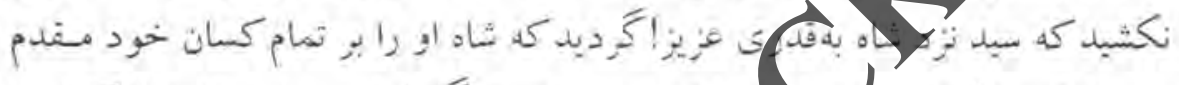

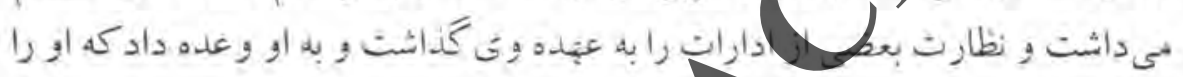

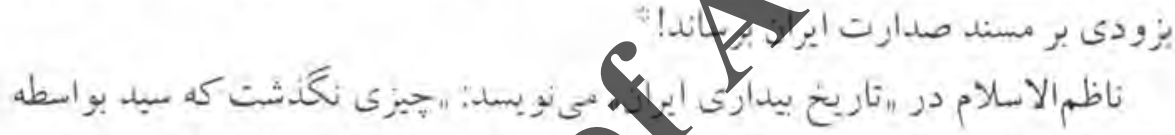

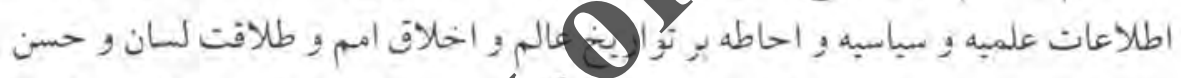

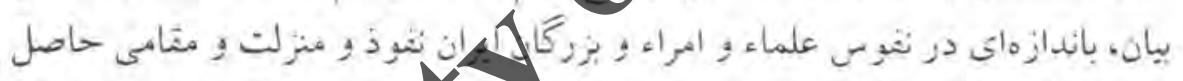

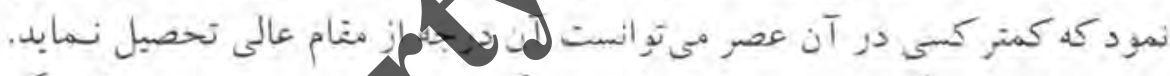

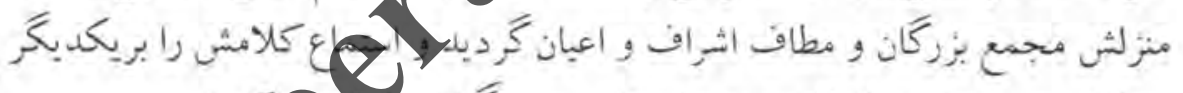

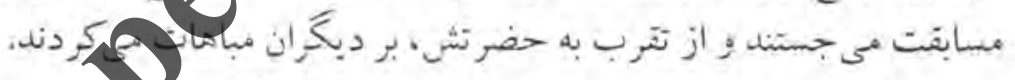

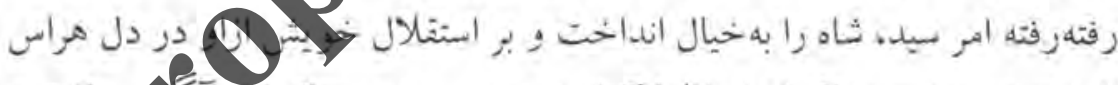

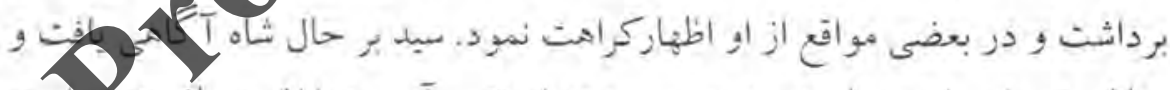

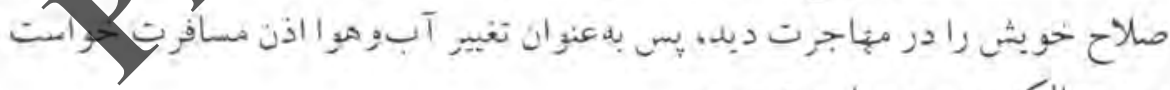

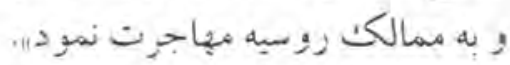

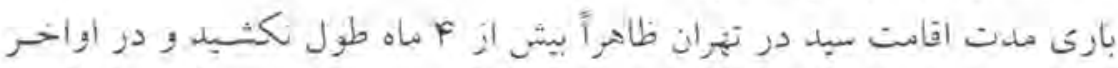

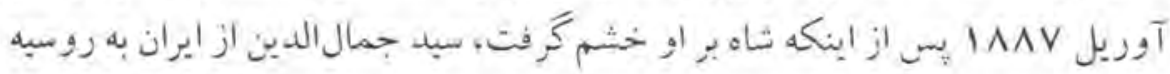

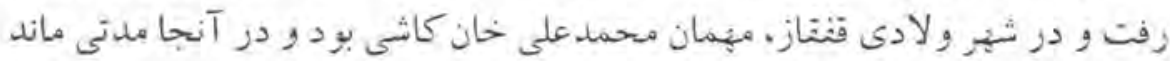


تا امين الضرب نيز از نهران رسبد و به اتفاق هم به مسكو رفتئن و در آنجا دو هفته در

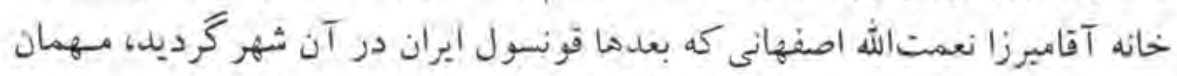

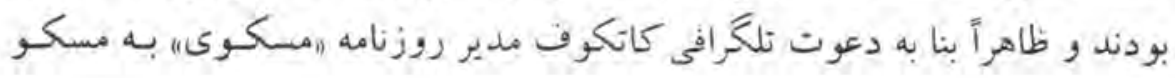
رفت...

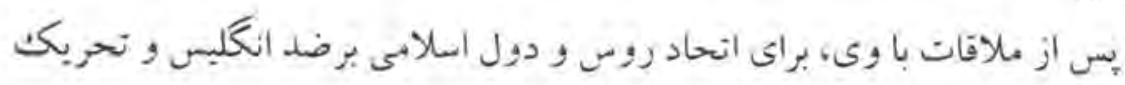

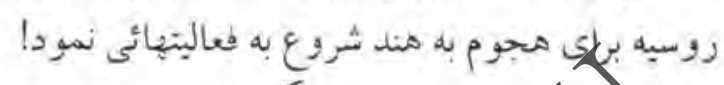

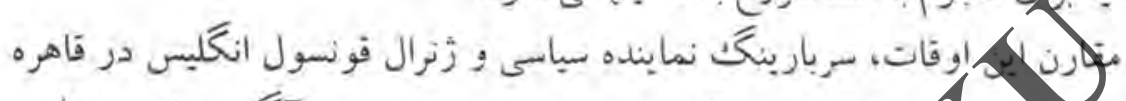

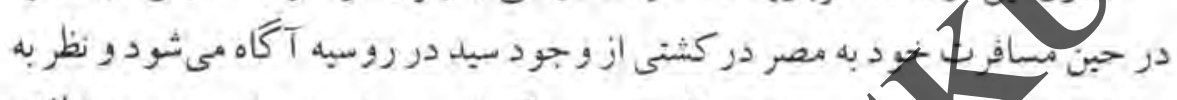

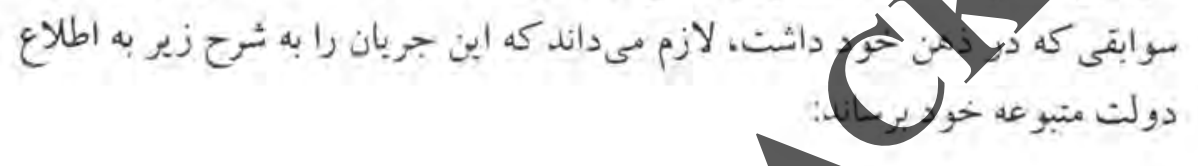

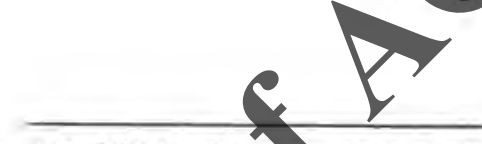

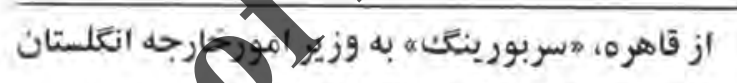

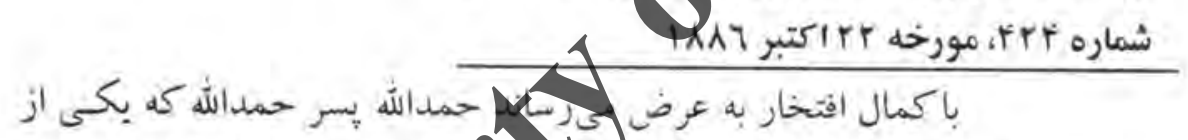

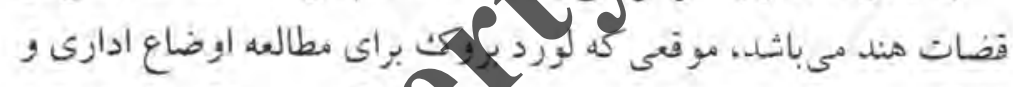

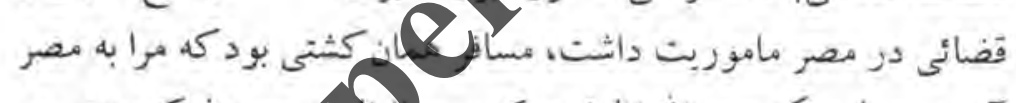

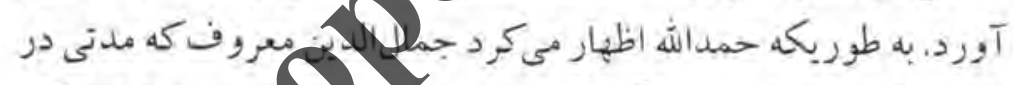

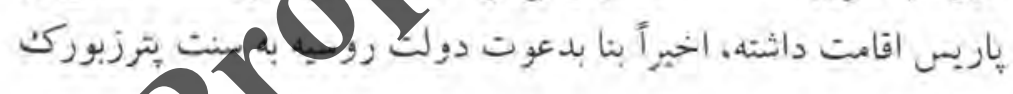
رفته است.

در اين جندسال اخير، دشتمنى هاى جمال الدين برعلية الوكليسها بيثي

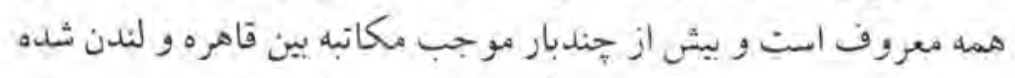

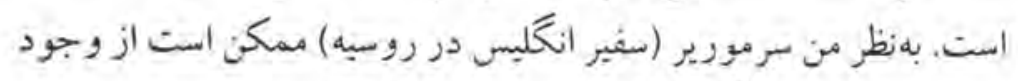
او در ستخ يترزبورك اطلاعات بيشترى داشنه باشد.

امضاء بارينك 
وزارت امور خارجه يس الز وصول نامه مزبور، با ارسال رونوشت آن، ضمن نامه

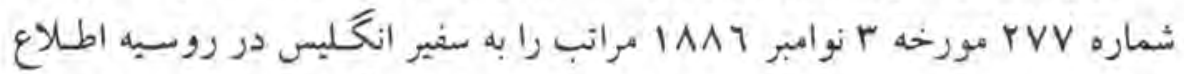

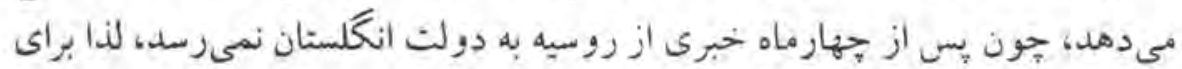

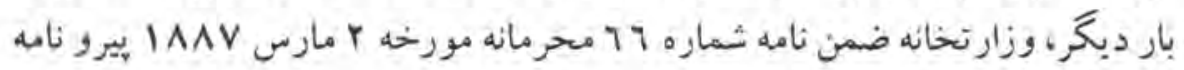

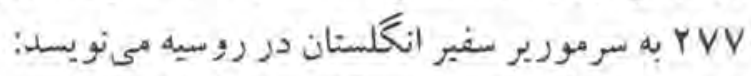

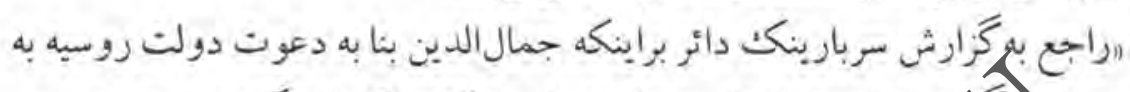

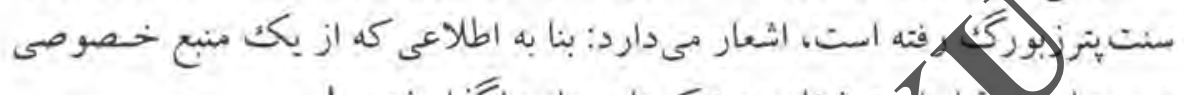

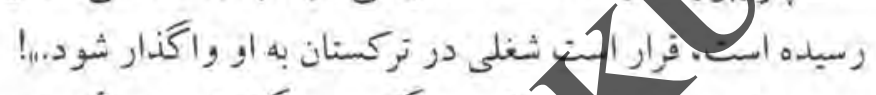

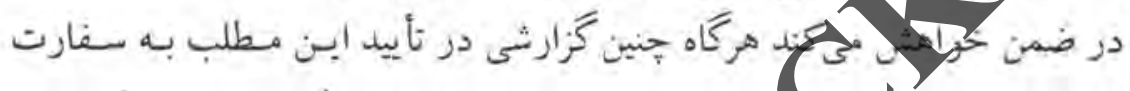

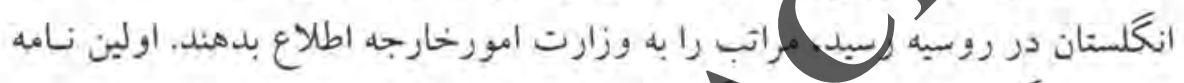

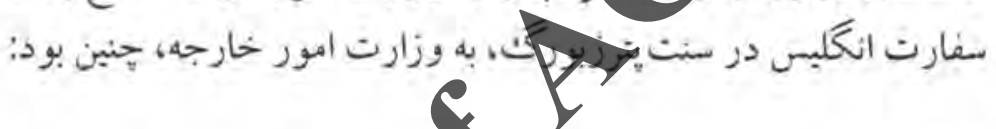

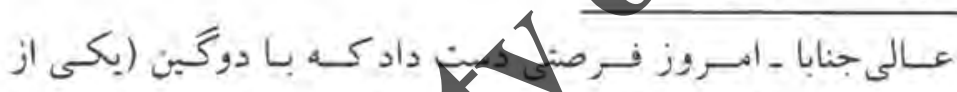

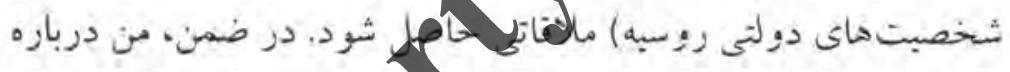

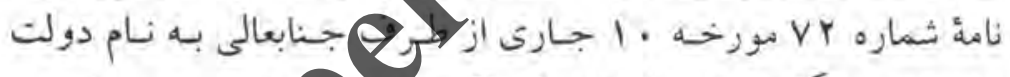

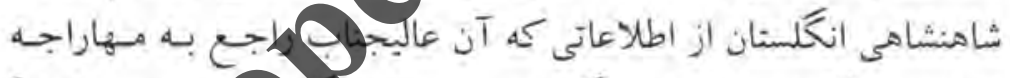

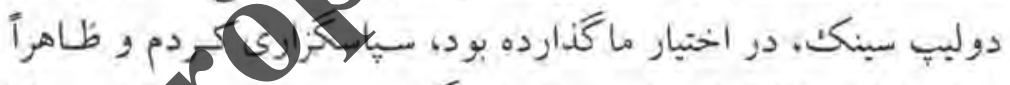

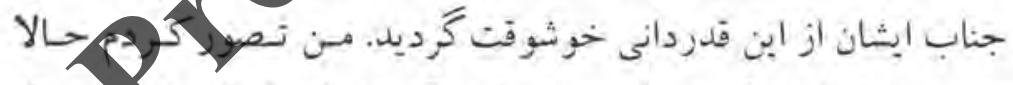

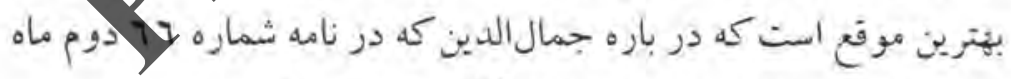

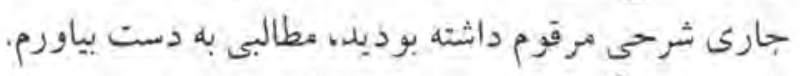

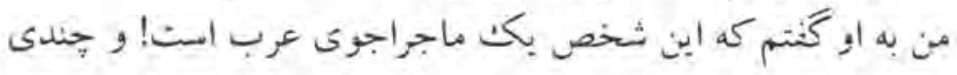

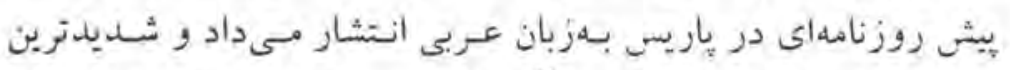

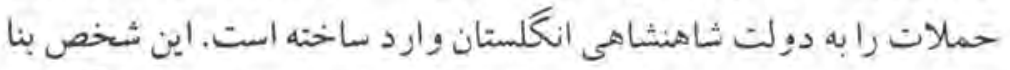
به أقرار خودش با مئدى (شخصى كمه در سودان برعليه مصر و انكلستان 
قيام تمود) و بعضى از رهبران اسلالمى، روابطى داشته و ثابت شده كه تمام

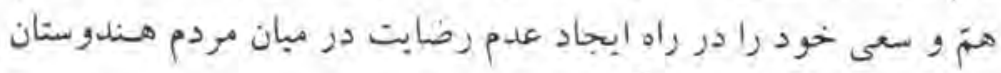

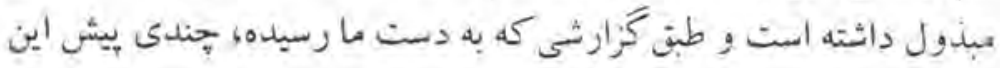

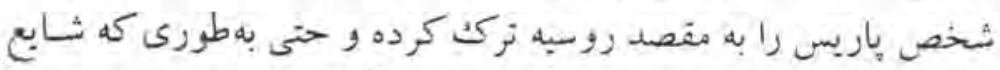

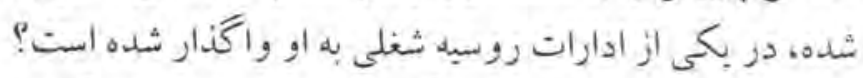

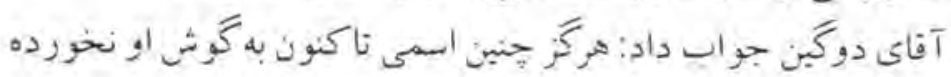

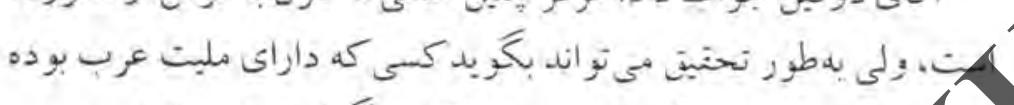

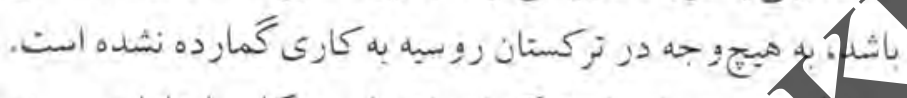

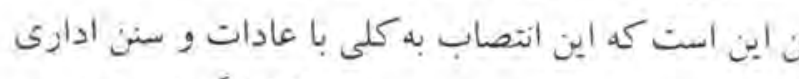

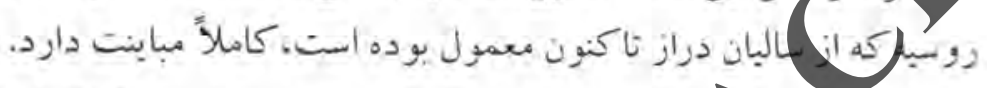
المضاء موريو

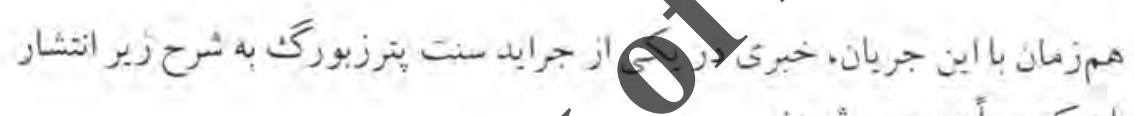

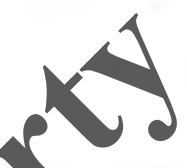
مىيابد كم عيناً دزج مئشود:

\section{شيخج جمال الدين در مسكو}

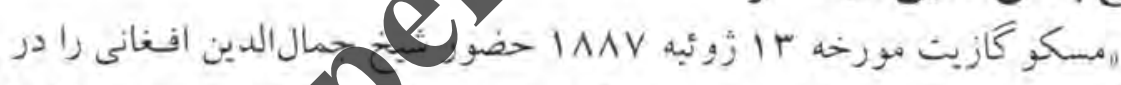

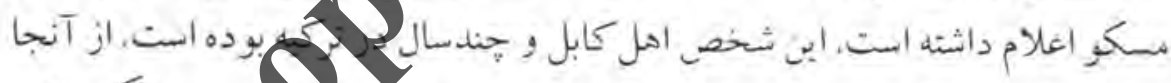

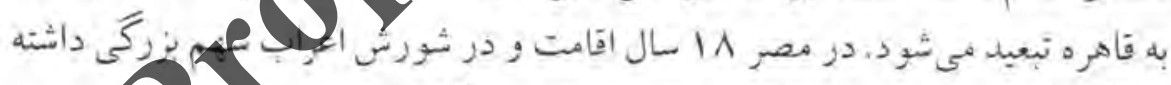

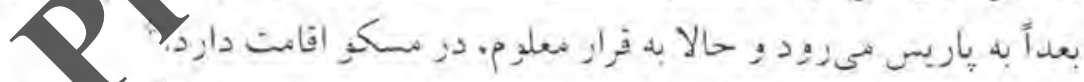

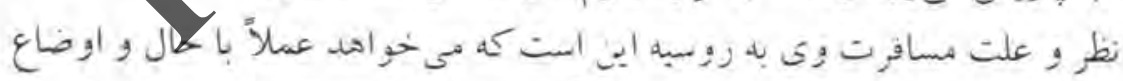

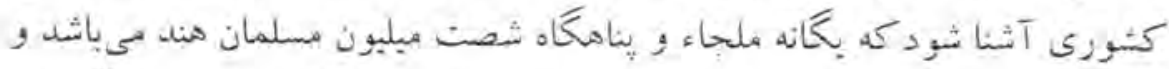

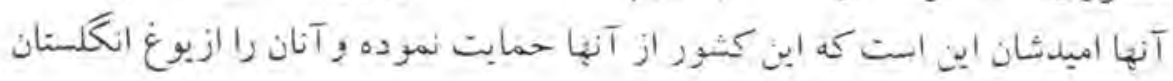

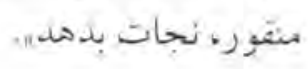




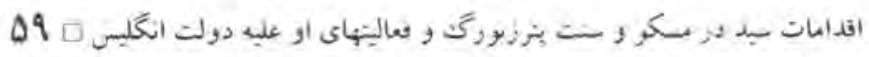

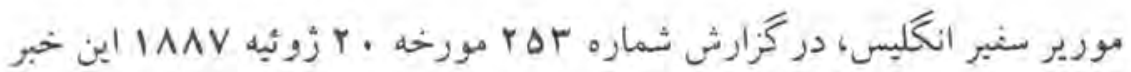

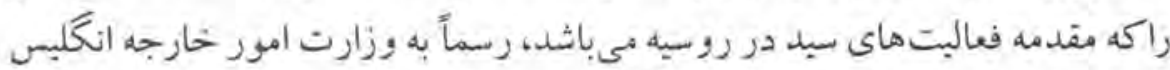

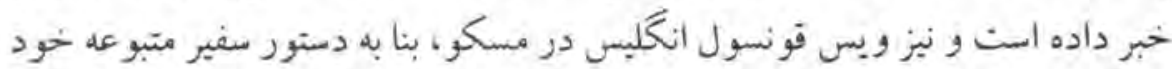

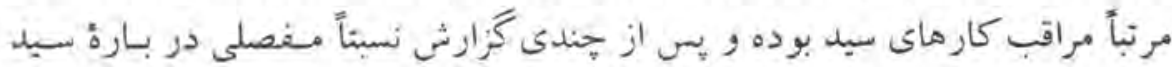

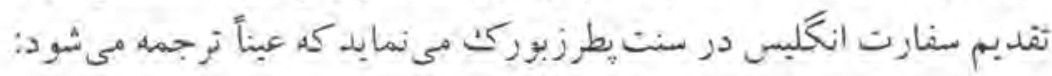

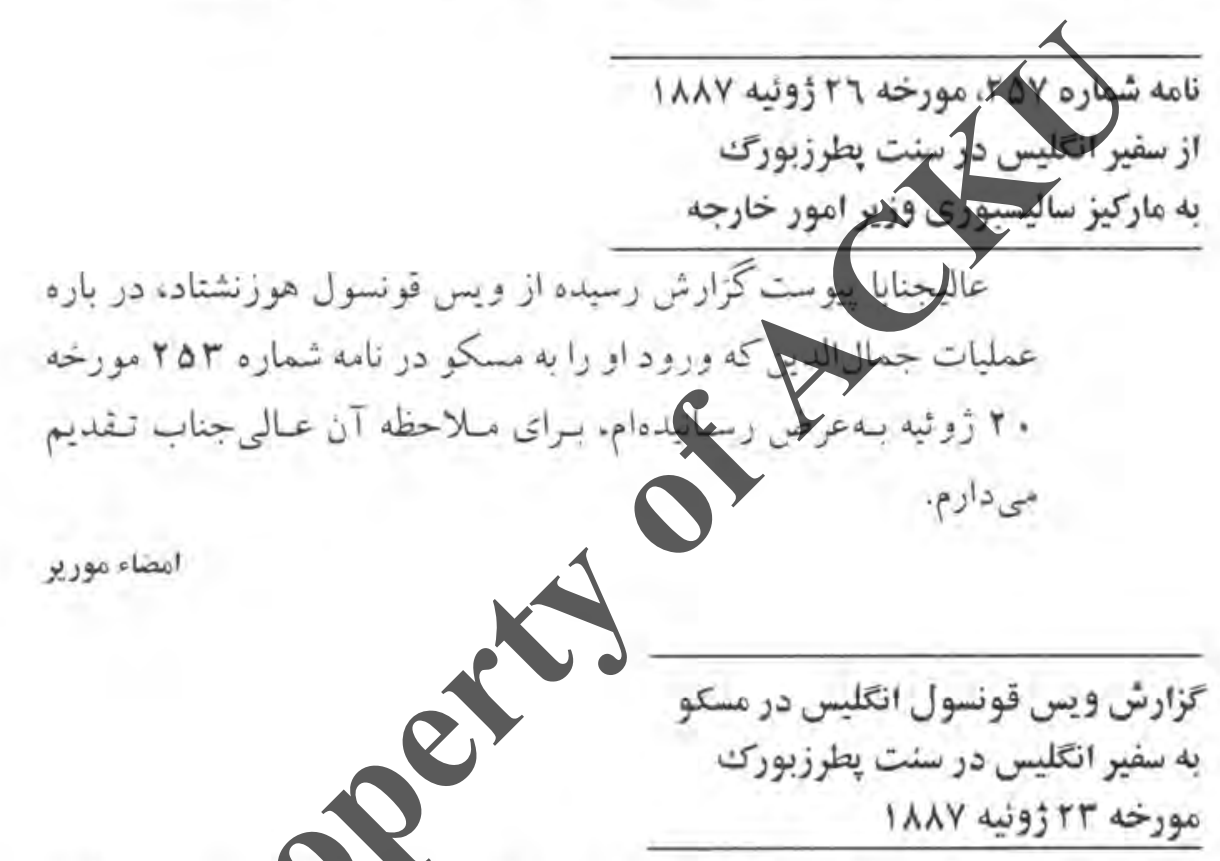

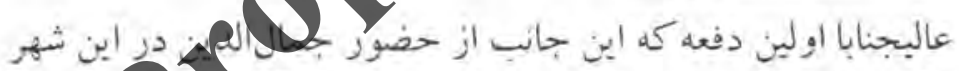

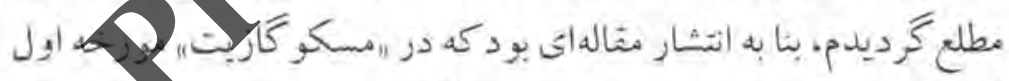

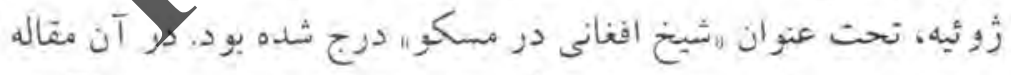

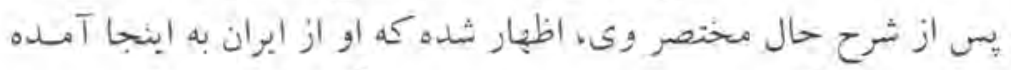

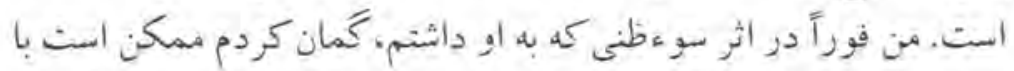

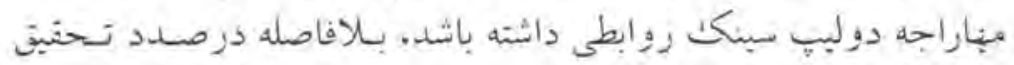

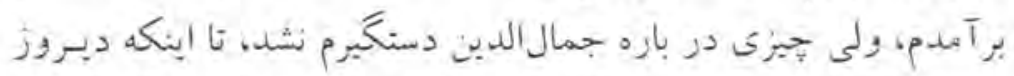

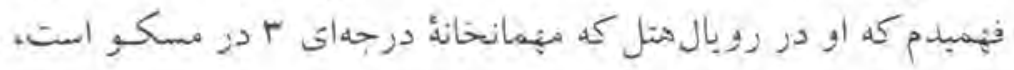




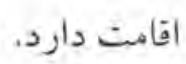

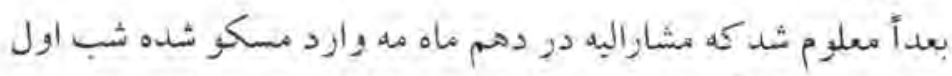

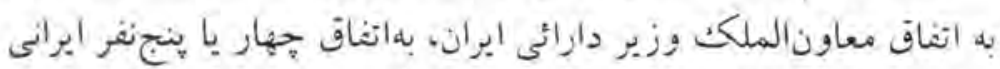

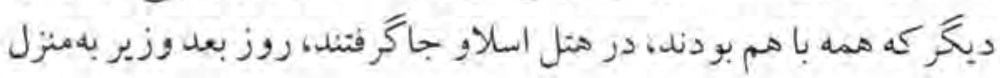

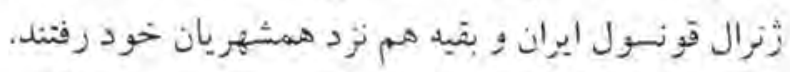

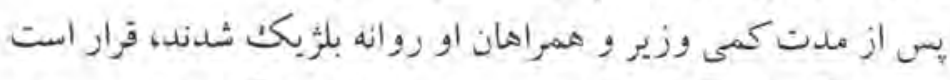

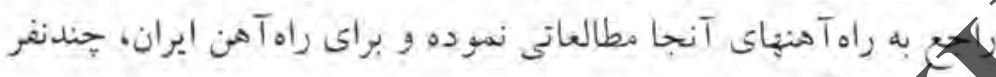

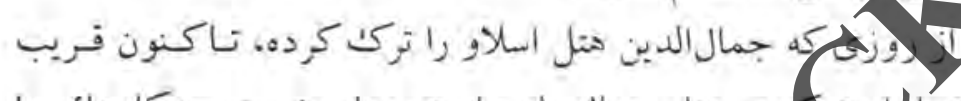

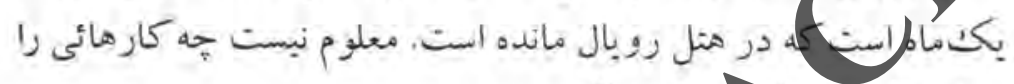

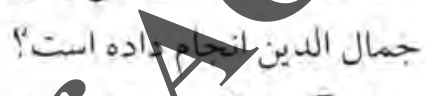

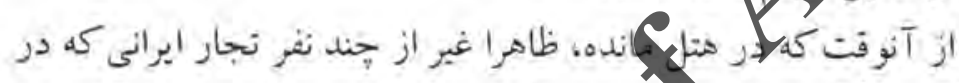

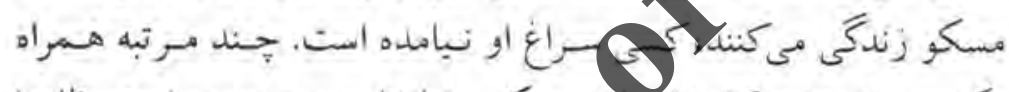

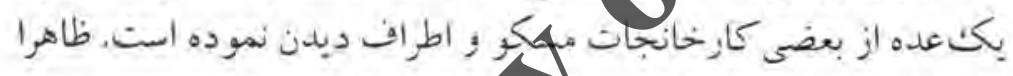

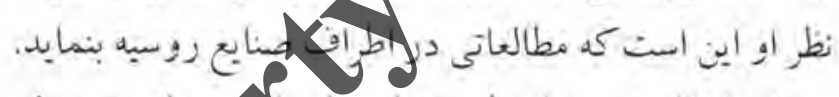

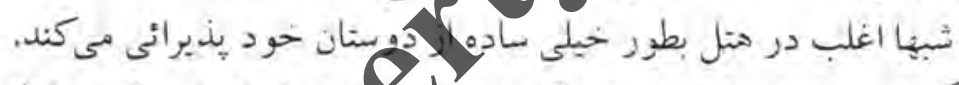

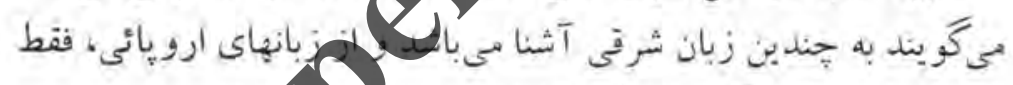

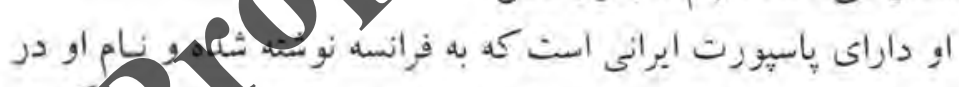

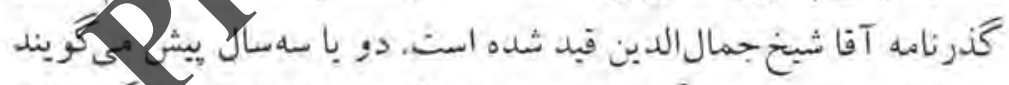

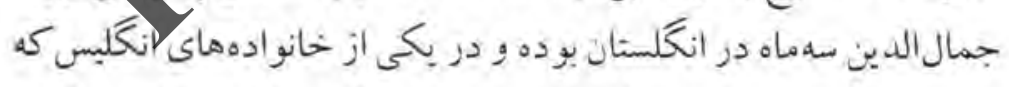

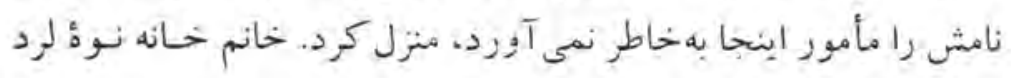

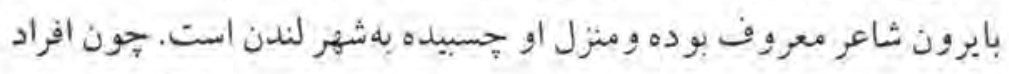

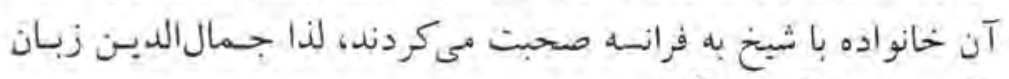

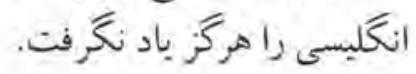




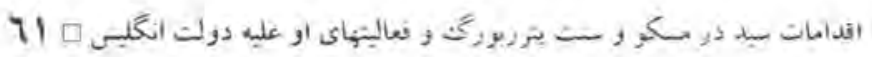

از آنجائيكه درليب سينك در بكت خانه يبلاقى نز ديك منسكو اقامت

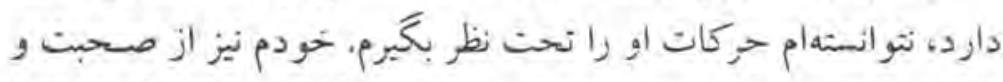

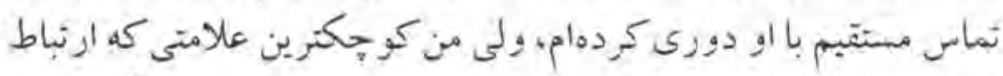

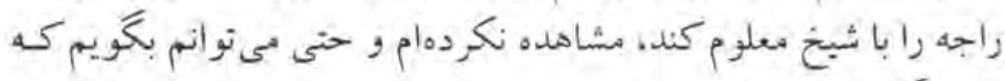

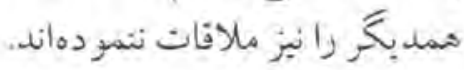

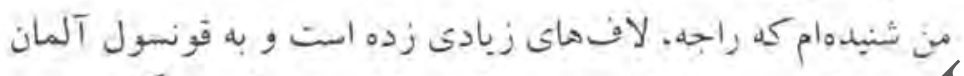

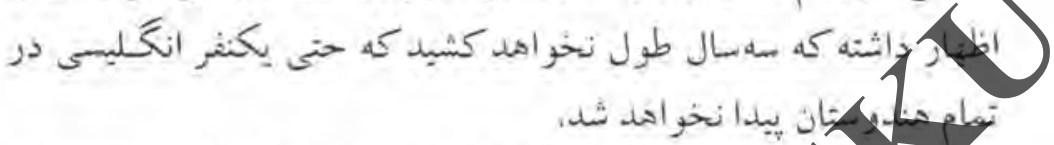

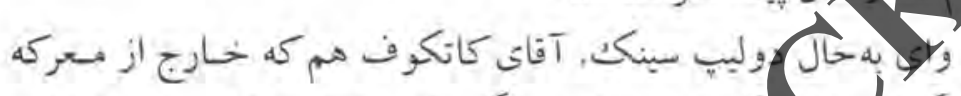

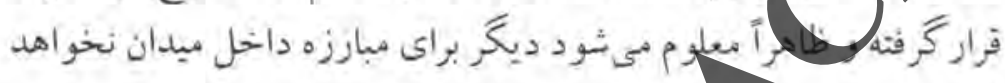

امضاء هورن استتاد

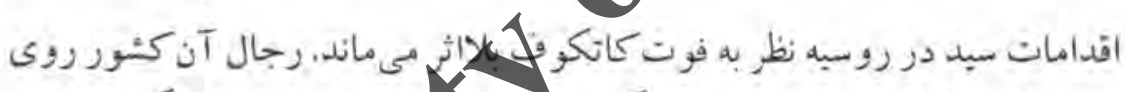

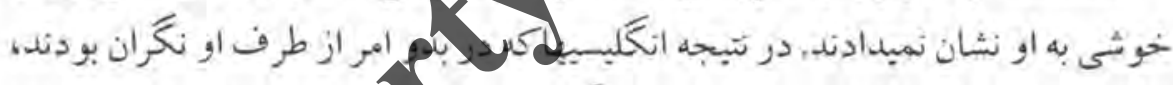

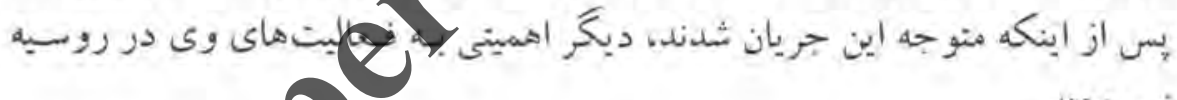

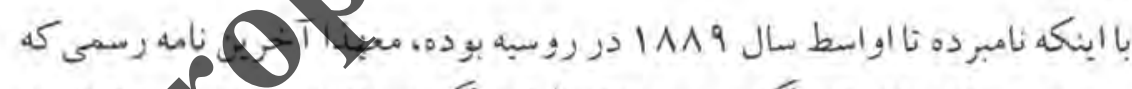

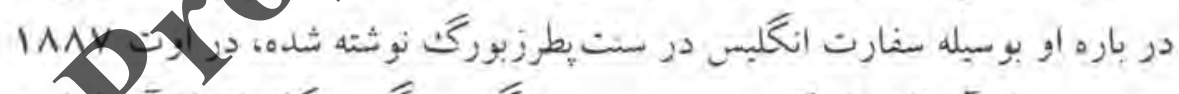

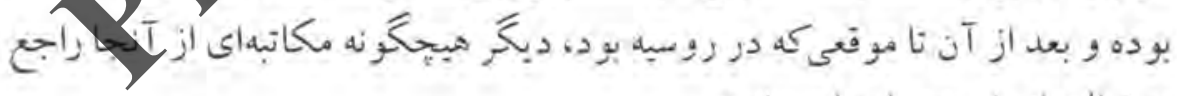

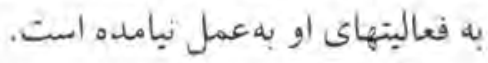

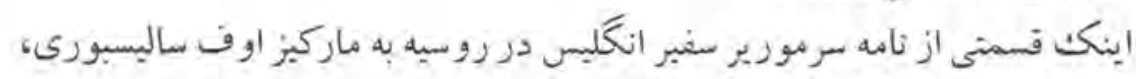
وزير امور خارجه انكلمنتان: 


\section{شAY- شماره}

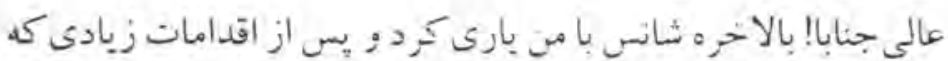

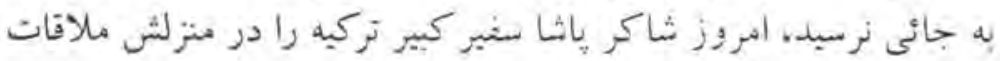

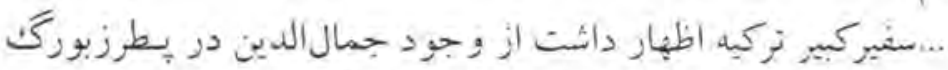

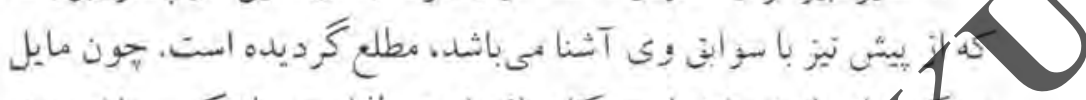

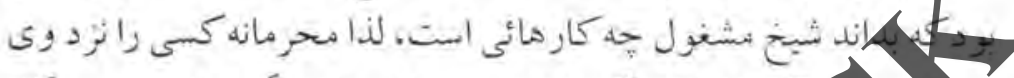

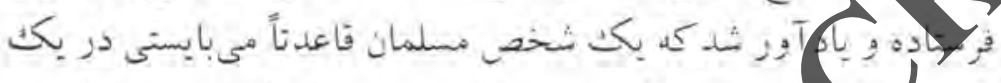

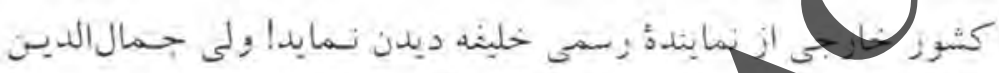

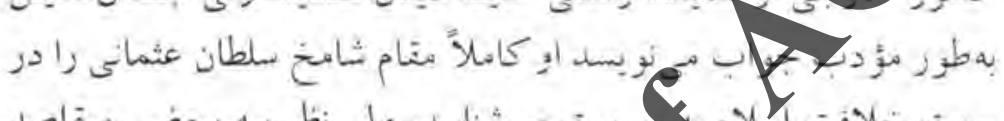

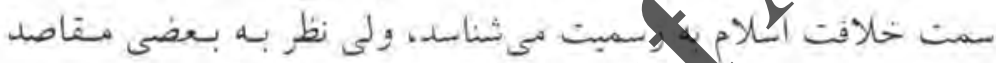

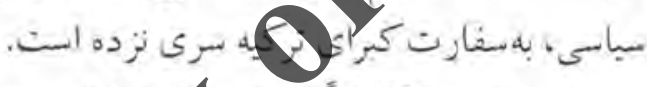

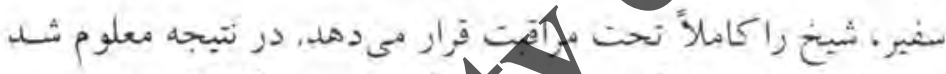

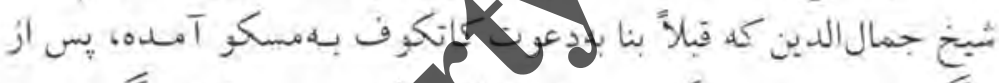

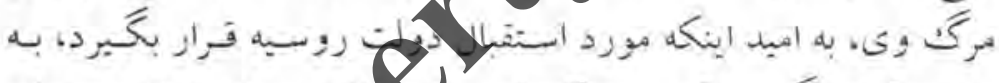

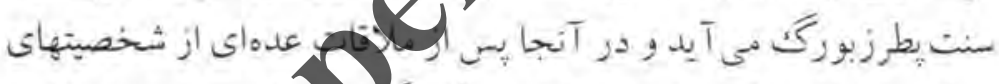

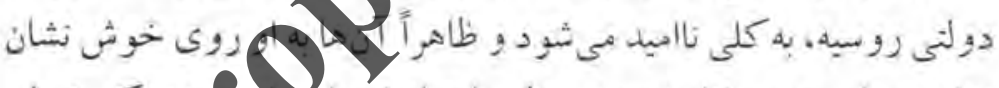

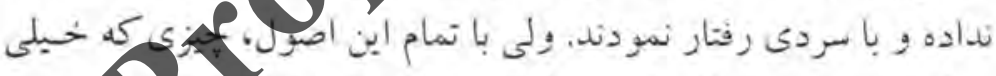

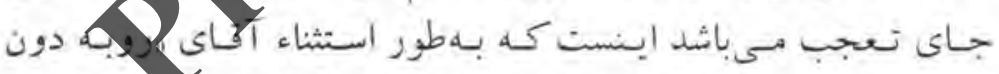

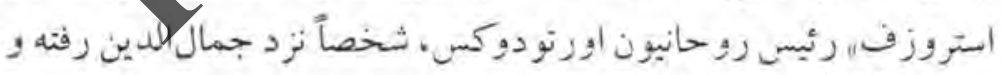

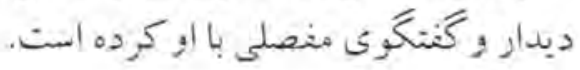

امضاء مورير

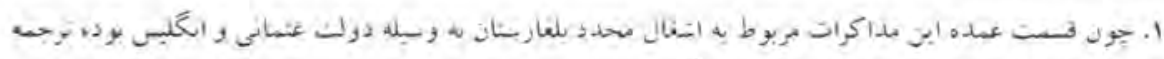

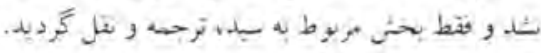




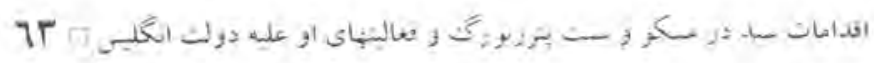

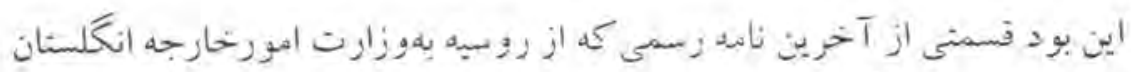

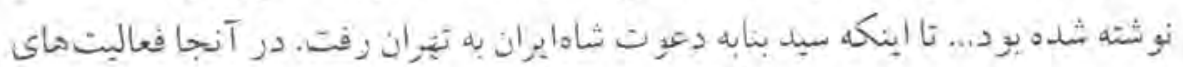

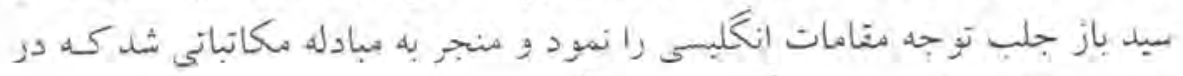
نتيجه سبد از تبران به وضع ناكوارئ تبعبد كرديد.

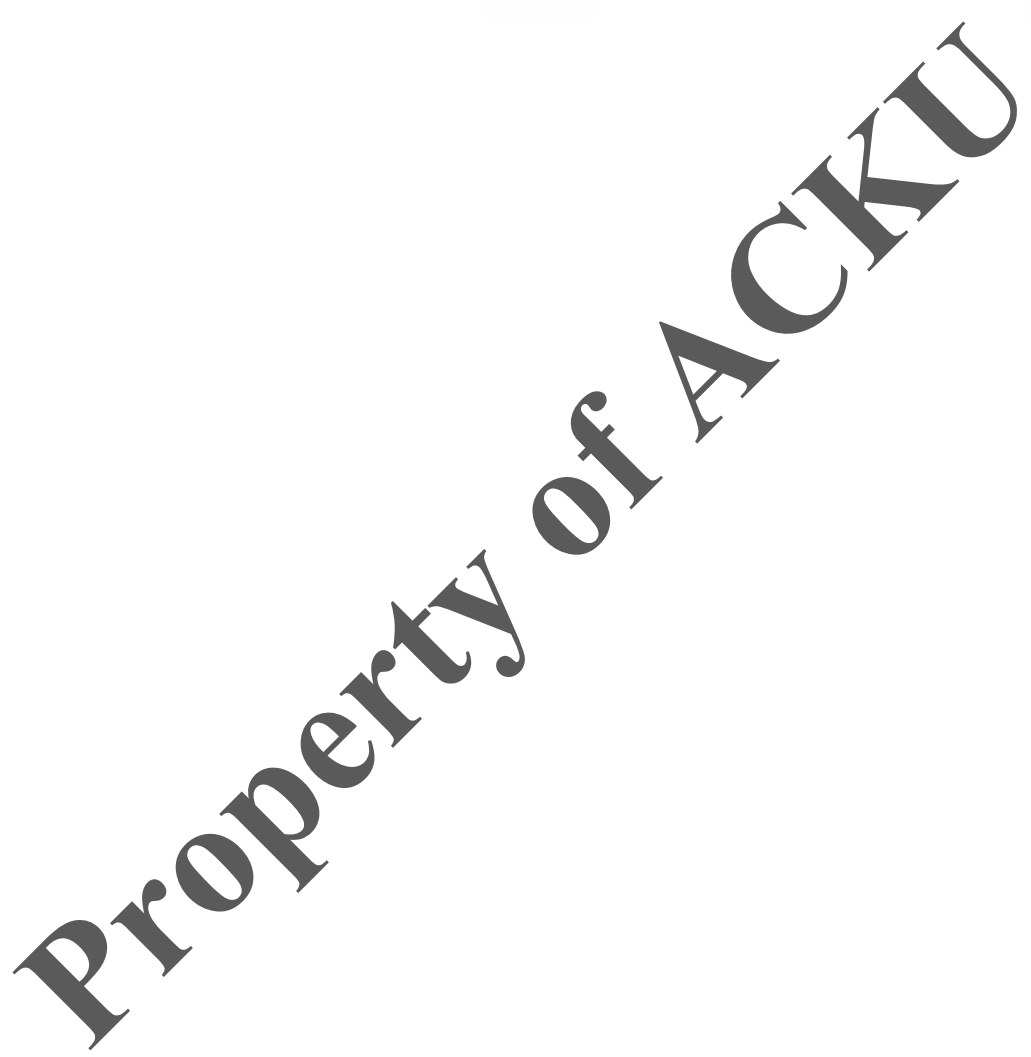




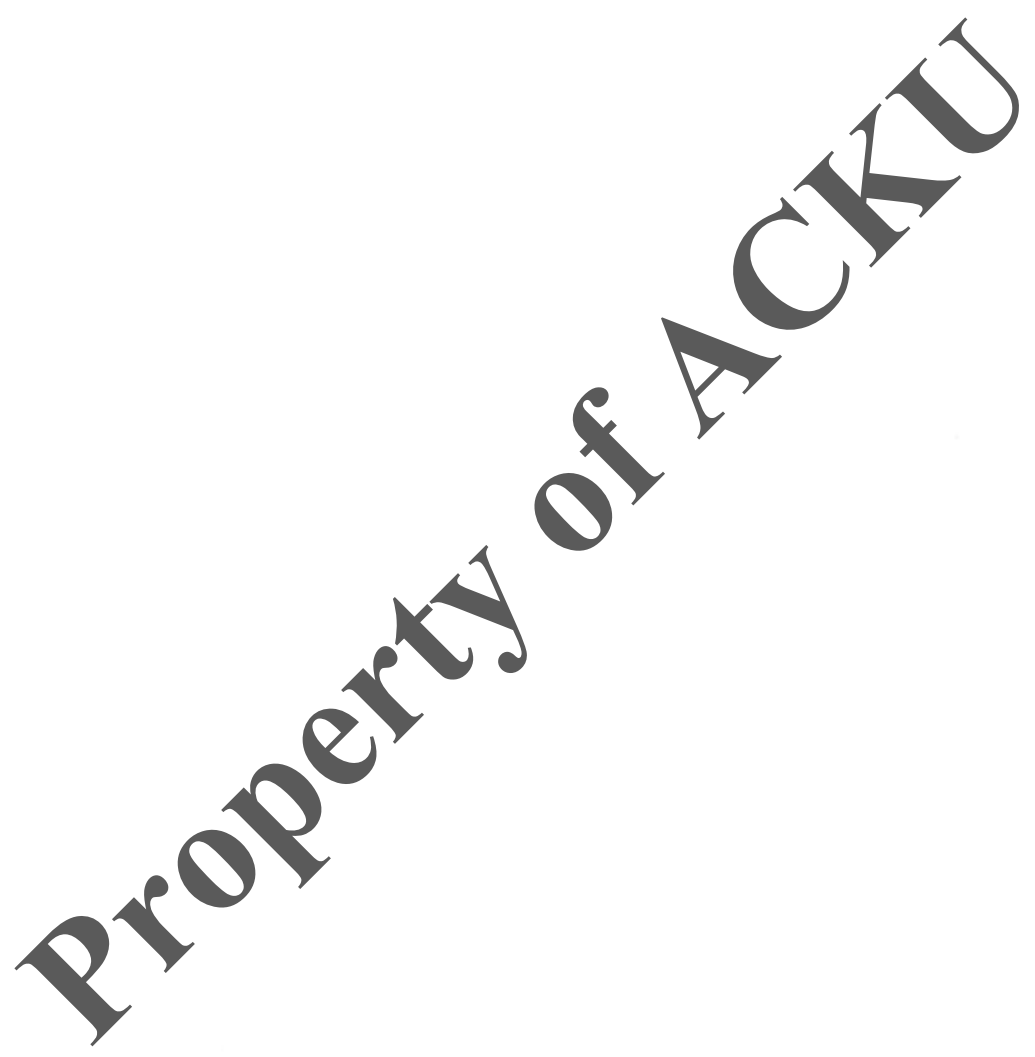




\section{$\frac{Y}{\text { دستعيرى و تبعيد ازايران }}$}

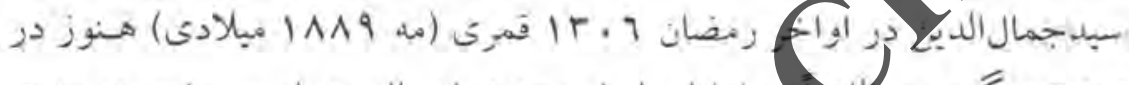

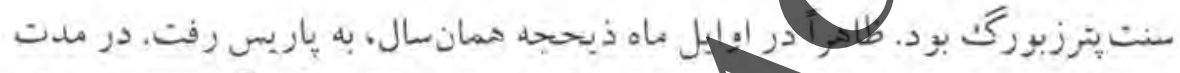

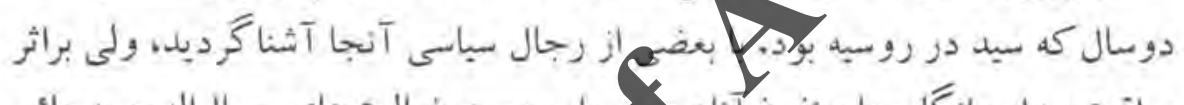

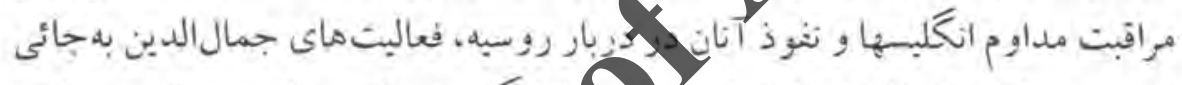

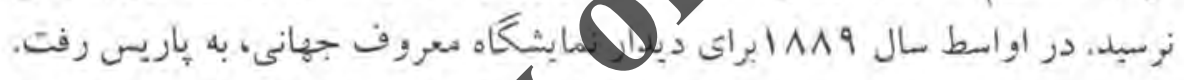

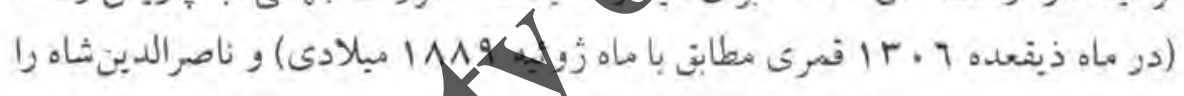

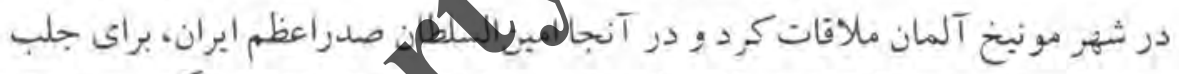

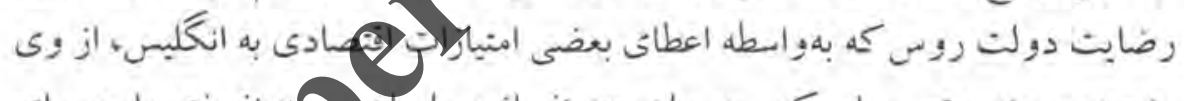

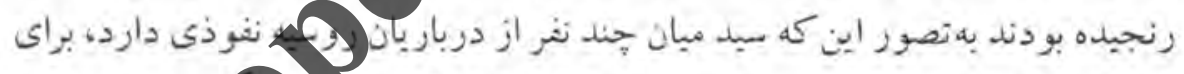

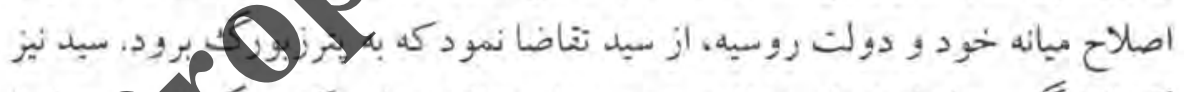

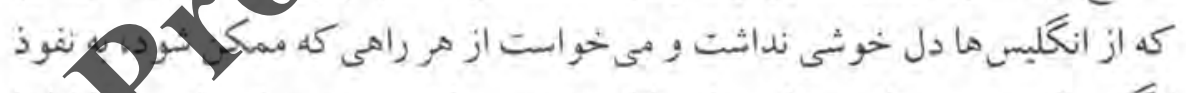

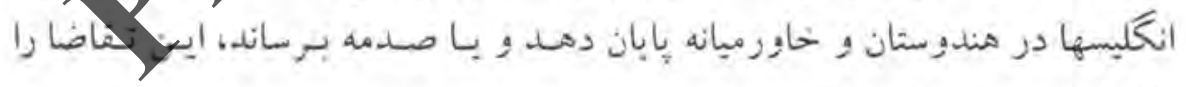

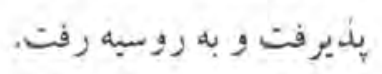

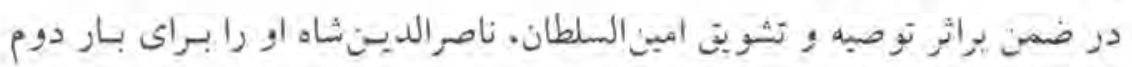

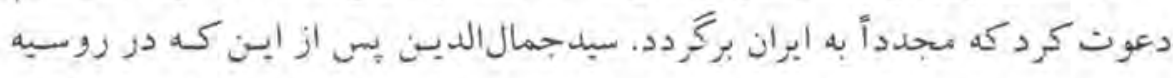

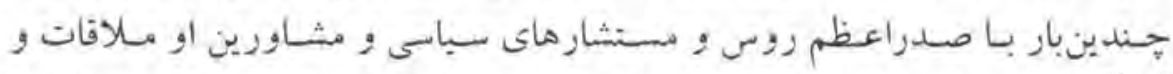

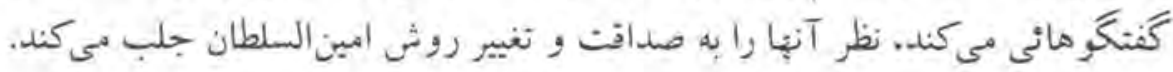




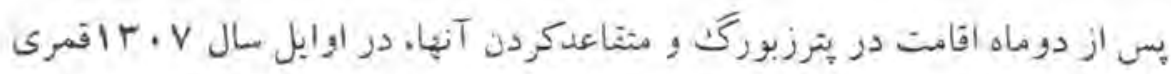

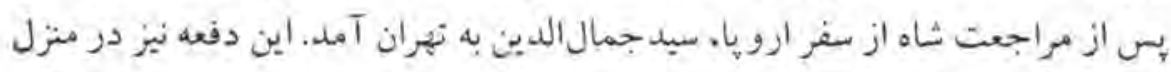

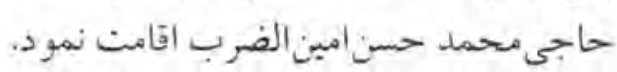

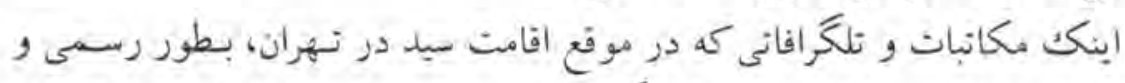

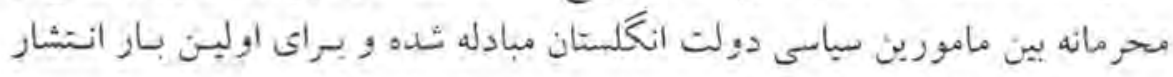

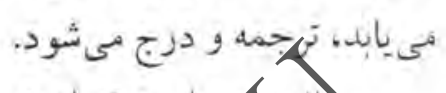

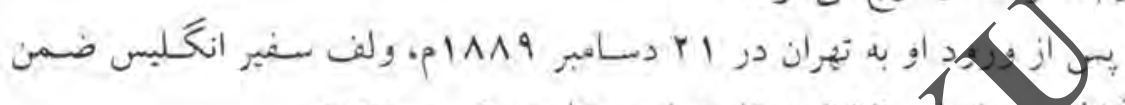

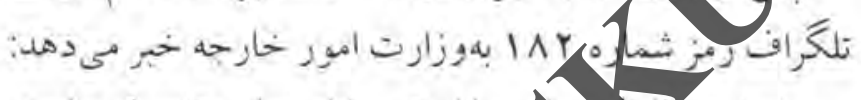

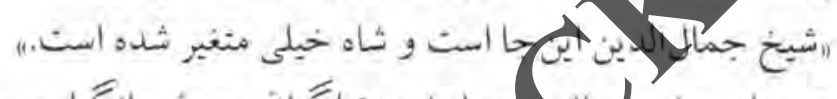

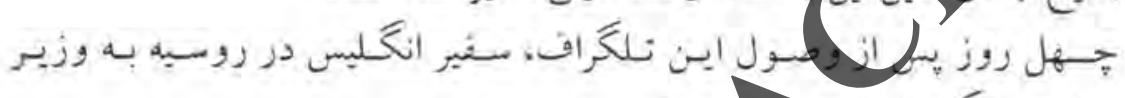

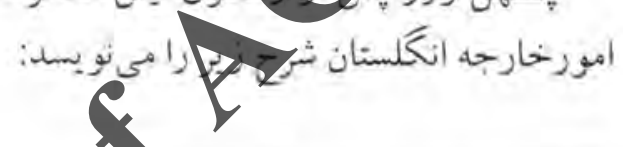

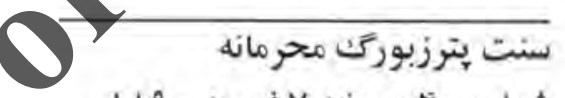

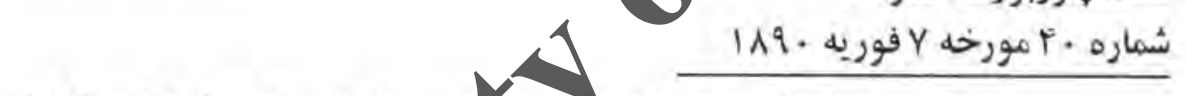

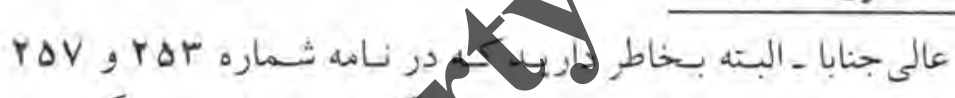

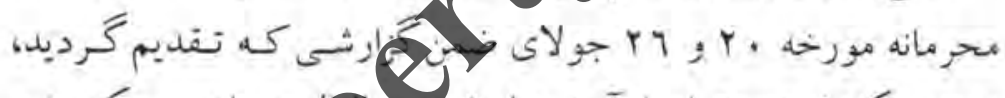

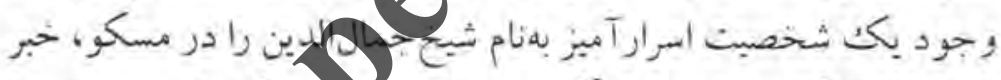

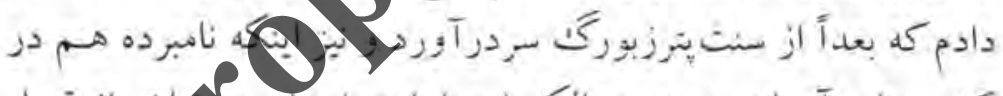

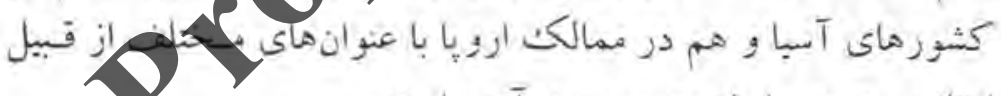

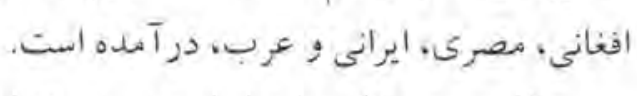

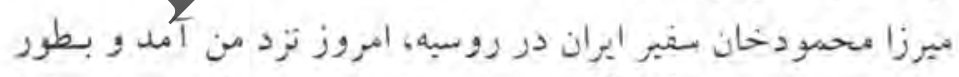

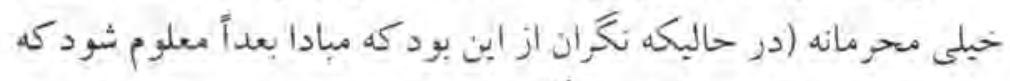

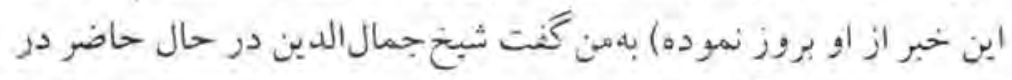

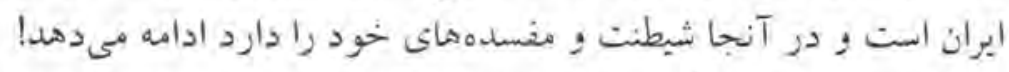

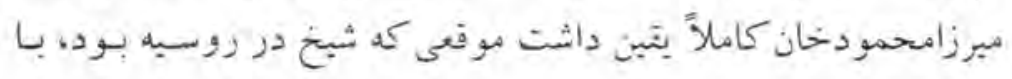


آفائ زينوريف جندين مر تبه ثماس كرفته و روابطى رابا او برقرار نهوده

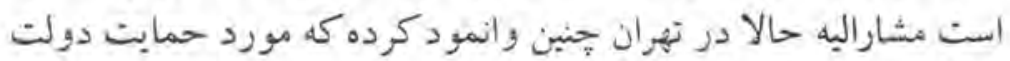
روسيه است. و بدون شكك درنظر دارد به نفع دولت روسيه فماليتهائى

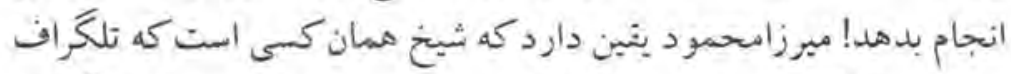

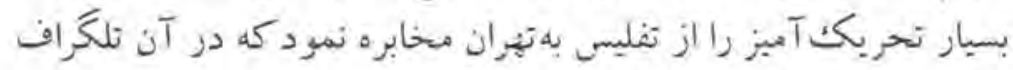

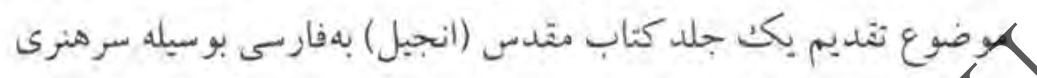

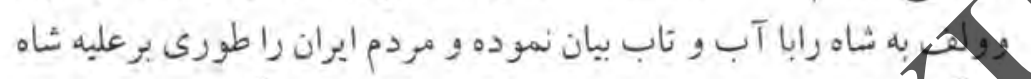

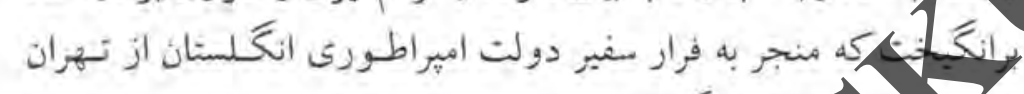

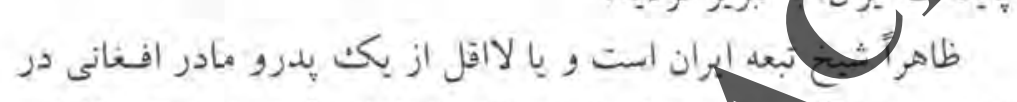

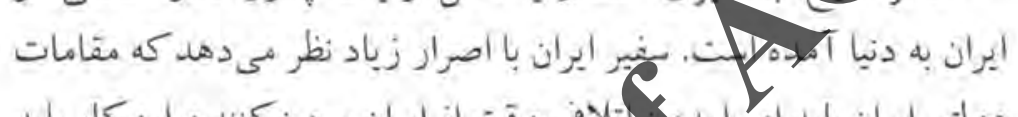

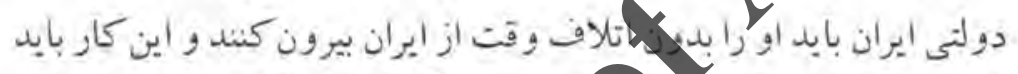

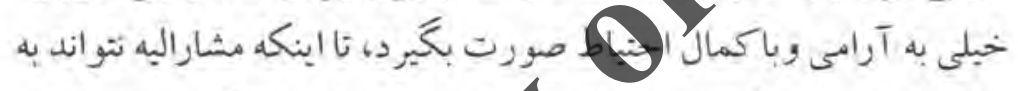

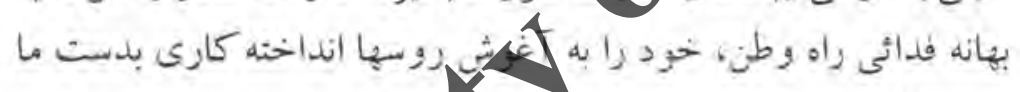

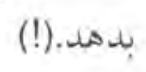

امضاء موريو

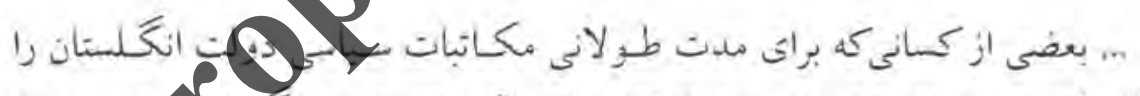

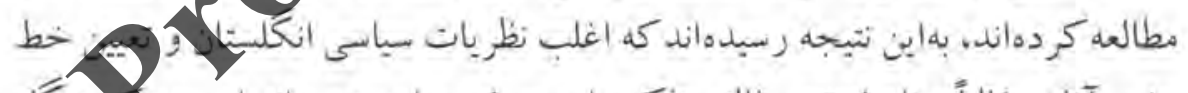

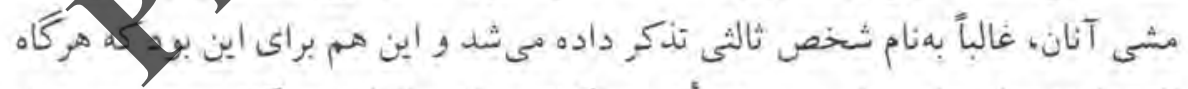

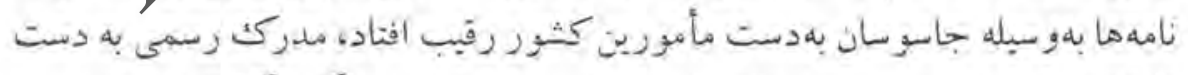

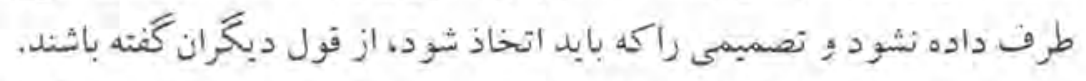

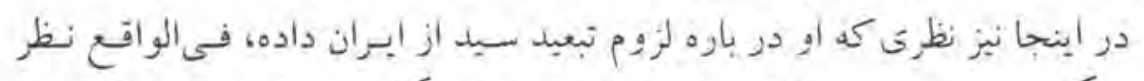

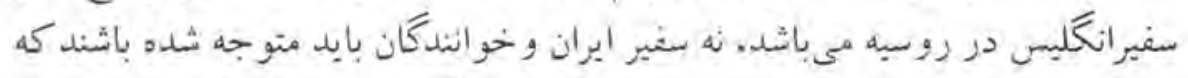

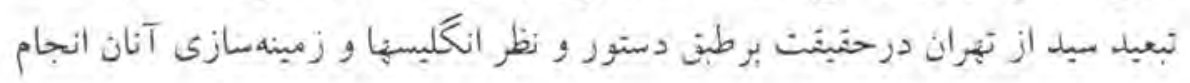




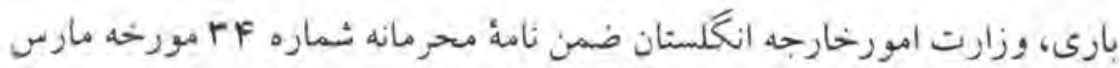

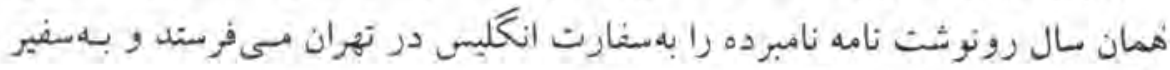

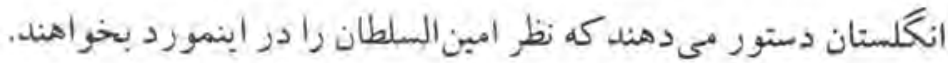

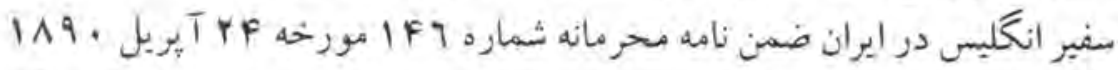

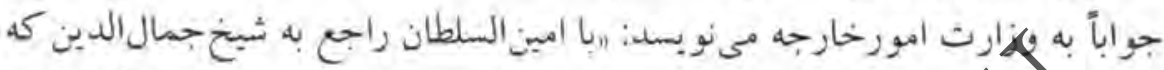

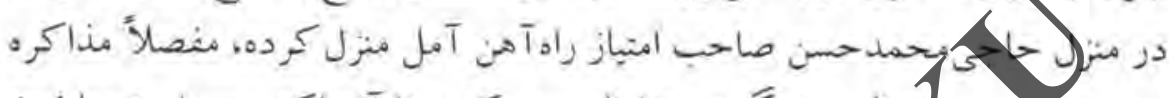

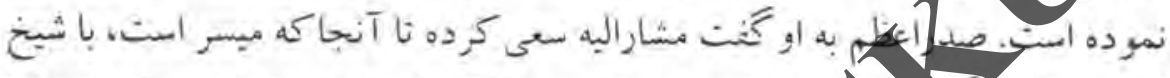

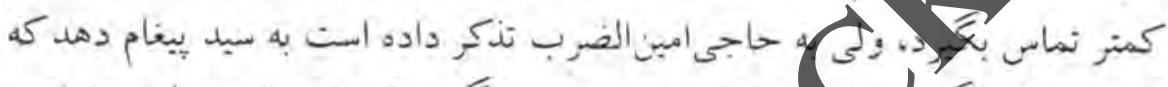

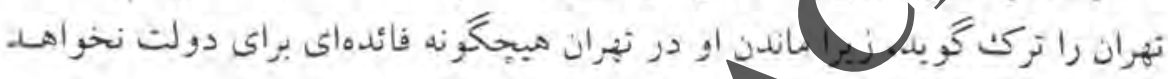
داشتخ:"
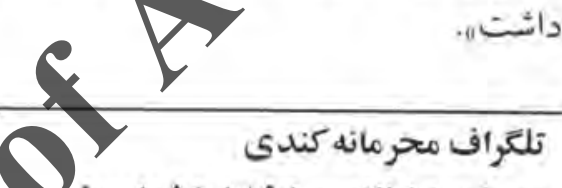

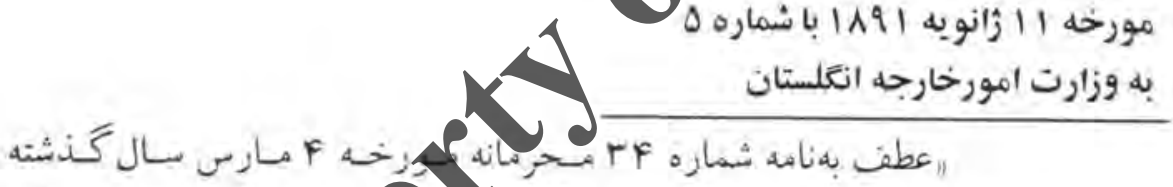

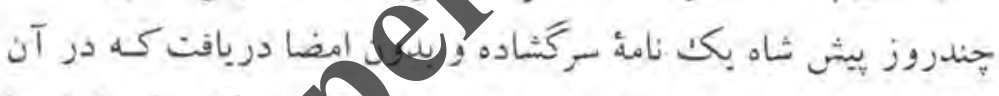

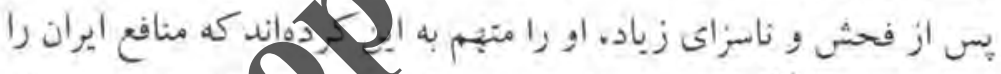

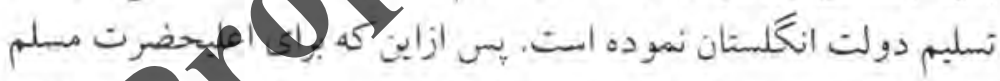

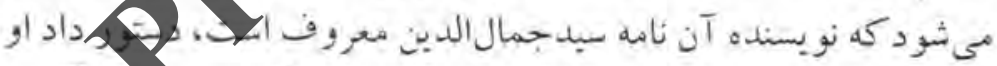

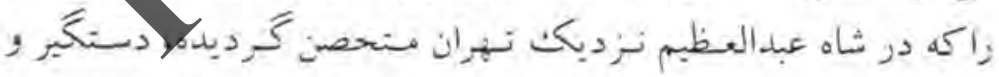

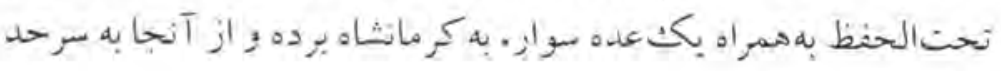
تركيه تبعبدث نمائن.

المقاء:كندى

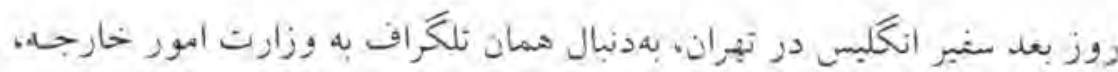


نتخِبرى و نبعيد ازايرانه 19

بةشرح زير نامه مغصلى مىنويسد:

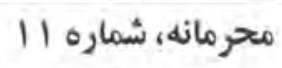

مورخه זا زائويه الهما الز تهران

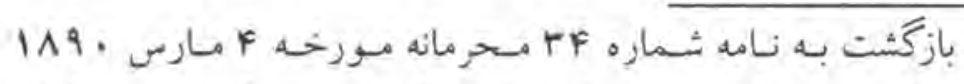

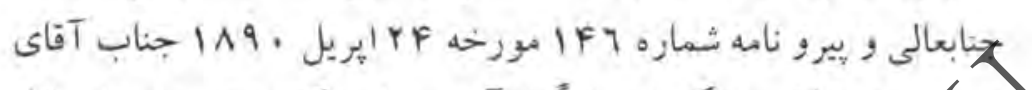

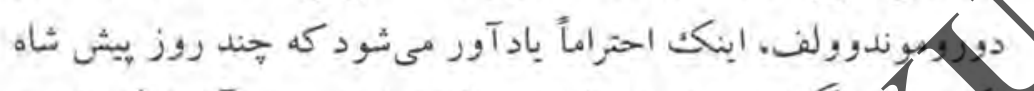

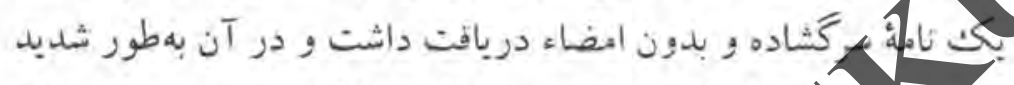

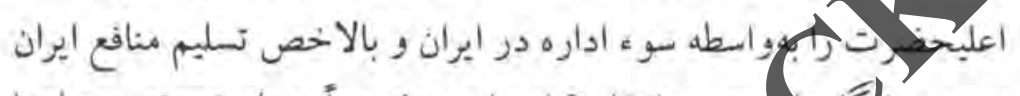

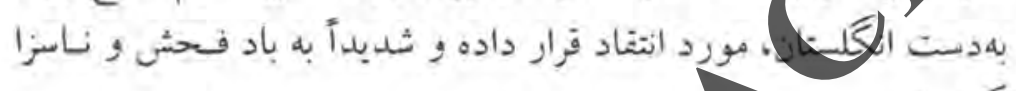

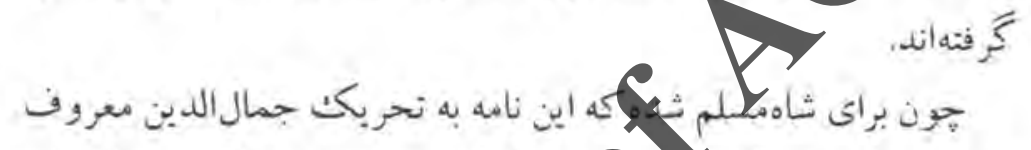

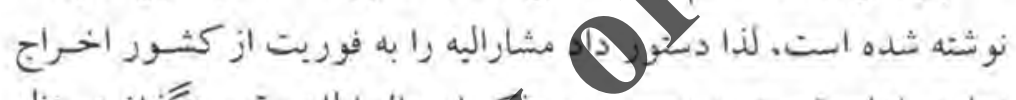

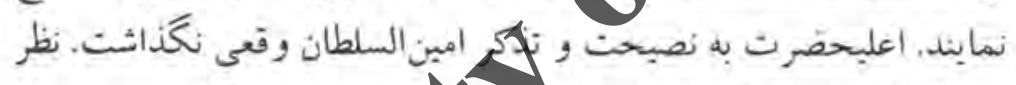

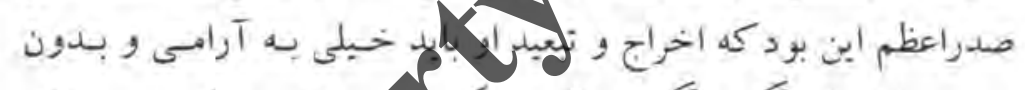

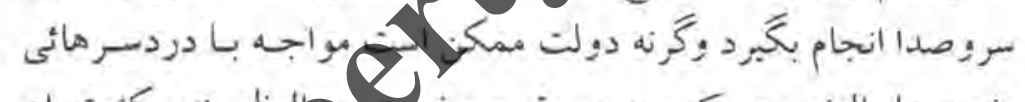

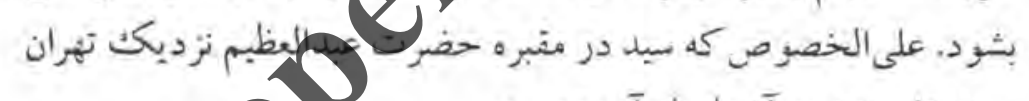

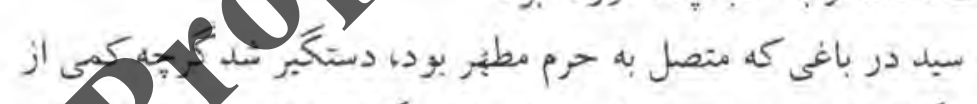

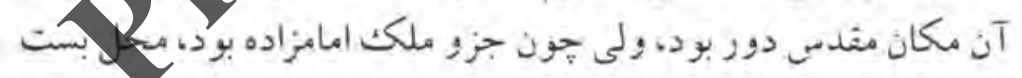

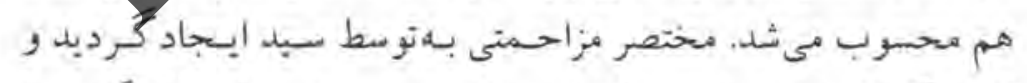

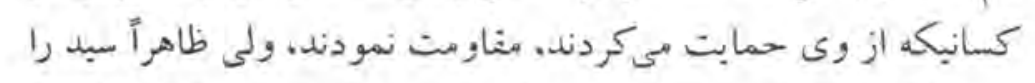

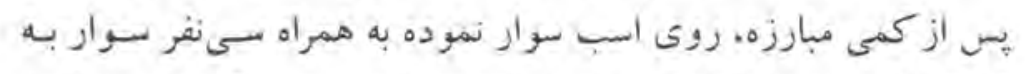

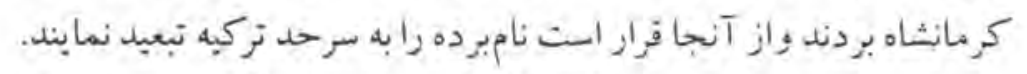

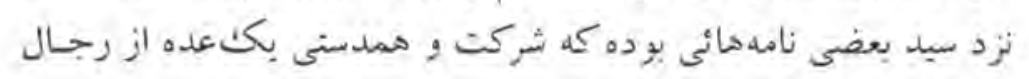


بزركت ابران را در نوطئه برغليه دولت ثابت مىنمابد. برجستهثرين آنها،

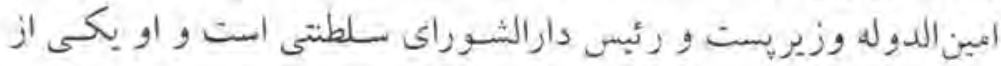

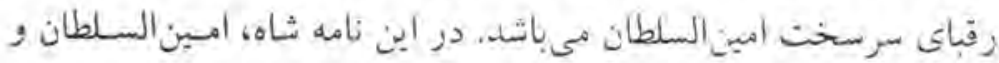

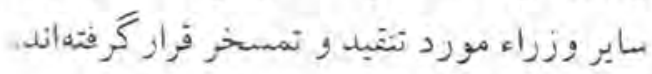

با باحترامات _كندى

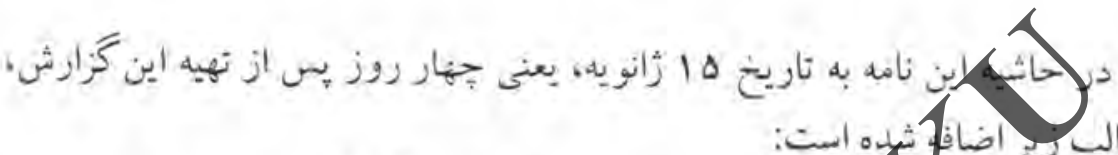

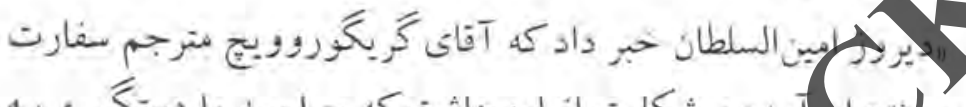

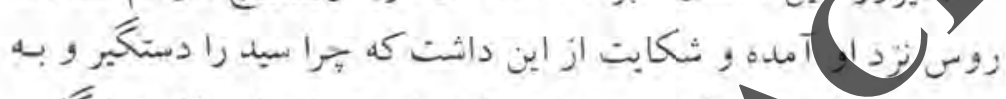

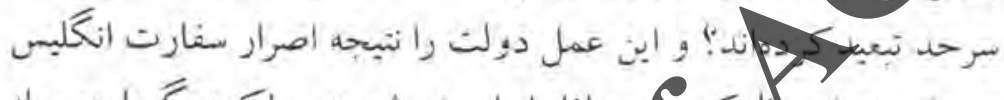

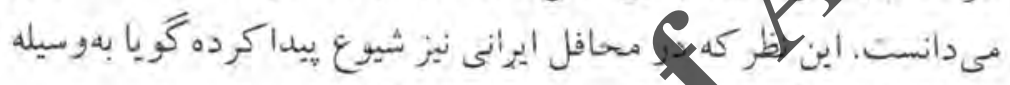

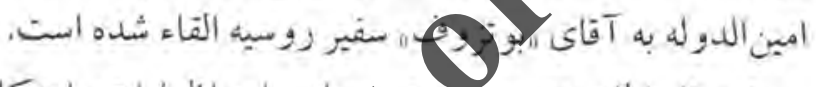

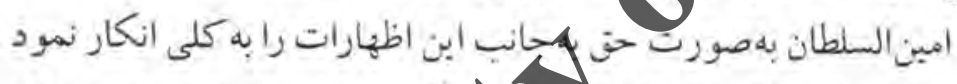

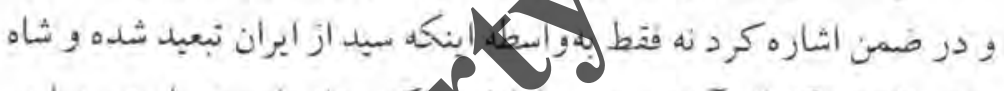

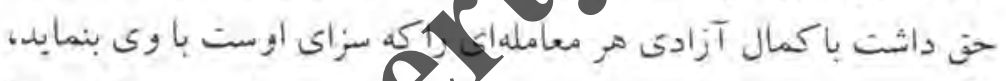

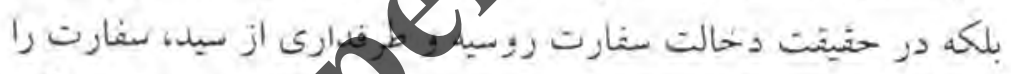

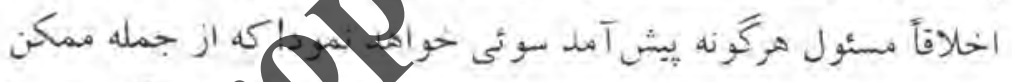

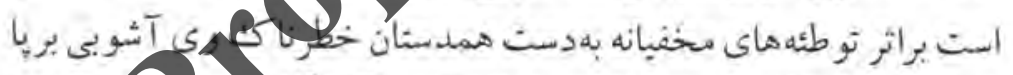

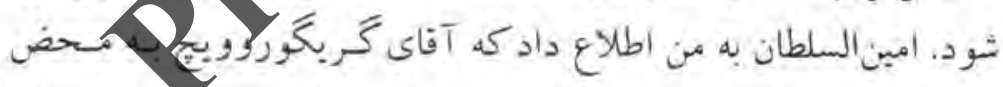

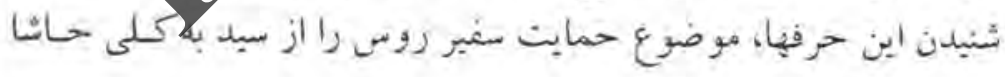
نمود.

المضاء_كندى

بس از دسثور تبعيد سيدجمال الدين، ير اثر فثار افكار عمومى و شـابد شكابت 


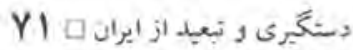

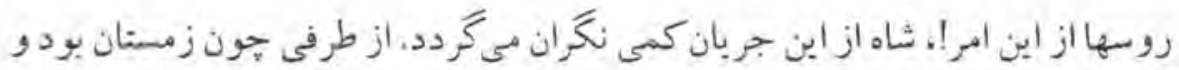

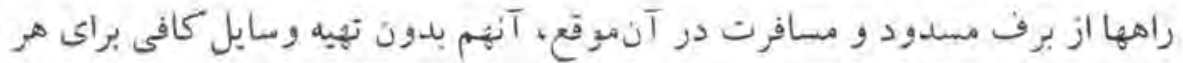

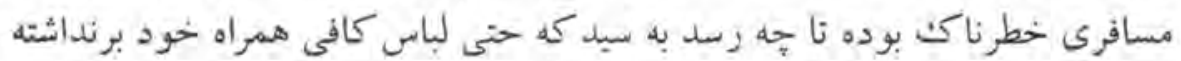

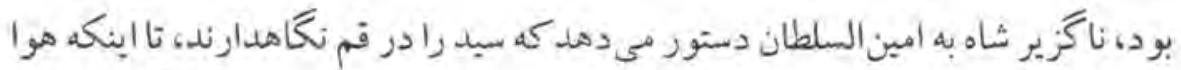

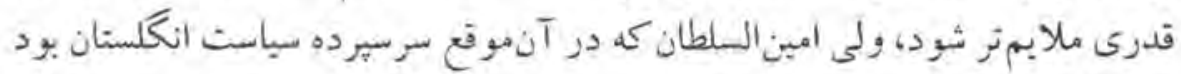

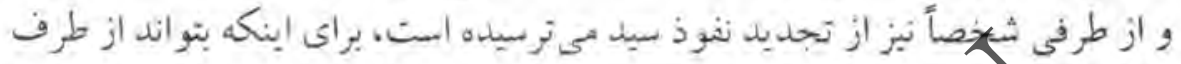

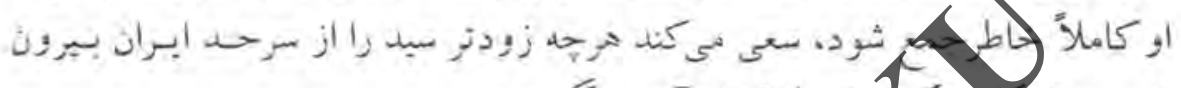

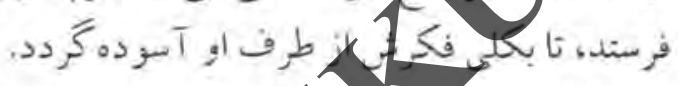

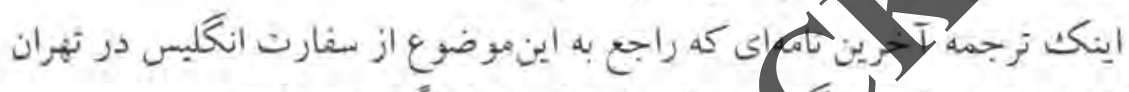

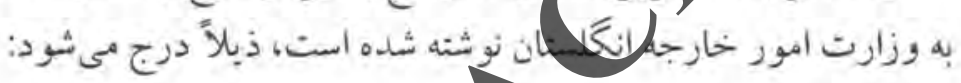

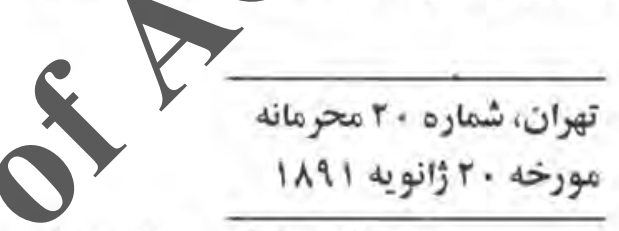

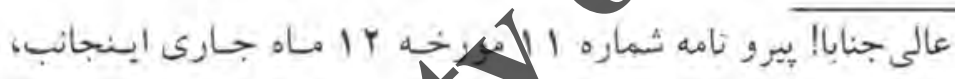

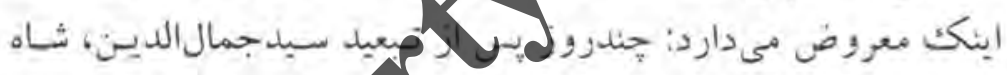

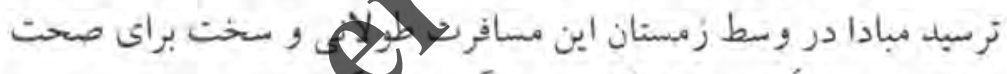

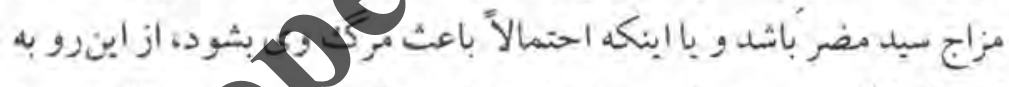

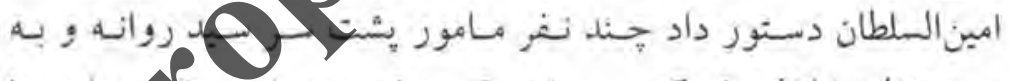

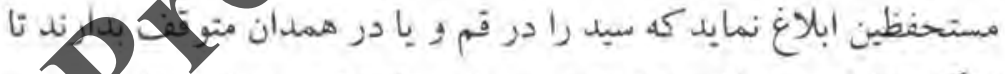

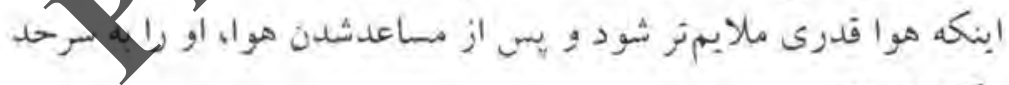
تركميه بنر سنتله.

ثناه مبل داشت كه بكت نامة دوستانه به سيد نوشته تُود و مقدارى يول

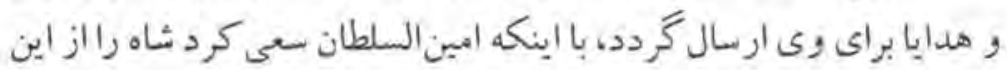

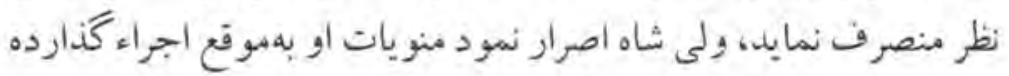
ثمود. 


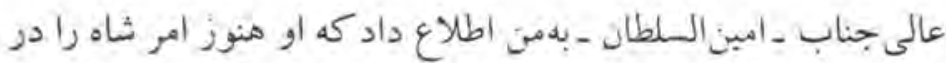

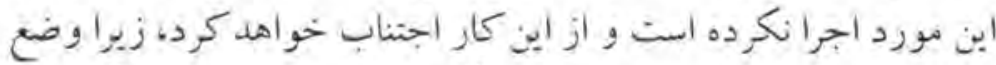

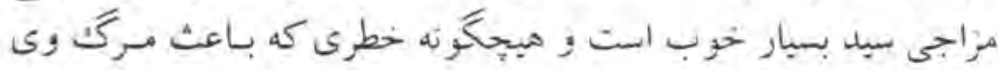

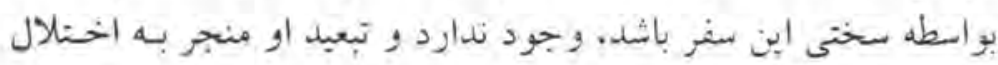

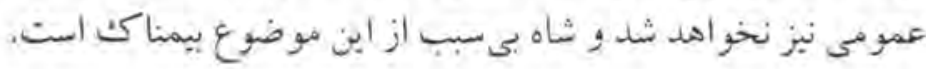
المضاء_كندي

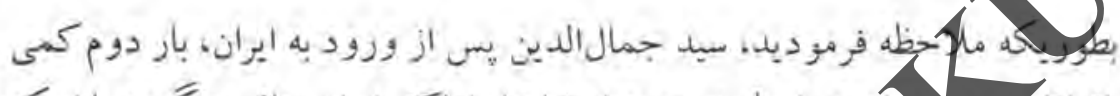

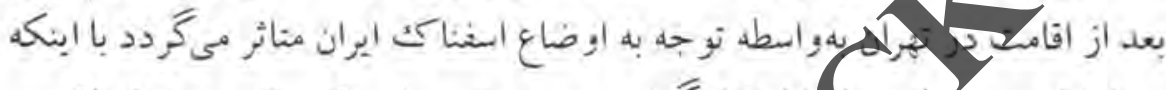

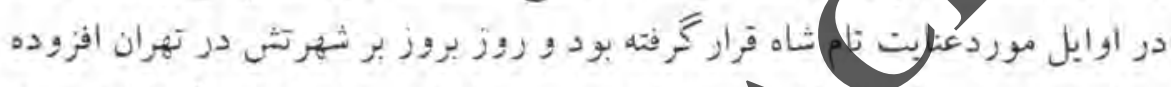

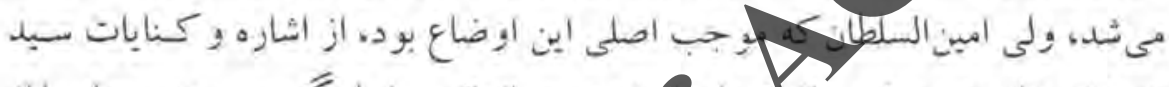

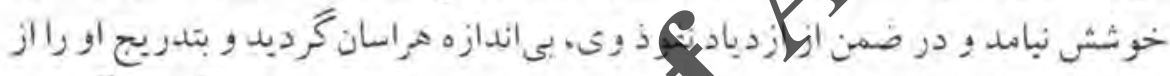

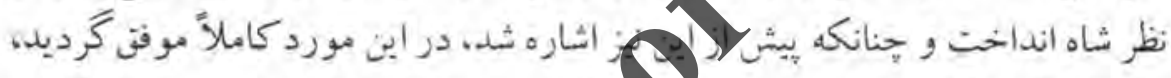

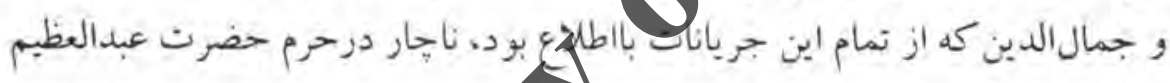
منحصن شد.

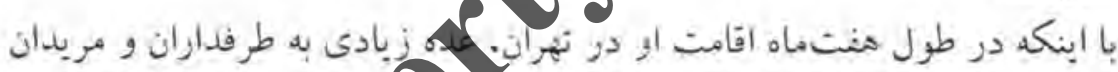

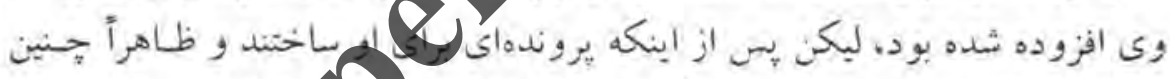

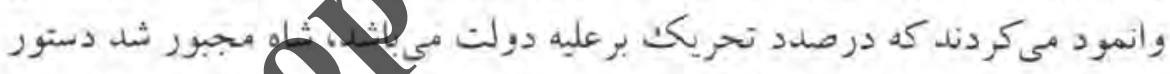

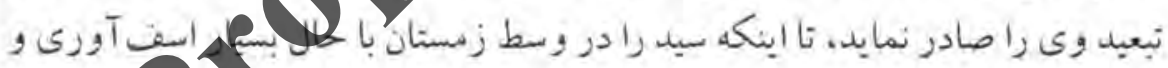

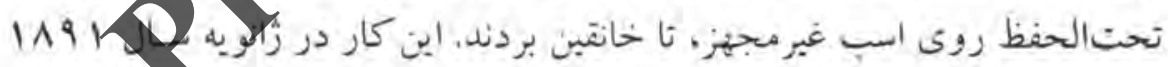

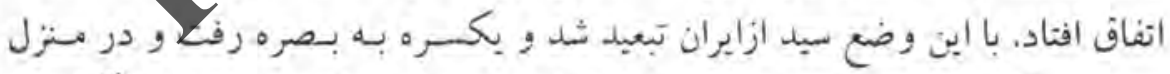

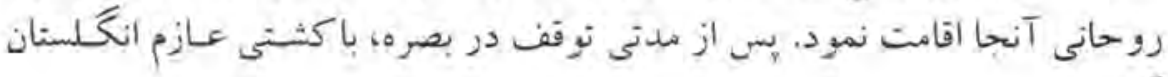
كرديد. 


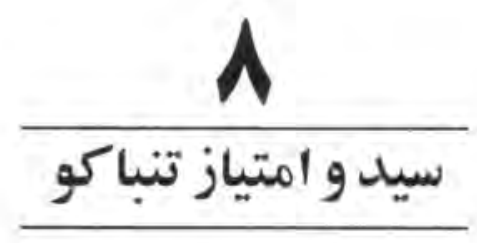

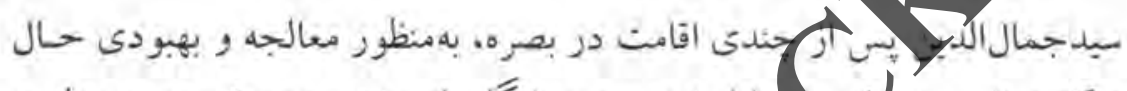

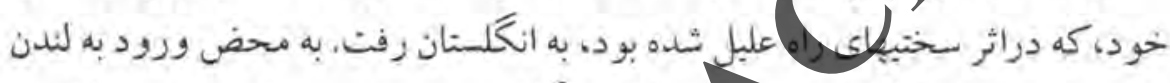

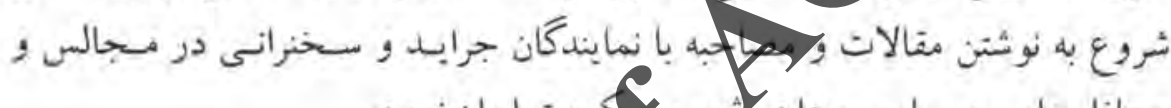

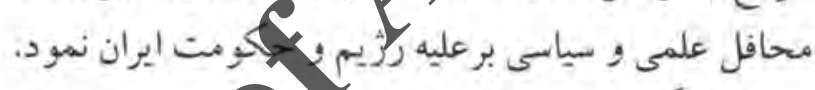

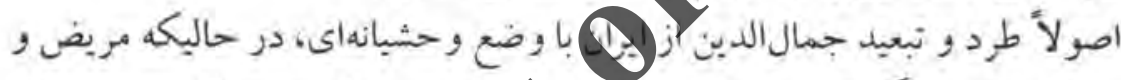

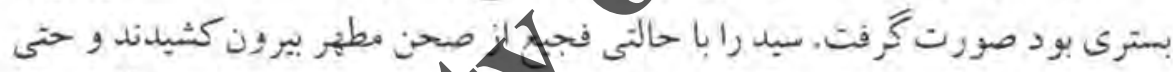

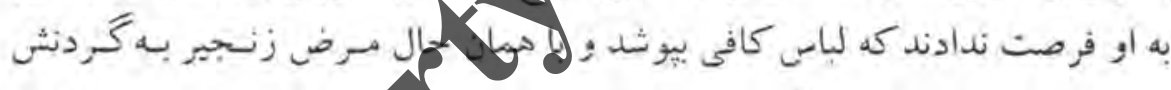

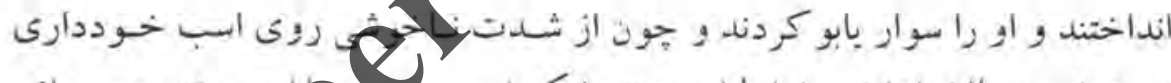

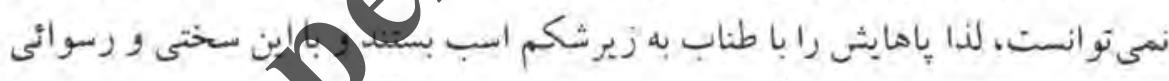

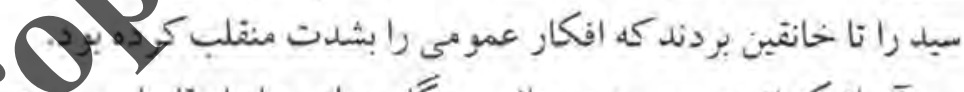

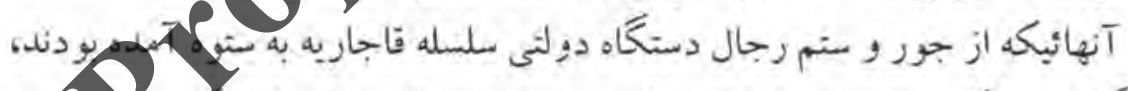

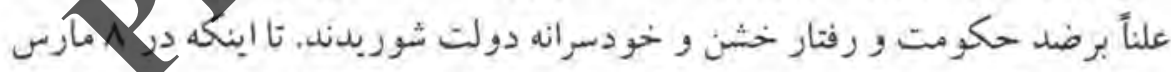

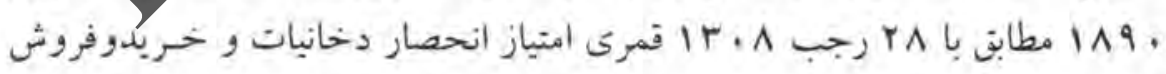

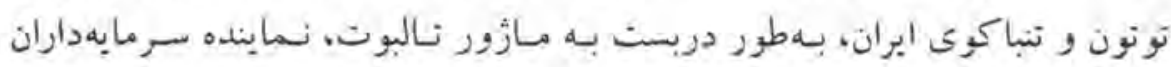
داده شد. انغليسي

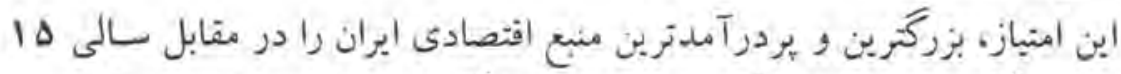

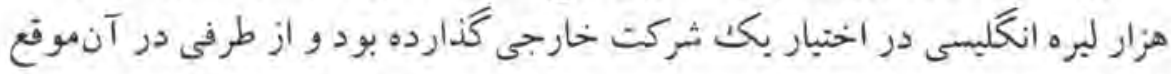


كم مردم داراي تعصبات شديد مذهبى بودند، بة ماهورين يكت دولت اجنبى اجسازه

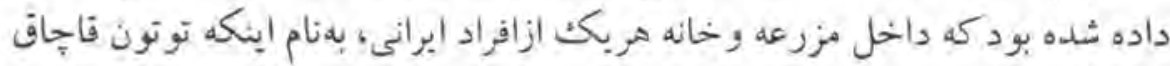

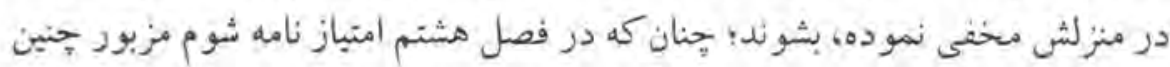
قيد شمده بودن

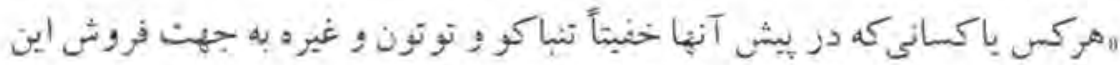

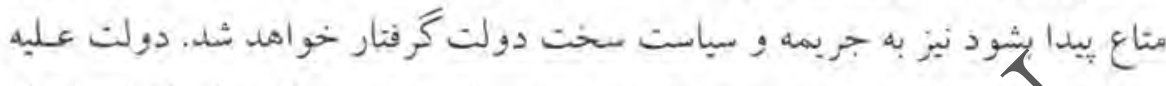

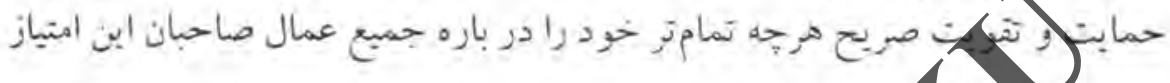

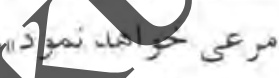

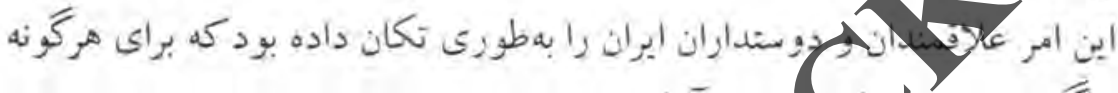

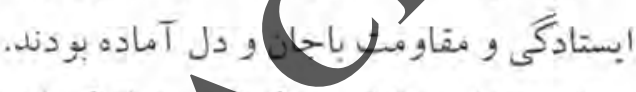

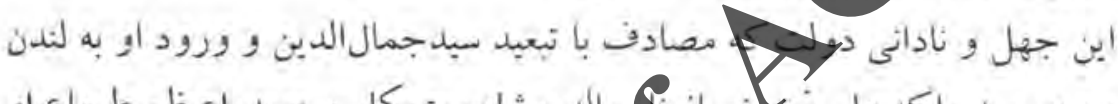

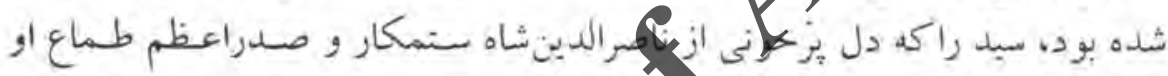

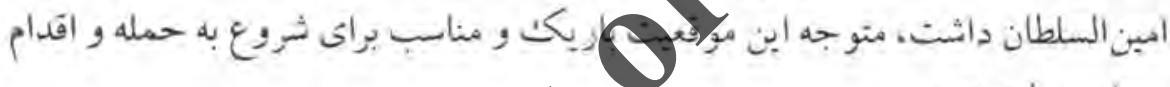

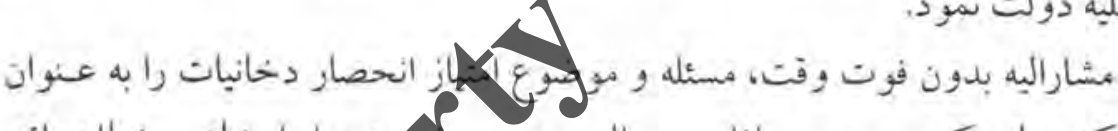

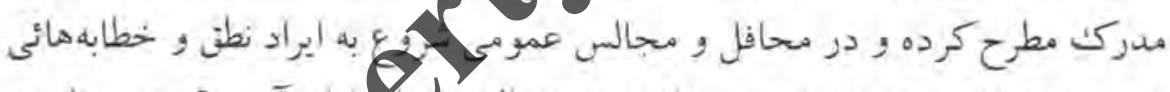

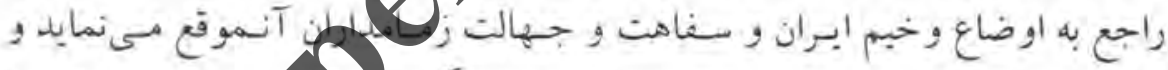

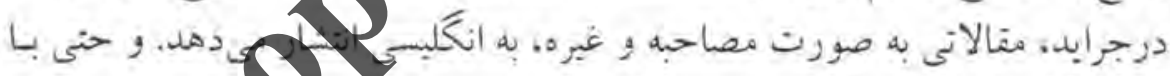

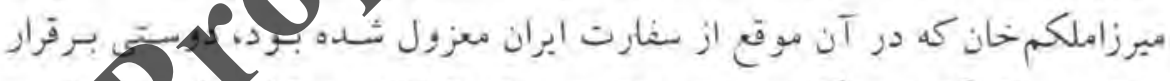

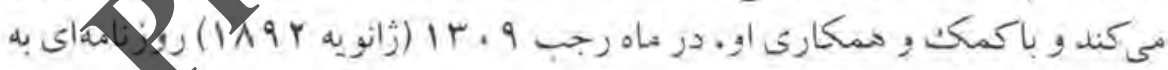

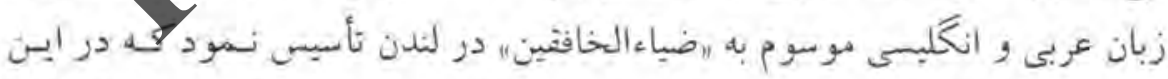

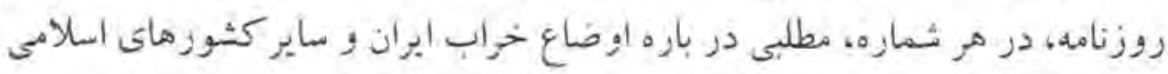

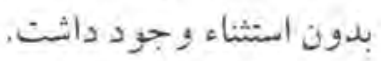

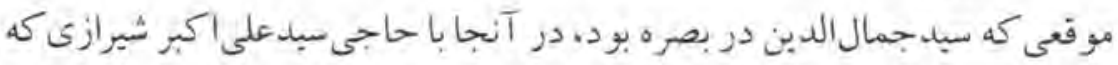

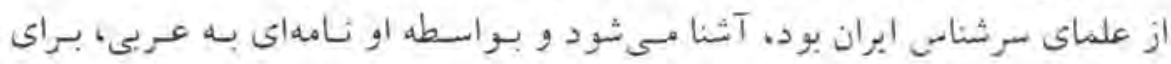




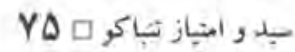

حاجى ميرزاحسن شيرازى مجتهد معروف و يكى از مراجع مهم تقليد ثشبعه كـه در

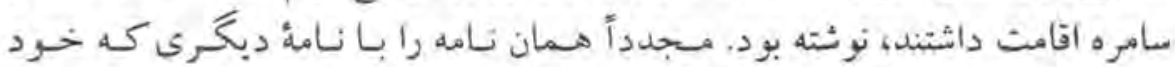

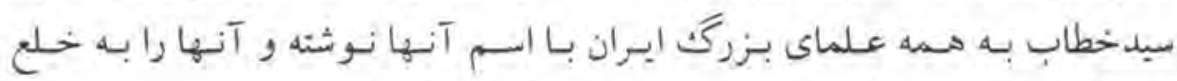

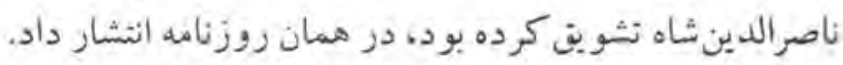

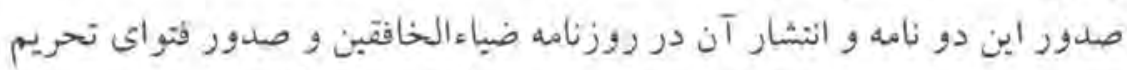

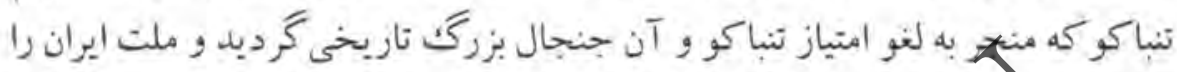

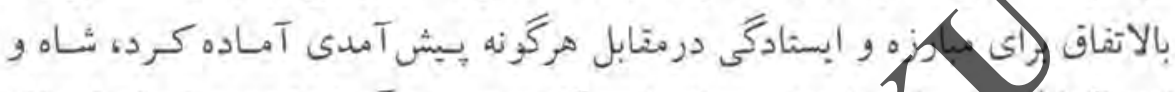

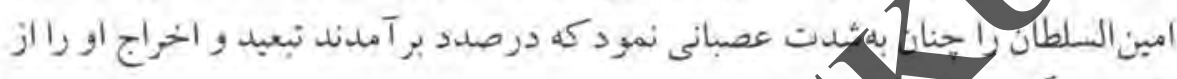

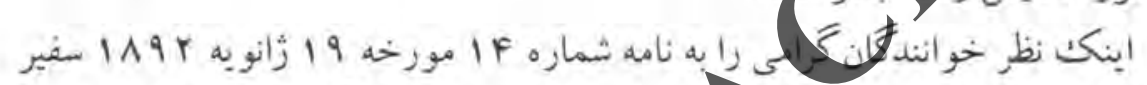

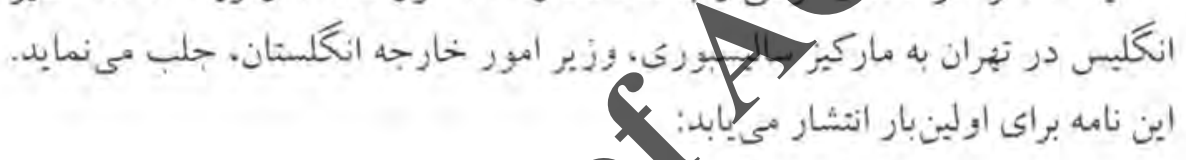

0

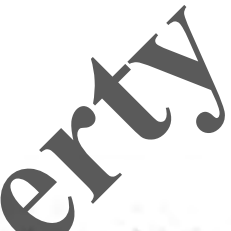

نامه فرانك لاسل سفير لندن در تهران به وزير المور خارجه انتليس لانس لنيس

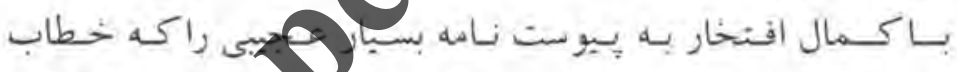

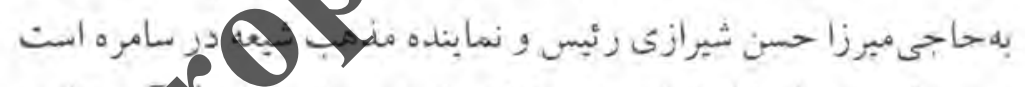

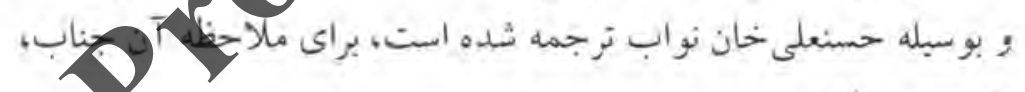
تقديم مي تواردم.

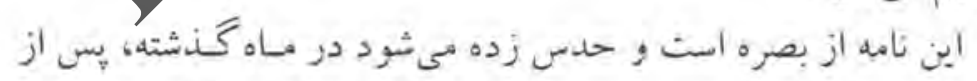

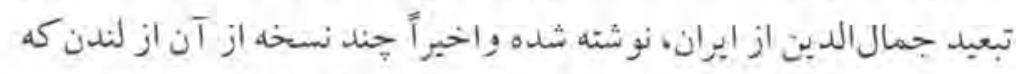

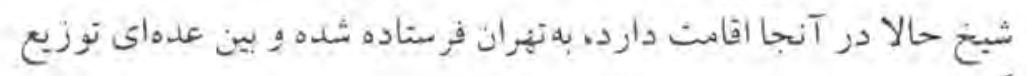

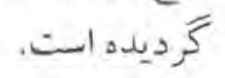

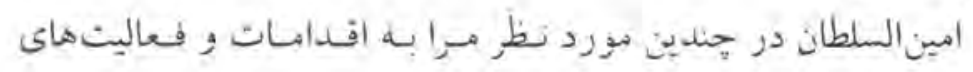


جمال الدين معطوف داشته است و مكرر موضوع جنش هائ سباسى اخير

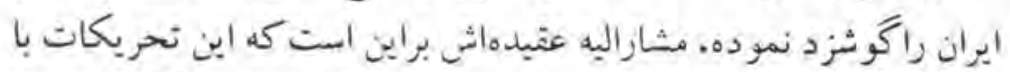

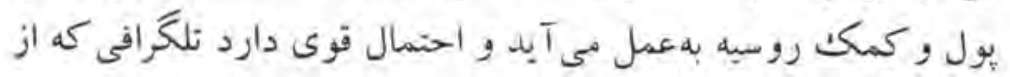

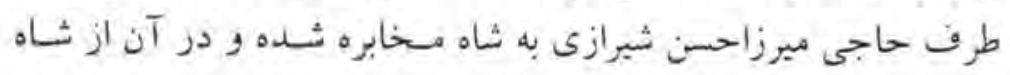

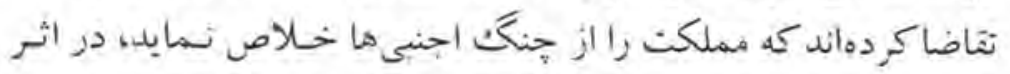

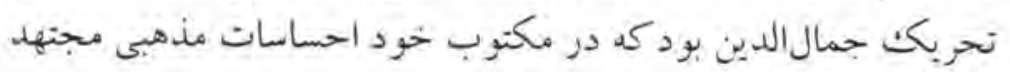

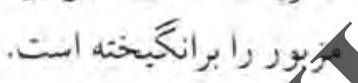

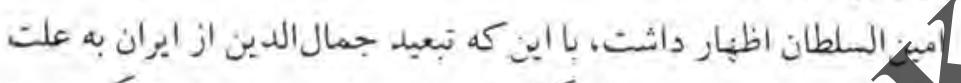

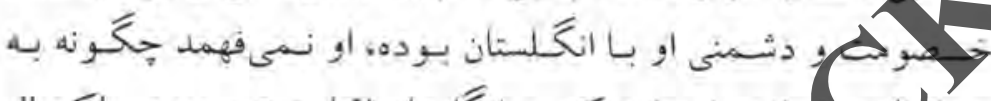

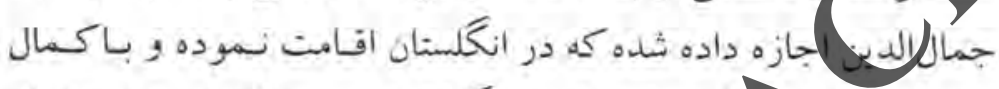

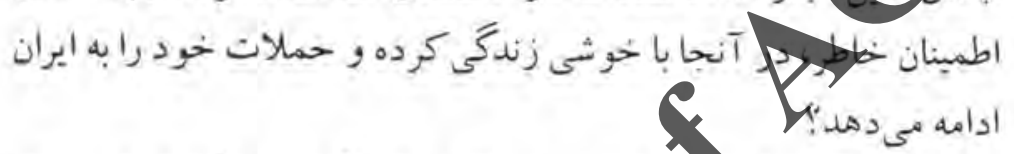

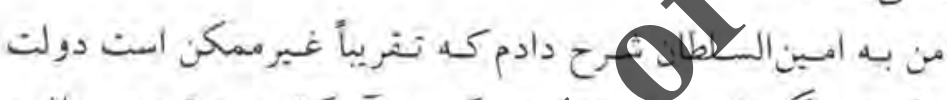

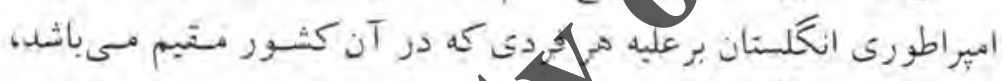

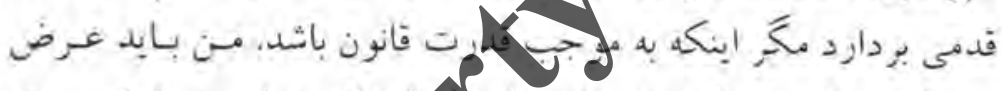

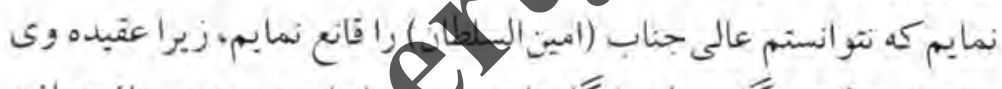

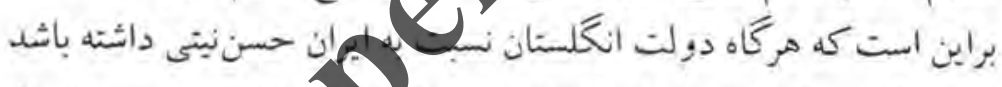

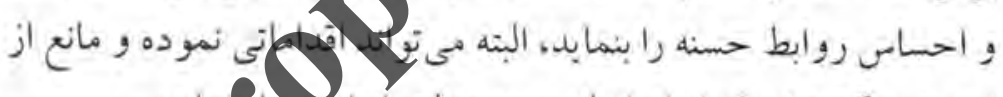

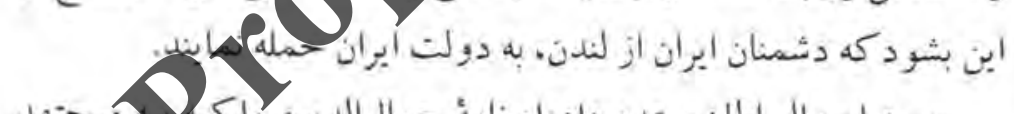

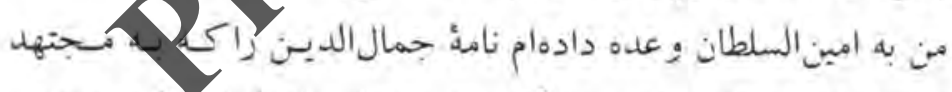

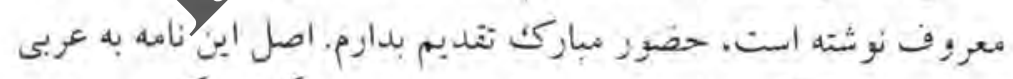

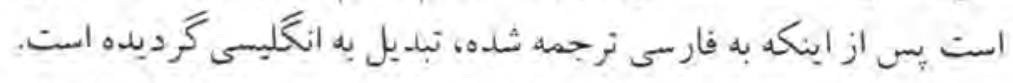

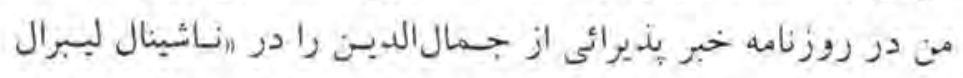

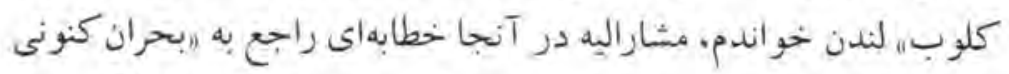

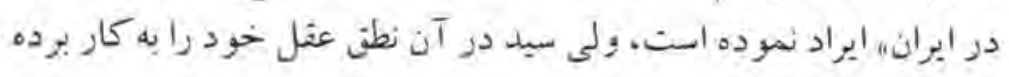


و از ذكر مطالبى كمه به انكلمتان و سباست خارجى آن برمى خورد، احتراز

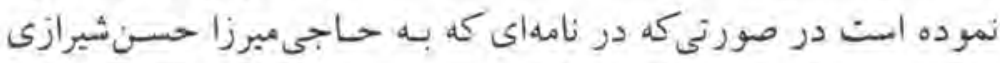

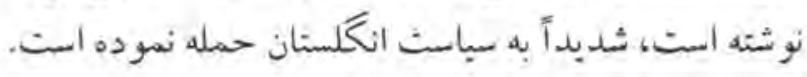

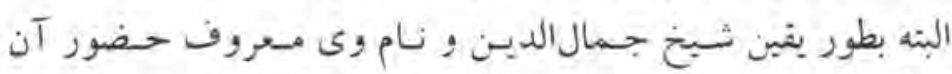
عاليجناب مي باثند.

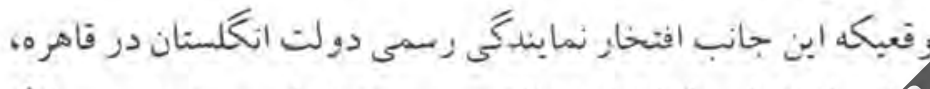

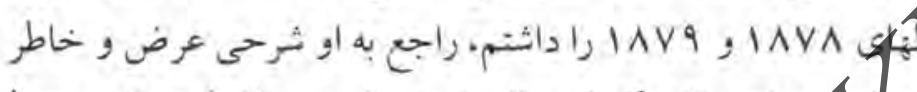

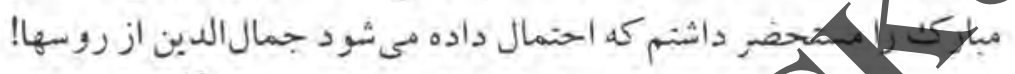

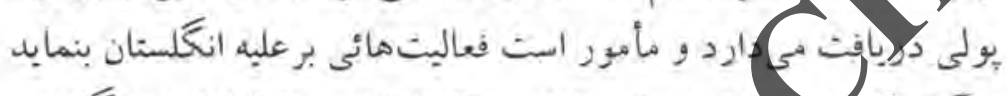

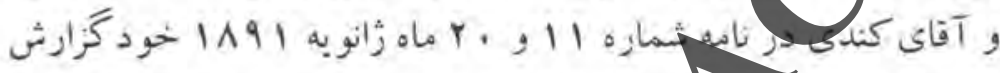

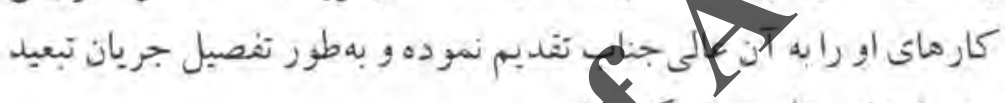

با تقديه احترامات ـ فرانك لاسل

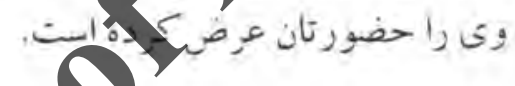

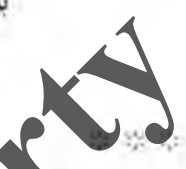

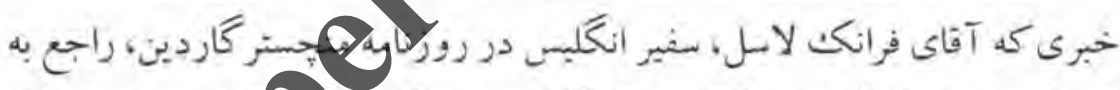

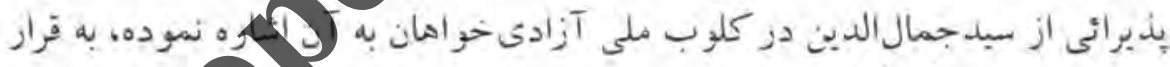

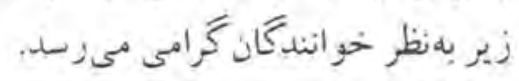

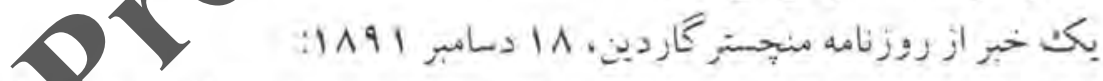

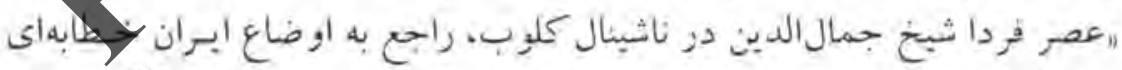

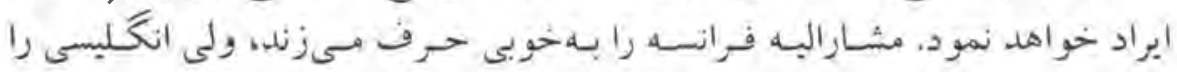

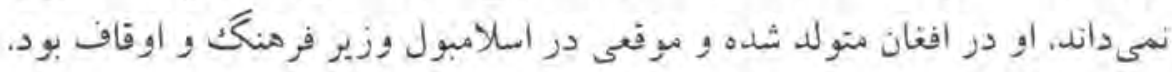

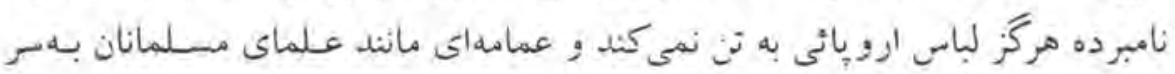

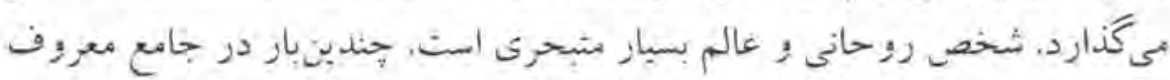

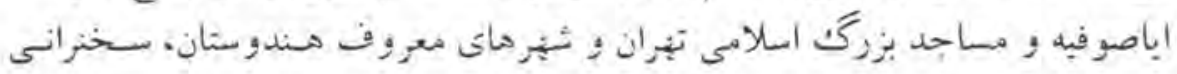




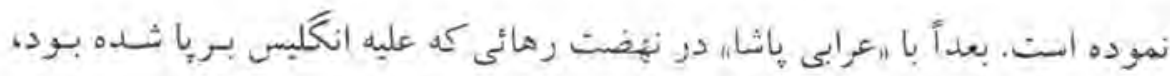

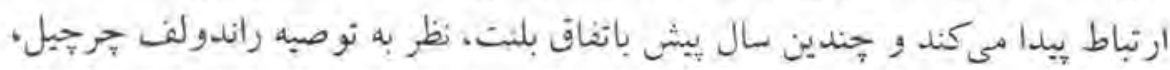

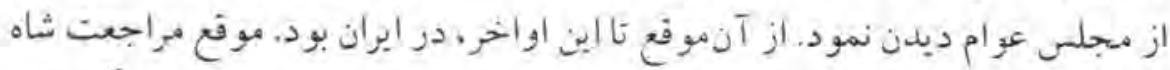

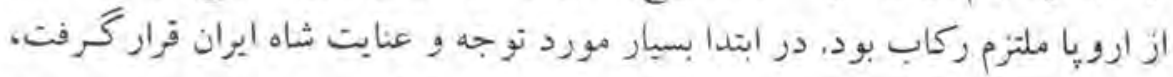

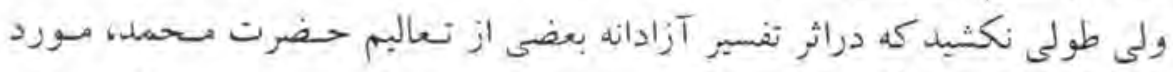

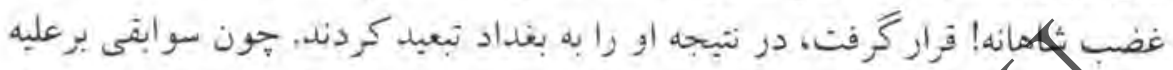

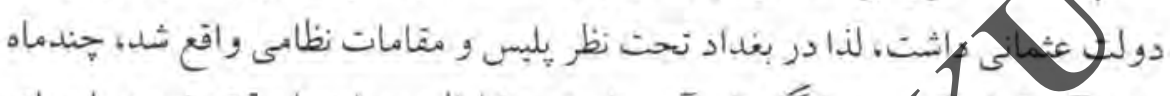

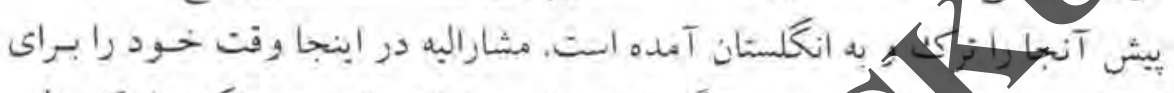

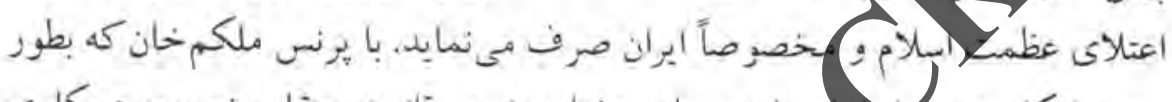

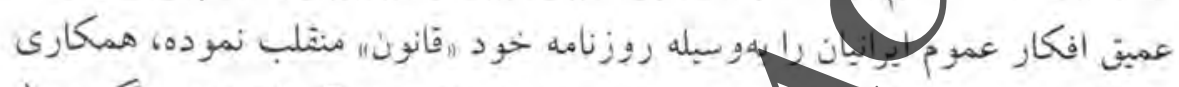

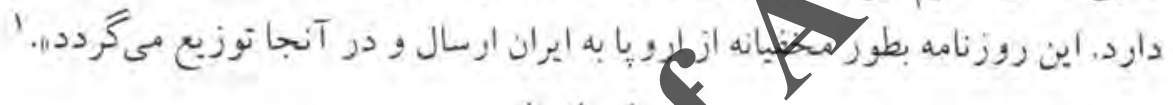

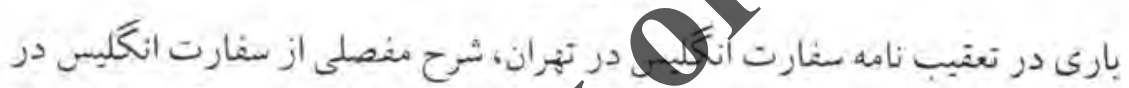

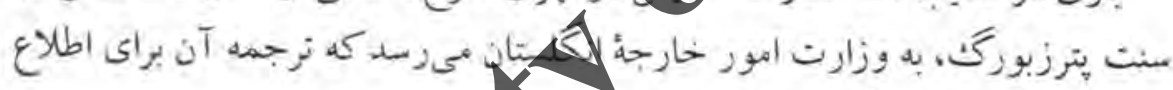

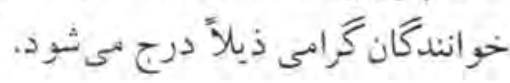
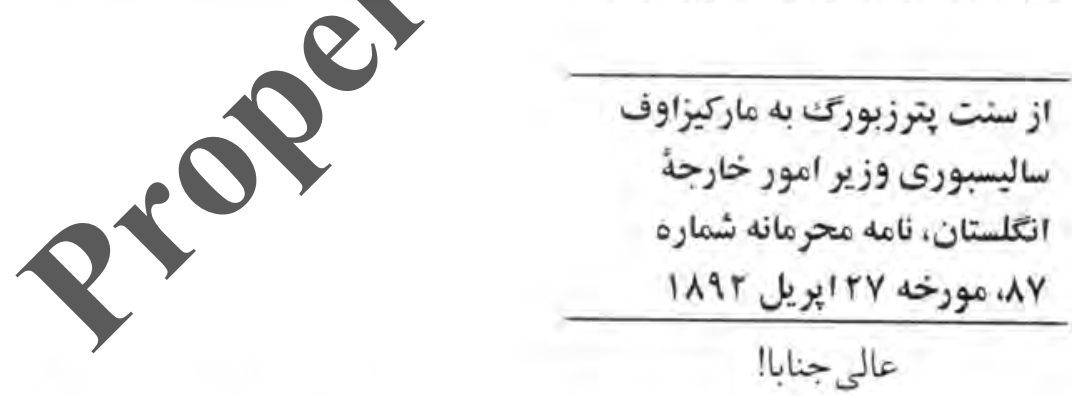

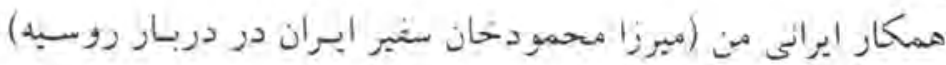

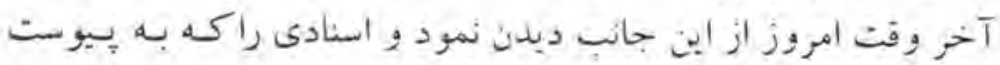

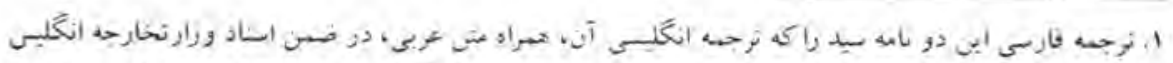

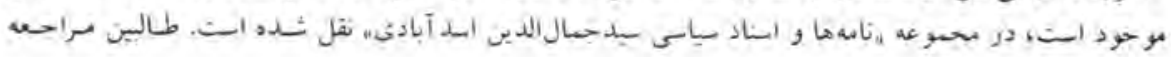




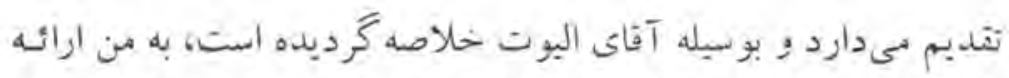

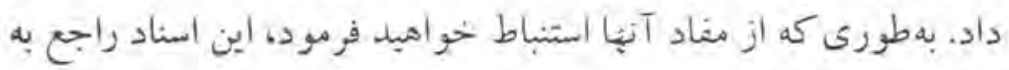

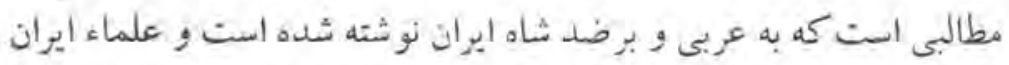

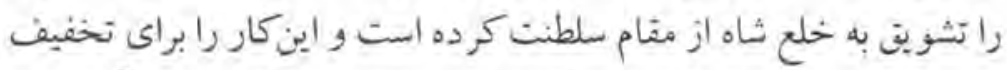

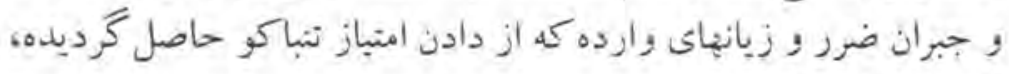

علاج دانسته استى.

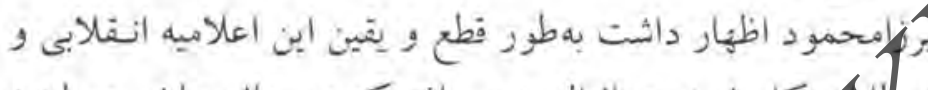

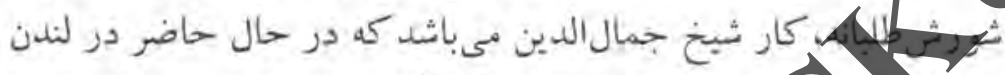

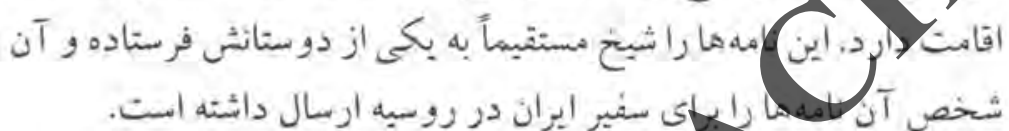

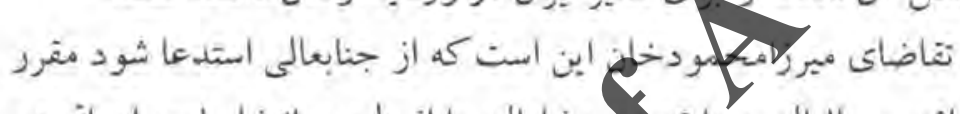

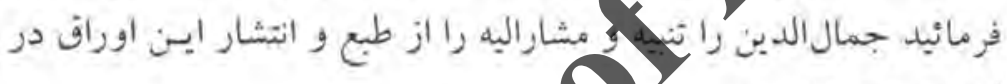

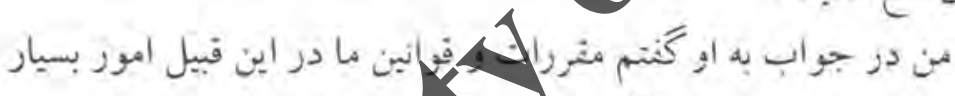

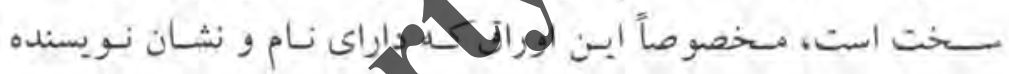

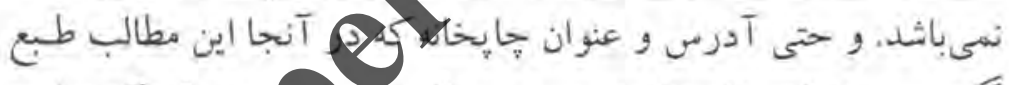

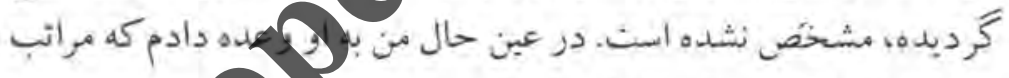
راحضور مباركت عرض خور اهم نمود 2.

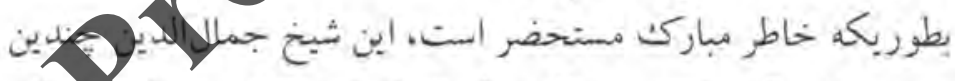

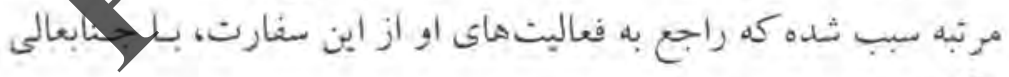
مكاتباتى ردوبدل شود.

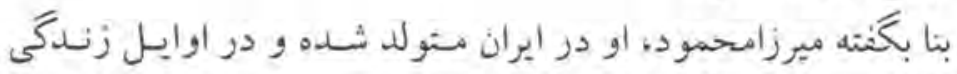

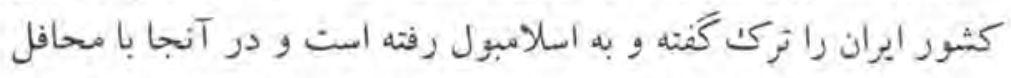

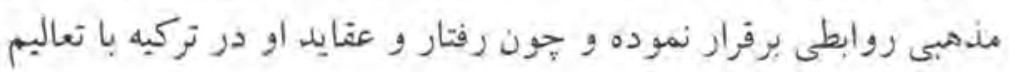

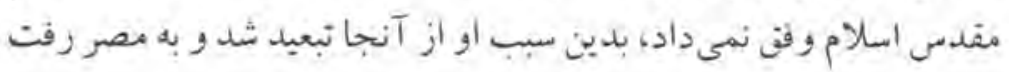




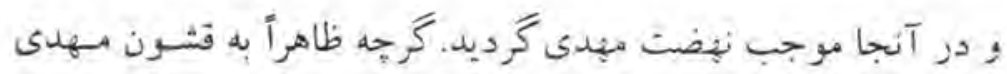

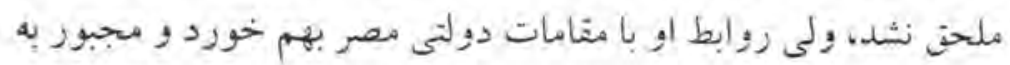

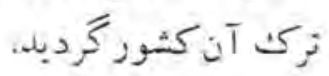

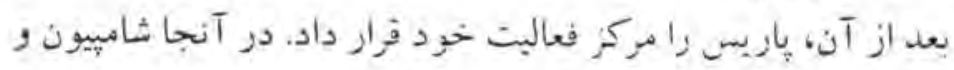

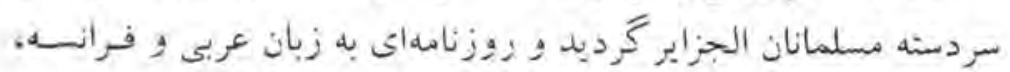

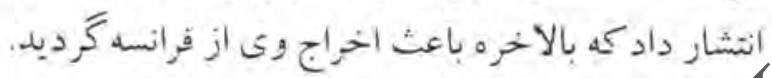

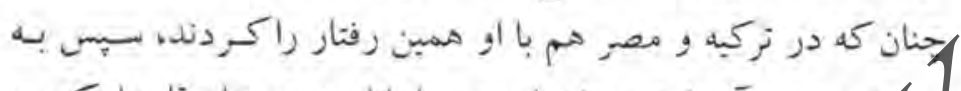

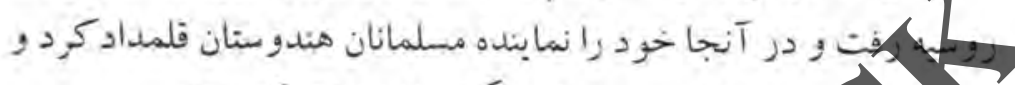

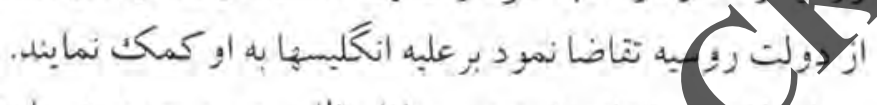

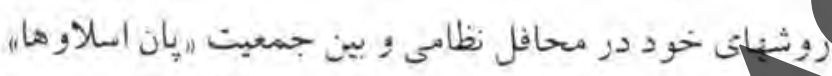

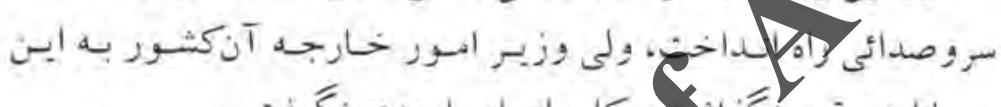

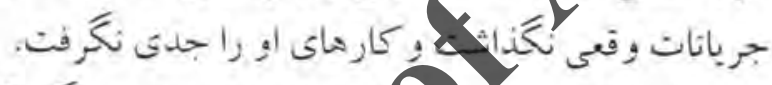

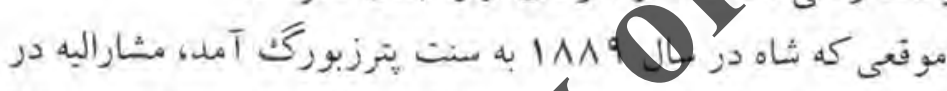

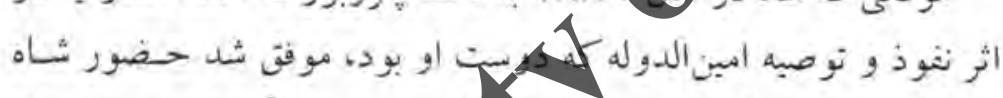

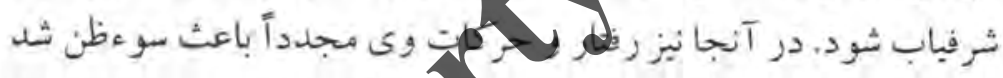

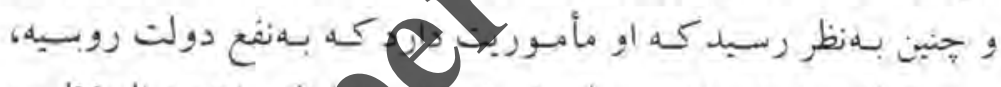

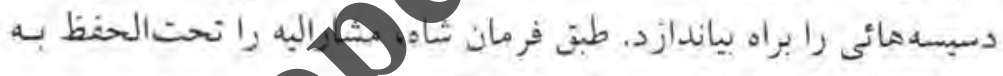

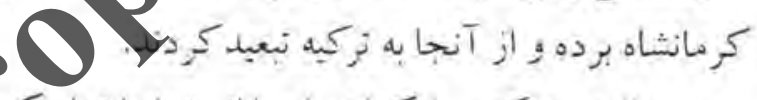

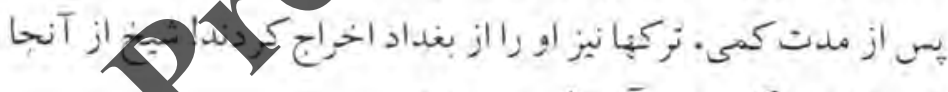

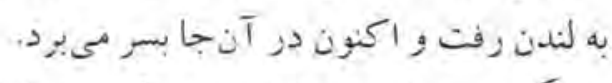

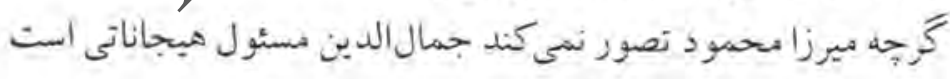

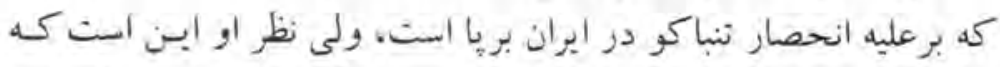

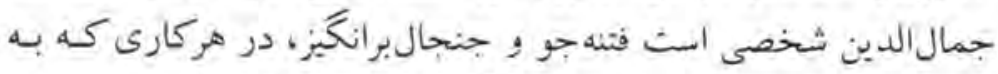

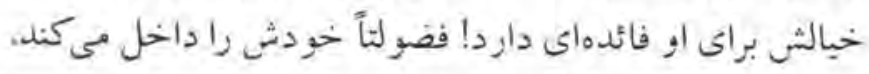

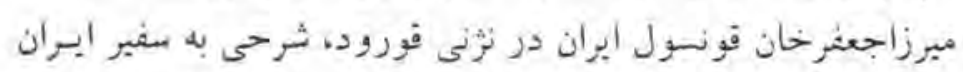




\section{ميد و امتباز تنباكو ما}

نوشته به ار تذكر داده امت. اخبارى راكه جرايد مهم روسيه راجهع بـه

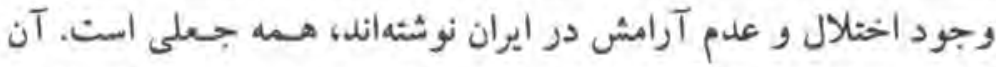

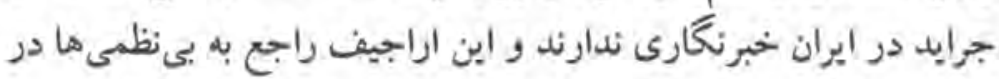

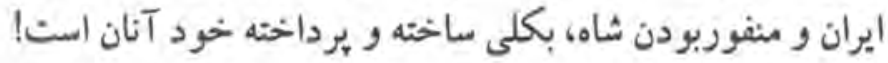

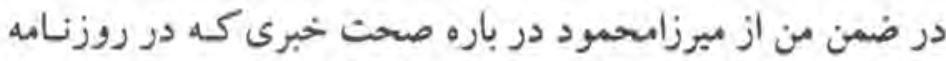
درج شده، داير براينكه روسها يِيشنهاد كردماند نيم مسبليون ليبره

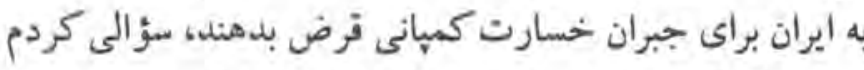

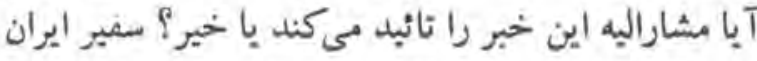

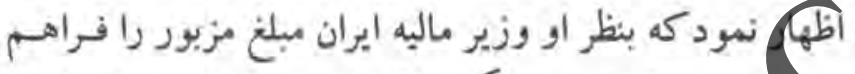

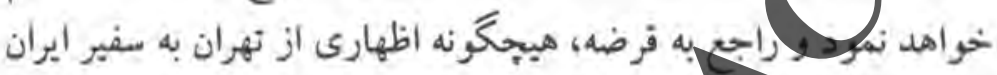
با احترامات - أمضاء مورير

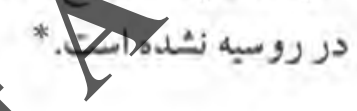

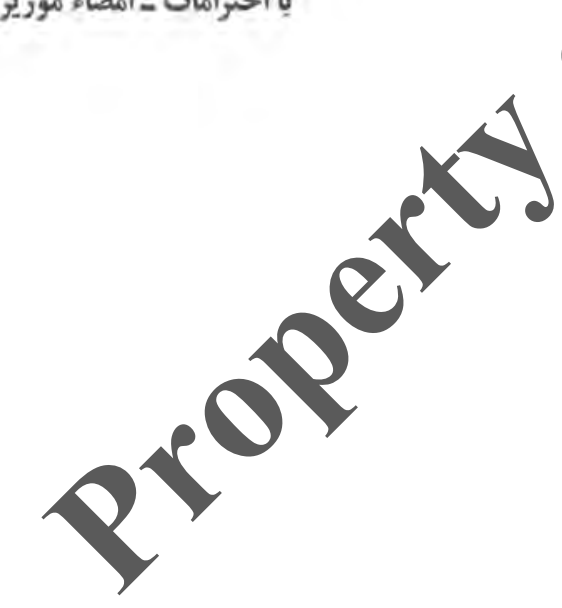

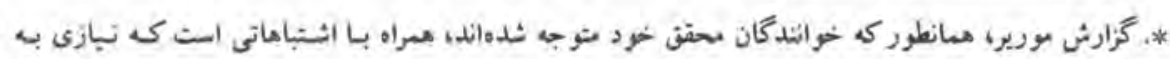

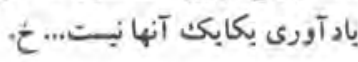




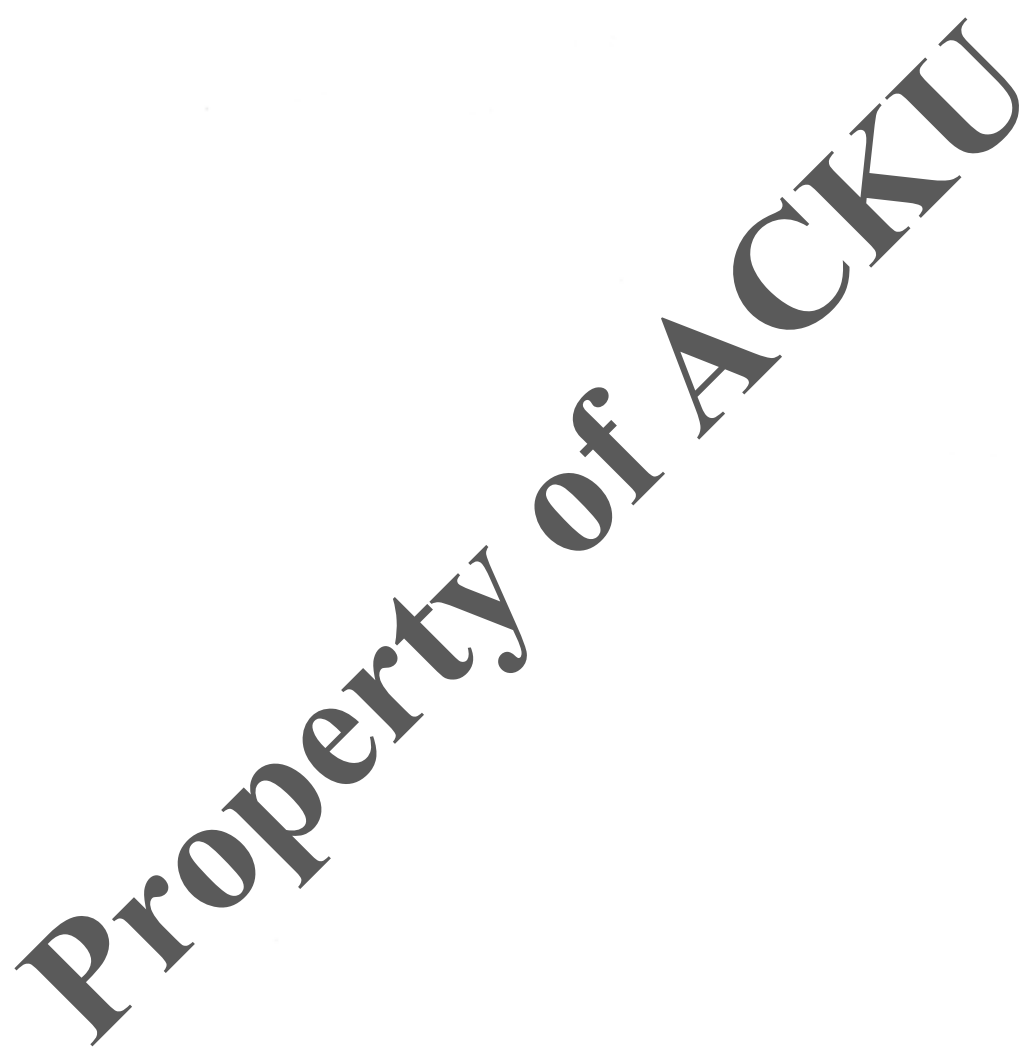




\section{9}

\section{بورخواست ناصرالدينشاه از انتليس!}

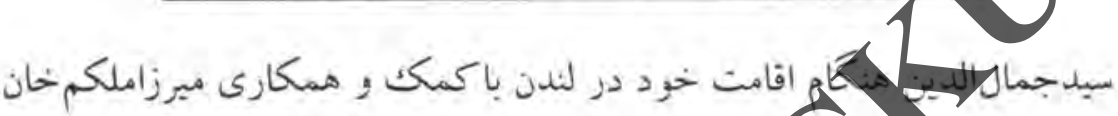

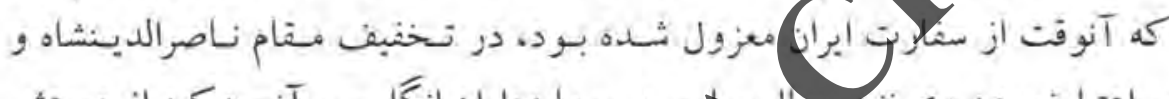

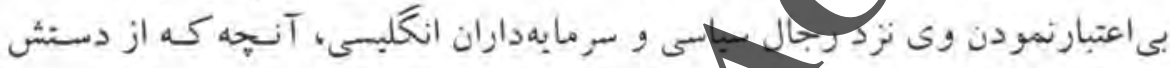

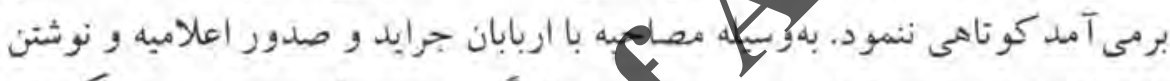

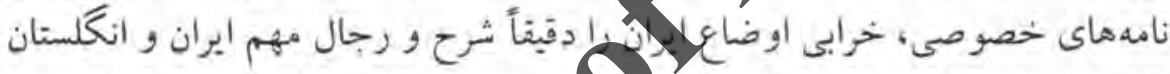

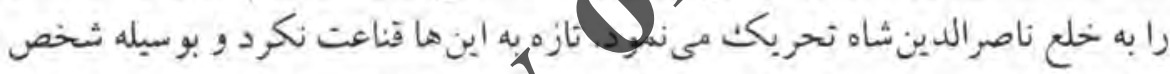

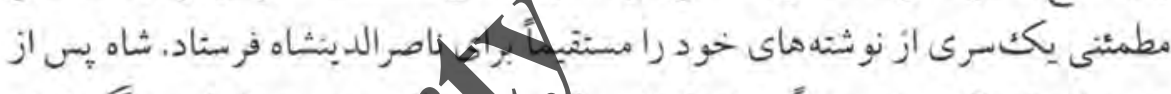

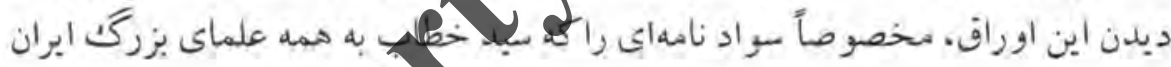

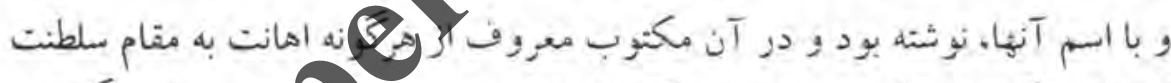

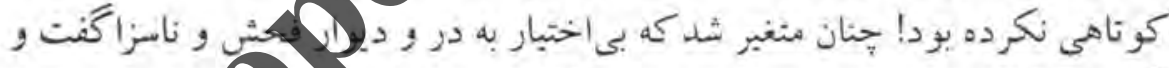

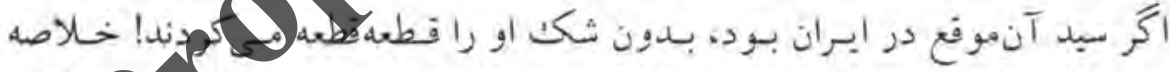

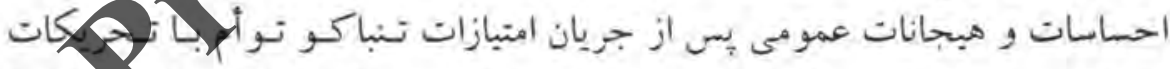

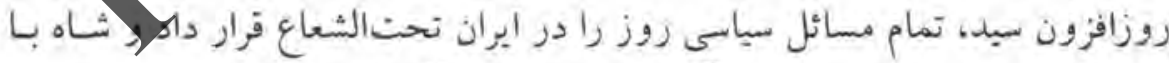

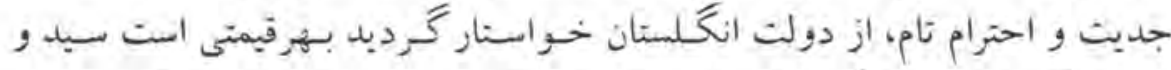

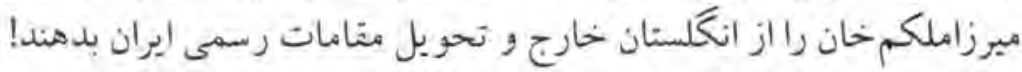

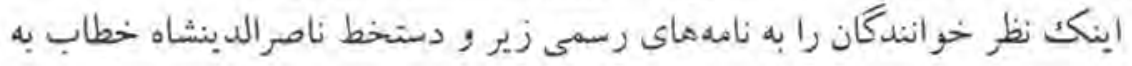

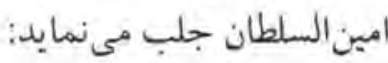




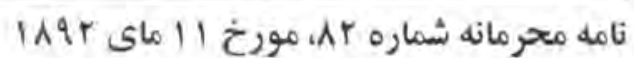

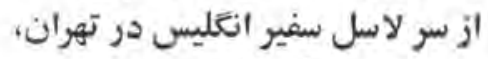

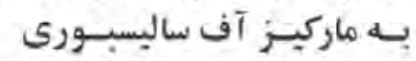

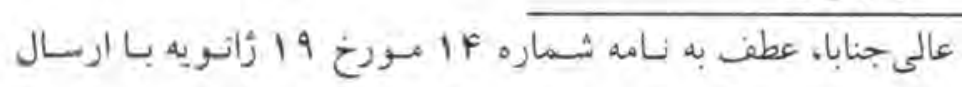

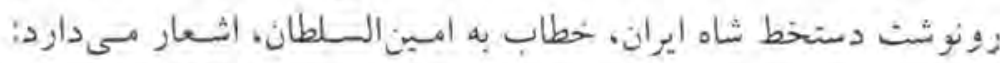

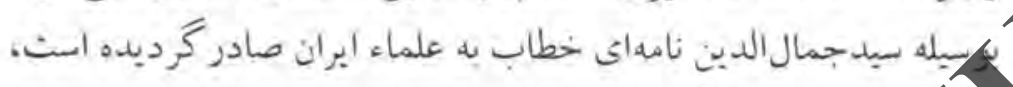

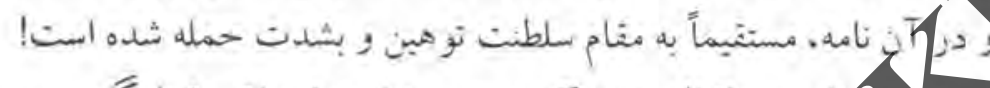

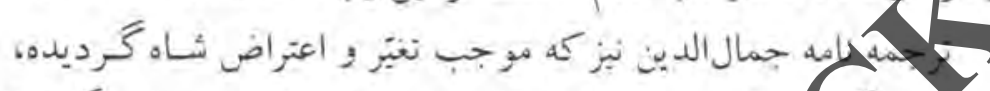

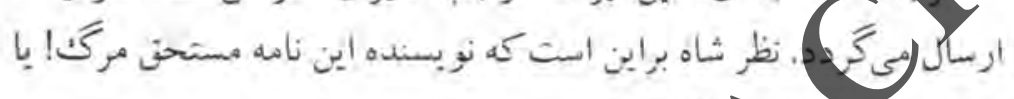

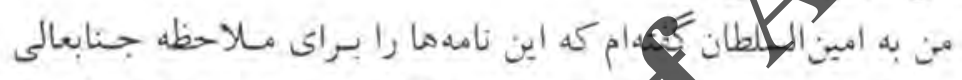

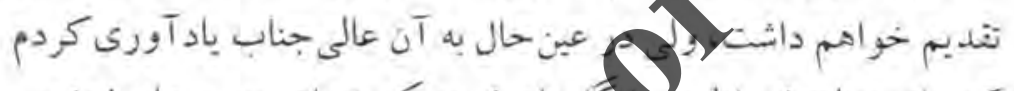

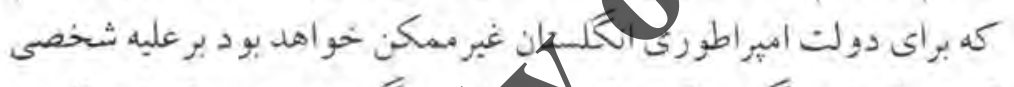

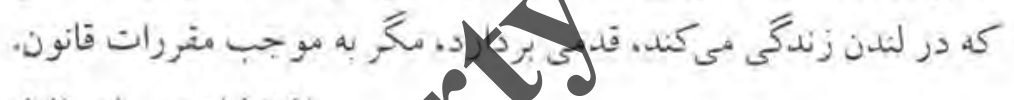
با أحترامات سر ـف - لاسل

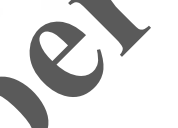

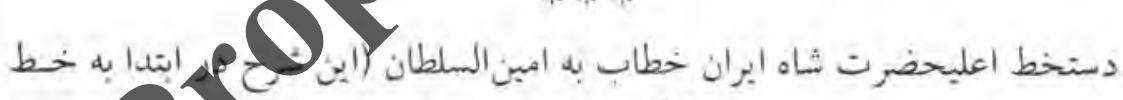

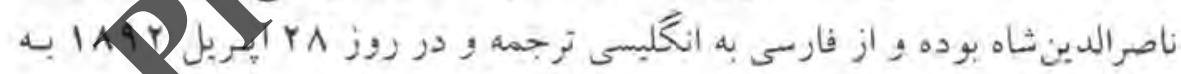

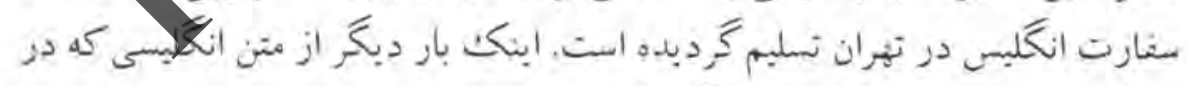

$$
\text { آرشيو دولثى است، به فارسى نتل كر ديد): }
$$

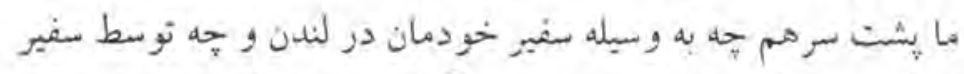

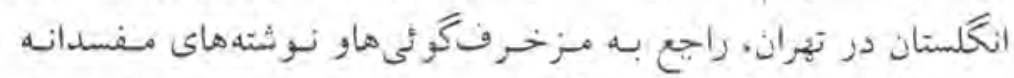

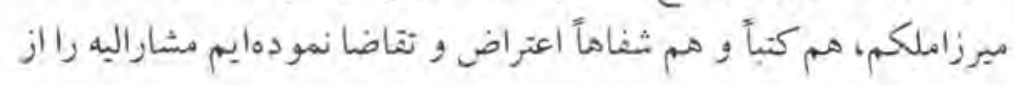




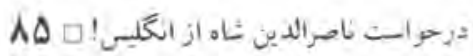

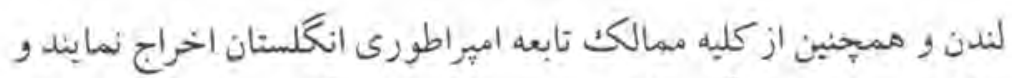

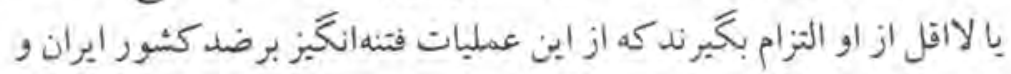

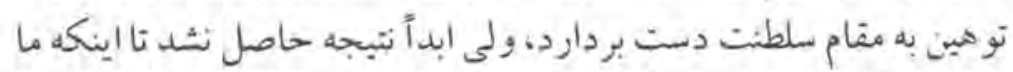

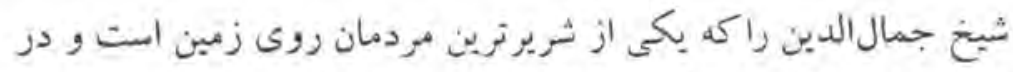

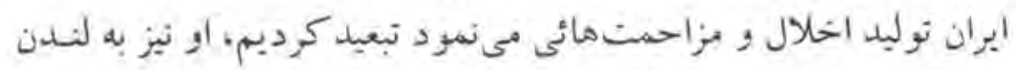

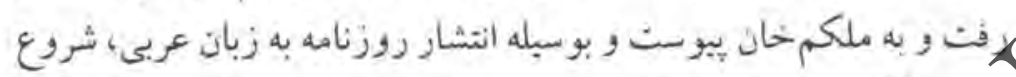

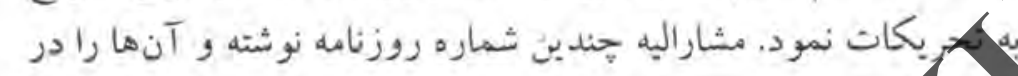

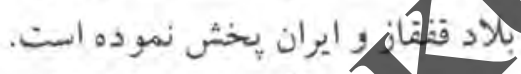

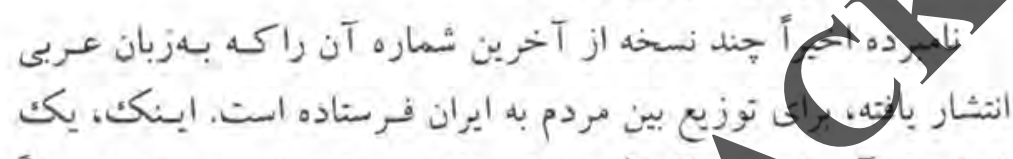

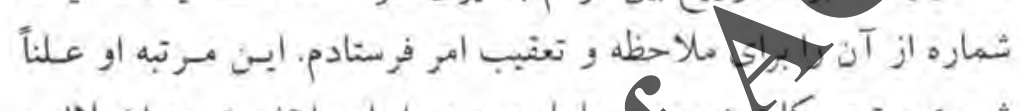

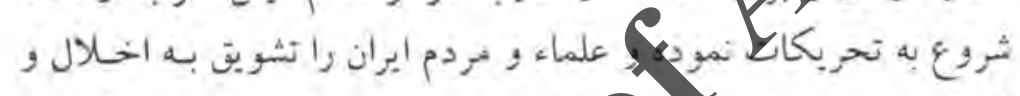

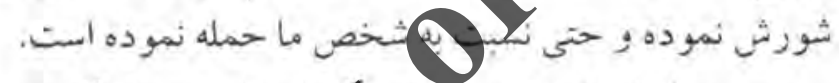

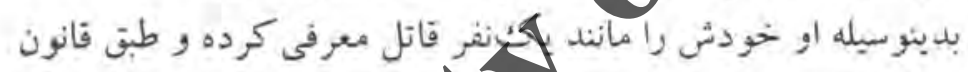

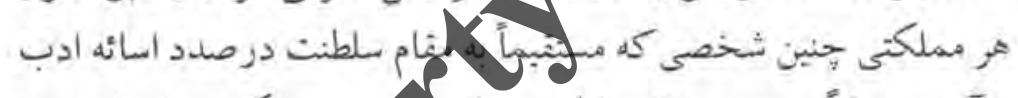

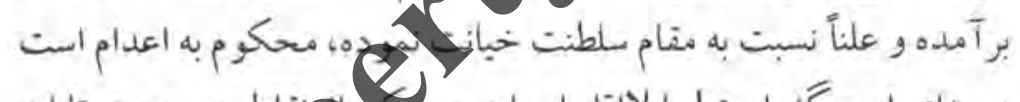

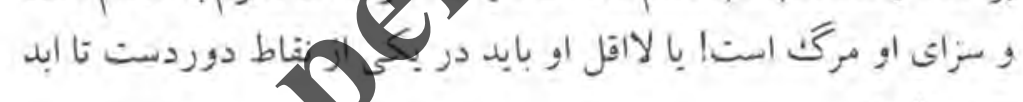
حبس شوند

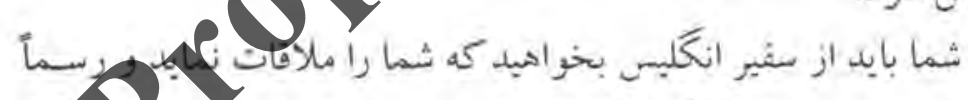

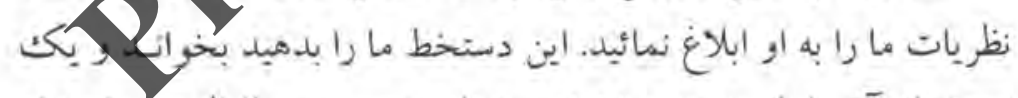

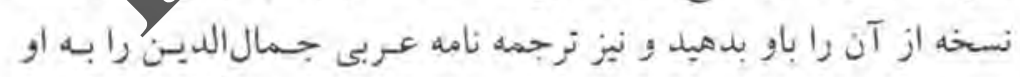

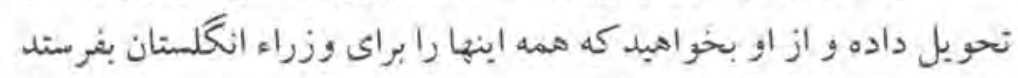

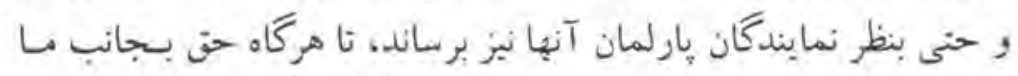

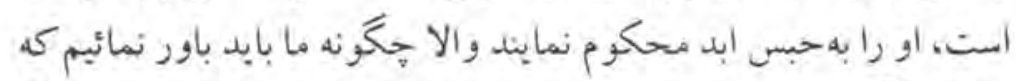

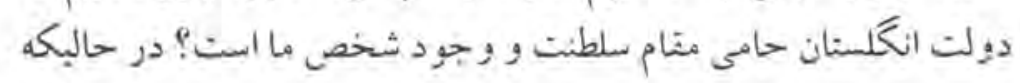




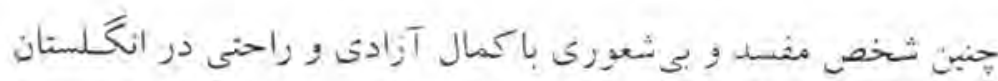

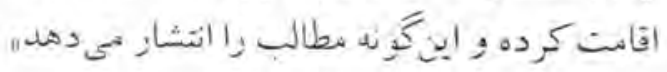

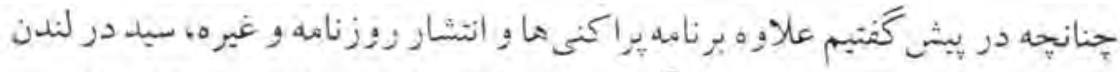

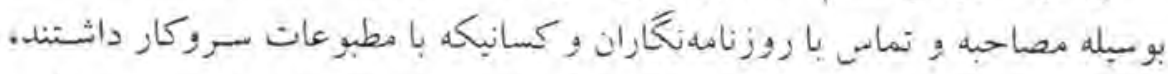

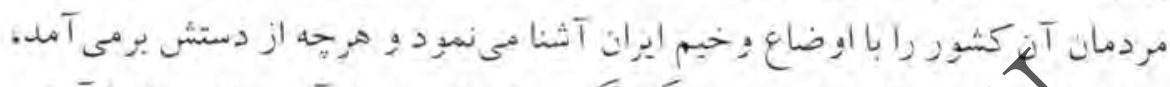

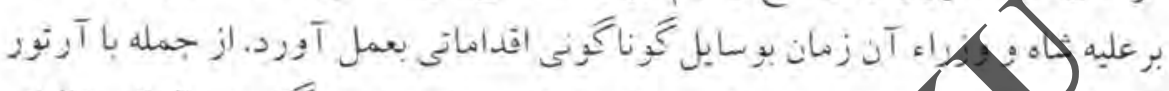

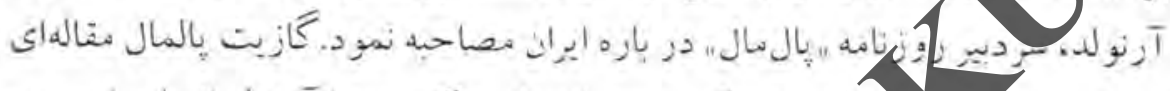

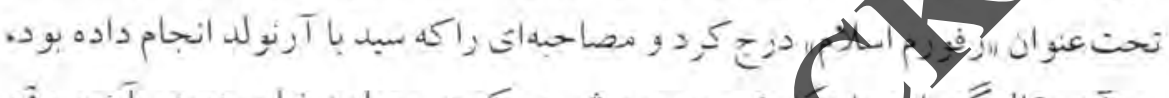

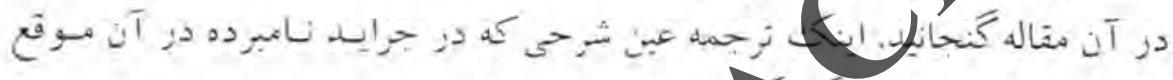

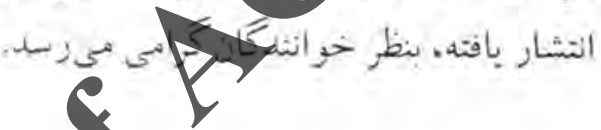

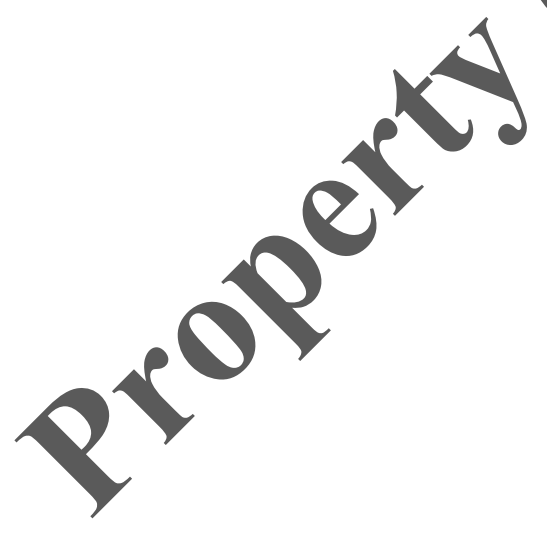




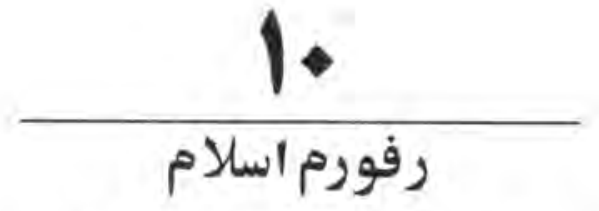

مصاحبه سيد با آرتود ـ آرنولد

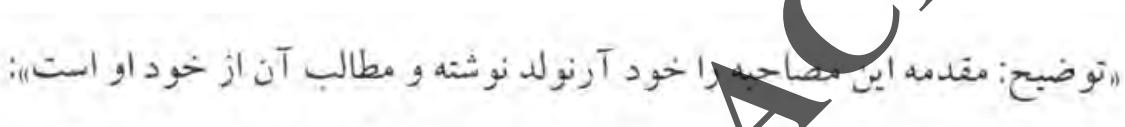

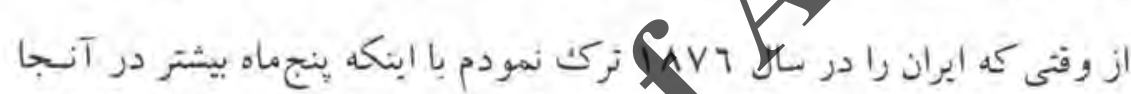

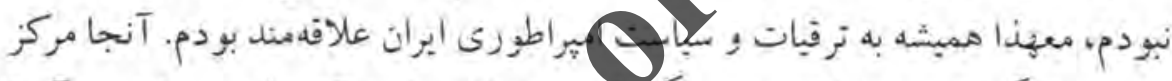

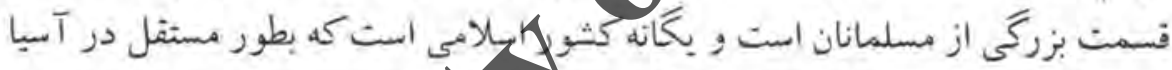

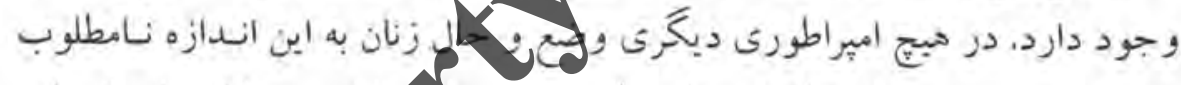

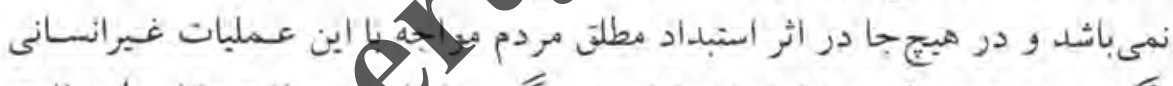

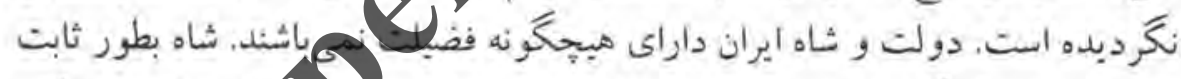

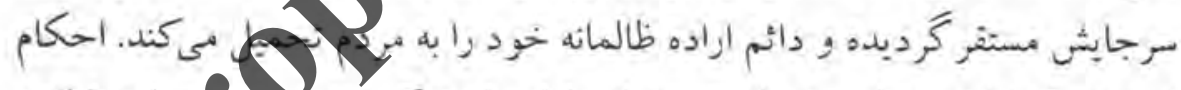

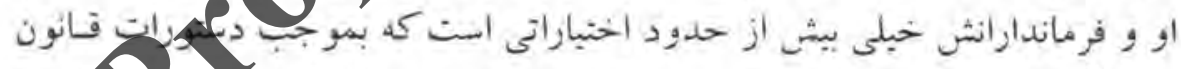

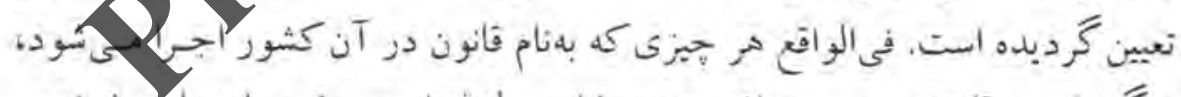

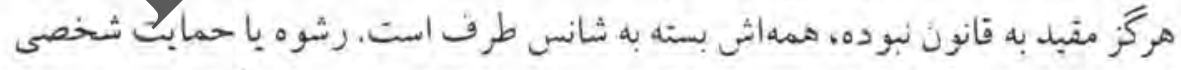

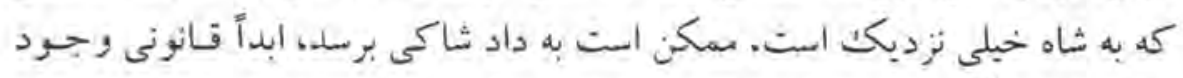

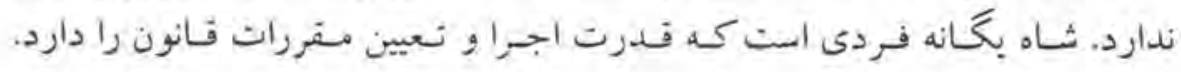

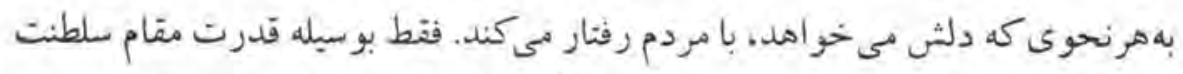

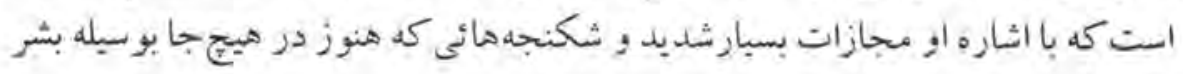




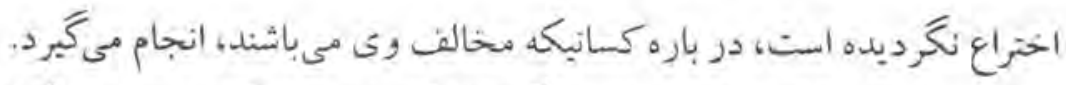

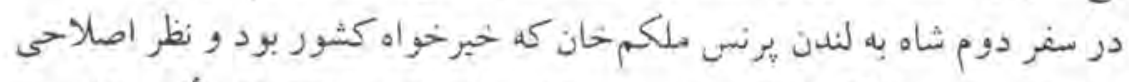

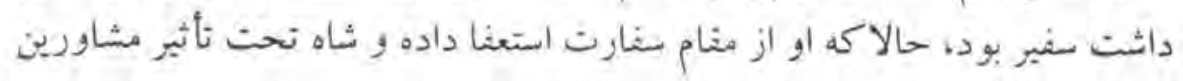

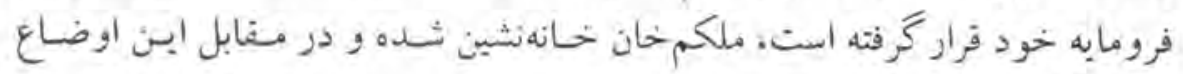

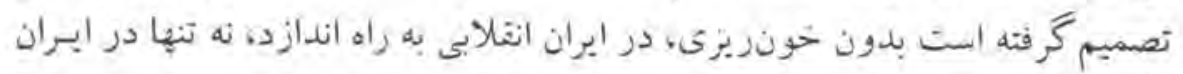

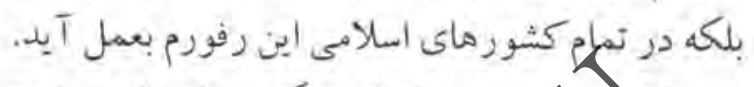

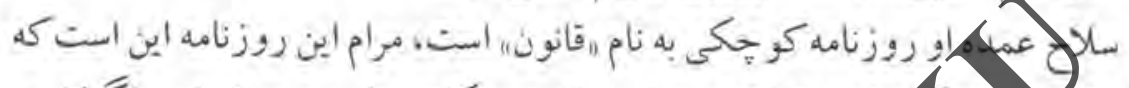

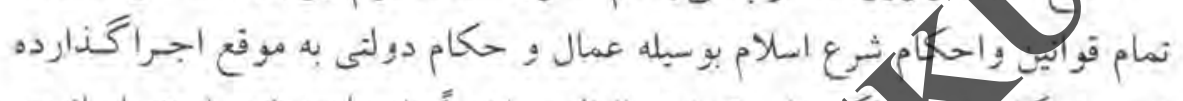

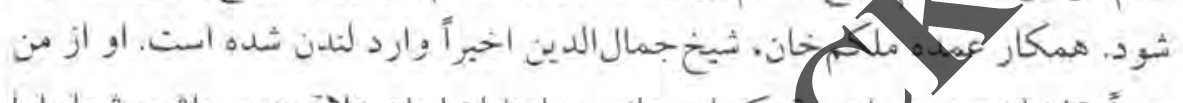

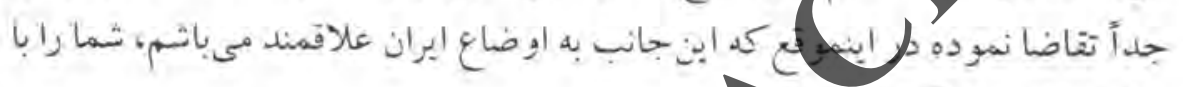

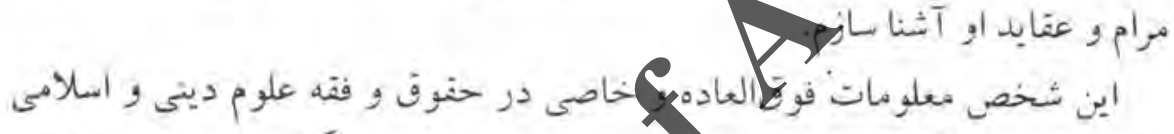

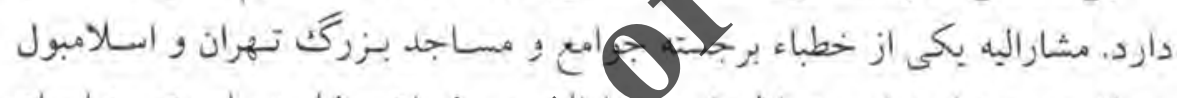

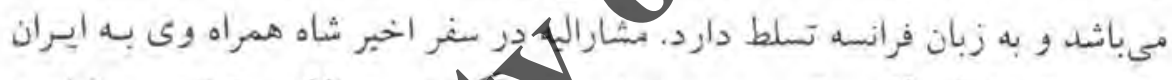

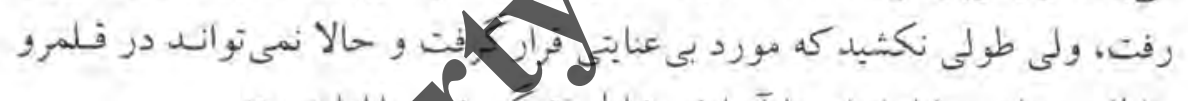

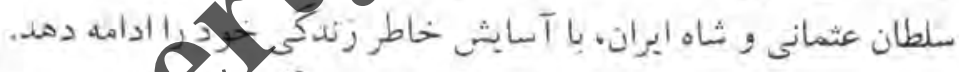

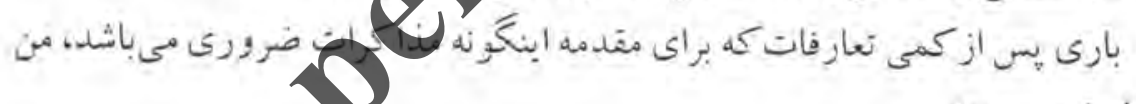

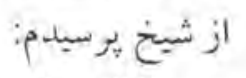

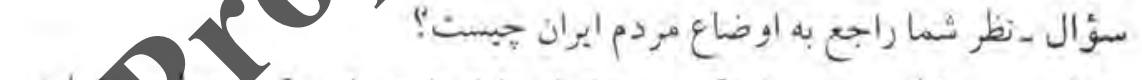

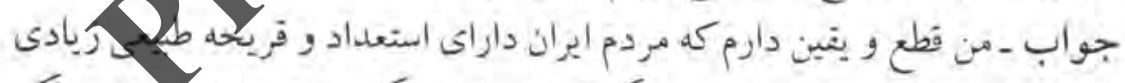

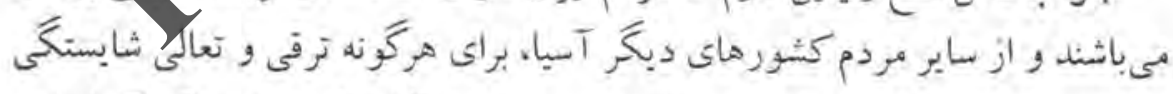

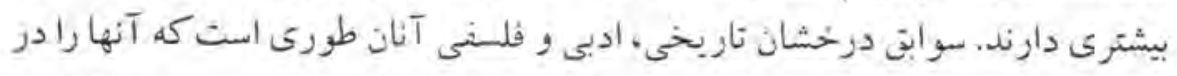

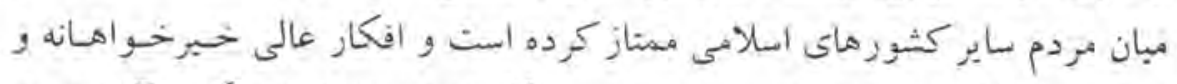

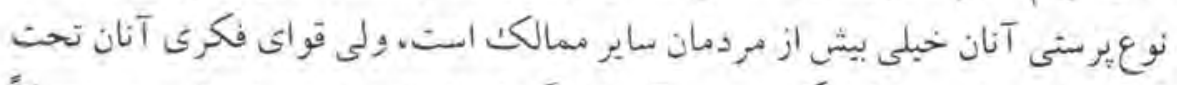

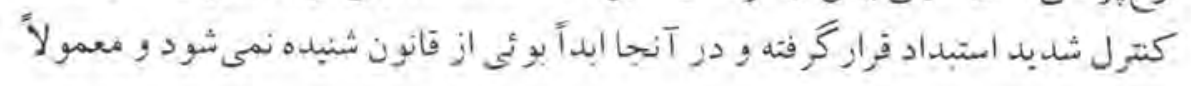




\section{رقورم اسلام}

بلهور كاملاًُ وحشيانه با آنان رفتار مىثود و آزادى عمل از دست آنهاكرفته شده.

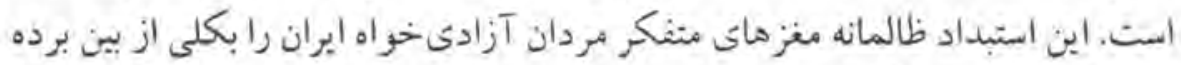

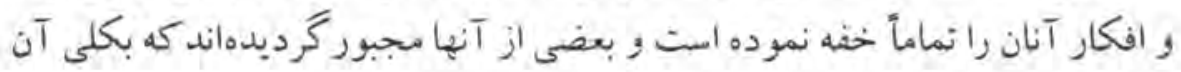

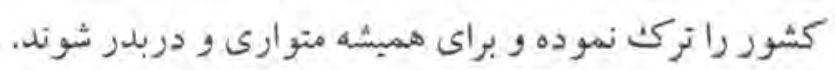

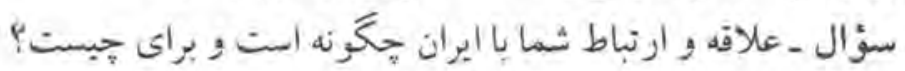

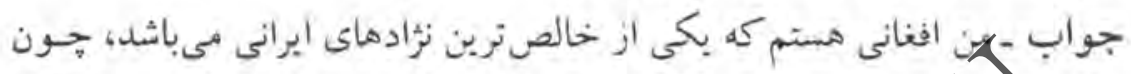

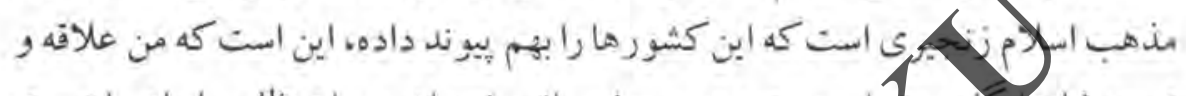

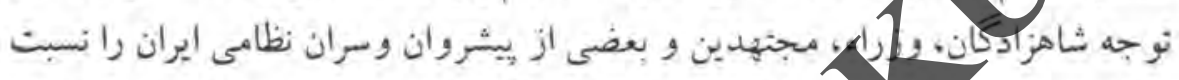

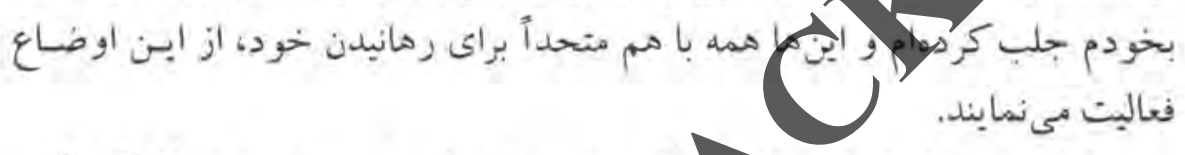

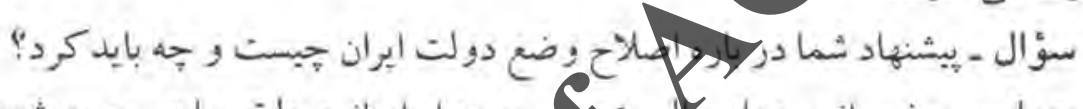

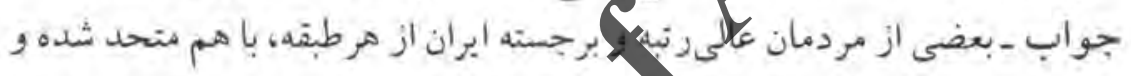

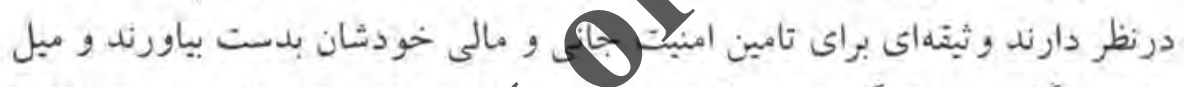

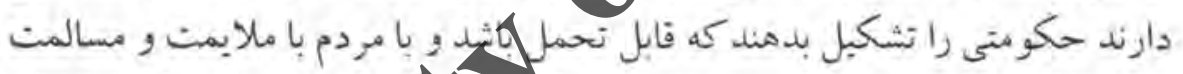

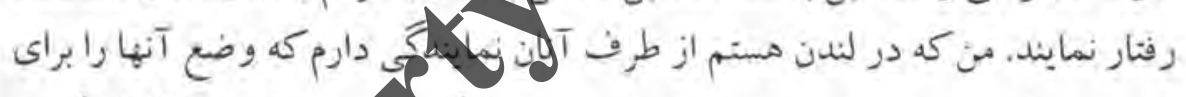

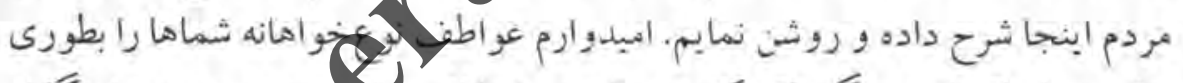

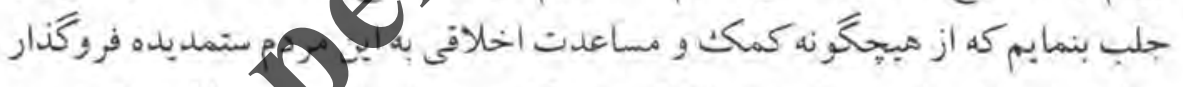

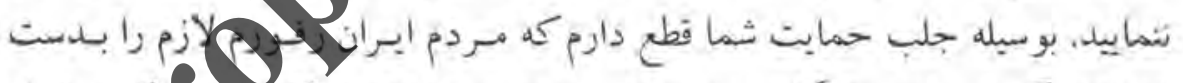

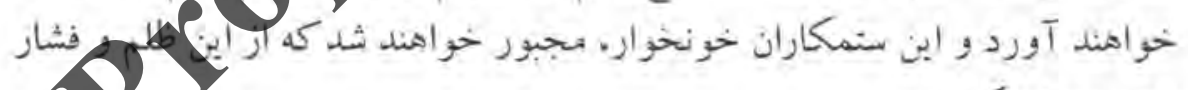
خود دمنت بكشند.

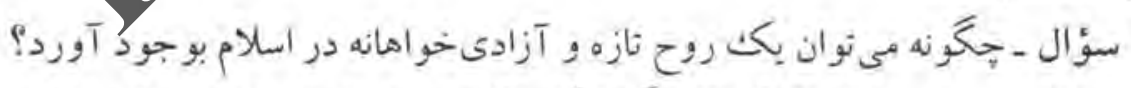

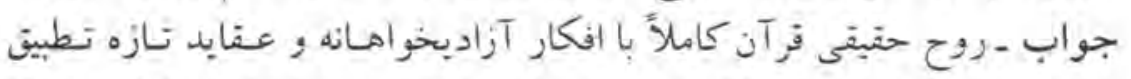

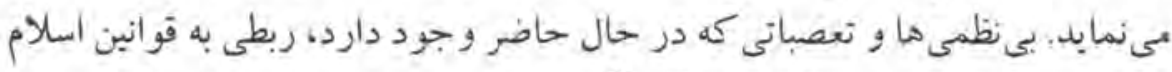

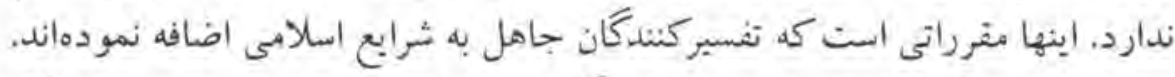

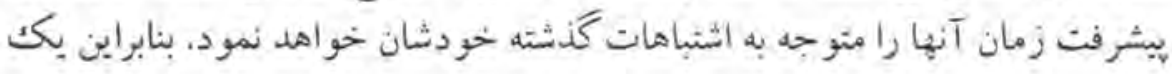




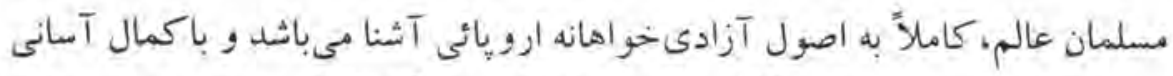

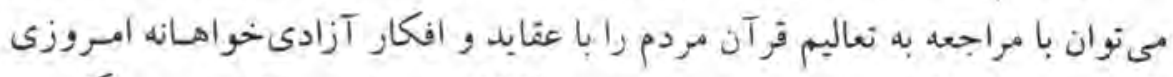

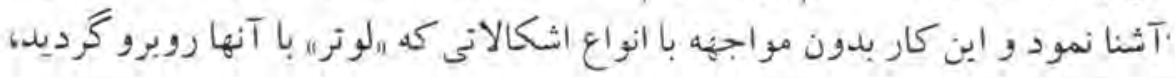

$$
\text { عملى خواهد شند. }
$$

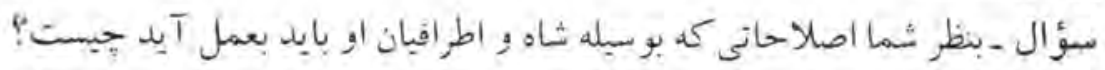

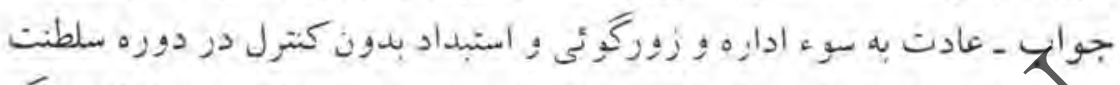

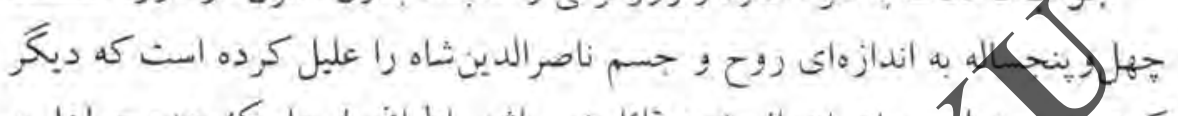

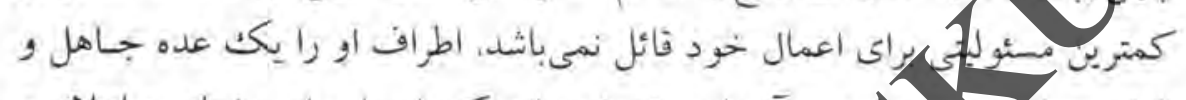

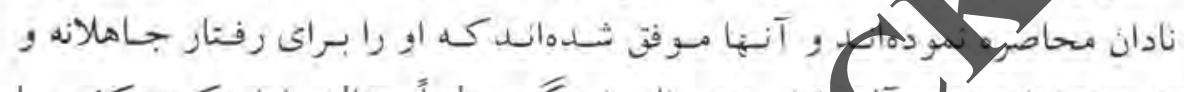

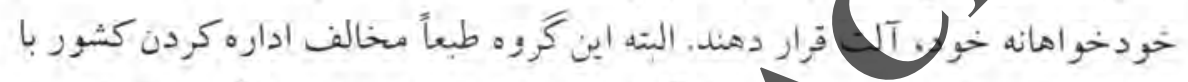

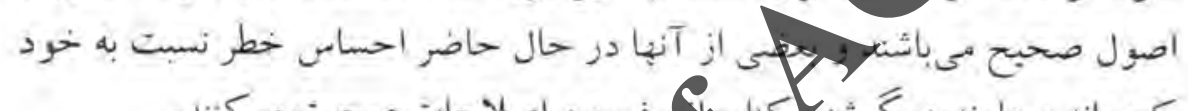

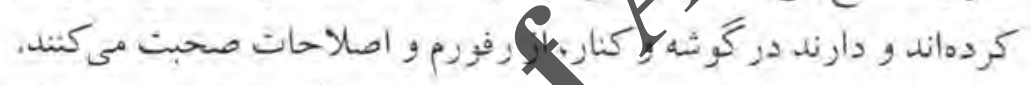

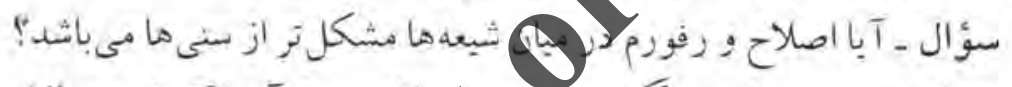

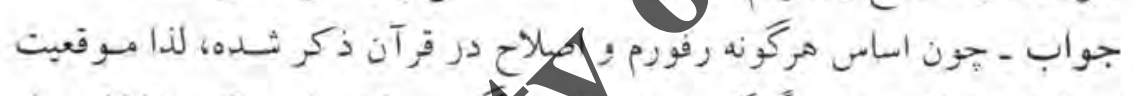

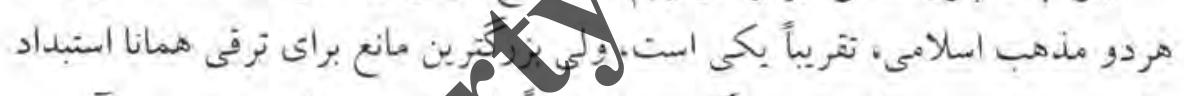

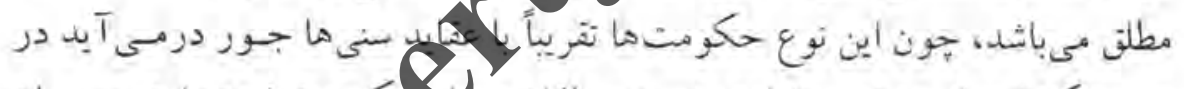

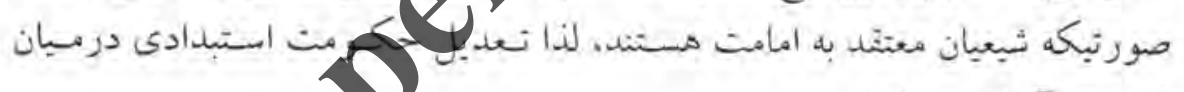

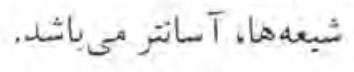

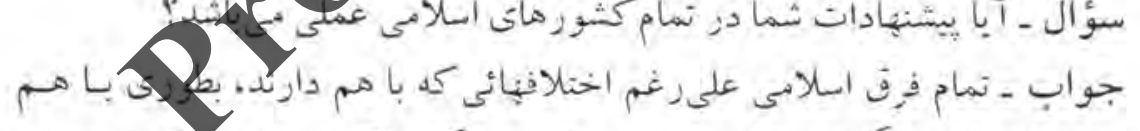

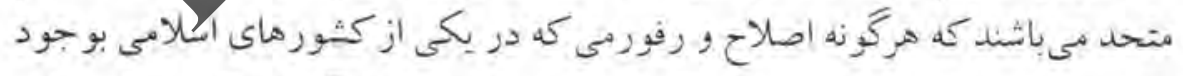

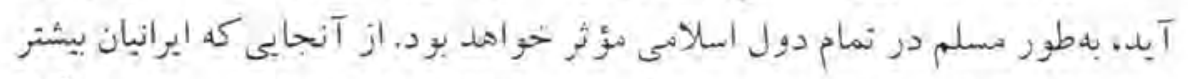

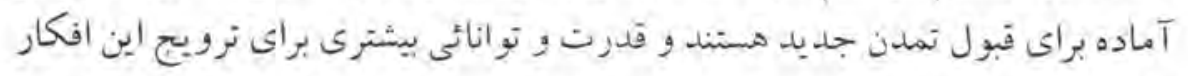

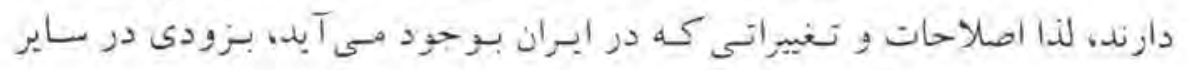

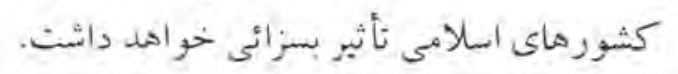




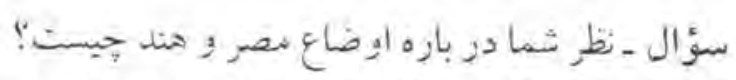

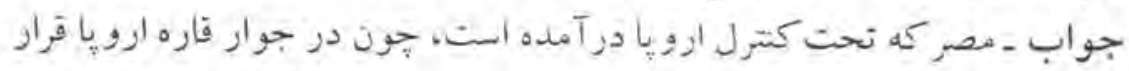

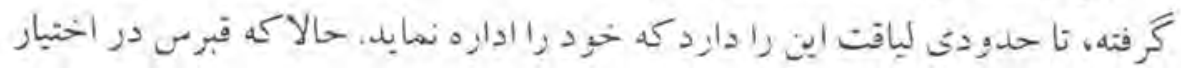

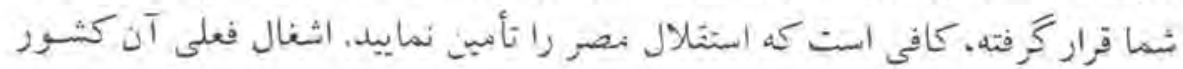

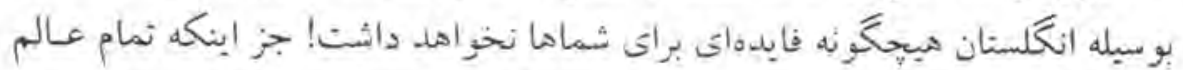

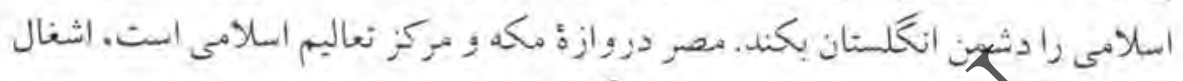

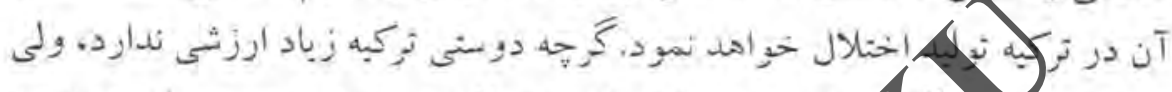

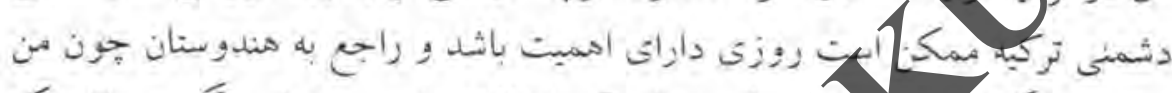

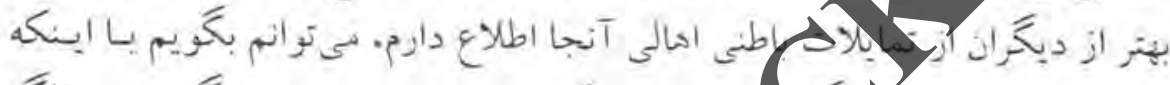

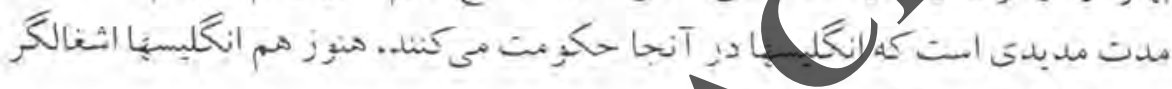

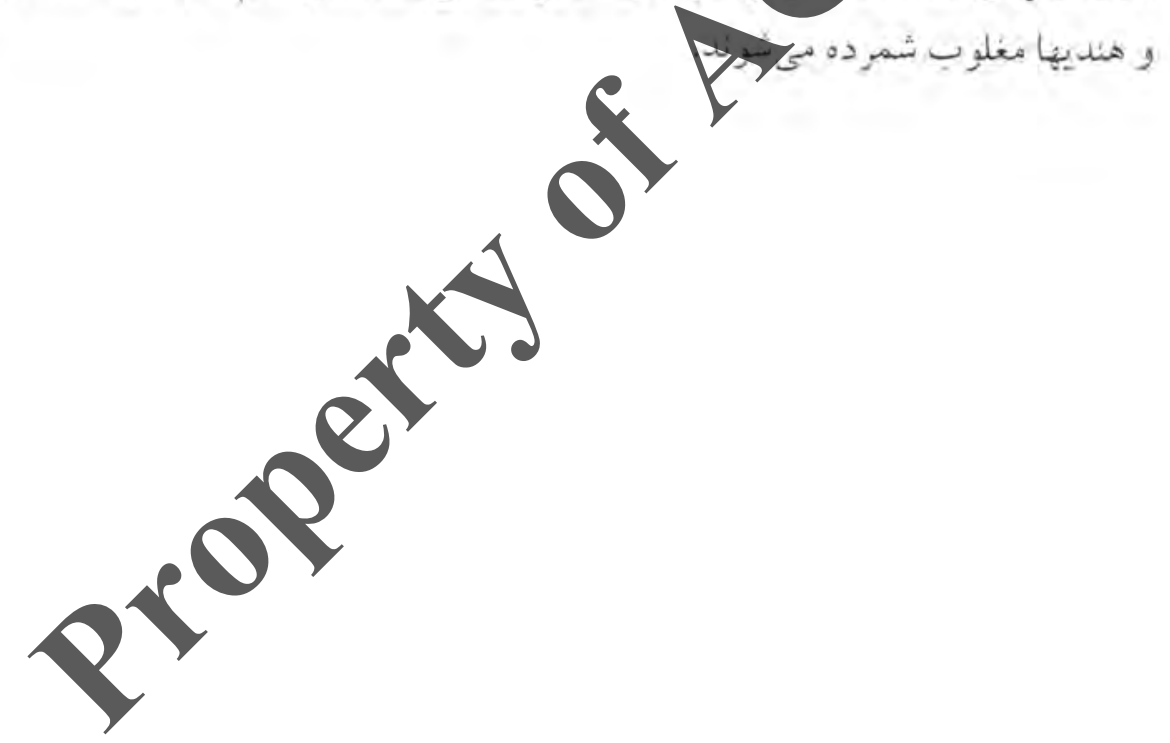




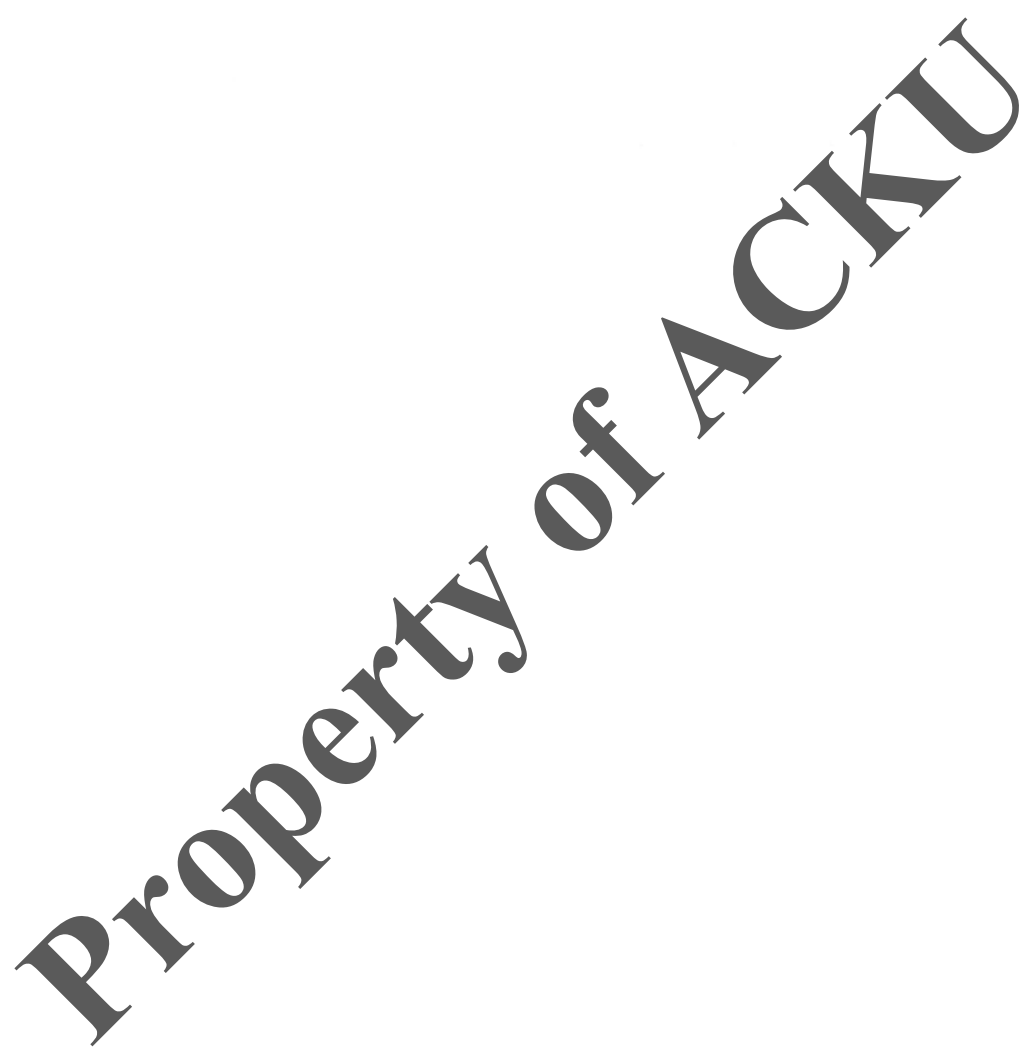




\section{1}

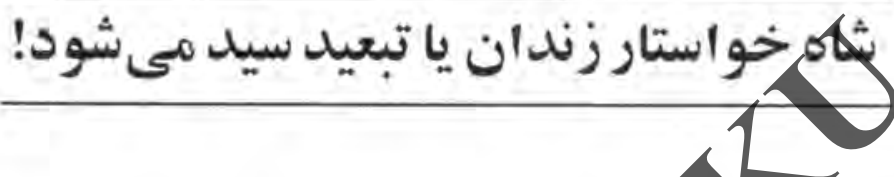

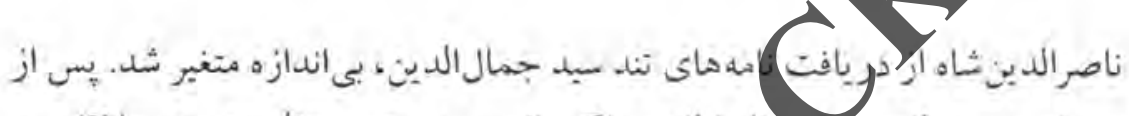

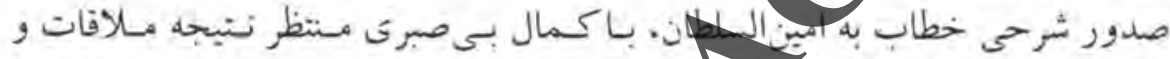

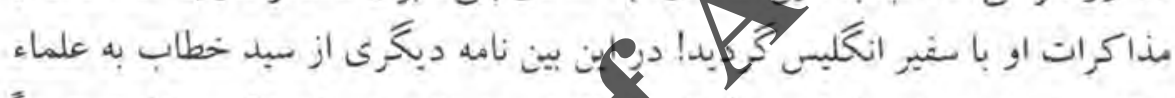

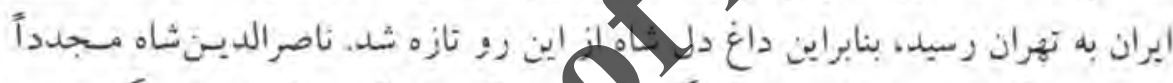

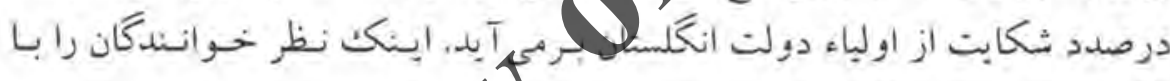
مكاتبات در اين رابطه جلب مى دئمايد.

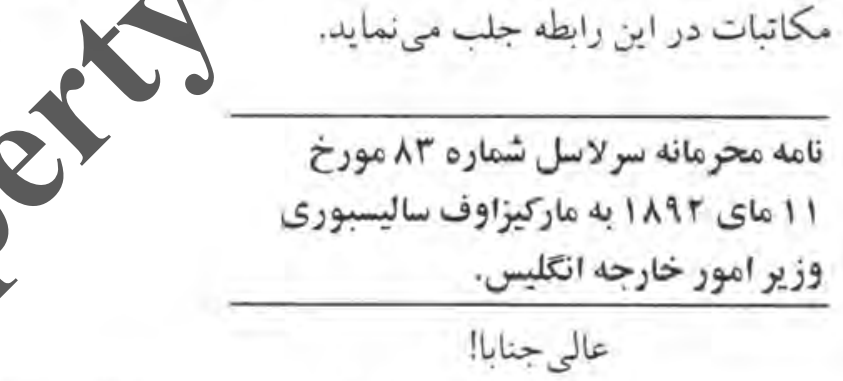

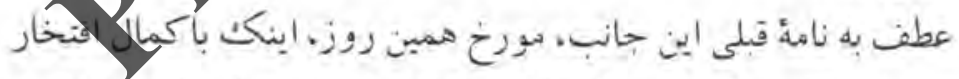

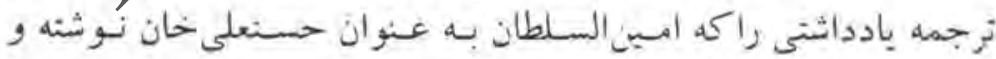

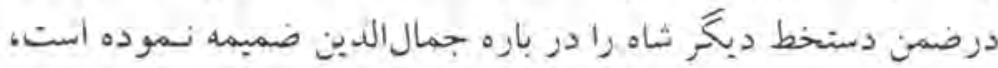

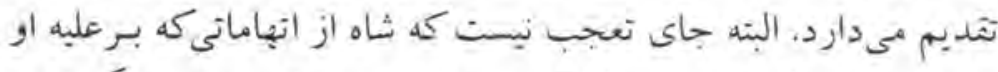

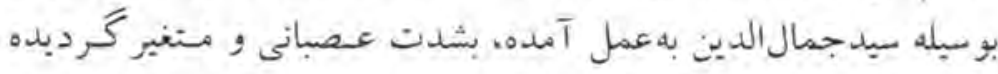

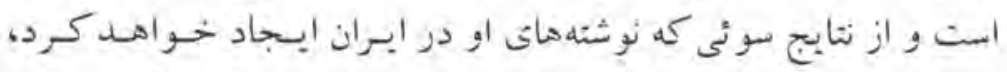

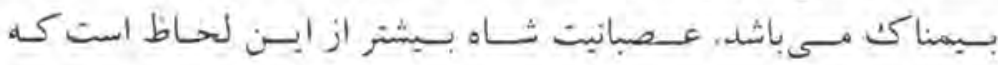


سبدجمال الدين حنائتى را در باره اوضاع فعلى كثور ايروان فاش نموده

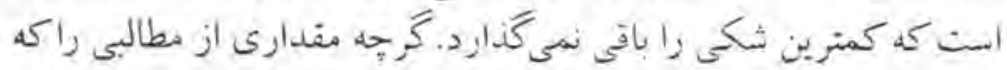

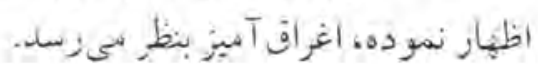

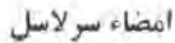

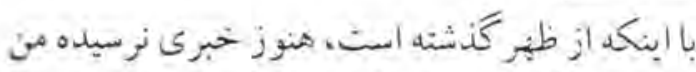

ترجمأ رونوشت نانه خصوصى امين السلطان

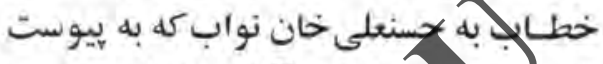

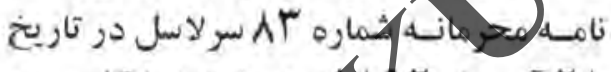

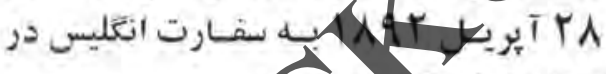

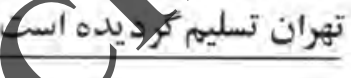

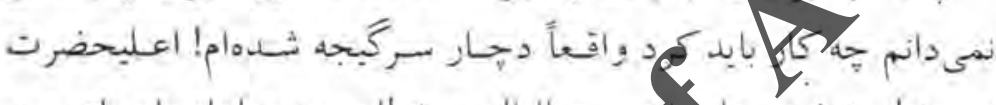

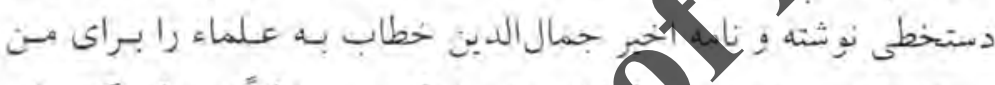

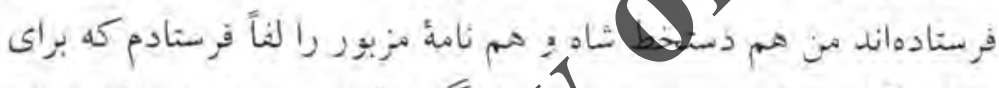

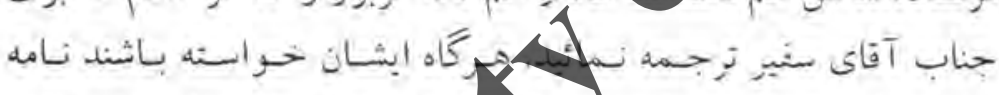

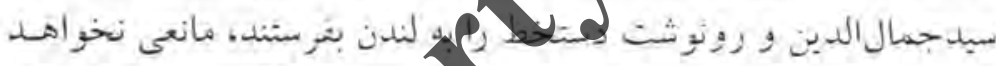

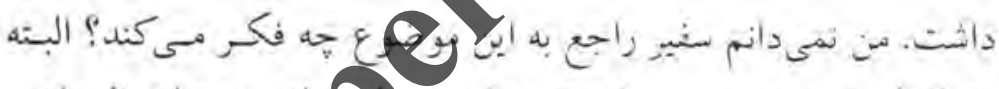

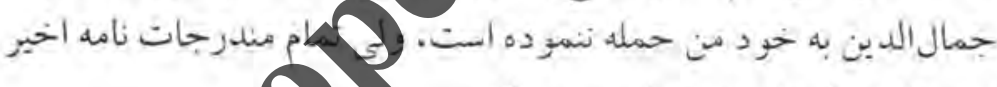

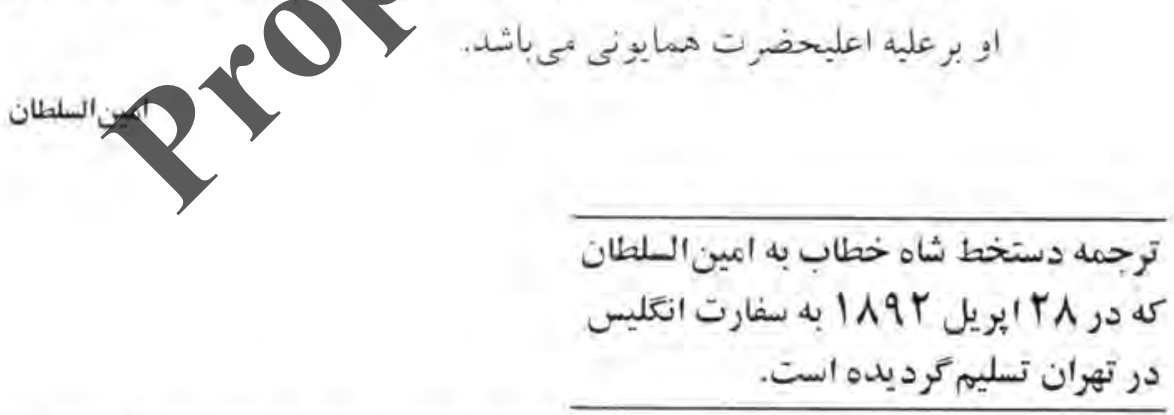

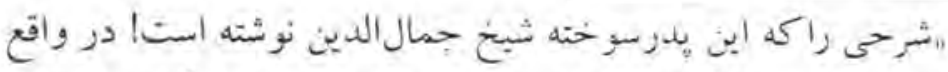

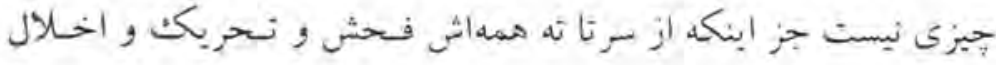




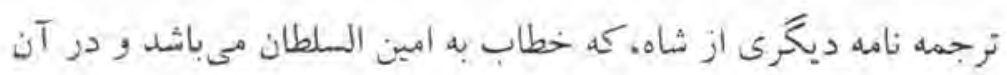

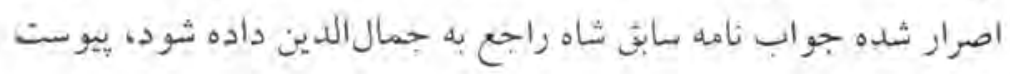

$$
\text { تخدبم مى دازد. }
$$

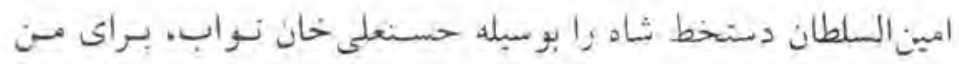

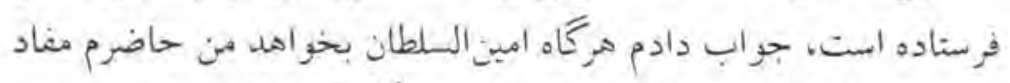

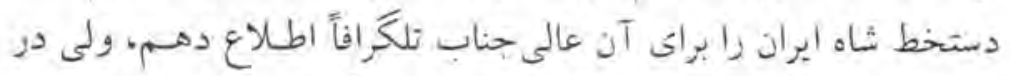

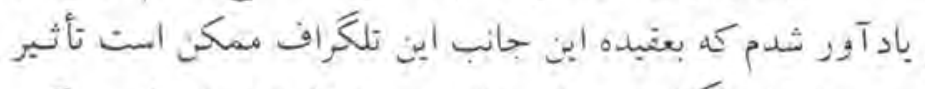

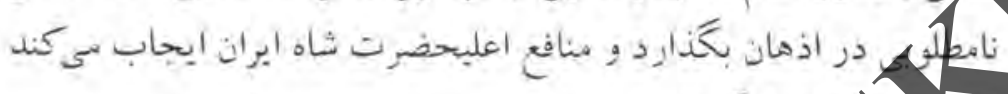

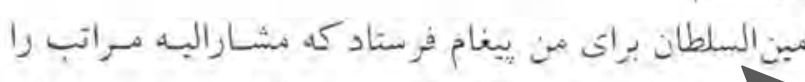

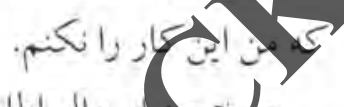

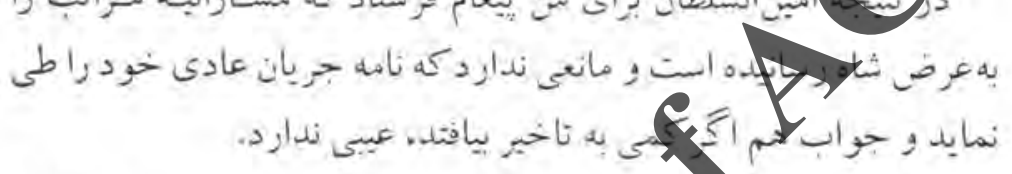

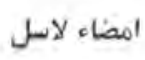
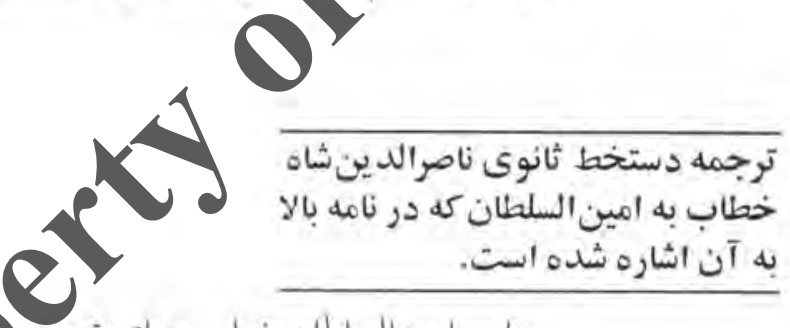

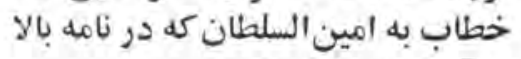

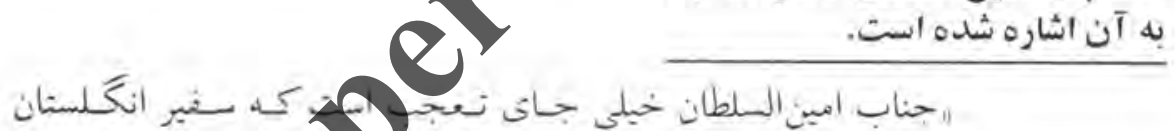

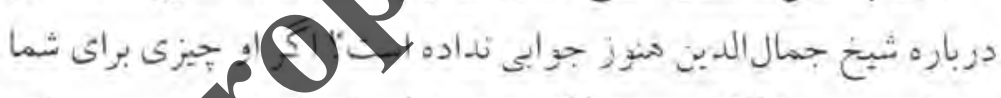

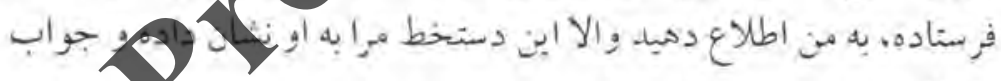
بخواهيد.

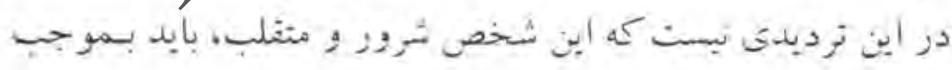

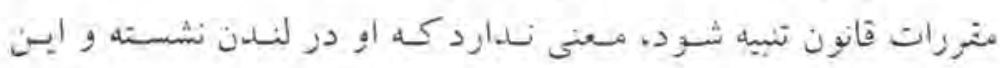

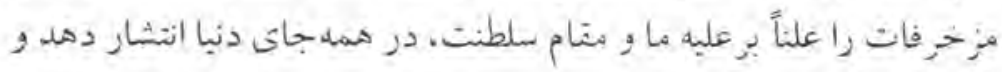

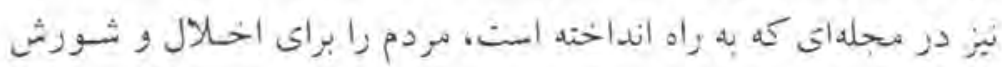

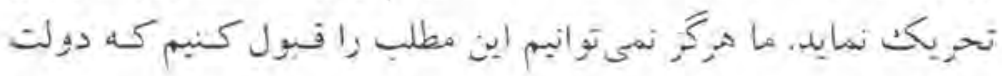




\section{Qناه خواستائز زينان يا تعبد سيد ميثودا1}

انكلمشتان دوست ما مى باشد با حامى منام سلطنت ما است با اينكه هبج كارى را نمى خواهند صورت بدهند و به اين شخص اجازه مى مدهند اين

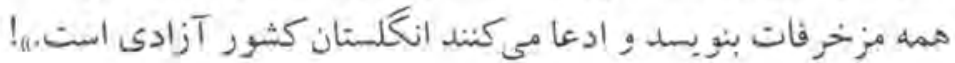

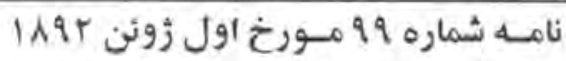

$$
\begin{aligned}
& \text { سر لاسل بعنوان وزير أمور خارجه انعلستان }
\end{aligned}
$$

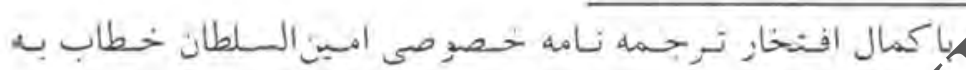

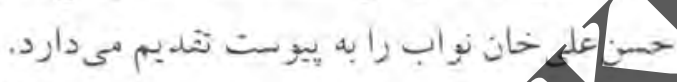

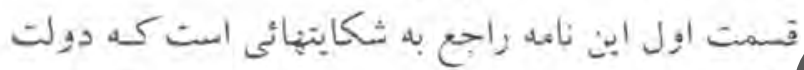

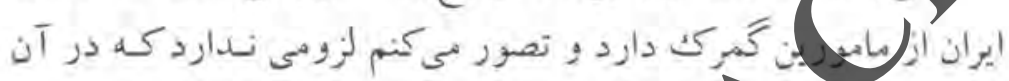

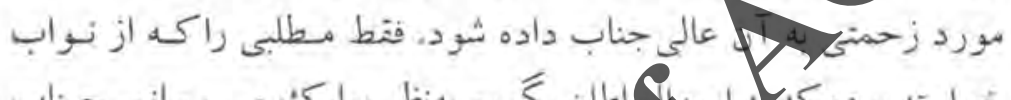

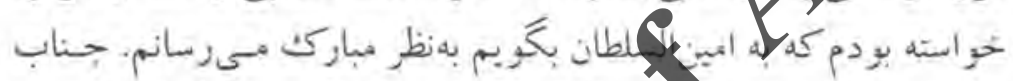

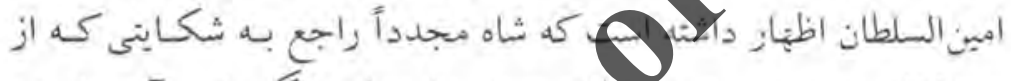

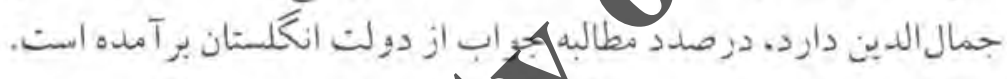

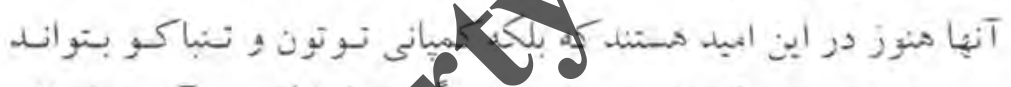

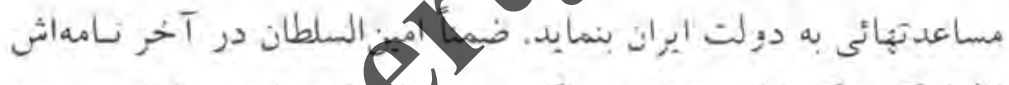

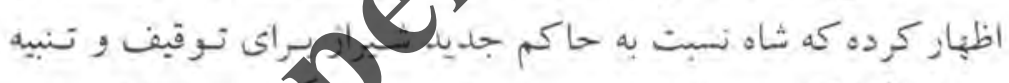

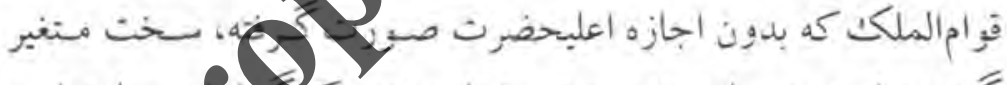

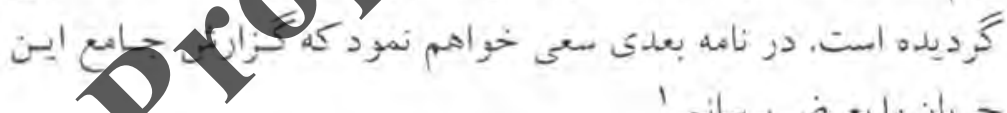

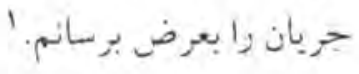

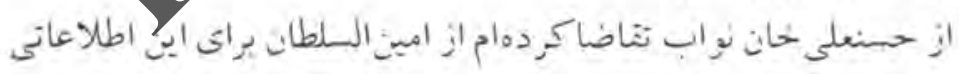

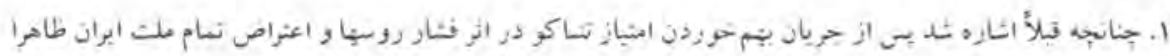

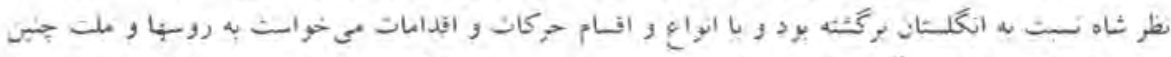

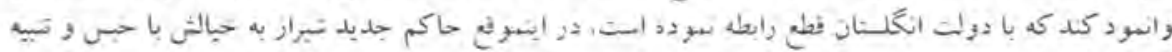

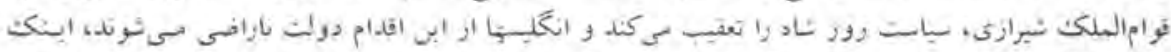

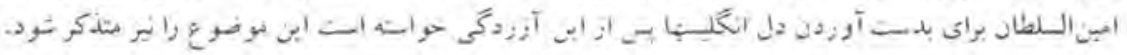


كم دز اختيار ماكنارارده است از جانب مد نشكر بنمابد و به ايشان بكوبد

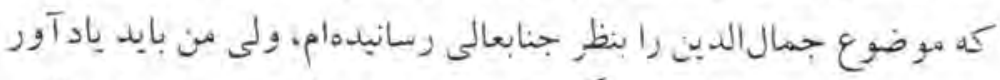

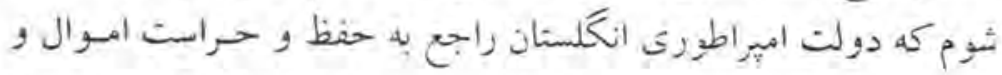

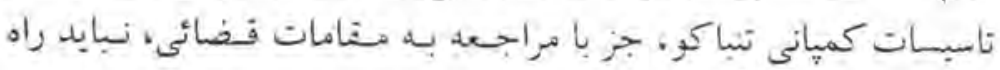

$$
\text { د بـ }
$$

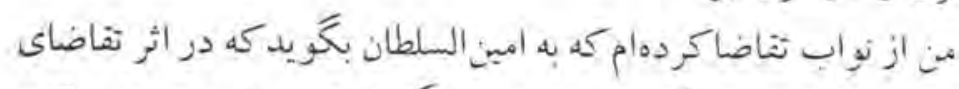

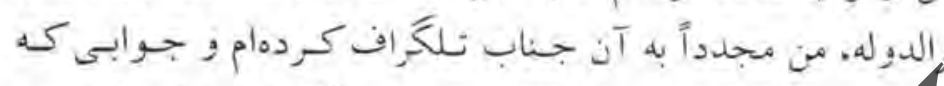

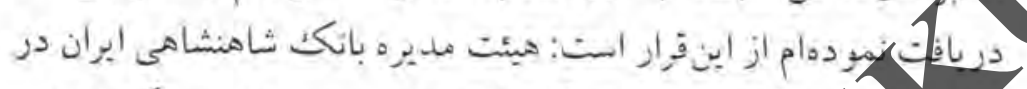

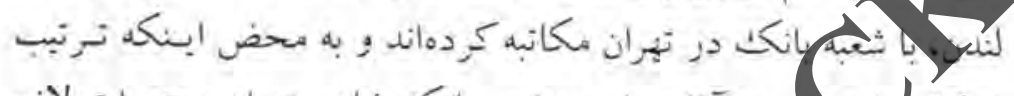

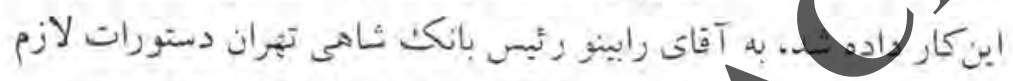

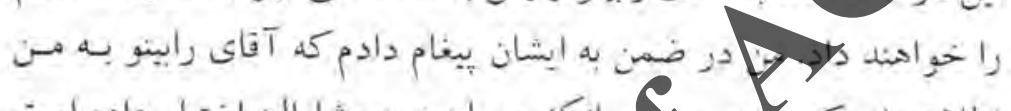

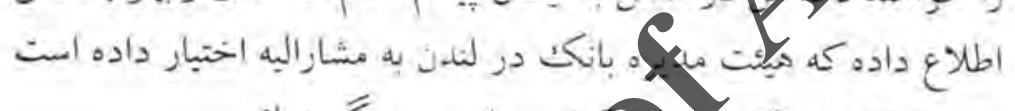

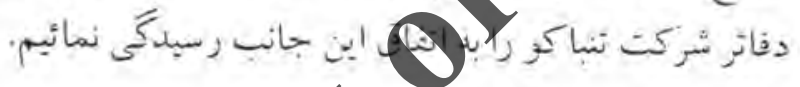

امقاء لانسل

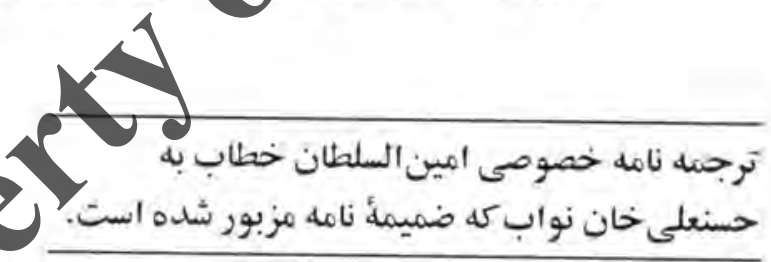

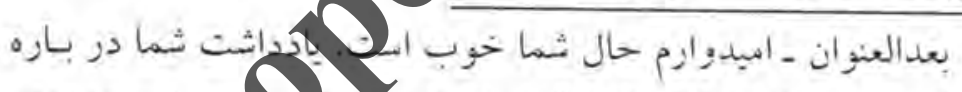

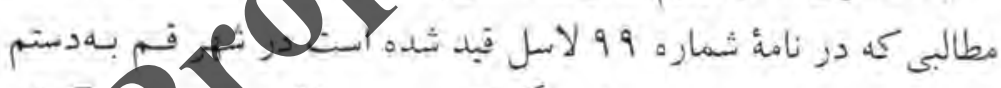

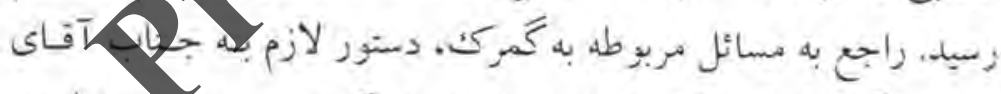

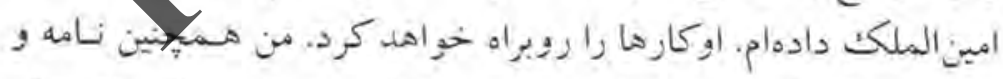

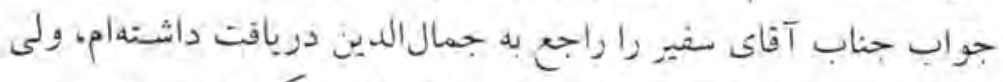

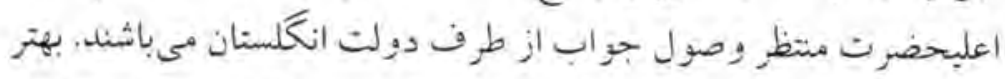

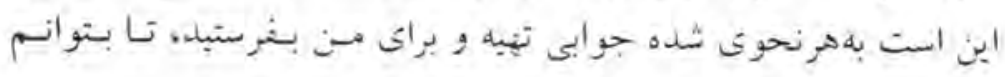

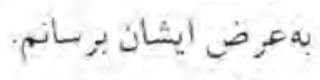

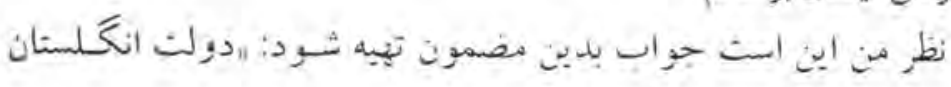




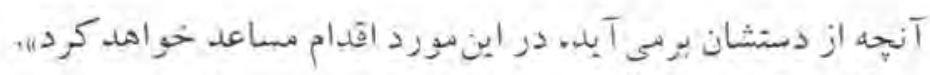

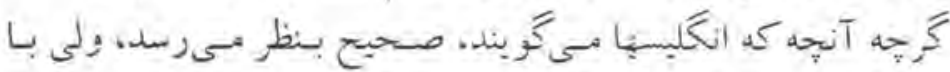

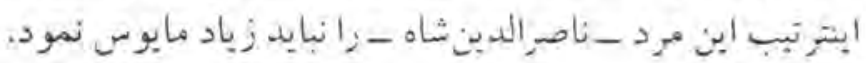

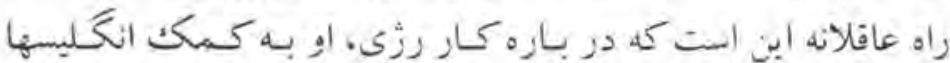

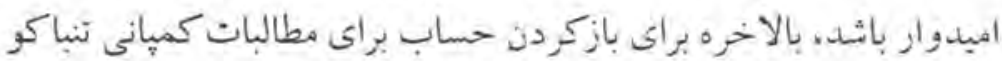

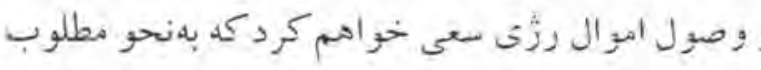

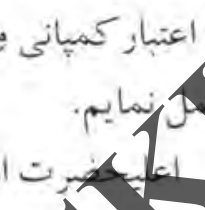

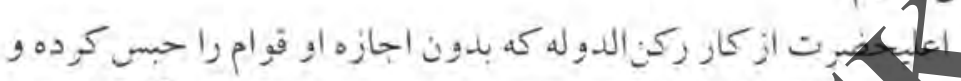

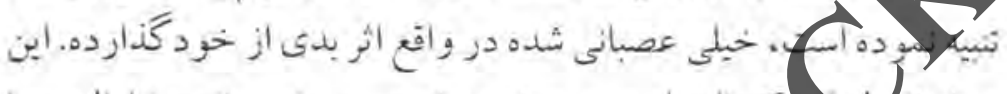

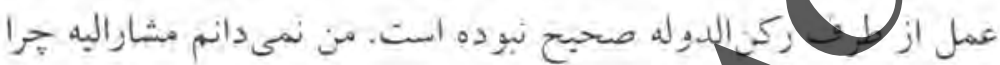

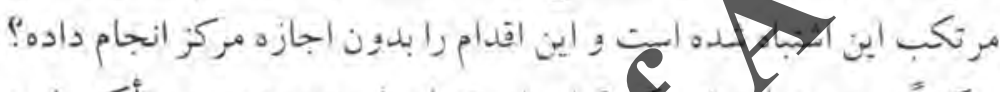

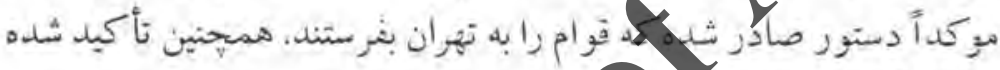
(امبن السلحلان

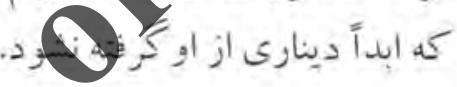

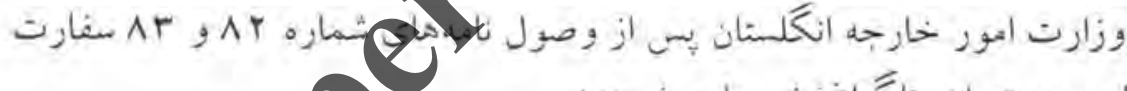

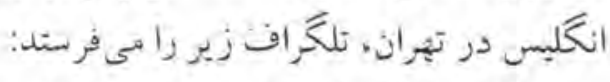

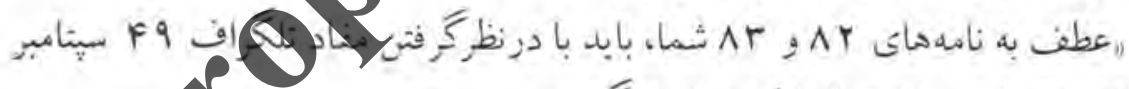

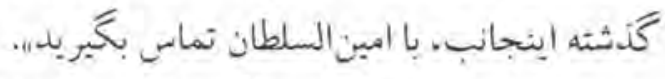

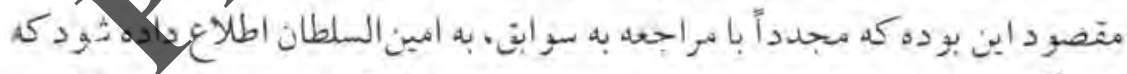

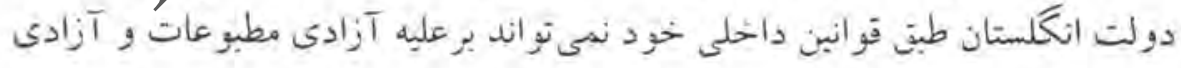
اشخاص، قدمى بردارد.

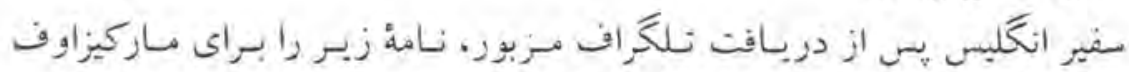

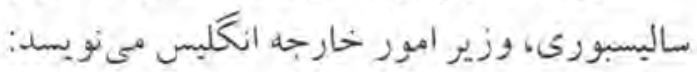




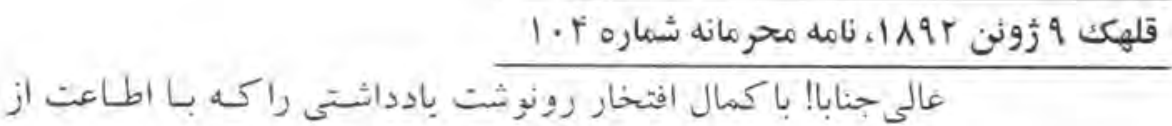

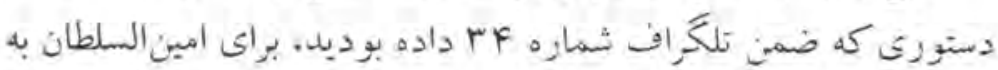

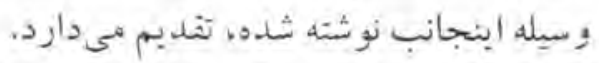

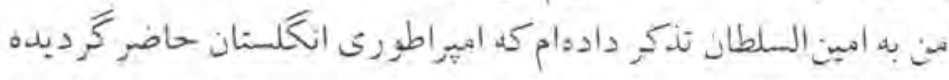

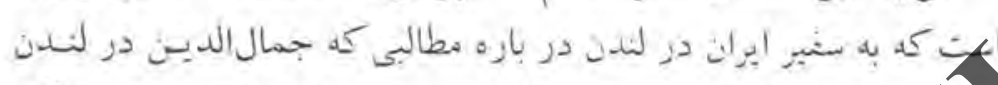

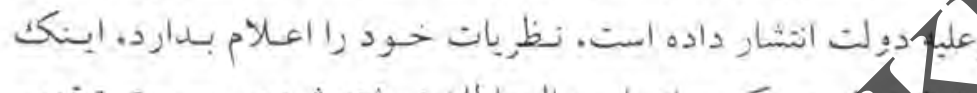

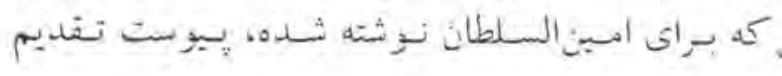

لاسل

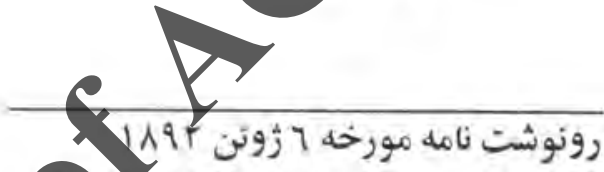

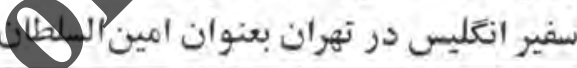

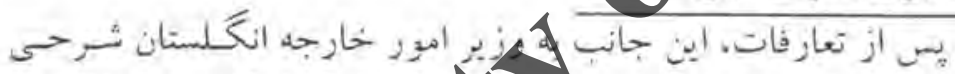

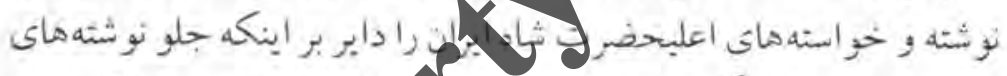

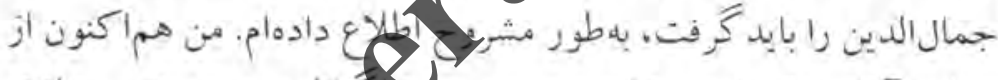

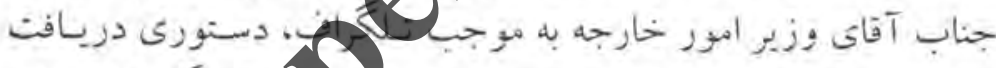

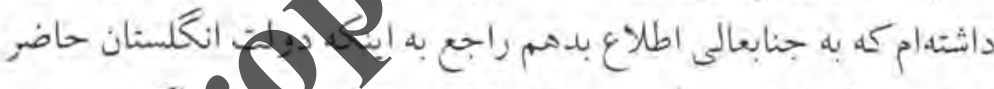

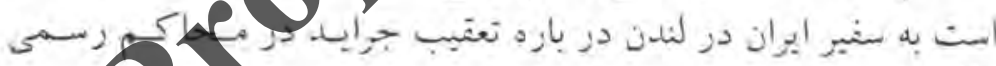

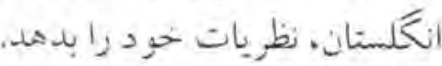

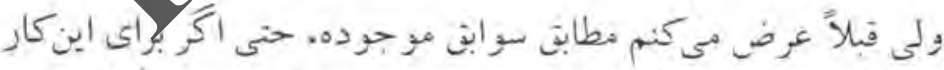

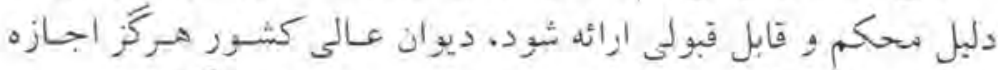

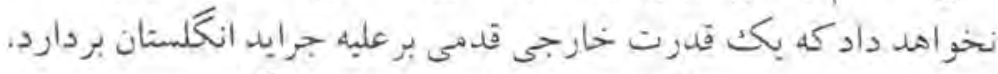

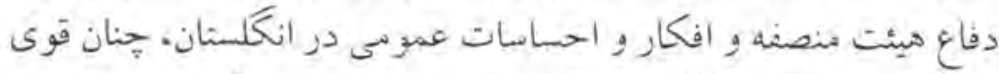

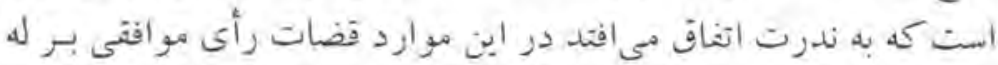
المضاء ـ لاسل لدعى بلدهن. 


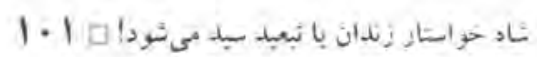

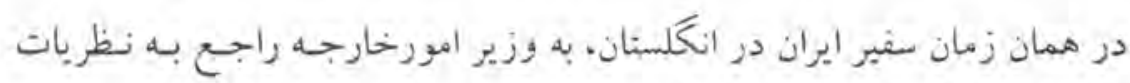

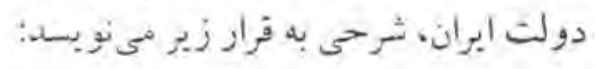

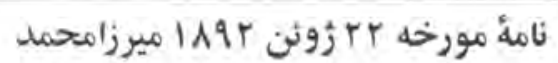

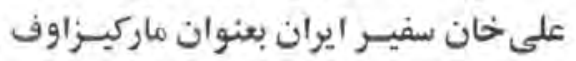
ساليسبورى

عالى جنابا باكمال افنخاز اسنادى راكمه ويروز راجع به آن با جنابعالى

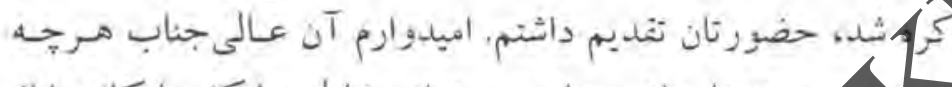

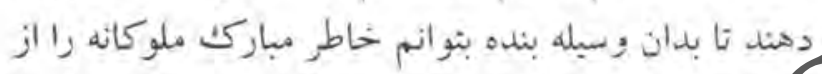

بأ ثفديم احترامات ـ محمد على

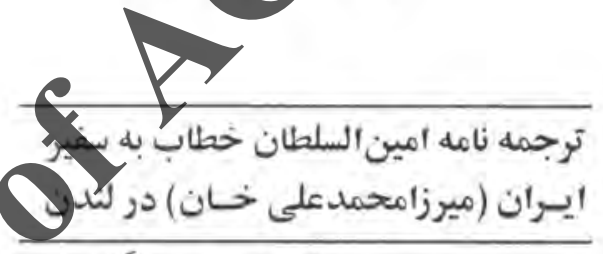

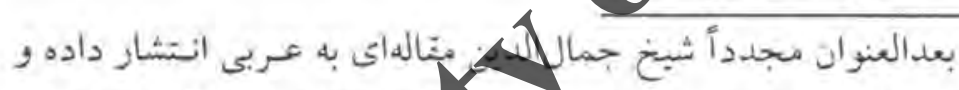

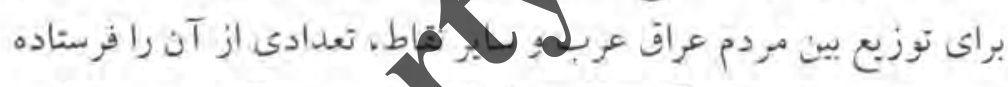

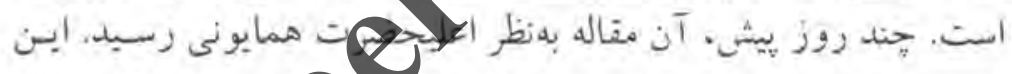

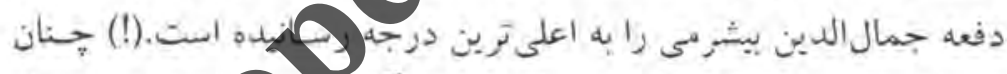

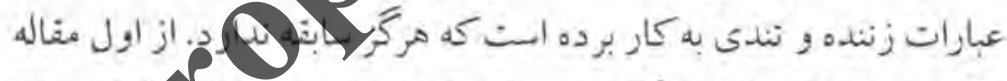

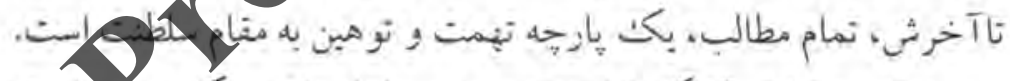

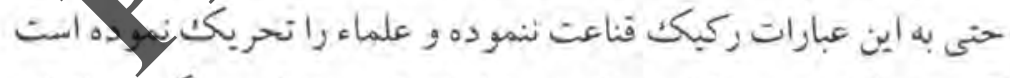

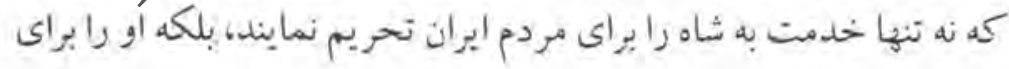

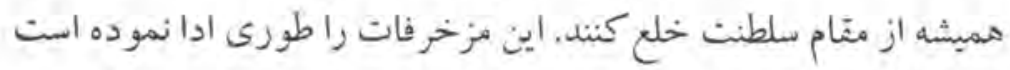

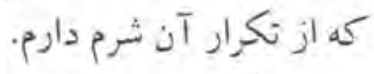

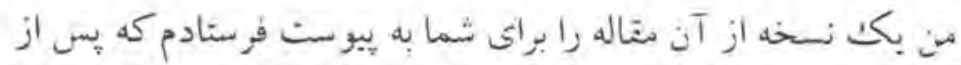

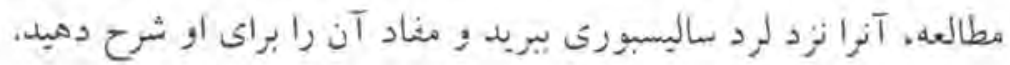




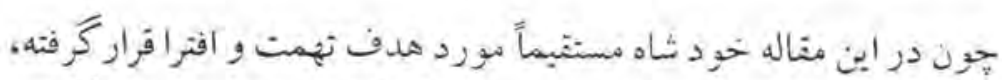

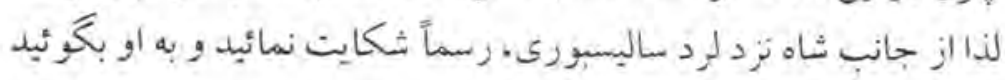

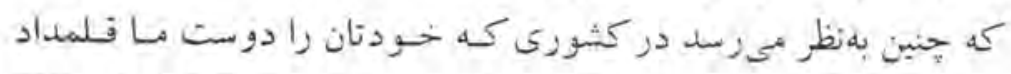

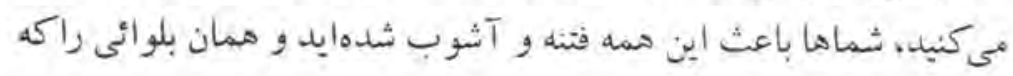

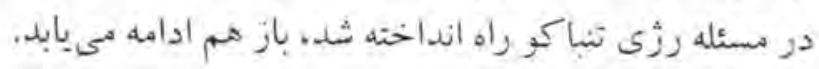

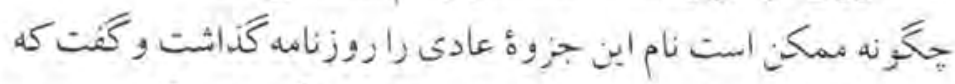

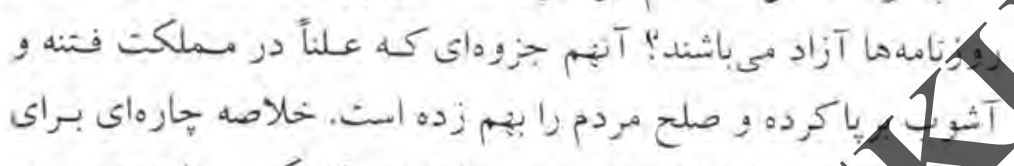

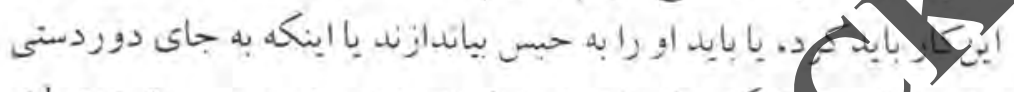

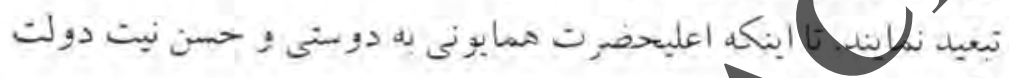
* امضاء امبين السلطظان

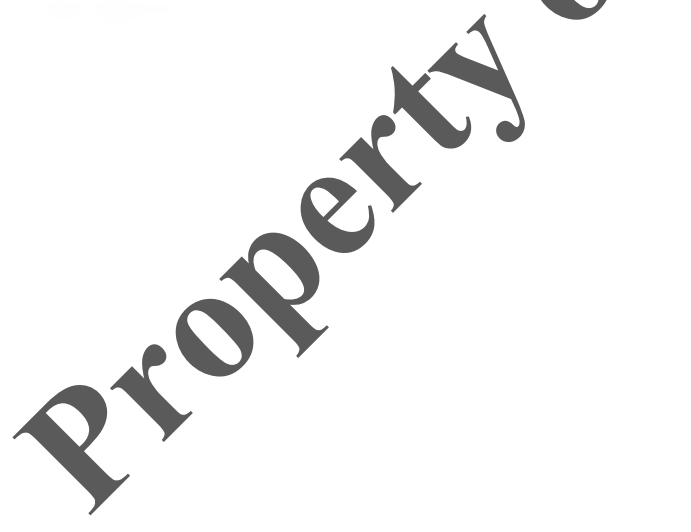

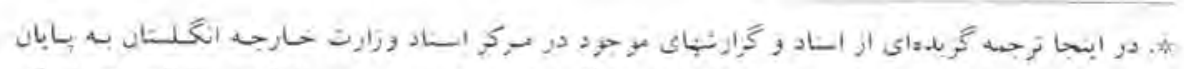

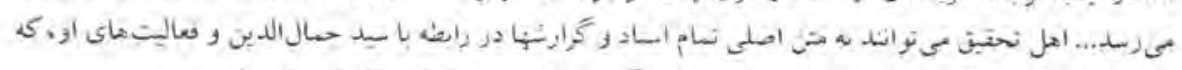

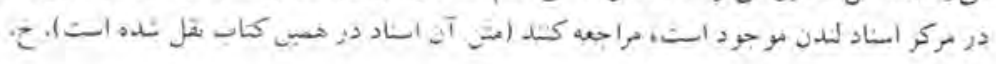




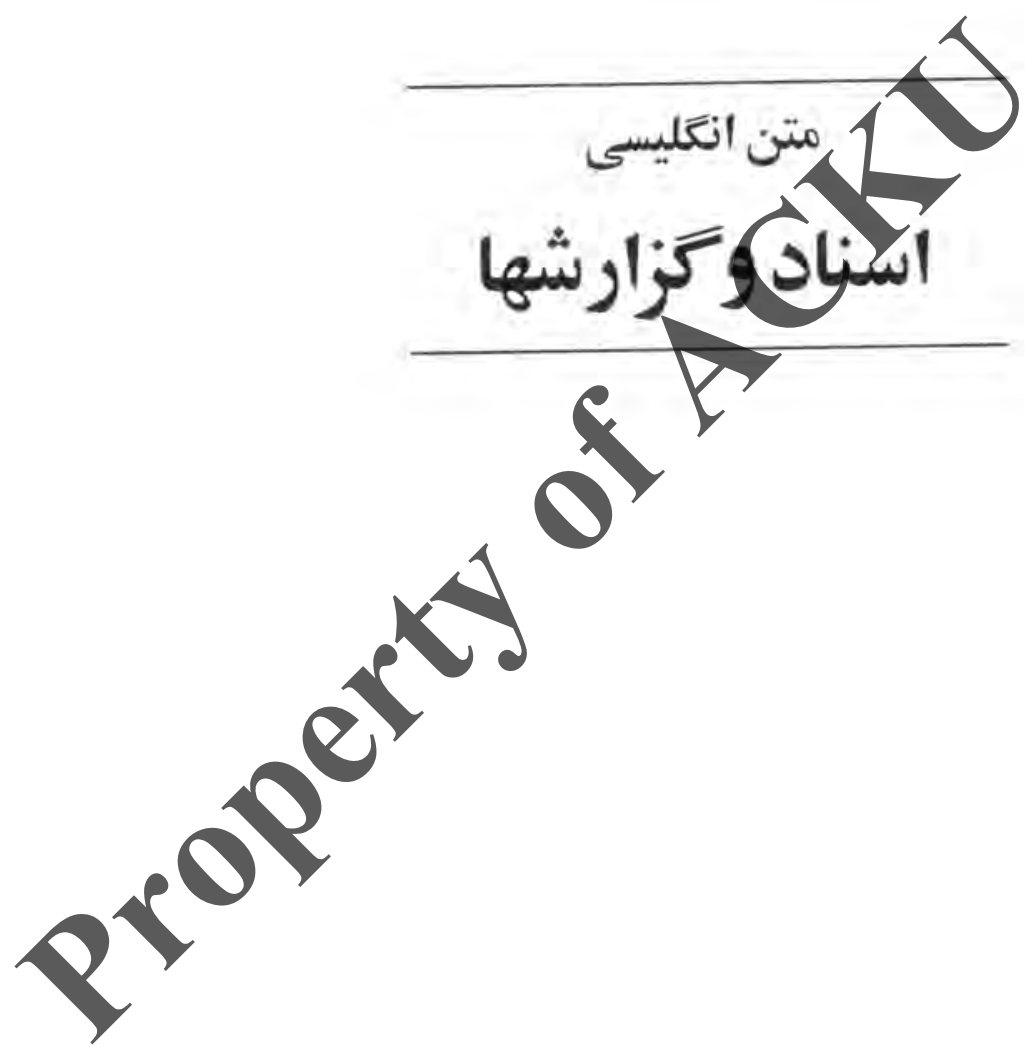

تهبه و تنظيهم:

سيلهادى خسروشاهى 


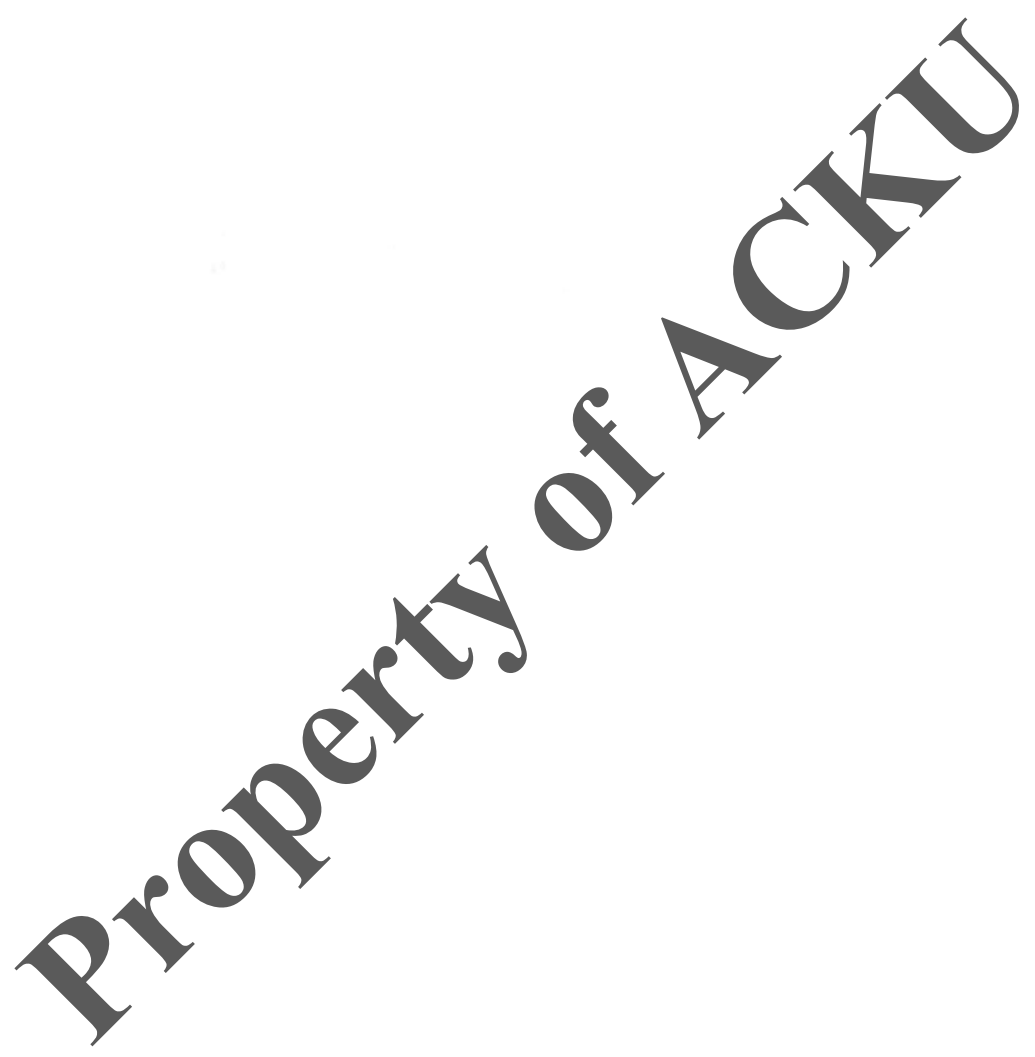




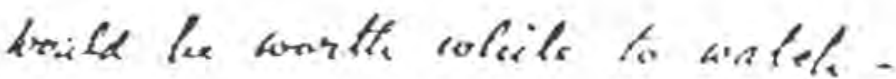

sleare the lesesenes to lies,

bith the frogkeds crofued,

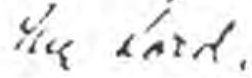

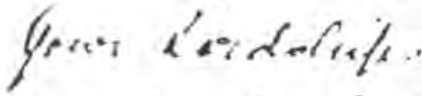

herot atedesint

kerulle serongat.

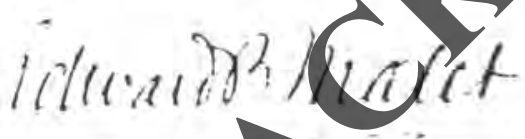

A

2

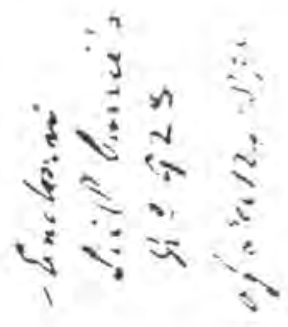

.

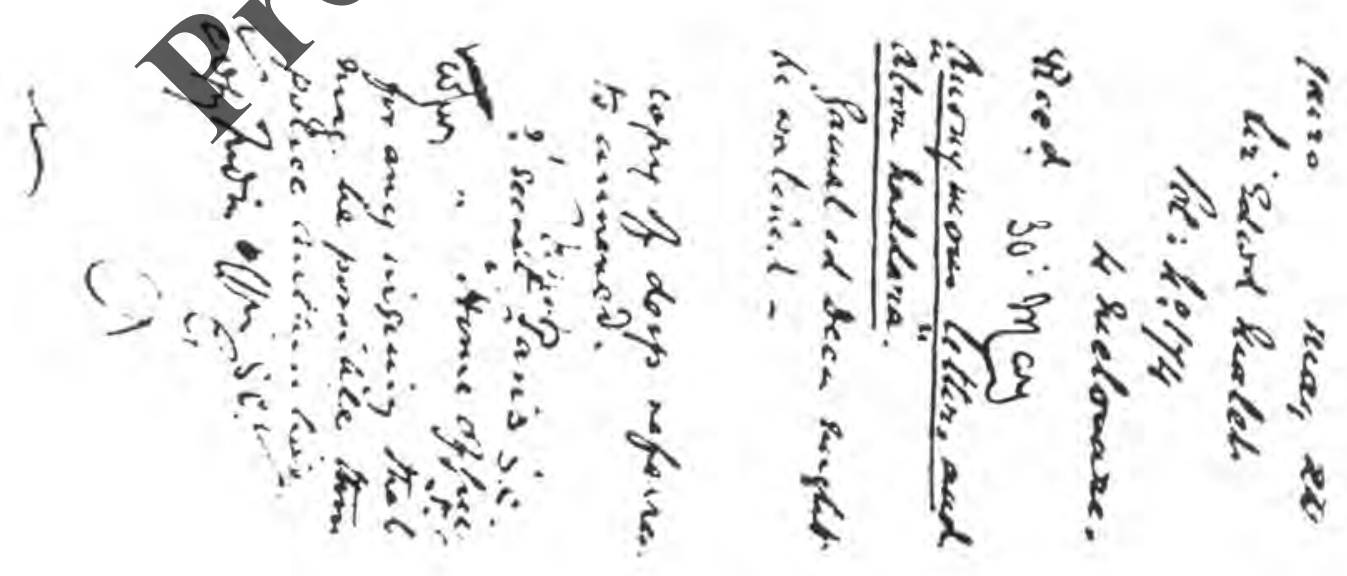


336

$1 \cdot 7$

holys

infidential lai:o

hury 22.1983

hry land

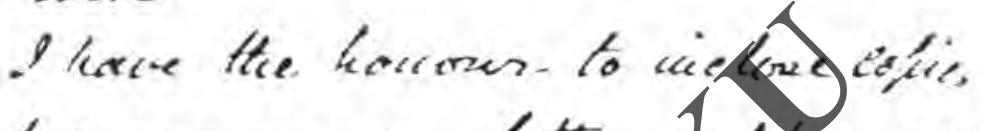

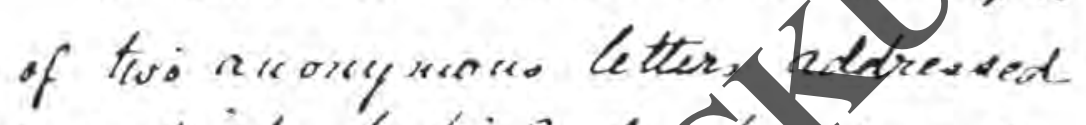
repectively to sir soelge legod aced.

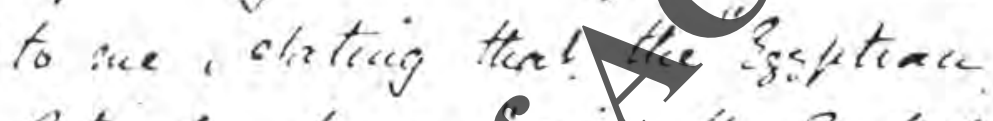

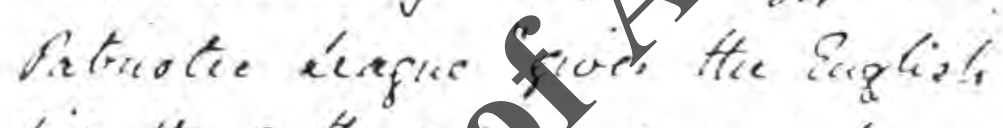

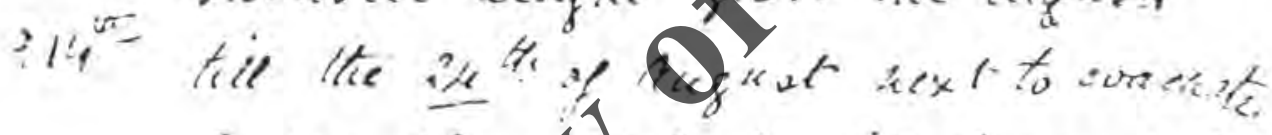

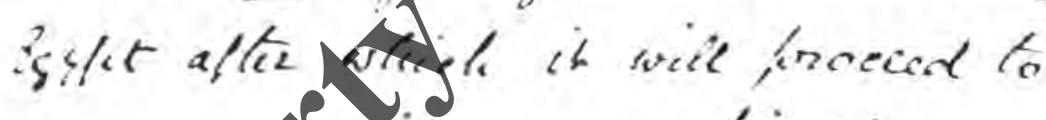

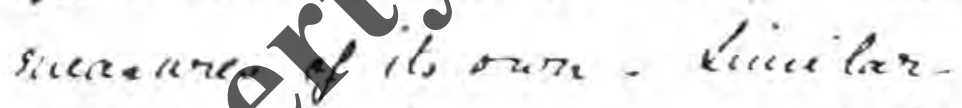

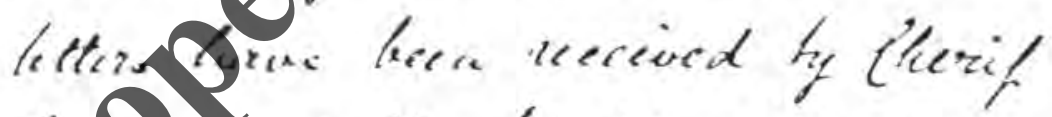

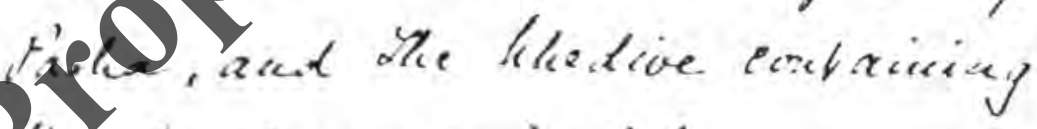
yerate of averuverienteris -

It in helivind that these letters

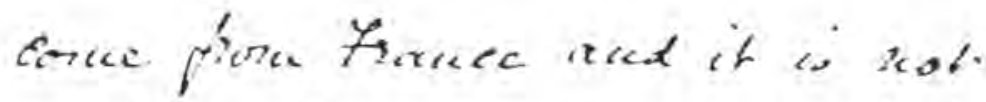

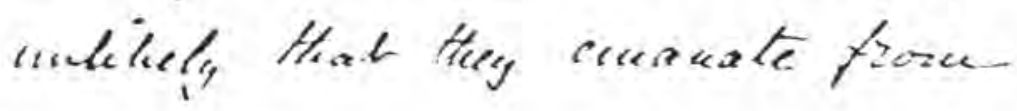
Eatc frawille kig.

$a$ 
I. $Y$

335

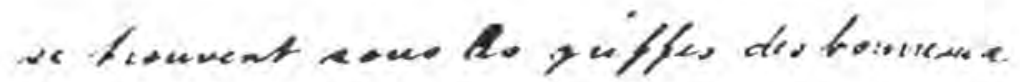

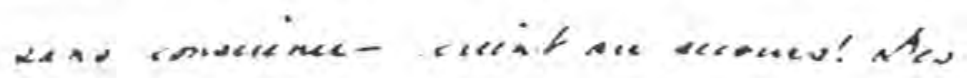

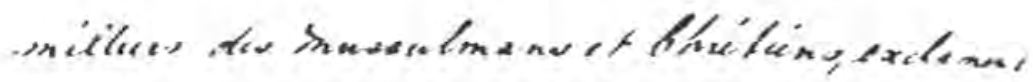

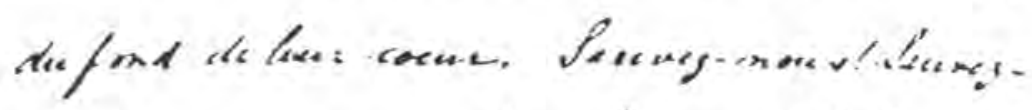

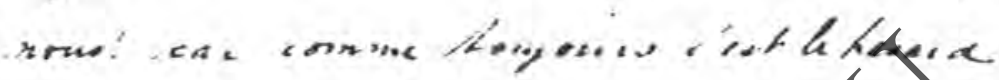

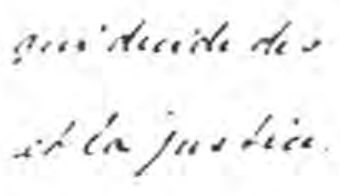

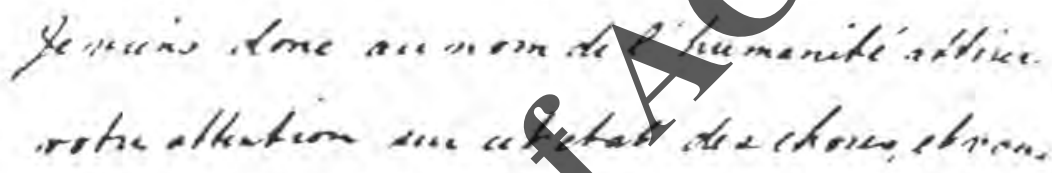

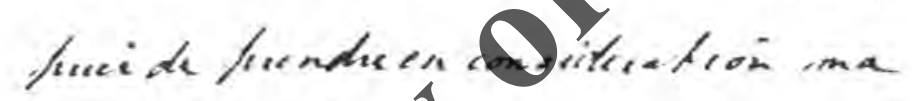
rlisuciéc.

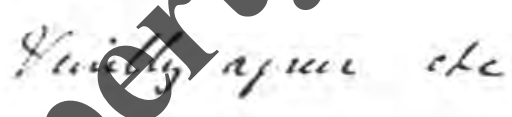

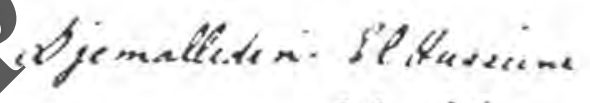

$$
\text { il afgare }
$$


334

$1 \cdot 1$

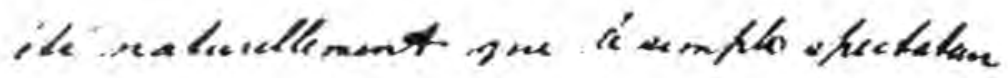

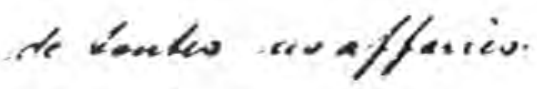

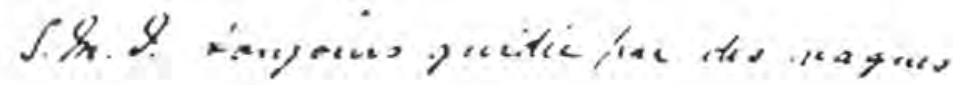

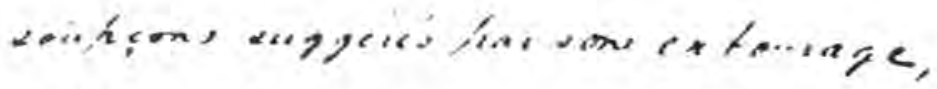

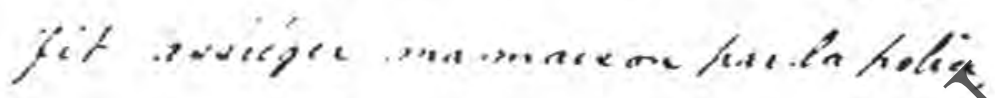

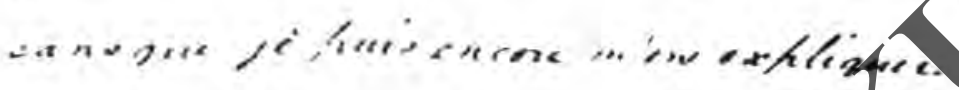

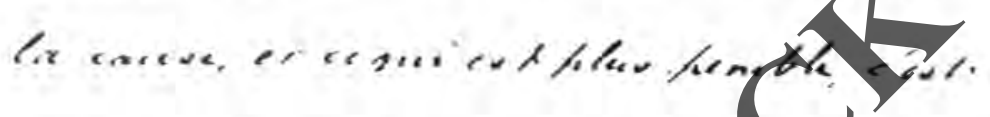

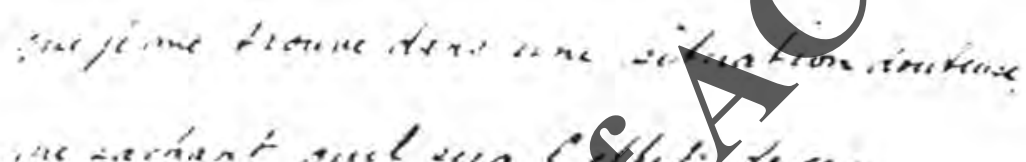

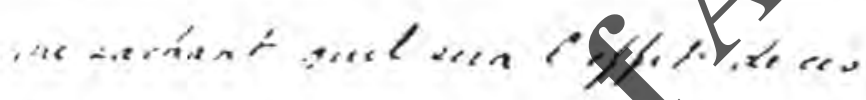

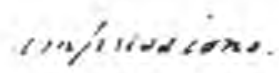

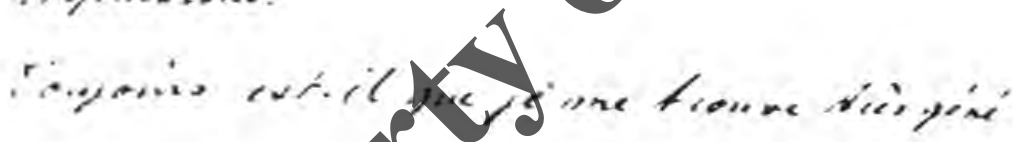

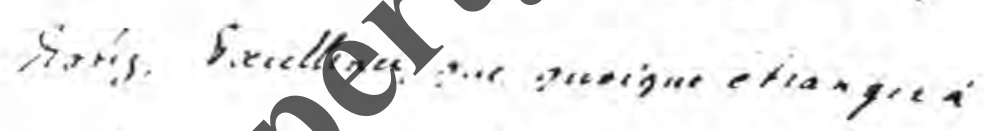

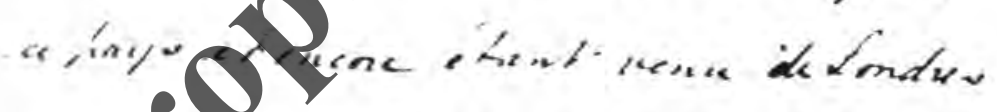

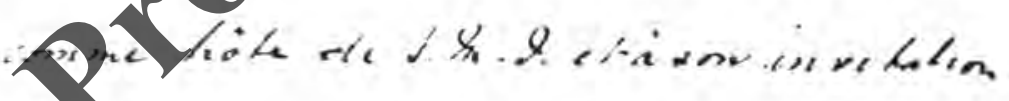

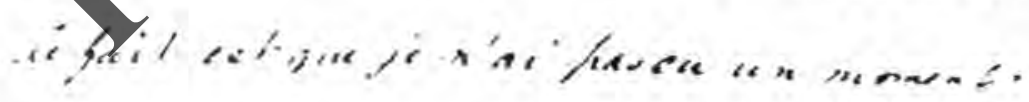

te ranguititi.

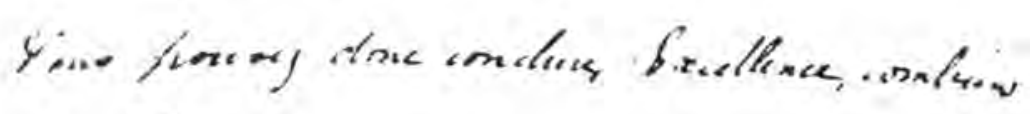

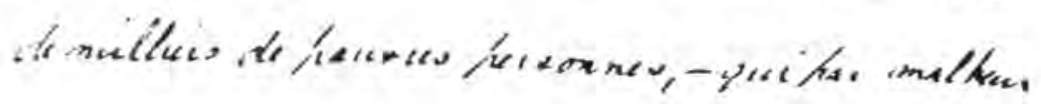

ec 
$1 \cdot 9$

333

iisitalioi numait pemaci fue henter

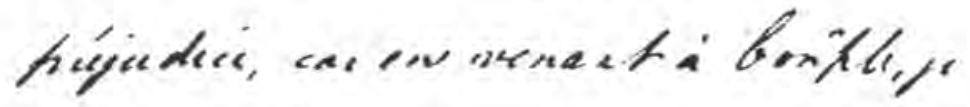

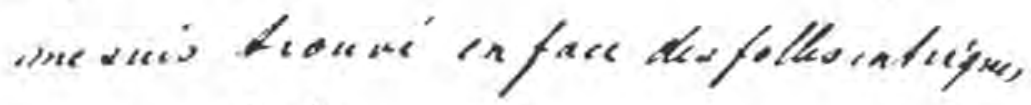

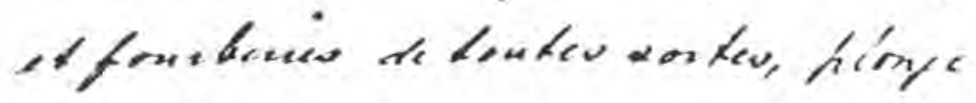

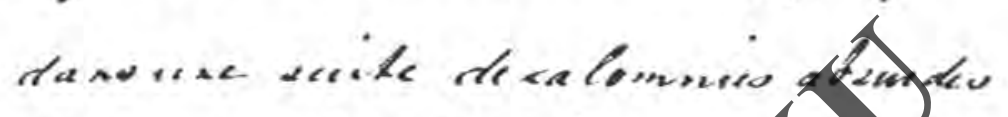

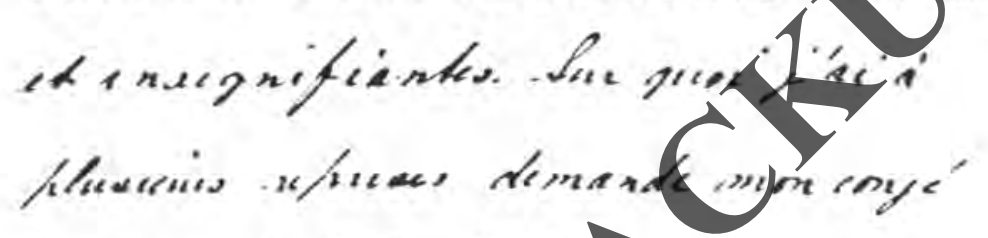

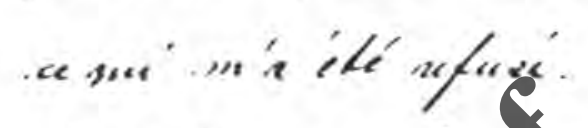

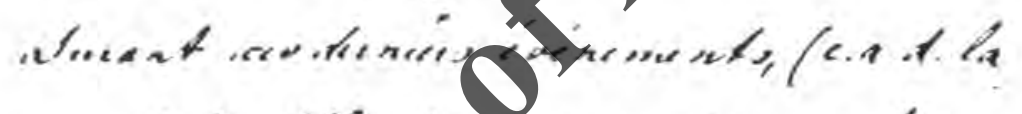

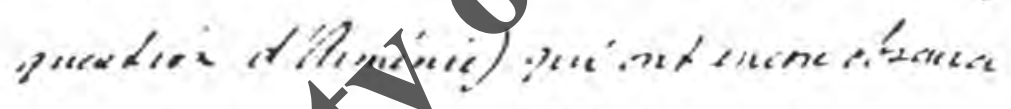

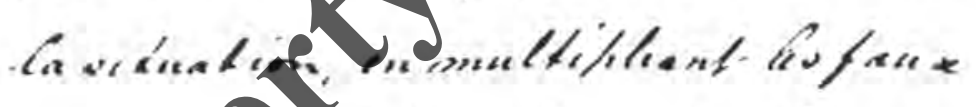

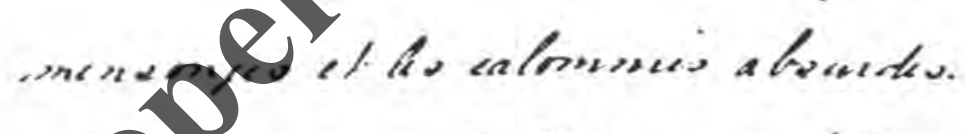

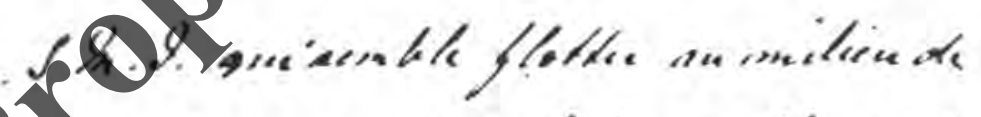

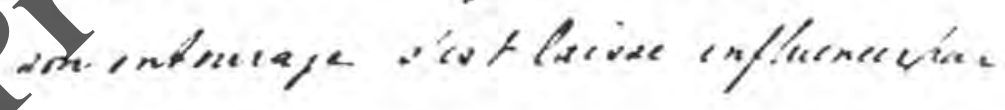

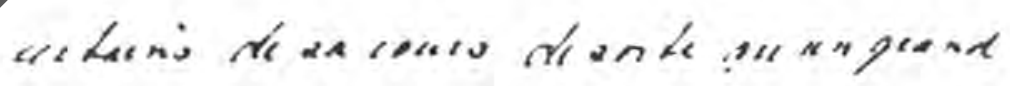

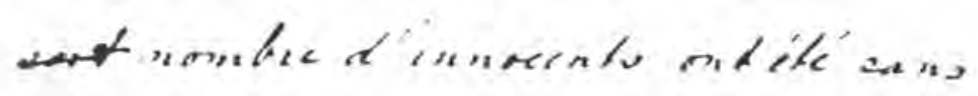

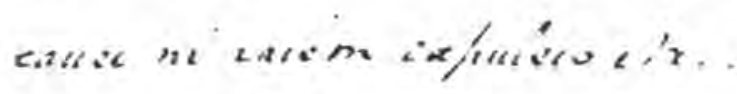

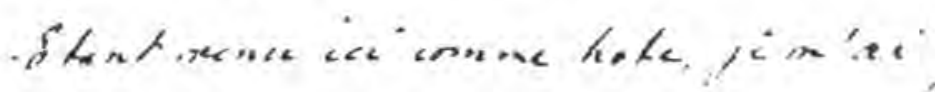


332

11.

de l'dnpleture. S'at heves anequede

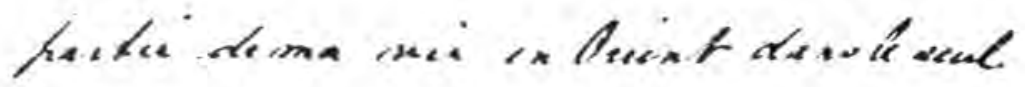

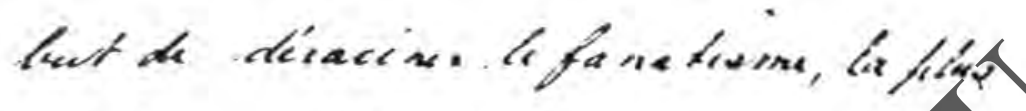

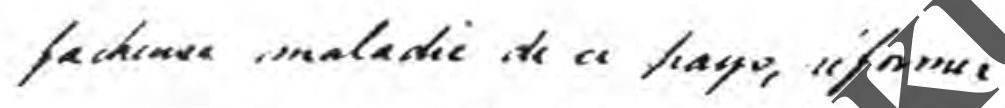

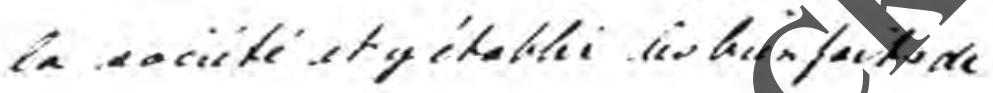

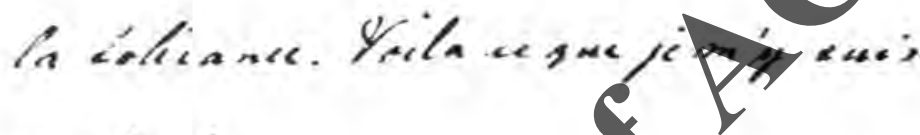
110

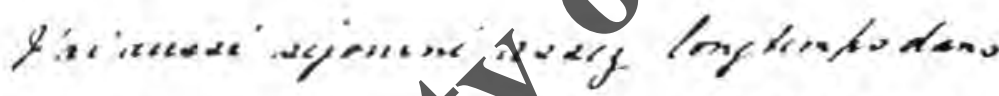

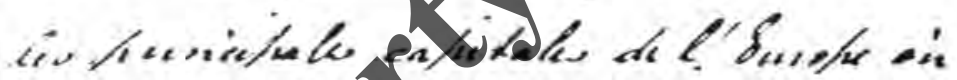

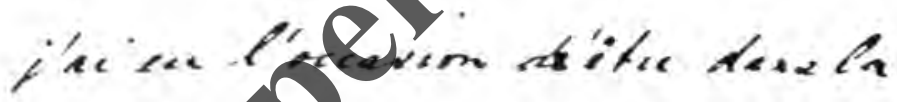

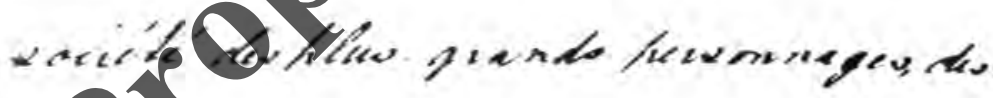

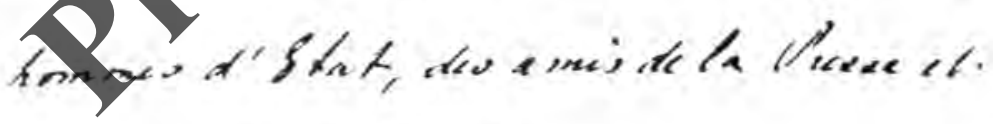

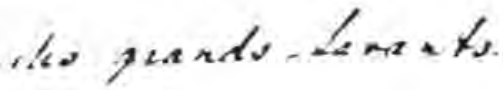

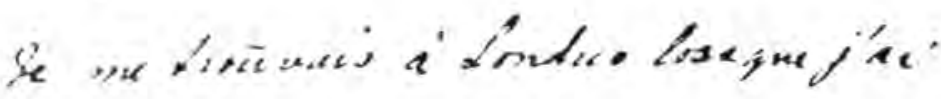

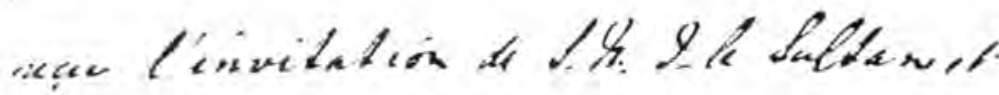

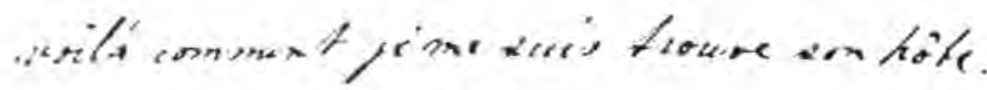


111

331

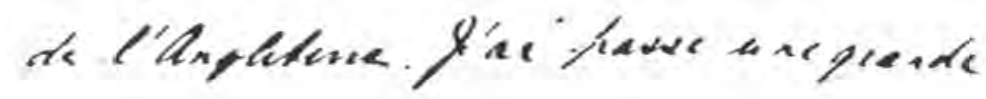

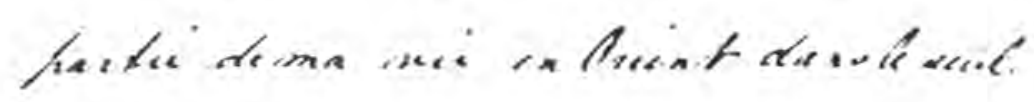

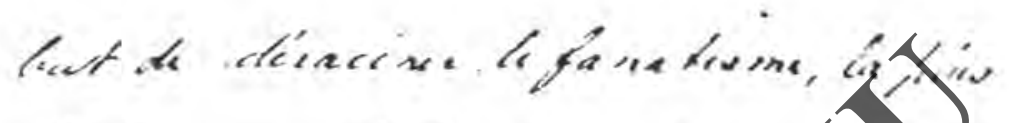

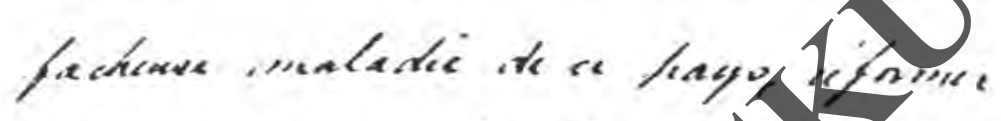

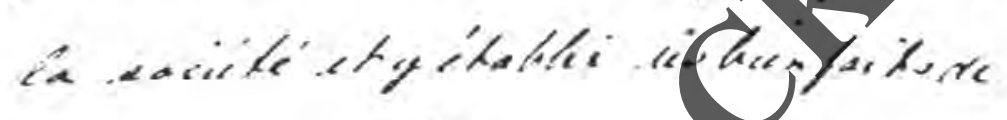

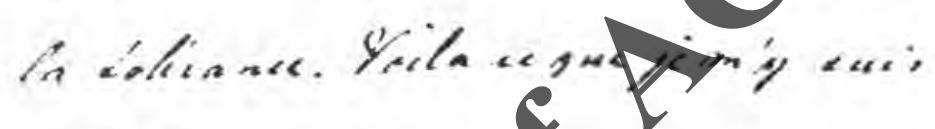

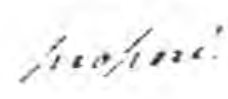

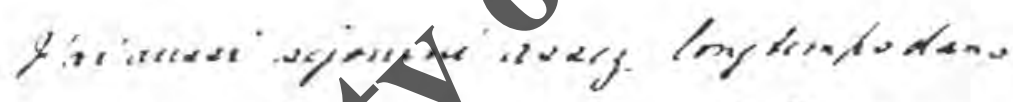

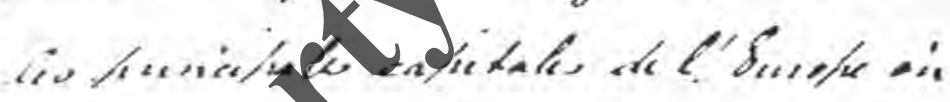

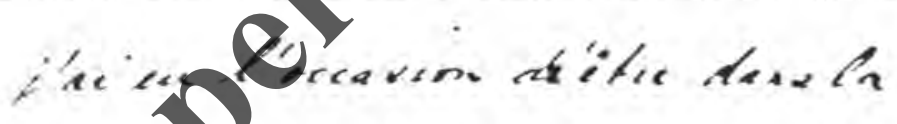

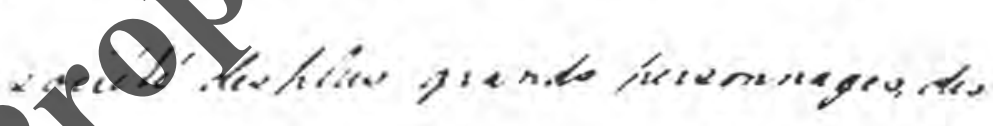

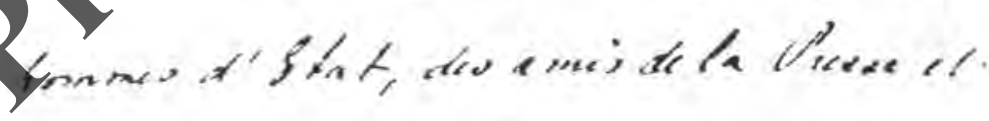

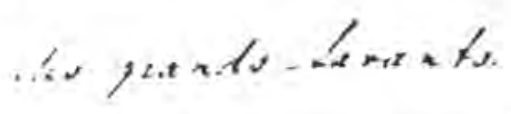

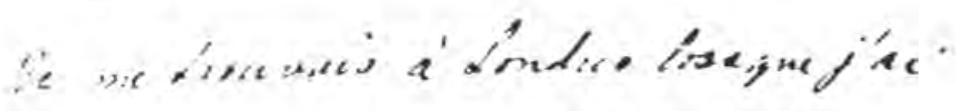

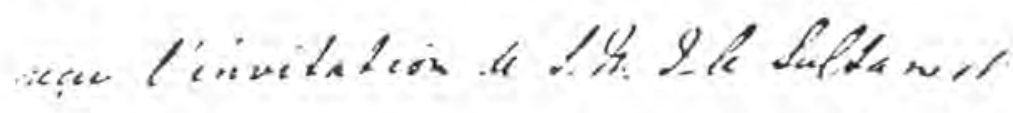

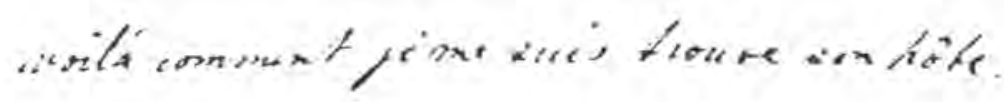


330

amkla

IIT

ie'ingors

tixellenes:

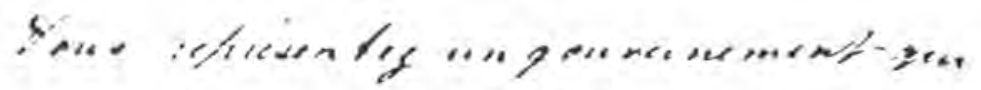

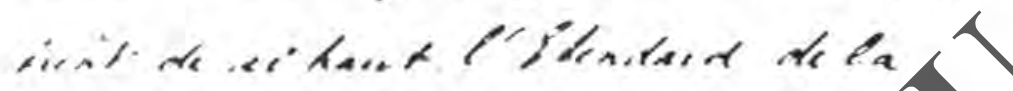

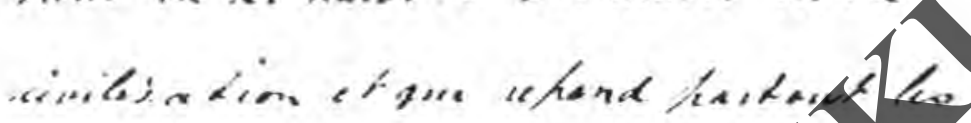

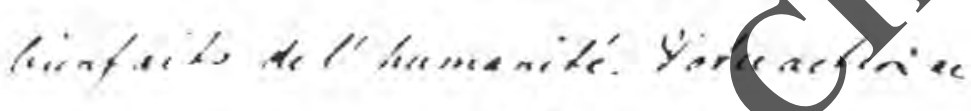

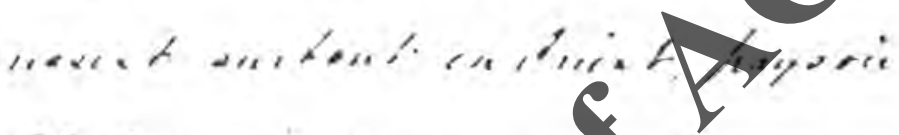

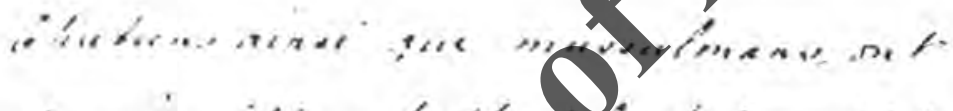

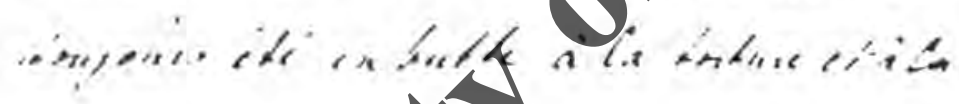

tyititnic.

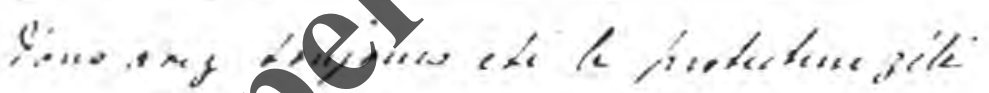

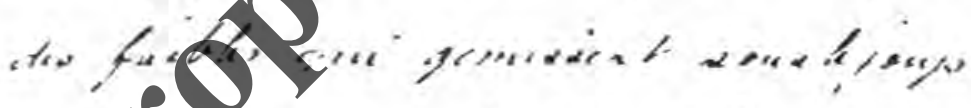

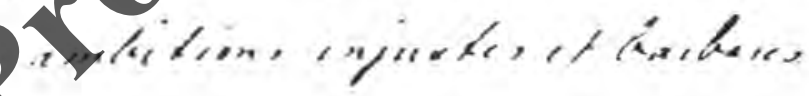

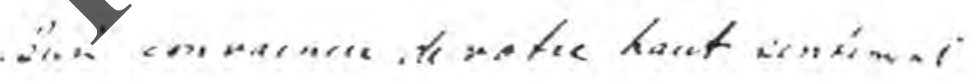

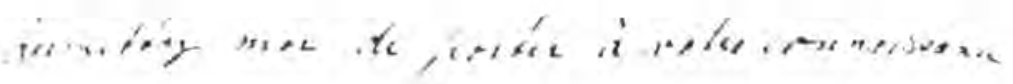

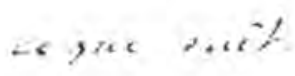

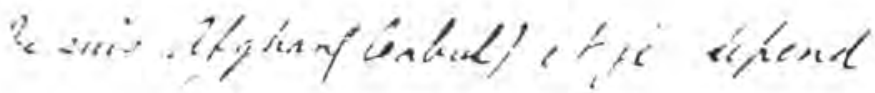

Ye, 
$11 \%$

329

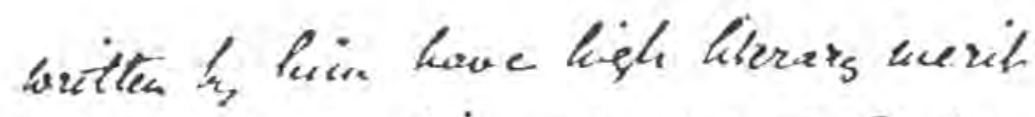

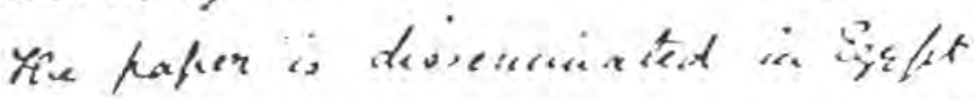

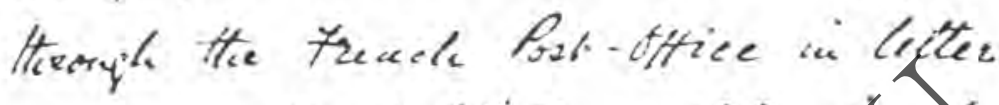
errets. He fublitieng ofiex

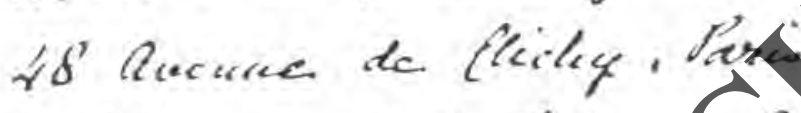

Hee corencetion betweres fajuic ce

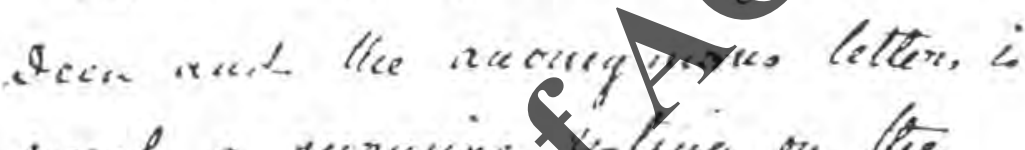

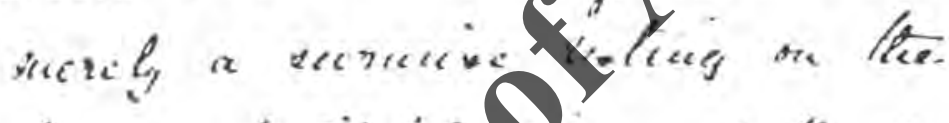

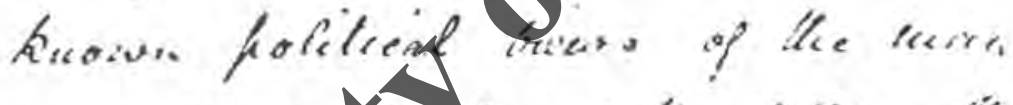

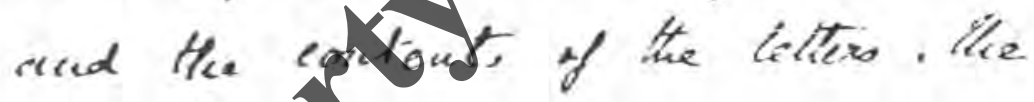

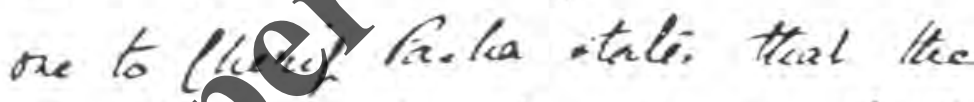
incular of the seaque are brotter

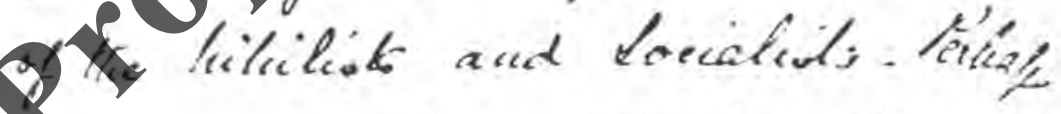

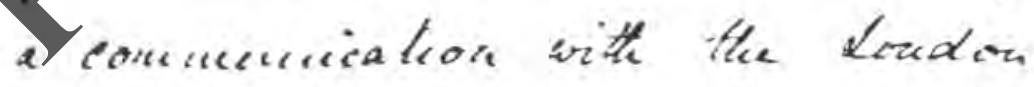
Porcie Ruthoritis night-elecit a

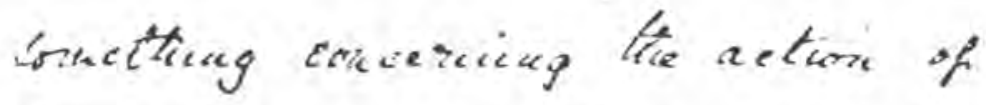

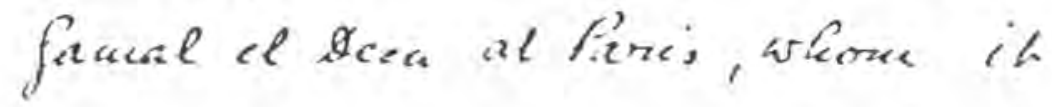

racel. 
328

$11 \%$

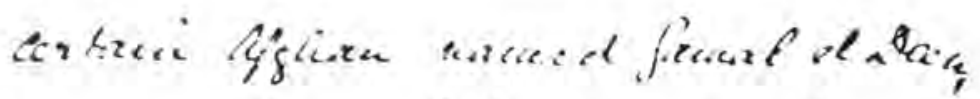

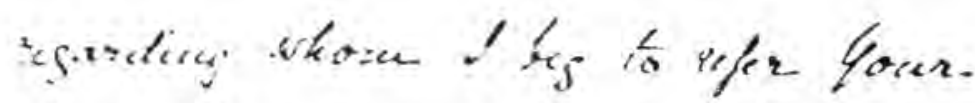

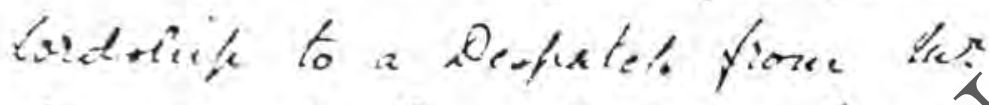

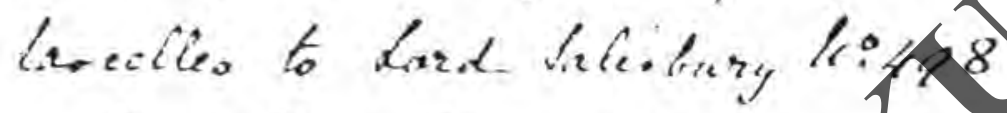
t the sols of Regional isp.

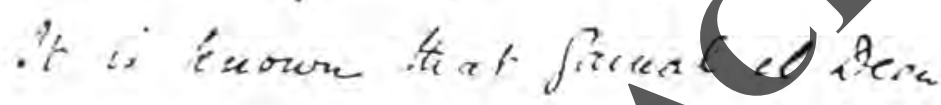

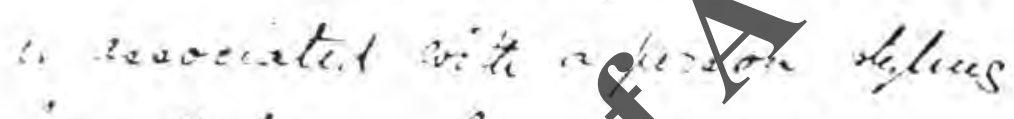

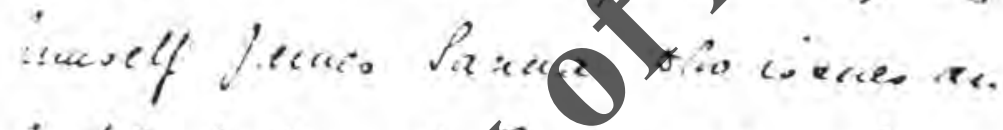

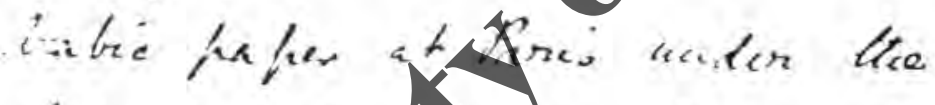

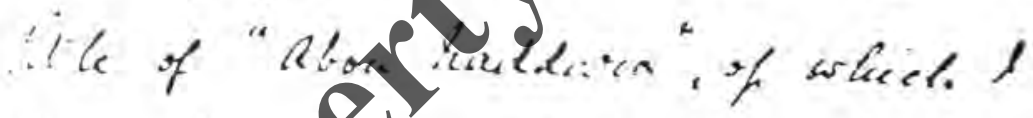

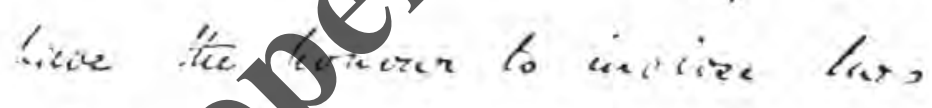

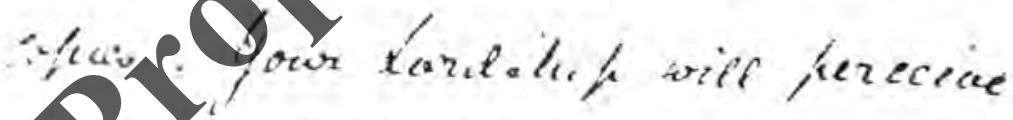

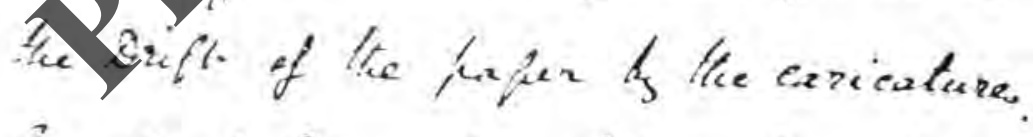

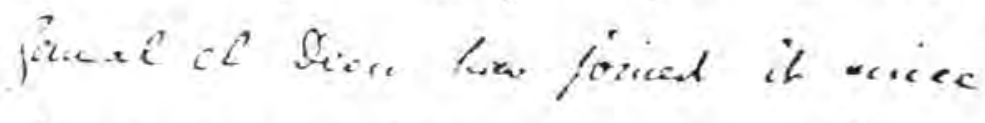

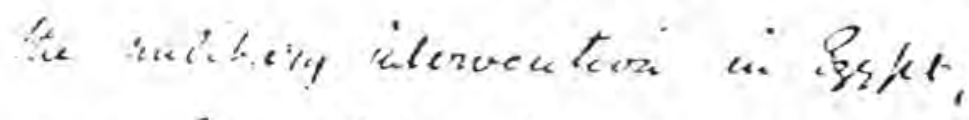
tar ? in told that the lentils witter. 
Appendix

ky dear Enat.

$$
\text { quac } 25^{2} 1 / 8
$$

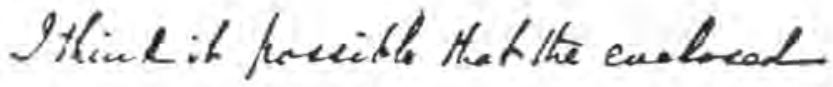
aceount of axilt-affector indivisub, who is kow cerving on his wate in lavis, jot th

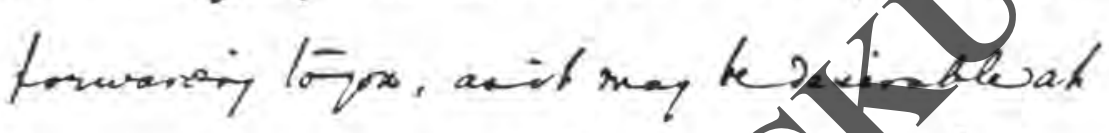
tome times or other theneve itith his

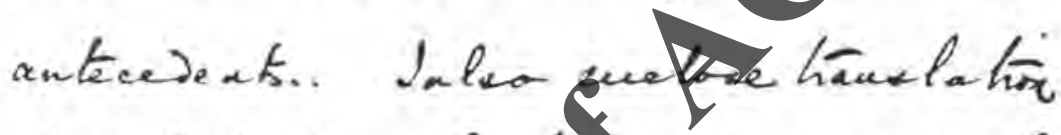

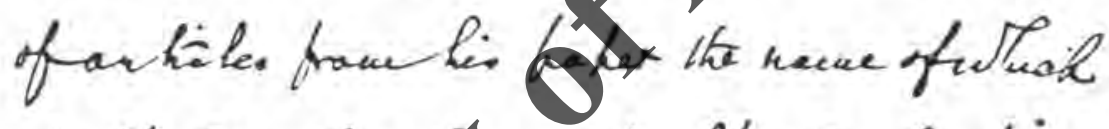
$\therefore$ als Yarkhar oferit. It-cisulation

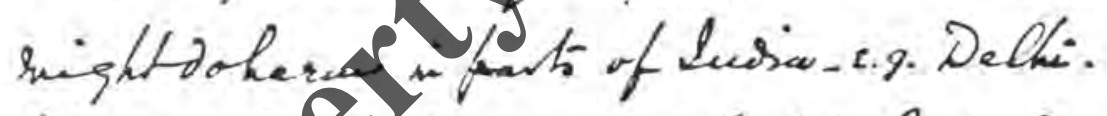
butherafor of eowron rot widely real

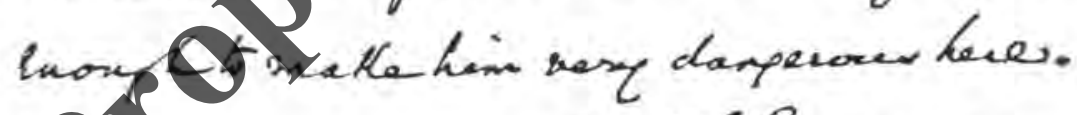

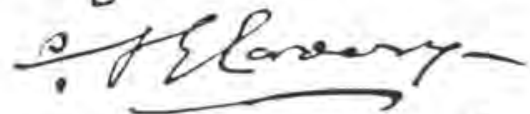
$\checkmark$. T. The slumberes of who the nan is may berkatos he asefful Whajor Baing axith is s Sylth heis now joing. 


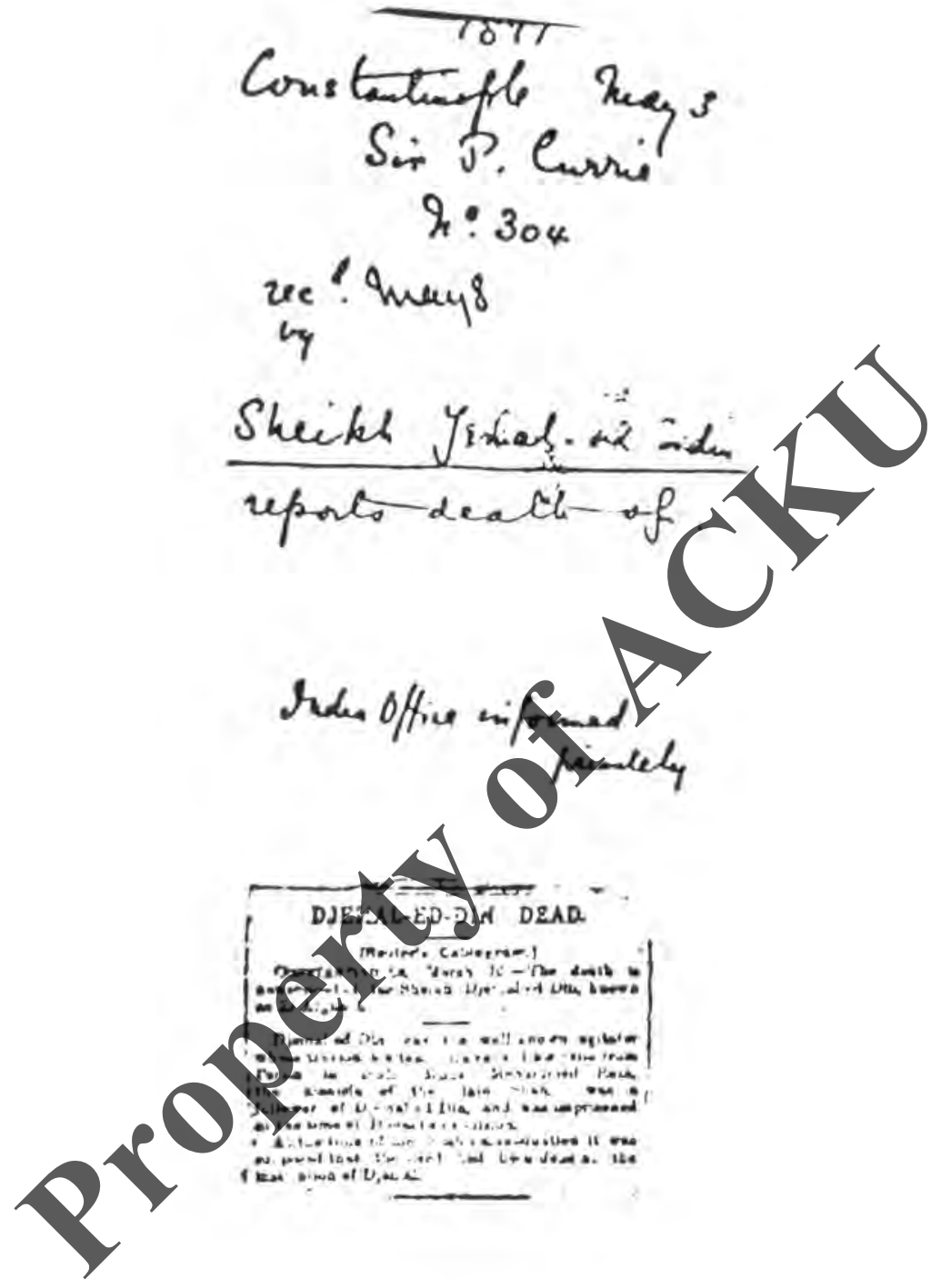


IIY

325

other Shimkls, styled the husafor or Suect of the Sultan from whome he recewid a monbly supsidy
y have the to the with the phent unpect, ny Lool,

your Lorfosfis nestobedient. Gevioble Lervant Philifuni 
324

111

Contant 197

Tway 31897

$h: S 04$

hy dord.

a. I manstand thal the Indei offic to have rewers of the fate of the sheiteh yenal - ud Din, y have the housur to uporttors the died at Constonturple on tuarch 9 las from the effect of a

cancer

The decered held no office butwes, the many

Le Tharquess of solition da. $g$.

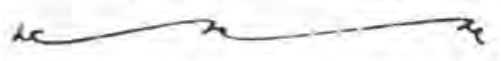


119

323

srap

Sir $P$ Cumie

Le. $1: 74$

7.0. Imay 6. 18g6 ( his Al. No 1/43)

Gmal-ed sin

of Percian sutionalito

Gegtion of umente bes

7 
322

15.

main 6. 1896

Ins thenan l? 143

S. P. Survic

Zi: $7 / 4$
(7 hag 4)

accuding

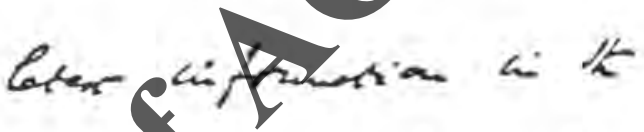

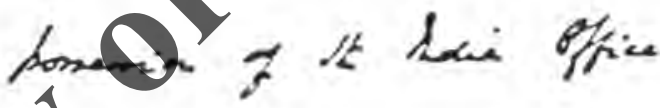

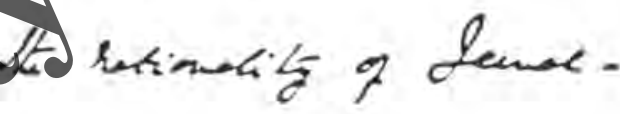

ex-din is Promian + k

Rutrian of his Anewares is

kenfere hot one thece

cancine a. S 
|r|

321

In diā officos

\&o. may 896

rationality of Icmal ex $\sin$

Hte is heleived to the a Prsian. teug makectot culeffare Nothis sumasorer.

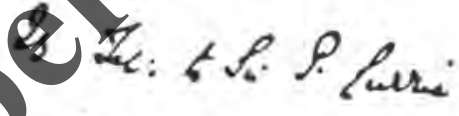

annerees.

Fel. $\min _{\text {Mog } 6.46}$

7415. 


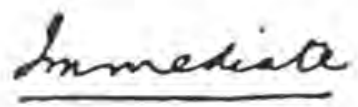

India orrice,

5 th May 1896.

81r,

I an dirocted by Lord Georgo Hanliton to acknowledgo the recelpt of your letter or tho att. $4 a y$, and to acqualnt you, for tho infuration of tho sedretary of state for Porelien affalre, trat as the naplonality of Jemal-ed-din 18, accerding to the latest informatfon in the possession or tisio orrice, fronounced egbe Persian, H15 Lordship ia that of oflation the question of hroburrender 1 s not one wilch concerne Her Kajesty s, Qoporment.

I have the honour to bo,

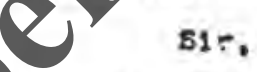

Vour most obedint servant.
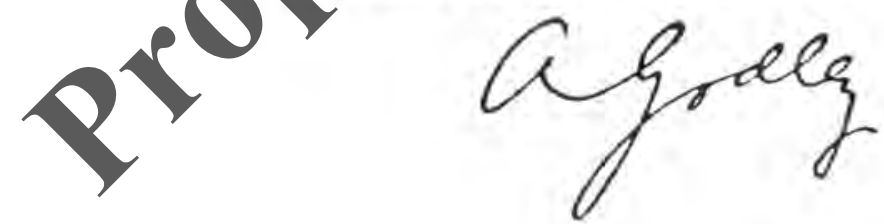

The Under Secretary of state,

Pore1gn orsice. 
IrT

319

oechinter

$\operatorname{Sin} 9$ Cunie

$$
h=143
$$

D \& Thay $189^{6}$

Arosimation of the Shath

Sheith Diclalleddin intered tolece

been inutenental in. 9emparg gout

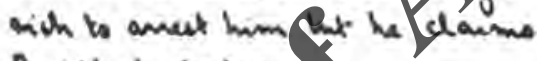

Britith protection.

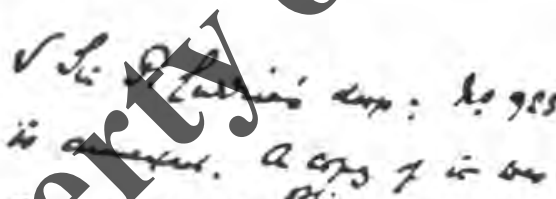

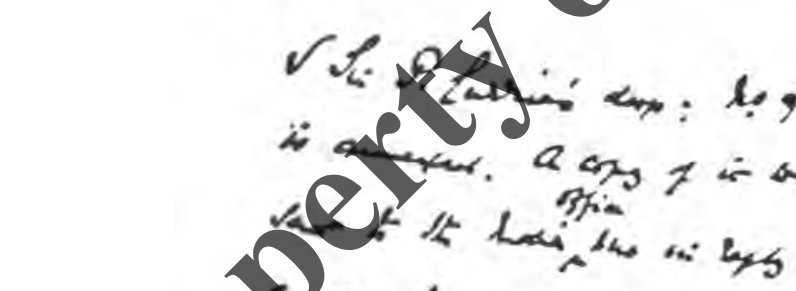

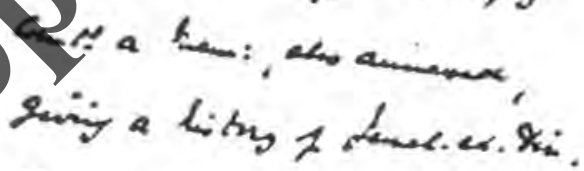

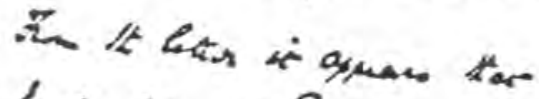

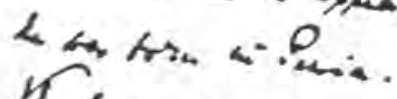

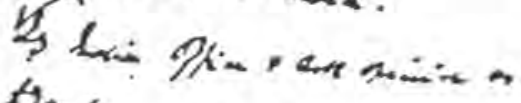
thas 
318

Irt

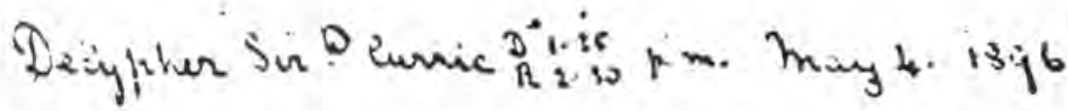

$h \div 143$

Qevian Antrassador vifiermu me that theve is reasen to bilieue that suasum of Shah nos an emissary.

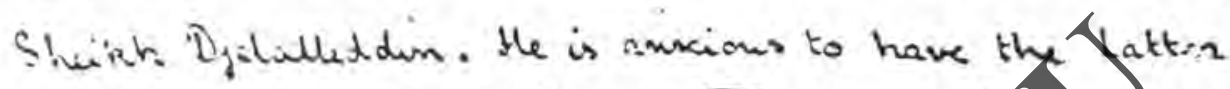
arrestict and sent to Paria. Tumbuts gout oldeing

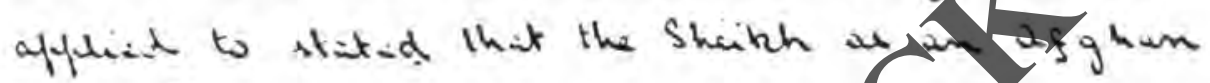
doims Sritish jurtection.

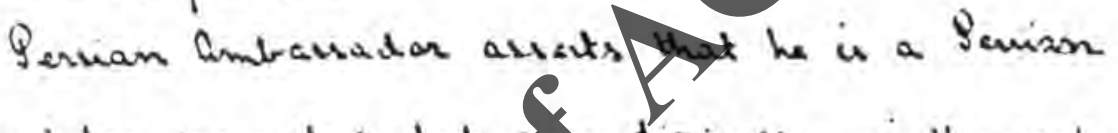

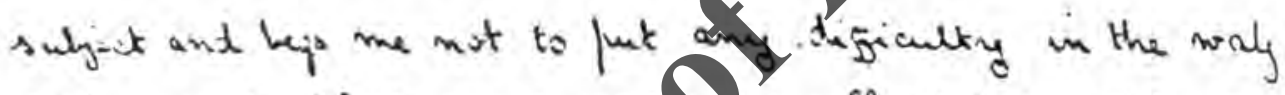
of lis irrest. What course wouldyewr Hindsing mish me

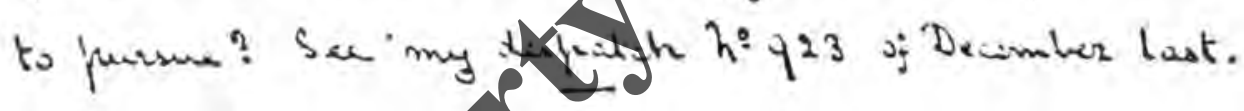


$1 \pi 0$

317

a his tersocedings suith was miclaed in yan tellen of the 6 th of Manch lant it is sialed that he was forn in Pernis, and novting xhmon to oulsint His claim stk an. a ruagi.

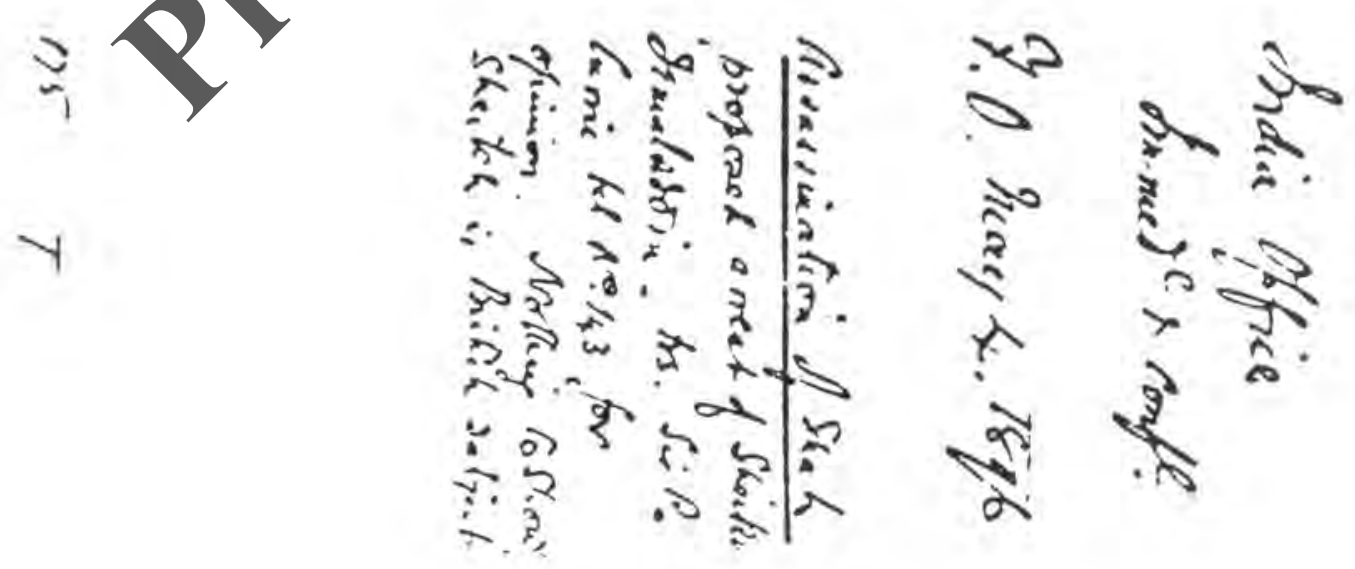


316

$1+7$

enfrued what sefly;

hanld, in Znd 19?

द. Hamiltan's

finim, le seat-

it Si ภ bainió

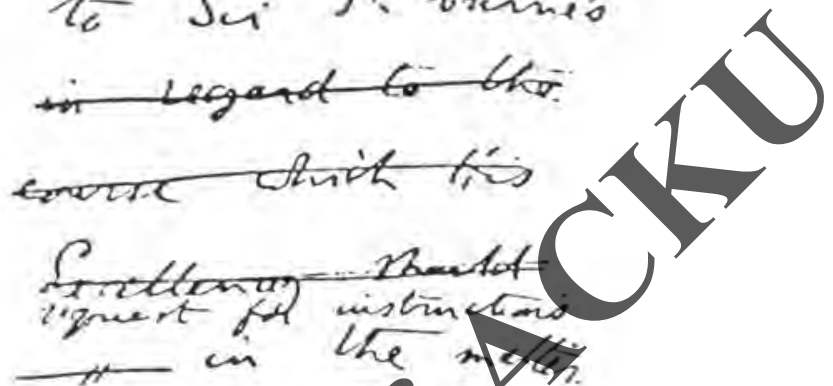

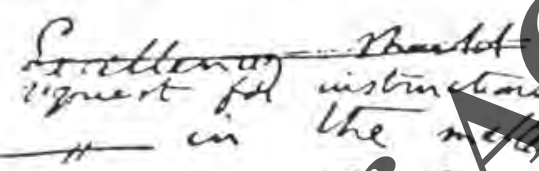

sis Planis

deshatich 20123

f Der. $k$ Tam.

refordes to in

the fetergiam. was

ofomminicalid to

- x al the $23^{\prime \prime}$

of Decemter.

In cronectors

wit the querhas

of sheikhfemelectdis

nationatis. Ind

Salistrung dreves

that, in the Hemanan 
ITY

315

The Perrim

Aubanaden wishes to have the sheith anited and sent Lo- Do, na, Int the Tunkish liv st hang 1 infonmed him the sheith

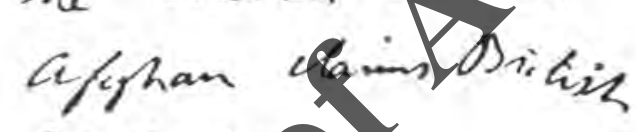
prrecka

We Tersicon Ampradar maintains ter he is a Passiam mbeit-and regs Sai P. Pasnie at-10 fant any etplacle in the way $f$ his aviest.

Ind Selishong ander be glad to he inifund 
Draft-.

F.

2ndia Office

India Office

Si,

May 1896

Si,

I am deriectod If the Mervis of Salistieng to ant

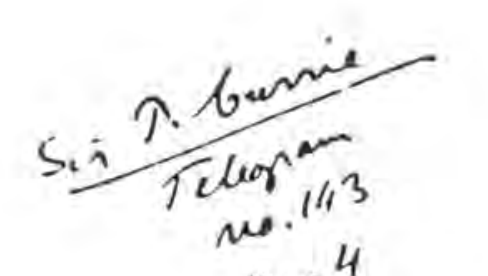

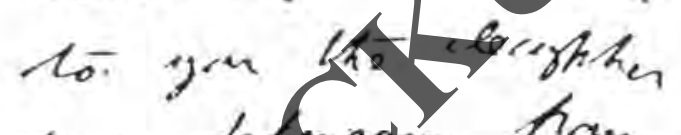
of a leforom fion

Mas, 4.-bi. Druburadn at tourteruhlingle. glativg that he 10 infund ly the et Aenrim Ambanaden ai Thke., ltat wive is reasen to brhere ctalthe arami of the tate Shate wes an emisong if Sheath Jomaleddin.

Re 
$1 \times 4$

313

Conio $18 y^{\circ}$ hay 2

Lino Cumen $N: 61$

Ro? $11=$ iby.

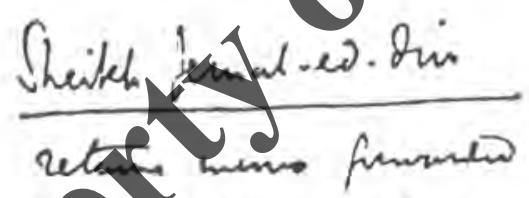

isto. si:60 y afmiezs. 
312

Canio.

15.

SO 61.

Inay 2. 1896.

Thy dord,

J have the houms loxeturn,

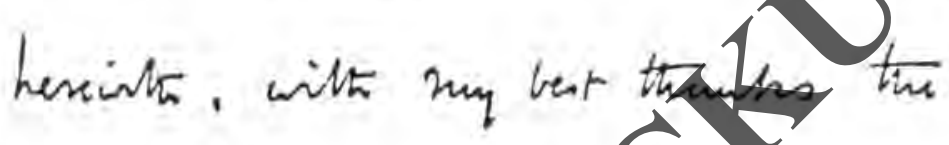
memorantum formanded the for

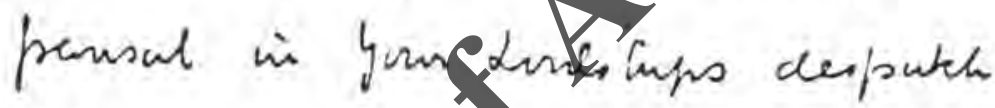

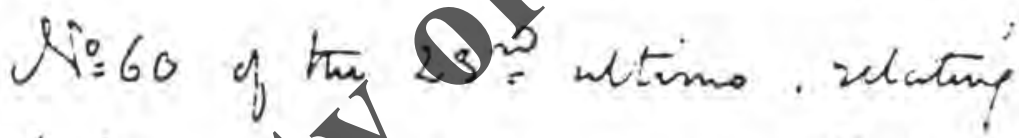
is the captestents of the Sheiks femated - din.

P I have the humour lobe with tion

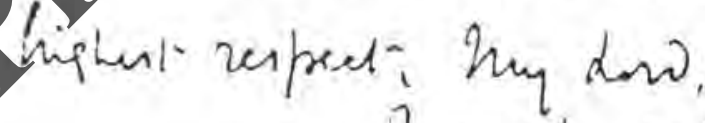

Znir desceshops nust Medrent

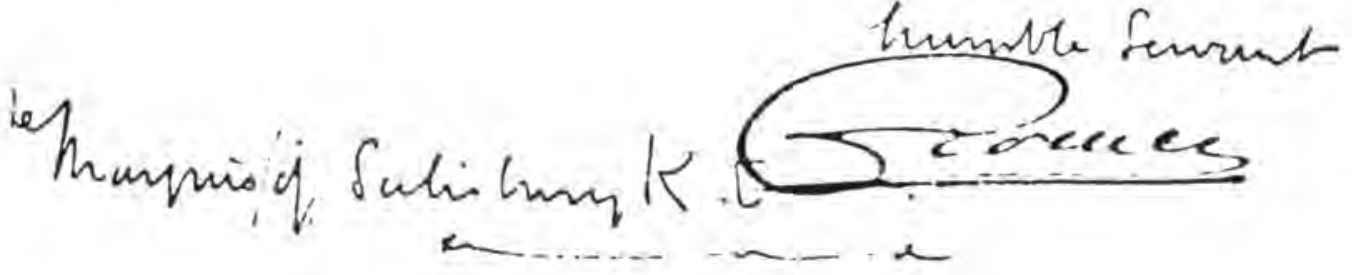




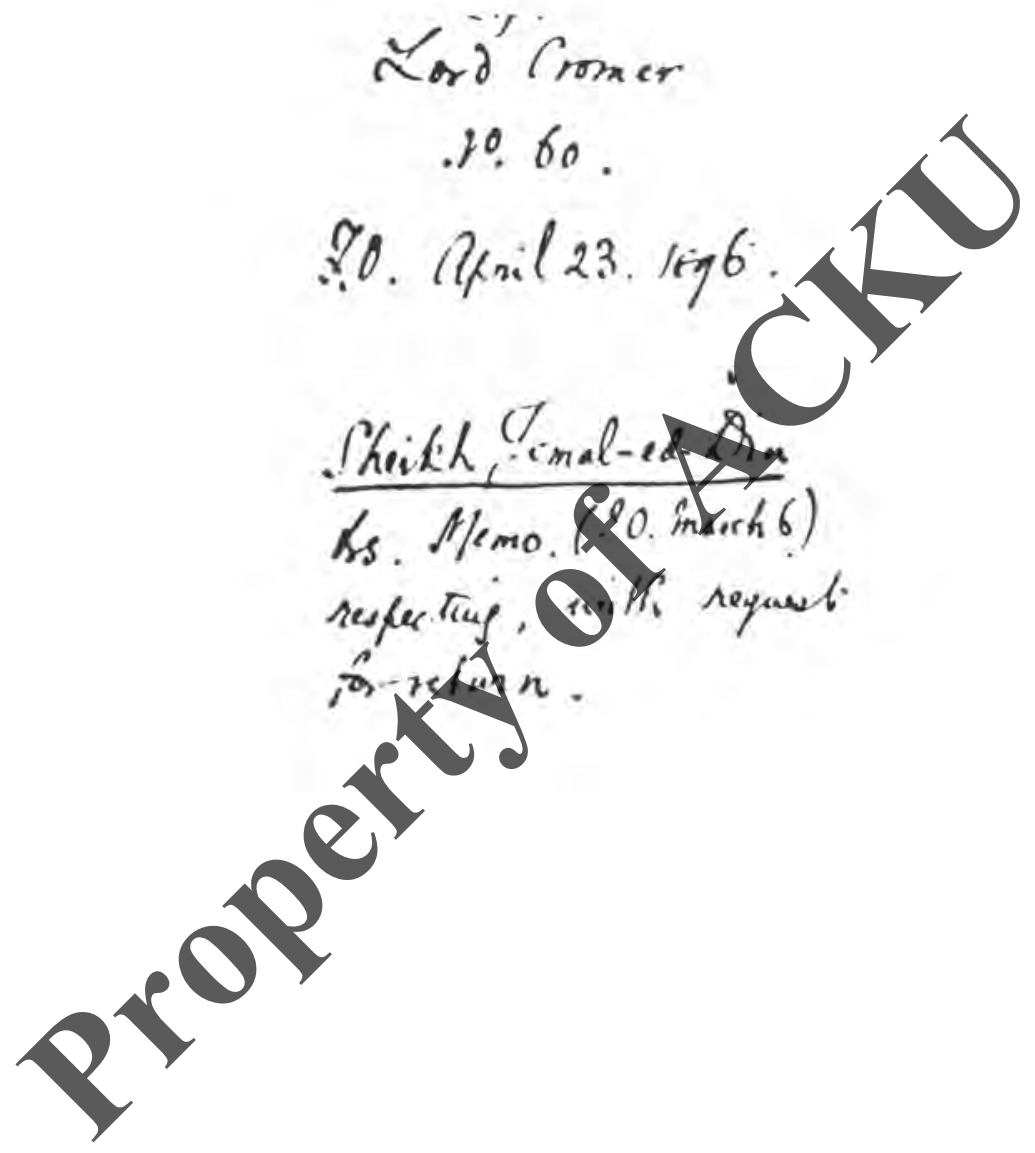


310

$\mathrm{Irt}$

$\frac{7}{20}$

safe

net truer

N. 60 lift 23 il 1896

key land.

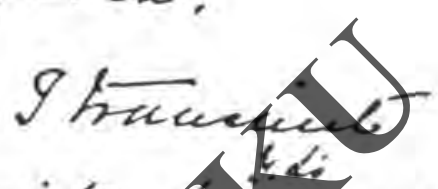

kencinte Árencel.

a merdaduar.

shese las buce

on Dnuncintide \& the

Dedia Dffices, ne the

sutindent of the

theikle friced-ed bice.

Liri munted the

the heewe neag, be

stincued to bai;

officie. 
IrT

309

Pine Hage.

Sir Pfanie

$\hat{i}: 285$

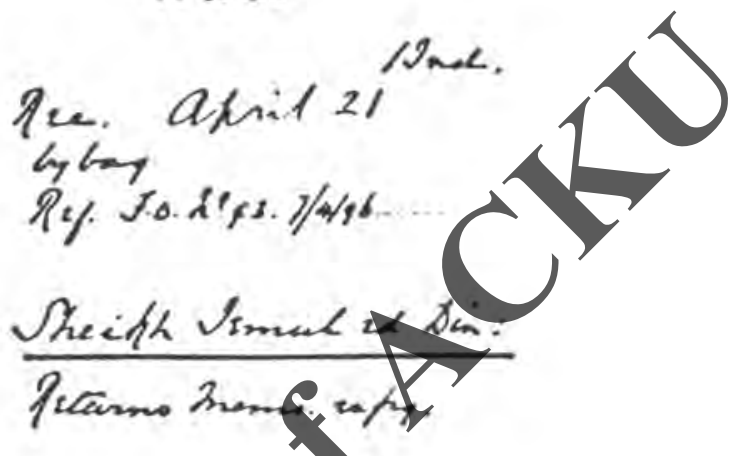

(a) $x e^{23} 19^{\circ}$

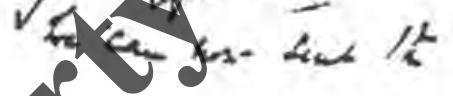

Qy2: t lain to hewere

* arum

Thos

299 
308

ITF

gs ofuie,

Sach fenther informalim -

neofrectiong this indicidual to

is in the frowessern of

Imbassy, will be parese in

iny desfritet 123 Precomber

$12^{*}$, plasinet?

Whapg te hamoun lote anth

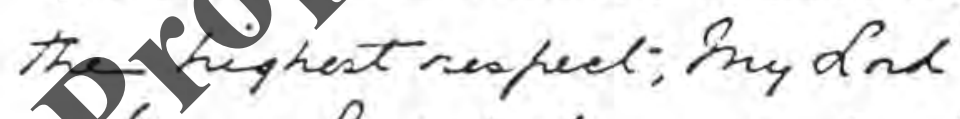

Yrun Sodstifis mons obedicint

humber vevent.

Philif funin 
110

307

14 Ginit 1896

185

$h: 283$

Byolad

to retum ourith the

usfertarn, the Skeith Imal

Q Dim, inclaed for my ferenal

in Gour Lodshifis deopratch ho

The

93

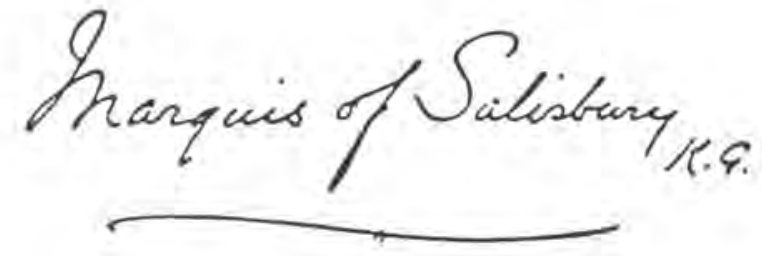




\title{
[3] I
}

In s despatch, dited 31bt Augunt 1889, from tho Indis Omce, London,

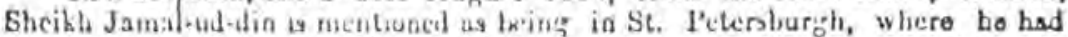
impressed upon some Kusinn atficials the prospects of a general rising in India wheneser the Kunvians chose to givo tho biznal. Whilo thero bo

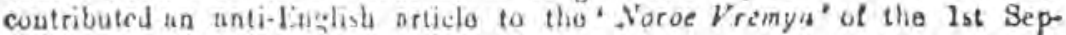
temkir 1bs7, but nothing way Learid of his Leing arpointed to any pose in Iusvian-Turkistan. In lecumber 14yl) the Shcikh went to Tetaran, much to the annoganee of the Stali, and endeavoured to gay his respets to the Amia. us-Sultan who put him off under sufur feretext. While lieco ho lived in tho bouso of Ilaji Jiuhammad llawan, Amin Dir-uz-7reh (SFint Master). Ito modus a number of converts to his asn particular faith which ho calls - Noturalism.' Whilat in l'enia he thought pruger to conform outwantly to the

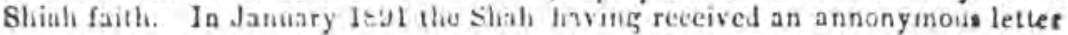

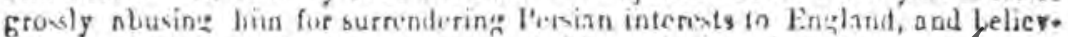

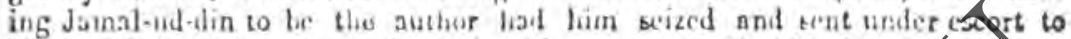
hermanshah, with a view to bathling him across the Turkish froutiek

Saynd Janal-ud-din after hinishment from Persia, found/Lit way to Bastab w Lero he stayed with the Jf ufti of that place.

Ho was next licaril of in London, where in December 1 sh ha was Tetively

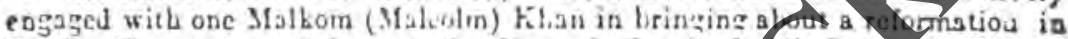
Persia. The war-cry of the party is "Kanun" (Law)I Paflic Safety and Indeo pendence I lig means of a pulitication called 'The Ranun" yralkom hiba's views were mado bnown in l'enia, and through Jumal-adetion the Ulems of Pensia wero being npproaclies nud stirred up azain the continuance of tho

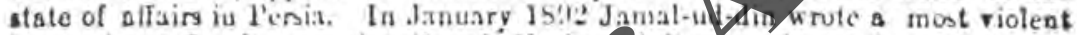
letter from London to the Cirand Vinzirt of P'ench the Amin-us-Sultan, a letter of such a charactie that the Sliah could hot un erstand how his request for the Streibli's expulsion fmm kneland couth not le complied with. Copies

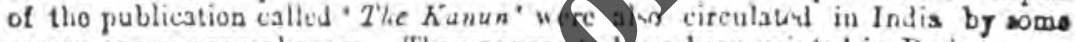
person or persong unbnown, Thicy appear to ave been privted in Faris.

In December 1593, wers was sectrod that one Abilulla Nedim had been givon s situation in tho Proshread at Constontinople, and had placed bimself under the protection of Jarnat-uil-gin. It is suid that the stieixh and

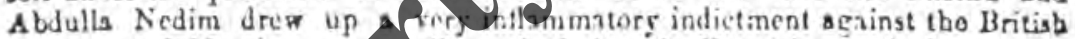
treatment of SIusalmans pearelli, and of thow in Eapt in particular, which

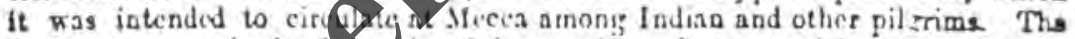
ducument nas submifted to tho Sultan, who, after consulting his adrison, recommended that re putter bo laid asida

Abdullo Netion is feverthel'as making way in the Imperial farour noder tho supives of tis triesyly sbeikh.

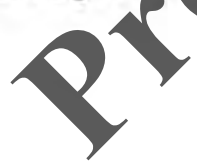

\author{
A. S. Lxtagridar, \\ General Superintendent. \\ Thagi of Dakaili Depardmeal.
}




\section{MEMORANDUM.}

The following is oll the information on reoon in the C.s.B. regarding Sbeikb Jamsl-ud-din of Persis.

Shcikh or Sarad Jamal-ud-din was born at Saidabad Framdan in Persis. At the anc of about 20 he went to Constantinorie, and there, through the influence of a l'nsha, who took an interest in him, he roso to tho position of a member of the Lducational Board. Through his diIferences with the Shaikb-ul. Islatn on relierious matlers he was kent away to lizypt, and for a lozg time was Professor of Ineic in tho Cairo University. While bere he became rege friendly with Iiaz l'a-ha and many otber E:sptian Frecthinkers. In IS79, be wa instrumental in forcing the Kledive Ismail Pastis to abdicze in forour of Tewfik.

During the Feyplian revolutionary movement in 1891, be was on tho sido of Arabi l'aslia nnd the Maldi. For this Le was deported be Heffedys. From this place, armed with introductory letters focm the cyetwe osten st Meces,

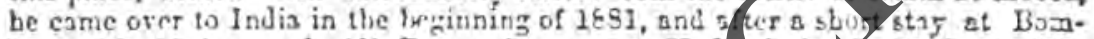

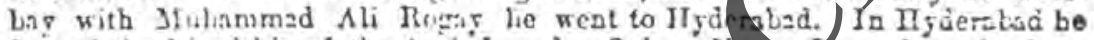
furmed the friendslip of the A rab Jemadar, Sultan Nawas Sarg, through whom be lad an interview with the late Sir Salar Jang. He was in Hyderabsd for about 20 months, and during his stay he assolpoed clieily with the rising Ecueration of freethinhers, tho followers Sayal A hmed of Aligerh. But in spite of all their bindness and hospitnlity otrards hm, Le fullistad a book in Fersian ngainst their dectrines. He peent to bold strong riens on such sulijects as ' lieviral of Istam 'and ' $L$ (ano is strength.' Ilis strry about hia expalsion from E-rpt was that lie was a Jinson, and that tho Etedire mistook him for a conspirator, and thus had him bualdealy deported.

Ile recciwe? Rs. 6,000 from lie late Sir Snlar Janz and come emall pecuniary liclp frem the otlicr polletacn of Hyderabad. With this money bo feft the Nizam's copital, and acetiviting Btopal on the rsy, be resched Calcutta carly in 15s2, ntefe lie remained for alout fire months and grve some Inctures in Feror of tlie Madrizs on 'I,lam.' While bere be offered bis services to Gorern (n)t, but they were declind with thas's. In September

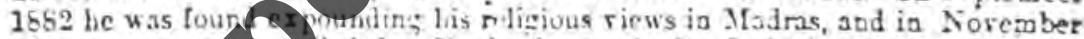

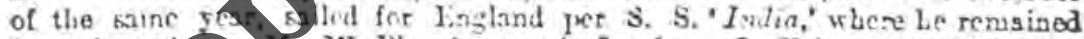
for a sliort tions HK. W. Blunt's guest in London. In February $1 \$ 23$ he was beard of in (ays and for a little while asoistad Janues Sanus, a Jew, to cdit the Ala Ausara, Aralic cotnie newspaper. Later on, in connection with oposthangad Eifendi, be started the 'Lreat-ul-It anga' Arabio newspaper. Mone for these venturis kere sujplied by Rine l'suba and his many other triendain Esyph

The next oews of him wos in $15 S 6$ when he went to Teheran, and oz his कay thither, ktopied at Buklire, Striraz nnd Ispuhan. Ho found I'cris to bot fer his advanced idens and was foried to leave it, rben to proceeded to Russia and juitied Dalip Singh and Ka:kofl at Moseow,

Ue made 3 rery food inprowinn in Russian Sucicty being a good trlker, and was som looked upon as a frat ruan amil yuite overobulowed linip Singh. This led to a quarrel betwen the 'wo whilh risulted in a complete estrangoment. Jastal-ud-din after this, thowed up lialies singh in hig true coloun to the liussian authurities, and when questimed ly M. de Giers about tho Lefalarajats inlluenec in India replied that - " Dalip singh bud not cven a dog with Inm in Indis, and that the rising foretation $\mathrm{knew}$ nothing about him." Jamal-ud-din is also rgorked to have bern connected with the lolisutio Leagua

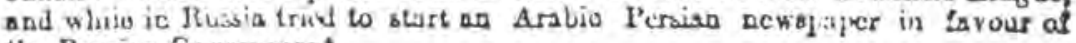
the Rusian Gurerameat. 
304

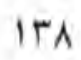

Sir. Samenter, or en ary treani Stion cons of the heen: fon th d. o.

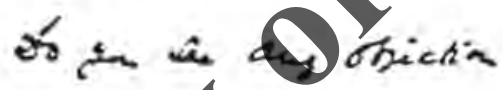
Ia $x$ and $=5$ - a,

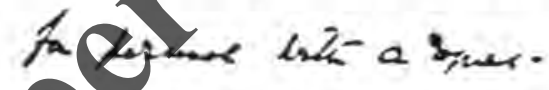

A- ki loy thatar

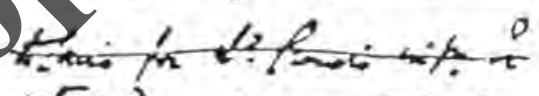
wheres cins Thes 


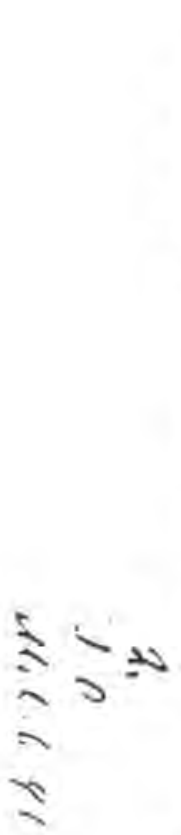

\section{[ \& ]}

In a despatch, dated 31st Angust 1893, from the Indis Ofico, Iondon. Sbeikh Jamal-ud-din is mentioned ns being in St. Petersburh, whero bo bad impressed upon some Russian oflicials the prospects of a geners rising in Indis whenerer the Rusians chose to givo tho signal. Whilo thero bo contributed an anti-English articlo to the "Noroe Vremya of the 1st. Beptember 1657, but nothing was heand of his being appointed to any post in Russian-Tarkistan. In December 1959 the Sheikh went to Tehran, mueh to the snanyance of the St:ah, and endexvoured to pay his respects to the Amirus-Sulian who put him ot under some pretext. While here ho lived in the

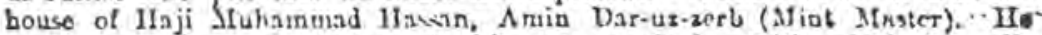
mado a number of converts to his own particulat faitb wlich bo callo "Natursilism." Whitat in Fersis le thought proper to conform od wardly to the Shiah faith. In January 15'1 tho Shah haviog received an Prnondwous letter

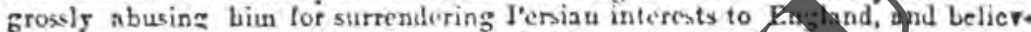
ing Jamal-ud-din to be the author had him seized and sent under escort to Kermanshah, with a viem to banisting him across the Turkish frosticr.

Sagad Jamal-ud-din after binisliment from, Terko found his oray to Basrob where be staged with the Ifulti of that phoes

Ho was next beard of in London, where in Acecmbgr 1991 be was actively engazcd rith one Jialhom (Maleo/m) Khan in frioging about a reformation in Penia. The war-cry of the parts is ' hanto (IAv) Public Salets l and Indo

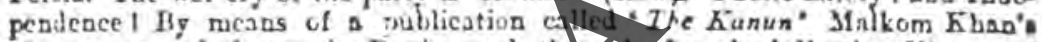
riews mere made knoms in Persin, and thryast Jamal-ud-din tho Vlems of Persis were being afproachel and sorred ur a gainst the contiuuance of tho state of aituirs in Peria. In Januner $15: 92$ Jamal-ud-din nrote a most violeat letter from Lndion to the Grand Pazer of Persia, the Amin-us-Sultan, a letter of sucb a character that troshabcould not understand how his request for the Sheibl'sexpulsion from Kavend could not be complicd with. Copies of the publication called. 'The Kantun' we re also circulated in Indis by somo person or persons uniknogh. Thieg appar to have kera printrd in Pario.

In December 16 ? news 25 received that one Ahdulls Nedim hod been giren a situztion ip trevrod Bureau at Constantinople, and had placed timvelt under tle protetion of Jainal-ud-din. It is sild that the Sheikb and Ablulla Nedipodruy a verg inflammatory indietment a gainst the Britisb trestment of thalmns fenerally, and of then in Egypt in particular, which

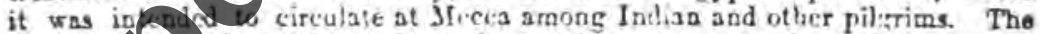
documct (n) submitud to the Sultan, who, sfur consulting his adrisers, recoparevel fust the matker be lsid asida

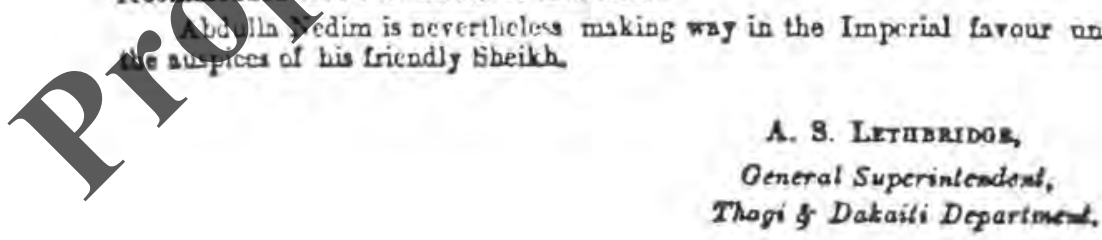




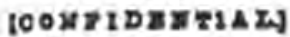

\section{MEMOR $\Lambda$ NDUM.}

The following in all the information on record in the C.8.B. regarding Sbeikb Jamal-ud-din of Peris.

Sheikb or Sayad Jamal-ud-din was born at Saidabad Hamdan in Pends At the age of about 20 be went to Constantinople, and thoro, through the induence of a Paslia, who took an inlerest in him, he rose to the position of a member of the Educstional Buard. Through his diferences with the Shaikh-ndIslam on relicious matters be was sent awoy to Ezzpt, ond for a Josz tiene pas Profeser of Logic in the Cairo Unirenity. While bere bo became ren fxiend)

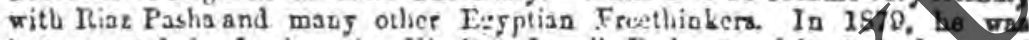
instrumental in forcing the Kliedive Ismail Pasba to abdicgle in favour of Tewfik.

During the Exsplian revolutionary morement in 1891 thens on tho cids

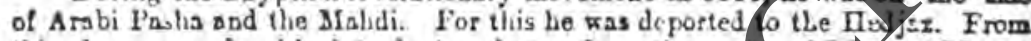
this place, armed with introjuctory fotters from the c:ntre of Is) on $x:$ Ifeces,

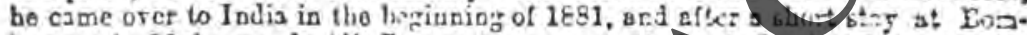

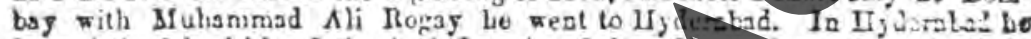

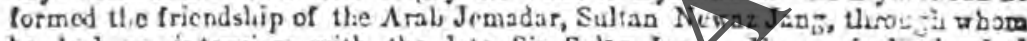
bo liad an intervie with the late Sir Sabor Jane. No was in lijcierabad for about 20 months, and during his stay he thociated Civfly with tho ricing generation of freethinbers, the followers of Syyd Atmed of Alizarh. But in

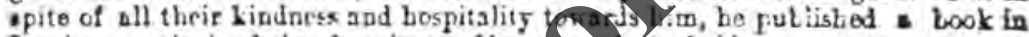
Yensian ngsinst their doctrines. IJe herny to hold stroug views on web

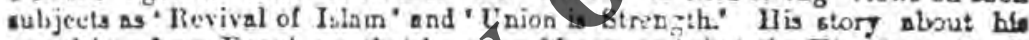

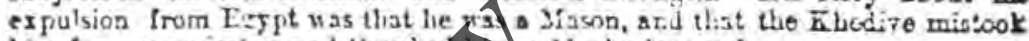
bim for a conspirator, and tisuswad him oudjealy deported.

He received Rs 5,000 from tise pro Sir Saiar Jang and wore mall

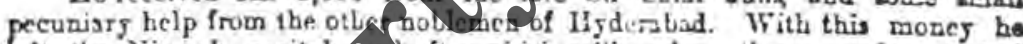
left the Nizare's eapital, and after visiting blucjal on the way, ho resched Calculla early in 18 2, (Byct lye remained for zbout fire months end garo nome lectures in Purin of the Madrass on 'Is'im." While here be offered

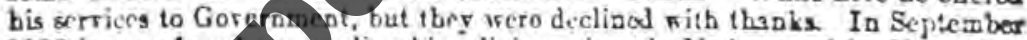

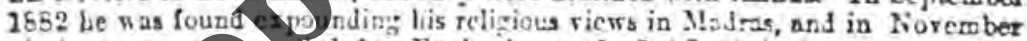

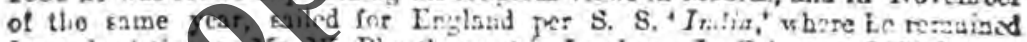

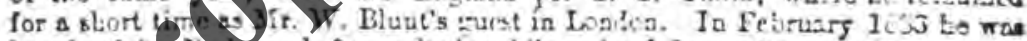

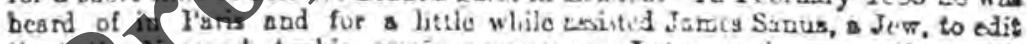

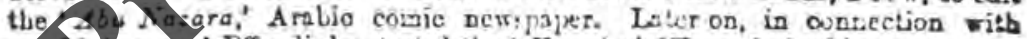

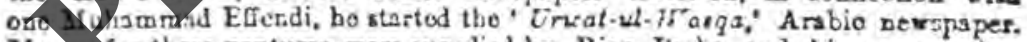
Afonty for these renture were supplied by Pins I'tits and bie many othor friends in Egtph

The sert new of him was in 1856 when ho went to Tekenen, asd on bis way thither, ktopped at Hustire, Stiraz and Ispaban. Ho found Peris too hot for his advanced ideas ond was forced to lesve it, when bo prococied to Ruraia sad joined Delip Siogh and Katkolf at Slowor.

Mo made a rery bood itursesion in Rusian Society boing a gnod terter, and was son looked upon as a ereat man and quite overshadoved Dalip Bingh. This led to a quarrel betirien the two which resulted in a corricto eatrnagoment. Jamal-ud-din after this, bliowed up Iolip Singh in his truo colous to

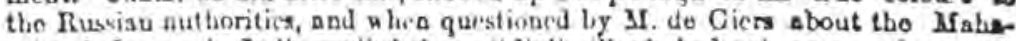
raja' intuence in India replied that - "1)alip singh had net erea a doz with lim in India, and that the rising sencration $\mathrm{knem}$ nothing about him=

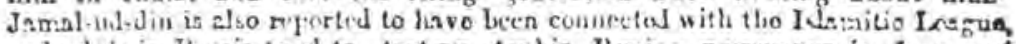

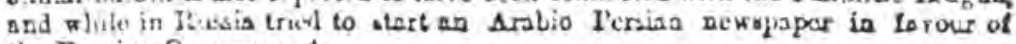
tho Russien Quterancot 


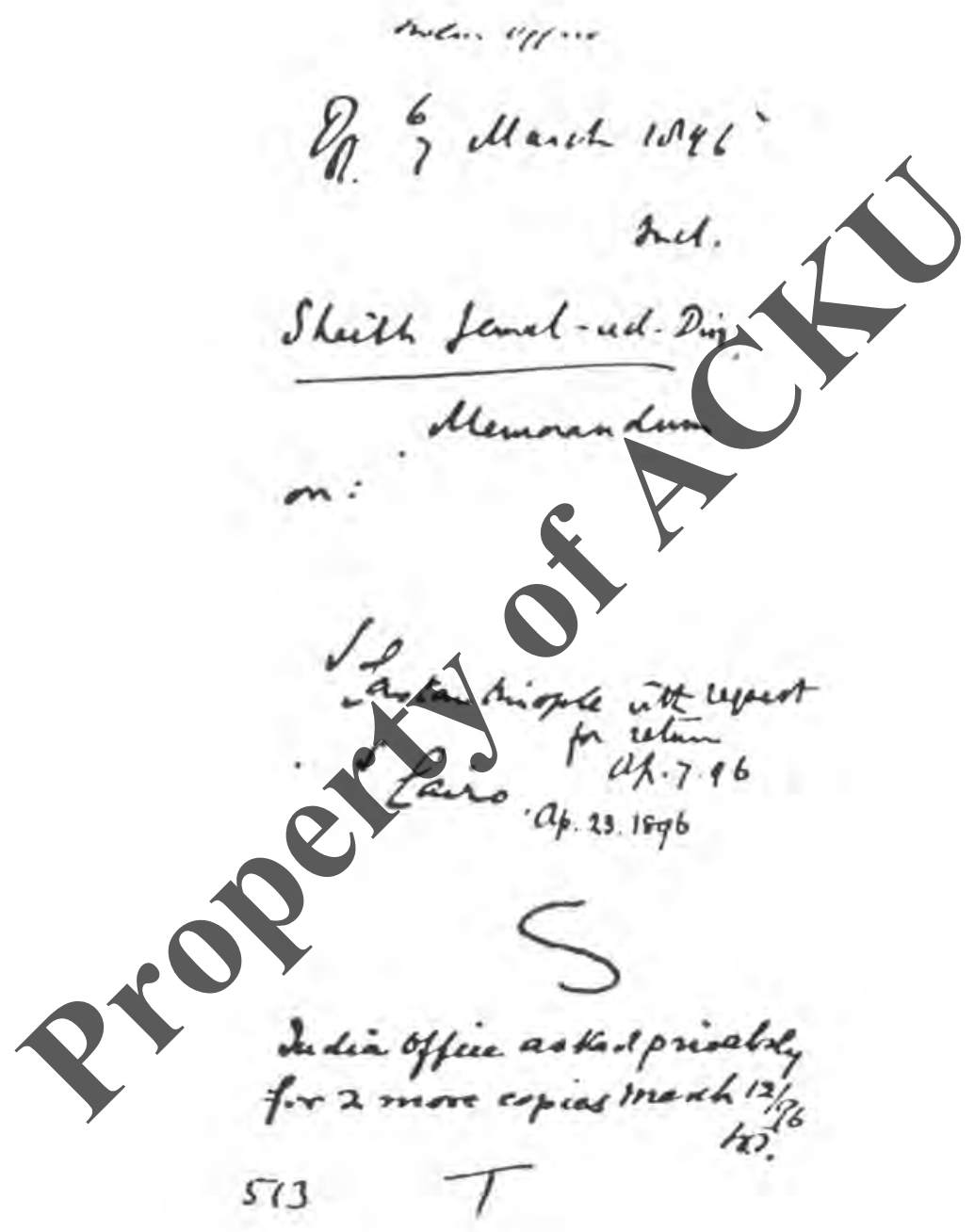


300

IF T

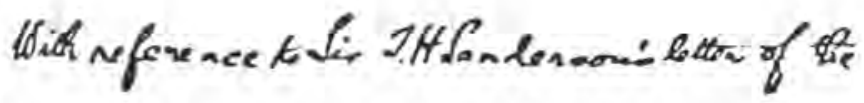
23.) Ak e. 19 , The Under Secretary of State for India presents his compliments to the Under Secretary of State for Foreign affairs, and, by direction of Lord george Jyatiliton, forwards herewith, for the is."mation of the Secretary of State,

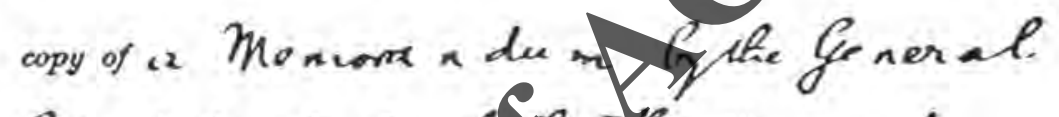
Superintendent of the the gi and Orkaiti Acparthent, ne gas ling fam al- a th

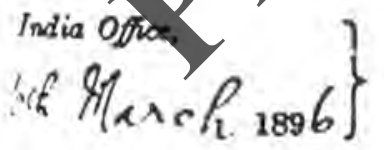

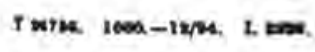


Srit (unie

70. Decumber 20.1095

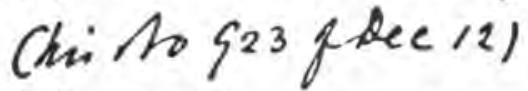

Sheith fernal es oige

semernents

Aptomes

ense respectse

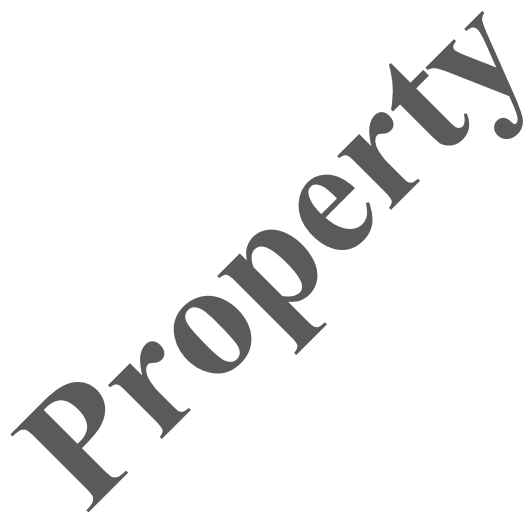


298

IfF

ช.

$\sec .20 \mathrm{gs}^{-}$

Drefe

P. Cunve

A. 410
Sir.

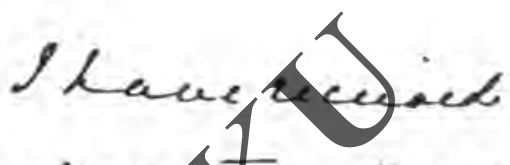

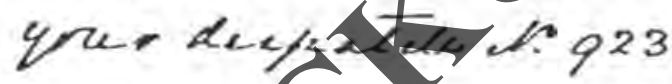

of the 12 sedened epplety no the popereris aced

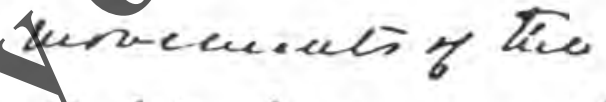
shibile feceral-ed-bied. ares lappure the enesse the ile Ener Backerey propes bpansae wite eyare \& this cincinkel

$$
\text { iff }
$$


$1+0$

297

Snew?

shaith deund-eat. dive

7.6. $\sec 17.1895$.

his w are accipest

年 mex in w

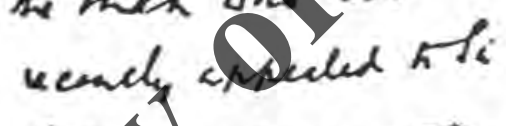

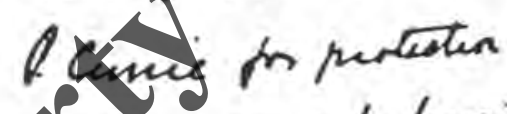

Exthe meen in lesmi

Costentiongle

$$
S_{\pi, 7}
$$


Pritide histineat of Busculueses, dracos op In cincaletios scunget pitgrian at kusces.

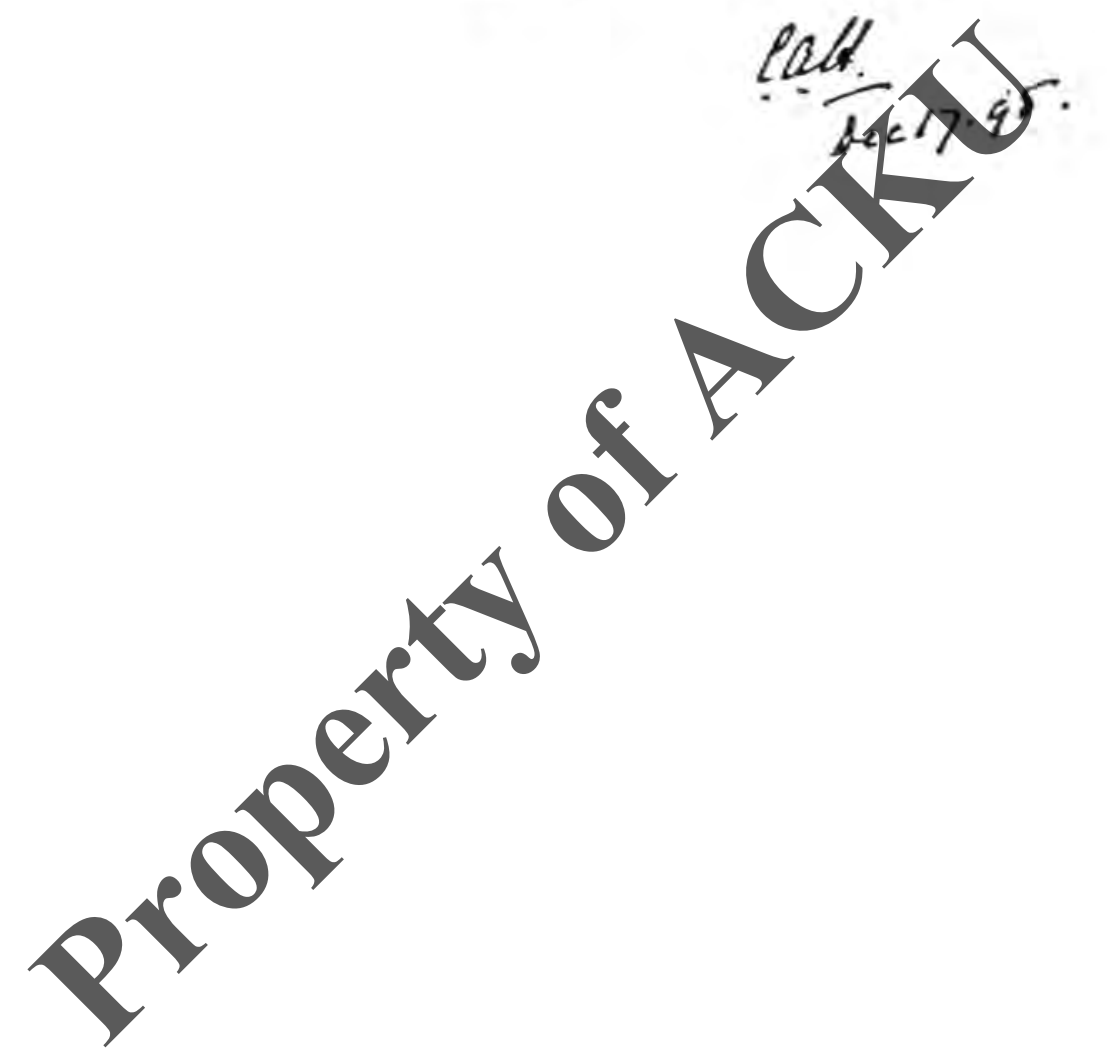


IFY

295

the affencall, lefe

147. Lescules

$2 x 114$ by $7 \cdot 9^{2}$

Sis C. Rod enfand hi laly 1892

for Creatifle thene he

waskaced cuarer

preci sarveifes ace.

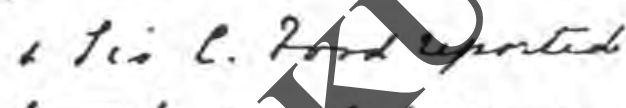

that he the enowed

to houdechera yortic

asipgier.

He nely Labreved

fis a. Liciolases

d. 5 g 6 Bec 22.931

2

Devarie of the theetse

encers br he cis a dospeata

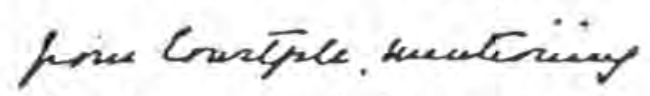

kis as a friet cattion

site mes absullale kexim

of ace cifflncumaling

midicturent agacerer tise

lititor 
294

$1+\lambda$

to bri si vativenuares

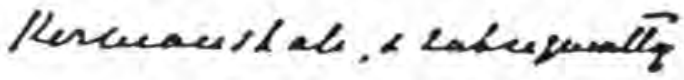

crecusted anres the

Twesile furti: Is

Tuster cypellew heis fores

Bagean, the chent.

Lrecese is 18 \&l.

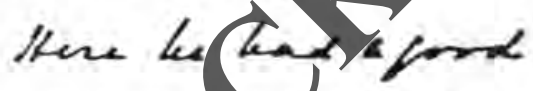

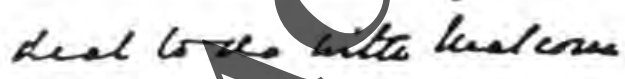

Heas. Ple isened

puppoleti a kates

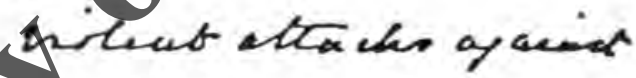

tha thats. the frequatis

sched theyp totates

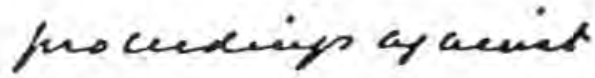

keis, sheile bey were unath $t h$.

Ke 
$1+9$

293

or a kefusuntation if tes bencaluens of hatir $\checkmark$ los besuen g an sppead for kelp aymaics the seyfick. He tauk wose unue vetrict tis Saurlacript teifiens

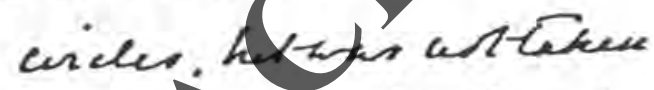
we firfory ly the Inerip muxile. He unarot QDar ca sadenesco of fur thale when H. Mn. weab blef ctencherge cix 1889. sar wintide hack to Pescie.

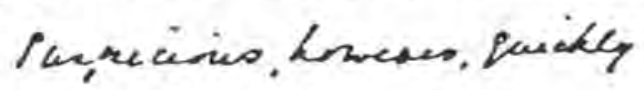
essae thers lleas he was intriguing si Resceare

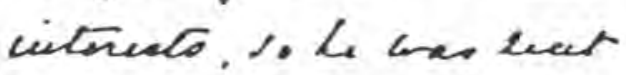

$c$ 
aced frivid a relyines

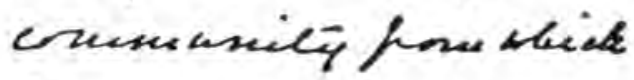
he war hose eyseled. the thens weentis sept. there he caprased $\bar{x}$

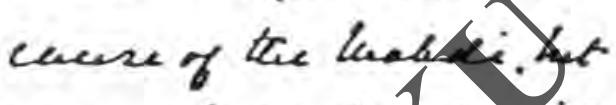
cakes cile chet ing site

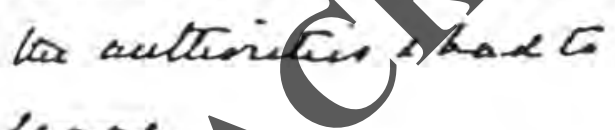
Leare.

Pars, LA his wes spitors of ofority. as the denenpin

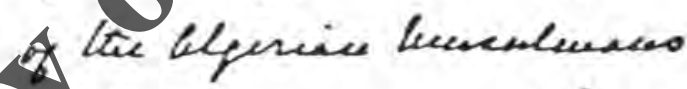
Y Dalito of a pepes wi treeds

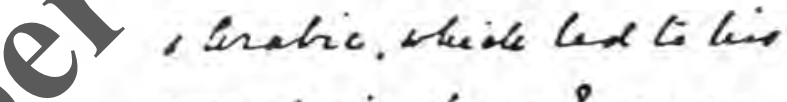
werelesin forse fraesce.

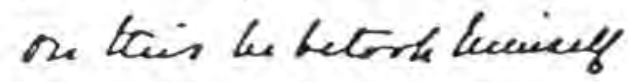

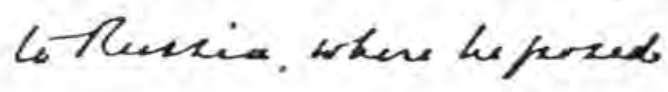
as 
Sit A. herver V.40 406.90

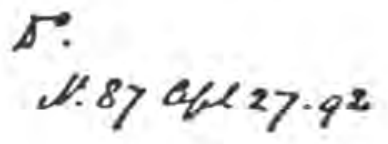

\section{knew:}

She theitile feculed. dice was bres ui Sencix 7 lfferes parcut. His kientes nio.

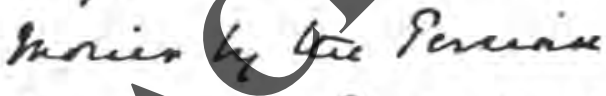

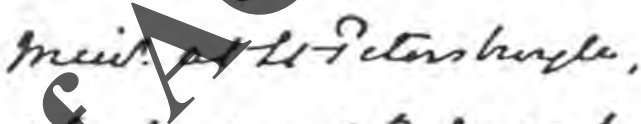
- kened to ryoud Dis marey ar a mincluienses citryoues almays hacy a cuelle cii any cause fors shick ke kyjht kap a moatoys. Lu zanly life fecued

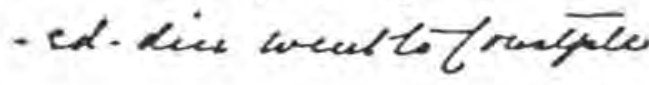
and 


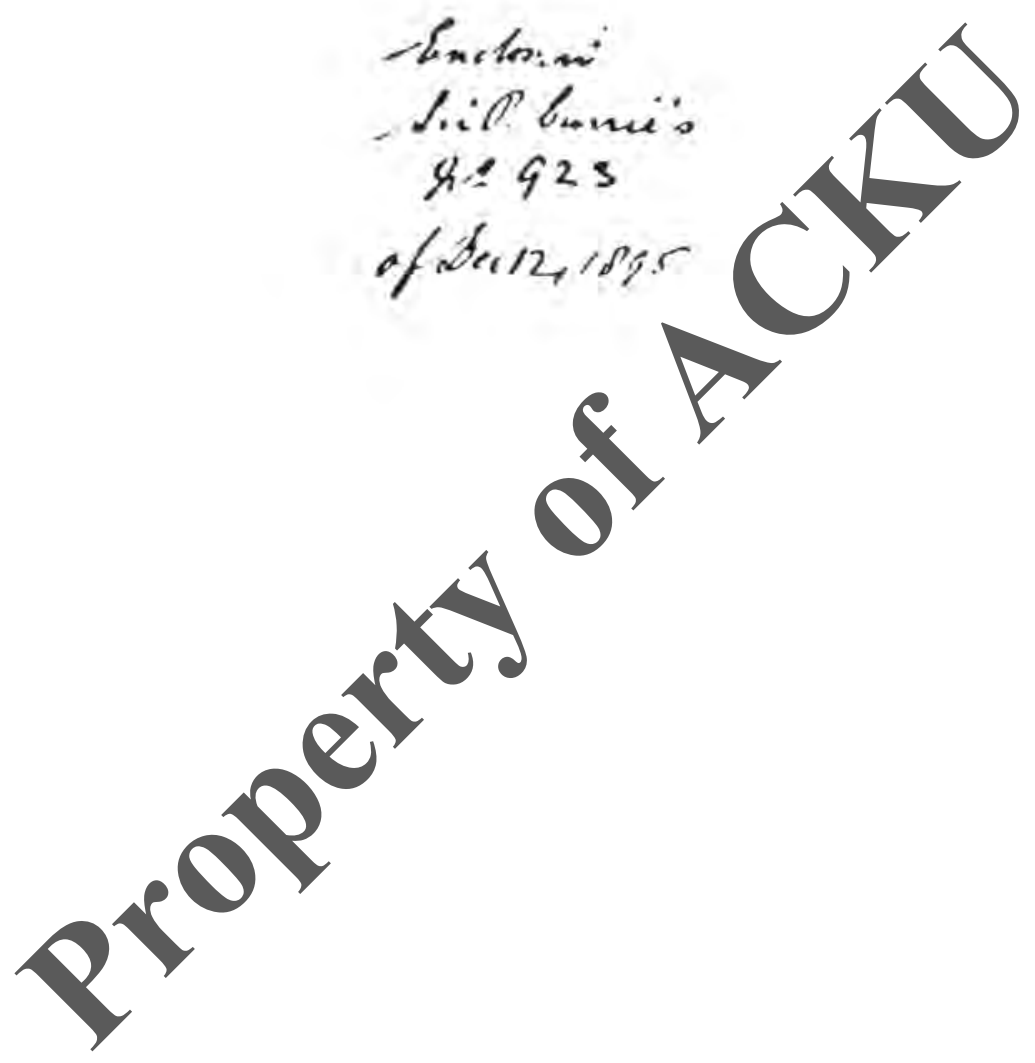


lor

289

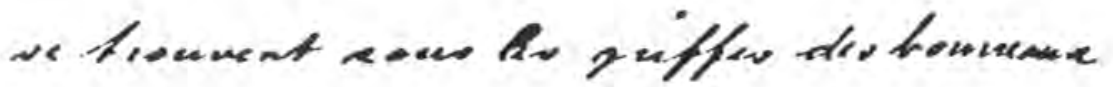

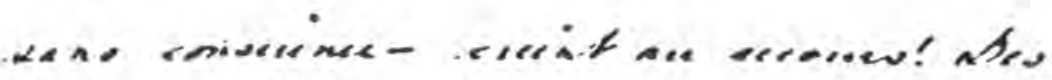

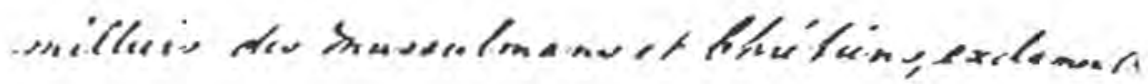

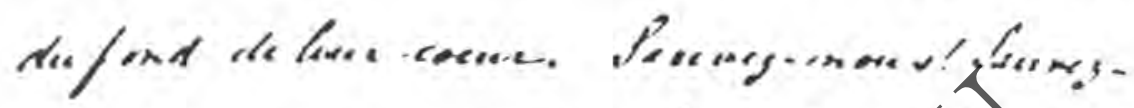

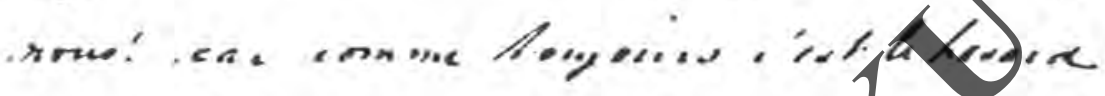

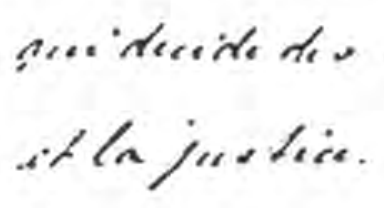

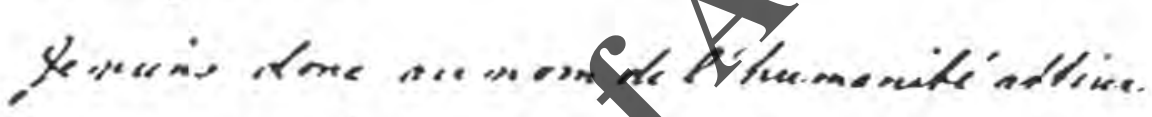

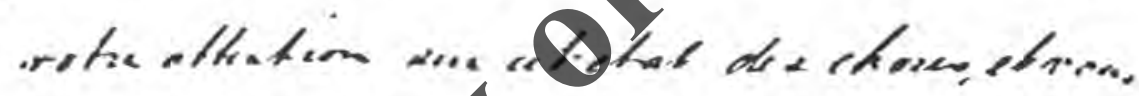

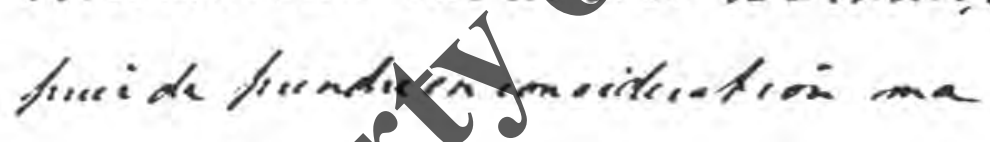
denesencor

exill, aree che

$Y(f)$ ifgemalfeden. Iltherserme il Rfgari 
288

$10 t$

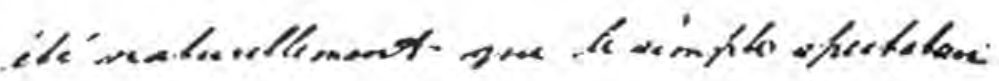

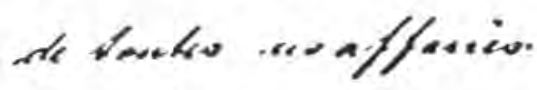

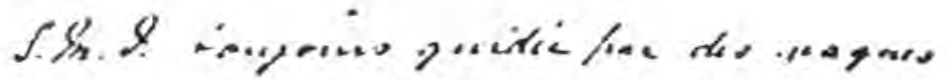

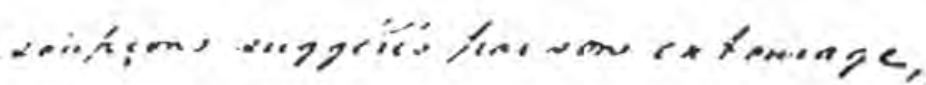

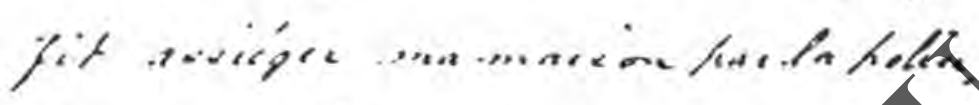

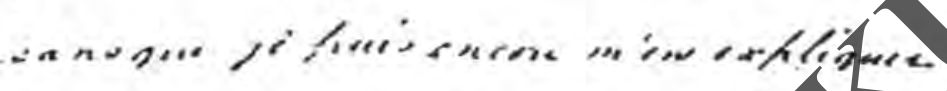

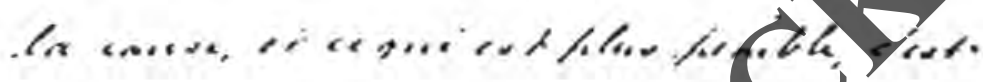

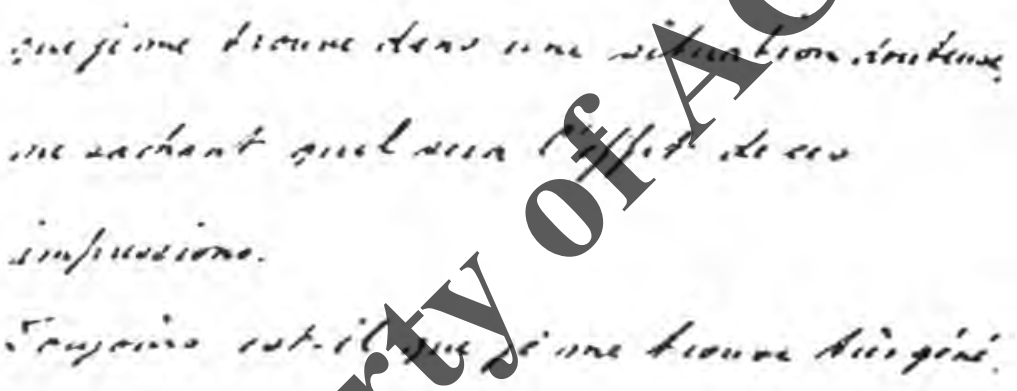

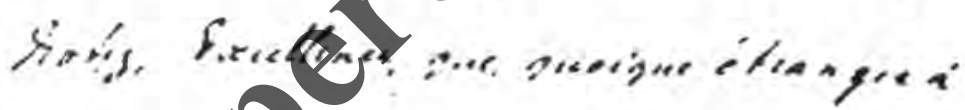

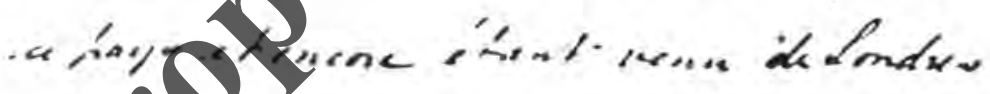

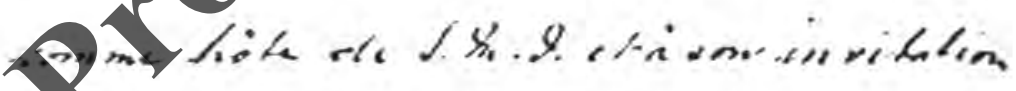

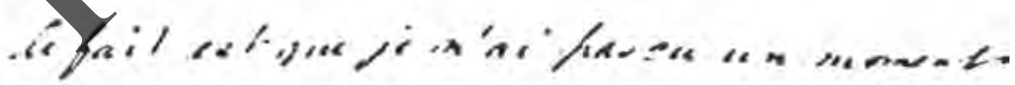

He reangellit:

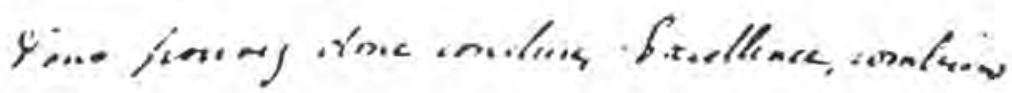

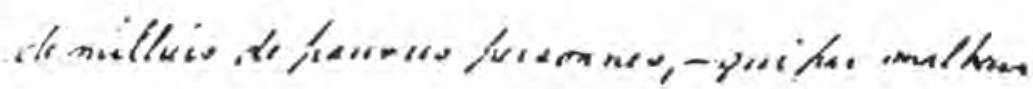

ec 
100

287

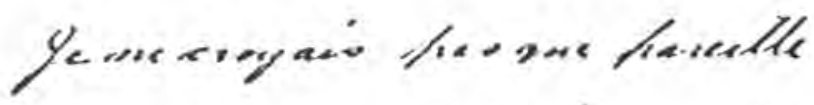

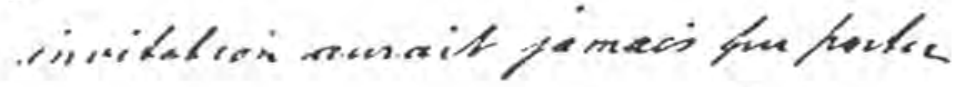

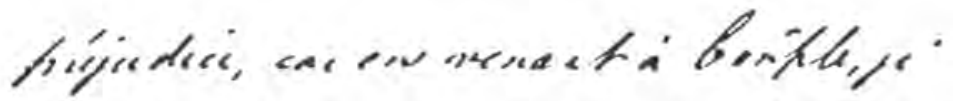

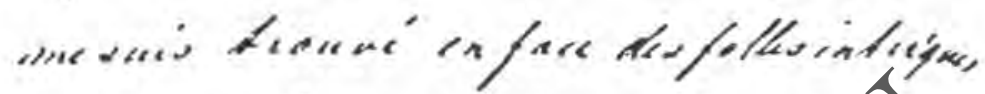

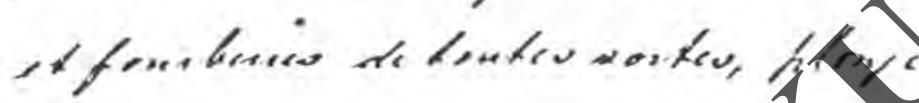

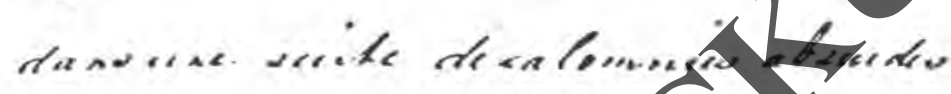

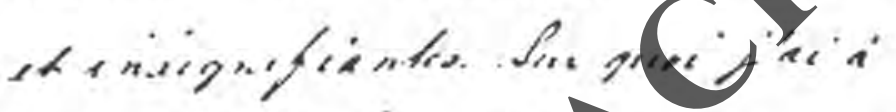

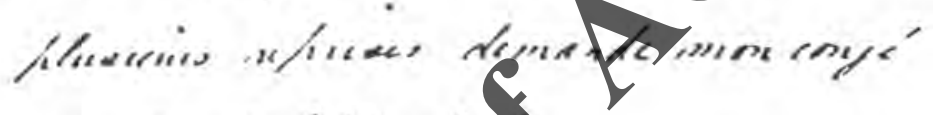

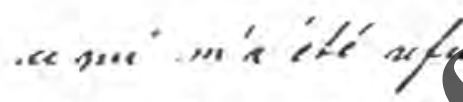

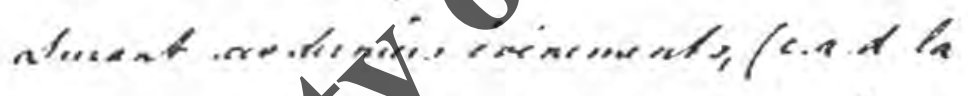

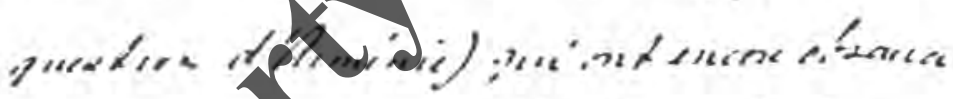

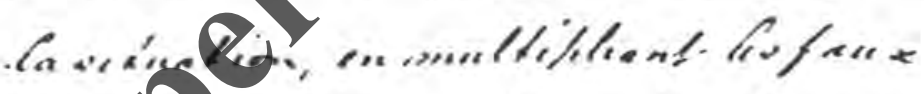

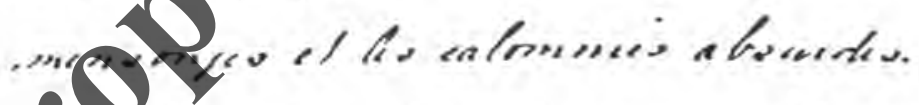

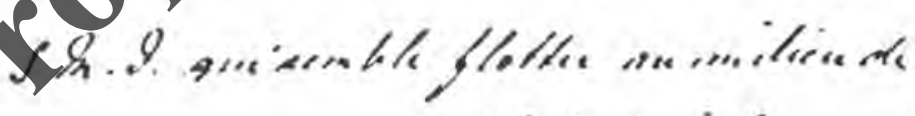

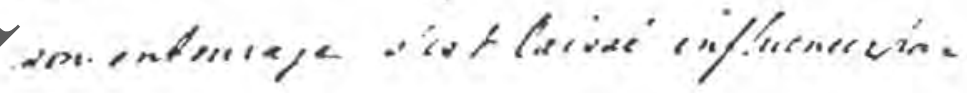

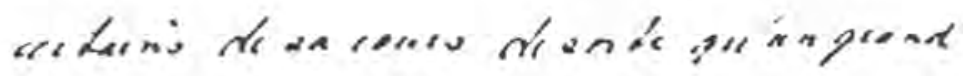

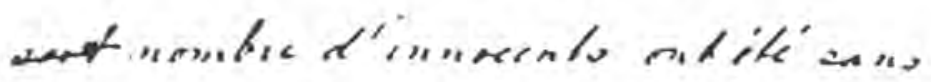

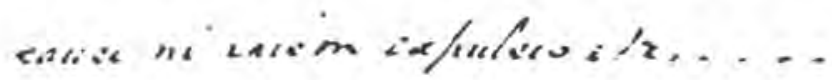

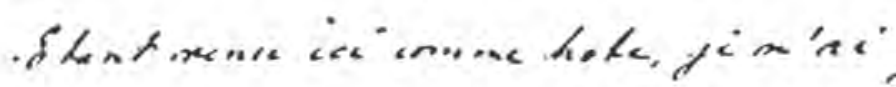
rice 
286

107

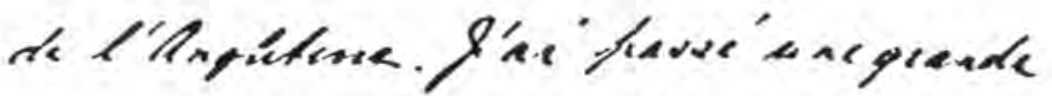

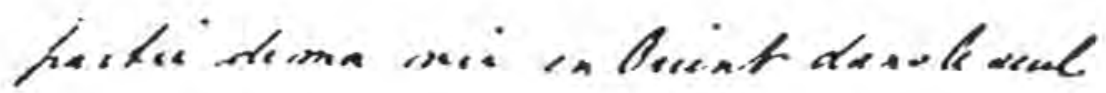
lust de diencine. le fanedroine, la filov

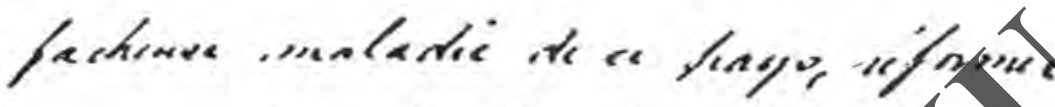

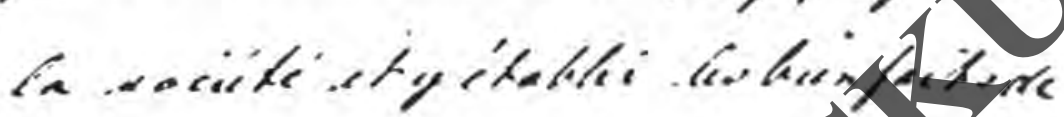

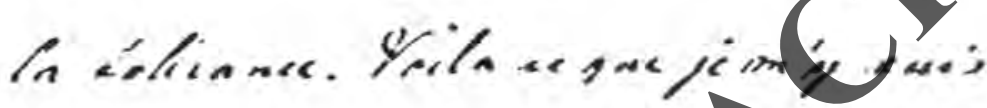
hiseprese:

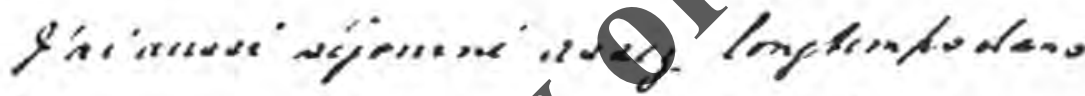

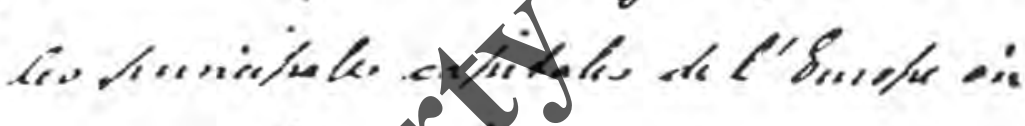

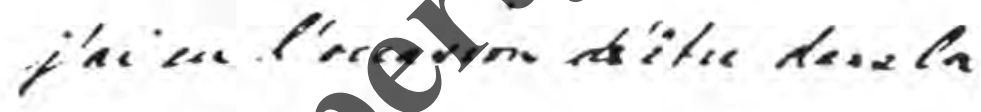

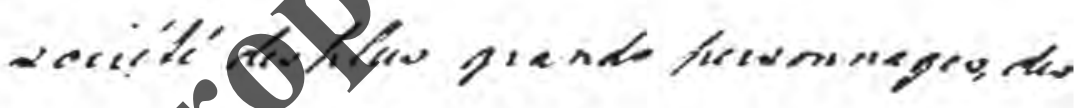

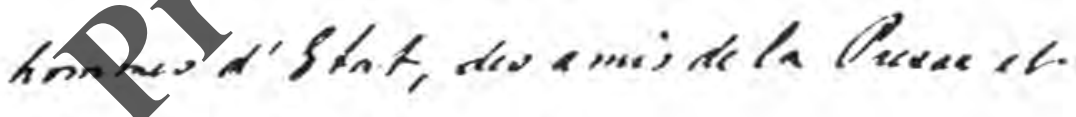
rico quends - Lenents.

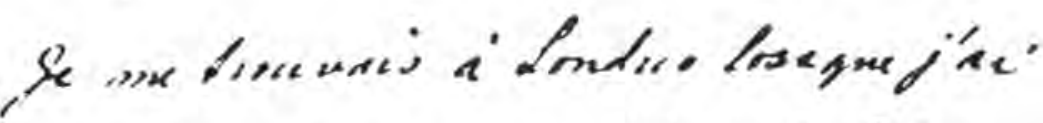

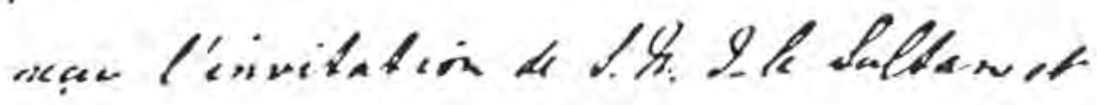

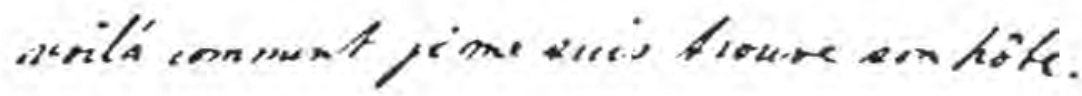


lomlla

le ignoons

- Hixellines:

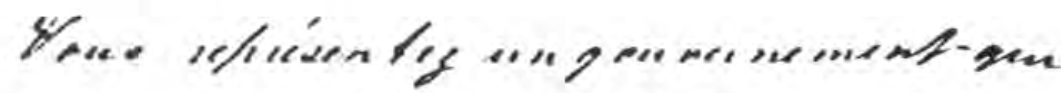

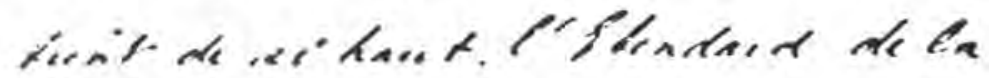

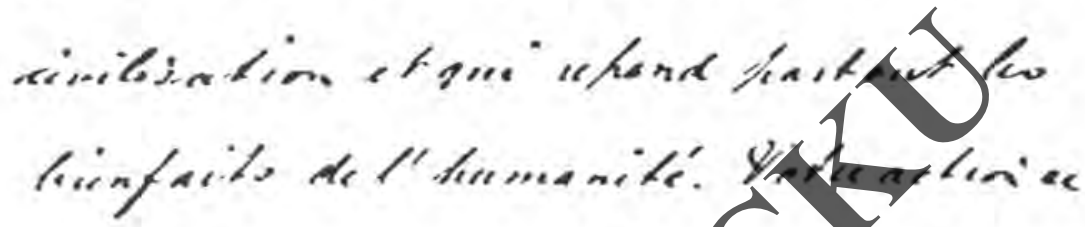

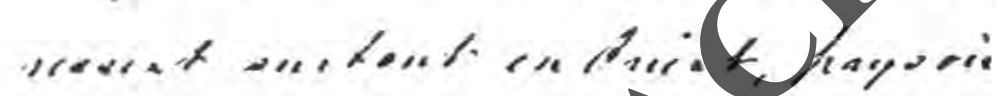

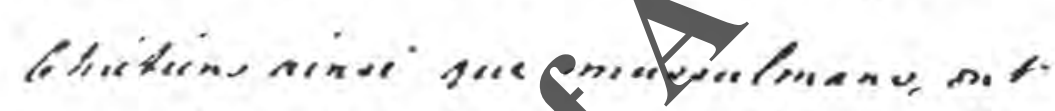

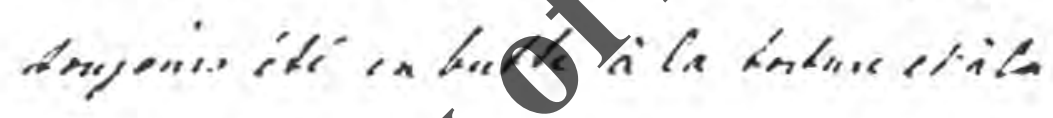
typenase.

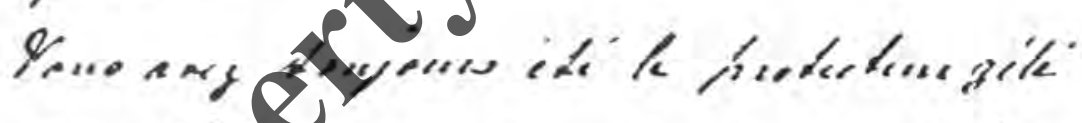

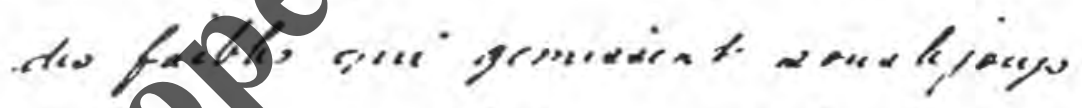

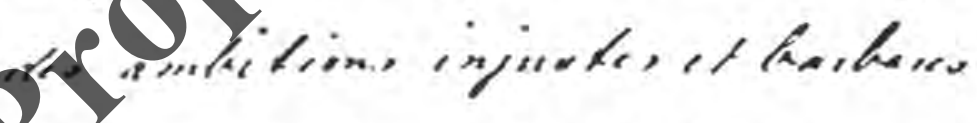

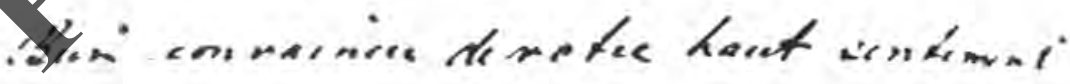

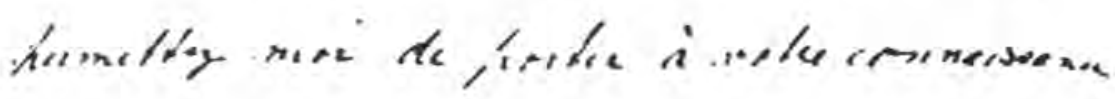
angestert.

se suiv Afghan Gabul) et fi defend.

Ne 
284

$1 \Delta \wedge$

Conatre Decis

SirPlurric

$$
\mathcal{N}=923 \text {. }
$$

encloure

tion $16 \%$

Sheibu femal-ed DC

Persion Qumbery

Pors office.

ate cheng hitura

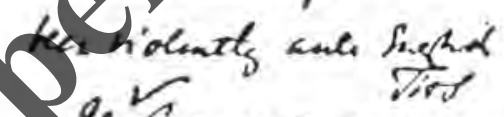

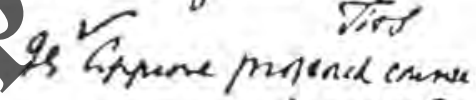

$\operatorname{dec} 20 / 9^{3}$

9.35 
104

283

Jpropose to take no

action in the matter and

with out further encouraging Jamal ed Dim, let then retrain possession of ty te British $p$ wesacafor Ido nor think it Grey that the Turki government will hand honorer to the Persians.

Johore be honour th be, wite tue highest respect ny y tare, Your at'ond ships moot obedient - humber Servant. Phiffunis 
282

17.

succeeded as yet in leaving the counting, the angle I understand he is anxious to do so.

The Persian Punbassaffor elaine s him as a Tropics

subject and on the ground. that he has roubletshed lireasenable prof em ate ins against tat shat, wishes to send drin under guard to lagan where the worked prot ably be rues to death.

Under the circumstances<smiles>CCOC</smiles> 
171

281

lim as a Gentreman resteut ar labul and procecoded to England via Viama Tlis $p$ ass is good for the rojage only and mextyy eurites the beares fo consular Mrorectipar.

The house inchlich femal = ed-Dir apt Jother Trohanmedan notrableptare lod ged ly the Suldar was burner the ofyer day and the 8heikh availed himself of the ofportunite to cocepe. Ite-has not howewn

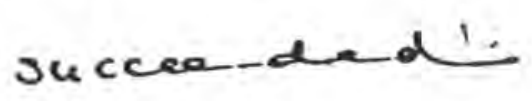


280

$17 r$

have the honour to enclose herewite and also sentan Indiar friend of his, Sayed hothanumed Padik Rlian, who descrives thenef of as" Judge of the Defcan to peead his eatpere To eucouragamapes how ower was gien tofthis incliendual Jengered-Din then applie to Hor Majesty's Conselate general and notbeing tizioun there. obtained a pase descriting him. 
$17 \pi$

279

among the softer hostile to the present Buchan and feat his bitter enemies the Sheikhs Ural tula and Ahmad Essay trailed thansalues of thin bring about his souffle. The was subjected to the strictest enrage and practicably made a prisoner in response. Under the circumstances the addressed One the letter which I have 
constantinople

$$
\sqrt{1923}
$$

December 12.1895

thy ord,

I have the honour to report to your epdotien the ar the Sheikyejal-ed Din whose history probe known to the Foreign Office, has recently papa ce overtures to this Pitas for British Protedgon as ar Af than. If would appear that the Sheikh was deeply implicated in a movement thin Marquis of Salisbury $\mathrm{kg}$. among the ate ot 


$$
\begin{aligned}
& \text { Cory } \\
& \text { Zelespon } \\
& t \\
& \text { Sin 7. Enscelles }
\end{aligned}
$$

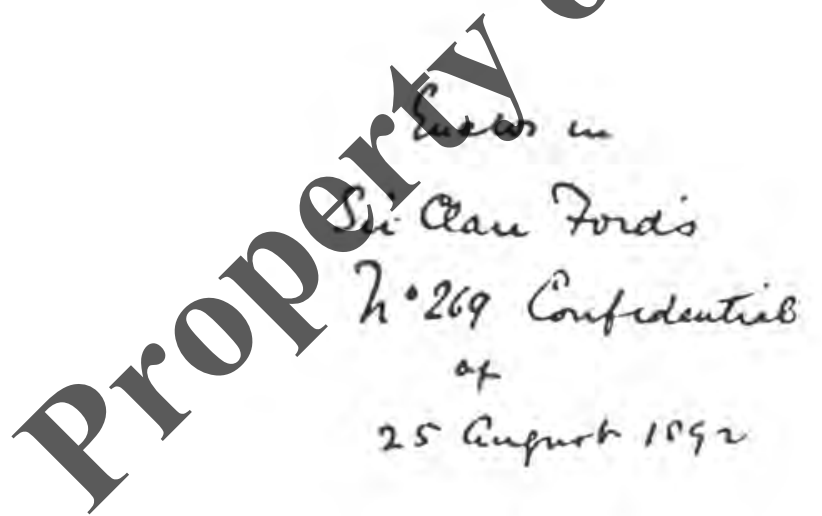


276

177

Jelevar

Constantinafle.

$25^{2}$ angush 1892

Gour teleprain of the ya inetant to Lod Saledury. Itam privafte informed by the enfond Plizir that the man in poryteris will be remoned by fuctani orders to some restete sfot in Ama hina Care wilg tee taken that any pubination -manating from the mite he devoid of all polatical on seditions matter.

Fond.

7. La:calces,

Tekeran. 
178

275

Compla Anguat 25

Sir Clare Ford

h:l6q Confidential

Res Sefut.2

thes

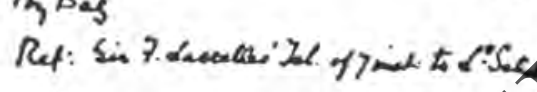

Alghan names Dyamalugf is

wile he esiles to a nomote $c t$

tranomiti cory telph

the is a sestris mita vense tofles frose reckes upuples, frach be at a towntance is the

QRider fort

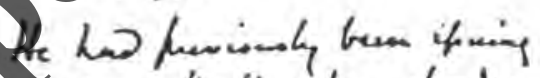

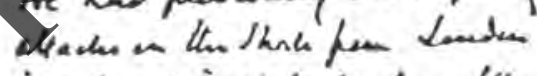

in crijuations withe mutam than

$\alpha$ egantefor his monore fer

thion comli wase mand b) the

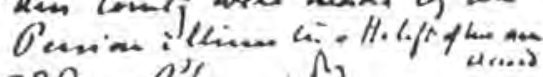
2S\& O'L Ul. 
$h: 26 q$.

sidential.
Constentinople.

254. Anguet 1892.

Iny Lord,

leith reference to tir 7. Laxcelles

Jeleqram dated Teheran Andyat $7^{\text {ta }}$ respecting an Afquan of the name of Djemaludin, I have the hovour to

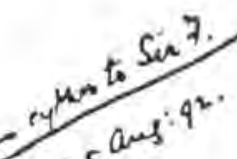

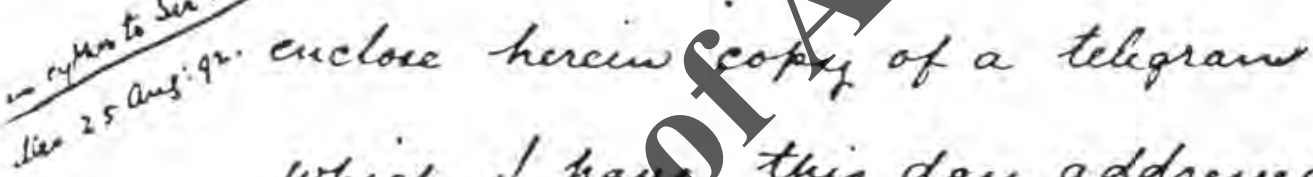
which thand this doy addressed

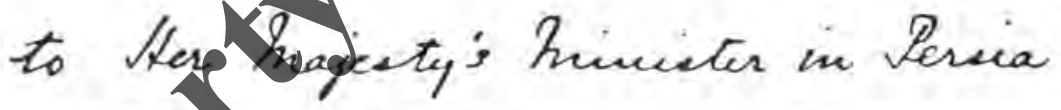

QRave the honam to be,

with the highent reoprect.

hey ford.

Your Lndelufis mort obdeciet.

humble ecruant,

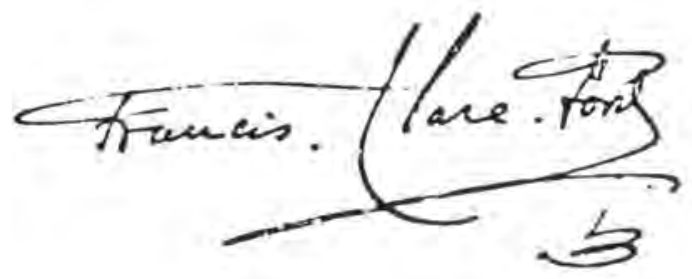

Easl of Roubery

$\mathrm{Ui}_{\mathrm{i}}$, to te te. 
179

273

if: 2: 11\%

Hewen if 7 Cugned 1PY2

Dienual.nd. Ai

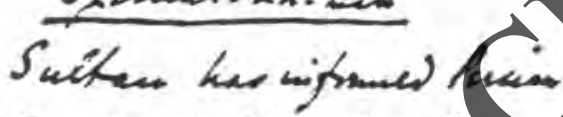

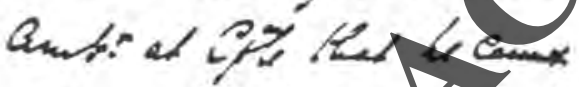

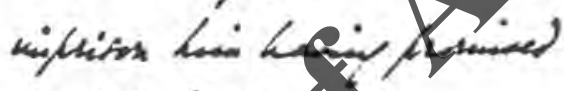

wox to sos.

ixugat 400

Qyencia Offece

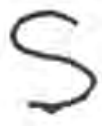


272

ir.

Deaypher. Sa 3.Sascalles. Tran. D 9.59 \& 9.159 pm. Aug $7 \mathrm{mpz}$ 10 114. Persian ambassador at constantinople trigraph that Sultan has informed him that he cannot imprison Djemaludin, as he had given hem promise not to do State for F.A., howeva, considered that he had dome shat a service in separating him from Malcom Khan, and he police surveillance, and hat ale owed to publish seditious writings. - fmen-co-Sultan has se me to ask my opinion parades ardently suspicion that the Sultan Pray Employ Djemaluain against Portal 1 replica : it appeared to the to be satrefation that he, was unary sur willard and separated from macon, and that I would the grape both to K. E. and J Th. anbassada at constantinople, in the hove that H.E. might be able to obtain information in regard to Dyemaludin's action at Constantine.

Repeated to Con stan anole. 
|VI

271

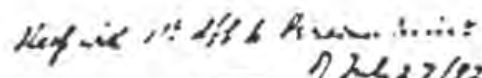

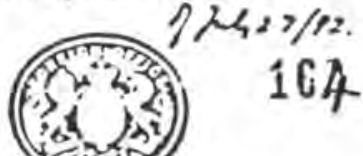

WDancera

the Pesias Heinita

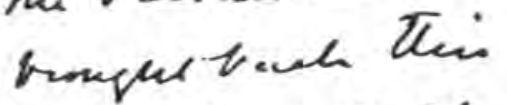
$\alpha$ artho if we coule madify s $^{-10}$ in Inf a way that biqum wall ne toe ther ifon hin to kes Ser. o kpany ran tew tript Pocestang Qgerat fined-e-din Qher arcondin tan Lan Hicen permen he nipurkame bose

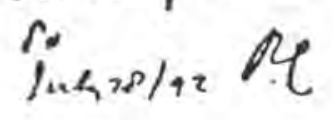


270

IVT

after 2 is aupraing the mulan andis th 2 mit 1 tumis su hed-

anse oxiftic a paproat are and antiont. way of meetiog th sulmittuy to ridoric to and olfaiving an firma Aom logat offonts of eminara lat a conicta Penian Huffuta. wither pisto the 4 was likely to resuet?

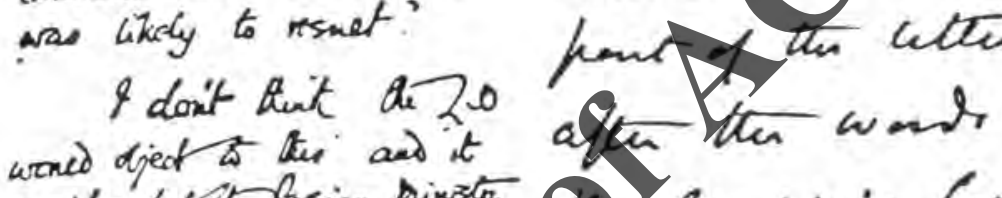
onit the cello

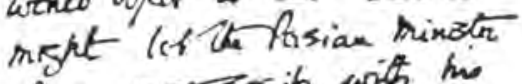
tionm mox zosity with hio

Q2 Ingites's fort. $\frac{\operatorname{CB} D}{29 / 7 / 9^{2}}$ ho Davisen cear en Inile now 2 w Plean have the 2nj - n-wai km danteryothin: arcenting

$\tau$

the s./pe

of 

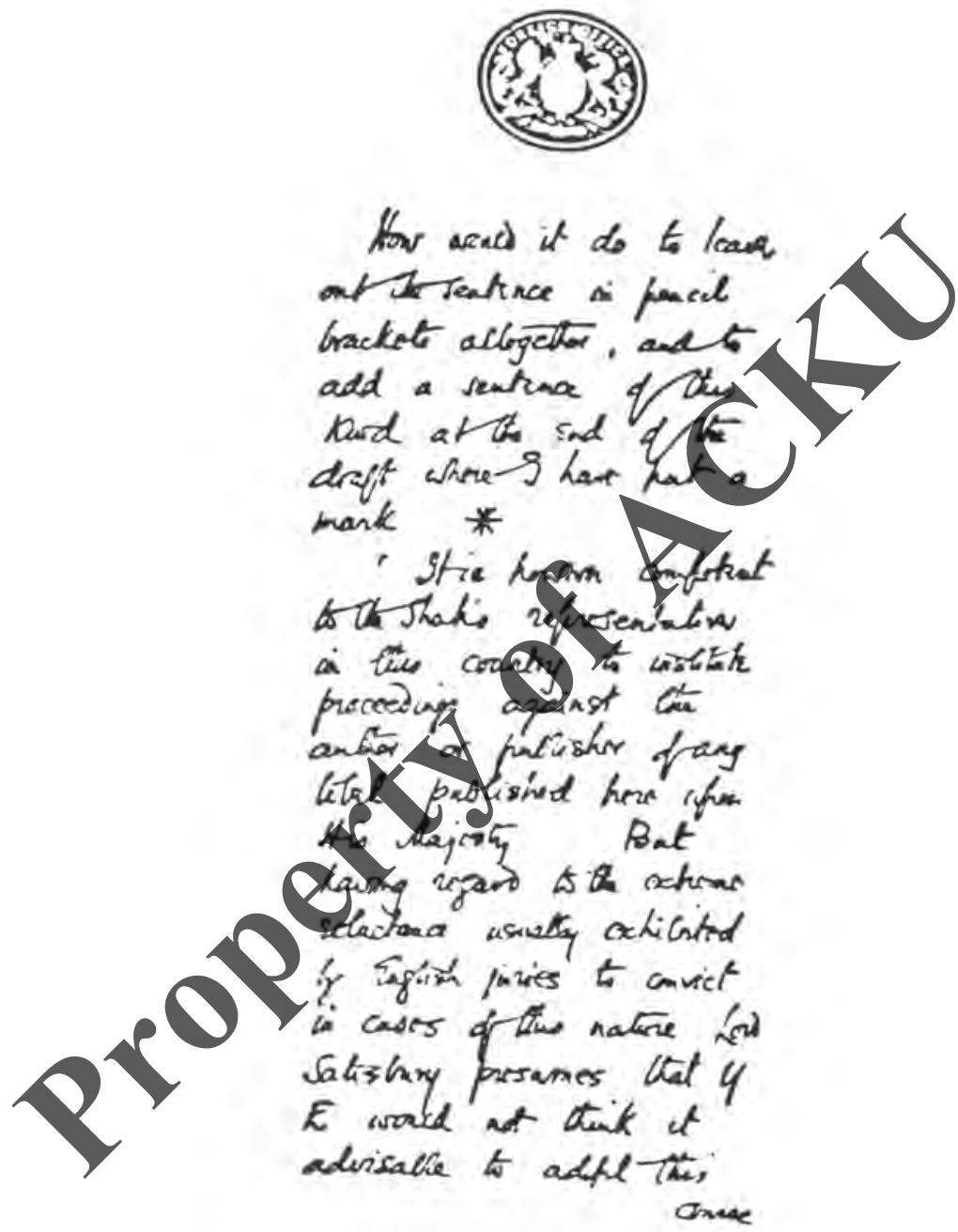
thiga horames all Kkian

Y. $0.942 \%+592$.

ref: his of gome 22.

Yamal ca bin

H. hr. Grof cacent withinde

trocerding apt or caftel hiof

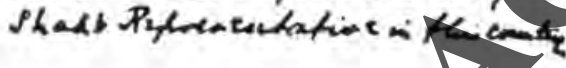

\&

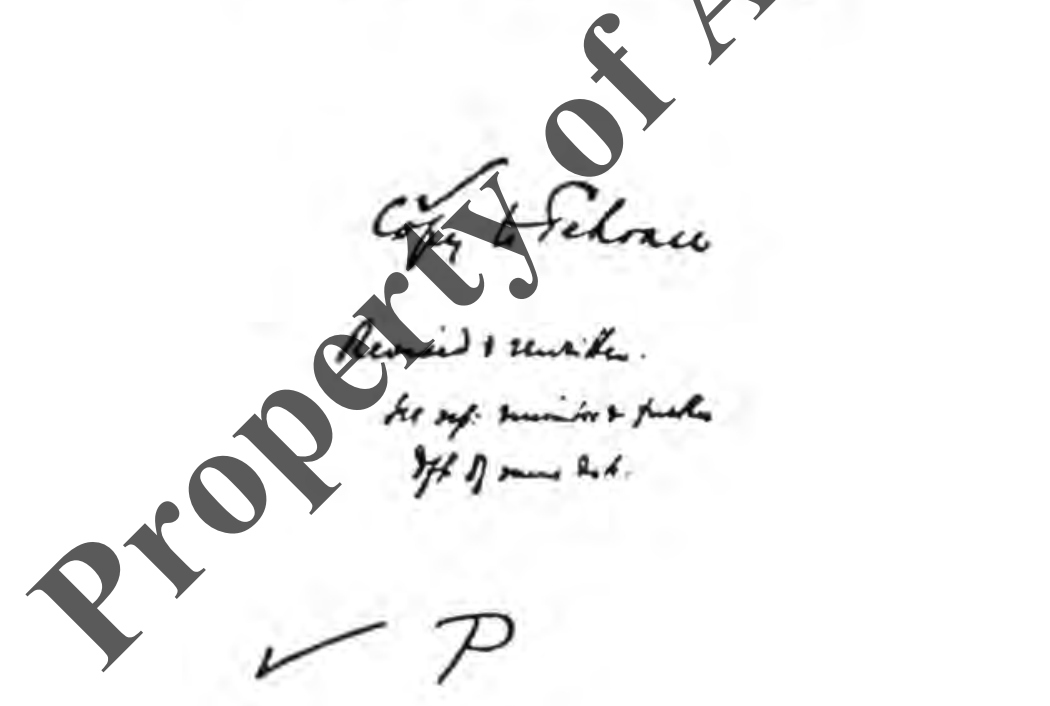


IYO

267

thetio

"Requefentodinci in this canafis to procuento the cuferer or quitliates faxy leter xuthated -here apap terejas ves are fust Qubrised that is a the ter cuy ectim

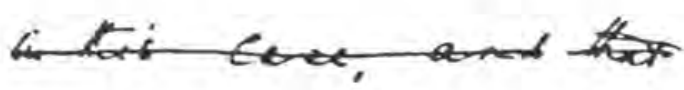
the have to stminestratio gerer of expetting the anthor of the thes complaind

$$
\frac{\operatorname{los} D}{25 / 1 q^{2}} \quad \delta
$$


Tientures

Srafs

$$
\begin{gathered}
\text { tho } \\
\text { She ey } \cos 2
\end{gathered}
$$

hije hak? alikhan

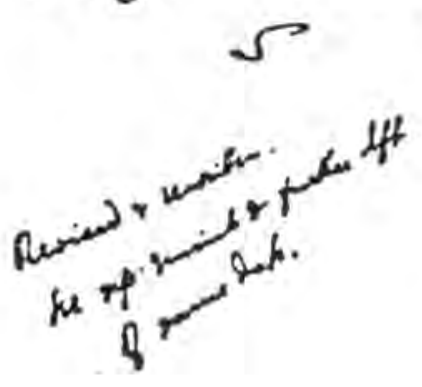

111. Le Rinistro

$Y$ her been :

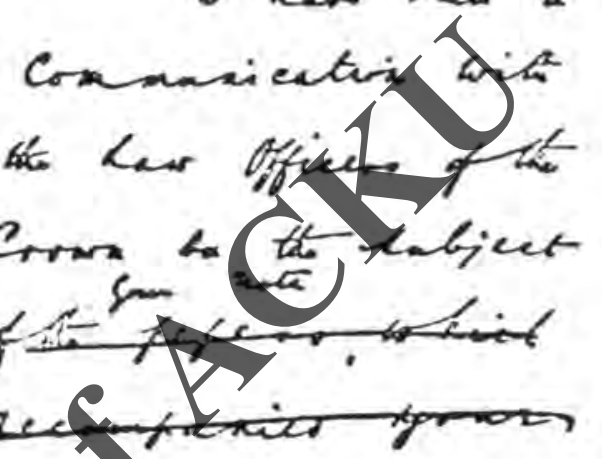

(Len ofivin ofly !)

if of 22 ald?

guepecting to purakings a the: conctor of theis Qjeima.ed-2in.

Yhen urs tor

Lonory to inform Vor

that" Lan edointe that

tifioct thated afford

is gromed for to

ciscitition ffegal froese

bo fixilyon.

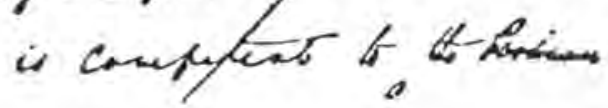


IYY

265

Anizgema? Acilchan

5.0. 7 oly $27 \cdot 18 q^{2}$

(dis2aculte)

Dianal.ed As

Hioprocending offirt

riggranud for higfe

brrecedaigforistsug.

cipytakran

$\checkmark \phi$ 


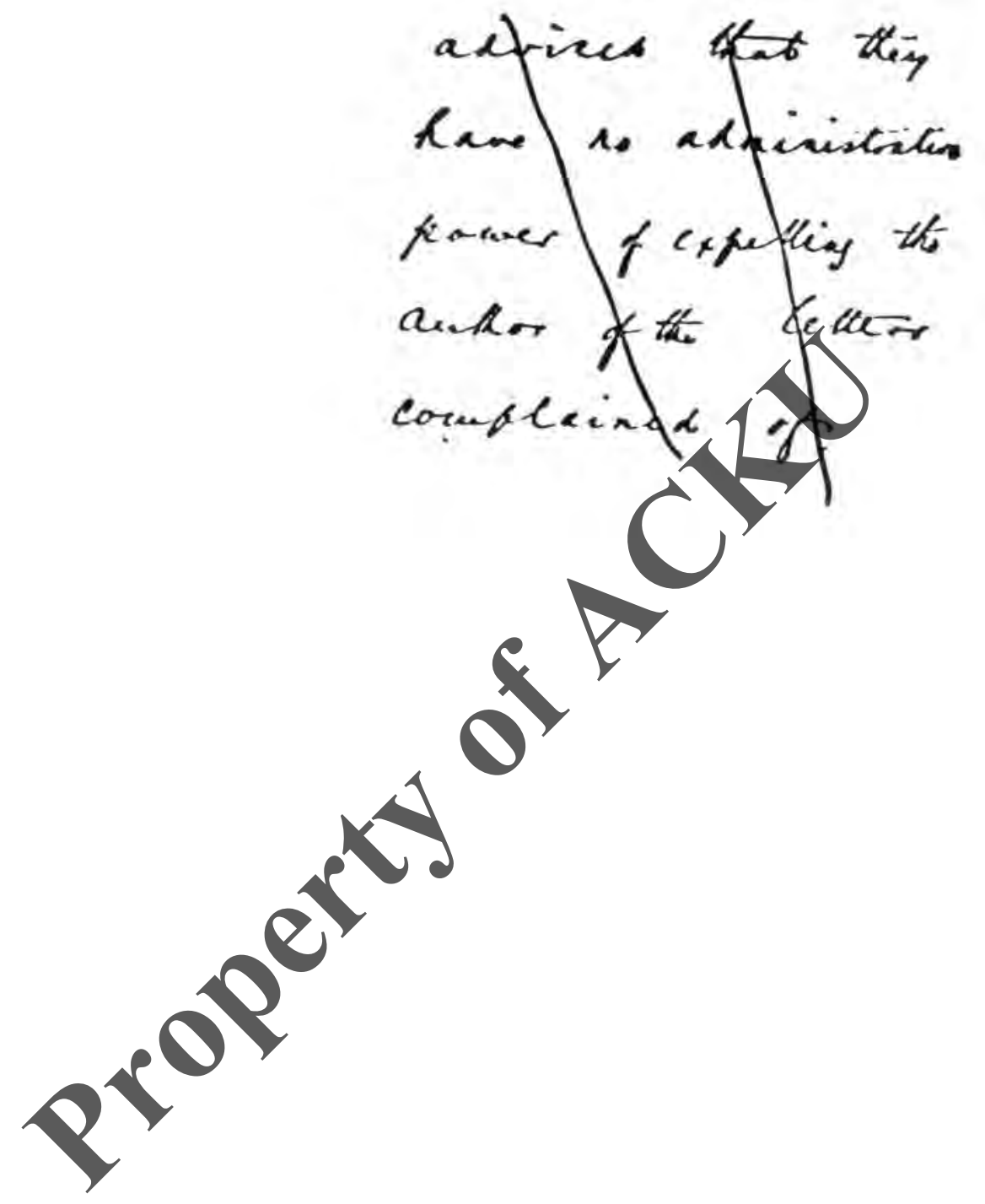


to.

Saly $77 . \mathrm{sog}$

Q.afs

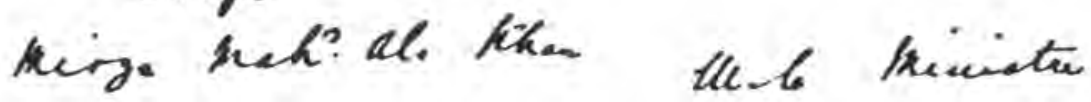

$S$.

Yhar been in

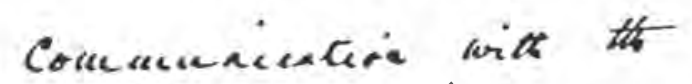

Las officer of Cirava.

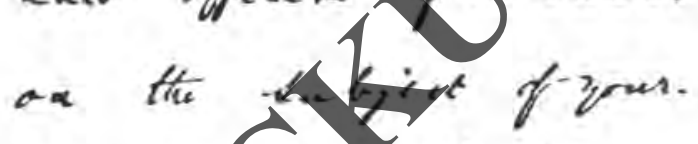

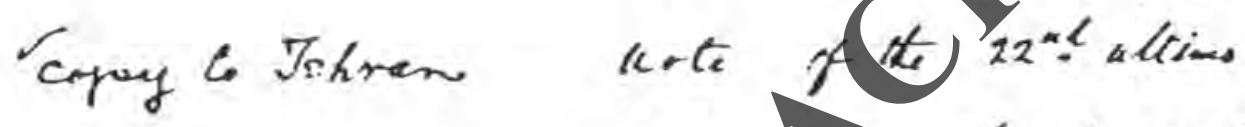

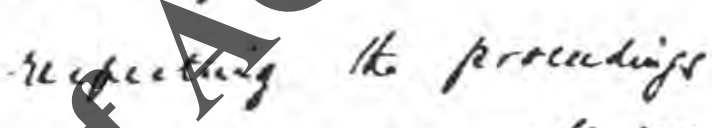
infer: conatis fitheikh Riémil-ed-Rein.

Yhave kear the

kouares to leifrea ceres

that Lean cheried that

thi- facte otatid afforx

tho granat for the

rietetution of teqel

procesdangs byttalyr.

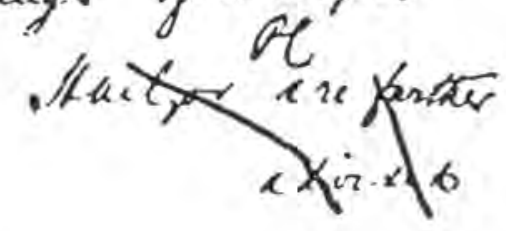




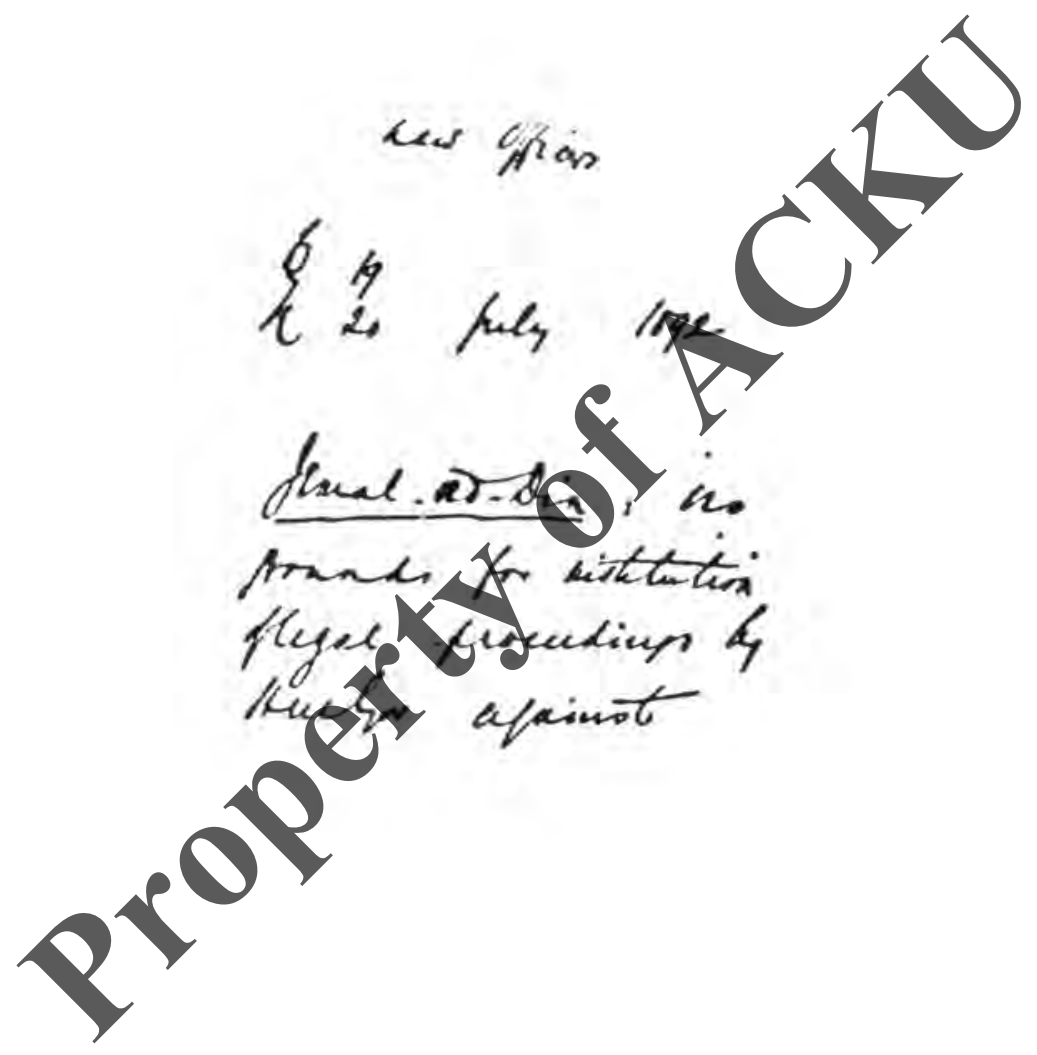


$|A|$

261

List of Papers

Mr. Kennedy Hel: $x^{0} 194$ Stors.109l. Jo do. do.

Sir F. Luscelles $X_{x 2} \div 82$ may 11.1872.

Yo do. ming matommel Alike $\times 22$, 
PBRSLA.

The Law Offeers of the Crown to the Mrarguis of Salisberg. - (Recriped Jnly 20.)

Yy Lord,

Royal Courte of Justice, July 10, 1892.

WD rere honoured with your Lordship's onmmands siznified in Sir Philip und

Curric $A$ letter of the 1 th iustant, statioz tivit he was directed by gour Lordehip to transmil to us a commenication which hal been allessed to you by the Persina Dinister at this Court. That it roiated to the procedsogs of a ecrtain Slicikh Jemal. ed-Iin, who was residing in lo:idon.

That that $n$ w was loom in T'ersia, his parents being natives of Afelianistan.

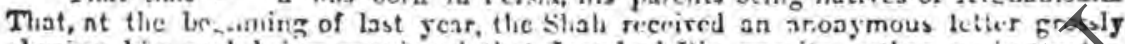

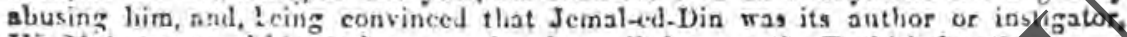

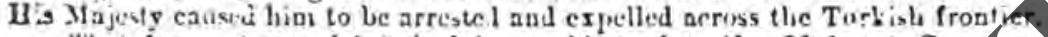

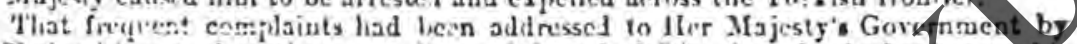

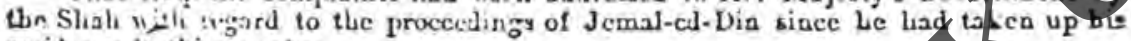
residence in this country.

That If is Mijesty alleged thint that person sends from hero te Resia mpers grossly pliusilig lime, aud inciting the priests and rethers to rolel a rinst his gerbority.

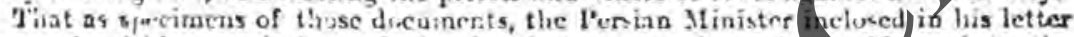

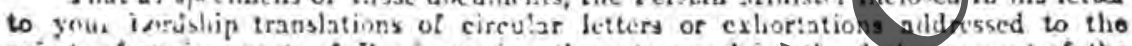

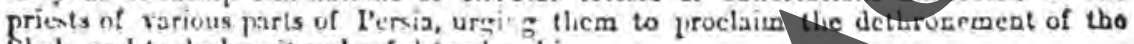
Slah, and to declore it unlawful to wl y him.

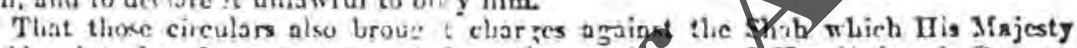
consilered to le of a nature to csic for action on the part of filer liajesty's Government.

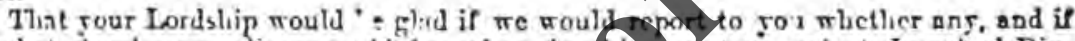
00 what, lezal proceeding could be taken in Ria country. a ainst Jemal-ed-Din,

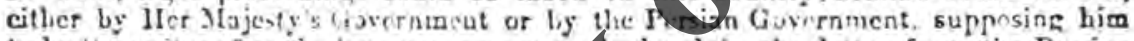
to be ilie wrike of euch doc acuts as werefuelosed in the letter from the Peraiza Mirister.

Tliat other papars, is marked in the masja." connected with the matter, wero inclowed fur our inforstition.

We lave tahes the pupeis intd nur consileration, and, in obedicaco to jour Lordslip's comiuabis, liave tue pangur is

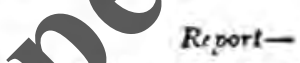

That, in our opinign, the frets stuted atord no ground for the institution of lezal n. $\mathbf{x}$ serer

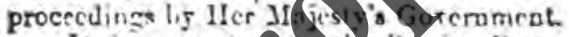

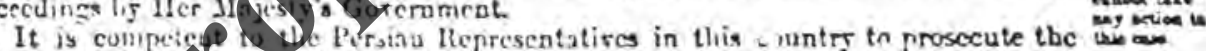

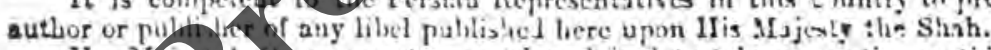

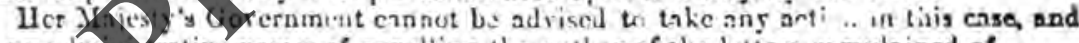

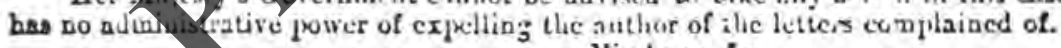

We have, sce.

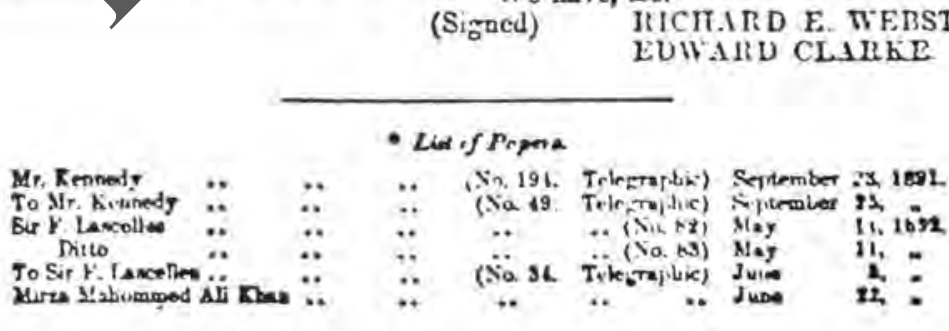

[151-28] 


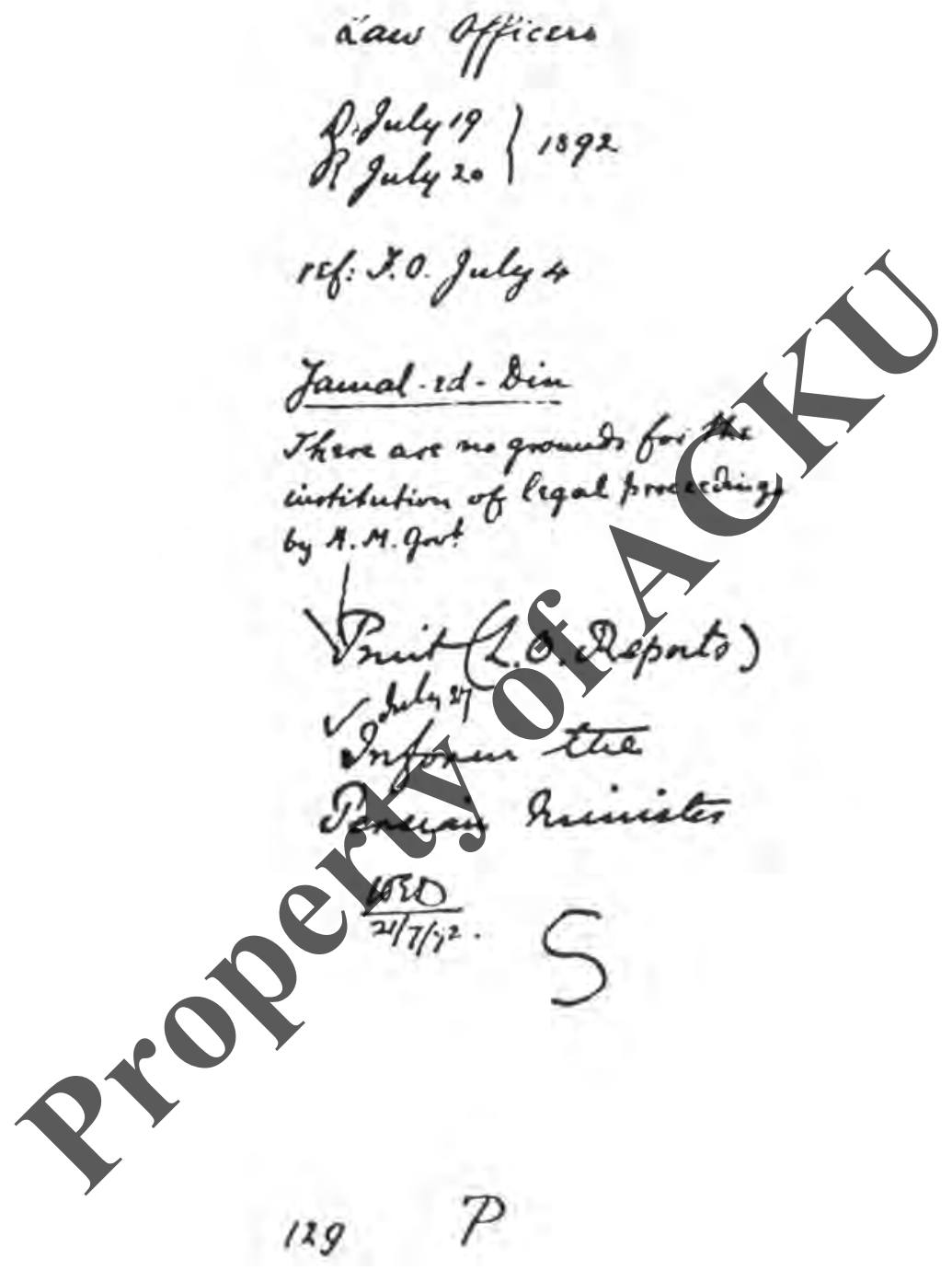


258

IAT

the authos of the letters confulanied of.

He have the homour to be, lley Lord,

Cow Lesdohifio most tedreint

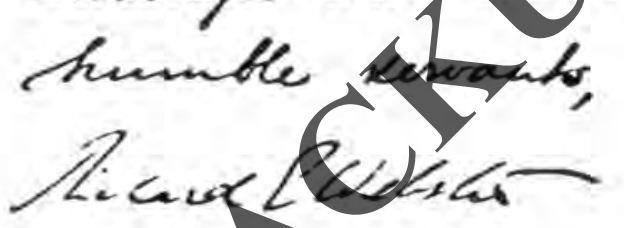

Ediagd blavias 
Reprot

That in our ofuicion the facts stateds afford no ground for the inctitution of legal froweedings by Her Majectyos Stovennment refresentatives in this connhy to prosente the antio or funtlebser of any libel fubliked here ifion thi Mayty the Shak.

Her Dethó bovermment canout betadived to take any ation in this case and has noadministrative frower of expelling 1.131

the 
crucidered to be $f$ a natienc if call for action on the part of the Hajectip Goverumal.

That your dordohif uned be flad if we mede refost 10 yow shether any, if so what, Gegal frowedingts conld be taten in this cosmety apainet fermat. ed. orit either in the Majuty the Pescian Sovenument kufferding kein to be the witer of ench digufents as were inclosed in the frow the Precian Stuvister.

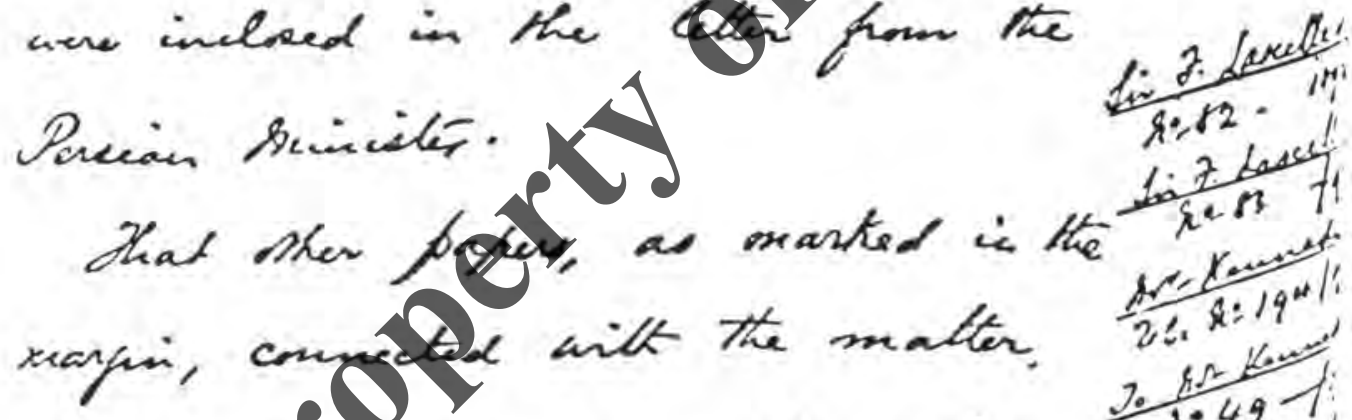
were foesed for oner niformation.$$
\text { ㄱ. }
$$
We have haken the papere cut 2el: $x: 14$ our consideration, and, in dbedience to How alondoligit's commande, have the henomer to

Pefort 
ry his secidence in this comeitry.

that thi Majesty alleyed that that herem seside frow here to Percia pafiers prosly aknoing him and inciting the foristo and phese to rebel againd his

that as ofucimens of the docuenensthe Pescian Mingilet enclosed ai

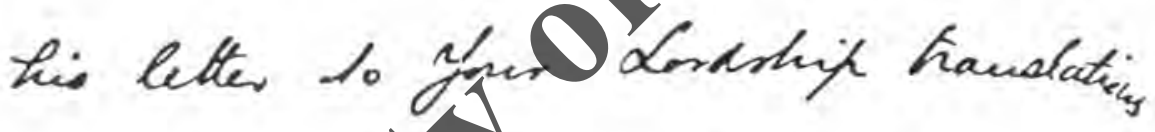

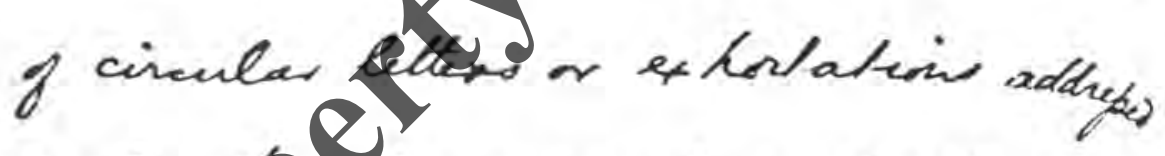
to the prets 1 rascion pasts of Doser unging then to provlarin the dethrovenument of the that 1 to dedare it eactangul to doy hin.

Trat those circulars ales brought charjes gavist the Shah which this Majedty crusideref 
254

111

ise Percia, his travente being reatives of Afshomitar. that at the beprining of laut year the that seceived an anomyroms letter giosely abusing cominiced that ferrat. C. Din was its author or sictifator, tho

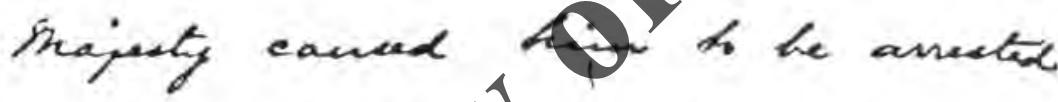
and expelled godss the. Inskin froutier.

Pet fregneat conflunits Kaxbbeen addresced to Ber snajesty Govermment by the that with regard to the provedize of fermal-ed- thin sines the hat takea 
119

253

Royal bourto of guatice,

$19=$ fuly $189^{2}$.

lly Lnd,

Fre were houndred with your dordshifis commang syritid in Siv Philix Anniex btter of the $4 \frac{\pi}{1}$ midant staling thet he was kivecte by yeur fordolife to N2yfer tranemil to a commenication which bof beew addresend to yom the Pescian sumieter at the coust. That it selated to the firoceedings of a cevain theikf ferral-.d. Din wh was sesideing in London.

' heat How the That that fureor sos bea The liearquis of alishorifes.

is. 
252

19.

lie fapers on have, ulokeng fo the mationicarel.

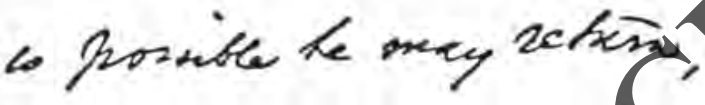

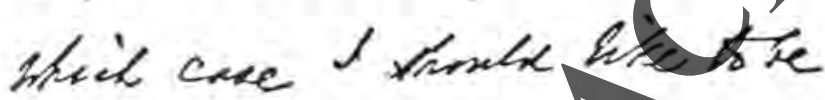
mefiared, frifiege thet the chenemi. Kase me the ocitiney pros to sh:

Cune K.C.N, 
191

251

dinga, Mothane atiktan tho Sin P Coming)

$L_{1}$ is July 1892

Iamal-el-dia

Heo legt England.

kweh negred.

not heoing eecher

in tives the ofinenos entertanised be bo about hime.
Prasian Lreation.

30. Ennisuone caroted.

Lech $x-1892$.

Dear tor Pratep funci:

Letoportate lite

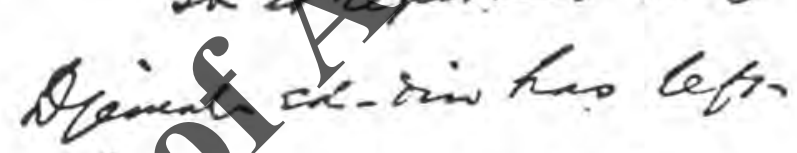

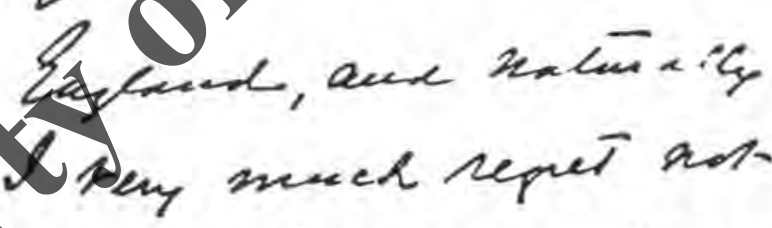

having keawr on forse

time, what: the cleoren

Intertacace, as kegrobs 
250

194

$\Delta / f$.

Law qian 7.0 . Inly 4. 1982.

famel de Sin

C. perow hivis

Wti. f pume 22. Et

pravine pators sinfiel:

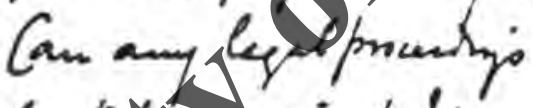

be likts dain! him,

wife what ?

$\sqrt{ }$ 
194

249

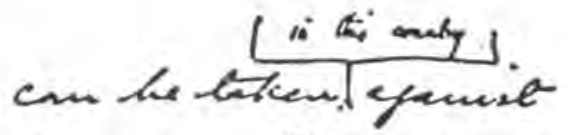
sameter Dai setiang s.tur fort or ty the Same: forte sapponigy han the the unter of suck beuments as ase michat in the LCOE.

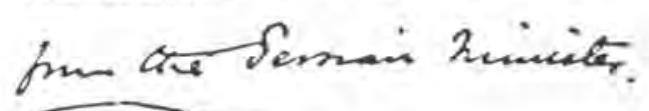
$\frac{6 \sqrt{2} 1}{3 / 6 / 7^{2}}$

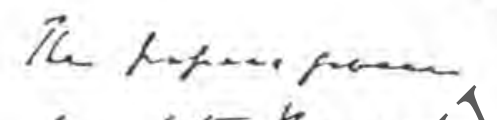
hae gita hangs thimis isflee Le typertracens?

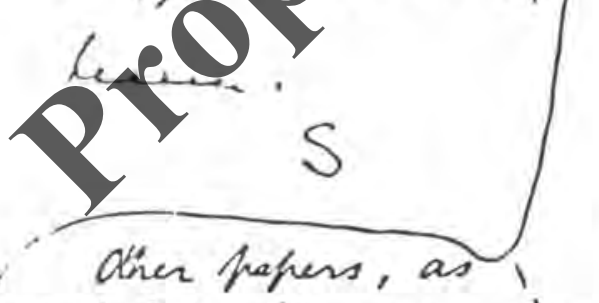
muaved in the mangri. conmeded with this malter, are sent hereunit for tganc. information

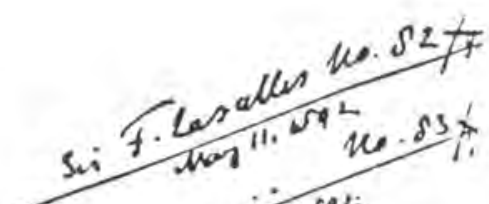
Sin wan "11. Ne. Ss?

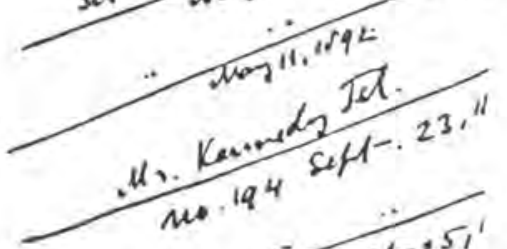
mo $\operatorname{set}^{2} 2^{51}$ me 44 , cas alles

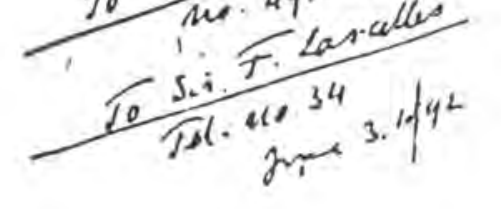


248

195

Os specuniens of these.

dacuments he Pexusin

Rimister nicleses an his

letar thand Lesshy

tranchtivens of Cimcer lecens or Ixhortions alenceer to the Srient of aerains beantes of Parag urgaig tean of procalo the dectinengenthe Shat, and floclane i. matawtiff or abey hani.

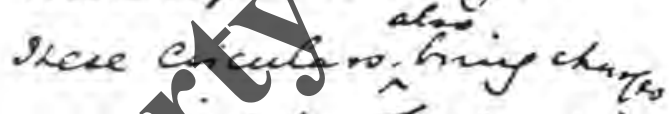
aegut time reath which

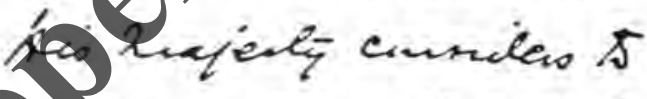
6 of a suture to callfor action on tappand of 3 ino finc

and Salistrang antic the plarlif for wile repont ts hine whether any oudif do whet lefal provededeng

can 
190

247

auniymuons letas frosely chuang him and haing cmuncied kistanul. 2h. Den wes its ancterer or usingater Ris hupeity canisd hum to the sureted ande sijucled acius ghel Derkinh proute: Irepant conpteste have heen apdrabete is

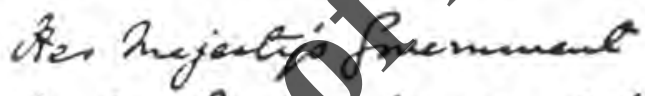
iy ime Saph mik igand to

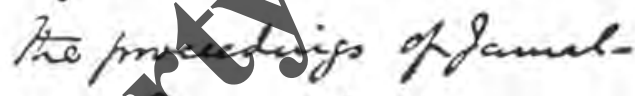

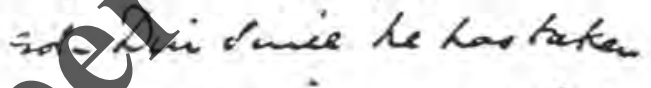

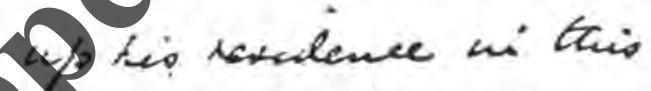
comenty. Kis hejesty alleger that that pesem deuls from tere to Sesua paperes frorsty aburiy tian and miciting the Divier mot atens of whel aprust his antionig.

Co 
4. Bin

Drafe.

Law officien

$$
\text { o.t. }
$$

70.

Suly 4. wryz.

Soutemenen.

By dinction Dese

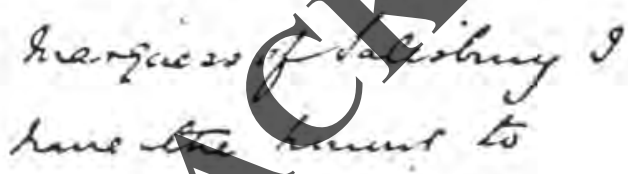

tranterts for a

coptrmioncisio whek hes

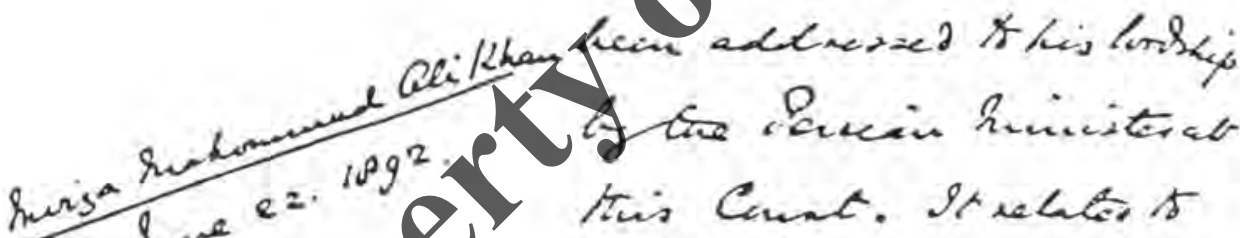
Q this Cunt. Iruehers teprocechloigs of a certai Shecte gamal-ers Din wso is at preach besting ai

s. 2 arulon.

This perene unobian ai Sersia his pansulo keige metrie of Affhomitin at the befmining of lare

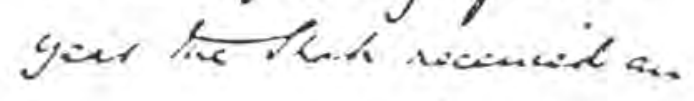




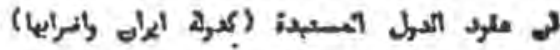

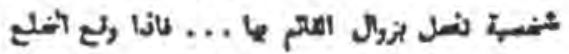

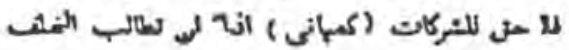
بزرامة التزمها على نلس الهبت

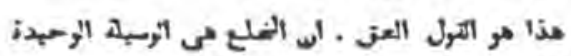

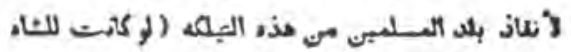

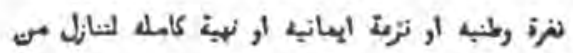

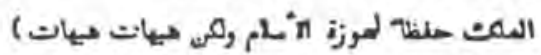

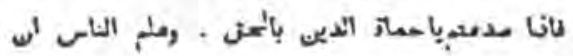

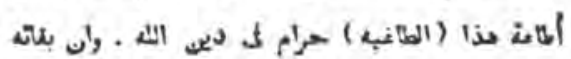

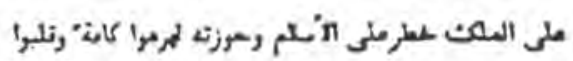

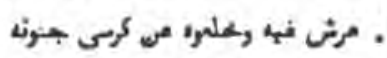

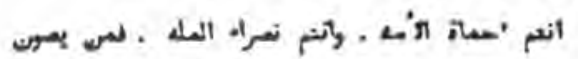

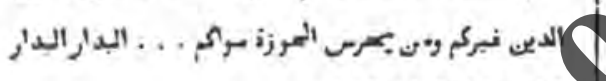

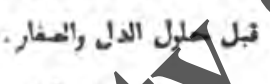

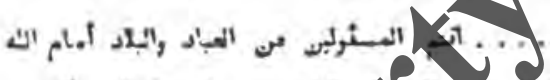

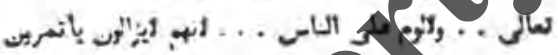

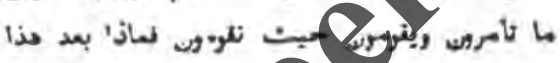

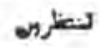

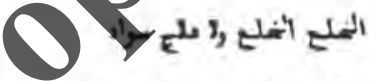

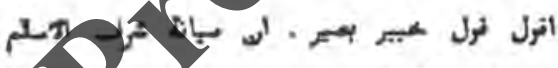

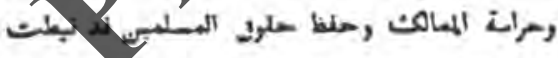

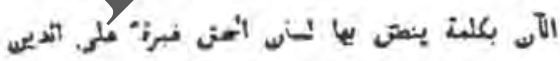

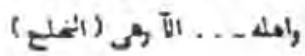

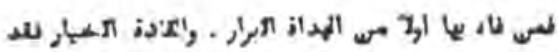

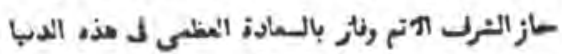

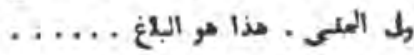

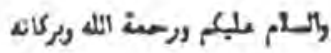

$$
\begin{aligned}
& \text { الהيد العستى }
\end{aligned}
$$

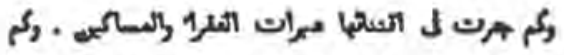

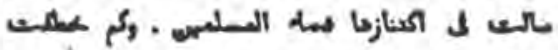

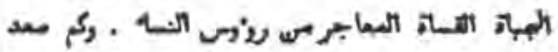

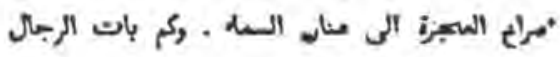

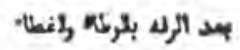

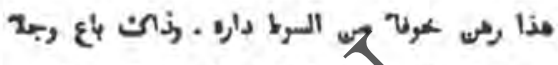

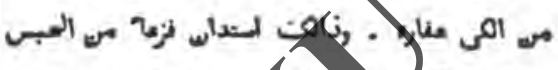

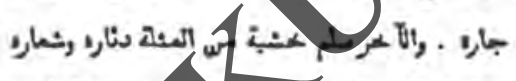

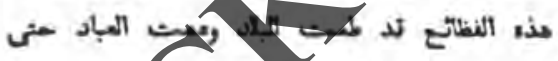

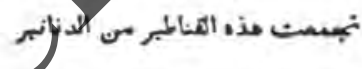

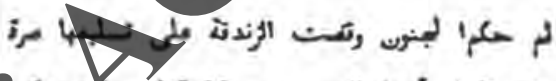

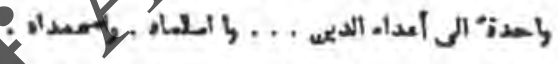

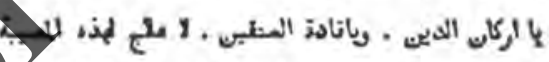

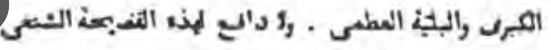

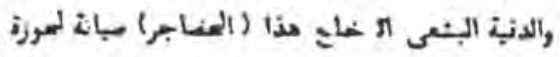

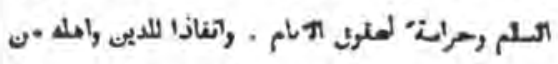

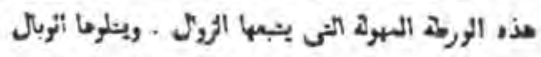

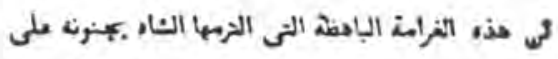
نلس ثبر اهلاد الرهبن مثبها مغادة" للانجلبز

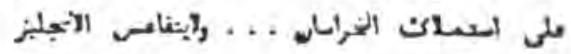

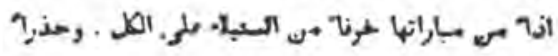

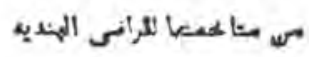

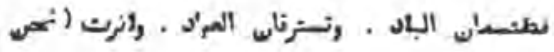

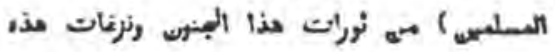

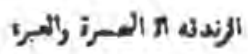

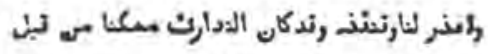

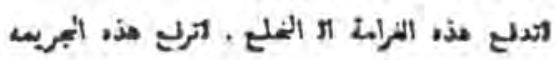

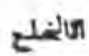




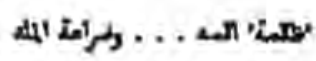

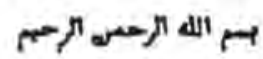

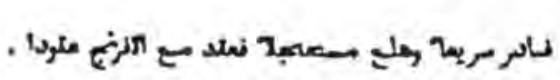

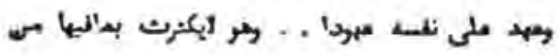

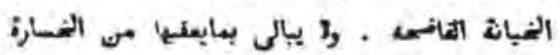

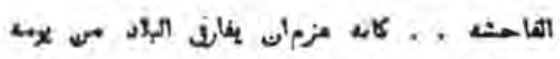
الر بـ

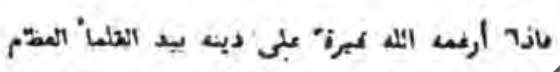

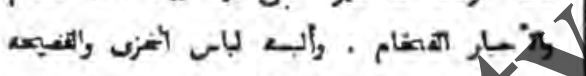

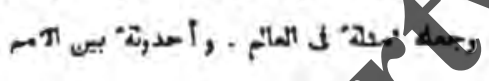

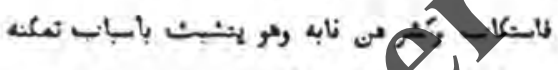

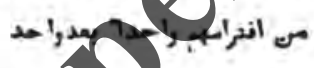

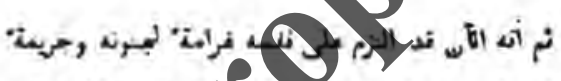

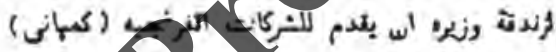

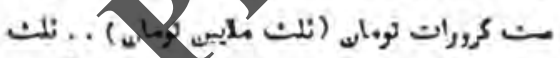

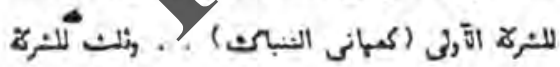

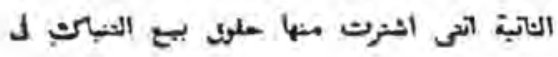
البلد العثانيه

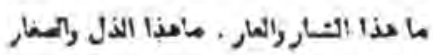

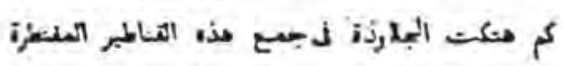

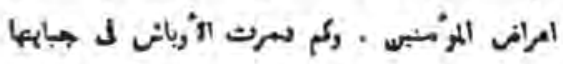

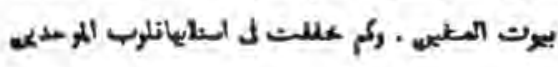

|

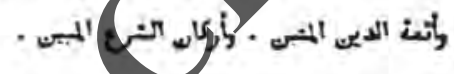

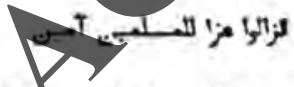

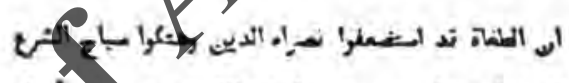

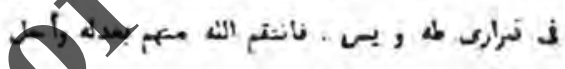

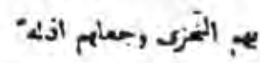
كلالمائميه

التاء لد زمس سلكاته ند جد حرطح مته على الدرأم

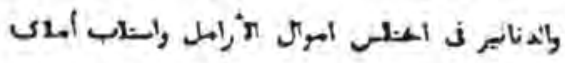

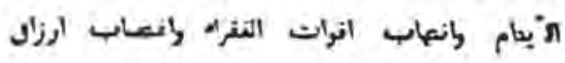
النساكب

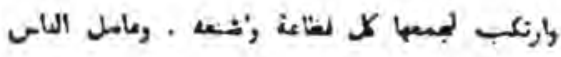

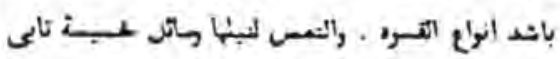

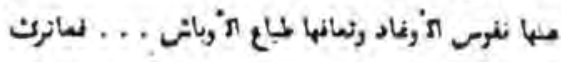

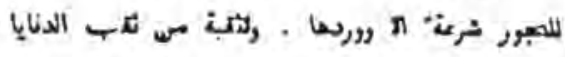
الرولجها

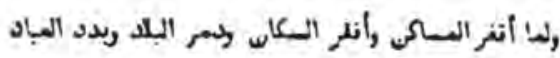

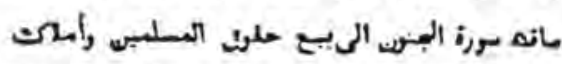

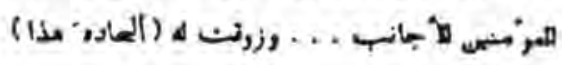
رندنة , رلغ العلرن 


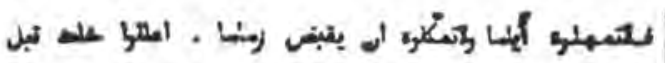

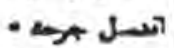

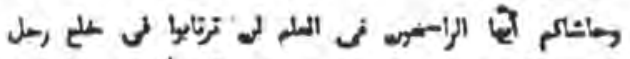

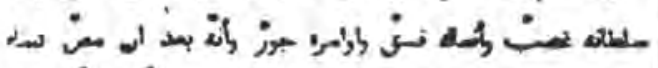

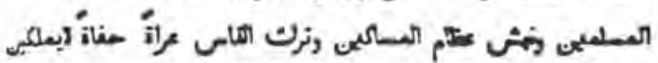

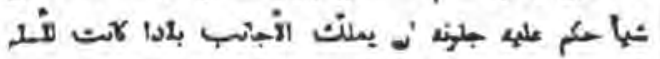

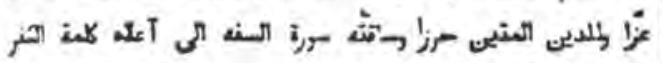

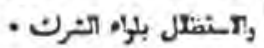

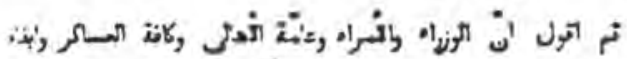

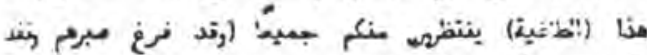

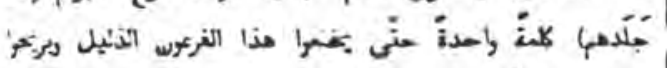

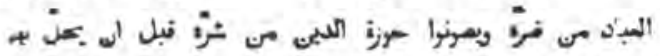

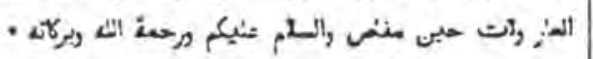
(المسيد "مهينى)

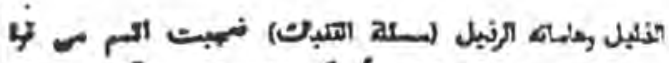

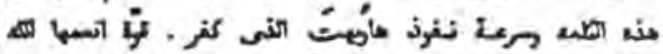

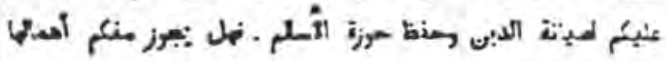

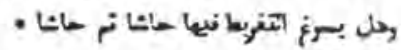

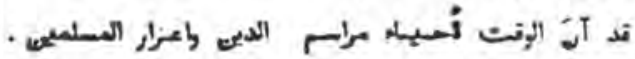

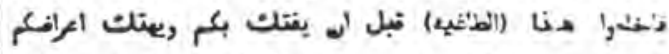

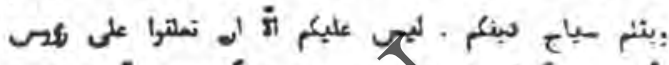

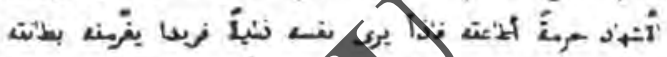
•

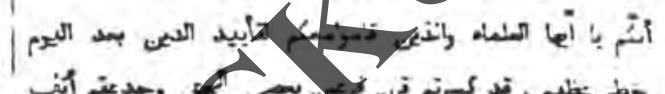

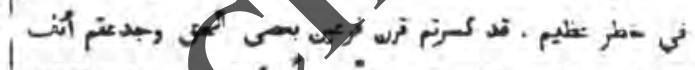

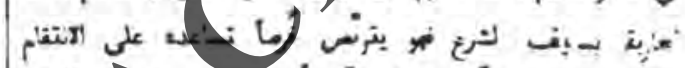

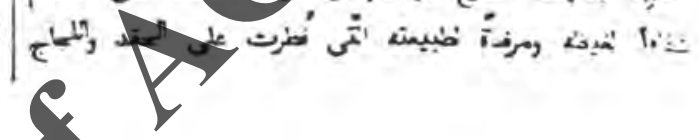




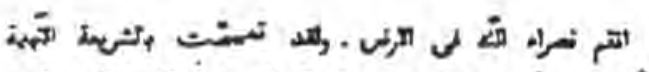

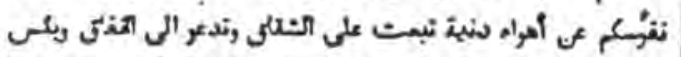

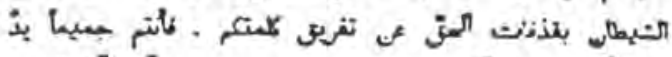

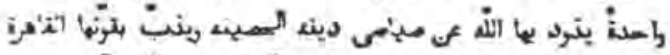

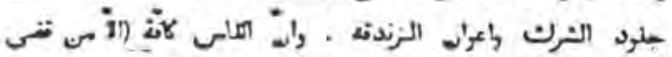

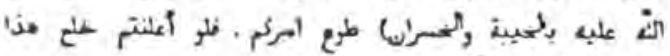

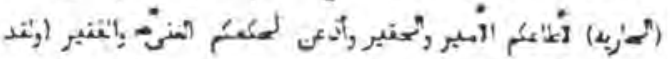

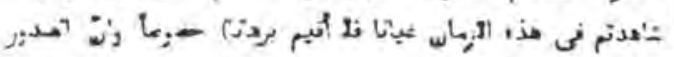

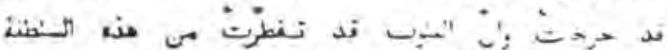

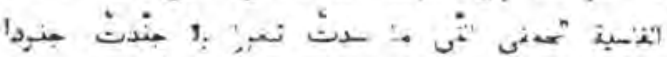

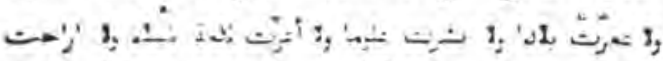

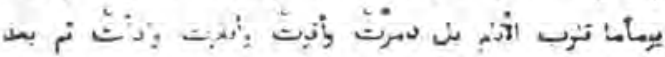

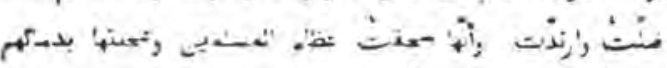

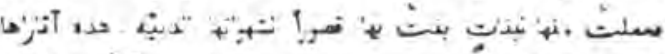

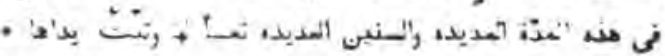

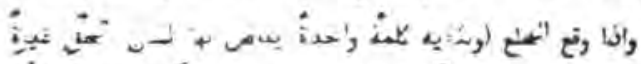

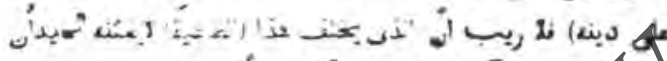

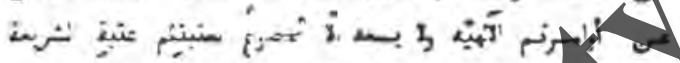

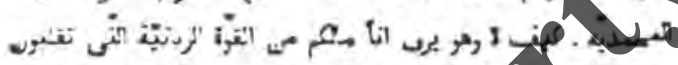

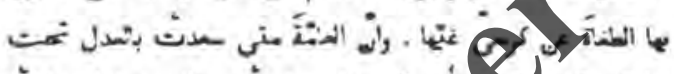

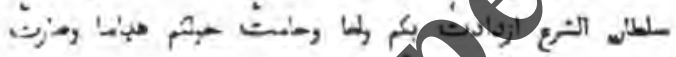

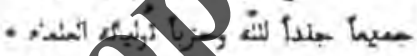

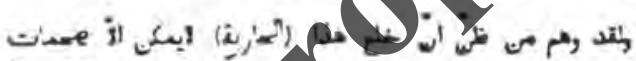

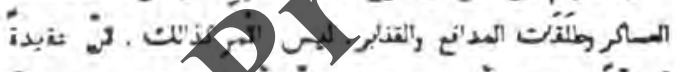

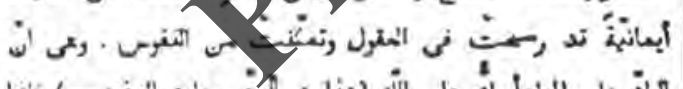

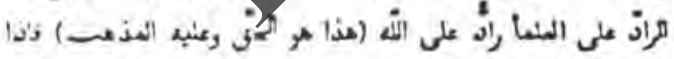

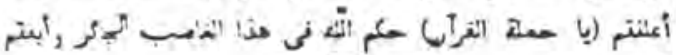

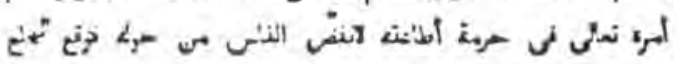
- 4 جدل 3

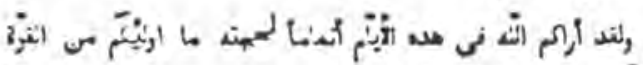

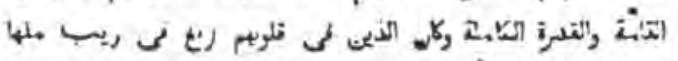

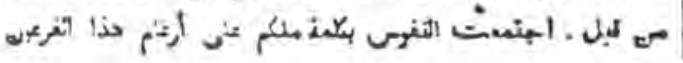

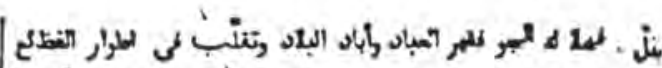

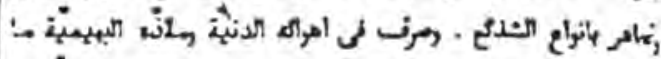

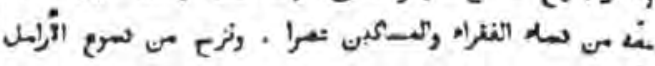

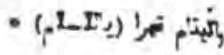

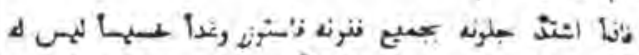

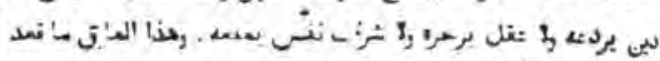

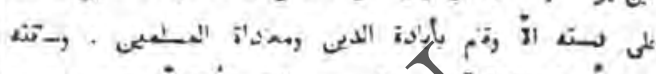

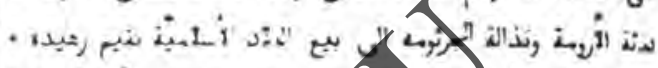

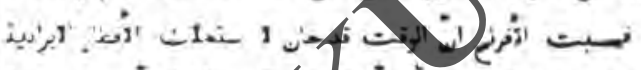

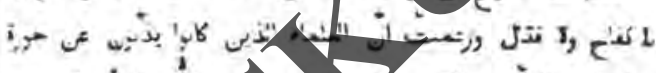

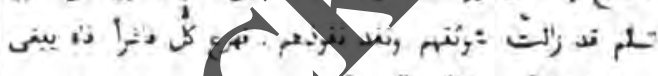

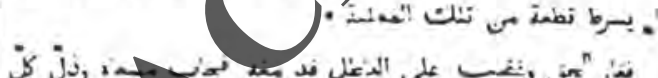

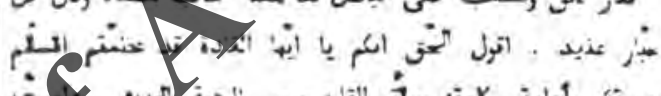

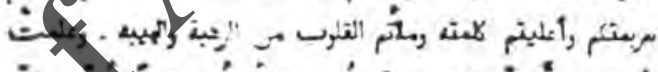

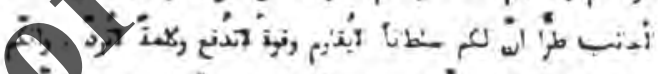

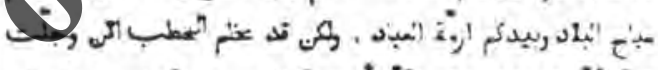

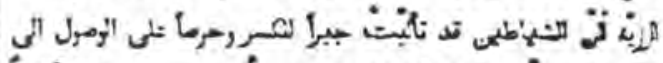

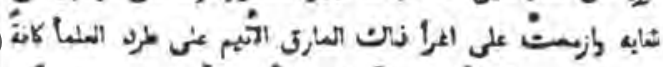

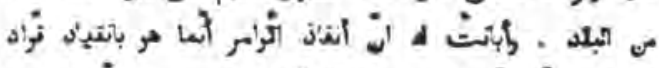

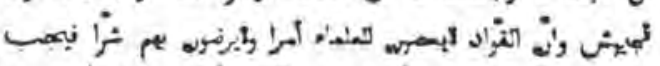

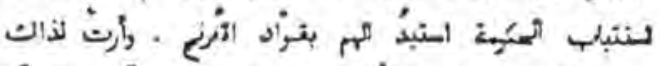

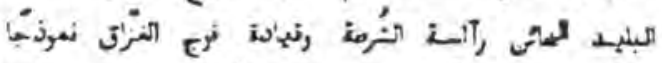

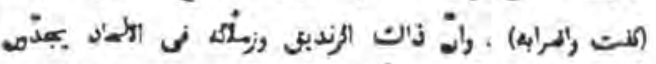

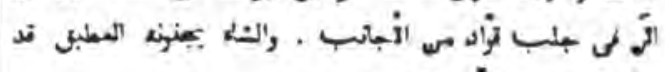

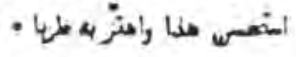

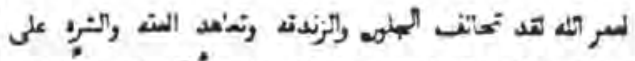

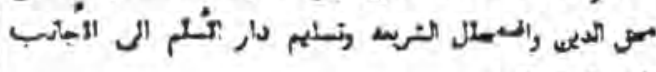

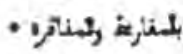

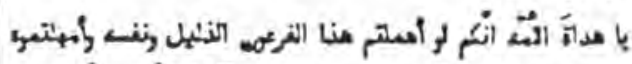

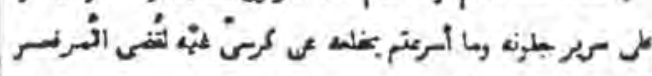

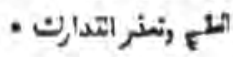




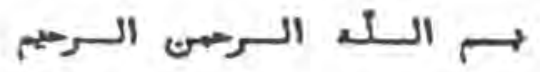

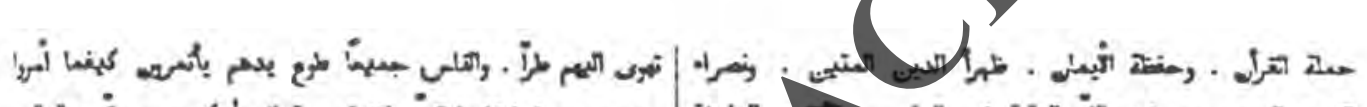

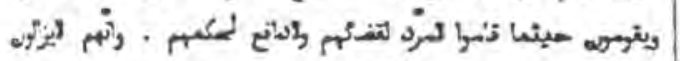

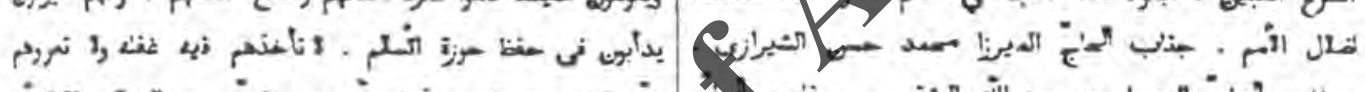

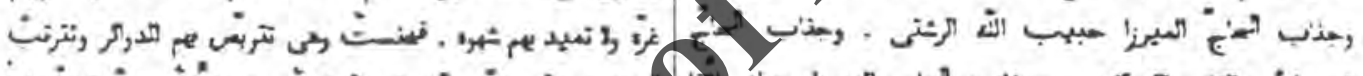

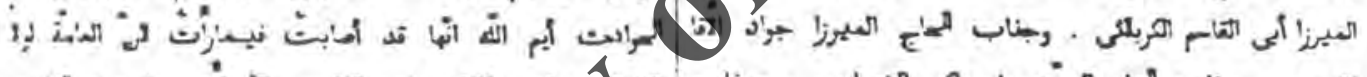

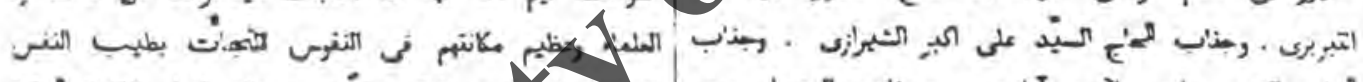

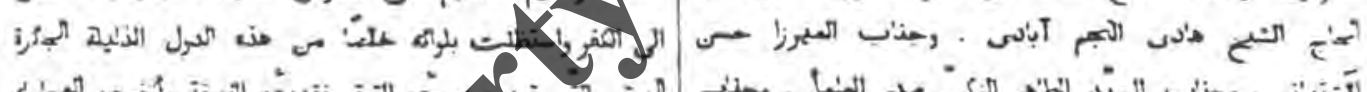

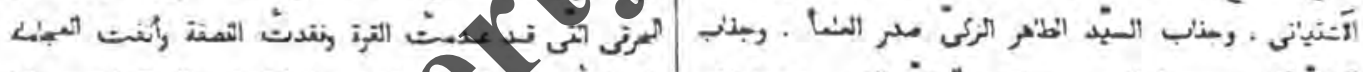

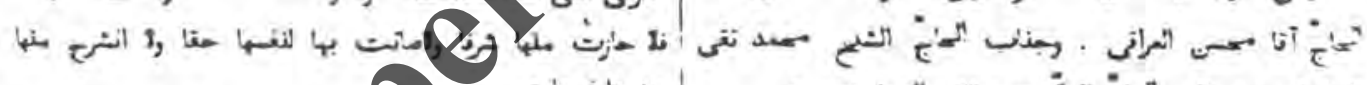

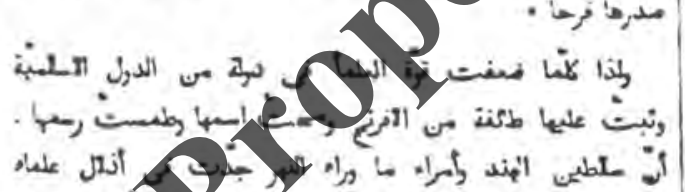

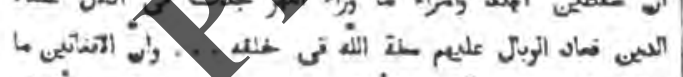
زأية

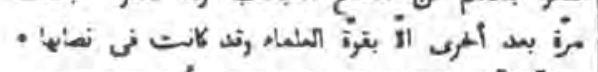

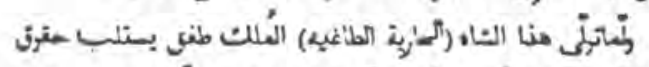

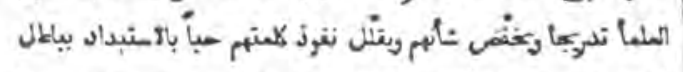

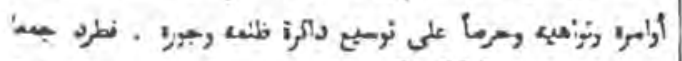

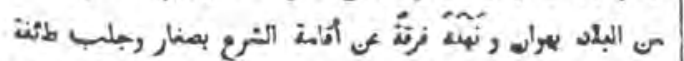

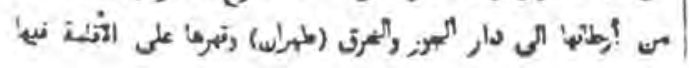

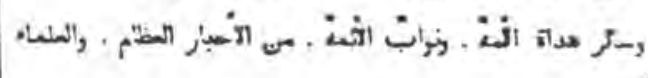
امران

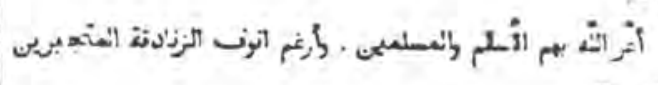

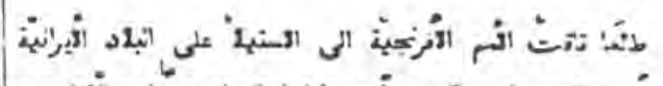

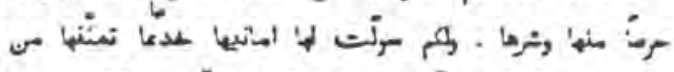

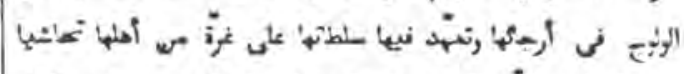

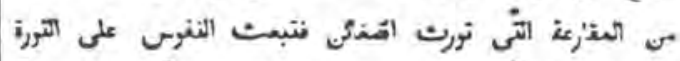

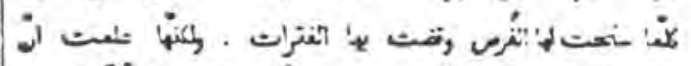

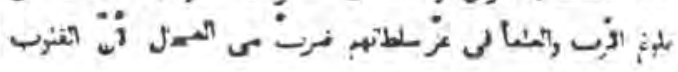




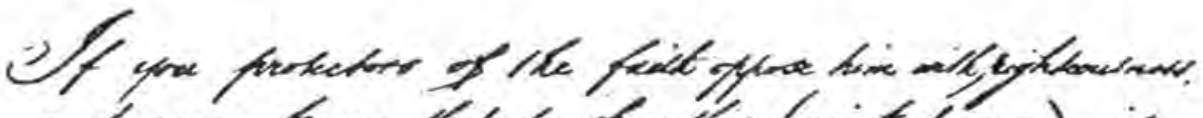

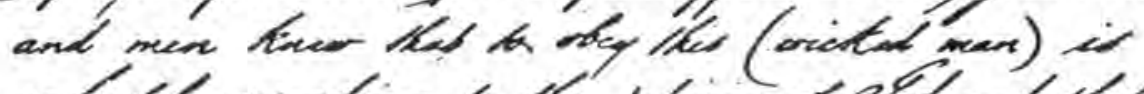

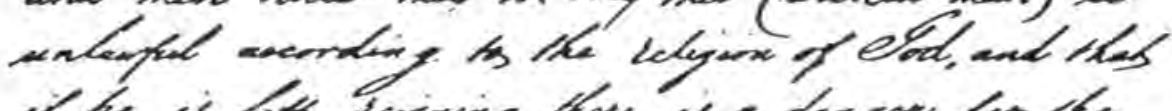
if he is lats lagineng thou is a dengor for the -

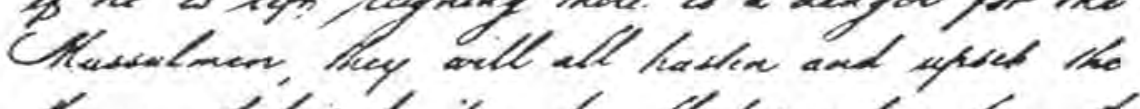

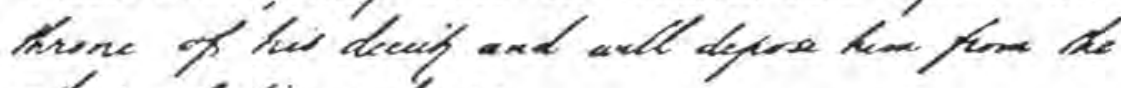
chase of his nedreas.

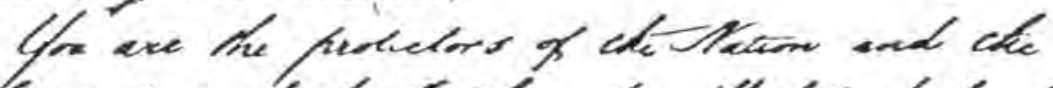

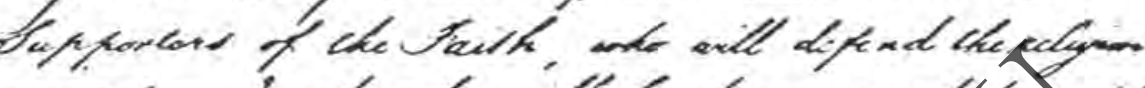

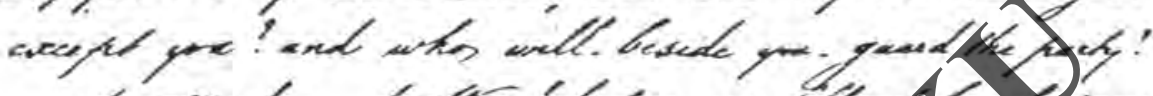

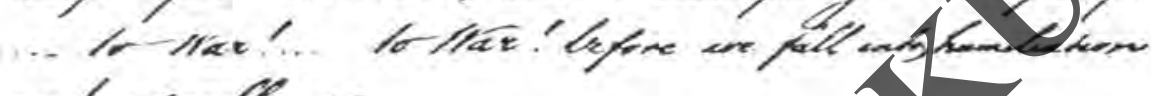
and armellaces.

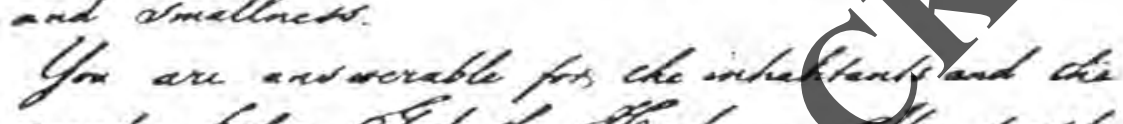

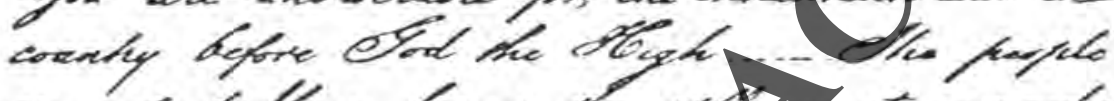

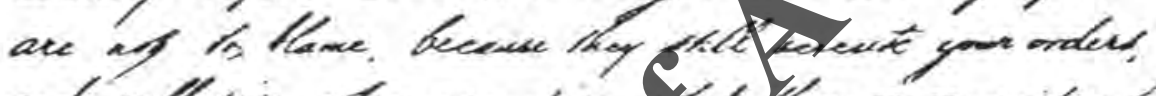

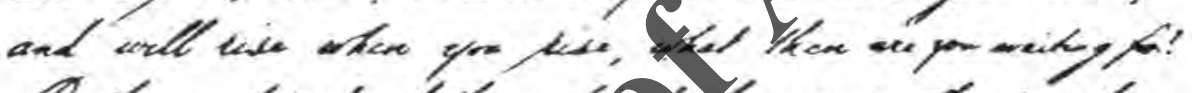

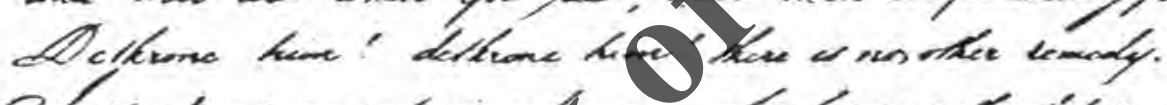

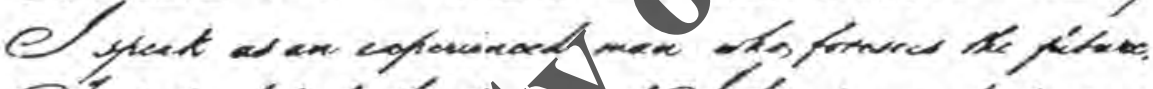

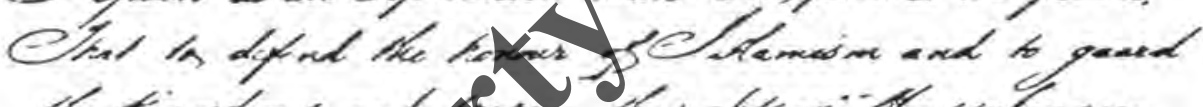

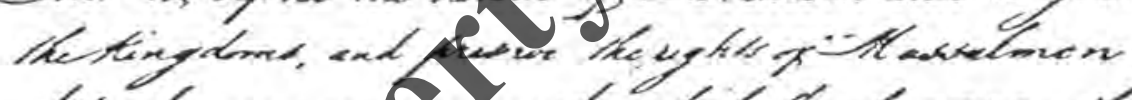

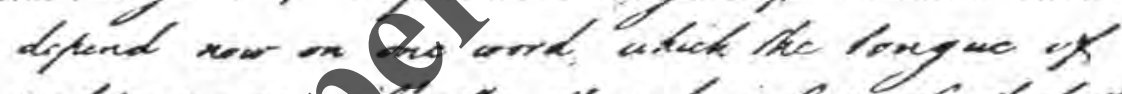

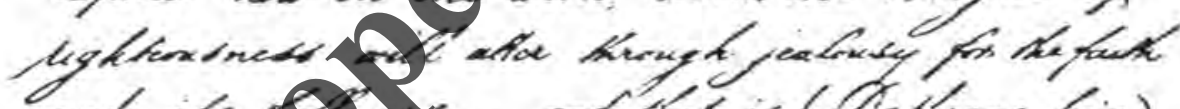

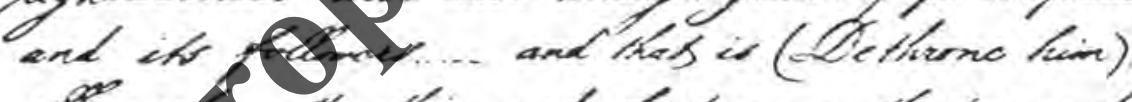

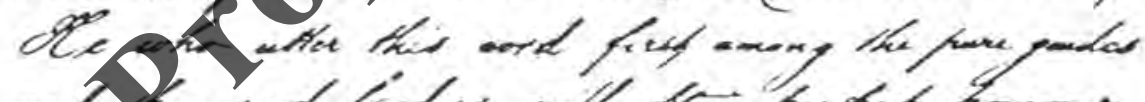

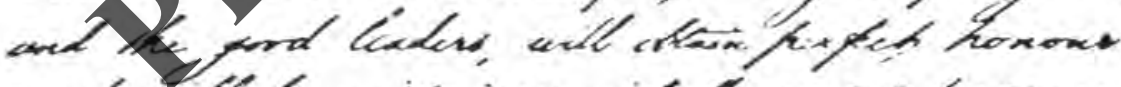

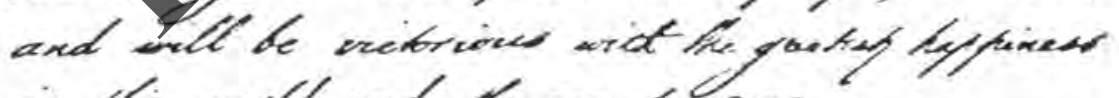

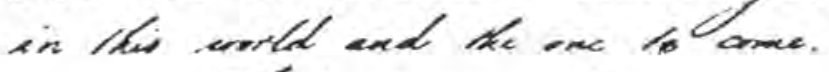

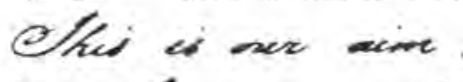

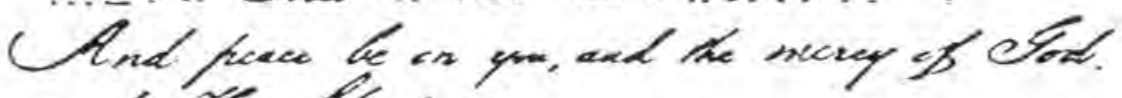

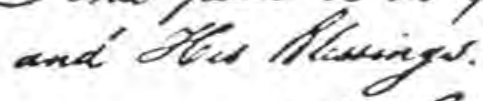

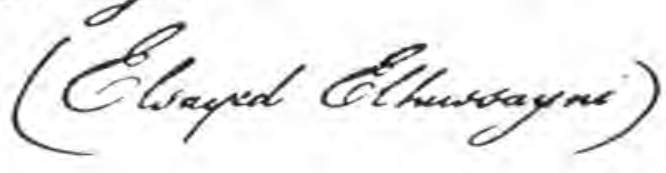




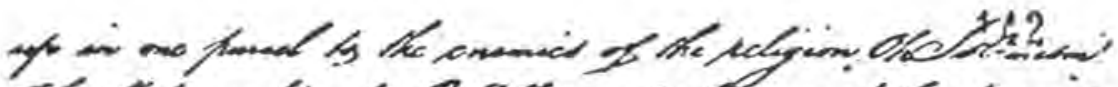

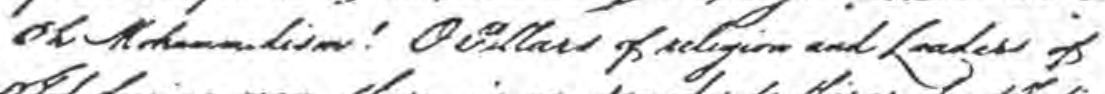

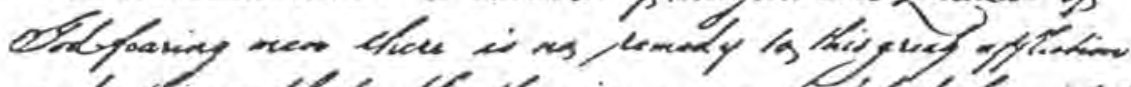

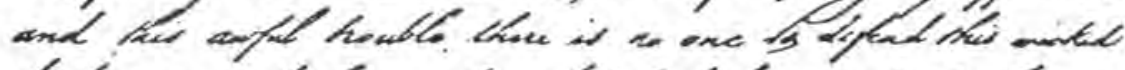

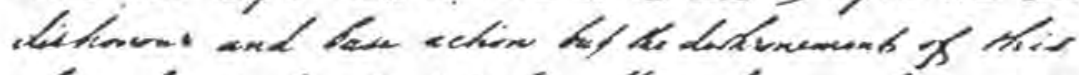

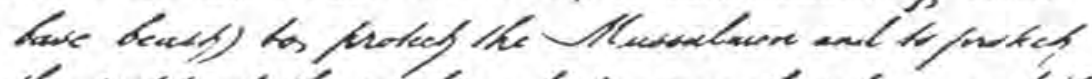

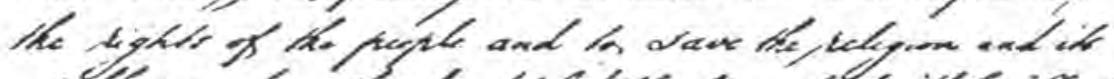

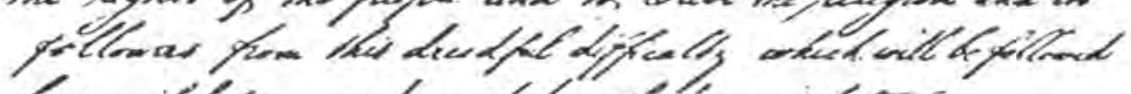

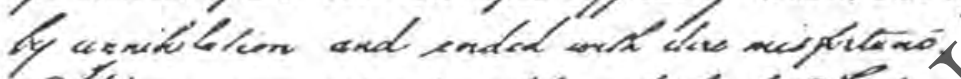

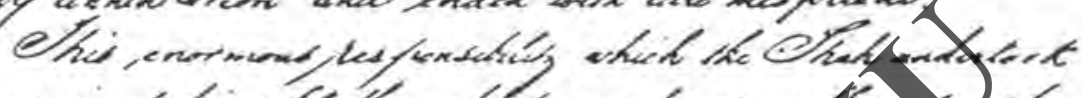

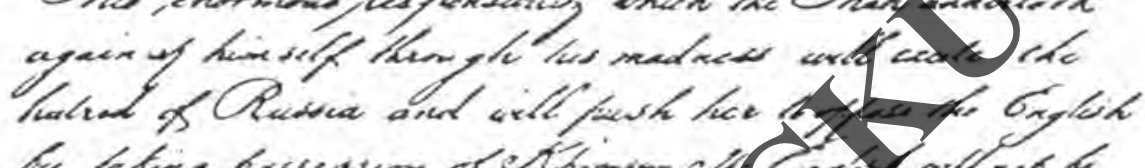

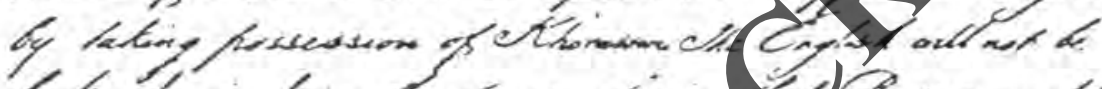

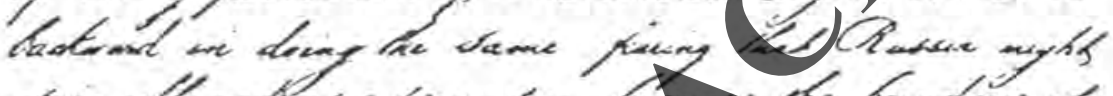

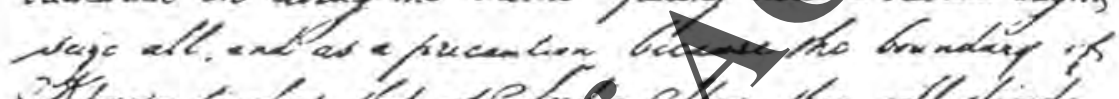

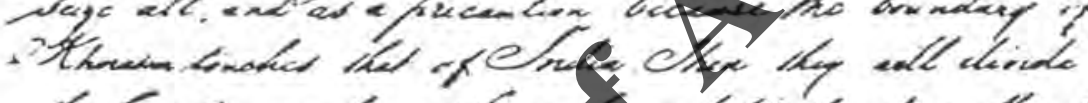

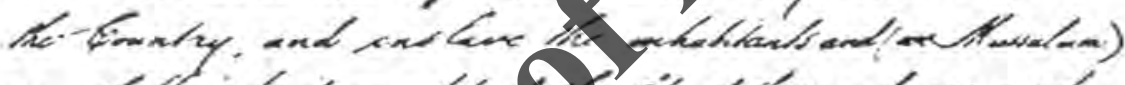

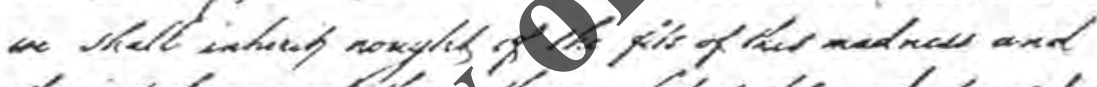

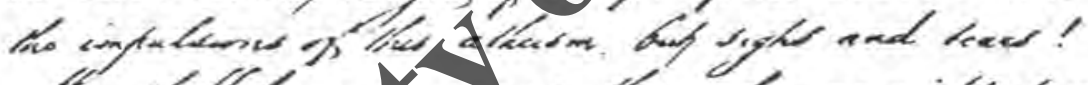

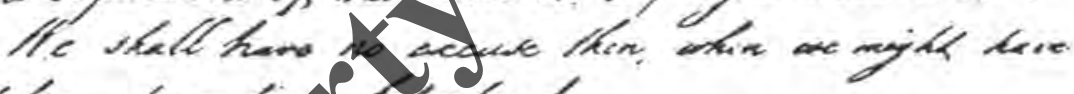

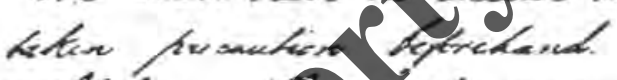

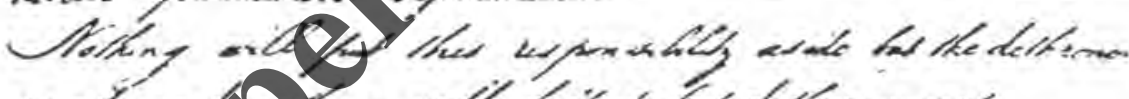

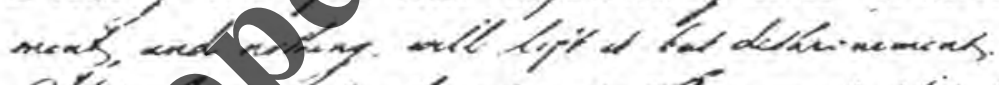

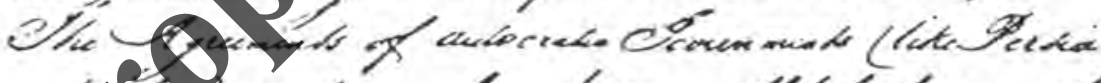

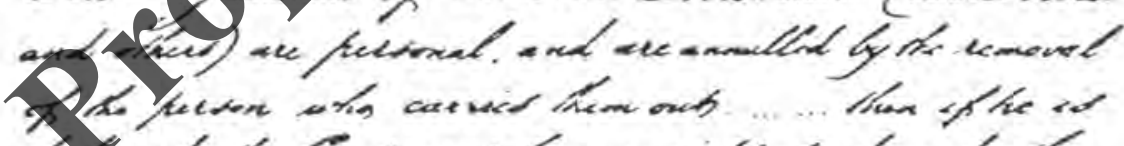

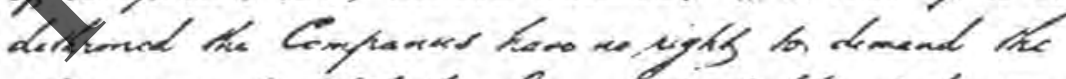

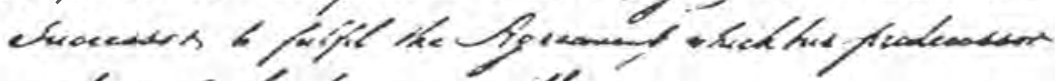
anditent ty his an anll.

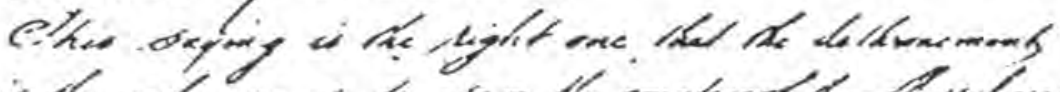

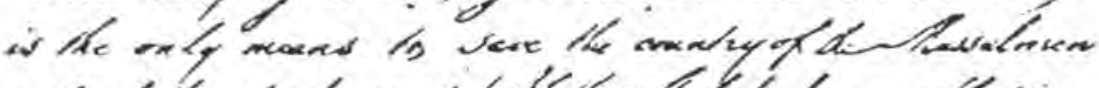

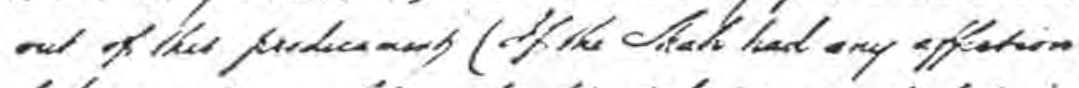

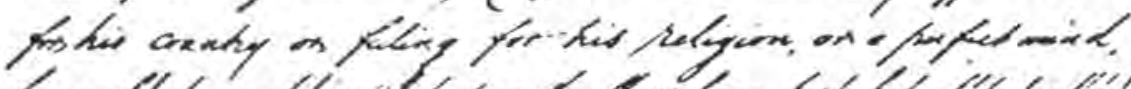

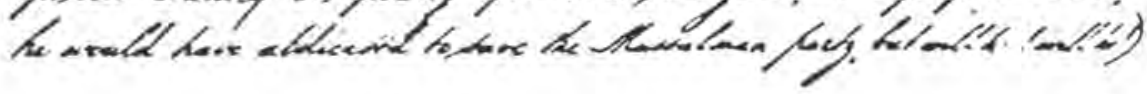




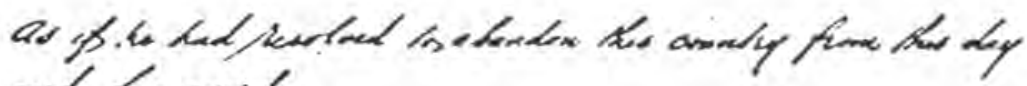

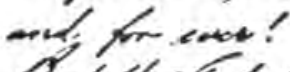

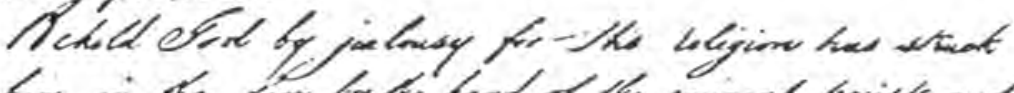

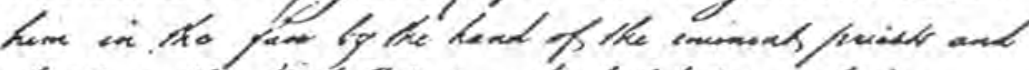

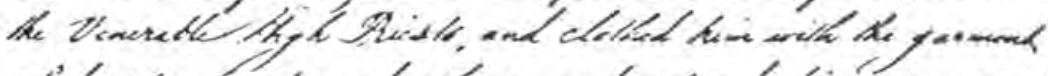

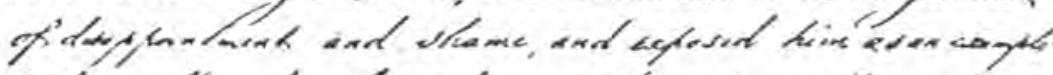

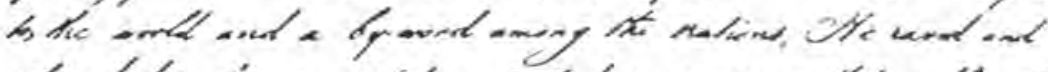

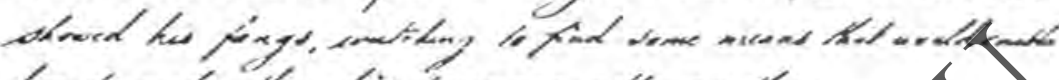

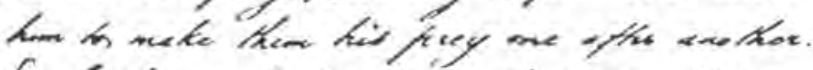

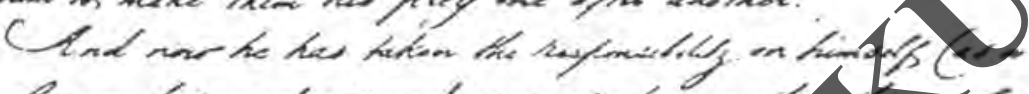

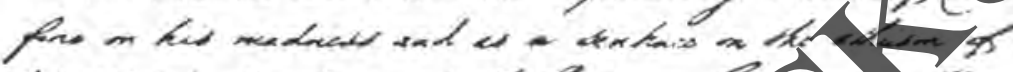

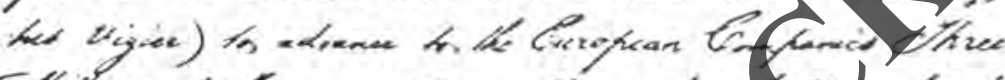

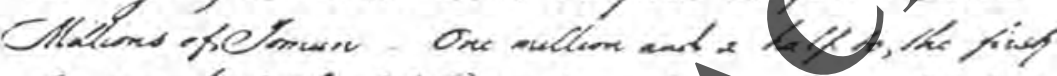

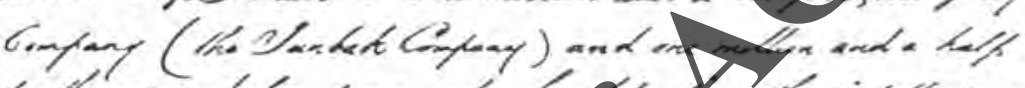

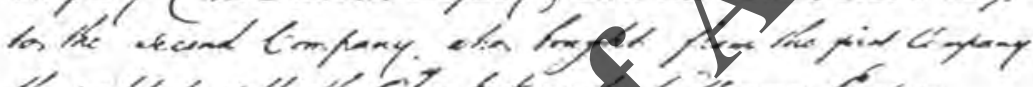

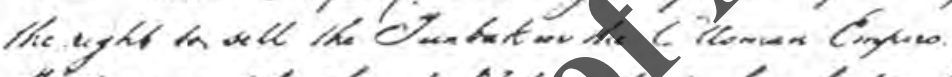

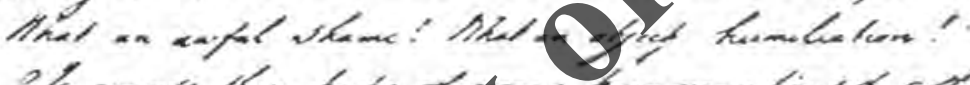

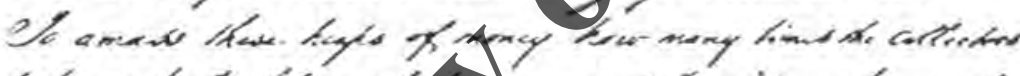

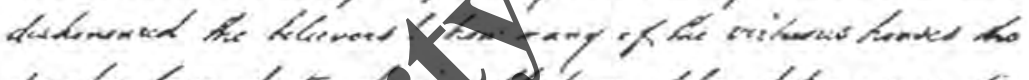

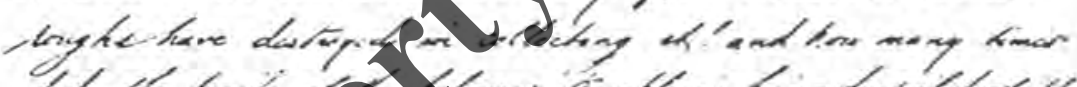

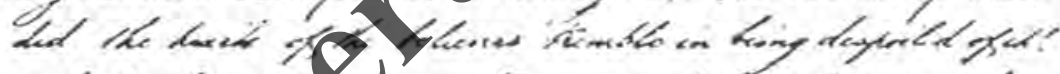

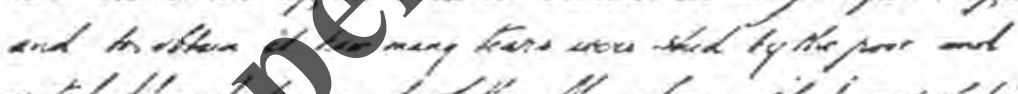

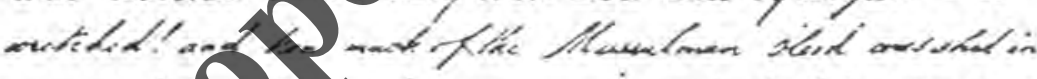

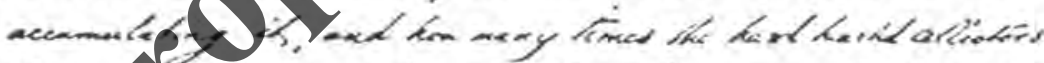

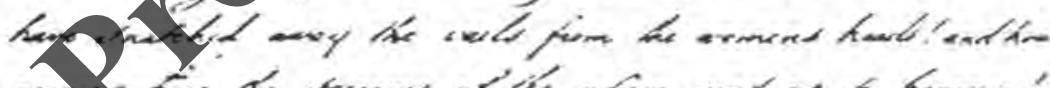

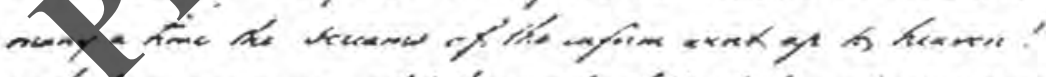

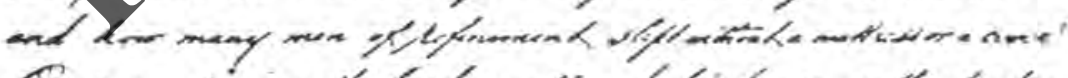

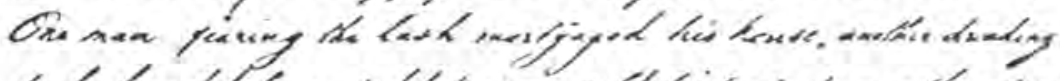

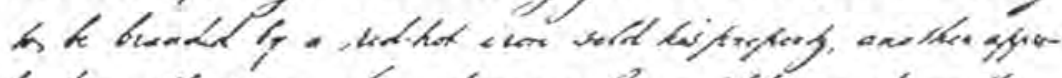

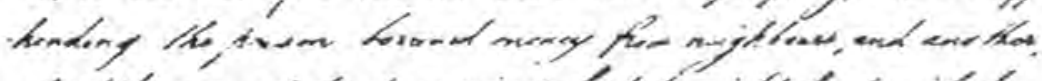

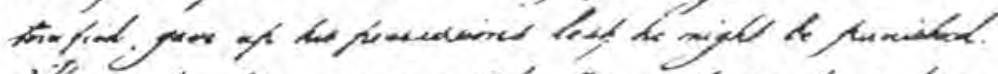

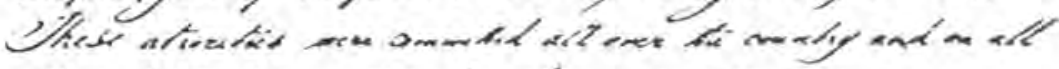

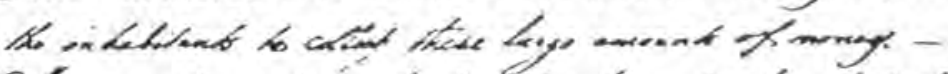

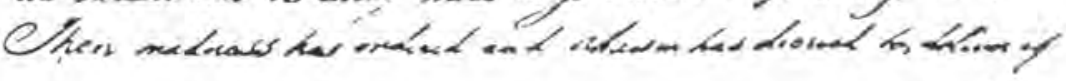




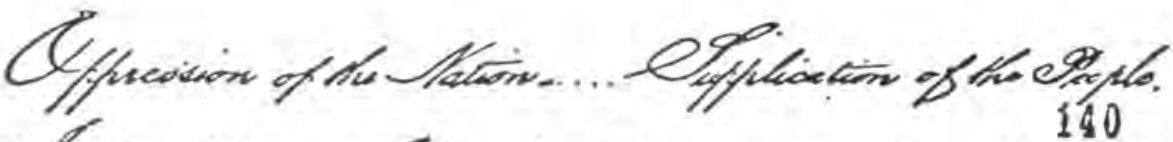

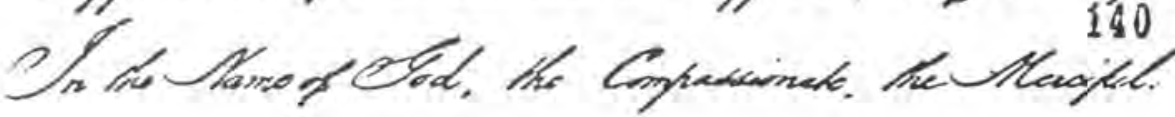

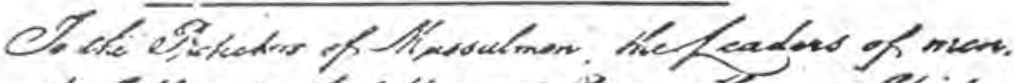

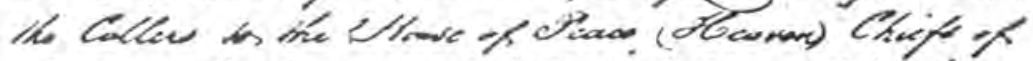

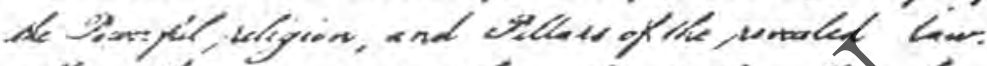

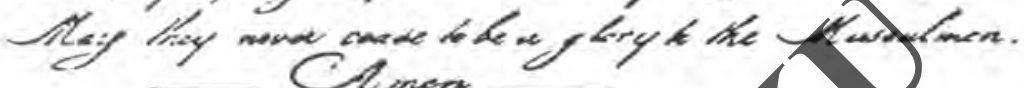

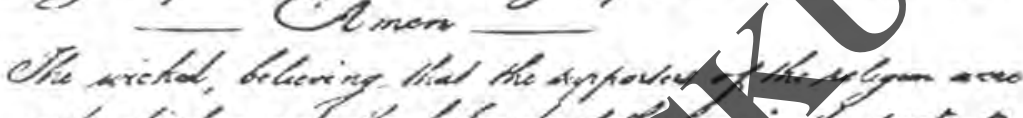

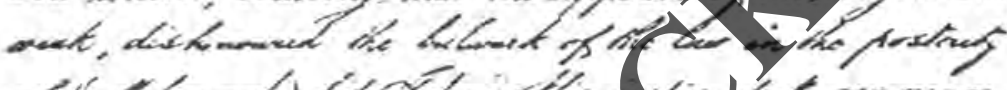

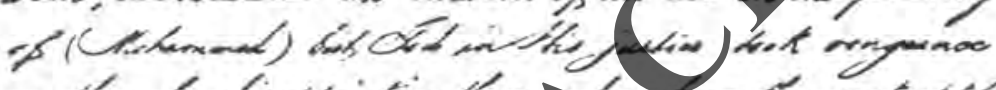

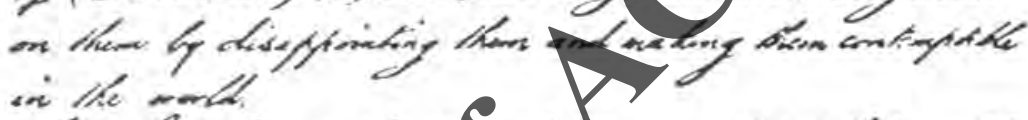

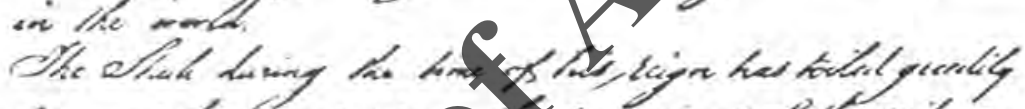

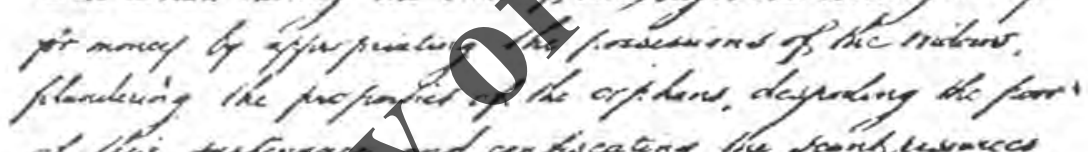

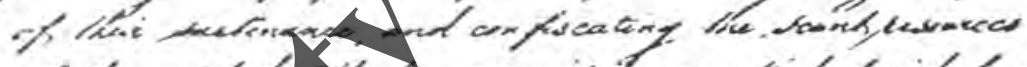

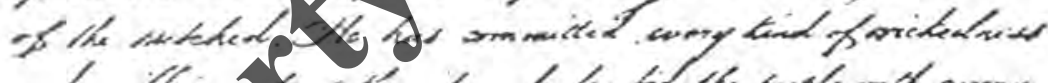

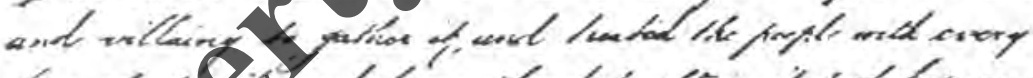

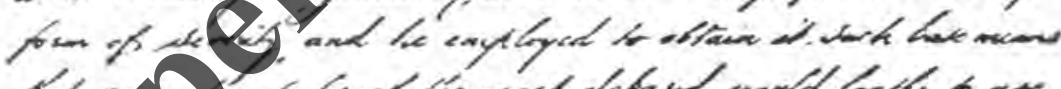

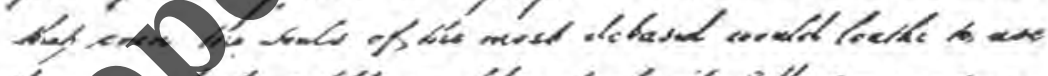

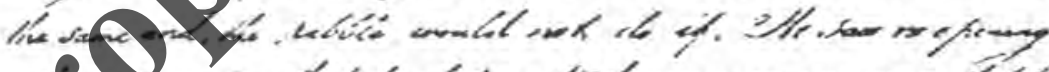

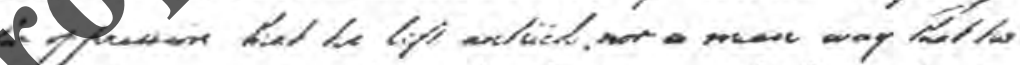

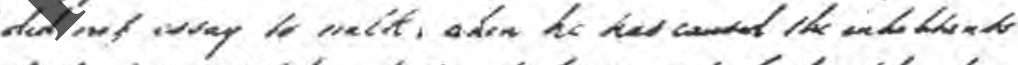

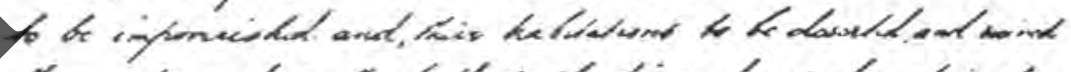

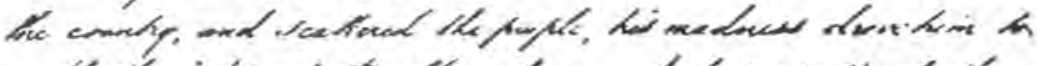

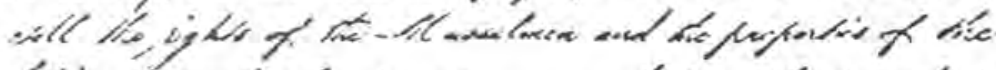

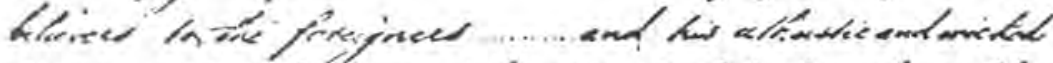

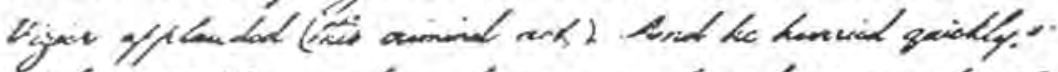

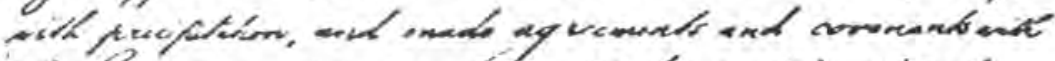

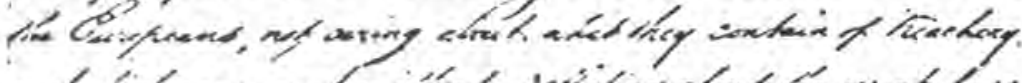

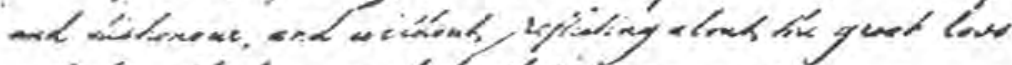

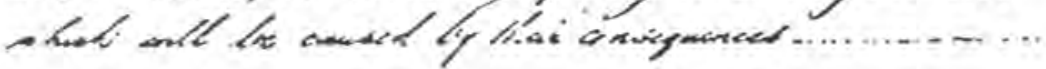


Frbarance is at are end) bo detheme this bace Ohavats, and giot vers the the fepple fine hes

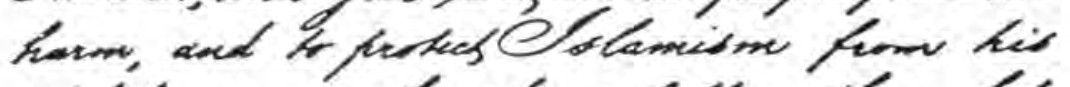

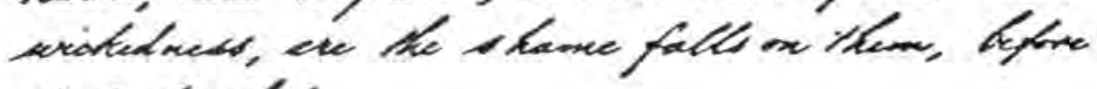
it is he late.

Clad freace be on you and the uneag of bod. and bies blewinge.

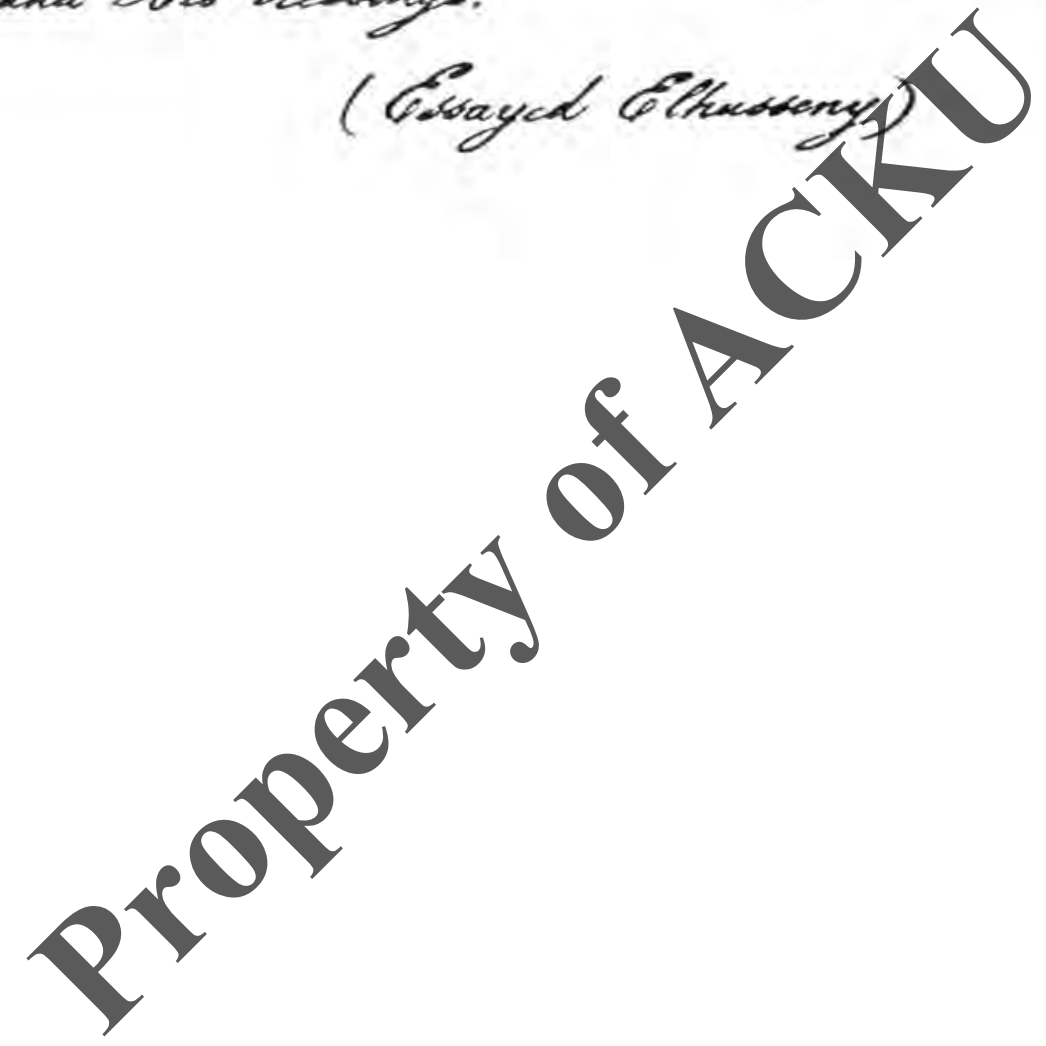




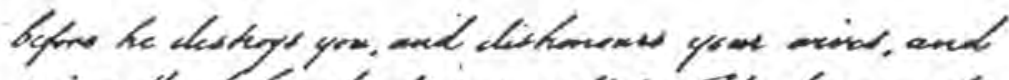

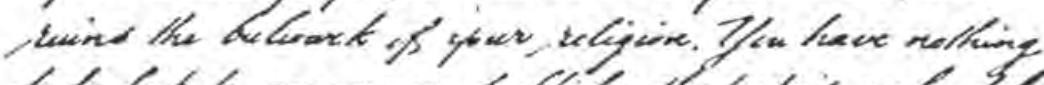

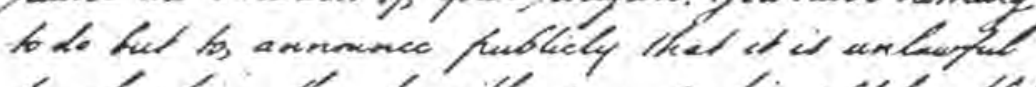

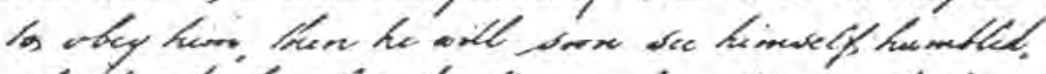
"tandered, thed fuivels yliping firme hive and hei

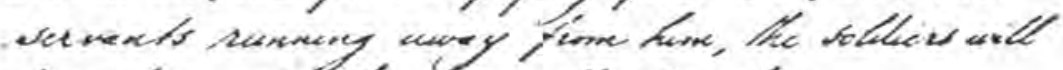

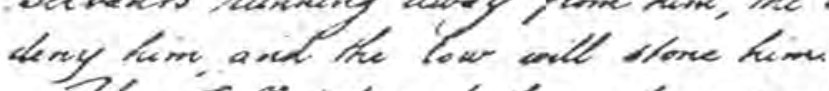

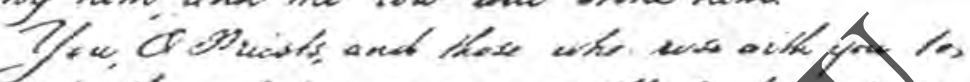

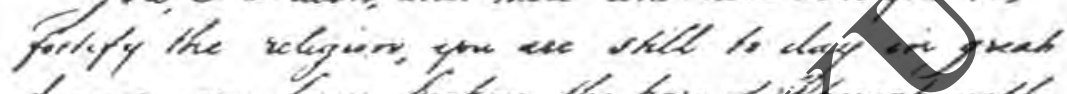

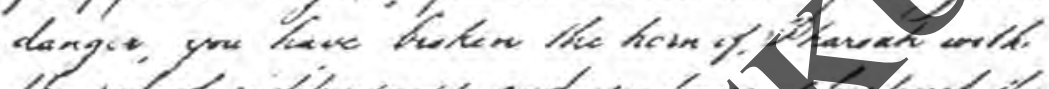

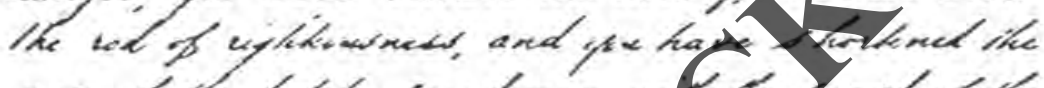

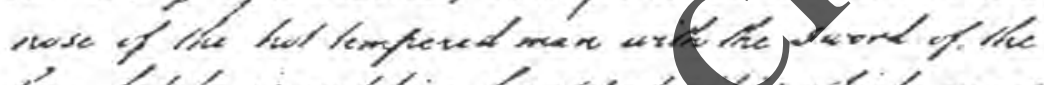

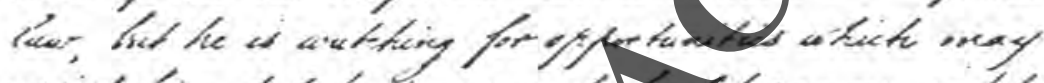

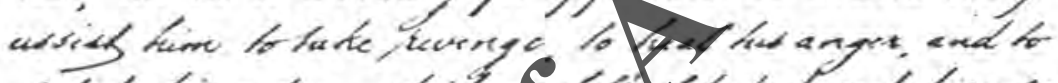

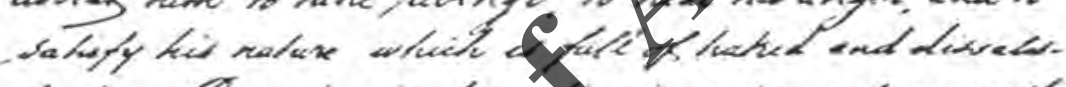

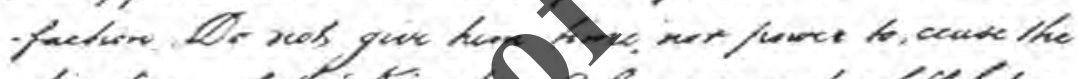

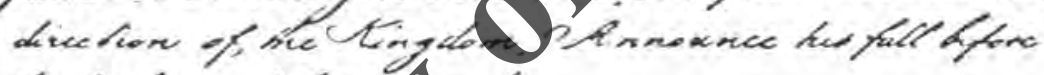
the hiakne of tie datad.

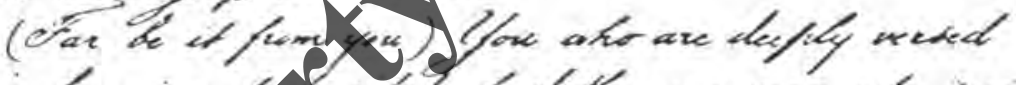

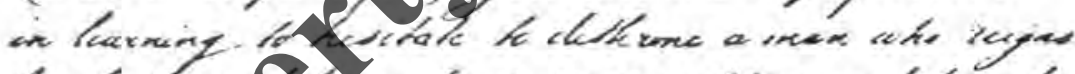

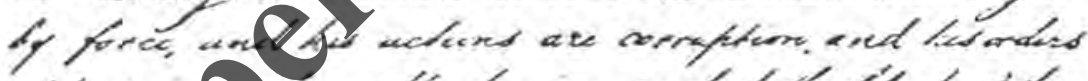

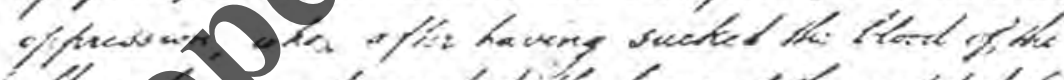

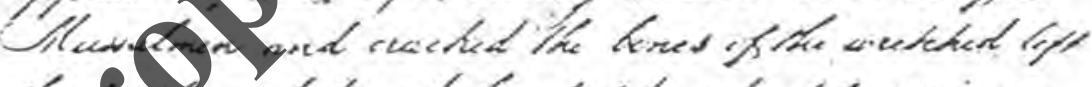

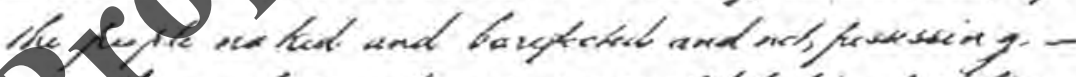

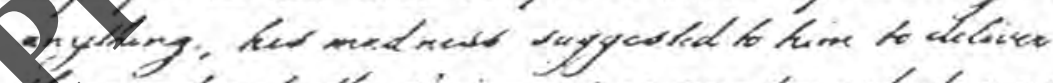

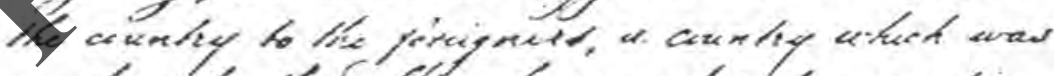

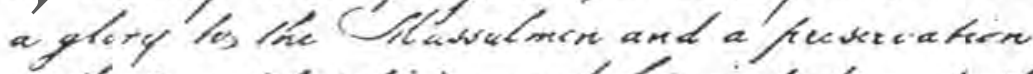

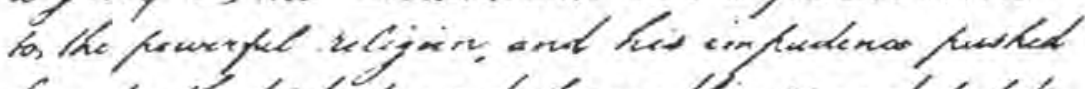

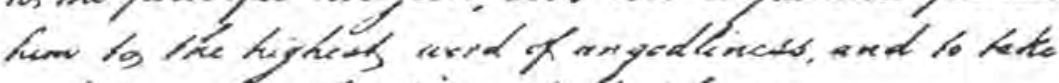

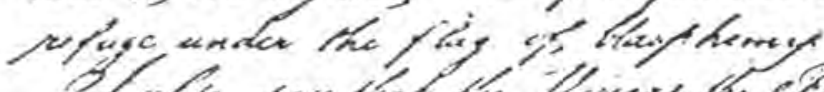

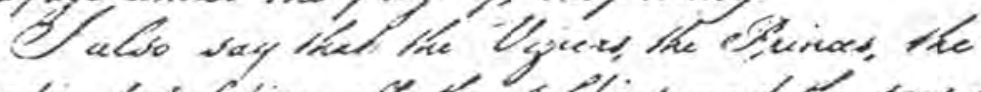

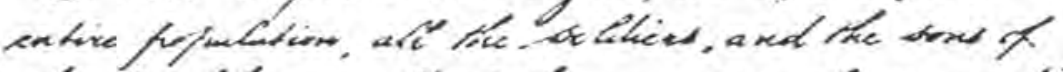

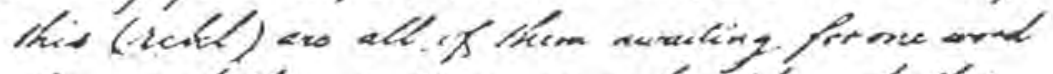

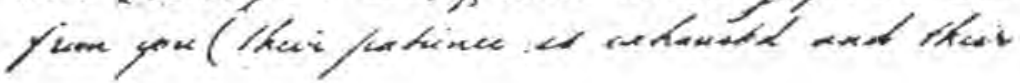




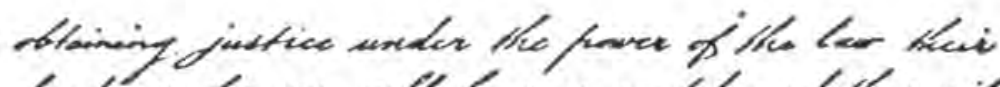

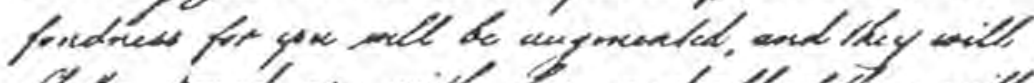

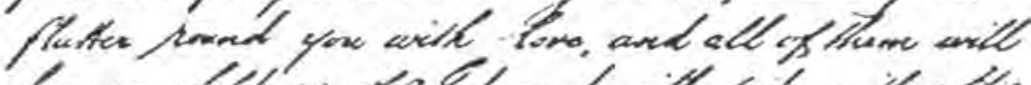

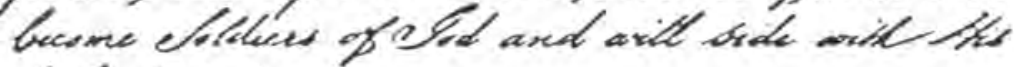
hory fuiess.

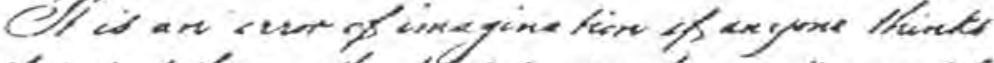

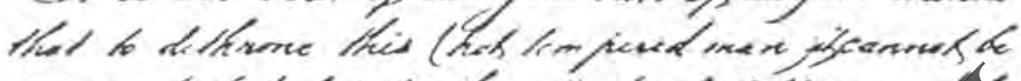
eccerplatied het \&y the attact of Stiflice and ox

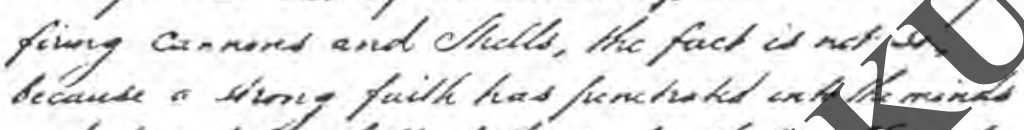

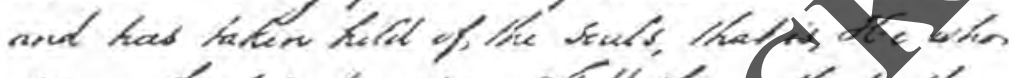

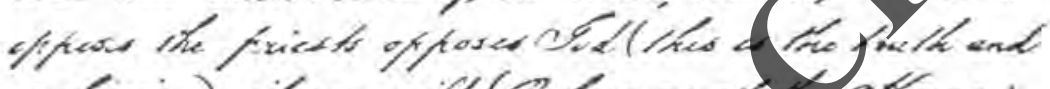

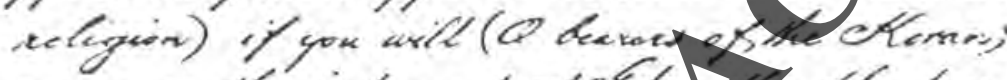

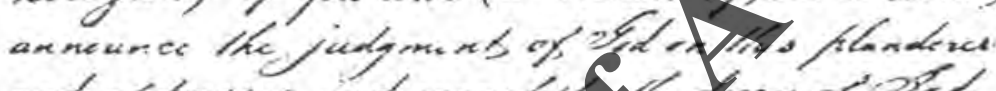

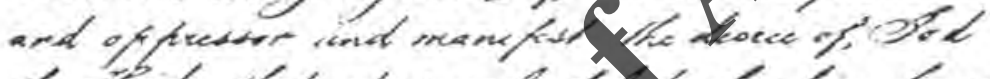

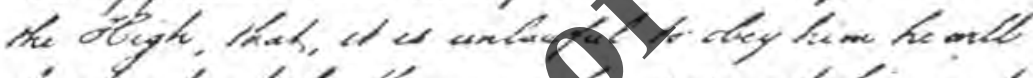

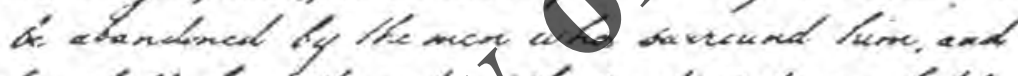

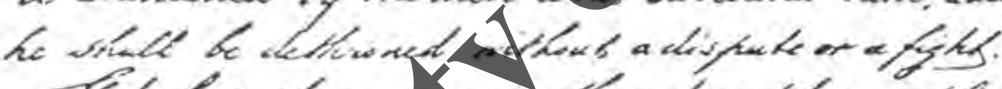

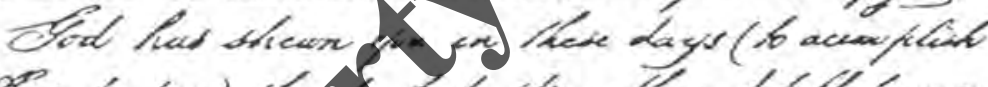

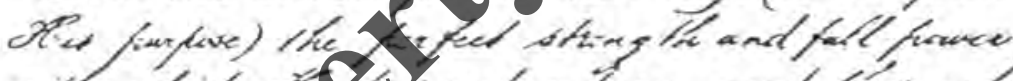

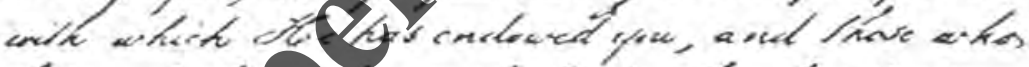

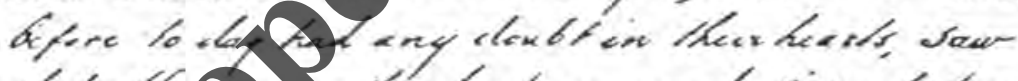

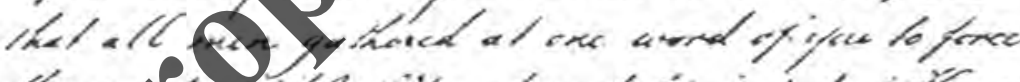

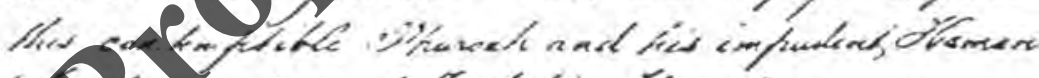

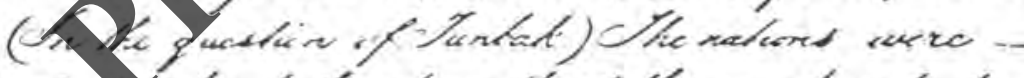

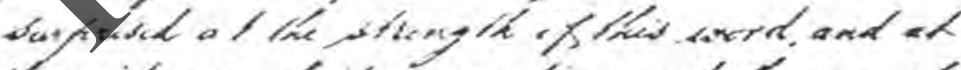

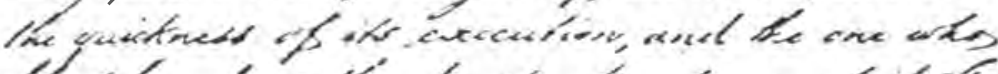

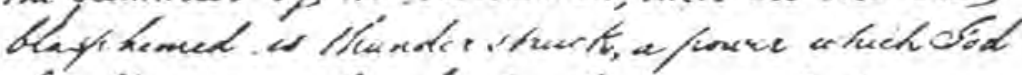

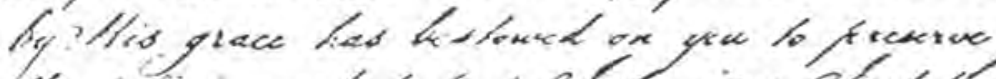

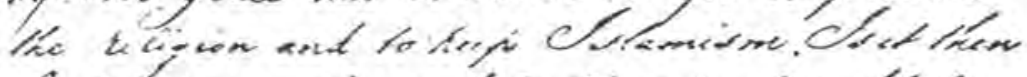

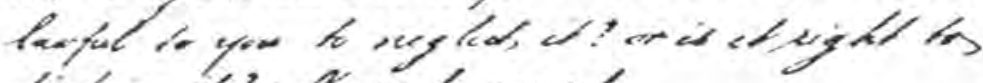
divdase if! Never! nead?

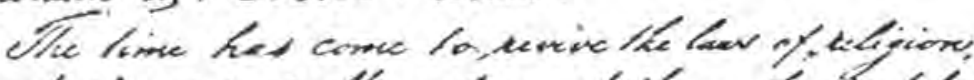

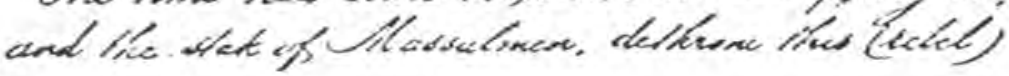




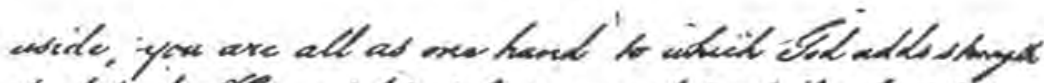

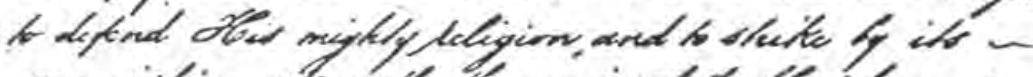

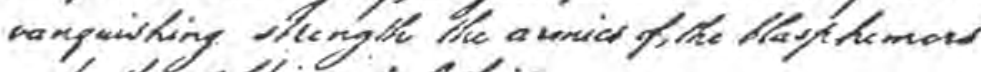

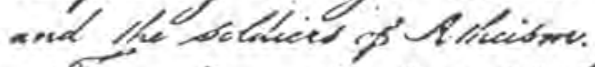

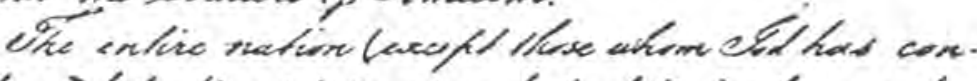

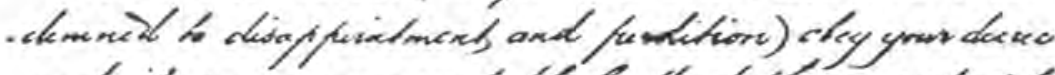

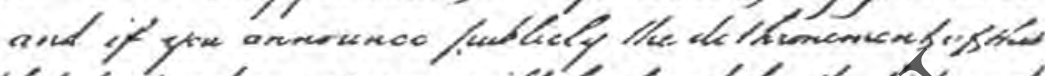
(hed kmplend mere) you will be obyed by the phend

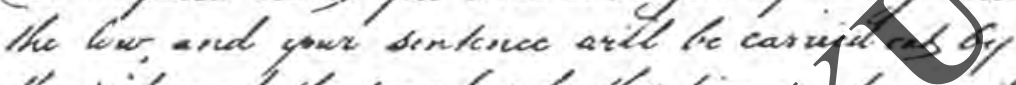
the vit and the piers (as by thes time pad have at.

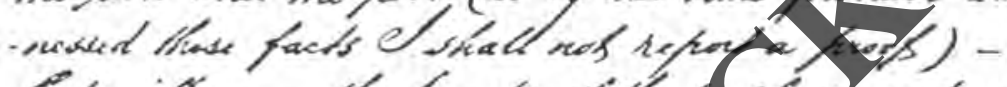

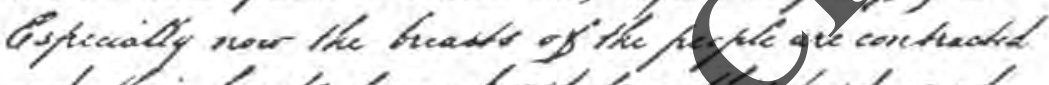

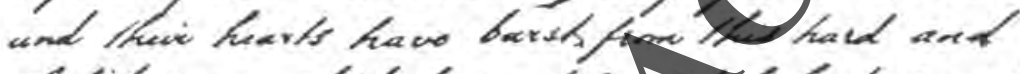

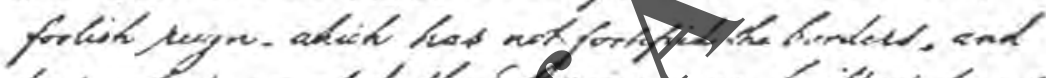

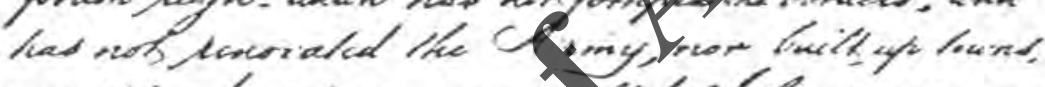

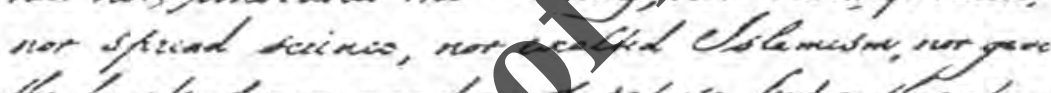

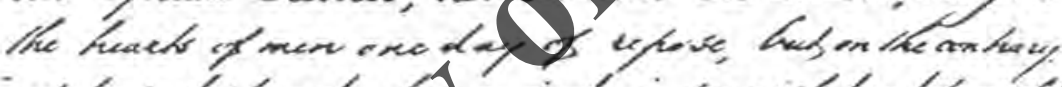

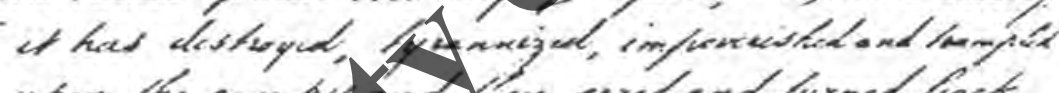

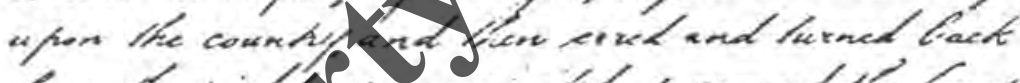

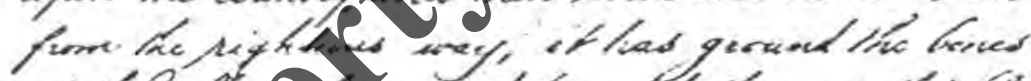

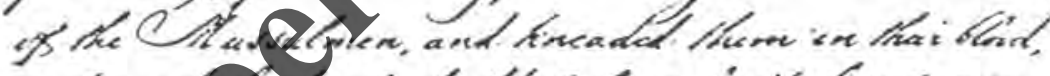

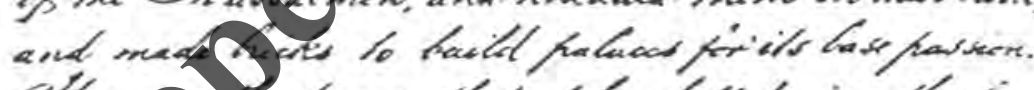

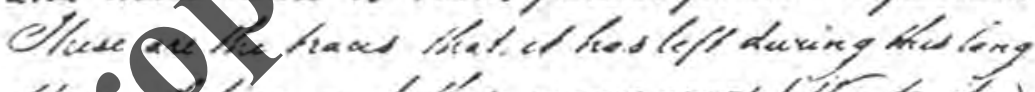

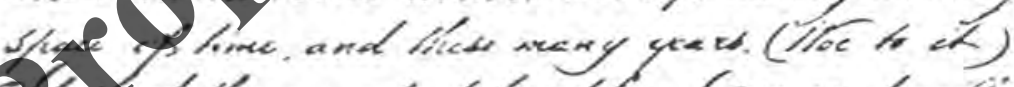

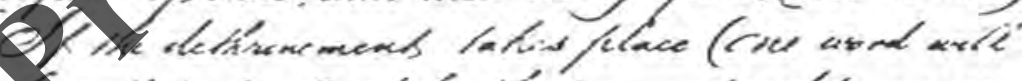

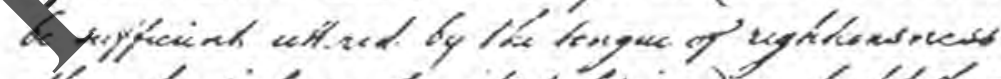

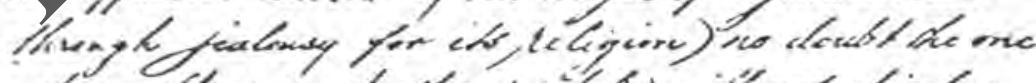

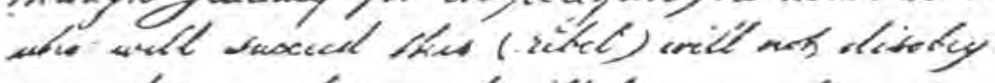

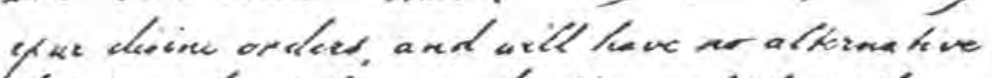

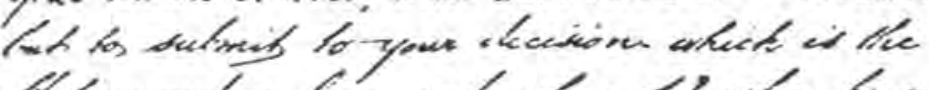

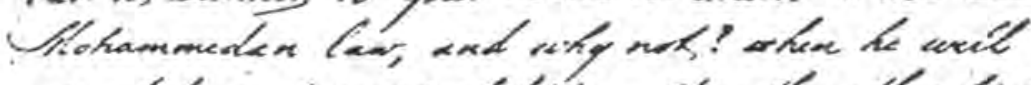

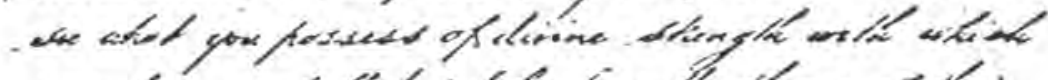

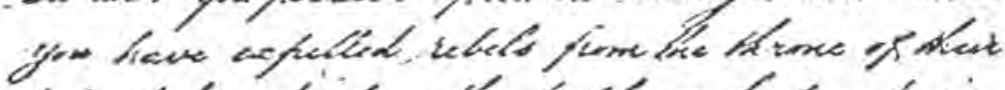

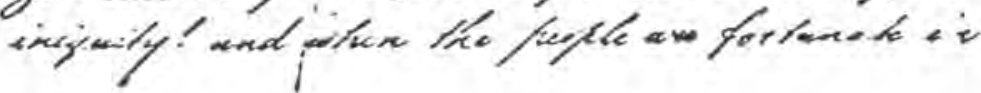




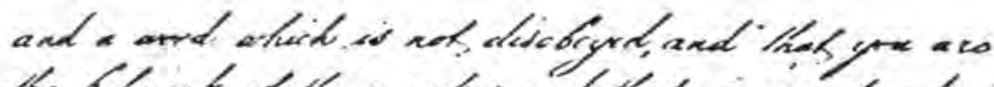

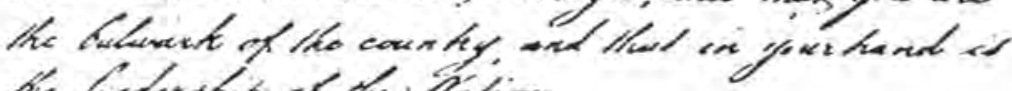

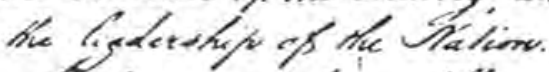

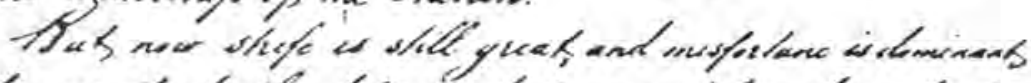

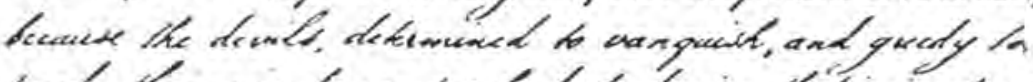

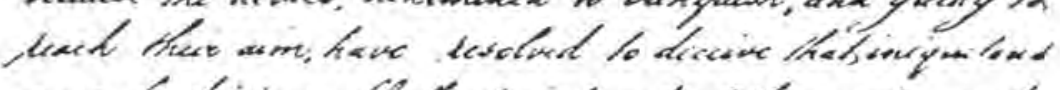

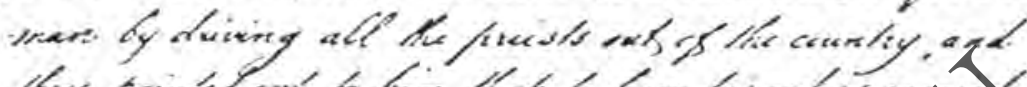

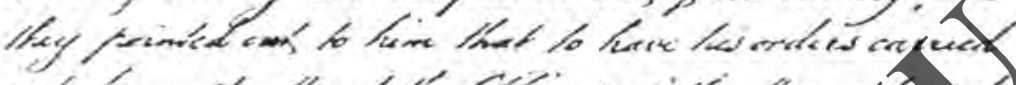

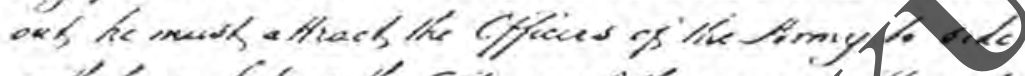

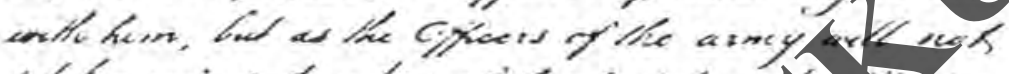

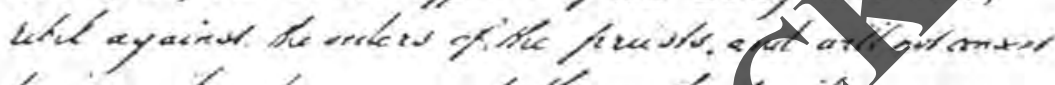

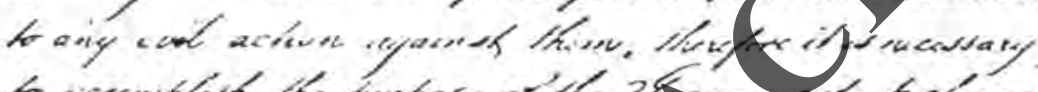

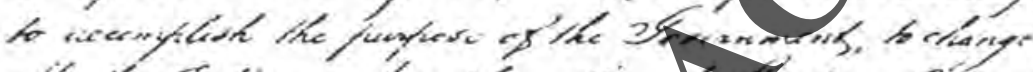

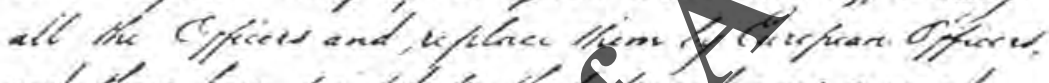

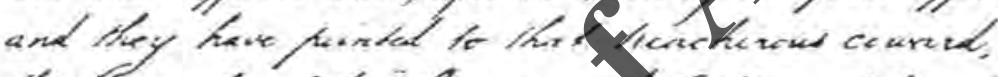

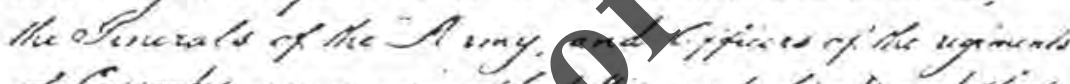

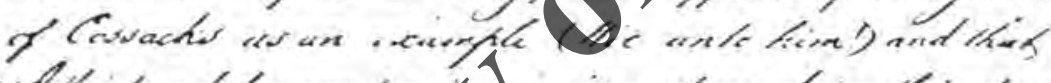

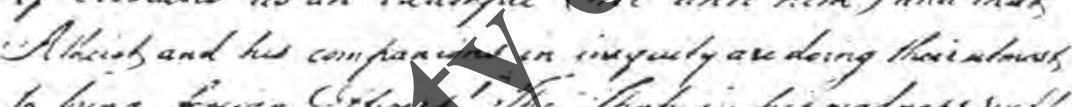

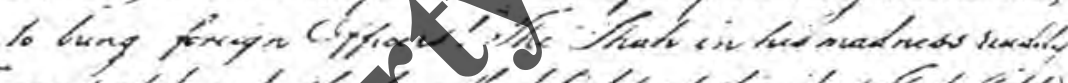

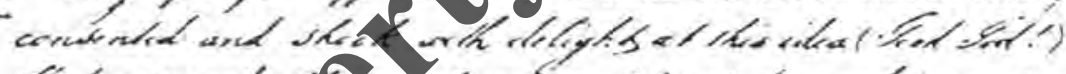

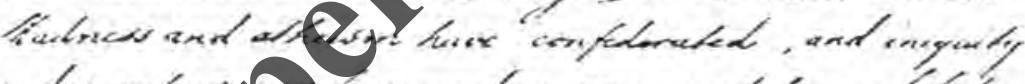

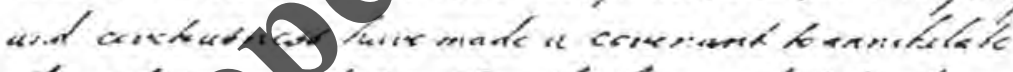

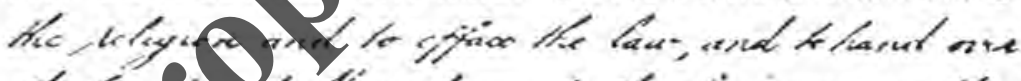

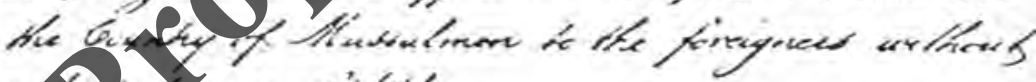

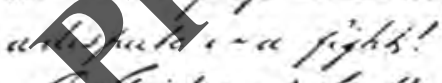

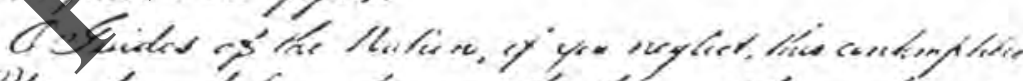

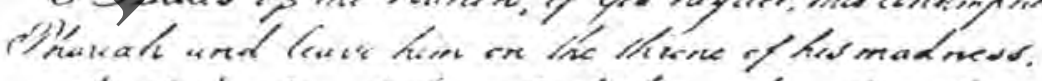

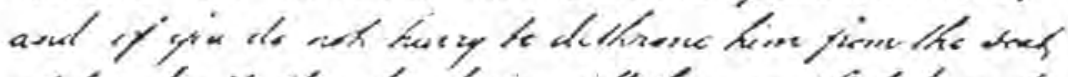

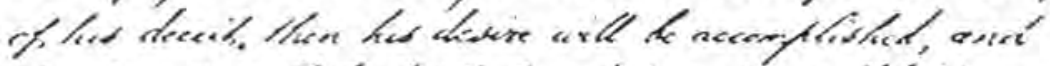

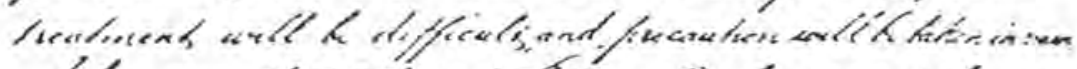

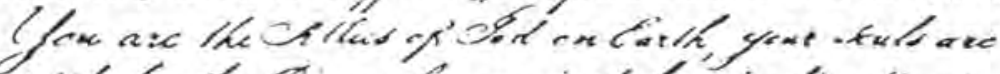

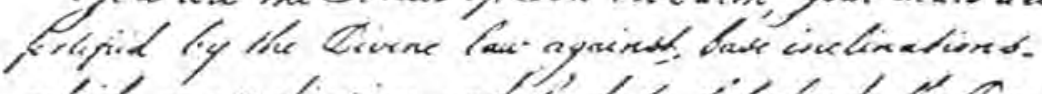

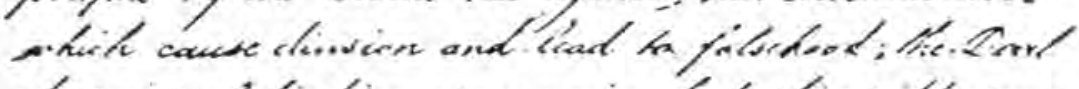

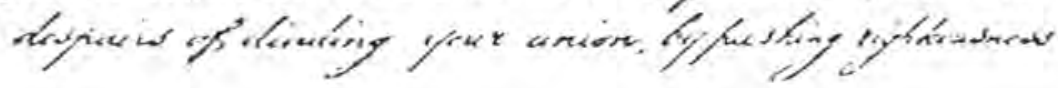




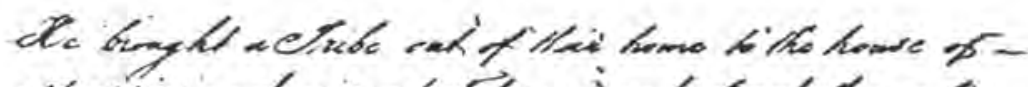

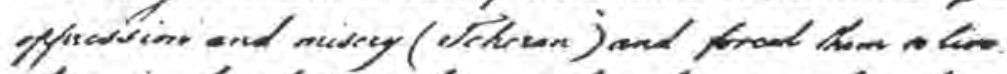

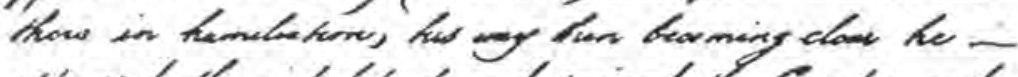

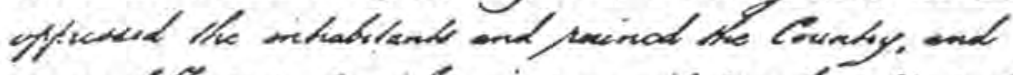

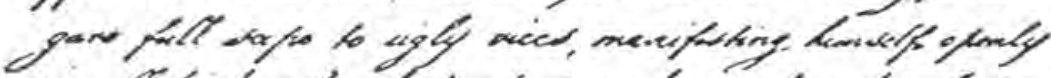

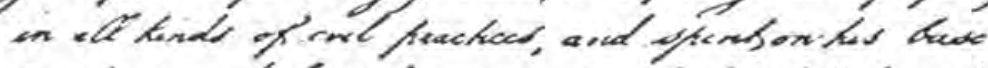

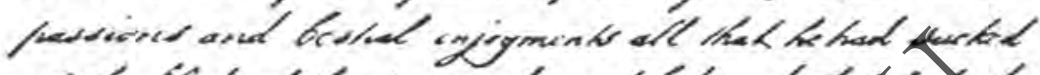

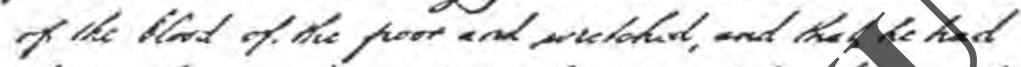

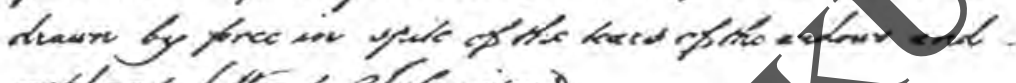

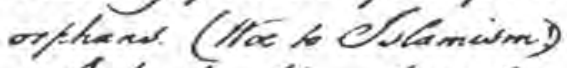

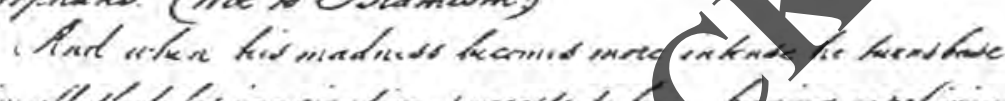

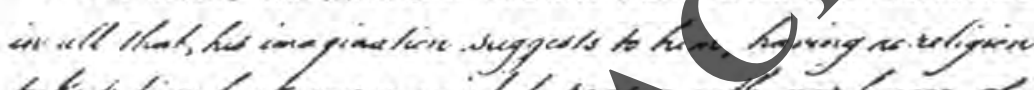

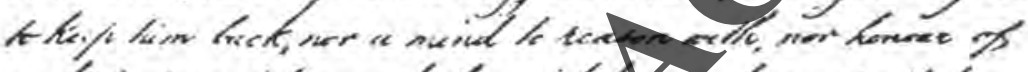

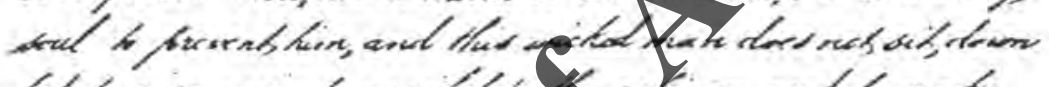

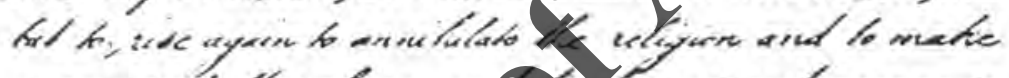

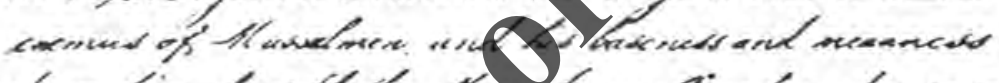

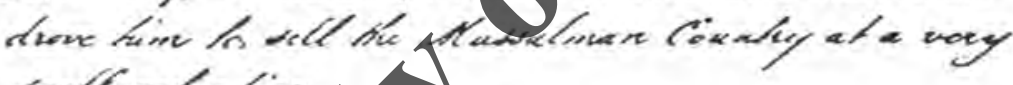
mall serkentinc.

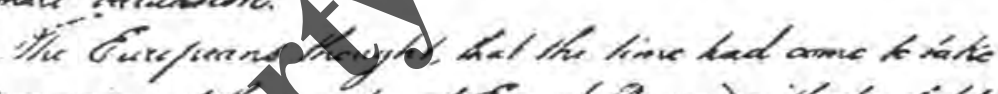

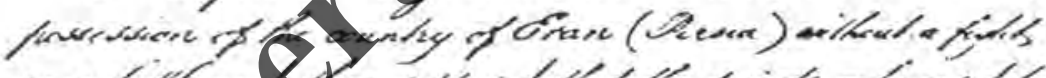

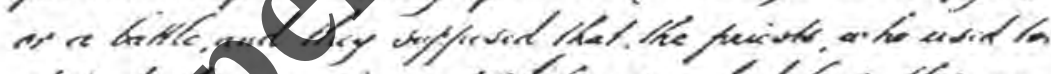

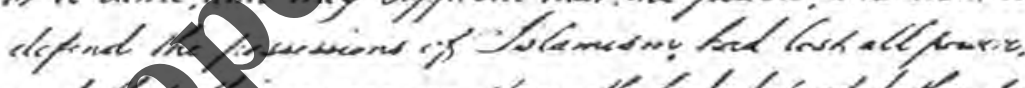

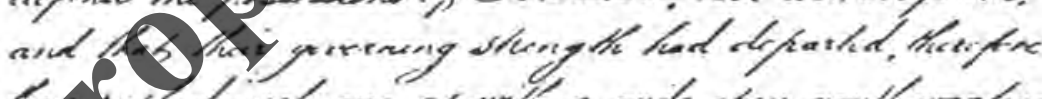

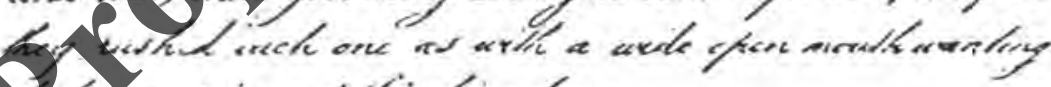

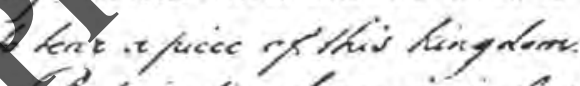

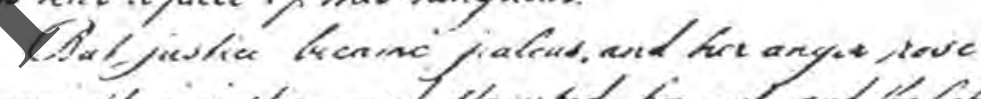

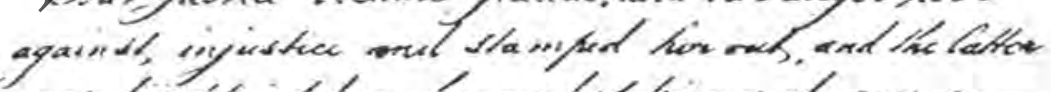

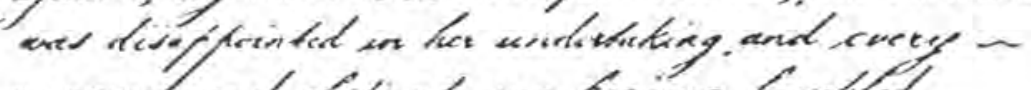

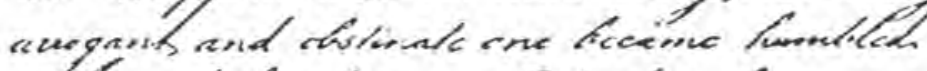

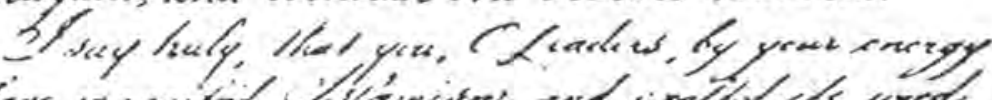

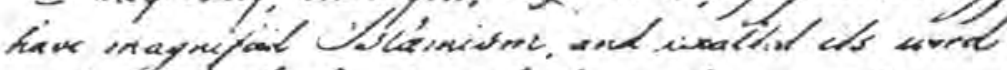

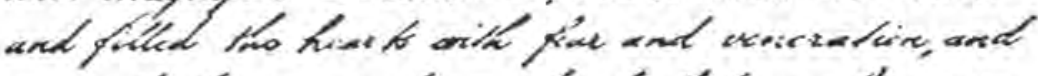

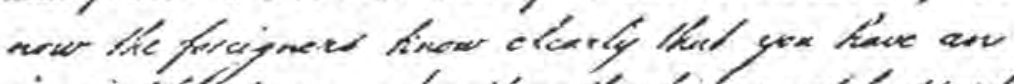

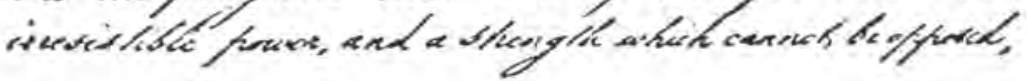




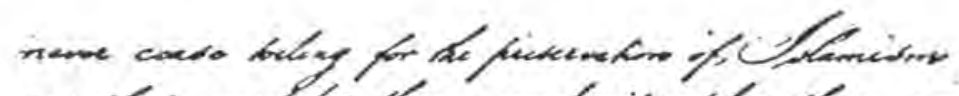

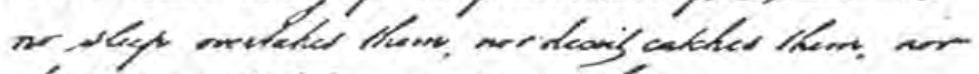

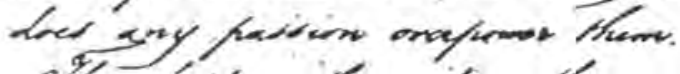

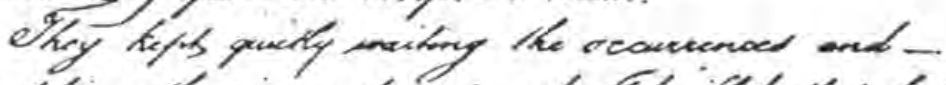

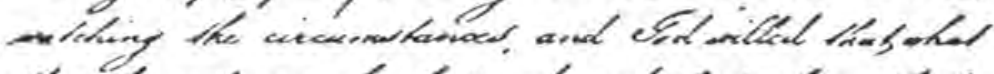

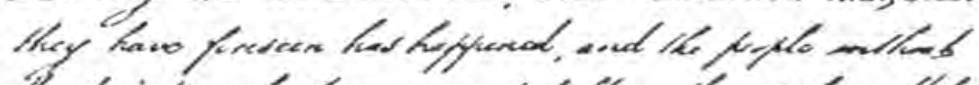

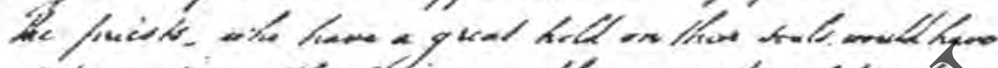

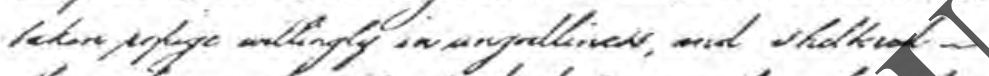

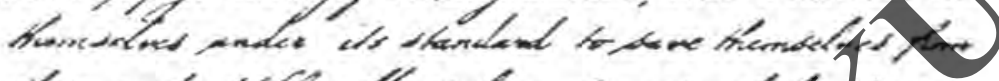

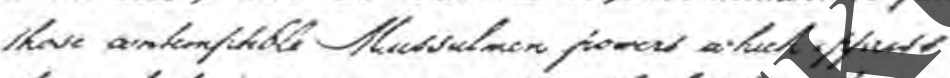

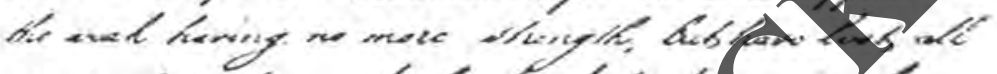

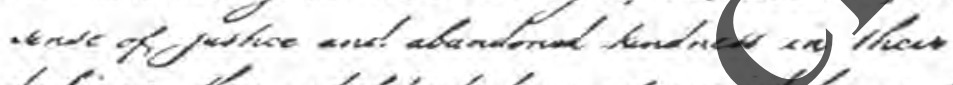

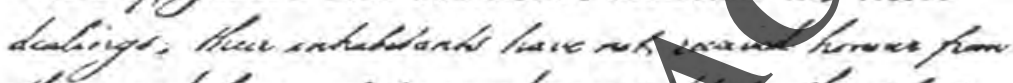

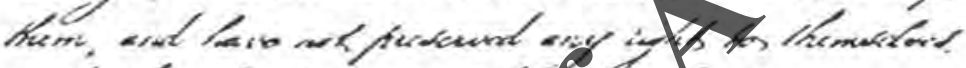

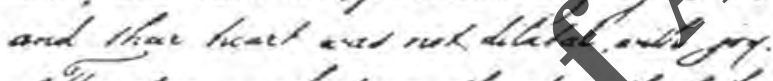

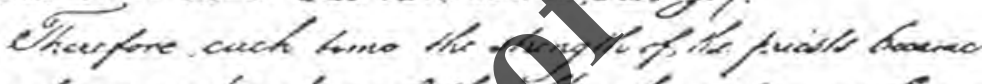

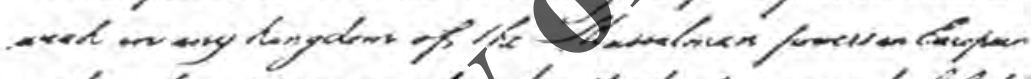

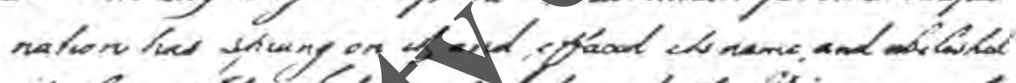

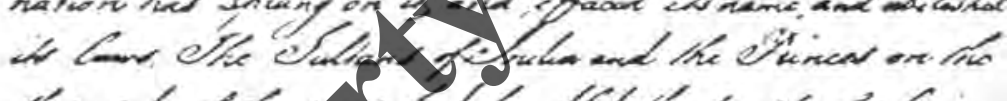

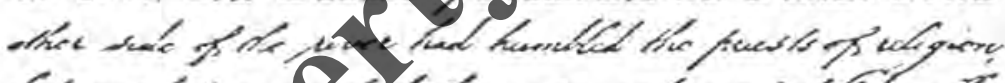

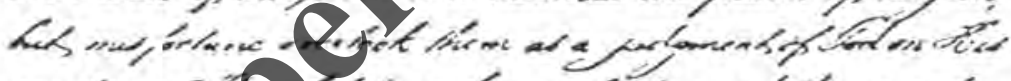

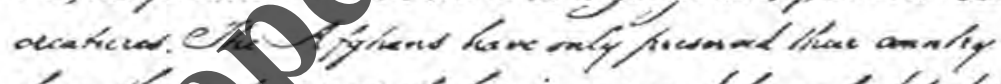

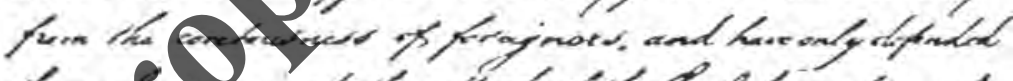

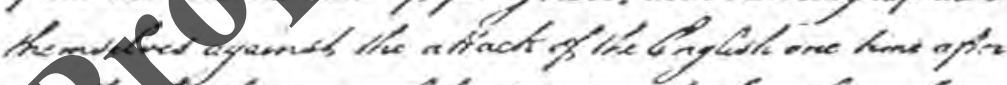

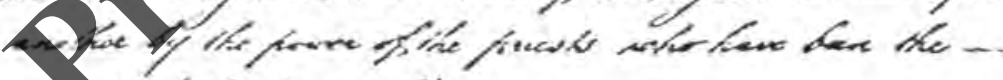

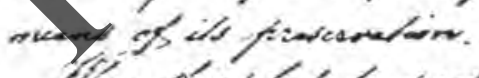

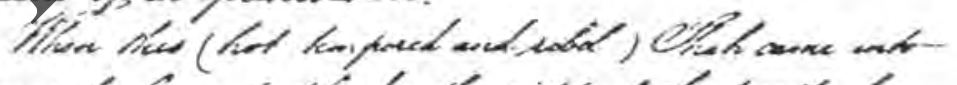

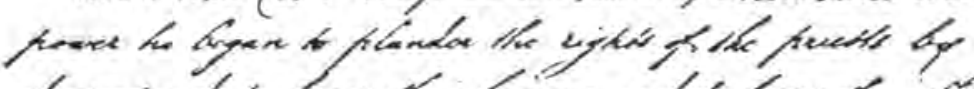

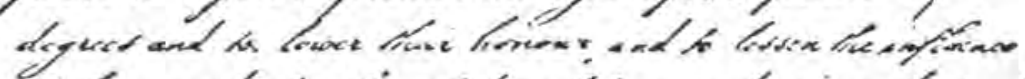

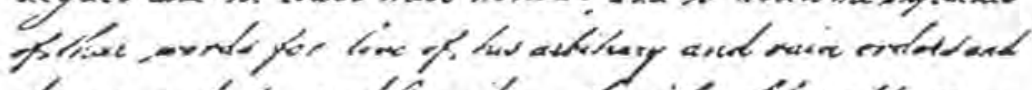

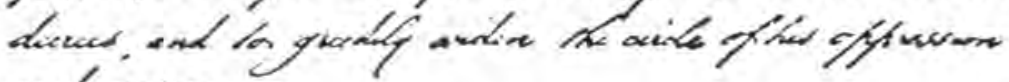
and enerenes.

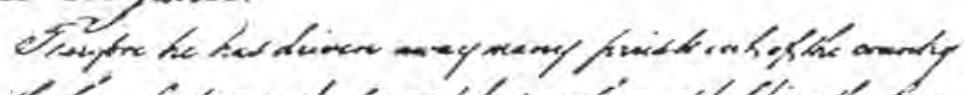

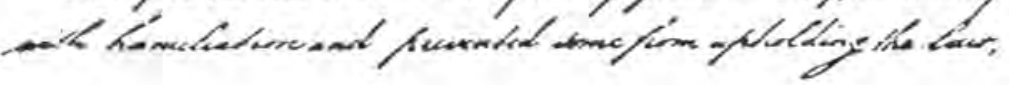




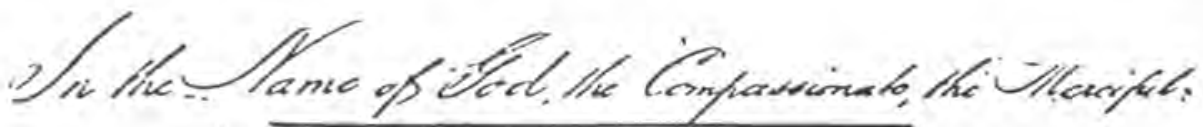

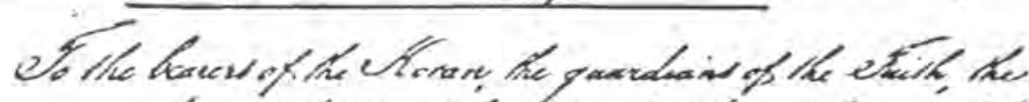

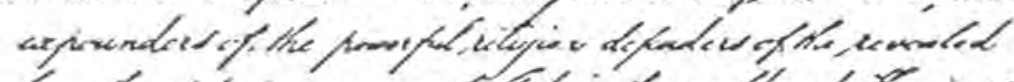

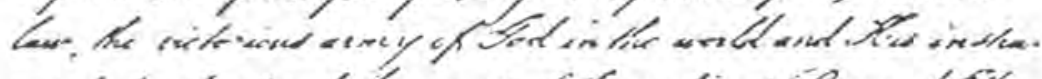

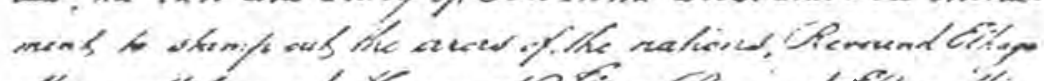

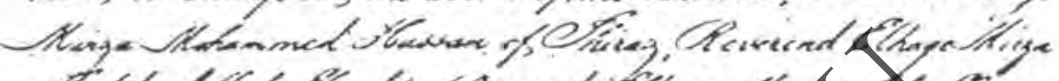

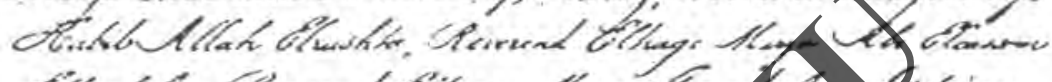

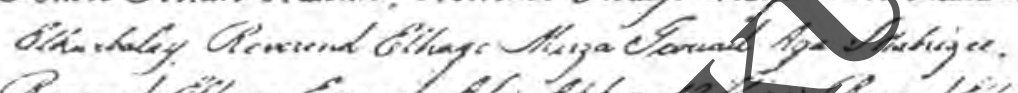

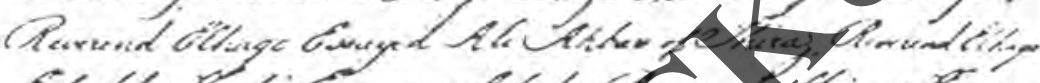

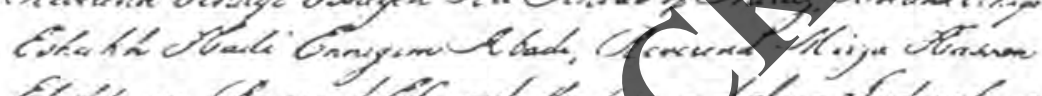

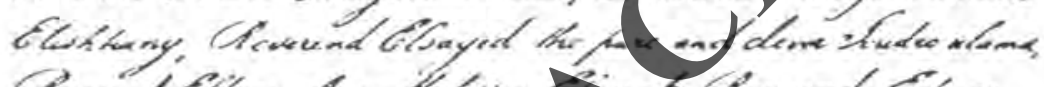

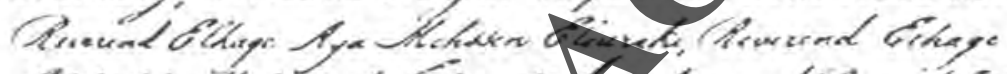

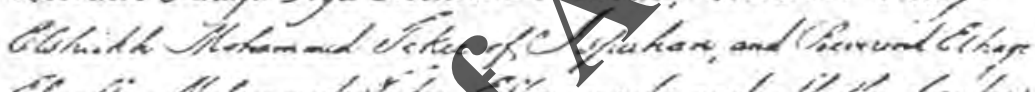

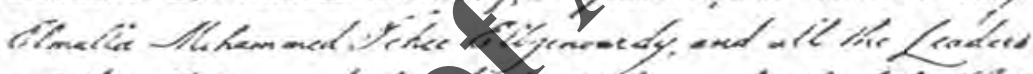

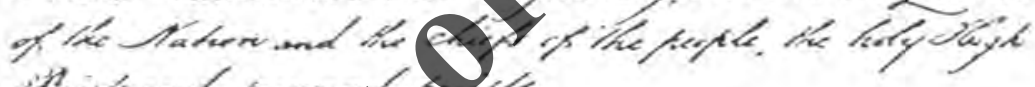

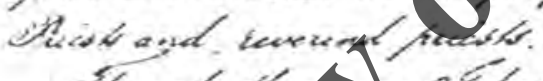

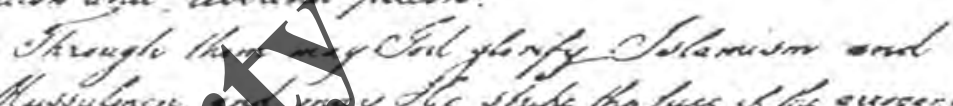

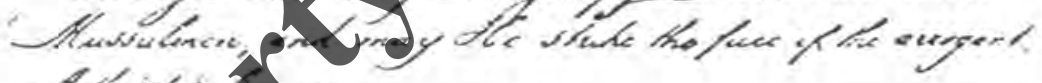
Alisish. Rerer.

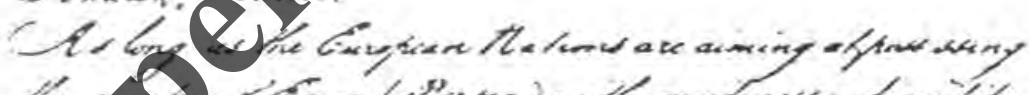

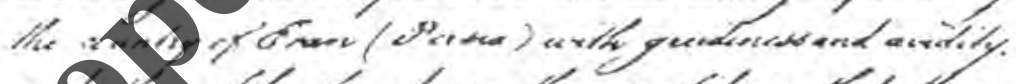

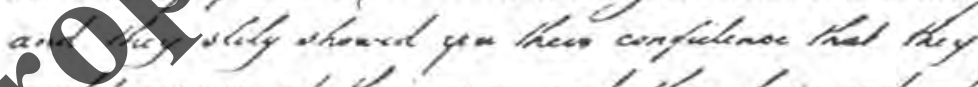

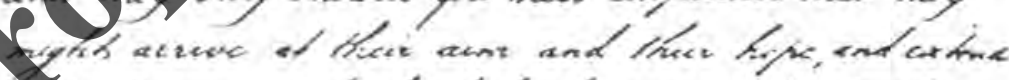

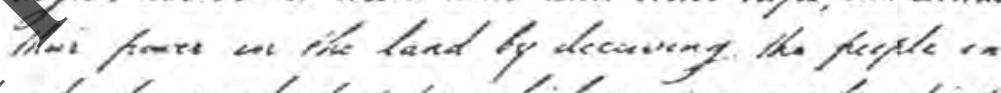

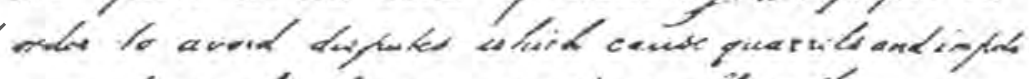

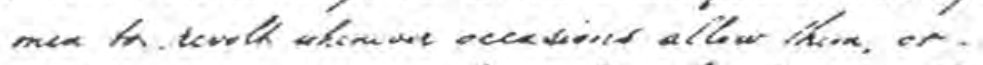

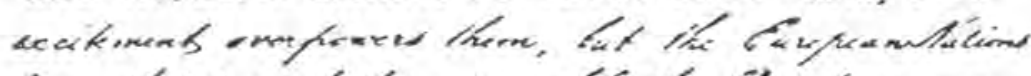

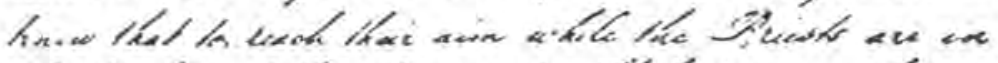

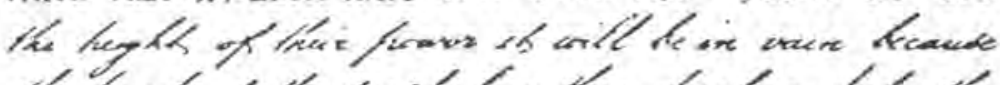

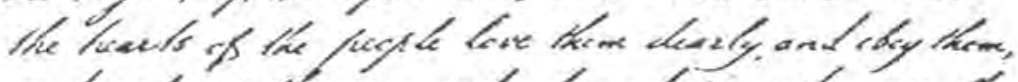

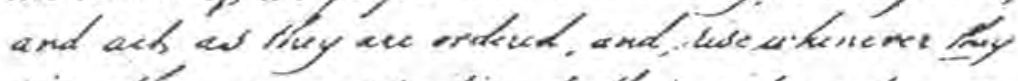

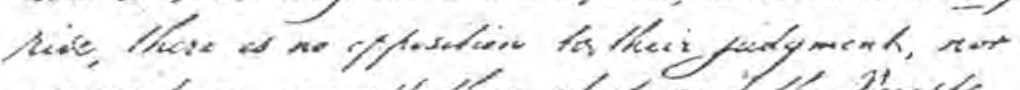

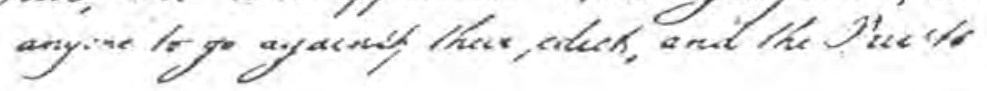



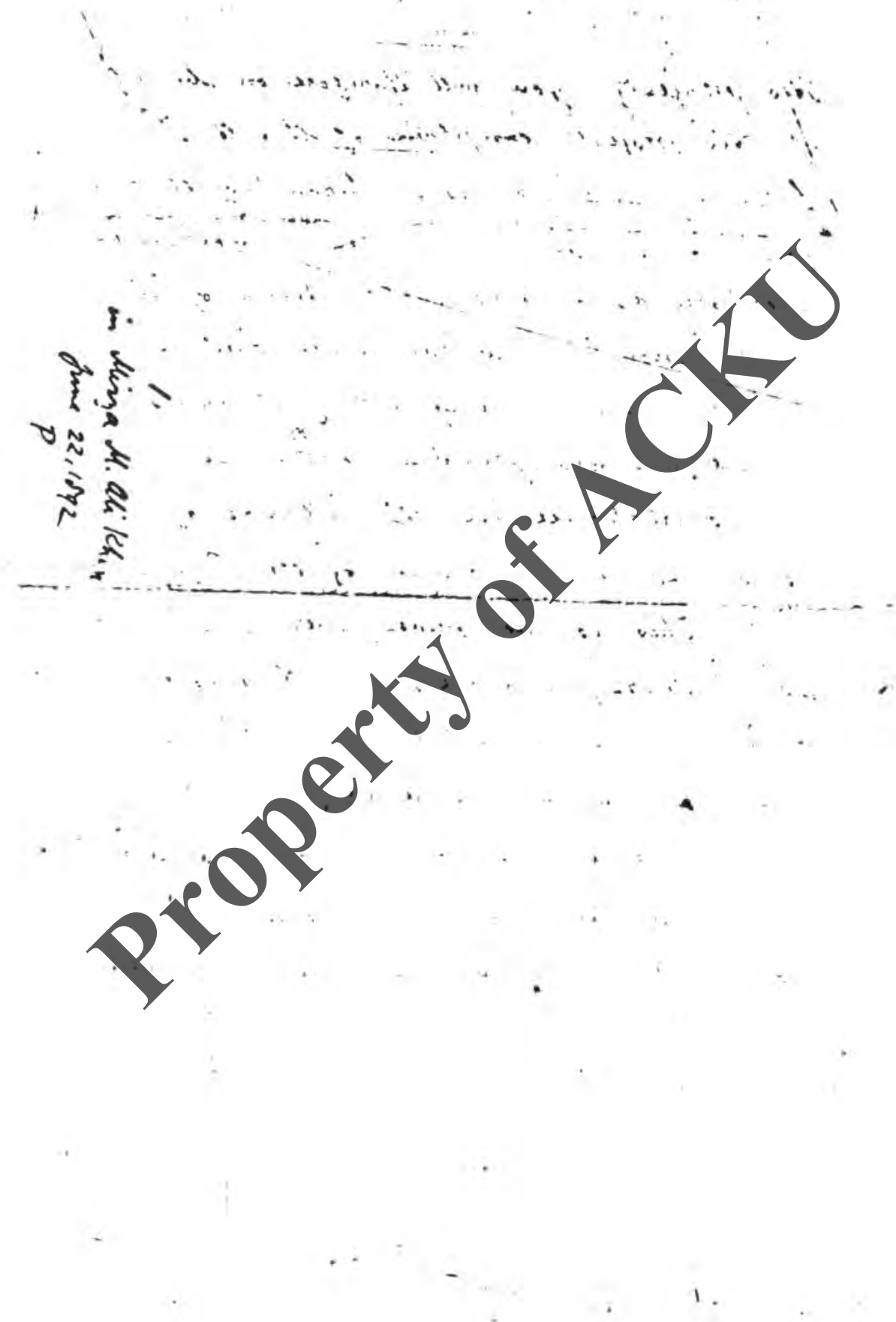
$+10$

227

thi majecty. you sill theregere on betey of thi majety complain of this to Lain Salibburg and say is it becoming tord in your county where you consider youraclues such friends of mers that arsicle. of dis natire strould be writan chere, aredsech incioility showed he practious tothares a Sovereign - your priend - creaty dexition and disturbence in the fouror of Persia, es it did in the meidets of the Pugie.

this is no has-paper beat- chey can pay - hero-paperp dre pree." this is a trampleselearly meant fo disterk the fuace and -breng

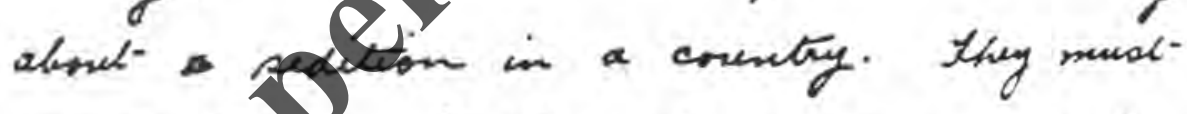

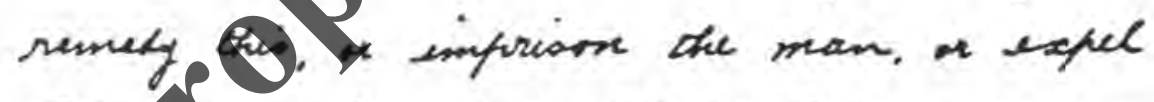
hot this Inferial hajesty may ga...

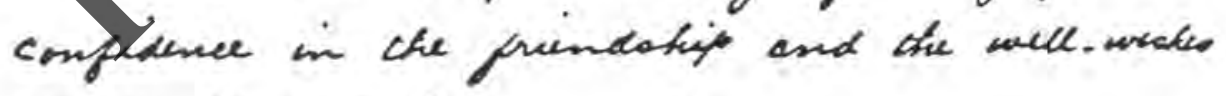
7 the Englisk Govermsint: 


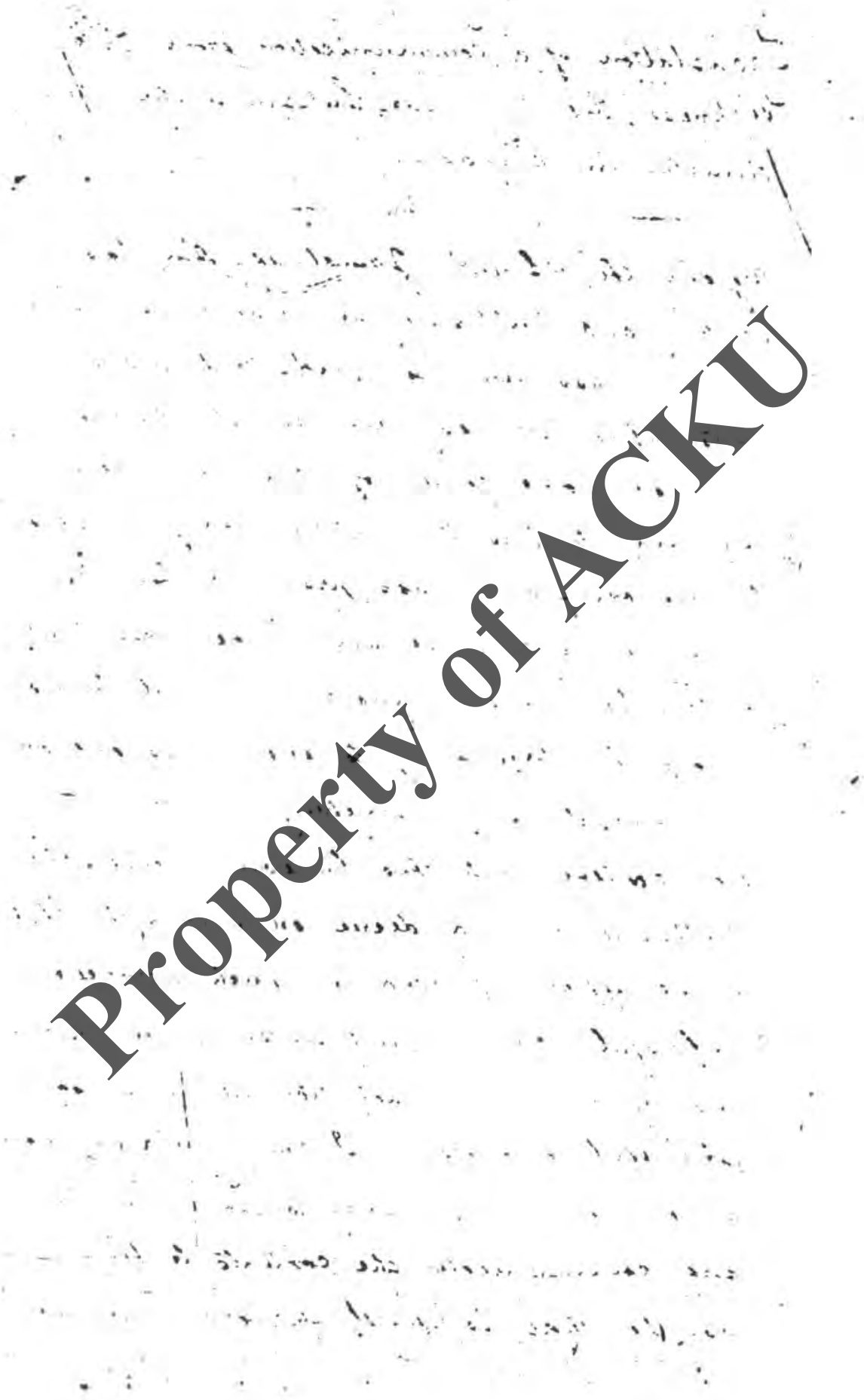




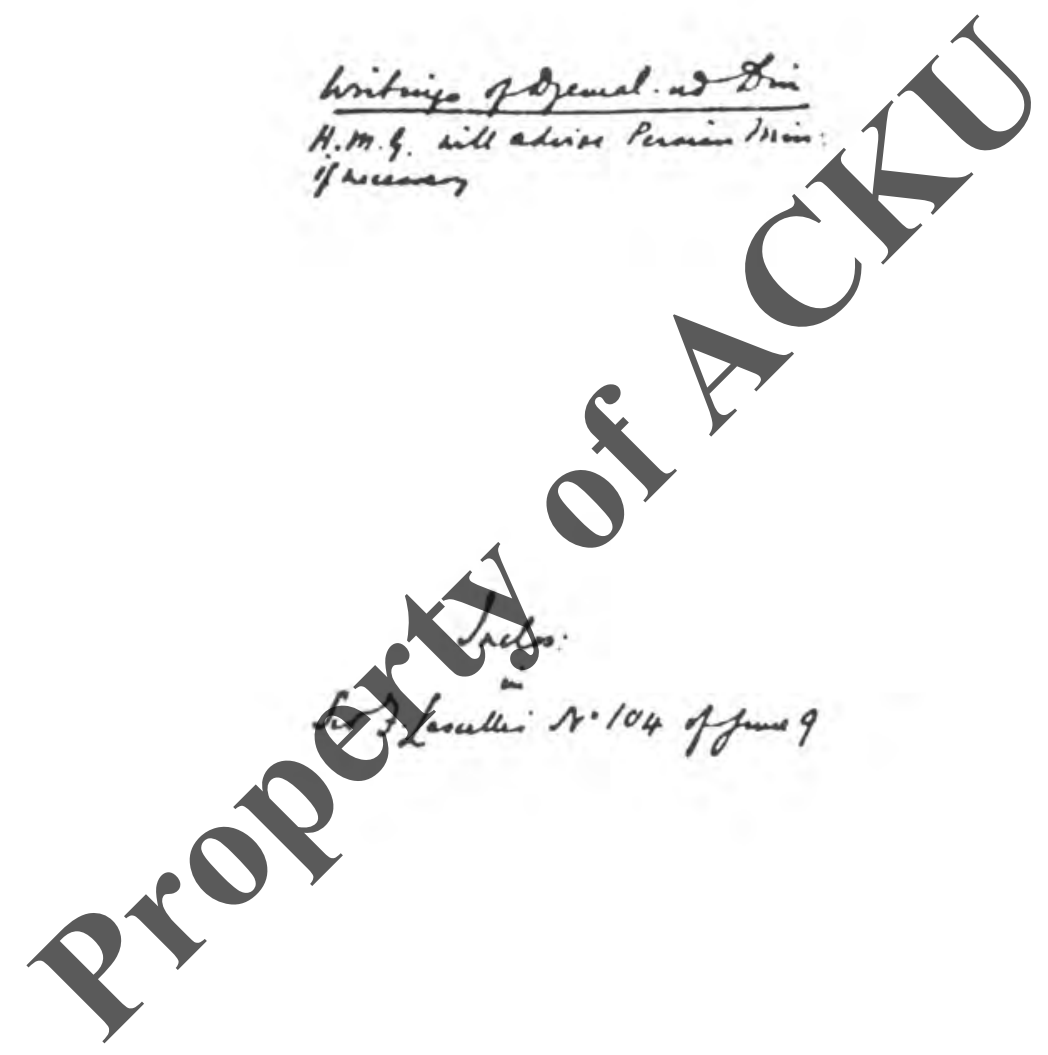


224

TIA

Transletion of a communicetion prom: thes Highness, the Amin.es. Lullern th the Ministar in London.

Again the theith Jawial es. Sin has writan and publistere in Crabie an arfies

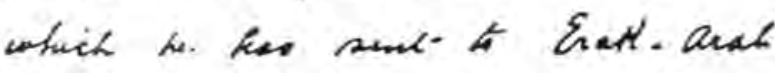

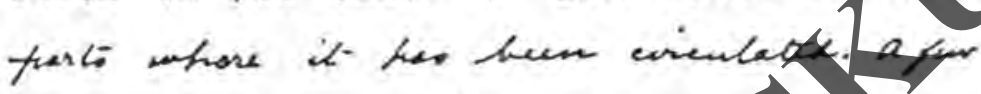

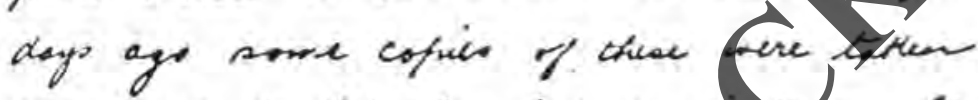

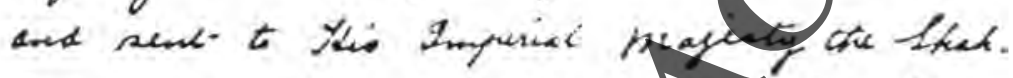
on this recasion the has practfert brun illess

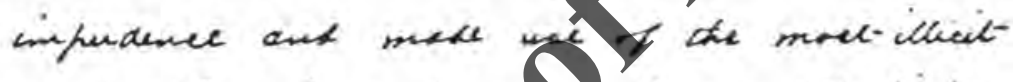

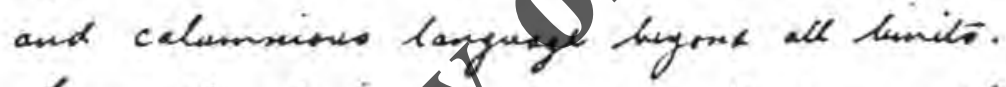

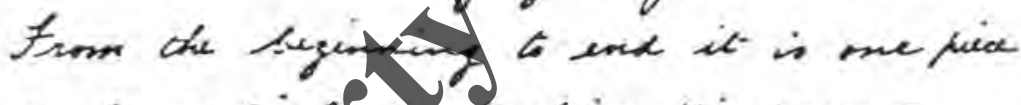

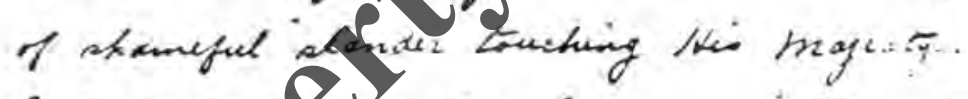

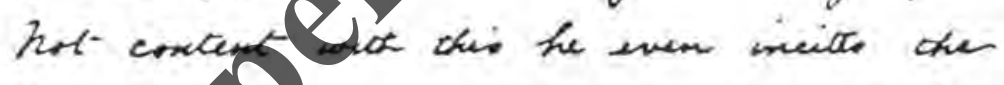
mullas foes a decue on sorving the thet

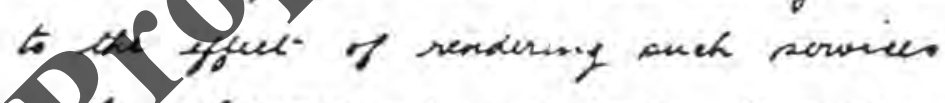
Danoful (xaram) and furcter to dactrone this hrajesty, and such like nonacerse to strameful it gerte. I am friwarding por a copy th ruad and tathe to Land Salideng and conmunisate the contents to firin, and ar the leat is furcly furamal tmething Win 


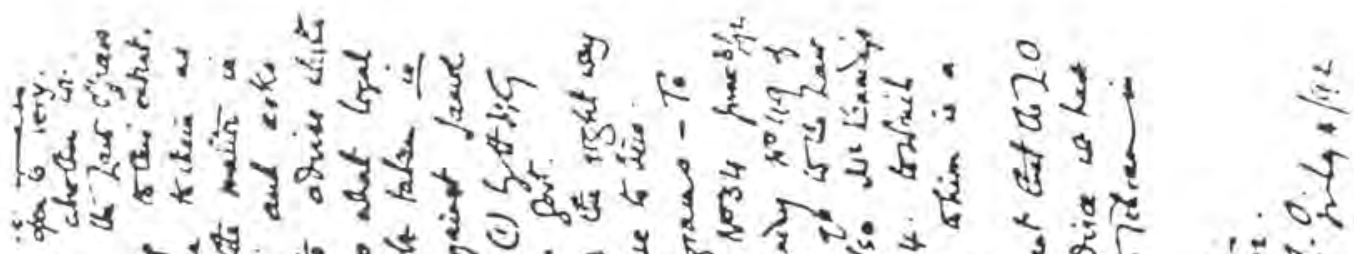

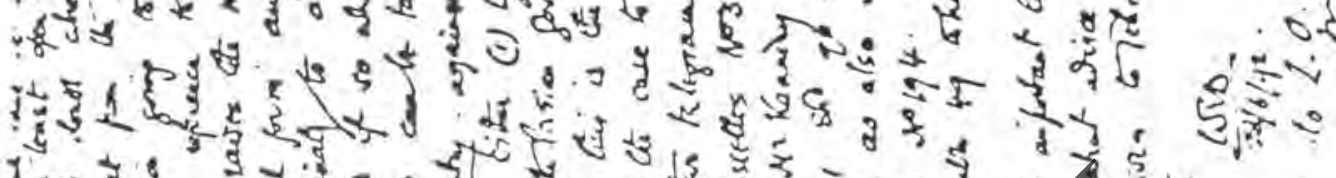

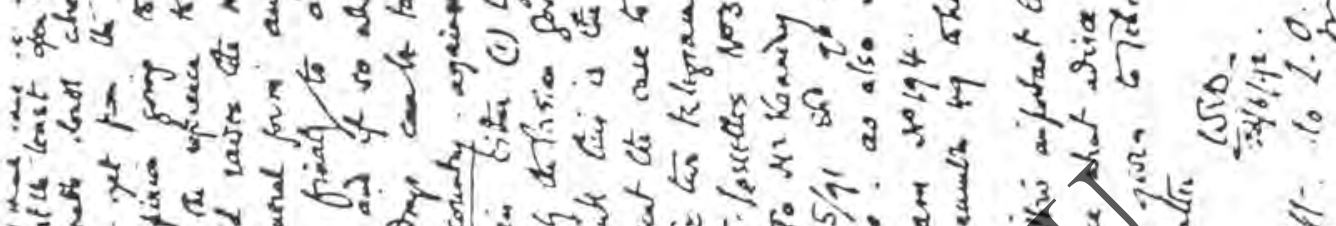

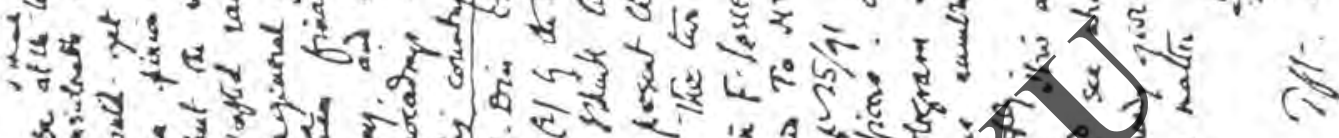

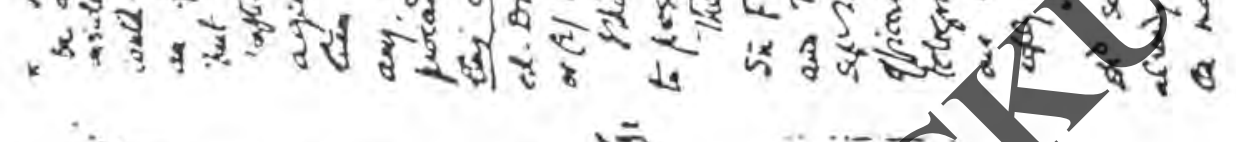

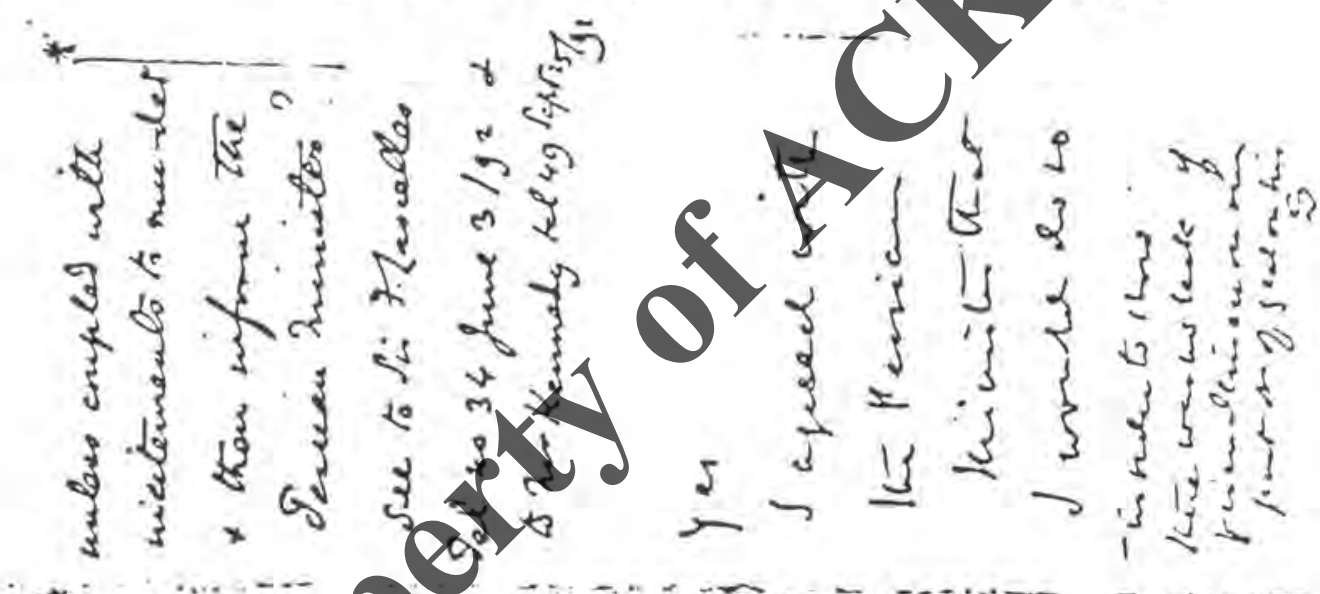

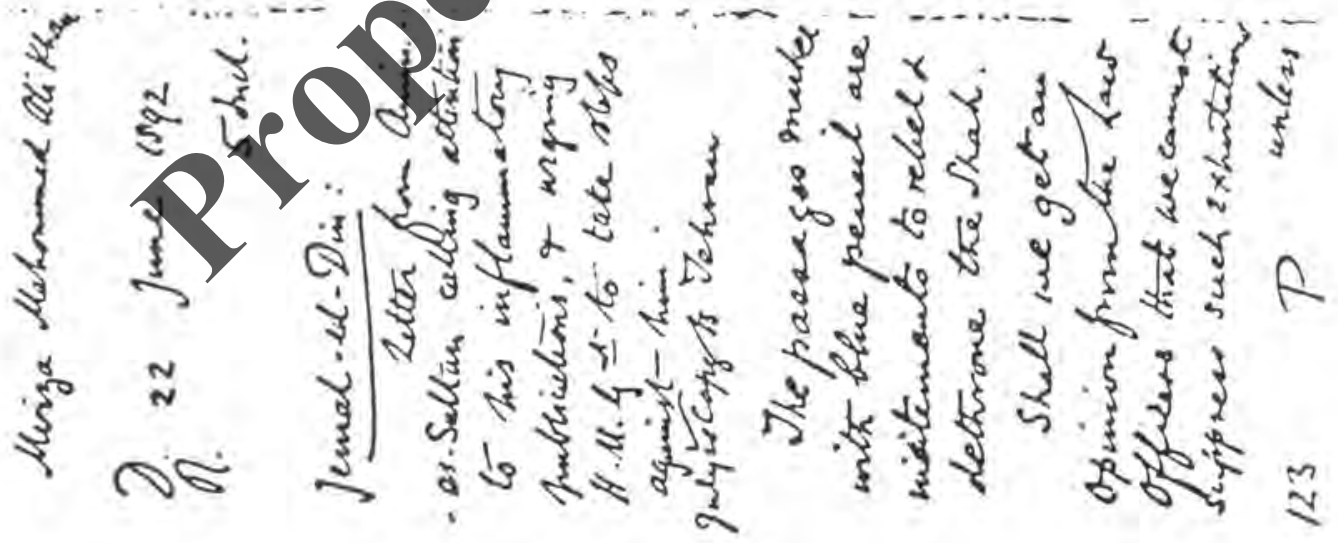




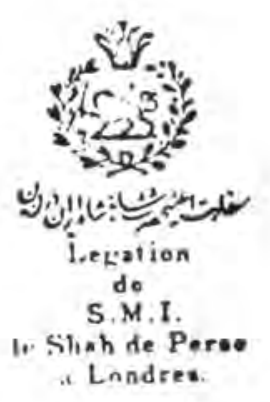

Lum 22. 1892

Sly Lanc

tham the hampre Caclowe the

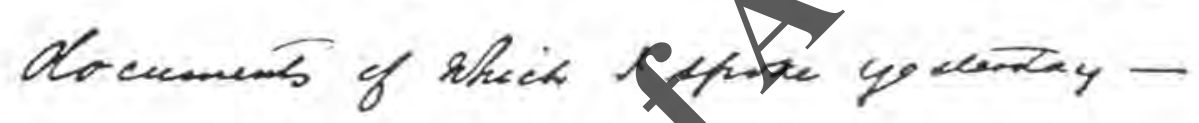

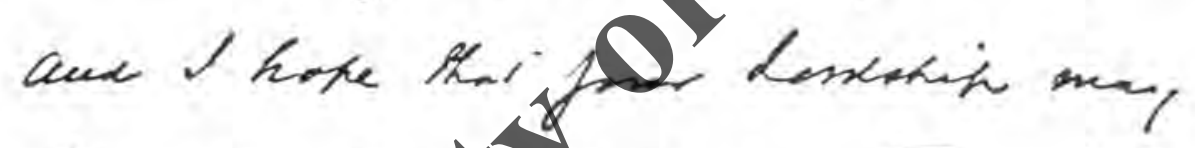
cork nuble do ecue a Topricing

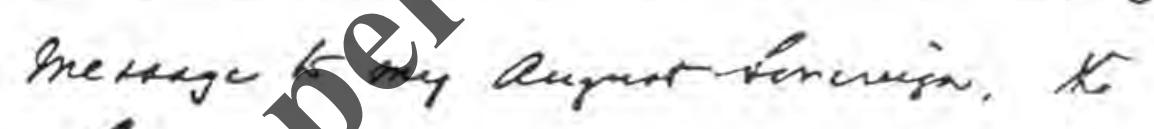
thate Thave to knorer wa.

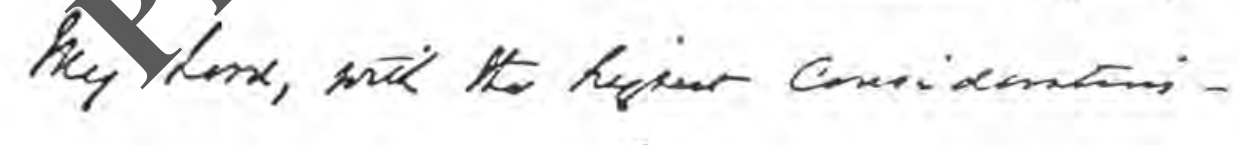

The Riga tomse

Your Basair Nemat

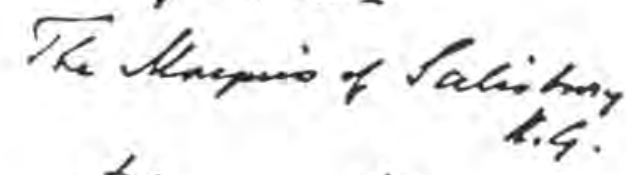

16. Sla'

in: 11 a, 
depens whock on the fury ant the

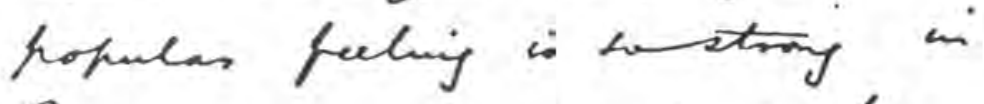
England agunis powtical preas presentem that funis can edtom be induces is comid.

(sd) 7.C.Lasufter

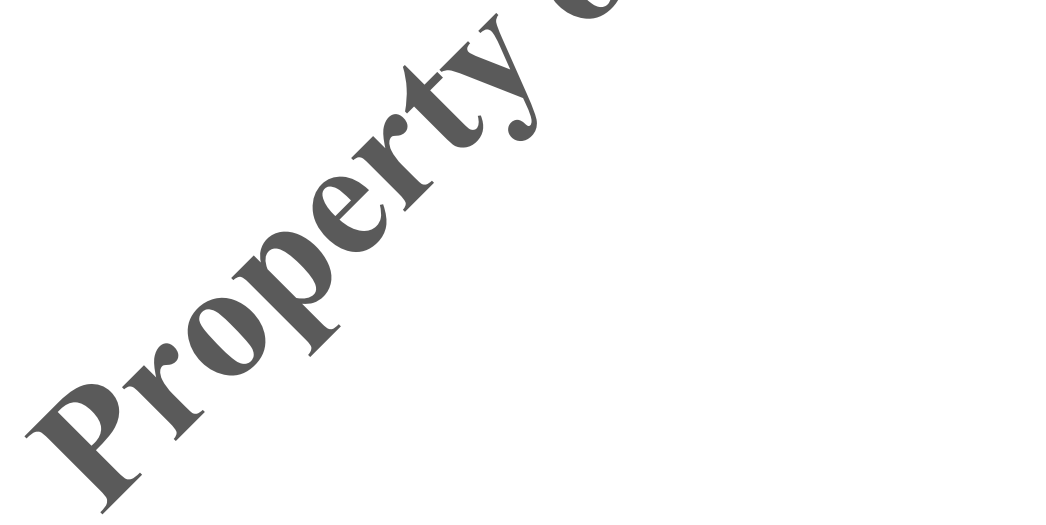


Euchet

fune. 6.92

A.C.

Shave not failed to commenicate 6

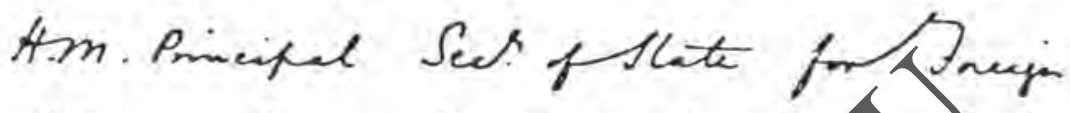
Offairs the wish which tim prosh hed expreseed that meen thened be taken to furt a stop (t) 花 untings

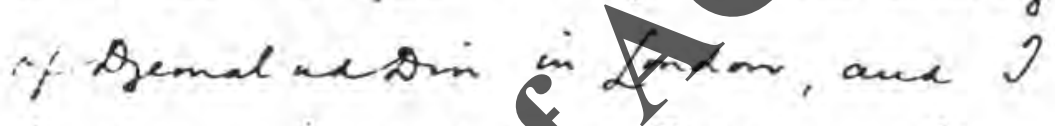
have now receniet' Jelegean from

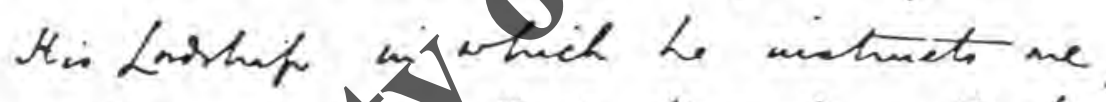
to inform that Hes wll be

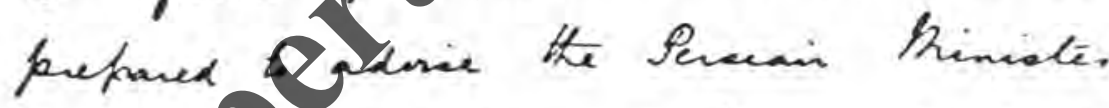
in Lros with regard to any fubtication ophes he thinks ongat to be provecuted. A.y ns a zale H.L. does wot aderiae any

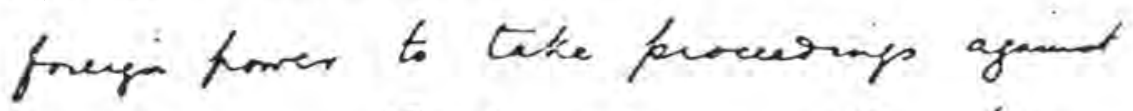
te Pues in England even when theie is good causs for doing 20 . The moncts

H.H.

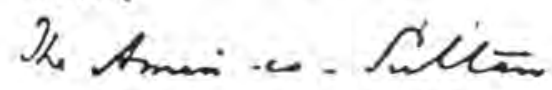
Lefears 
219

rTr

Queket goos 9

Sirs. Cascelles

s:104

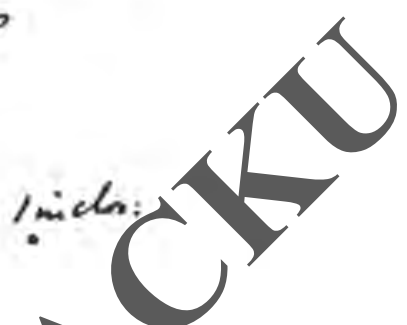

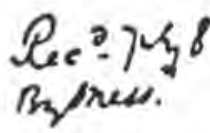

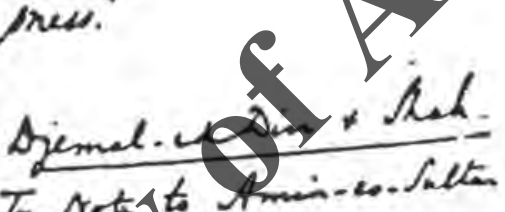

Tr. Noth suter

copies Contia.

Zu thes Lmen 0

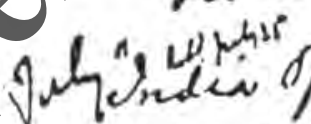

perueal

122 


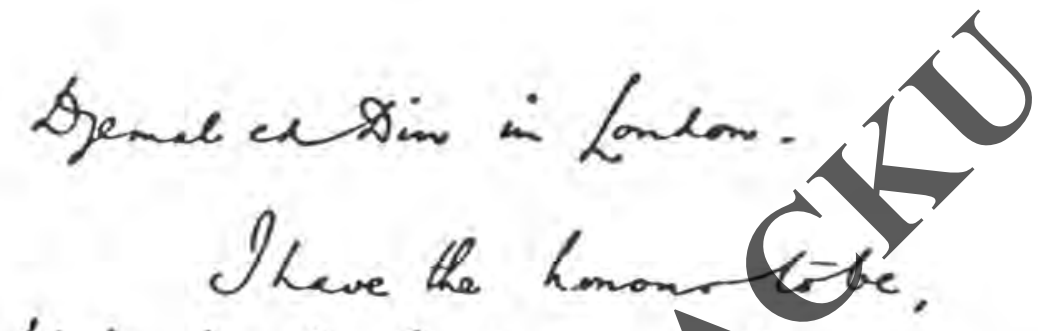

Lith the nigheat zogfent.

Yomforstifs ly.

Yonforshifos motobevinh
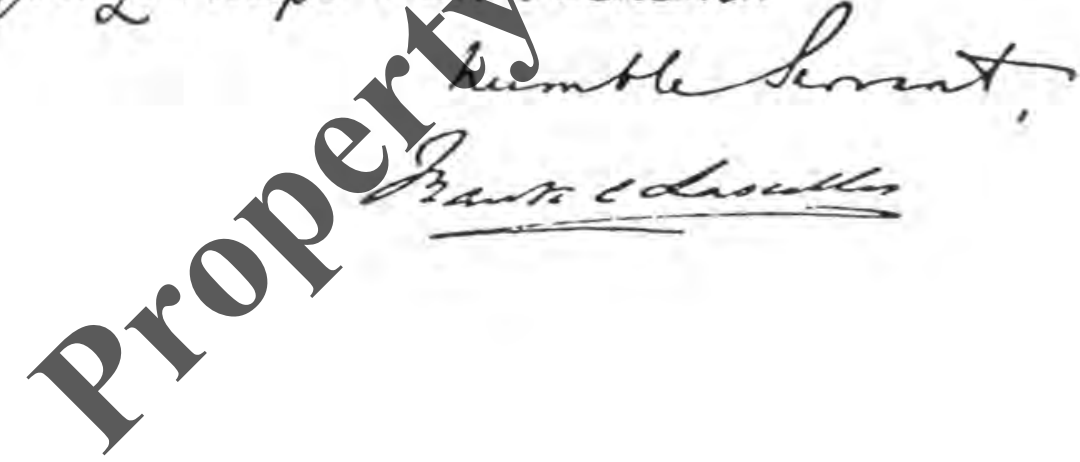
$\mathrm{CSg}_{\mathrm{O}} / \mathrm{O}$

Eulakek forme. 9.1892

Myfore.

I have the honom to ficlone a copy of a vote why atyi obehance

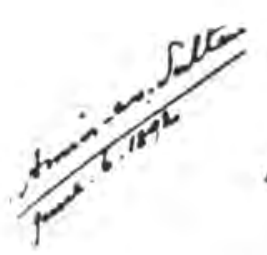

contagued in Yous

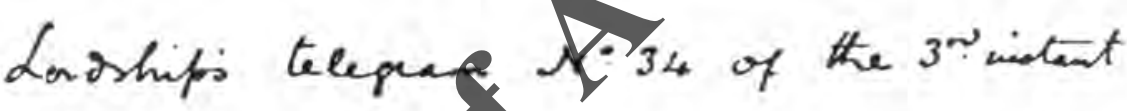
Thave achesed to the Aman as Sultan on the tropel of the alinice whech Her Regetigi Evvermment are prepared to grite to the Presian Mnnider in Fondon with regand to the $;$ sedetions witeripe frublished by Spenal

the

Marzan of actherztes. 


\section{DRAFT.}

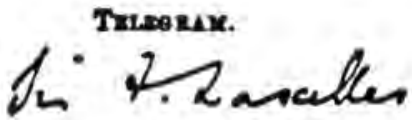 S. 34}

r. a Sume 3 .

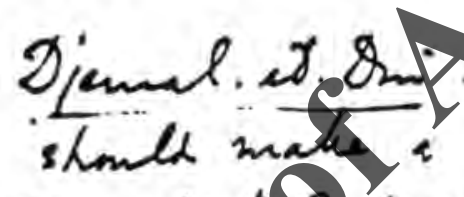

cominn "s Olinim is che tr. 4 . $1>$

$$
\begin{aligned}
& \text { Poreptim } \\
& \text { India Offie sout- }
\end{aligned}
$$

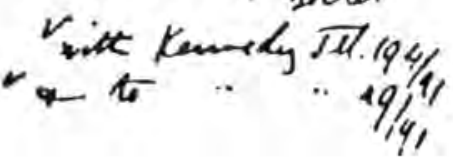




\section{Drafi.}

Sin T. Lascelles

Telegram.

$$
\text { Na } 34
$$

F. 0 .

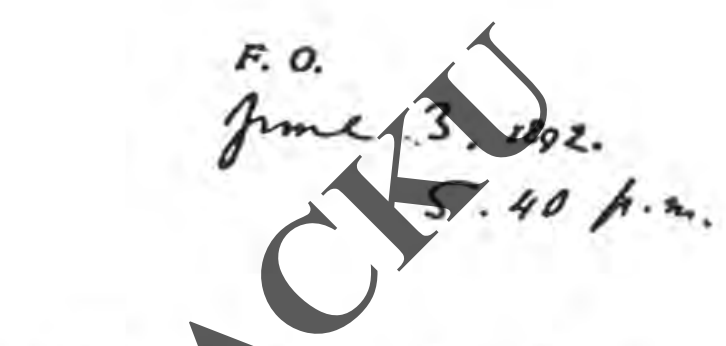

$$
\text { [Qjempled. Din]. }
$$

- zanes deptatches 82 fo 83 . Zon sould make 1 a commonialion 60 the

Q Anvin-es. Sultan in accordance with my Iet. to Kennedy No. 49 of Seft- 25 last 
214

res

Private better from ke

Amin-es. Sultan $t$

trowat Hasoen thithen

Demal. Dn:

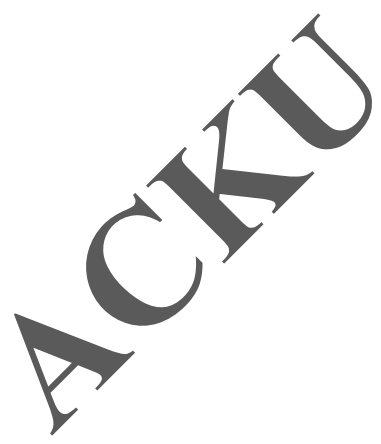

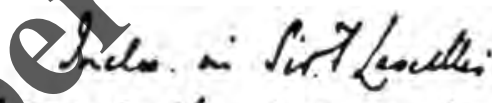

Q6 99 cont? of jume ! 
rra

213

arrected ano-funinded the tharem and infect whas werebt effect. In Reken-n. Dowleh, it Las not a good act whin kut.

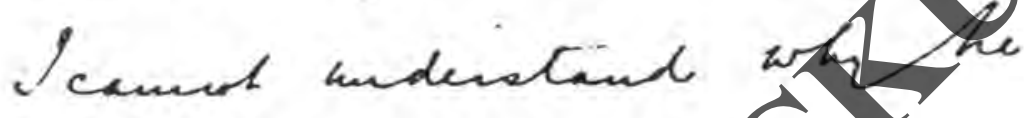
inale ruch a mustake afd did

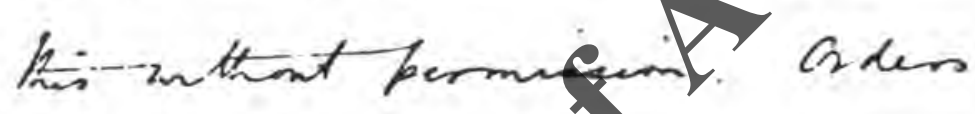
Lave-heen coipel L-wand the Caram top Dohiom and not -

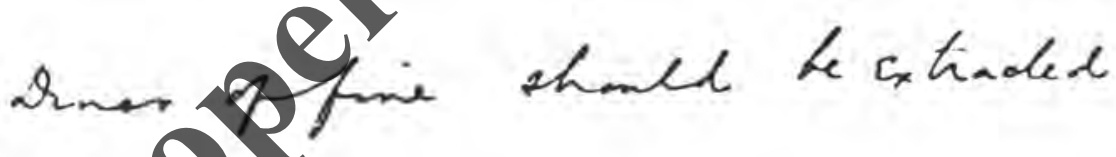


give even a fiommasery auswes luct as $t \mathrm{ta}$ that the (the Euffirt lori.) will do what ther can. Alttongh the Eughah are suite right in what the say man (thethah) thaver risafformted. It is befter that he shomer have hober at hulid Lits regard the Regie, Shote the sill oty zomething in stes to begrolith the cocomats $x$ recere the asects.

4.7A. Las bey much ameres wis Rukn.wo Dowleh for Laving sittent perminarion ensodid 
latin

a.c.

Ihope you are well. Your reached me at Kunn. Lith reference to the Customs quen Ihave mislencted tik. we Multe the will focthe matter right. Ithere alf heceived an answer-form f4.8 The livister Lith rezote to Sued fumal -o Dom and 2.4 letters $4.2 n$ again Qter for a refly. It is well that an auswer shomed be given thel Inar refuesent it to heri. In me opinion it is belter $t$ Sove 
210

rrt

Selven forme!

Sorj. Lascelles

se.99

Comft

I viclos

Relore

ingless:

Diemal na Din

9. private eate from the Ams

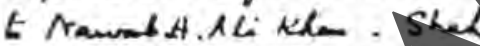

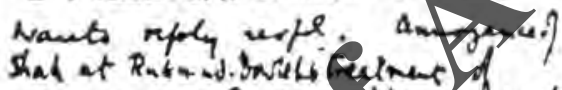

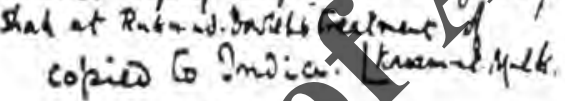

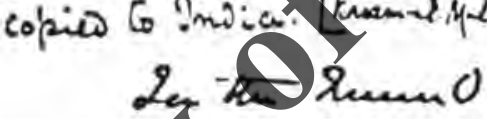

7 
rue

209

If asked that der Robins had informed mes that he had been authorizes by his Directors to examine is Boots of the $1 \div$ Corporation with me.

I have the honours, Lith the highest repent

Your ford ships most obivint

humble Servants

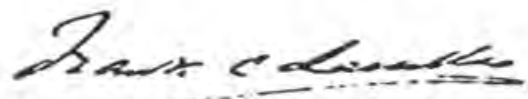


miter except by process of law

Lith regard to the exacts of the Jobeces Corfantion. It hers sequedied the salas to inform the amines. Sulla n that at Ko request of the Mortar. Do pah

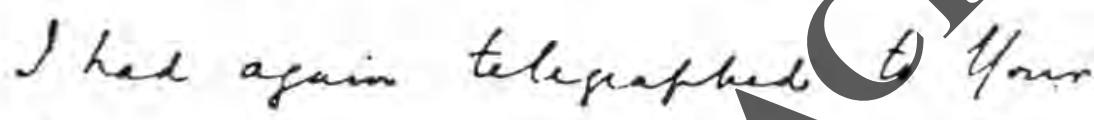
Lashio on the enbiet! and had

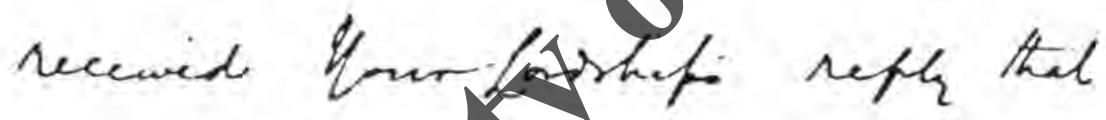
the Burictiof of the Bank were in competition with the Conation and would no doubt Lend metructions to Mr Matins as tom as an arrangement has been arrived at $\mathcal{\jmath}$ 
rro

207

and frimishel." Kavann we thuck withont tis Mejesty fiemisain.

Sohale art fiil to epat full tyourfarshif when this matter in a entrequent Despatcl.

J have requested ife Yravab Hacean ali khain pemver to ine Amini-es. Sullang ony thanks for the informatesy contance in, Ito Highness Oatter and to clate thet haporofened the case of-Diemab Qx. Din to Upur forshiff, fuet that I am ofraid kat Her deyeats Eroument will at zee their way to tating action in the mater 
206

דיד

low which Shad iequenters the saivas to irring to Asi Arighenefo. rotice.

Hir Aighucfs states that the fhe wh Les again asted for an quivor with regard to the confferaito Le had made againg promal celoin, expresere the Lope that the ropolo Corperation may hane over thai acut to sac Devain Eovemment, and Concluder by expreseing the thath annoyance with the new-lyoveror of Shiras for having anected and 
freatial

i弥.

Thrien

fune. 1. 1892

diyford.

Shave the honour thelove a Irandetion of a parate lete. acheacel by the dymin-es. Sullain

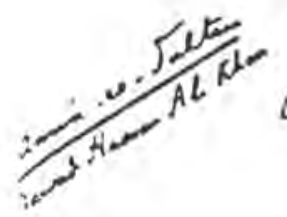
the Nannd Hasean ali Khar.

the fint furtion of the lelte. refersot certain complarinto againat

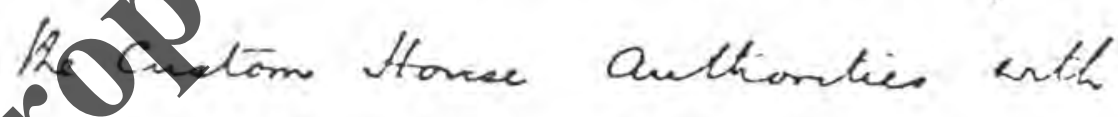
Which Shave not thought it receuery to tronti Vourforshif, bath

The

Marquis of Saliabury tel.

$$
\text { re, }
$$


hempapers and siciles firper $t$ disturbances

$$
\text { is cannes acaps or }
$$

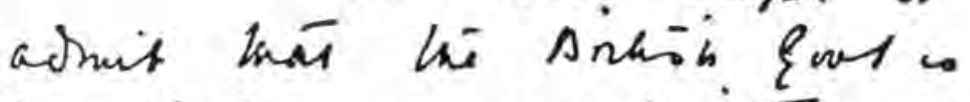
Ons freind os the proiecter of Oar

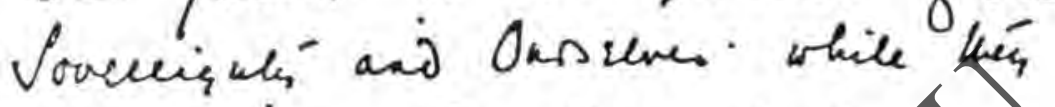
do rolinies and cleos vack po ferson twrite vech rongturese lumes and kin vas. lunotEngland is a free couserp

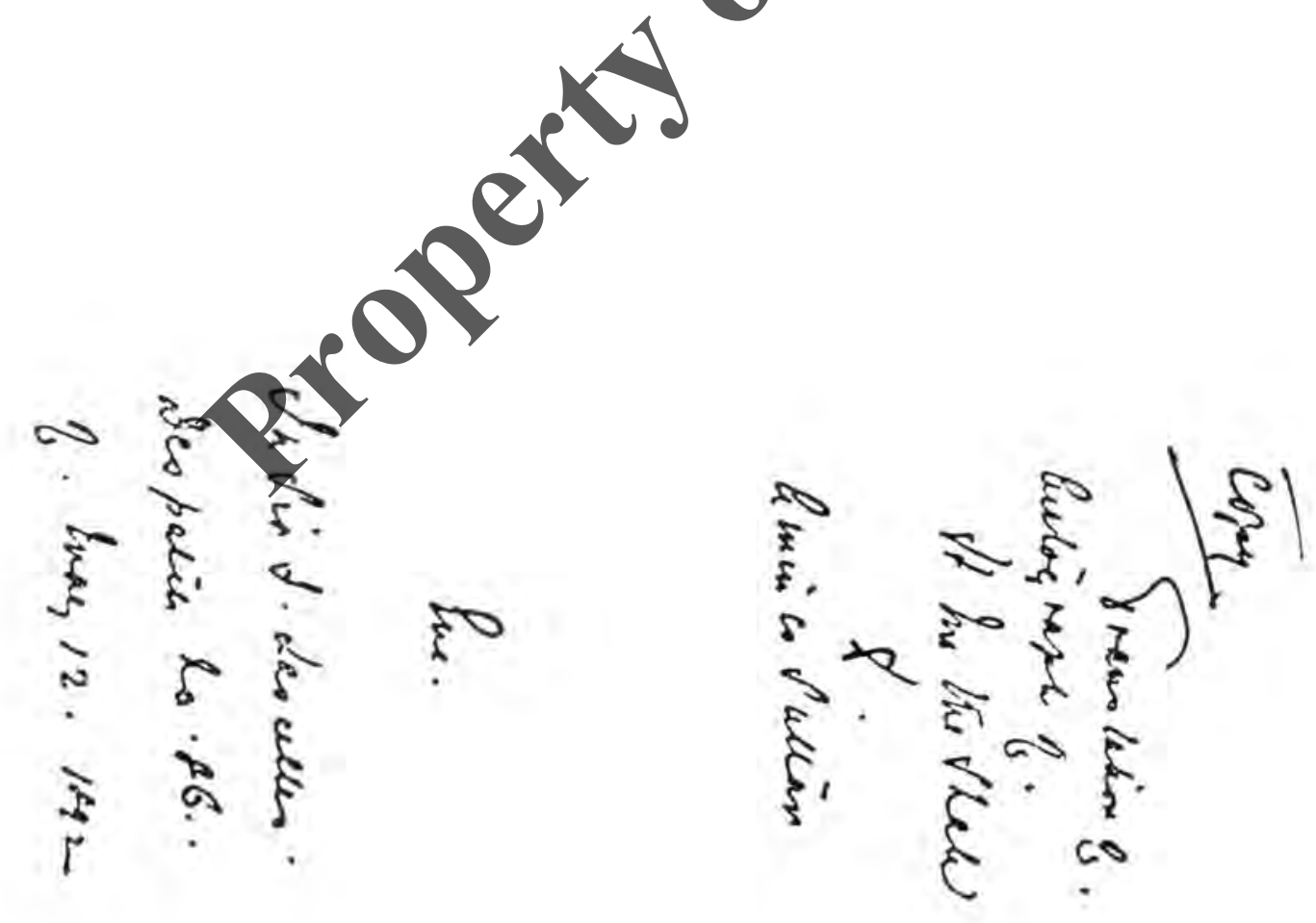


Equss

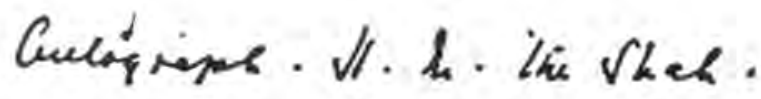

renlation

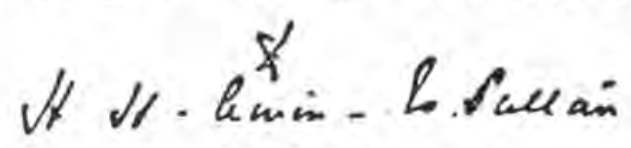

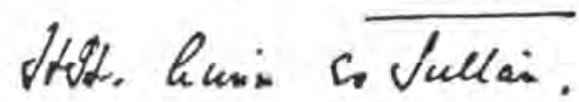

If is $t$ be wondered at thes ins Arbin Ininite has ar quin lis an answes regarotins Shakis flumene Din.

If he hes ofy yox an answed, inform Othersis sine for the elentele and exthim see lim antaras and abl for a . rexos

Ef pumis mens y plin's siched and franticens inan is cisideat. Qh ang law. Nhe kivis in toxton and write: lnea inportivences

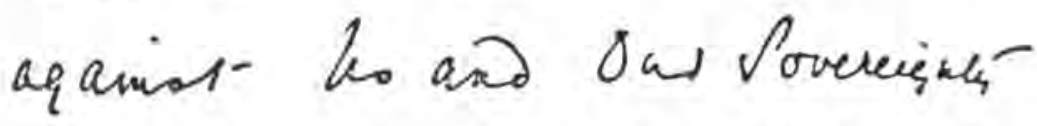
publich, and prabiskes liem \& ale, lis Wored. Jeacos erie: news papen 
202

rr.

Debrau. Tray 12

Si.f. Lavectles 2.:8f.

Des frove 1 luelo Q4. (24.1.8.83).

Sinal.us oin.

Trautograph from

the Snath artanig when tir

Qumin is qunig inget of uply to tivis Eruplates.

copief to tria.

y, 2 een 0

sepprinte an ko

Q

J.0. for ponesal 
ris

201

the uob to Jo so.

The Amii es Suctian cent ine back word that he had explanied to one shat tiali souce delay nual vecissarily elapse befine an ancuver sucd be received.

Yhave the donour bog with the higheat respect huy dord.

Your dorsitip;. nust ofopient, hymbe servaut, 
Jelinace.

h.86.

Ray 12, 1842

my doon.

hith reference to any despatch $x: 83$ of yesterday' date Thave the havour to inclose Tiaulation of a funther better addidpeed iy

$\log ^{\infty}$ the Shal to the Qumin es Suctaly watructing His titigluess to asts for a firy, the temamis whick tis hajesty had wabe witer equand to Shikh ojemal es oin.

the amin soldetan hamig sent we

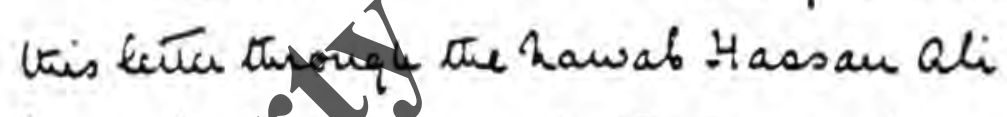
Than, Inf Plicd through the seme chanuct

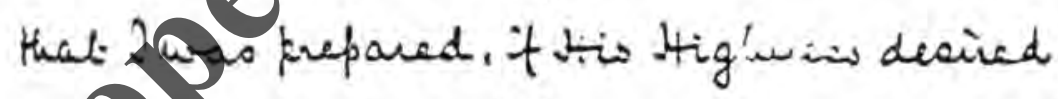

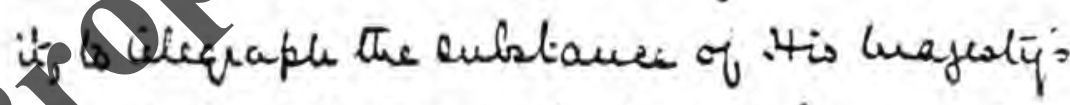
tetter to four Lorskip, but that I was of opsinion that it wented profonce a very sufavonable iupuescion, and that in this luajistip interests it would be bethe fis

2 inquess of Salisbury $r q$.

ure El etri, et 
be ovet the infirioneiv for ble.

Othemis. Ws mad be Ereally disppointe in ino frient his phelead.
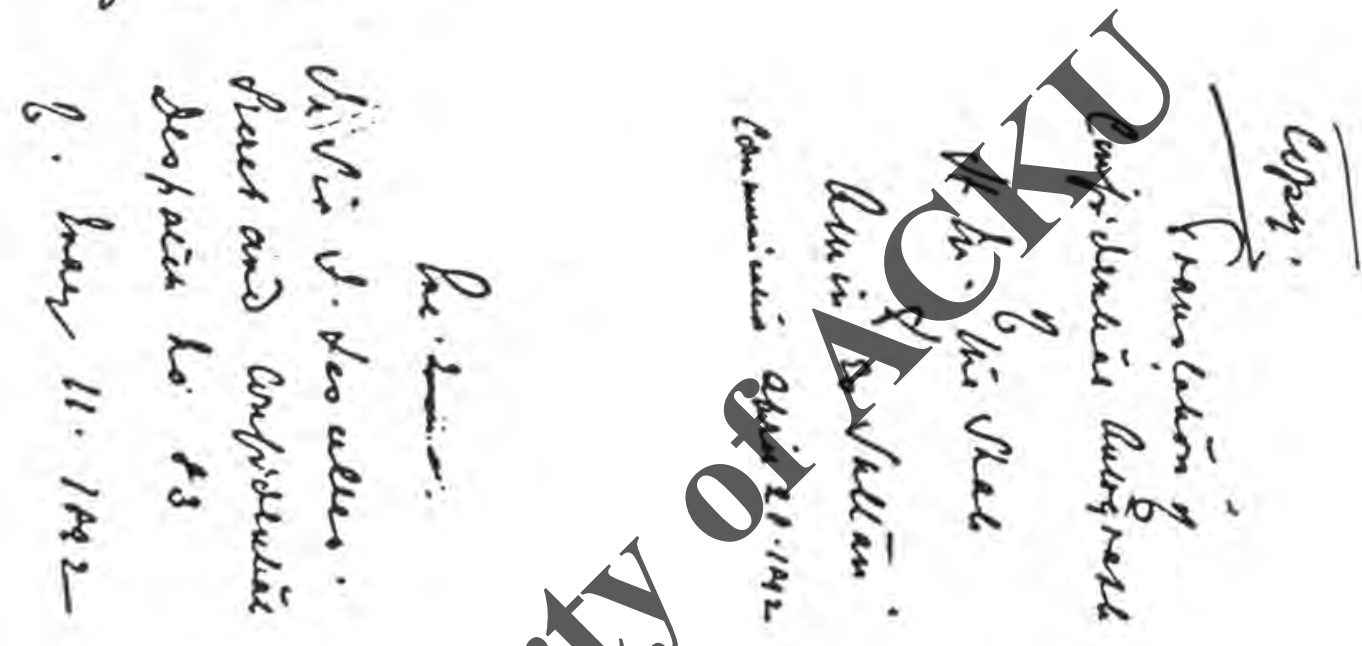
198

rete

Cops.

remicain: Confidenteins.

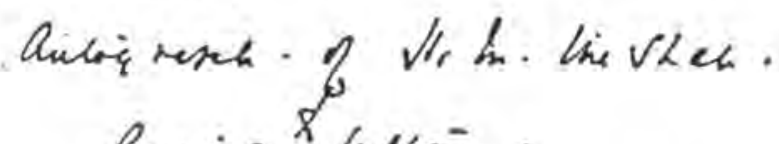

Cimen is "Vwellan.

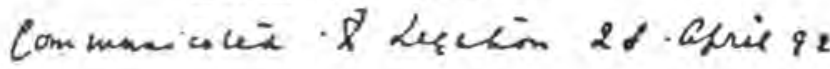
H.J. Cimin so vullain.

Tho brekei uhat plat

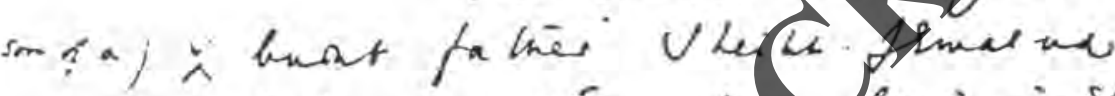

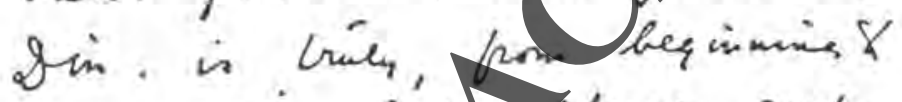
End, wolinies gus frakus and inciturent odirbirbances.

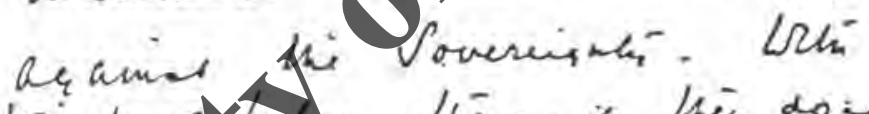

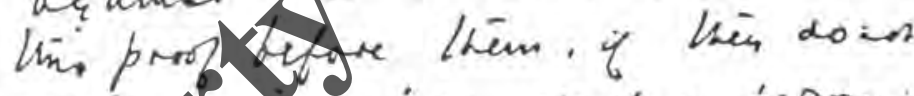
at knat unginion vach a jerson,

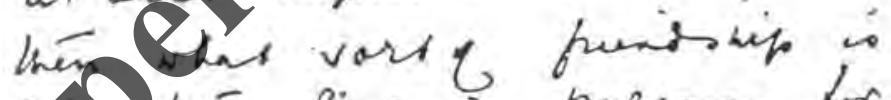
Inás lisecand progeass. for Itow an $\&$ theleni bien frumbly worts you vined kin orz day ade

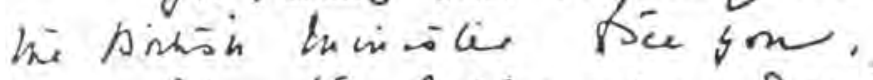

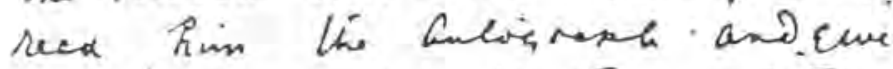

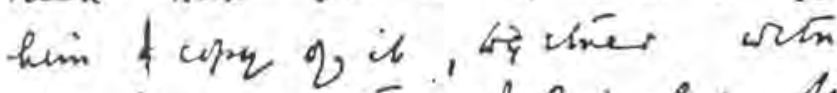
hi Grebi ectue. Meforion him tries, sis his tron before him $\mathcal{L}$.

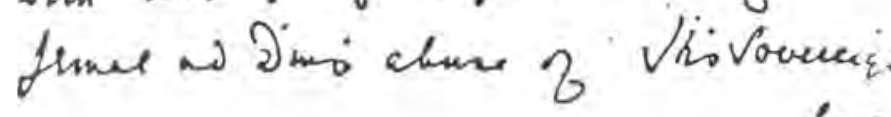
the 
rTo

197

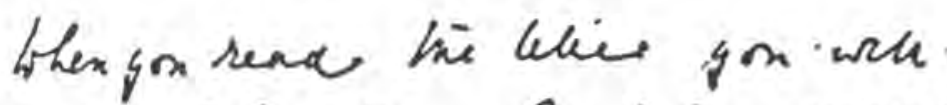
underseand is Any barz woreec reshat it mick. Inole a poverazs. Udoit knos whes. Lis heminitir

thinks atront this mater I, know only thas vece eleien are fue censer 8 mach diskrobanes. Ghe is hor ditcute aqamist fue pessonelly. She whole wateme.

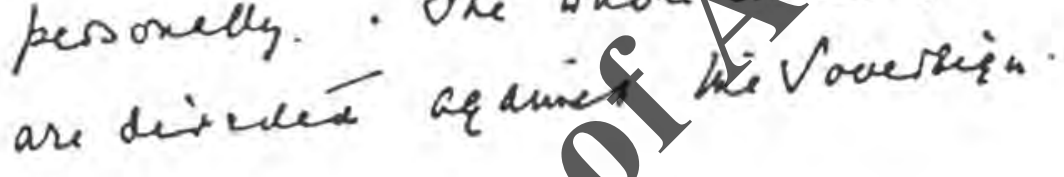

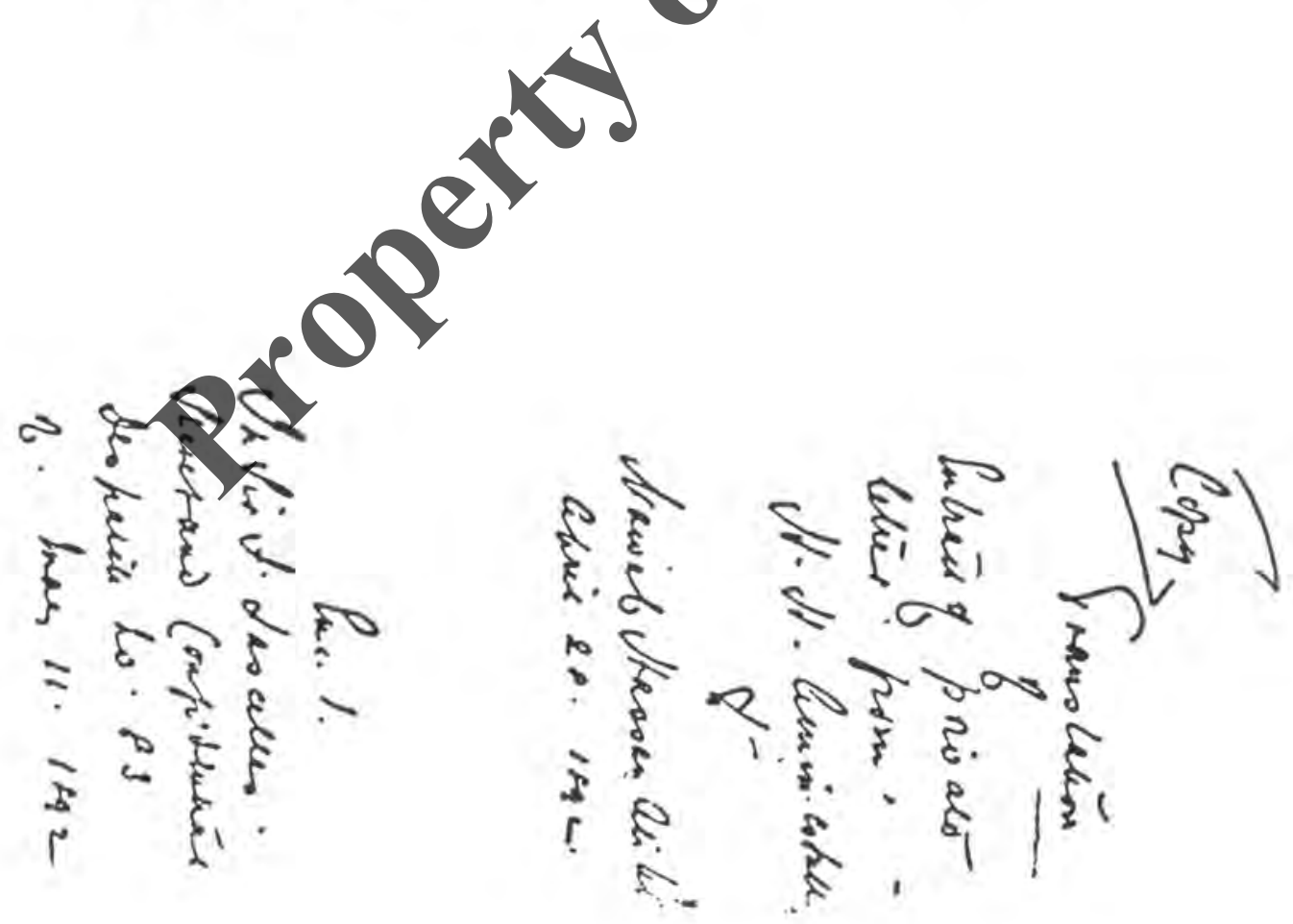


Copy. Pulses of violate cosier.

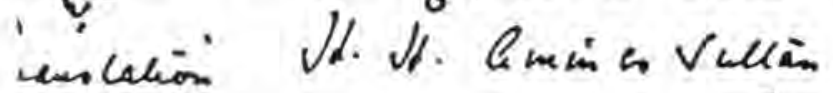

ه

Havel. Meson Gi than.

varied. 28.4 s.

Fharsdas has come and is is pas es noon and no news. Odis thy what

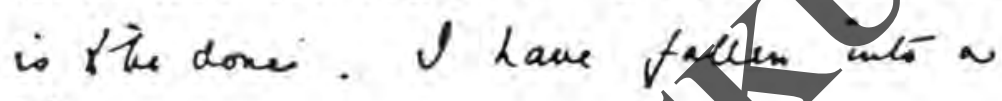
diftivery

Ni s treicali hep water time

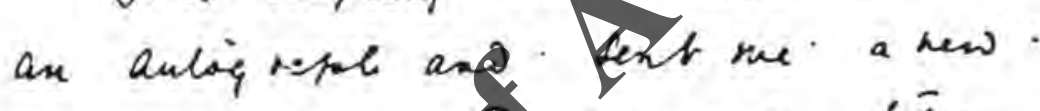

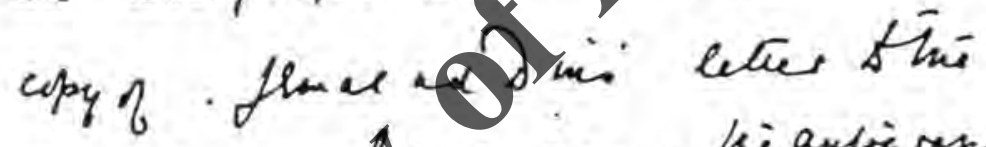

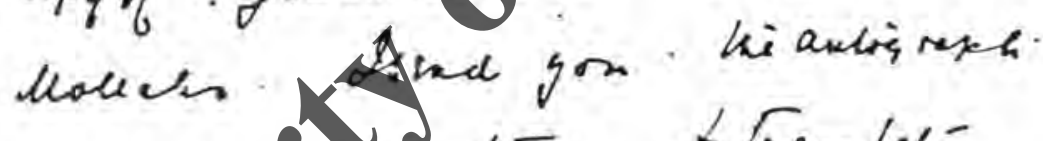
sol ween the liter trawler for the envisice. There is or. if he sister in voting the lever and a coper 2 this aubirape thomson.

Sis heisole is r ez much ar ed and ingres abodes. His mater and he has reason the then 
Selvan. (') Mayll.

Si F Lascellio

83. Ifrestoncd metee

$$
\text { lo. } 83
$$

Senet an confintertial

a camonentication is the

Su? trae'

(Ret. precoving ons?!

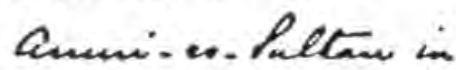

ginal.ei. Sin.

Trjunthen autuagapio fim suath, coupplarining about.

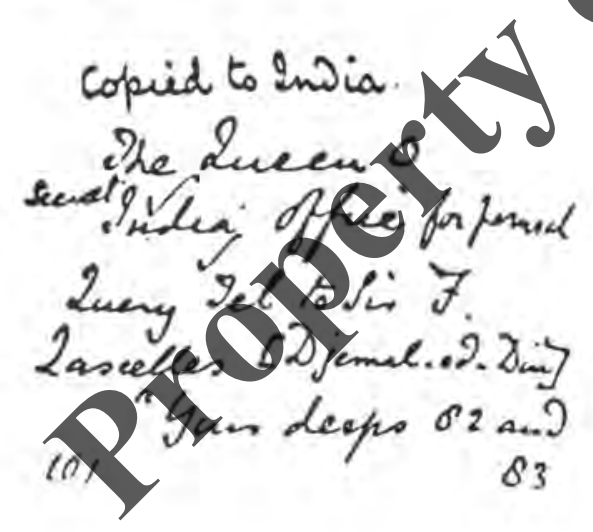


194

tris

Selnaw.

20.83

hay II. 1892 .

حسن tal

Linfidential. Any Lord.

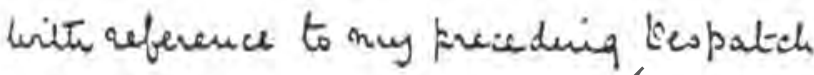
Sothis days date, Thave the houour to

6 enclose a trauslation of an ixyitit from a

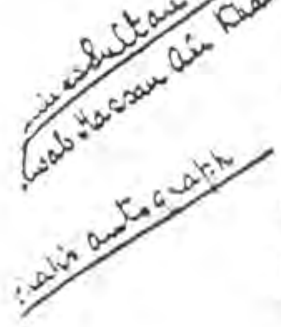
hote addreased if the Gurif coldtear to the

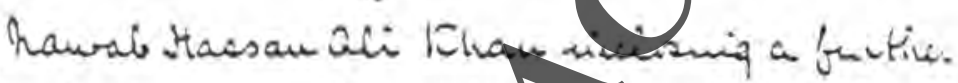
autograph furm the siale of Ete inizict of Bimal a vinis betre

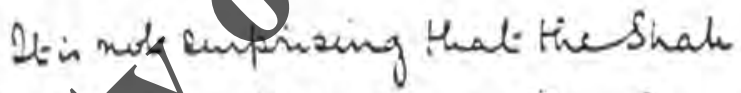
showid diegfy degut the aimsations inought aganist toy oginal es $\sin$ and fear the itfectopich they may provence in the ponthrimase especiaily as, aithough there Day be orut exaggenation of Cinfuage, there can be kiltê doubt of thin buistiantiai $\sqrt{2 u} \pi$.

Shave the horower to be with the higheat cespect:

huy dord.

Your Lerishifis

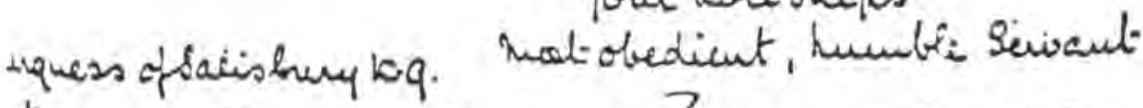
elis et 7 tax $<x<10$ 
ris

193

Coper

Srauslacion.

Sheike fince ad sn' $f$.

Wermes

Pue 2.

Ul Vir g-Lesculu.

Inspolits ho 82

of luee 11.1492 
192 TQ.

Sho sucks hi bluad and the brees $q$. bostems.

Sosue troclamateon for his. removes. Vhased bis counly be ounsiio by forcisines, 1 wir remain no restectifor Slam.

lasoure you tuat all the Eniniten, ling Oncin. he Lond, liexargue and ine

perpe cost infretine you I revogr hin shech and geterese hi vaves o Ea. from the wicheites pain man, cre le lesmei Inewo torn 8 shame - 


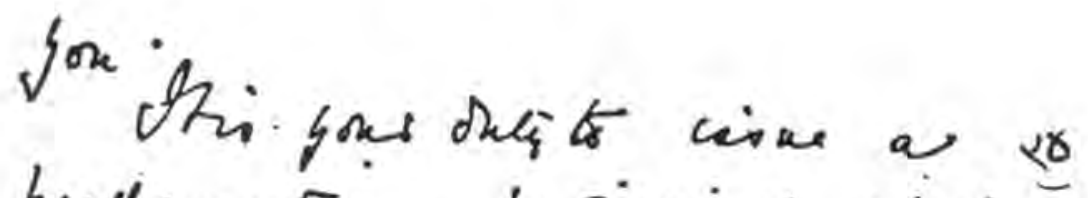
prodametion, prosiciencis alleciance It lis Wheh - when be is acome and wrectiver then ile ki. followes flee arot ine arony win fire walles at linj and lis cheiten wiev vlonet em.'

0. I? Llewars Cyreah. dose kni otherpy wis lue rot o reinleos and heck bis an whe line smord This thot daw . She waice for an propunitu it retseraic on

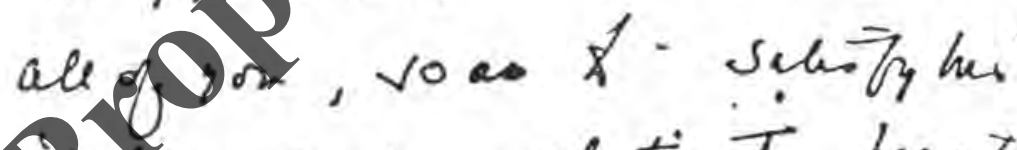
Potom and abotinate heart:

Do hot que such a man a momens' crear, his hourter Ypoverenintis - Sore dails wesk it hovest but orie and oppresinem 15 who 
hio pinesoses:

Loreyinen sodered as mot

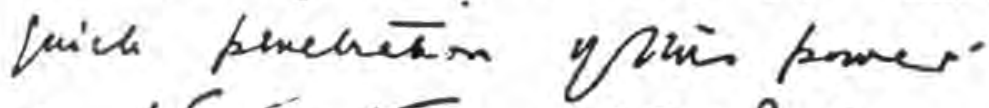
in lnis inalecr Vine vieqce, hut athees has go ho ho graveré his power tizin for his probetion $y$ sscary Cherefore - it is man refs hais yos stoones hesciar

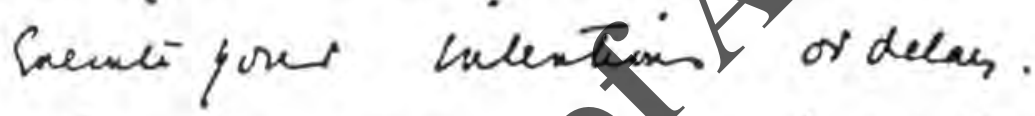
the rime hes conse. for lue

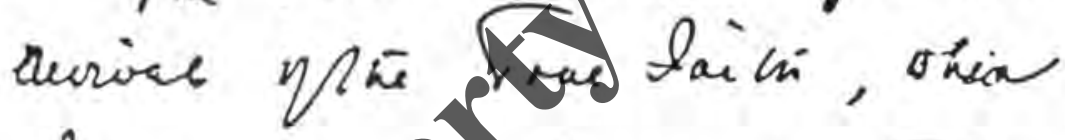
Enstems be ciensited ax reopedst on line fau g pur. caras.

- vernore lini oppresesor, Mis slak, from his Mrone, Qre he arrenti and deqrebs

is you 
armien or the Hash powaed. alone - lo - in has. The belief in this trae taikis: frimly unberesed in bikears-

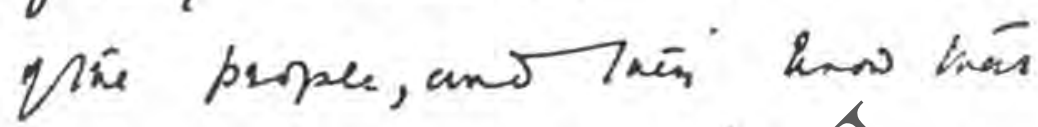

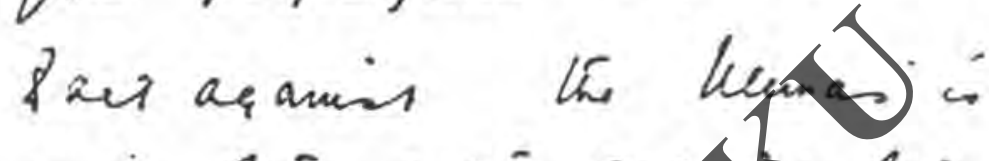

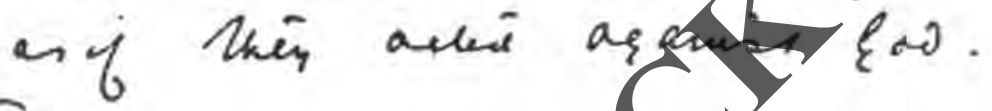
Fherefsee, when 被to are tié

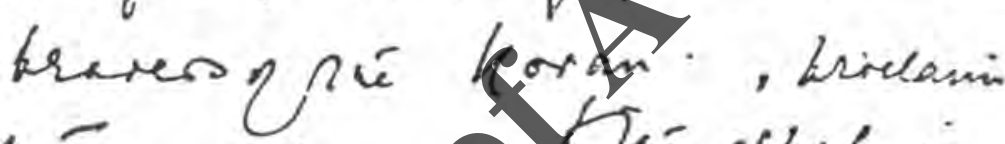
has aleigianen 0 thi shata is

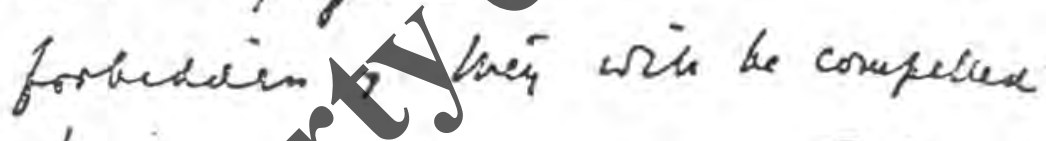

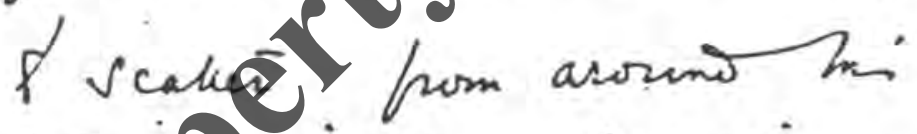
laroglcimone ficheri, nor anes one remain on the Side - hay. God tha a humber of sainti beenare doubs and hes lateon from tho muns

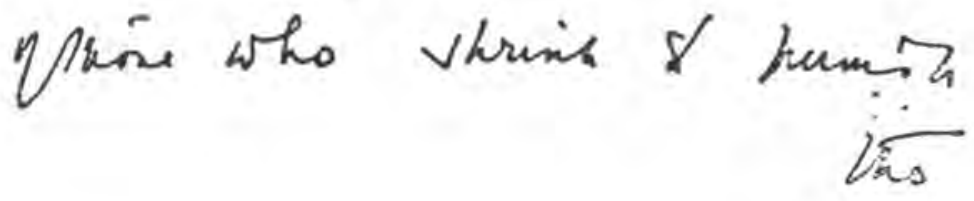


188

wis be inforsice for he

rAP

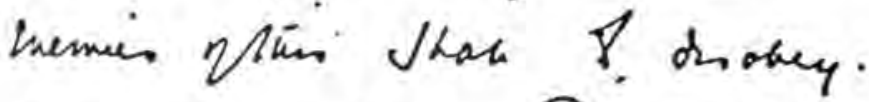
gou commans. Shey mess bow at your thrinkoed, shic $\therefore$ hair jiní Whole Law 2 . hahomes.

Yi can the kow lingatby. hi lower $q$ goo, \&2 ca renome the rebeluing form has lisone of oppeniog

then the poleceare in birinear Vini comforito perees, ander

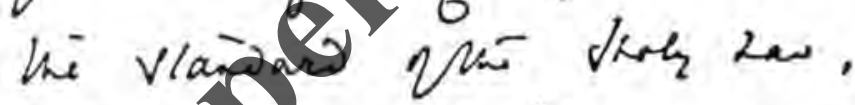
hin wer wai folew zon, Q. I vere his thi kang? $\xi o$, and vapposcer yor saine:

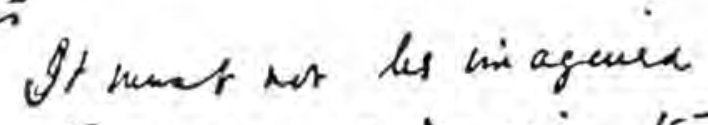

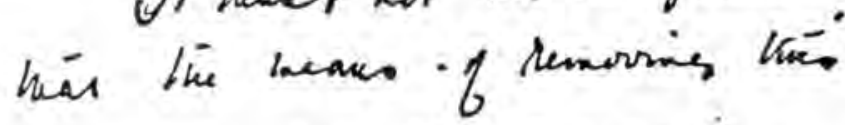

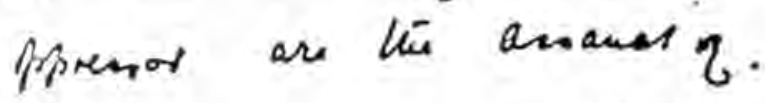

asmes. 
than', as y2 have isfercenced is lati, when rich and poor, oes and youme, were oberines bo Thio itis necters $\&$ prome.

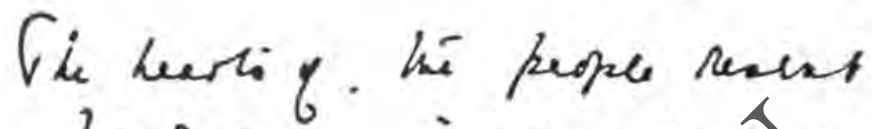
hii, haro and iquerant Sofereig, forth hes reithe vhengthenco bie

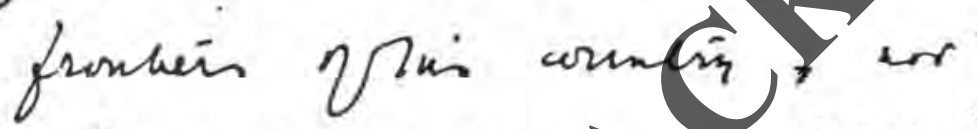
sictibithes an arme hor repaires

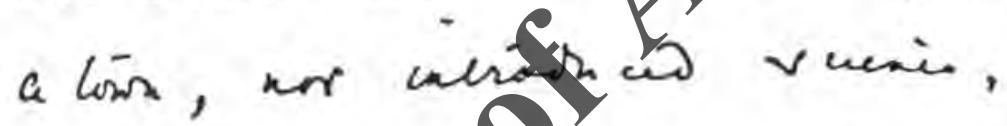
art restecenty the preapto Slam. ber ded he, even for a

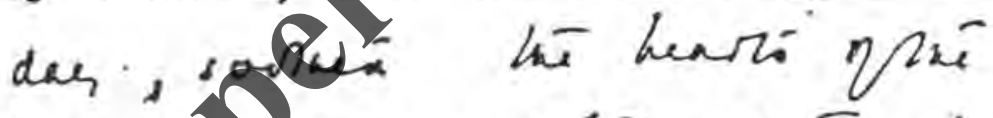
pepar, late, ox thi concrats, the 20. coved, imporesintes and stoked kien from he veght. pasta and turise hem 7 nesequer.

Hodoust is there leas is wis 
of ys beve his rebevionstherare.

themiry, ans est-hein remain on his thisoce of mestrees, and do Lot hester tremove hein from.

his reque livanos, hi misctiog

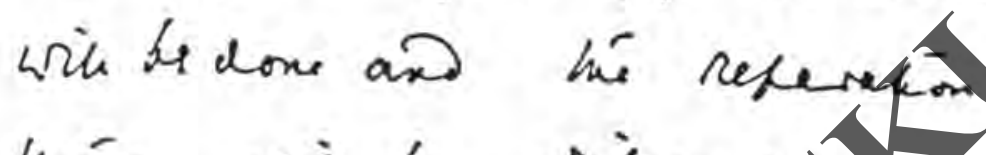
hering wite he rifowet.

$y_{5}$ are ale lim copsusion?

godor saris: Gouns vore are. birluint and purfiom ale ire

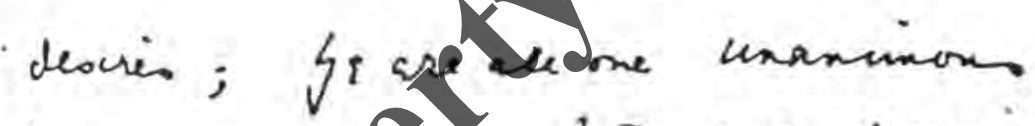

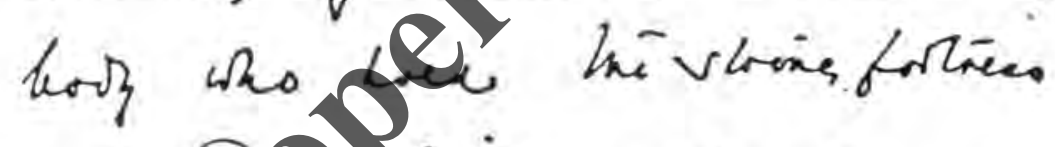
Yhis fou Yait and repuere.

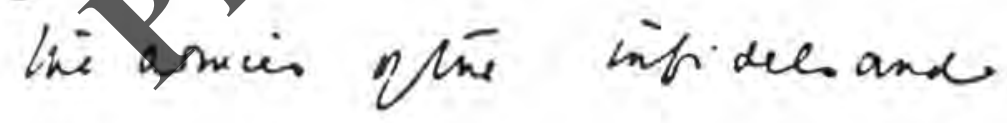
lini unbeberien; berzou -

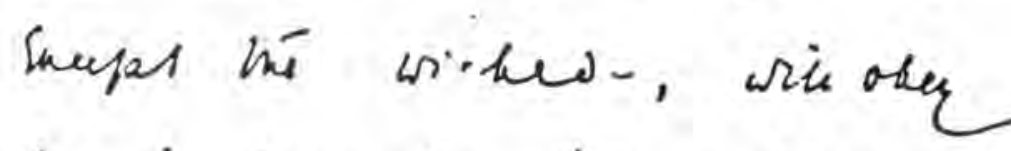
you in reunosing his rebeleutis.

maxi 
roY

185

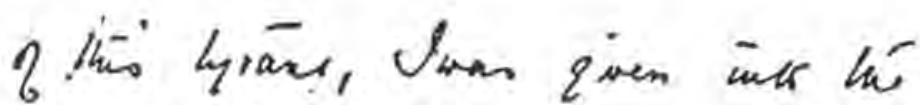
charee a koop of (oroseces who beat we with whips and

caresent for a feyged cike mesty.

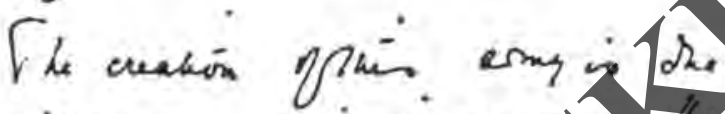

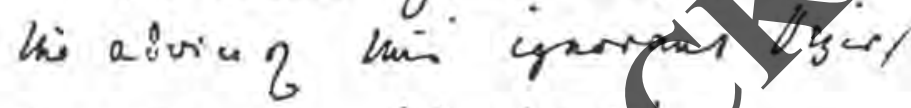
and some?. hi paratho.

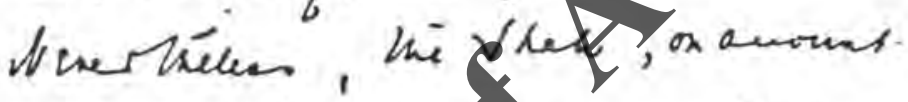

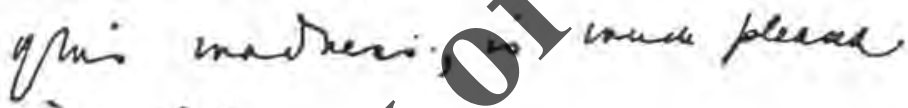
sitn Lim.

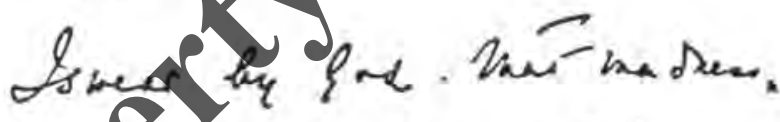
wicheiras, lumees and ciñiame

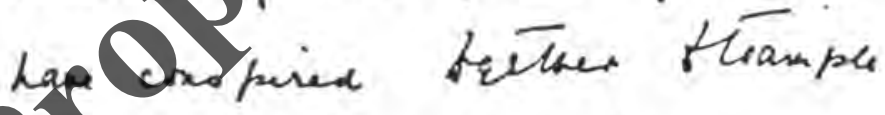
Lin Shavias under foos and Phelines is emaen olstam vinn's ficke, \& the forceigres.

0 ys leates of rekision

9

भf. 
184

Thé peoperare is yourtand.

ros

lhis minchiog hes lon be come Gleat, ss certan devis, govenment.

officiace; conform to and vuppost.

the vhats inlention againas laie

Wlemes; as regard their eapation

from the courers, and

impreare huin. with int ges

liás his power dependeron lne

obestion qrai dost vaí

arme. Dutrof tié lates wis

in conount the hoske axd vin

against themas, theis have

airing she th forme grescely

Qxernes 2 . Foreigurs, his ardes

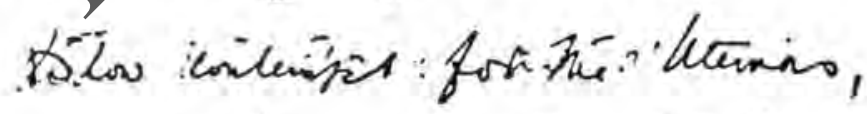

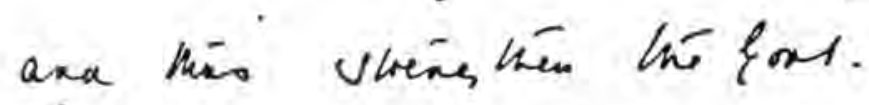

Jinessy have Ledentersures ofini loor, when, by tis ondes:

?. 
roq

183

beenase bie bebine has tho infleves ifnis Kemar, who-were his proseton if Jecam, is infaried,

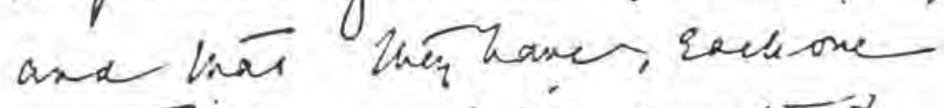

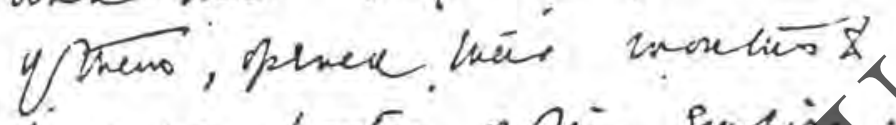
devons a protion iphis sunsid.

0 . Is Lleman: yicara

hoof wo have virene, Eneges Islane wir jout An nuention, Gsulane Eloripios ward of Islam and pare pies the heriso q formignes wis prat and gure.

Fonare welle aware loat yoldeverein canas Levir liem vinele handed, What $y_{n}$ are lue hor g. Wo Leve a porer lnat hexsus unvabrue, luat gon are vio definders Vai fails an Stria, arolnas lis remi

2 
182

and his bers your poor and his ophans.

then tho medreas of tius Whek was as ies heigit, to

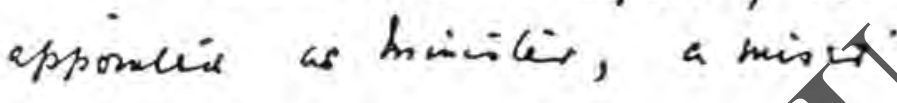
and a man 8 cors kirst. the

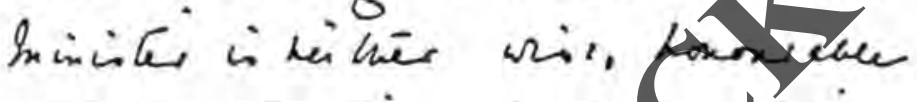
hor conocientim in preatives the vovereic prome far core acti. Thin foorst max

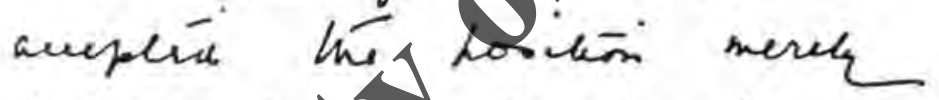
ppres forporo descichion $q$

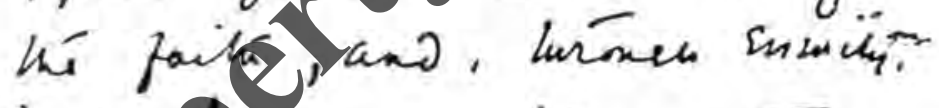

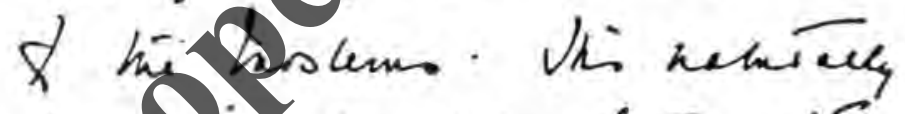
trotheleer les him tora he

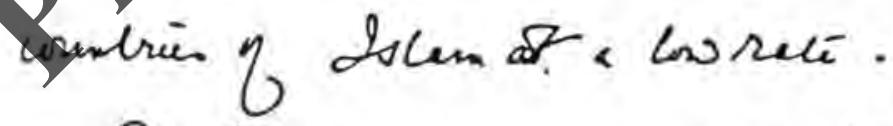

The furporeses row. lliming has thi lin for ouspueses frreen, shont a shaire, is as haxd, 6 benenge 
ril

181

were the pensioners glnelanes that line shele hes, vinis bes. aceosion the terome, fressored his intertion of arnulemes, by

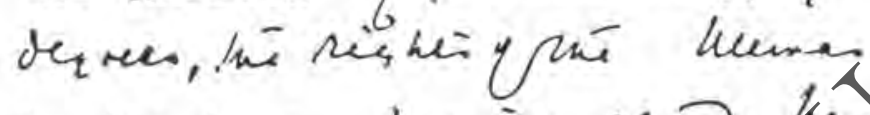
as wach as posocines, and keo

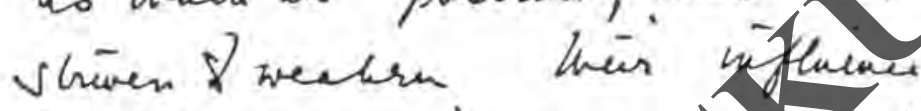
for his ow purposet. porder Hábina lis circle op reasin, bherefore he has afutece de he heemes from his copantri and has forbeiven hē Pmain leim lié lars Y pin pteyine, and hes

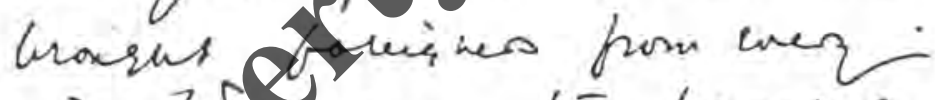
ocie zofla ar, line hose ? opprest an lyranay, and Q⿱ $)$ suborad the pesper, and

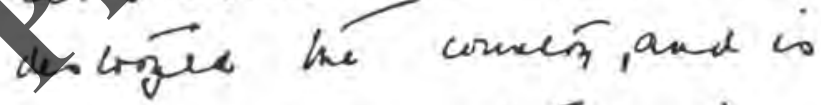
engaged in unoortuer action, presines his low descres os whes le hes solorkex from bis beos 5

an 
180

leyt to hinoxs for in gout or in Lation, ent has selavien, for

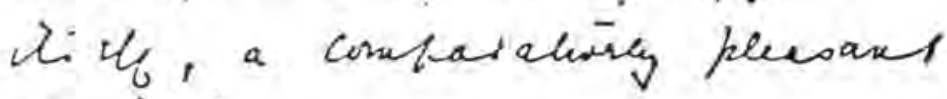
prition.

Hence, overgwere where hí

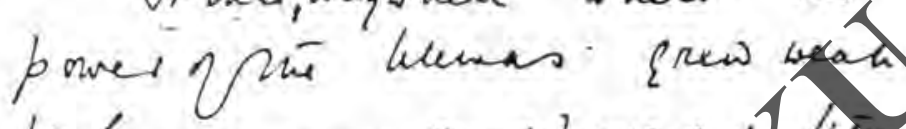

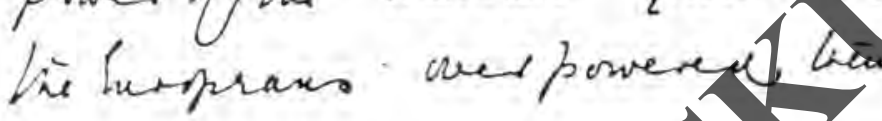
Wogut was best quif yomes axolié cincum oblinion.

Ch himig Plucis and he viutery orato Jxance Whoie \& oceran lat pover? hi luenos of aber fartis - ano

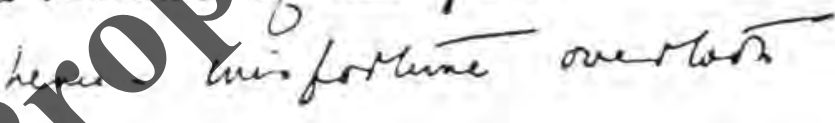

The Chenans proteder

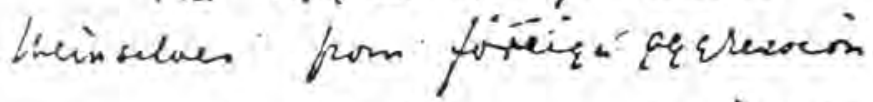
and is theminces of bugliok. spreacion siurnise hi power

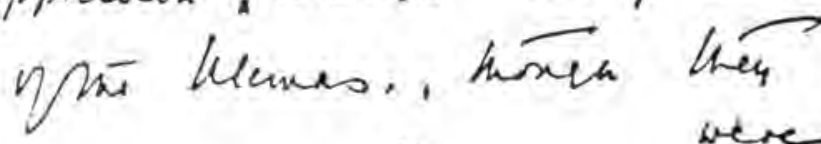

4 were 
rit

179

are in pern 7 corruption.

Under these circamslanes, ine

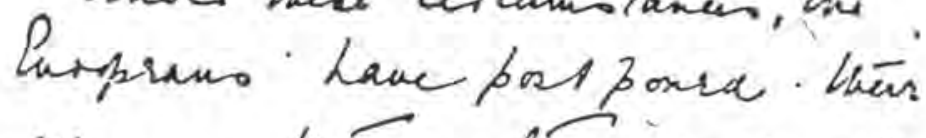
schence, kouter they are deves. and asle \& vertuñ evezk soliberilea milito hion.

Aswear by \&oo have formed a tius seifion pom whes hen hateen axe inferma. fork th Ereas hemei vereforsin insovis, this peopleprongs enlandy axd

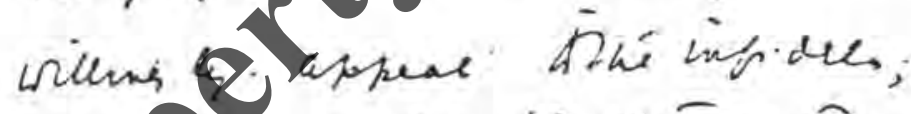
and Poly round hi vLationo oteophemes, is arder the HeknoD from lin lase bat, Which hes oblicirated and. destricto the powe and jualie Yone peple, axd he ifs 
178

by your smin lais

The Eumpran are parezone homse $X$ ocups $v$ isci siant

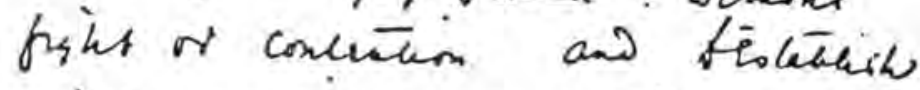

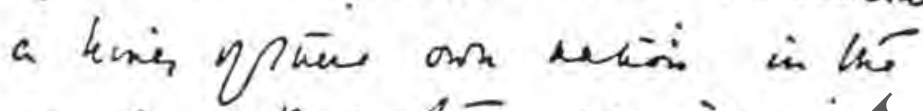
cosucion. Dut thes consider is

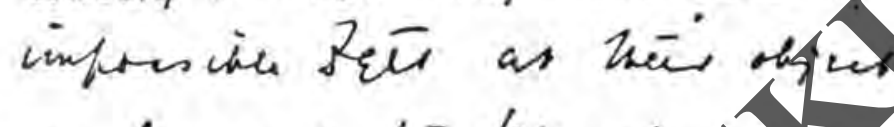
as love an hi kemen ar bor

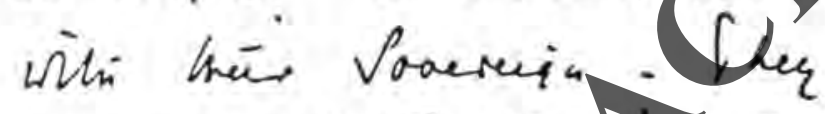
are aware Inas porting hi perpe are mings thars the

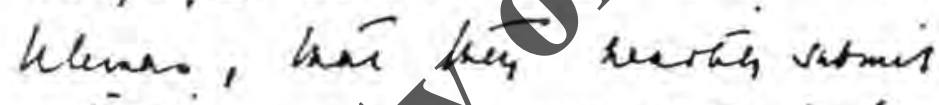

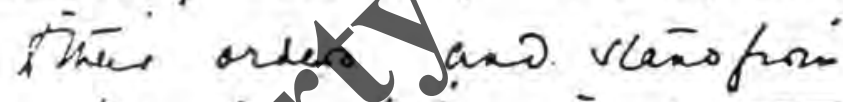

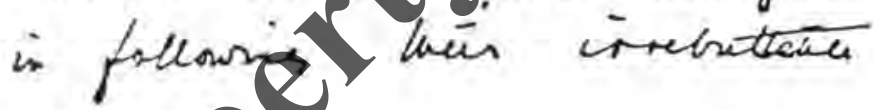
comenes.

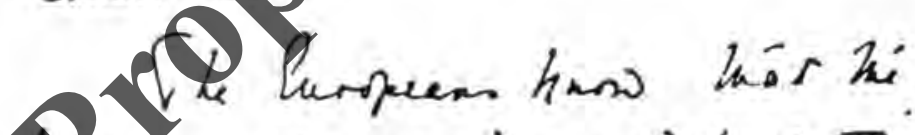
Qheras are ascithons in protectives and mainceriming hi cidcle? Istan; and rever realect if ginsteres in theis delis and are 
r7o

177

Letier adoresose this (hie hailateis at Jamera and thi prisaper

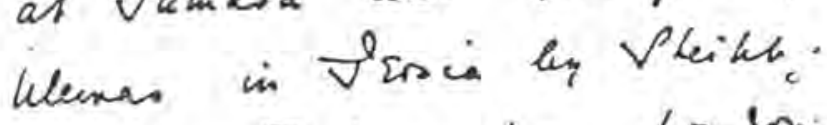
froverad Din from Lartor.

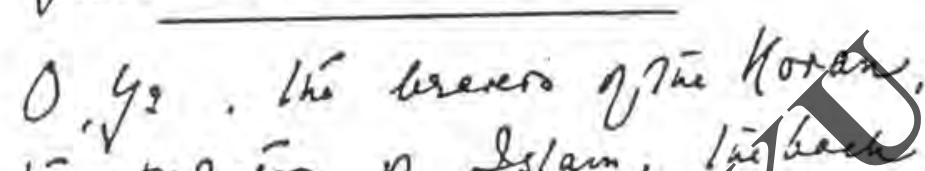

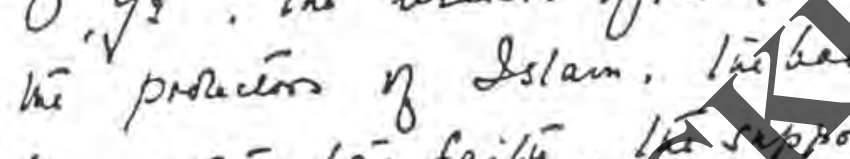

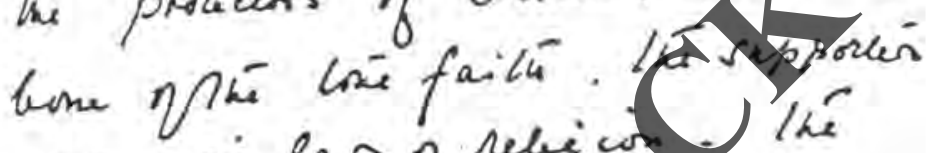
Thi volis laws of releich the armes goo whe prangench he thi Wores. thissorno g god:

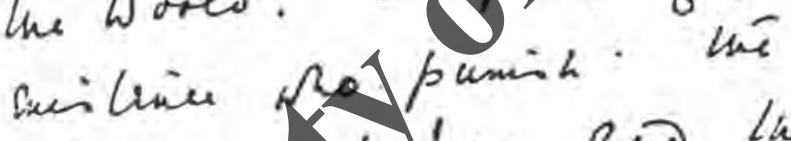
Laingreases lines goo laroin yon Istam and Thé

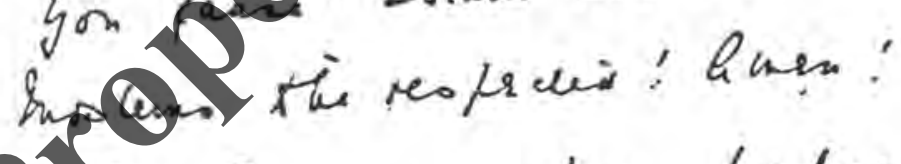

Cho Europarams Lave for lones brieo, with Ancessive avareic and qrew, dominate 8 sois, bes kneis ofjut has been delaced by. 
176

ry

$\overline{\cos 4}$

franclation ?.

Gutrequph. "?.

It h. Jie Shat

of

Cumin so vactain

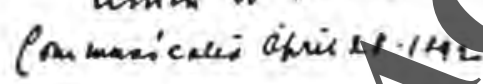

R. 1

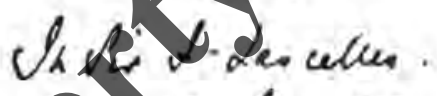

Destatic lo 82 .

\%. has "I. IHL 
rาy

175

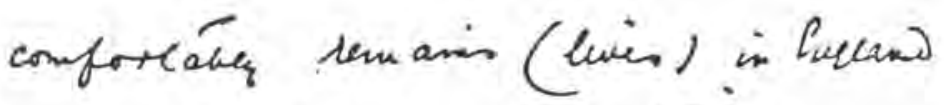
and pubaibles skek lemes vily. 
174

ro

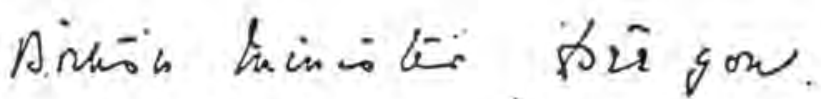
and lete him pincialle on ox betef. and Eive him hin autograph thend, aloo cui him a coper of is also. have Sheike flmel we Dins arobs leter Lrañcatés laves. the ale there \& tondro t' the

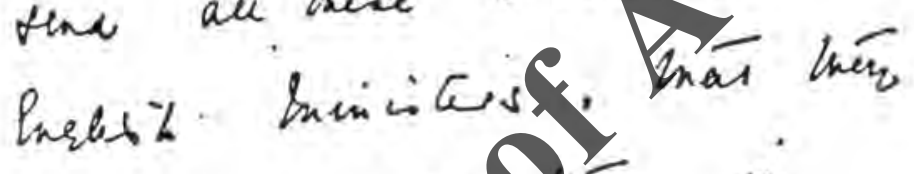
vloned raen gae them in Iarliamente we are vight

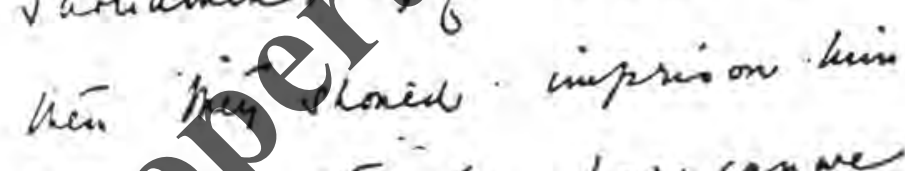

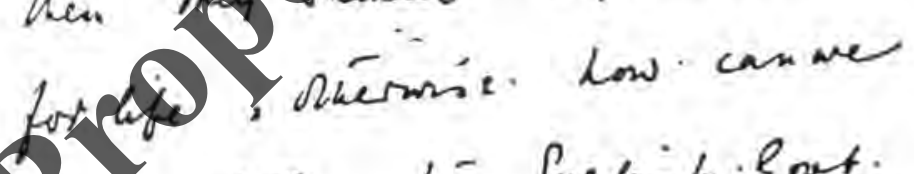

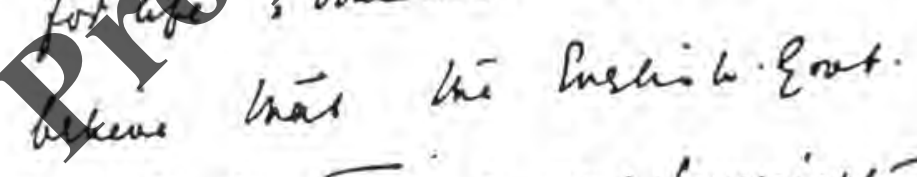

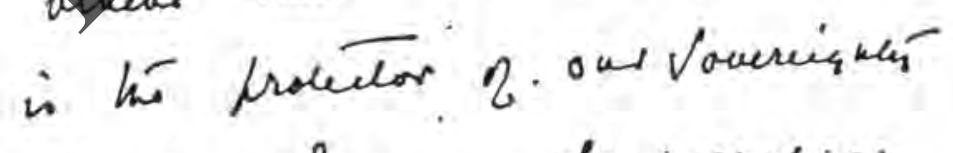
and Out versor then sackia wiche axd le disposere van tous orom so preele, ano. comforcents 
179

173

is. Ucuceose a ceppy for cone persuale. Fhis lime he diskanters instiakis and arees the bolles. ance the pepal it creati clear.

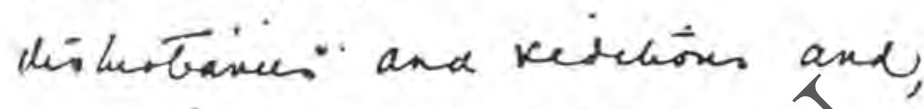
in 'reacien', he has mader fon aliach on Out Visont. The has vhows hemis cy

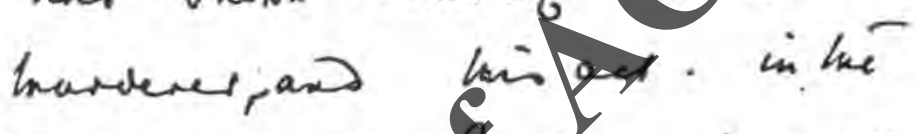

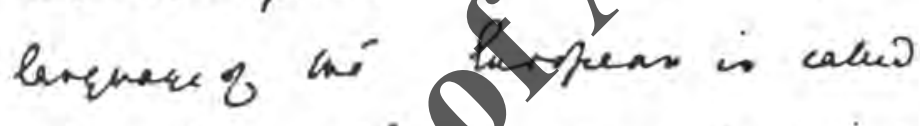

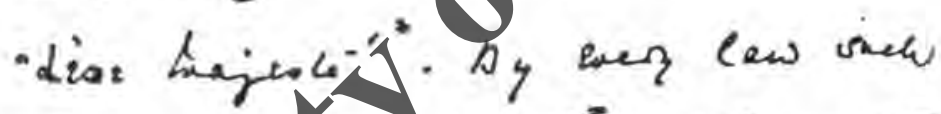

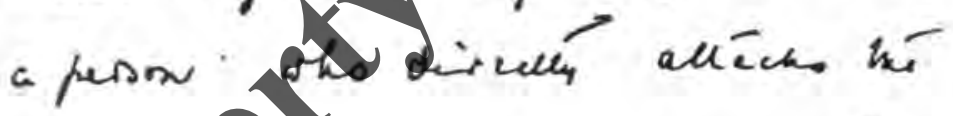

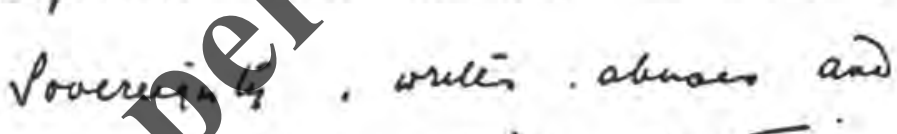

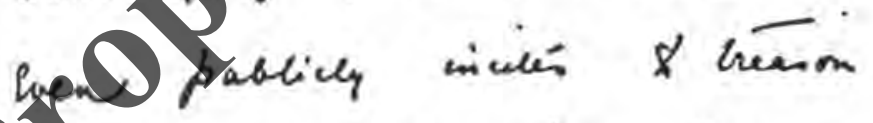
aganias lis voveruiz. destraes deatr. at lend: he is inafrioned for life in renarer plever.

Gow vioued. Los arber the Asein 
172

ry.

aquinst the versian Eoveramert ancer vorereianty 8 versia, but. here was no reoved.

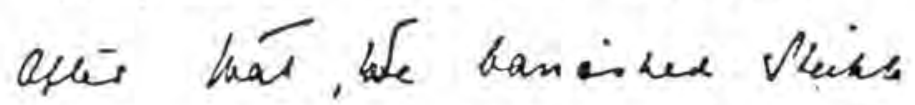
ferae no Jin, who is ne 2 var sicked man optien

put his hand tereas af funfthans in torocis. Whane ferts London, jomed tacm than ans bever put king retrais

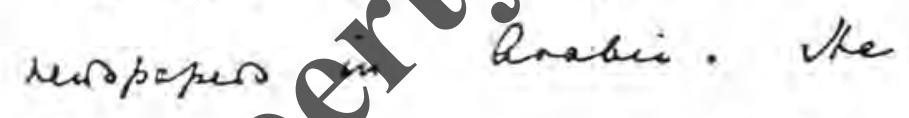
his severac numbers colat promaleated hem in

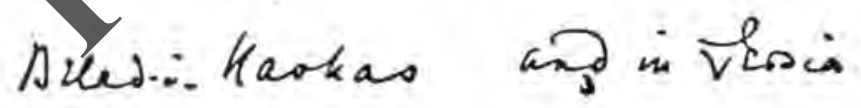

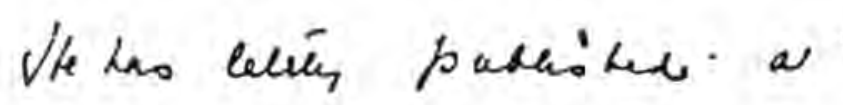
number in Araki and disferas 
TYI

171

wry....

tansalión

Qucipians H. is. The shas.

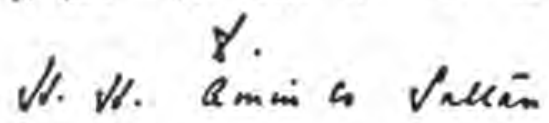

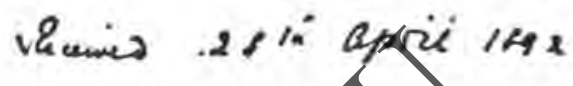

ItTh. Bunin a Pallis.

Ws latud repercist

wituen and complainger, born

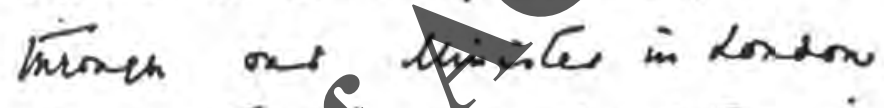
and the Eario luinite in furant veractine he cose chalserds and ari wretiacs Wthem than. in Laton

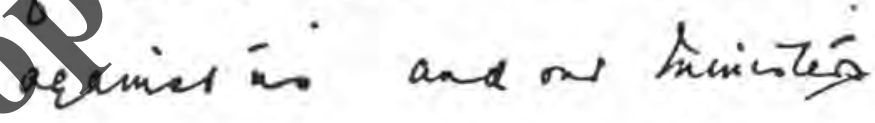
and haver. requested hat.he

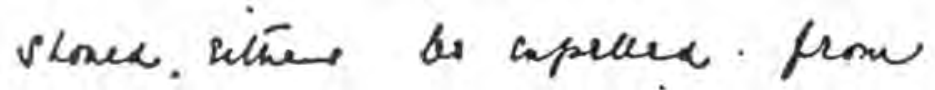
Losbor ance the Enclest Subiri, or he vhoued be forbidem 8 icerry on these sedition abunts. atainal 
170

rYY

अभव

Ecleran. hay "I.

S. F. dascelles $x ! 82$.

Beng fruel 2 Ynelo

Sy.

(Rit. 2.14 of Jan. Ig)

Beival is oin

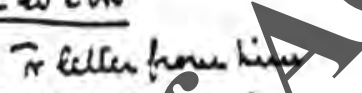

to Pescain hollabsend

autograpis from

comptaining of

Goped to inia.

le guean 0

aurs intrioffici

senual S S

100 
rur

169

I would not fail to kiansmit these papers ti Your Lussidip, but Pacuminded ttis Highuess at the same inve that it was imposaible for Sevinaigitip Govermment to proceed agginit

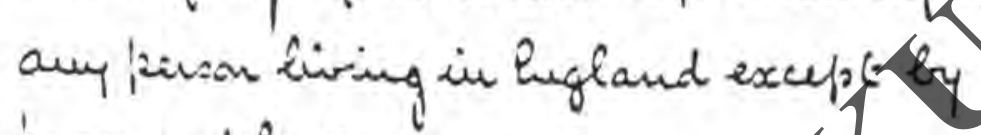
fiosiss of law.

Shave the honour to b urit the highiat reopect.

ing g

yougdonshipi

orobedient,

$\sum$ tumble serwant,<smiles>C#CC#CCCCC(C)(C)C</smiles> 
168

TYF

Seluan,

$x \div 82$.

Mor 11. 1892.

hug doud.

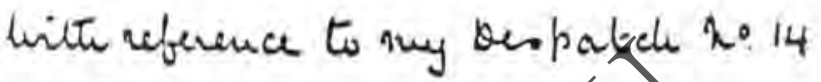
of January 19, Thave the havorp E. Silose .

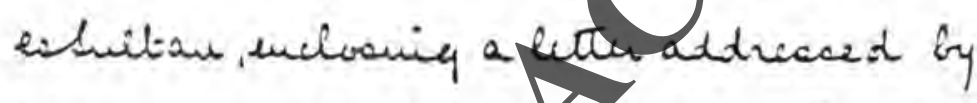
bjemal.ed. Sin to the Afollaks of Persia, in which the dieit getacles the kisson of

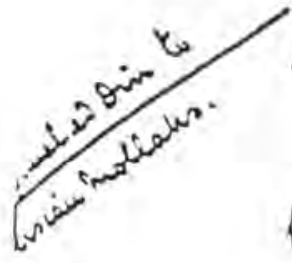
Itis hragisty

Inafe also the hovour to inclese a

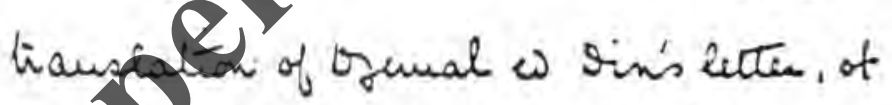
why hach couplaim and which, it Hxs lnajestep opmion anders the wiiter Desewning of death or at least simcrisonment for rife.

Sheve bed the Qunivel Sultan that

4 ! 9 narquess of salisbury k. 9 .

eli eti elis 
monachy, says the acteren, andy thes sineresirn who activally makes an engerpent $\therefore$ buende by 1 . Henes if the shach were dothinged thes

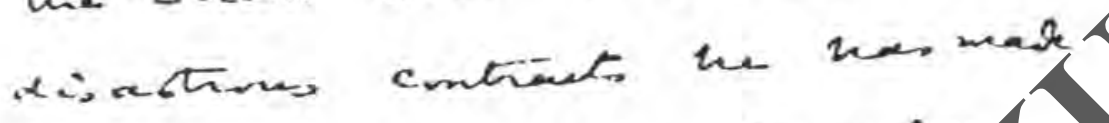

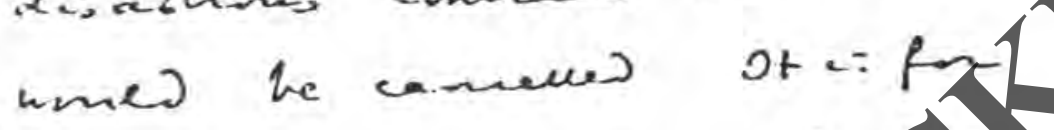
lue monah to vies. infor in dethimement. Of tufforo int rale the le.cetroy

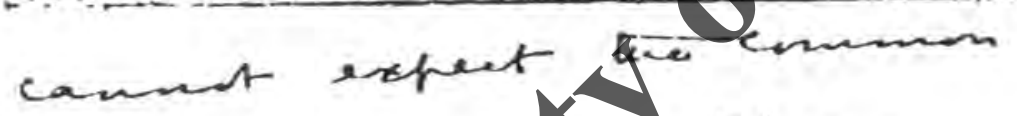
people to evengthing.

The profonation, wich $\therefore$ ostenpsidy sinftinal $1=$ Theraerror, end, with twe und 15 ive pereen "owa i lies meforge"-

It irened sead et theini

aquil. 2). (Bq, C.w. E. Elid 
166

ryT

weakinegs of Rugland means the stiengte of Rugpias, and tor eater will sciges Khoraforin, when England wiles nat dave to ofpose hen being ofraid of be deaigns on sngia.

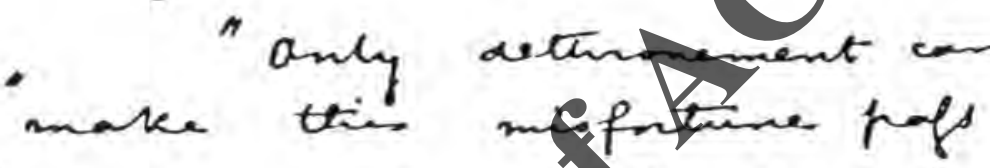
"amay : only gethromenent. can make effic sim pags "away - plyed und "dethremement $\therefore$ the plitura of tar ust of tas peclamation wich itenges mase ot of such

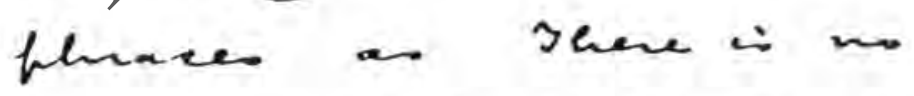
"remedy but detionemement" "The watch-unde is dithonement eta ect. In un alsmite menarthy 
ryY

165

and enfidity of tha shah ame cilis verses from ther-Corain in faurm of the repusition of unjust monarah. Shen it the subject of thporbaces Company. The joh has not arly, made frues tar interes of his ginting to infides. bint now to hay as the hoet a heavy fine which is young out of this pesple. the fecet that the Englishs have feen defeated is no ackentage, thecanere thes weatnefs 
164

rYA

The hand uniting at the to po is -.

The witref of Islam and "the pillars of the Lour Mirza "Ismail $a_{y}$. my the yod "High make him an to to "religion + the velfrift.

virgo complice is the Mnjtehide of tabriz to whom the drunkest was sent.

The furdemation : headed:-

"zeN Ifprefoirn of religion and the humiliation of - faith.

After sire religions formulae it begins by denouncing the rapacity $6-135 \%$ 


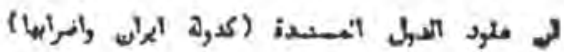

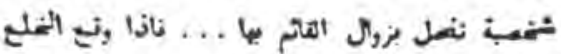

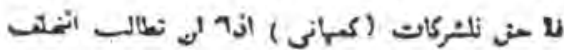

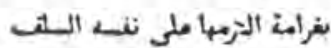

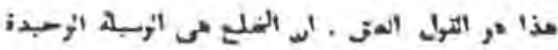

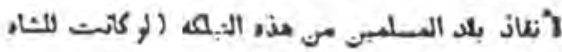

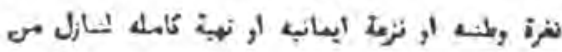

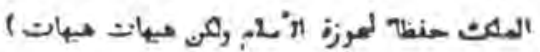

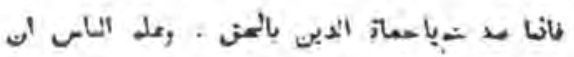

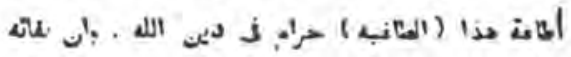

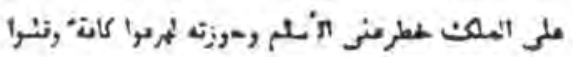
مرن في بحلموا عس كريى جنونك

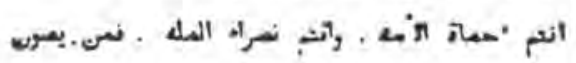

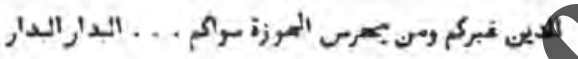
كبل كلاب الذال والمفار.

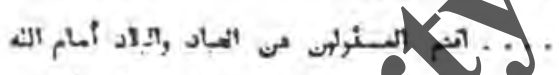

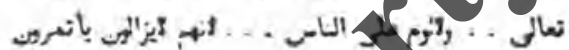

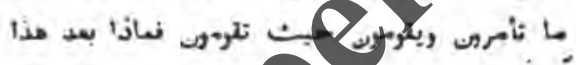
تنيغرمه

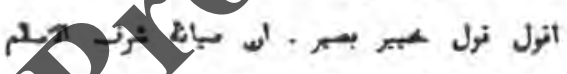

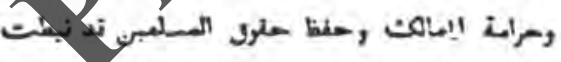

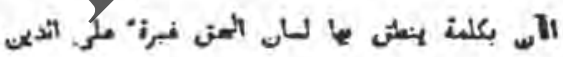

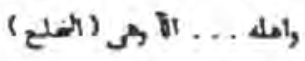

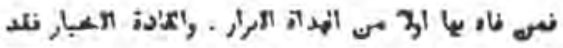

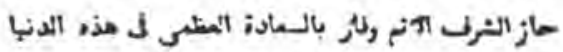

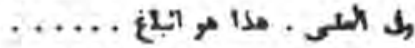

$$
\begin{aligned}
& \text { לـلم ملبكم ريعة اللد وركانه } \\
& \text { هب }
\end{aligned}
$$

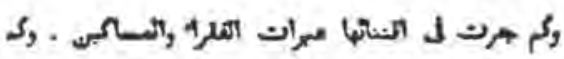

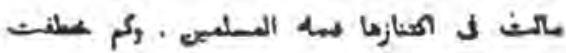

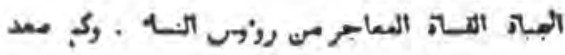

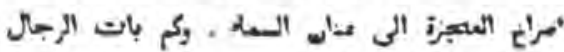

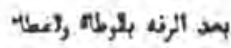

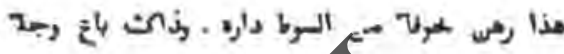

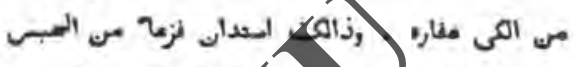

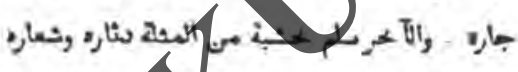

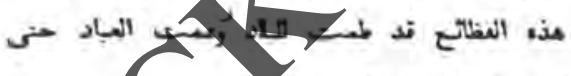

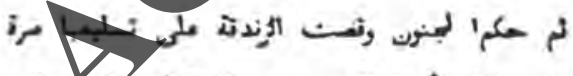

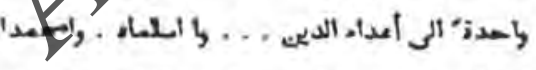

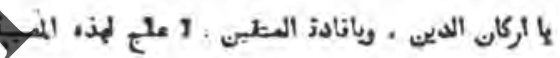

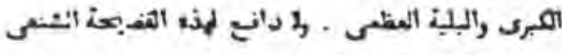

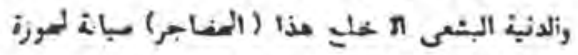

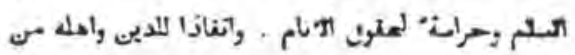

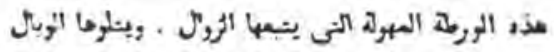

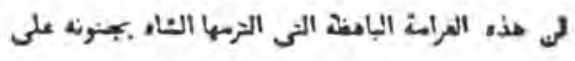

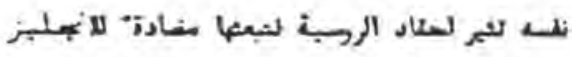

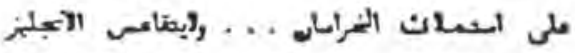

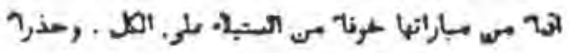

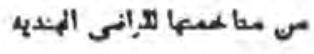

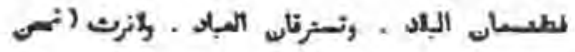

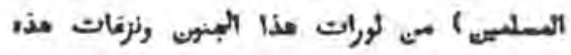

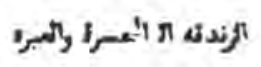

$$
\text { رمنر كارنفنـ رتدكال الدأرت معنا مه كبل }
$$

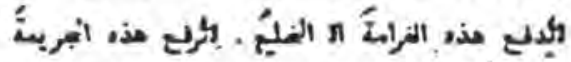
हैंक 


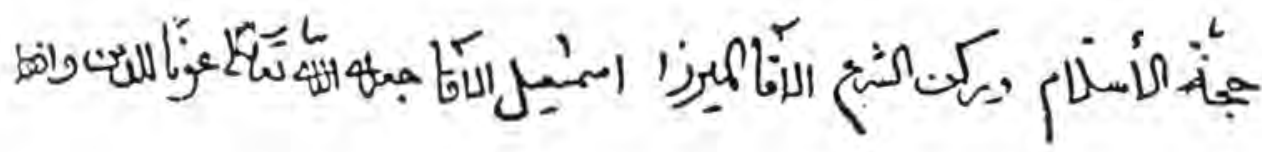

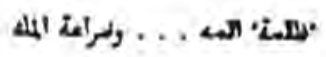

$$
\text { بـ الله الرحمى الرحبم }
$$

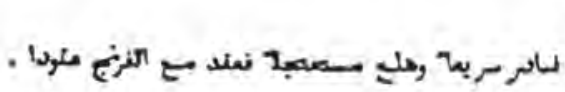

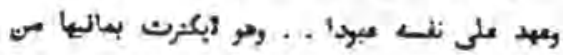

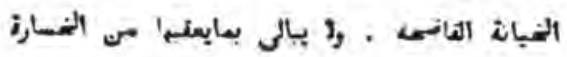

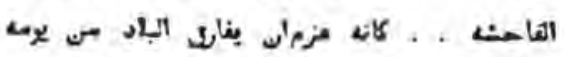

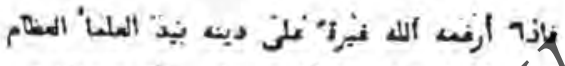

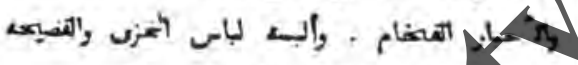

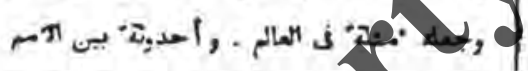

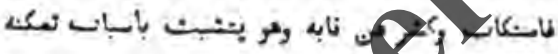

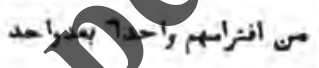

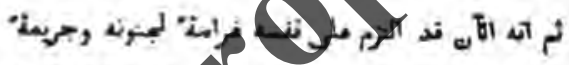

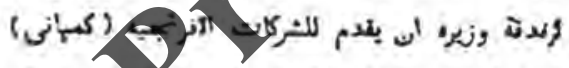

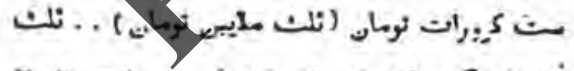

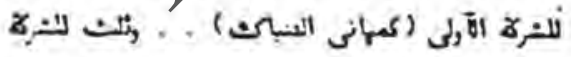

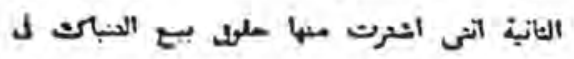

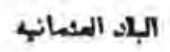

ما هذا التشاروالمار . ماهذا الذل رالهفار

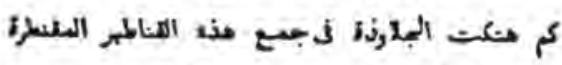

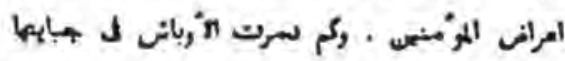

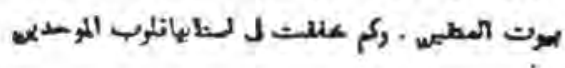$$
\text { (1) }
$$

.

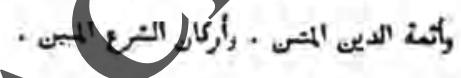

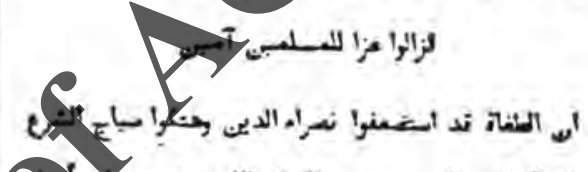

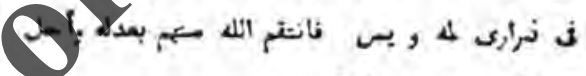
بـ المزي , حسله أذله زل الماليهن

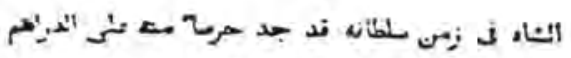

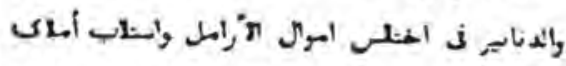

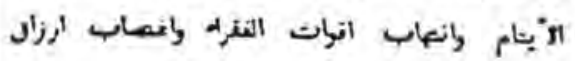

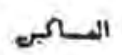

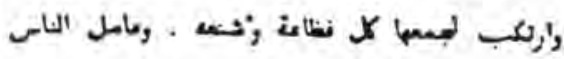

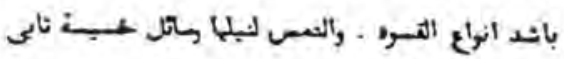

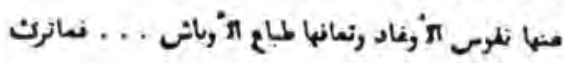

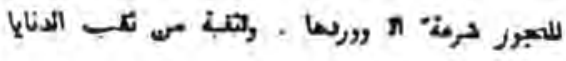
"المورجبيا

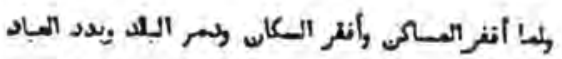

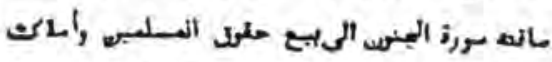

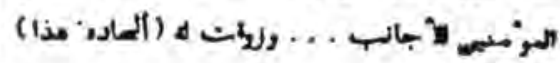

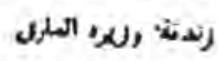


- Itow ene the reforma you surteil recarded by tha Shah a od his present advimen ?"- All the abuses and excesset of unrestrained despotism in a reign of ferty-five yearn have a destroyed the intellectual and phyaical foculties of the Stath that there re,nains little responsibility for his pernonsl actions. lle hes surrounded himself with some yourg men and boys, who will him and have suceeded in mating him llicinstrume of of their uwn ignorant and capricinus mneuct. This band is natarally opposed to rezular edministration. But the Mtinisters end resperabie people ceneritty are to favour of re!orta, and rome are now in dibzrace aod cteal danger beisise they apeak of reforme"

"Ia retorin note daticult amorg the Shishe than acong the Sunnia?* - As the bavs of uiy refurm muat be found in the Koran, the posicioo of fliese two tratulies of latsin is altnest the same. But as the chief obstaile ta progress is despotisin, and as that furnt of governmetit has

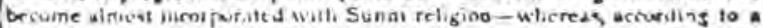
fundamezital Jogma of Shiah religion, any monarichy is a isurpation: - the niod,riction of despotism is more casy ainorig the Stidile:

"Do your prupusals affect the whole of istian?"- All the serta Islam, in spite of their differences, furm such a snity that any arnour

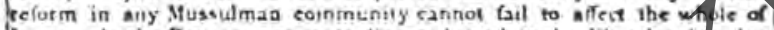
lsiam As the Persans are more disposed to adupt the liberal pritipies of inodern civilization aud have great power of pnipigatiog those prustives. reforms adopted by Persis will swoil becoine reforms of laters

"W'bat has been your observation in F Eyft and the the neightourhood and uniter the control of Luruge, is ra cersin 4 gree more abie to govern herself, and, as jour possession of $C$, frus is a duficieat

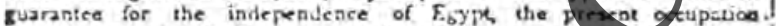
of that ceuntry has for Ingtand no orher ellect twa to indispor the whate

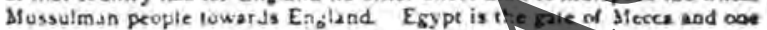

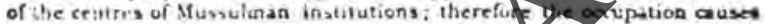
much ireutic in Tuskey, and, thousth licefriendst raal oo be of much ralue, the hostilaty of Turbey may pon: Oy at some Unie be of importance As fir India, I know tetier it:an many the secret disponition of the peoples and can say that, in spite of the lomg perted of English rule, the English remin the couquerors and the lodsh the conquered The whole

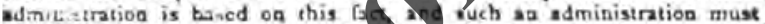
Datiarally be without reot It the actaistration be clanged, then 250 milltuns of reople would be willing partisans of England"

"What is jour view of hie future of Porsia ?"-" If the refuras that $t$ and the best of our Wusted std uiemas, with all the respectable clases, now ask are earried out, Fersia mill eater upon a new en and will be able to maintain hetgupeofened But it the present miserabie situation continues the peopto themselies fill brirg stout a dissolution, the resula of which wit be in

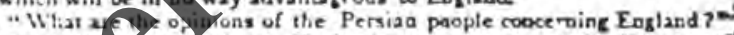

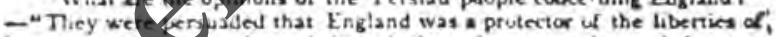
humationd hase lung b-lieved that the sympaiky and irterest of Enylid were working fur the pristess and welcie of Persians abd

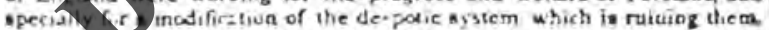
Yatakl there has been disappcintmeat, when they saw that some of tha

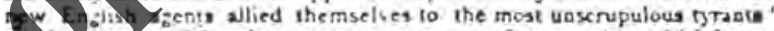
and bale invented 6 ir these men dew means of oppresaica, which have o dieply enajerated the people that inatead di relying on Englina yppori they are joukurg for oiher asisiabce" 
mE IMEIXn NISMAT-ED.DIX.

ingeouity of makind bas eve iovested

The Statia secood visit to Lzodon gave to Perialscarn and eapable teformer in Priace Malom Kban wboraigned 1 p pace of Minister in Lordon ohea the Shab foll usier the dezision of ren

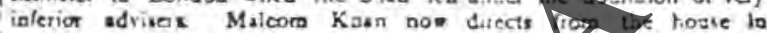
l-bich be reseived bis Sovereign a agra:ios the arra of whicb ta

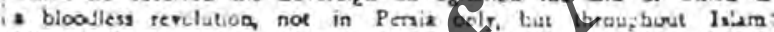

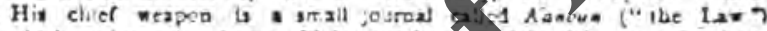

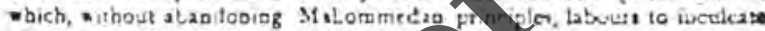

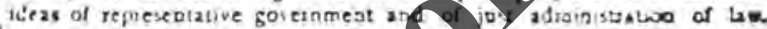
'Hir chiet zusociate, Sbeikb D,emaled-dik bar La:cly arried in Londoa,

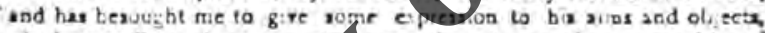

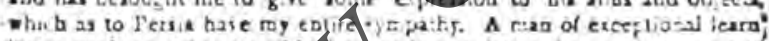

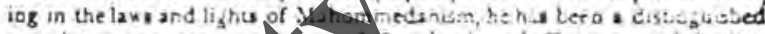
preacher is the great mos ues of Sumbuat and Teseras and be bas ecquied mucb cosmand of, the Freacb langlage lle tratelled *ith the Shab on ho Laskbovemard jource, bat sood fell unda displeasure at Tebeat abd cragu safely returo to the reaias of

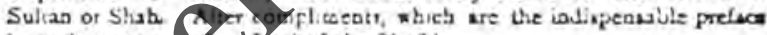
to such e conruratan, I'asked the Stalk :-

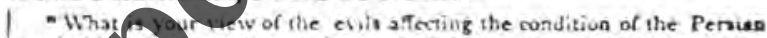
people 32- I Jun ronvinced," he rejlied, "that the people have greater

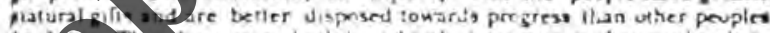

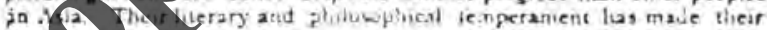

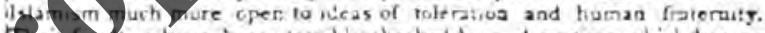
freindanties liave keen terribly clecked by a deapouism which has no

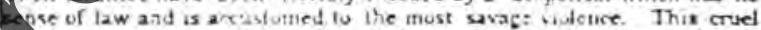
despoism persecutes all hiserdi miaded niea, wao are perating is Perais, borke forces to emigrate.

"What nas been your cunnection with thein?-"I am Nighag belonging 10 , the purest Iraitall rare, and, the Mussaician Gith being a stronk bonat, i have tial the suad foitivic to altrart the sympattiy of Pernas

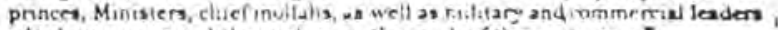
who liave aswociated tiemseives in the wort of their saisatum=

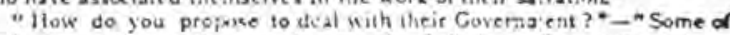

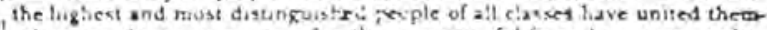
selves to obtuin guarantecs fior the serunty of life a d property ad to

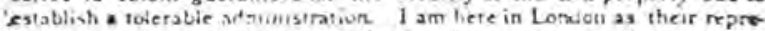
sentailive to expose their situalion, teping itien generoun oud hutramianas mind, will not Lill to help by mival supper ir our oppressed peopte. Witb

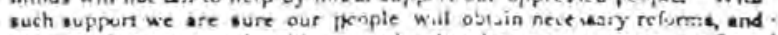

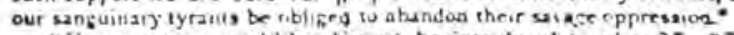

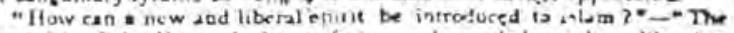

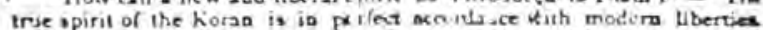

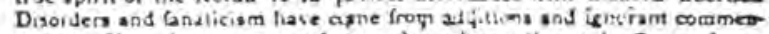
uries Now the picgress of firne has stiowa the evil effecu of past miniakes Therefore, - leamed, Sfussulnian, well acquinied with the liberal principiea of Europe, can rasily runvey them to his propie wis the autiority of the Kong, wiqiout tise ditculcea which ours inded Latbe.: 
TAT

159

1892.

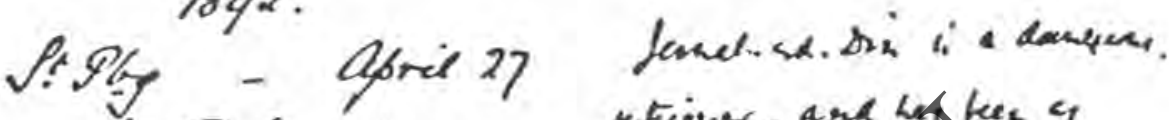
Ier-R.horier notugiver - and boy bees at NPS7.

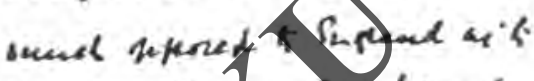
any ithen gent ont les ind

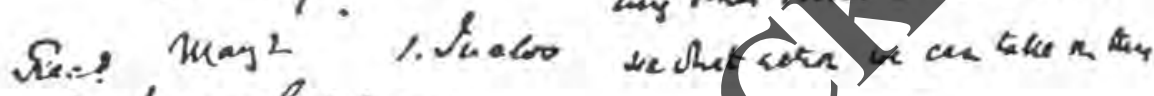
ty kueser Godedend pree. Dits

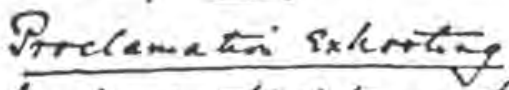

halleaks 6 delitume tar

Jhak: 5 .

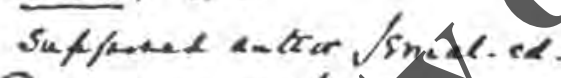

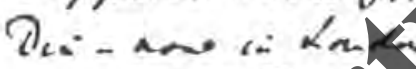

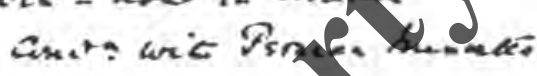

whe know aty a 4

Suruai: 40 Hodyment

finter temwity.

p Ne. etberla:

The Qneen 0

Irisi fffice

Tehmon

oftlligenec Durioin

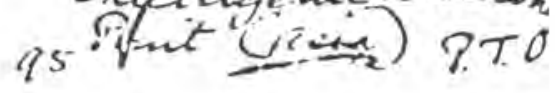


158

TAF

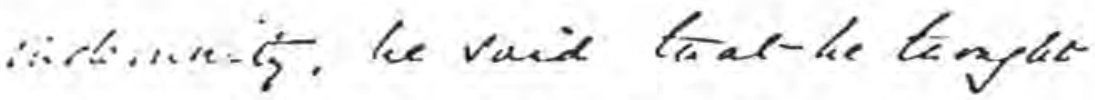

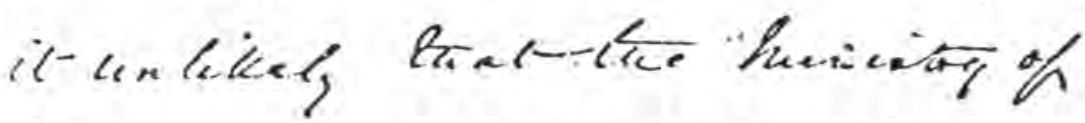
grianee amed furedece the noney wail tad at eny rate ho hention

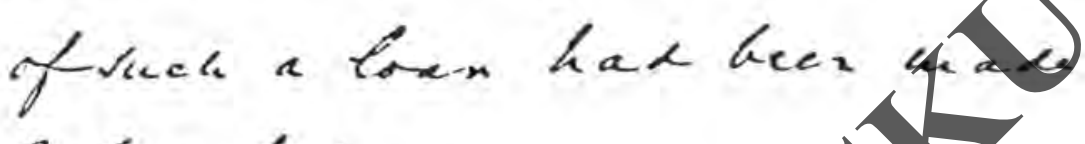
2then: hene.

lhane the konorpt he arit ene lighert reapecent

hy hord

botrotorescifa:

Paxtrabeliexe-

hamale teranint,

Resomoricer 
Jafar Khan, probally civeneid by tue Pirseain Legatar. hes beem

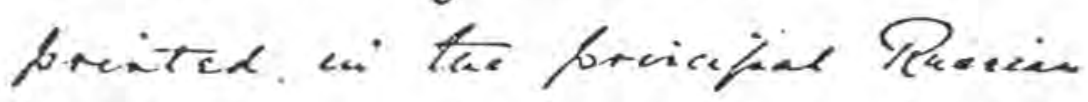

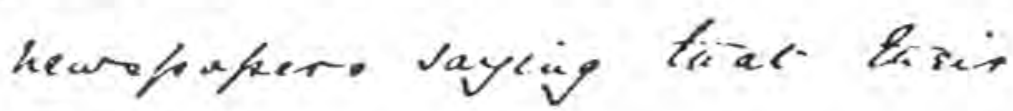
Porkeai correofendence lo kuroty ancretid la St Pelarbut oud

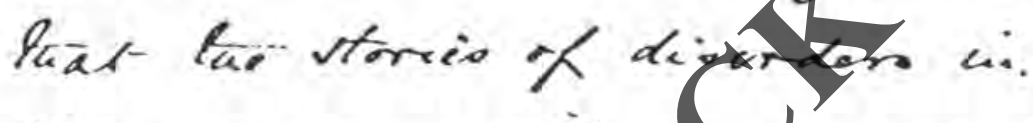
Tehren ane of toxplapalaris

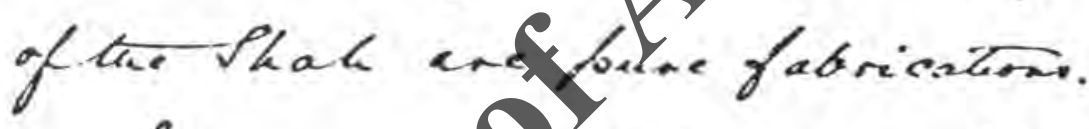
I way apd linat when $y$ a.ked buto thakmond if he knew foltuing reafuicting the

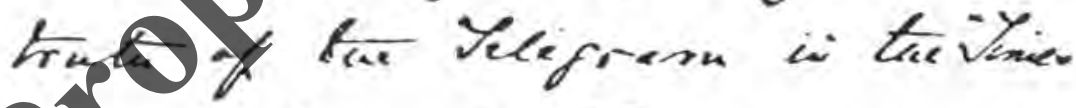

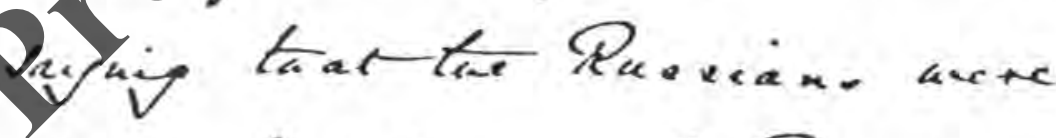
abrier to alovance to Proved a lian of half a nullein Norling fortue kayiuent of the todecces kidentis 
156

rA7

subegreatly war sontaver tae

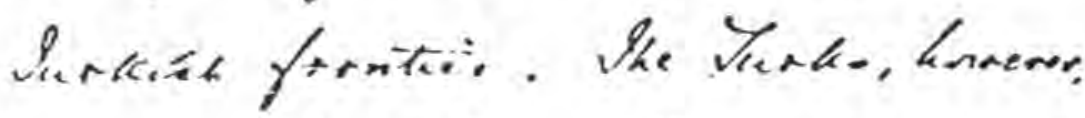

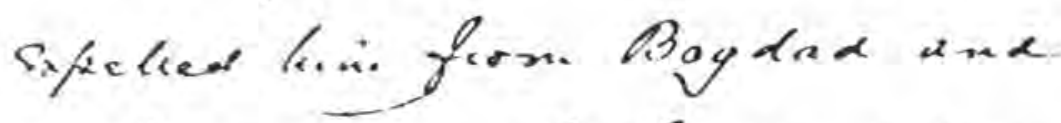

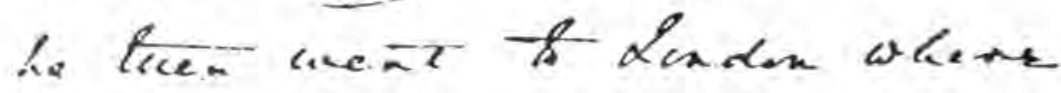
le lava is.

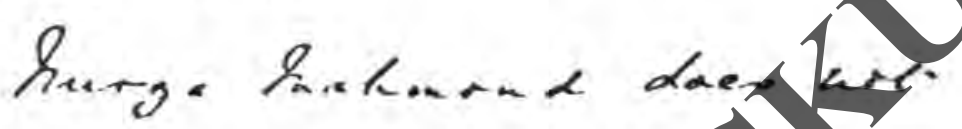

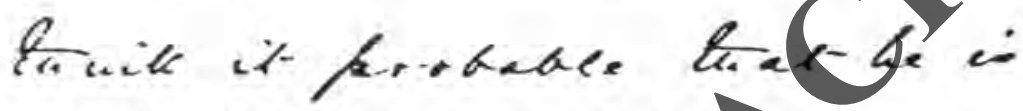

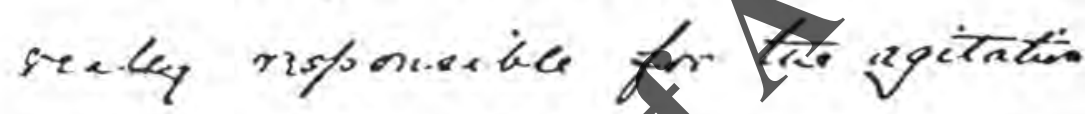
afac.... the totary nowrlaty

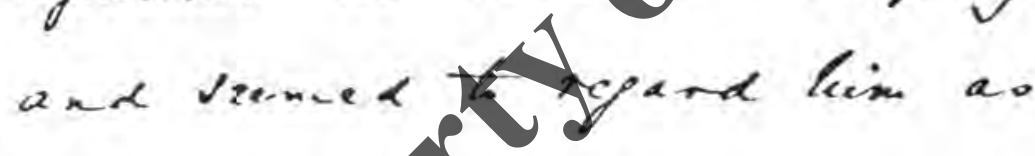

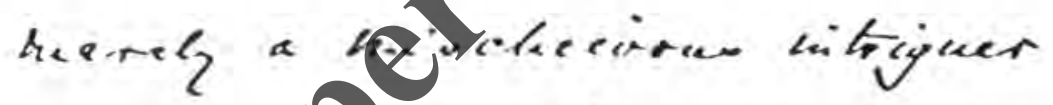

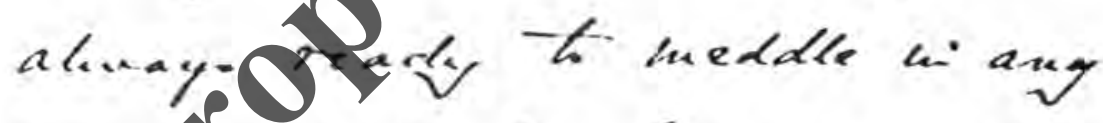
conoryolued be tionght ence bring he mi: aderetrige.

A lelter feow tar Piracen Cinene at Mijus-Mrogand, huige

Japan 
TAY

155

obtaned sone artarieg in Pavdrovis and uilitary circles hot we-kot

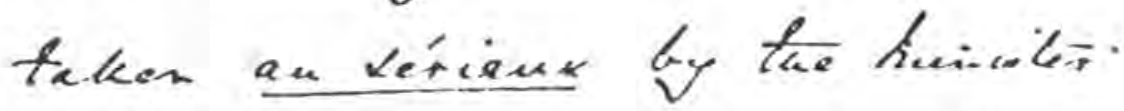
for Ioreyis Offacis. When the shat cance to Pe Prerclank losp ha cantraied to hayk on

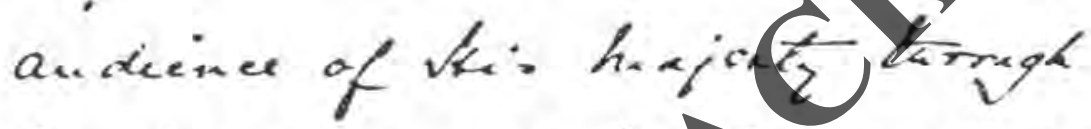

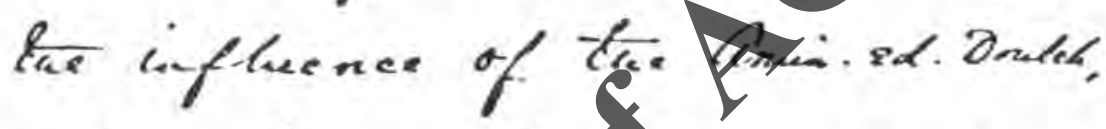
whone freenit he pata., ext recened anploitates to retes:

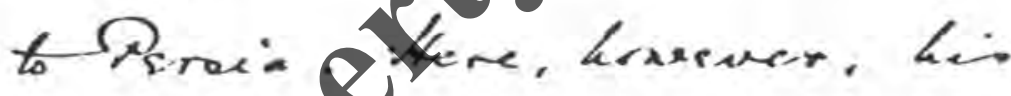

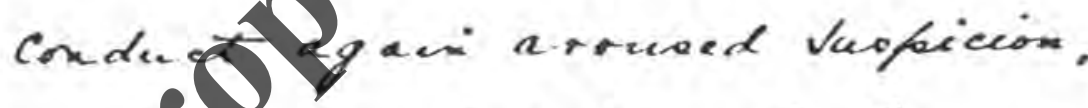
ar. was belevied to b.

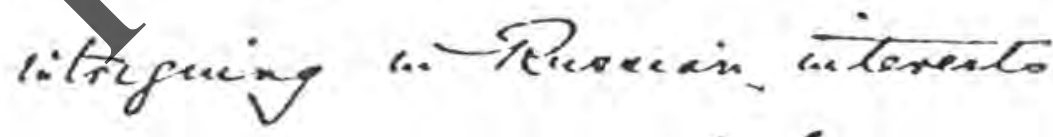
and war ardereed to hie cir teterimest at Karmanokala ancé sachegunthy 3.- $1352 a$ 


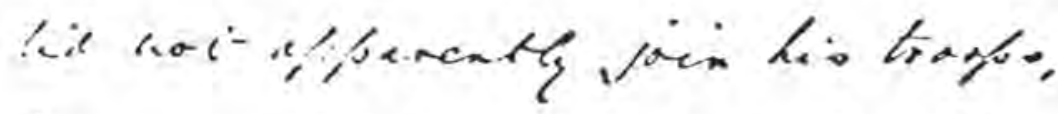

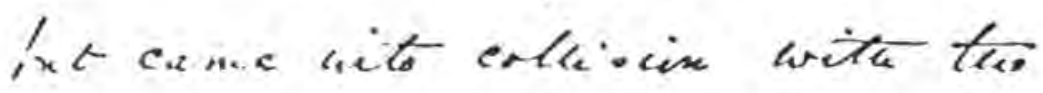
autcoriter and hac to lowe the coutry. Partis wathi aekt soluere of ackivily, where be

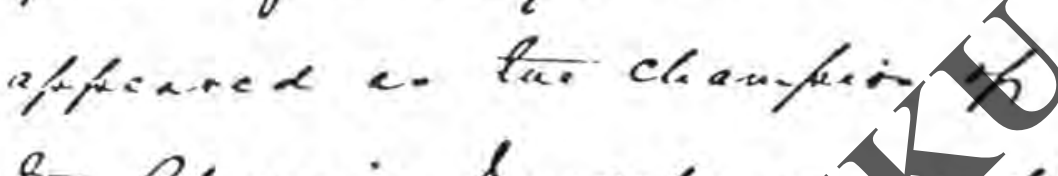

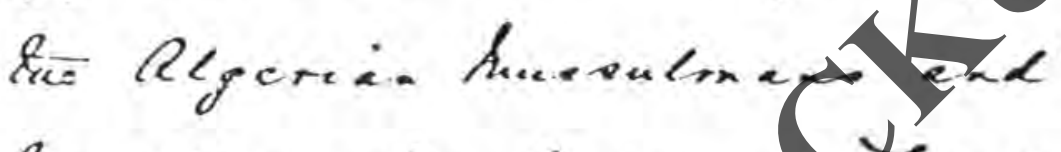
lia editor of a fafuer theack and arabic. Whack ted to hi: hivg Expelieed feon Hanee as ie lade beexperod Tuxkey and

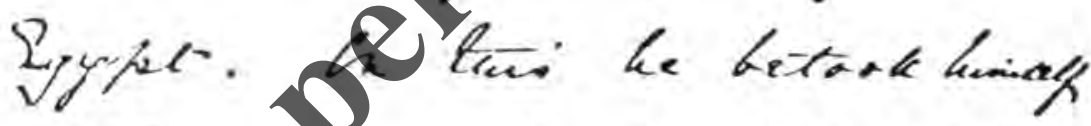

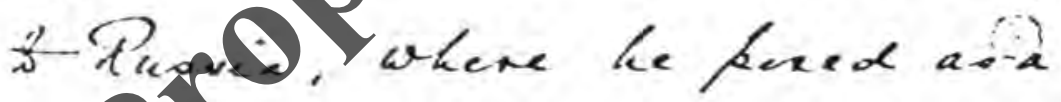

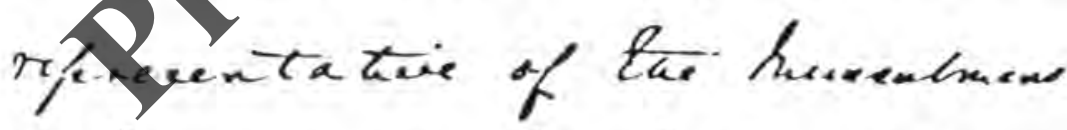
of India and the bearer of an aspeale for help aganis tue Sugliti. St tasi character he obtruned 
rA9 Lordalifo.

153

Shis Sheikh fonal-ed. Din kes. as low Lenkelujb co arrene, formed on keveral deeasiones the subject

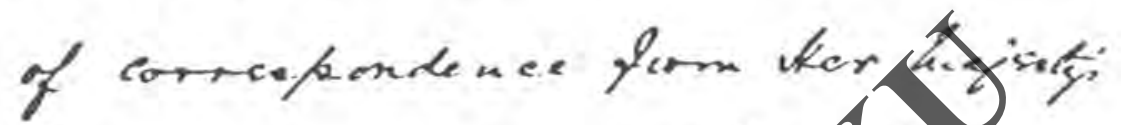

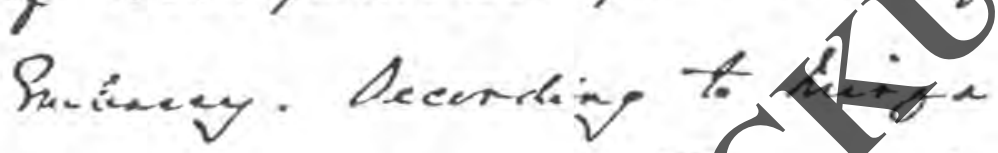
hakinond he is a Propkin by bith

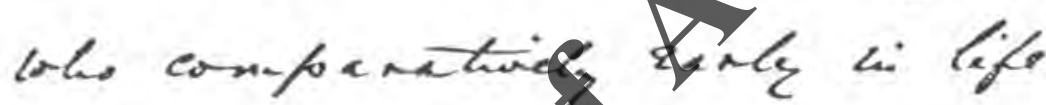

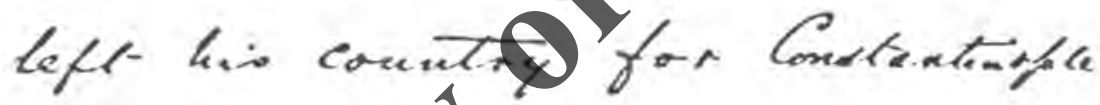

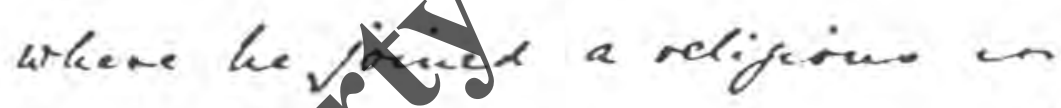
conmunity? kis manuer of life, kningon fole below the lavel of

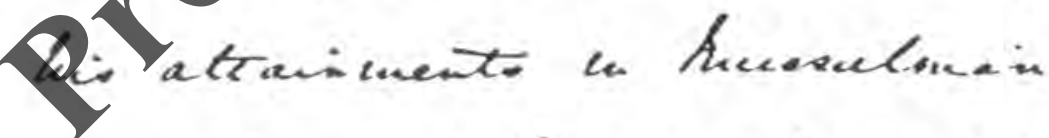

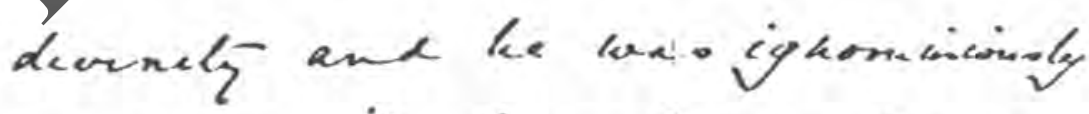
sapelued. He tuen betark kankely

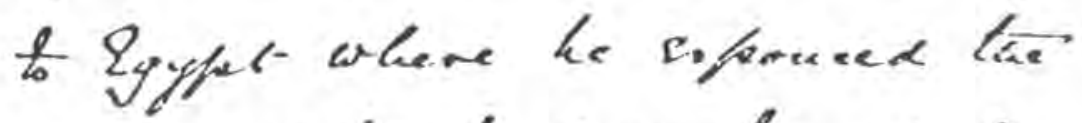
canne of tur hackdi. thongh he ded 
152

rq.

work of tar- Miceile fomal. ed. Dim at frecent dormiciled is doudru. whe hat vint it to a friend fiom weom ik: Freelkney had it dinger. He beyged une to requed foud Lordacip th take menenfergfor banioking fromal-ed. Pi and for ferevertivip king from kaving

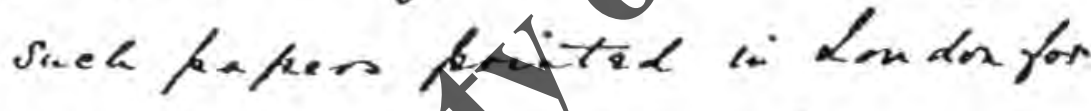

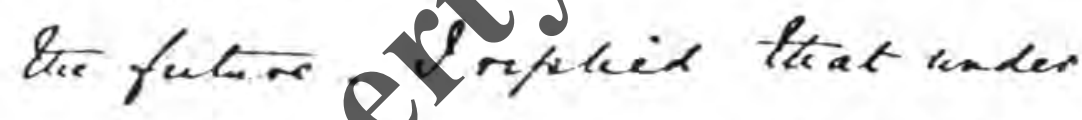
our lawo the: ara- acry difficale, kyoficule-ly ar tae dreament ded are beer. tas arme of tur writer or taat of tar hrose where it bax-bristed. Iforracied. invever. to forware it to four Q'ardokefo 
19. 87

Sit Petretury

Qforie 27, 1892.

hydore.

hy Proseis collergue called on we litely axid fave fye tar

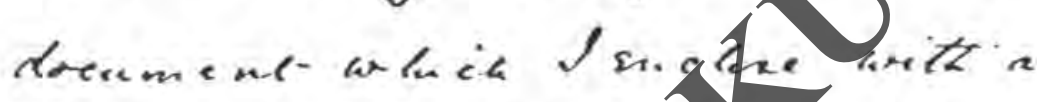

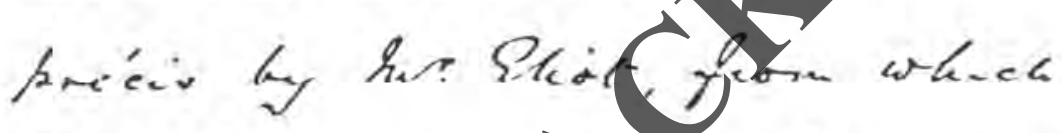

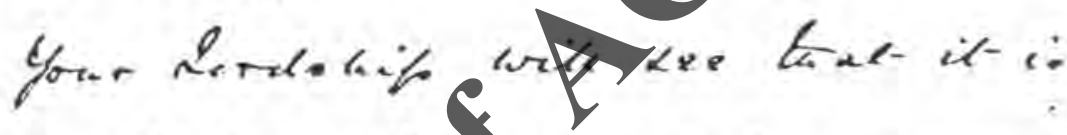

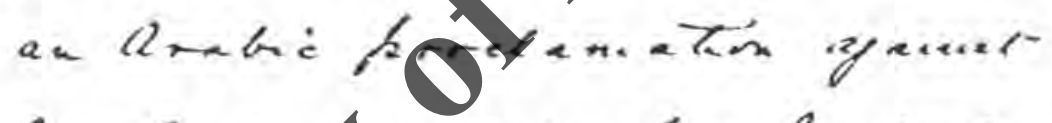

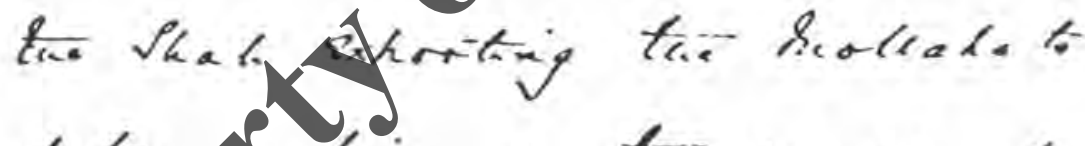
deterfithin a. the one romely

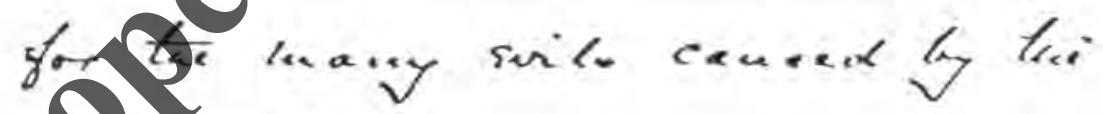
Padace. Company.

huiga haklwowe said ke exke provitueg avenre are that luar She, rooknterian hasifest wes the herenis f falibury coratk

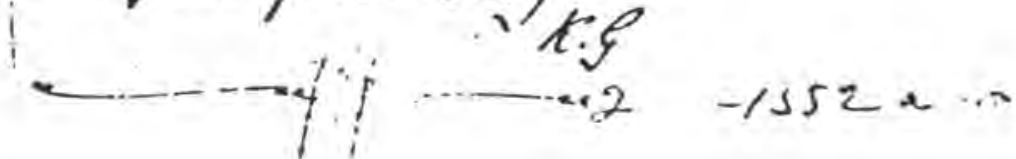




\section{Tramuntion}

Syew ryemal.now. Xin

criep maytisw of

Sarmara.
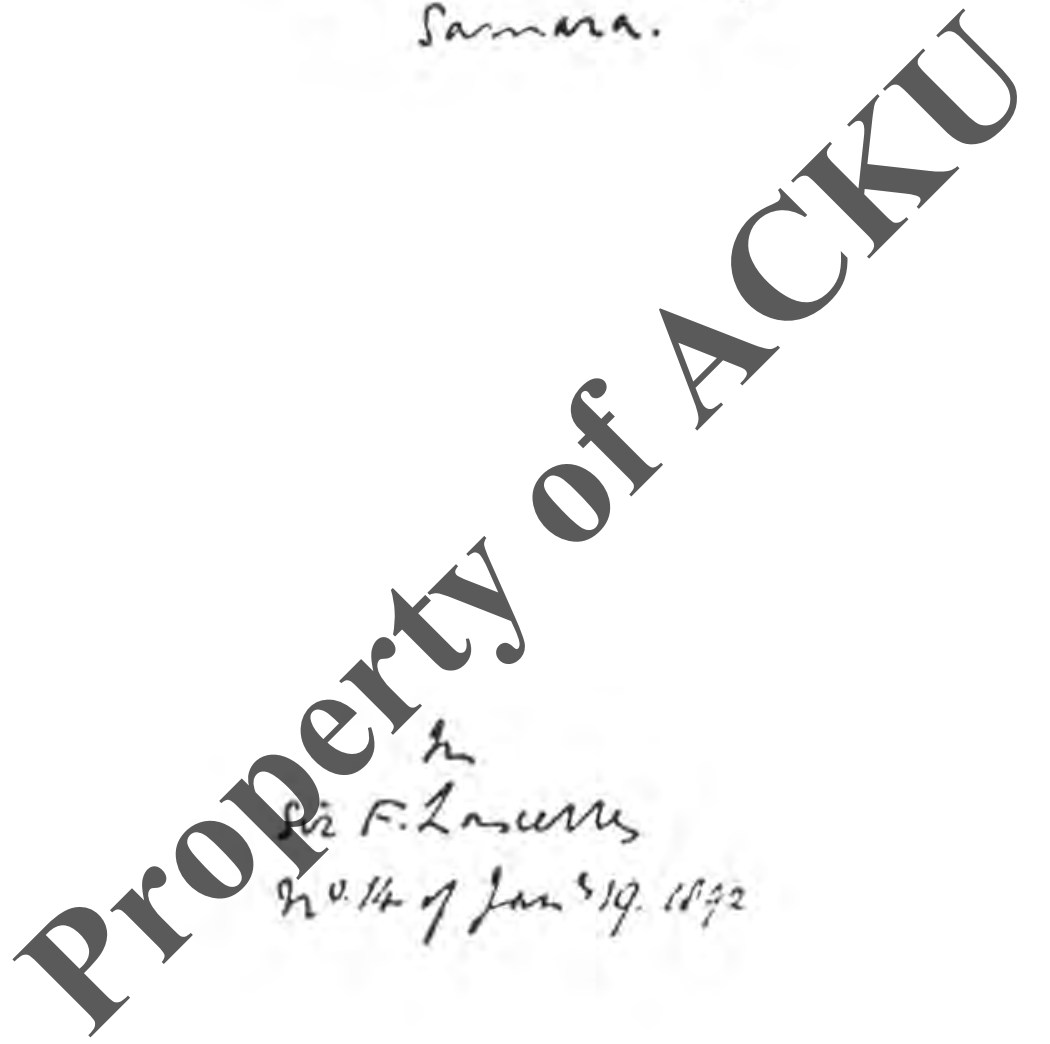
rar

149

lung loch grent the hoostemes the forrer of retaliate on thisis. wivted siefidel.

Ao I have liot the hommer to pay hy respecto and a long waz off Thareenterte epenal anggation of byed ati allbar, wutt in detail on the entyeat Dit-aty kotle fruid maye Sacquaricted ritit these eviterucidento aned of tusto tod had hay correat ort tumigh thy hanito. (dd) Sigyeh-al. kasecinci 
148

rat

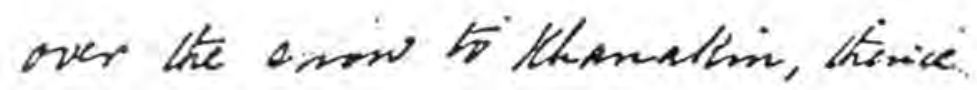
the bali of Buarech, whes linew bie

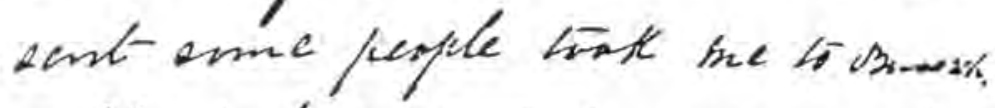
tradlic hot eme to ling seocus I dimuld have pariotid by this trink.

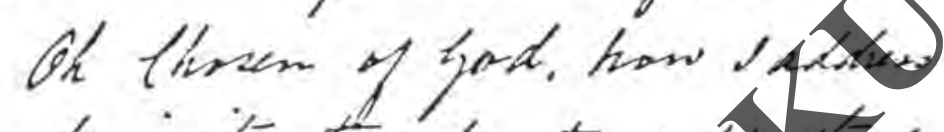

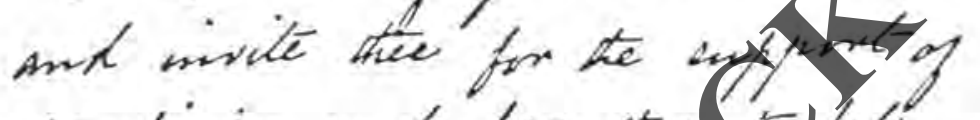
be selinion and bey thec (v) belat

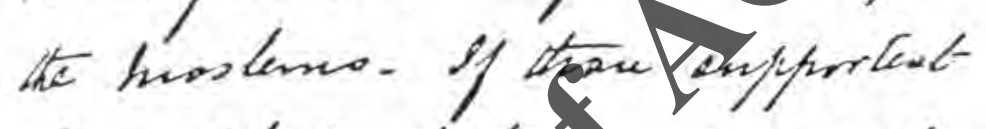
them thope that ist vign of thio

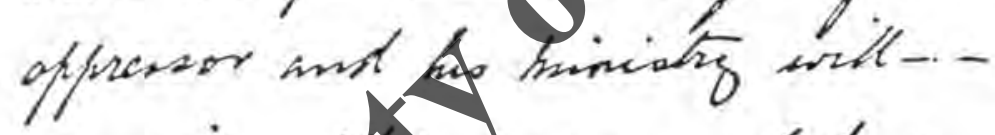

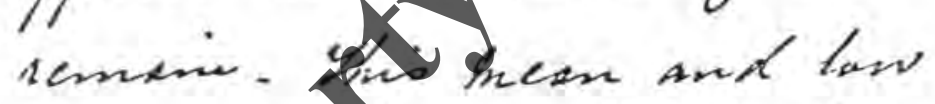

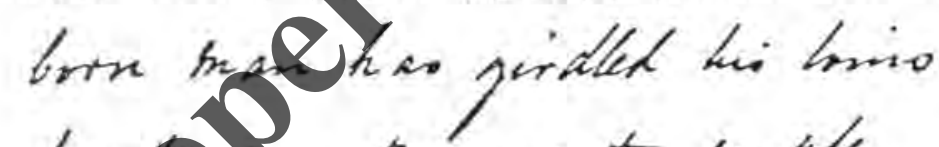
for toxentirn of the reapte. For man, as it is oeid, is -. Impacotinably a Babi: the weatincases of delaras axe ath canced ly thiti inciare inan solup wetho the hastions, afpecoves the felarwes, and entement the sypeto. frasy 
ras

147

leyi byid ali altere threspe

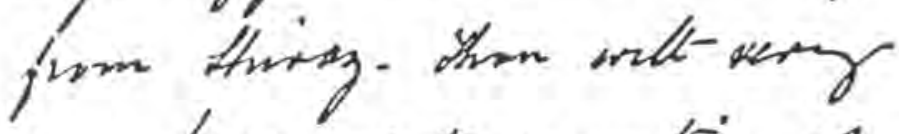
soon herr, of is exention of a

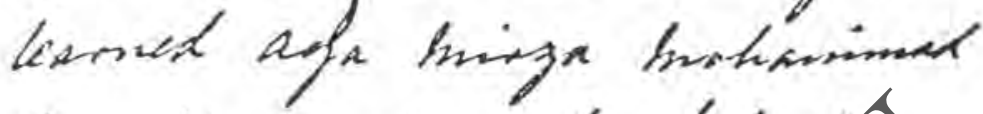
Dege tirmanci, and what have donce to hiriza trorgtion

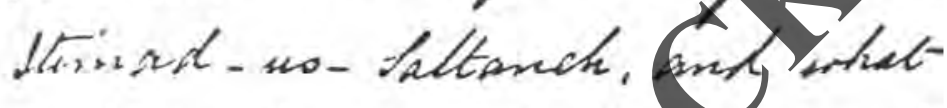

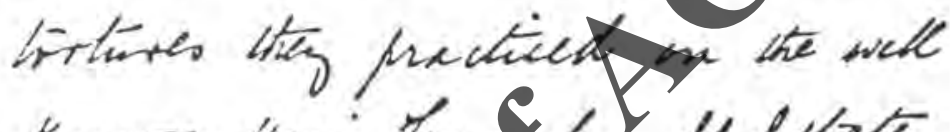

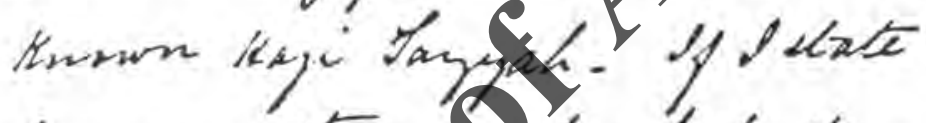
fing arr atory $d$ what the have done ace the hearts y the fulting of the fritt will trats.

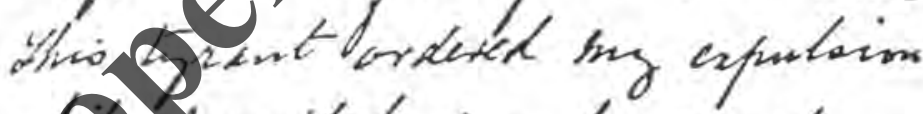
no Wrexidet in a howace at .. Trongetiabidod Angin and was cotremely ith in ing bed. A unure of fluolame fromed on the and frich sie. on an juoble say to got m inruetack, when the wintes

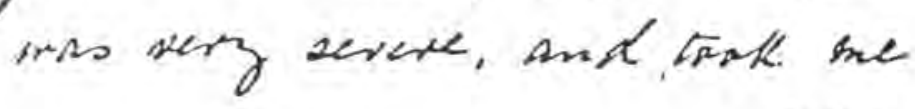
wier 
the witled and will proted the wort and cirile of hlarn (them sll afforio axt from tae to thee and lowerdo thee).

Itrm art responsible is te bax and this proptuti for the hiter in the diy of fudgmente.

the Ulamias alone axe Nable so

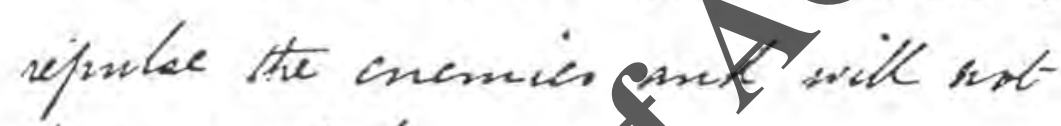
be ancesefal.

lendrobledty int-eage of tho hation has leard how igmonusivi the oxacing foremmant betared

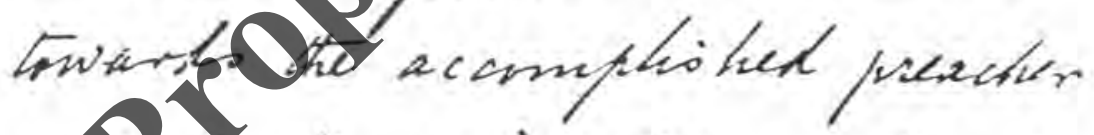
trage hustle taizullek berbande: in expelling form fom Yerivan, and whint afipizasenes the did in banciomig the kerened tyyed. kye. 
which they enffer from the treemo of this traitis, are exty so colledtogetter at apprisited spot. They cemort one iz one un ant for furtic mbleso the place $y$ incetring io kamet Afe con-entrlated, fy whic Stam

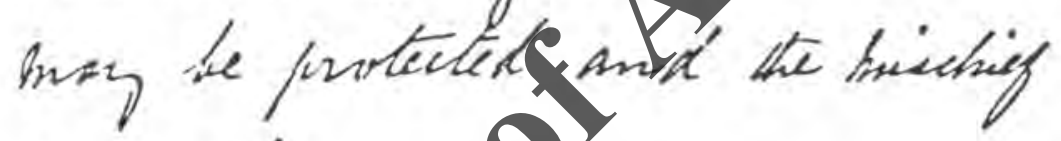
umoved.

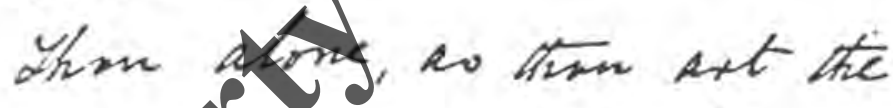
thosen Ay fod and haldeat a higkastion, canat qive -.. Qun mank and they will abey ing arders, they will fain ctiee at different flacer. A word from thec, sifwit may he for te watke of the treator, will seresure ine 
becanae they throm that the -. interference of the morean gors: in the smeties of Selam will... aloo be detrimantal to the camon jovermonent:

Then again the ministers of

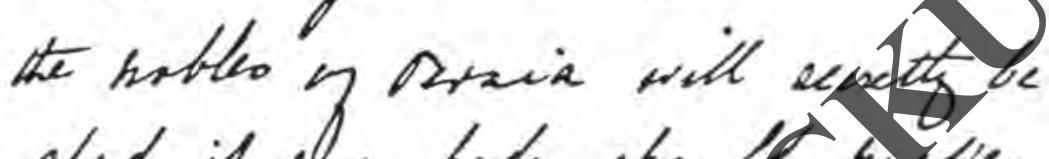
glad if aly body stonelf Goxtic... umartls and tatle hepereses in theer sinatieno, they Dranimanoly exonorate therefires from the creation of conts and adinst the hiotos. They are vearly f.-

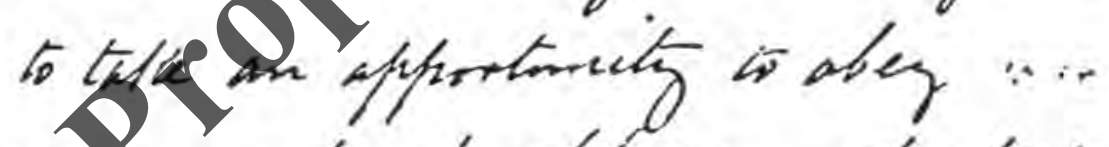
comprands dronte zan dartefor three parto.

Inoweat there that he Hema. of Detaix, on acrount of the Geandis which 
Shromld tion reyledt this-.. opportinnity and tert welte thes afferis while toun ant ahie..tiviting with hat hereafler give thee the grod hame twan hase has.

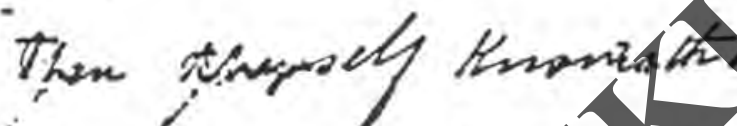

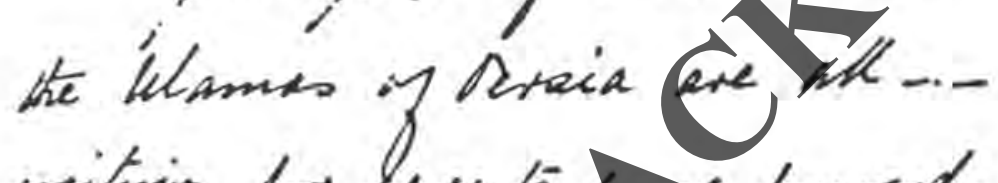

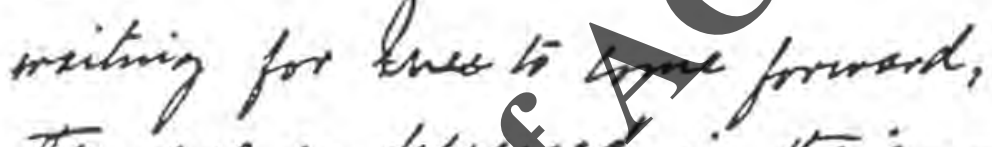
The are es defuched in thar-.hearto and minito thet they eay

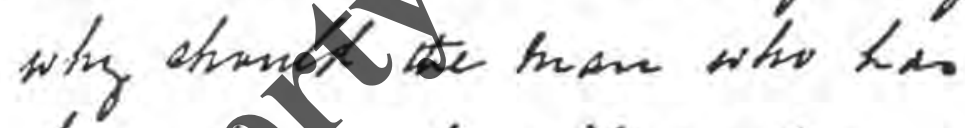
ken formed wits ench promers by allow tho hation to dafjer calamaties to taio extent. Sdedare to thas, throen of bod.

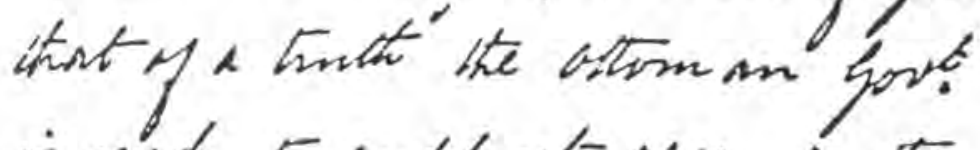
is reasy 5 anfiport tive in the olepo which toon buagtalle,

becuose 
Orsuix mite the hand of the hearer, we has old the combines of Salem and the we ign of thothammad and ho followers, and so the is been. he has mot com sold them at:.hight prices, lest at a very lapps

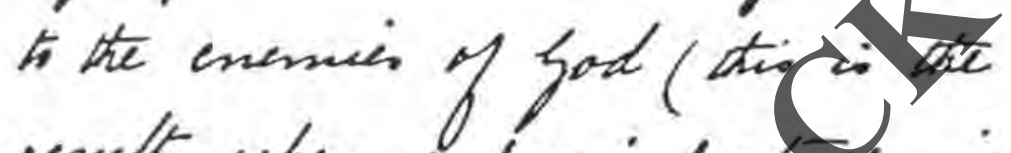
result when a basis macule io

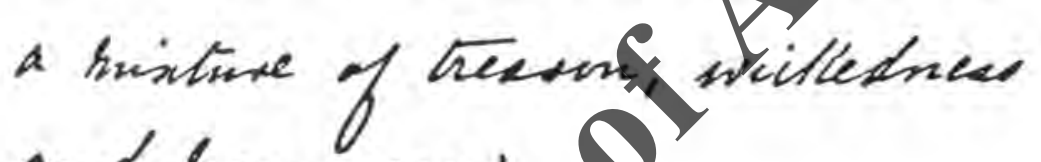
and hesnmess of

of thoompty bod if there...

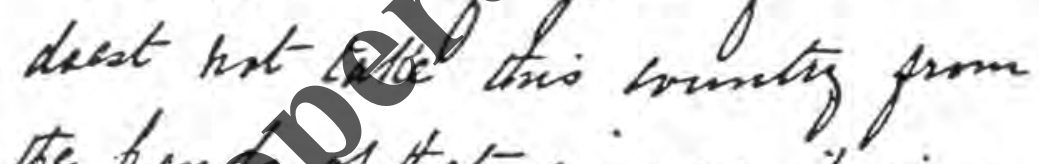
在 hon of that amer, it: as if hast left the wite of Selim mater the statute prover of the Foreigners (they will wary eros carry but the rr mitintion. whiloverer they bay be).

Htracth 
$r \cdot 1$

141

Inch as the neabt tajoon, the sivers of Yaberiotan, the

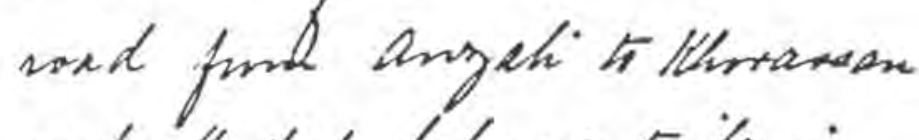
and all that belmyo to it. the aray of canalo and lisas

Pont the Ansican pratrunt turn up their hapeltho tho and comvider teat lall thio is to libte, therr Oitentem is if all thase wrougoions which -. dedtare entomionion of the comes to the menciso of fod,

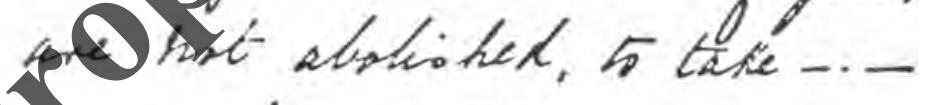
Travesaion of theoracasm, -.. azerbijan. and busyandersen. In short this wicled sman has, so thangh ot were, a furstie ante given all the prosimicesy
peracia 
140

$r \cdot r$

artides sucusery is savtle seme. the Buntl shect mististioni w watity besno giving the reins of of the enernzy and timo bucerso

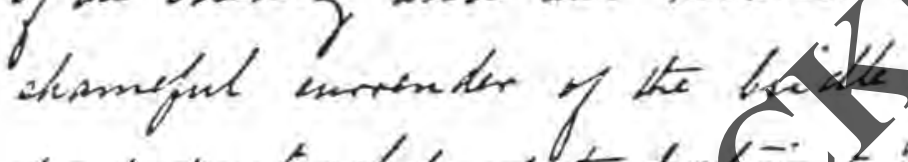

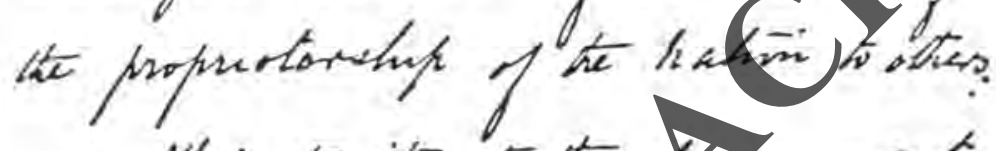
this traition to the formment by plisusible nesomensiffic the perple by eaynd itat thexe -. -

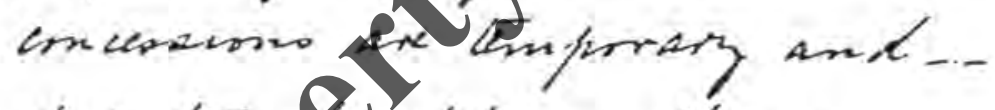

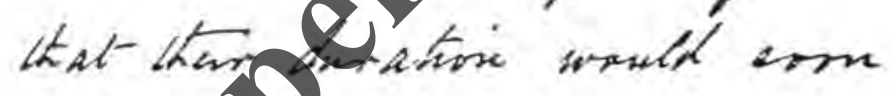

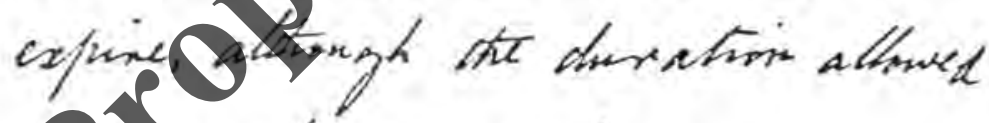
Qsirore tian a cuntarz.

the has gusen to the Amaviones the umaining fording of the lowatis in oris titat they should keep quet (It the semini estent?). tow 
$r \cdot r$

139

Lifferent provimices and wichates srialeover lands and yertion tattiney be an the weys ithe esiver kermen and. it- tribulinis from the inoth to ket Amare.

Iiv Rrabistan saxd

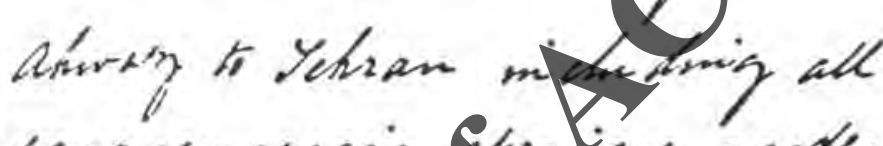

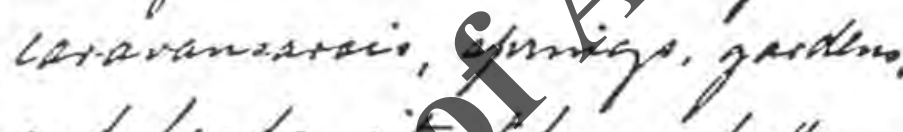
and. land, irioed in batt.. wites of the sefat.

Tobace and all that helonge

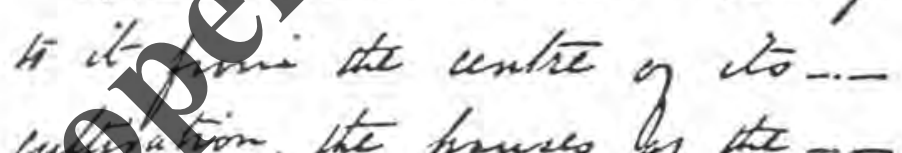
uptestain, the houses of the - -

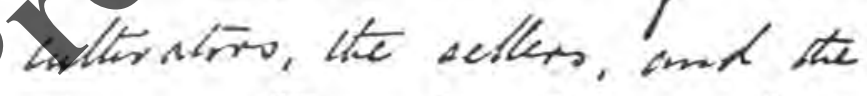

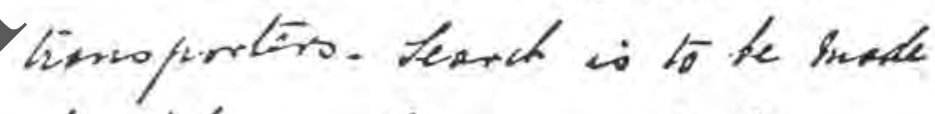
for totaces attevery spot. Katies for batlonis arie and.

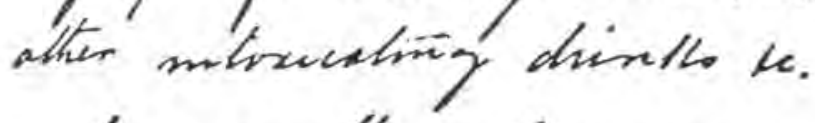

torpe, candles and enger eat 
he hes berme freely addicted of mitoricationg drovilo, he kusces wite the ingudelo, and dismes sumasely Fwards the holy incon. (These are ite inguricis ttatt the widked mas has done to hemesty)

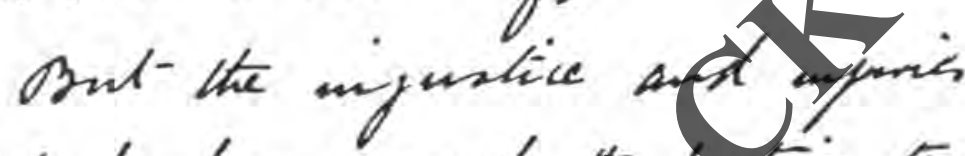
ital- he has coused tration to enffer are as followis.

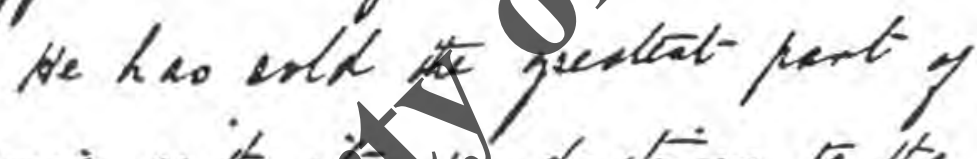

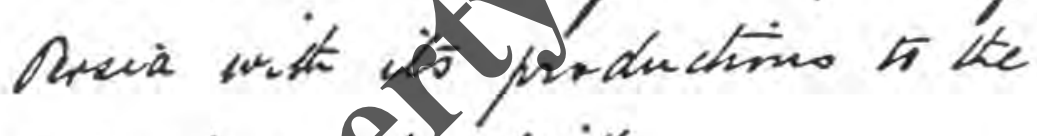
enemies fritt.

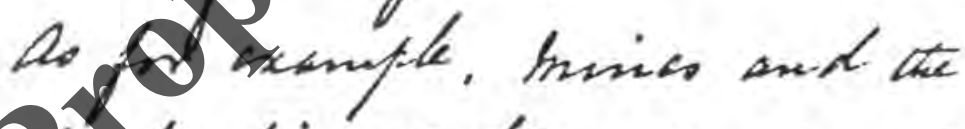

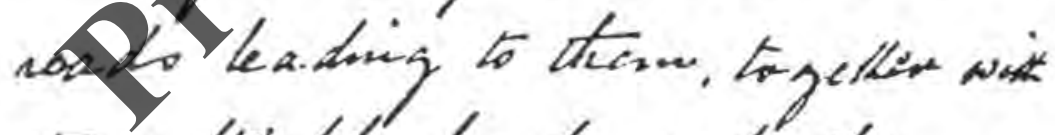
the nuliriable lando and the -. cararumaseer sitivaled on thee

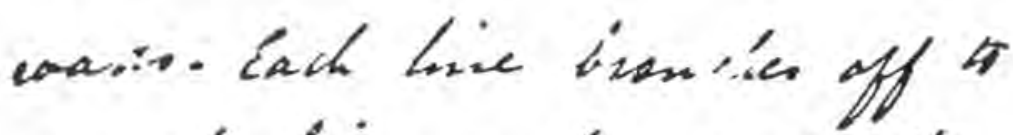
everal lines mot goes tif the 
$r \cdot \Delta$

heme witlext, emouft; and.-

137 weat inmided. Ao for motsece

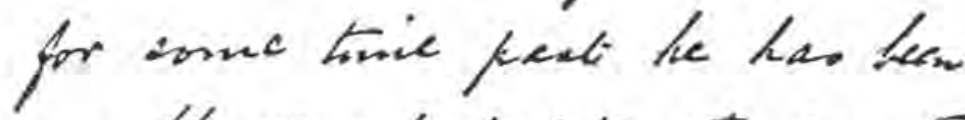

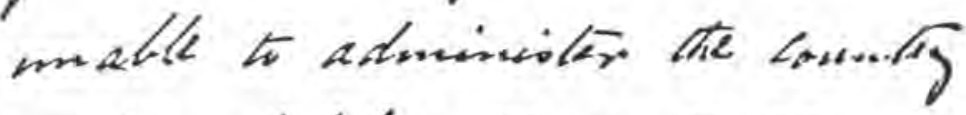

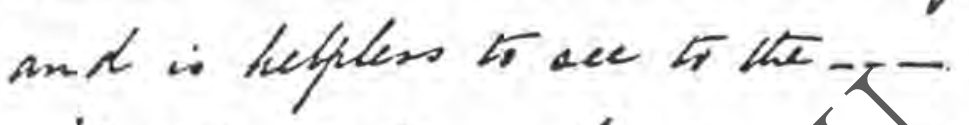

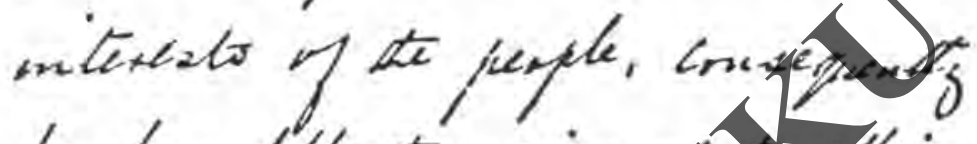
he has left the wive of the fflais

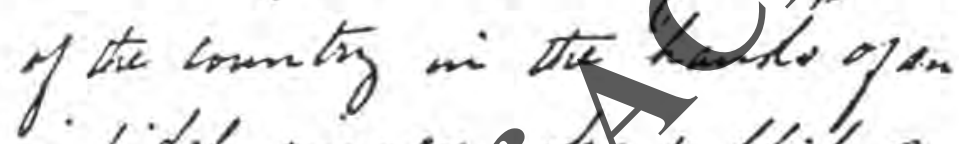

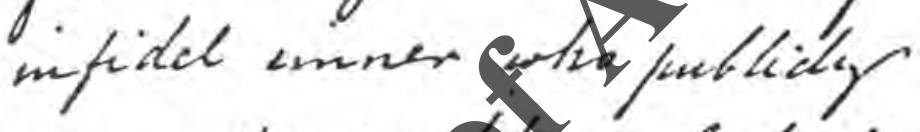
unse. the jumpers of God in wery liwat and kever citer sivilio lete thase crivts a seljyin of hopenmad. He dac hot:we ont the chifs of the factis, -

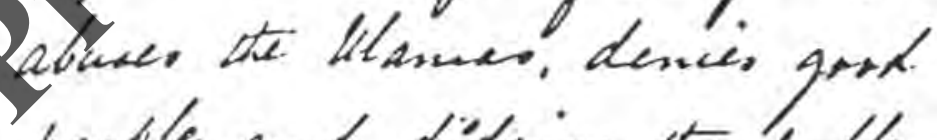
perfile and distame the leate

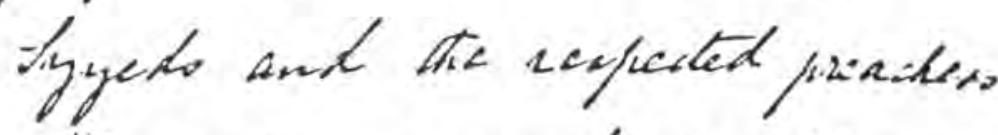
ufter the inames of hew timided fermos.

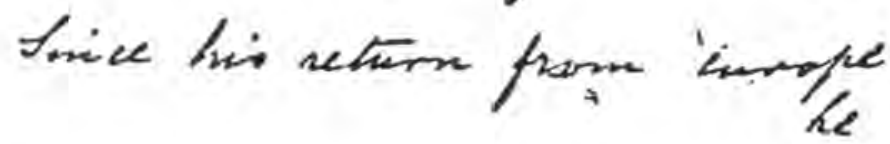


136

$r \cdot 7$

Slane a hijk parition.

St is clear to all pemerns that hion axt de lead of the borly of patrotion, and the and of the life of this katim. Weituren of then can be of ang wat sacept 1

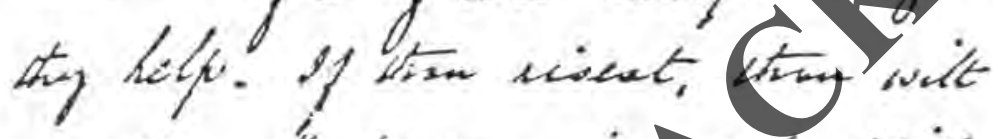

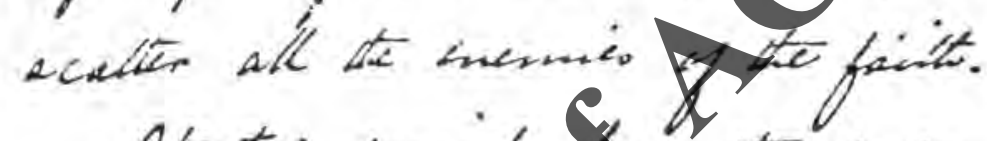

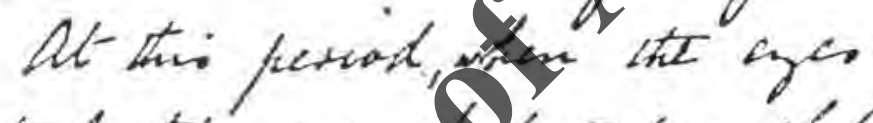
of the hation anegent- 5 lempolity

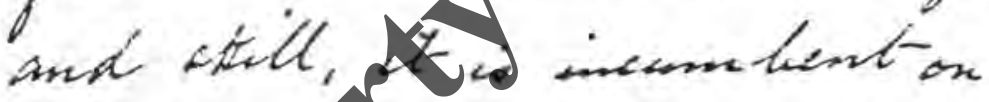
otice, whopost the protector of

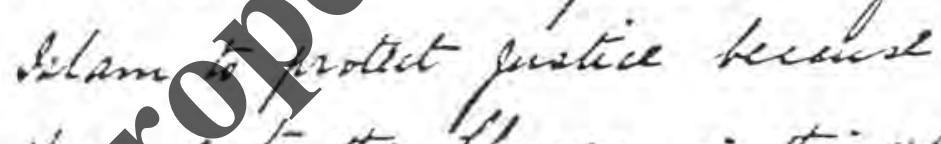

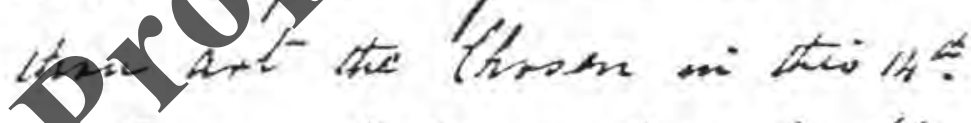
arfary and the poof of torti

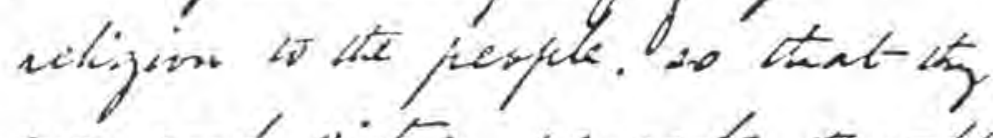

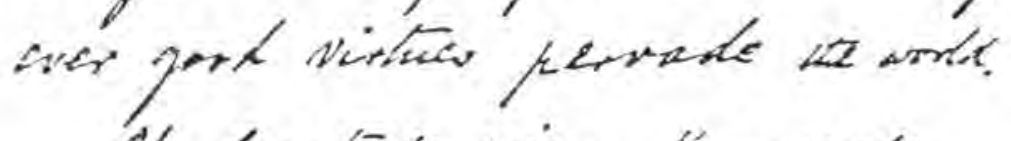

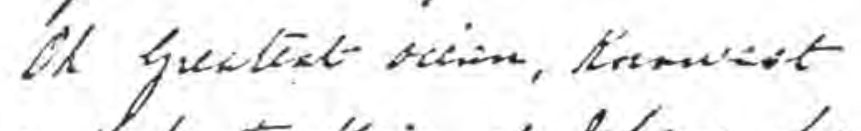

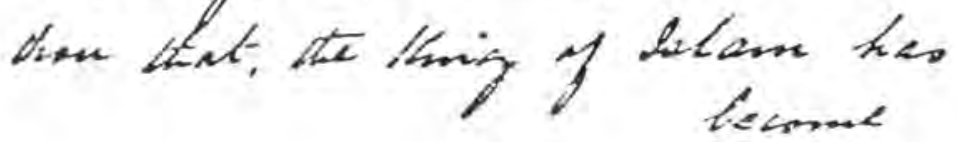


$r \cdot Y$

135

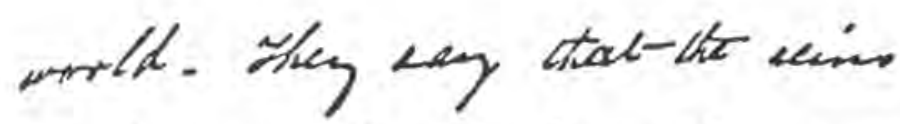

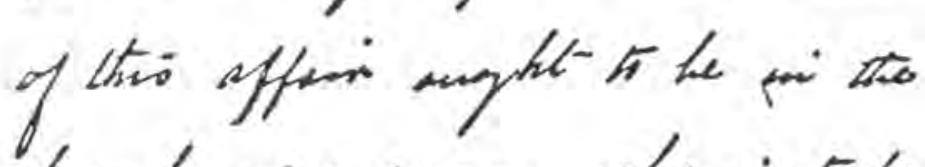
teando of a pram whos it tody wive, stivful, sud a good speater.

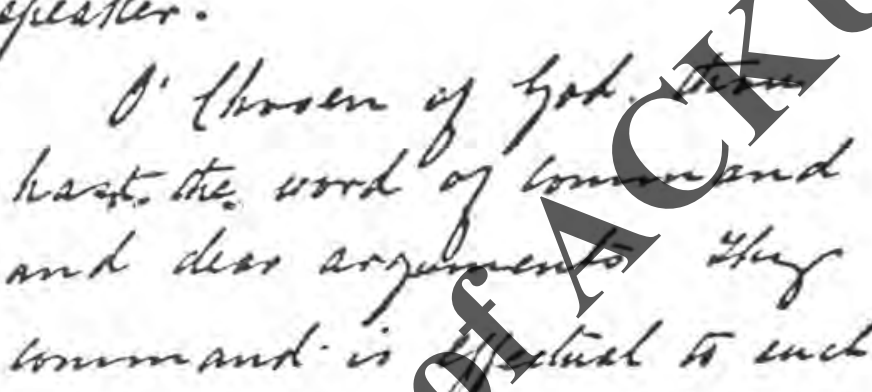

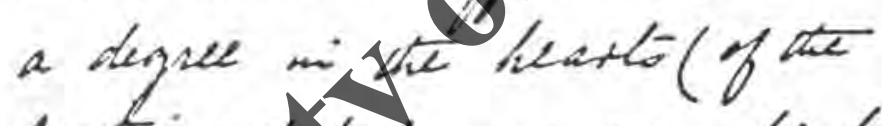
hatori sers do ne can dioney t. by eroord of tammant tion St alle to werd off ite pertedureer of the nifidets and

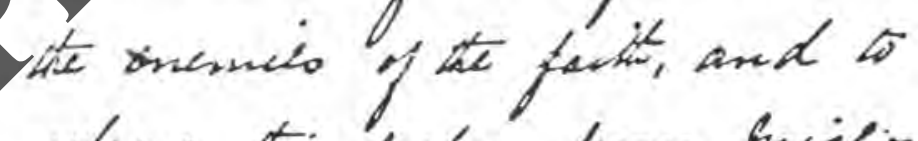

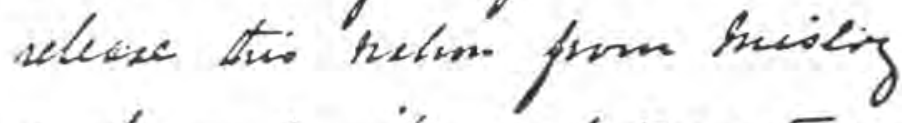

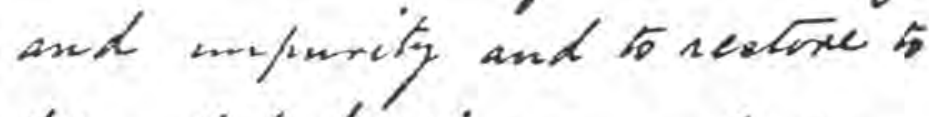
them ttat hafipineas, and to -. strengthen therr factit sud sender 
firm the throen of bod (chane art their object) that me aecundof their excesone exfuctationes chis uges ahrumet staxt out of theer orckets; but on the contrary thay we hathing hut cilence anp

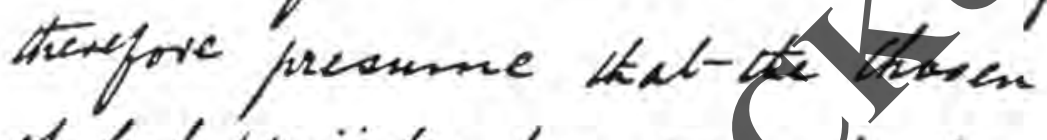

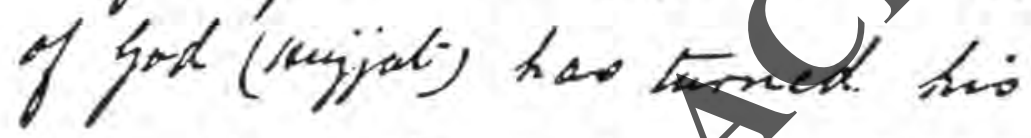
eyes from the enteroly of the tare fritit and is phed to abandon ate thara hecuge he hao no... ather emetog and scheme to -.. prevent tet invesion of te infickes axyoly the mation:

theng furor ferfle of the hation werv magine that lat thorea of bod has not a hand in the regulation of the affarro of thas 
$r \cdot q$

133

and we mi a dilememin dasbling

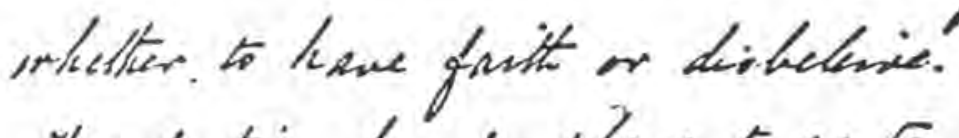
sthis hation has he place to go 5 and are helyters in the dewent of kegligance and enopense. thiere is an me to lead and to cativate them fin the fectings of dectein to bebic thim mmito have beamec teycy and. t5 apen to themp yatio of hope.

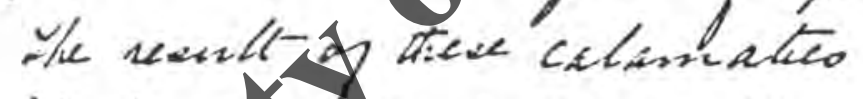
will be ratim will... follow Qturiated ding who-.ont 10 oro the vice of anbition antrl unowalitis and who would antivily lead this hatimi surey -..-

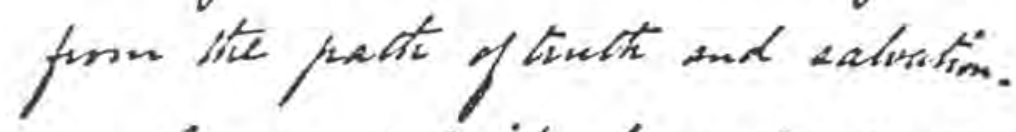

lvery mitividual of tion kation $\therefore$ is anviones of clek' a commesud frm 
132

ri.

than dorthot enppout thene, their old extativilued hust in thee sill be changid to fomel doublo and expluicoms and ane by are they will eqearate and will diverges from theri twe fait and unithen out of the strang fit parth.

In short the paffe feravi

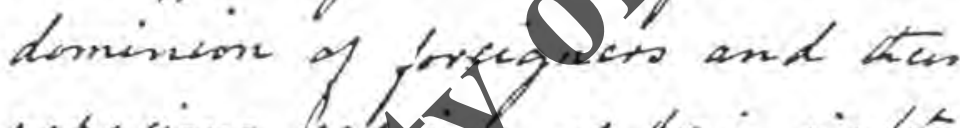

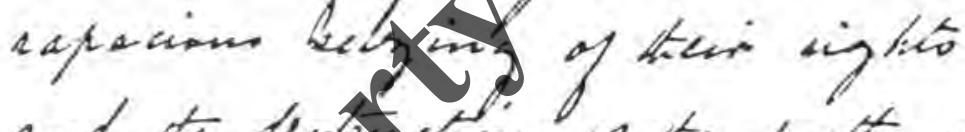
and the depylan of the pate of

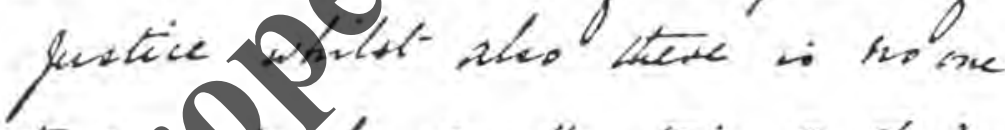
to kaf lic kaot atterition of thea:

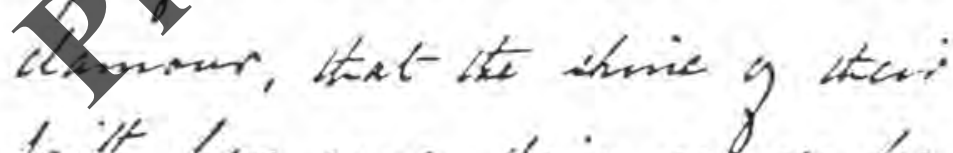
favith ho gone, their semis tine fecome dartiened, weir beley and disblip have beonne ferierted

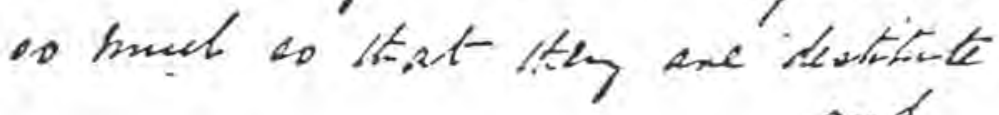


ril

131

all tisy had will he beotioned... "flem theme. The gas of this kation are lver Fanced Fonserdo thee..patkintarly at the linoment withen

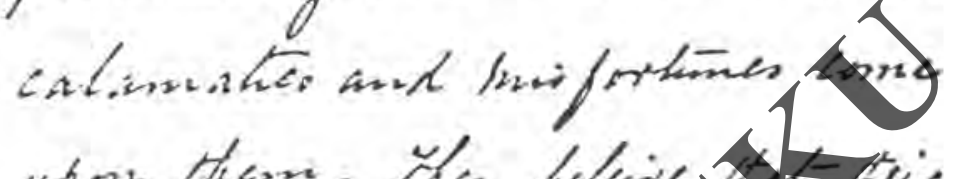

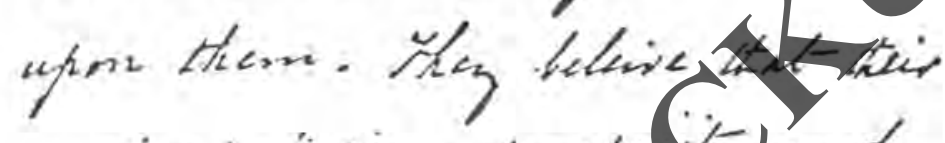

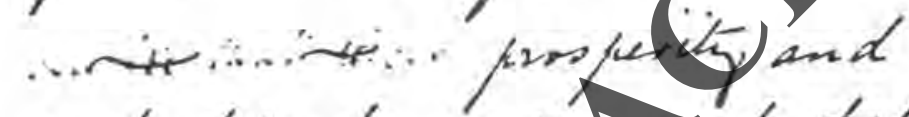
gorit degiend on thepand dat

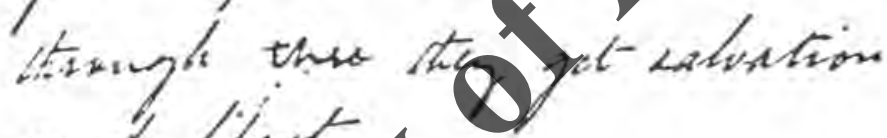
inest kiturtion

kizdeptor cirimetance

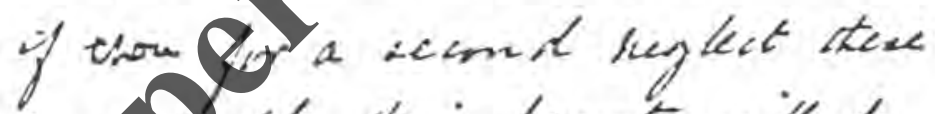

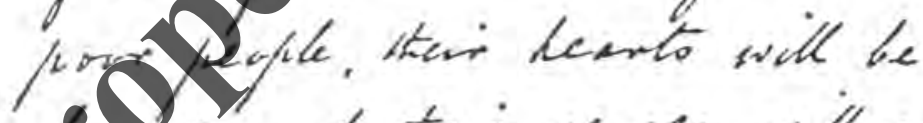
Pernt and ters semes will jet

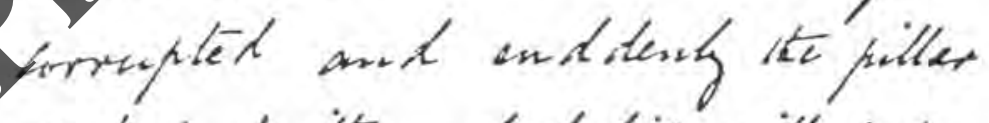

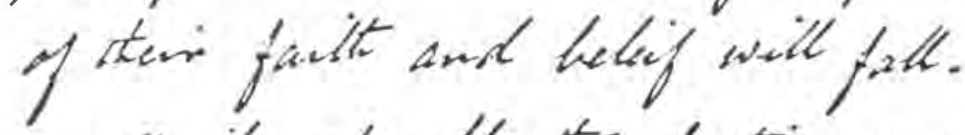
Verity stwoll hiso kition soer leconce mable of remone not the diobluivers of the celugion and 
dear of derifinces. He hith minoted

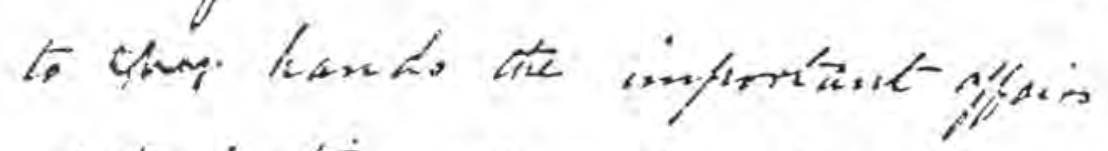

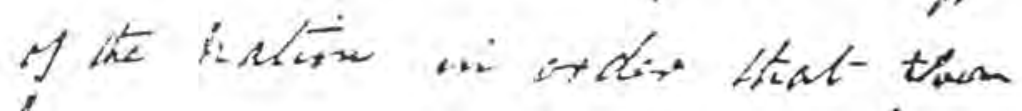

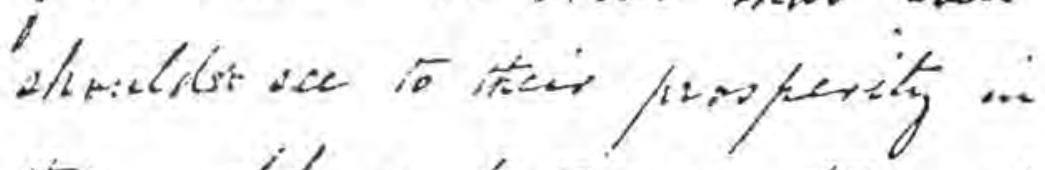

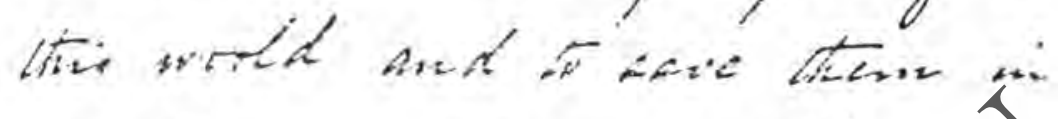

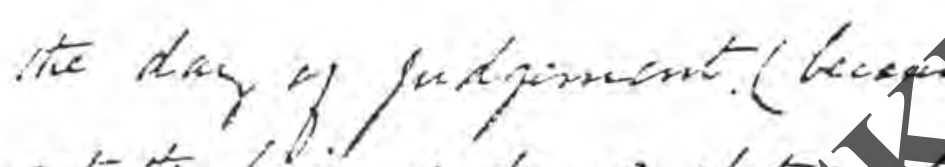

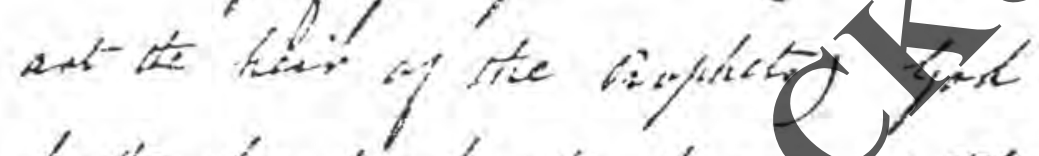
hat

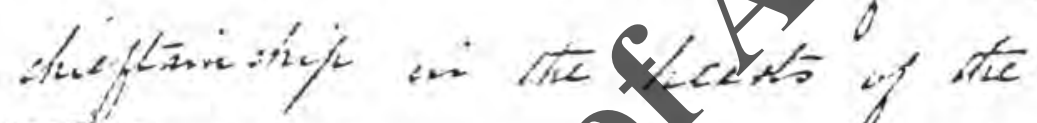
monte

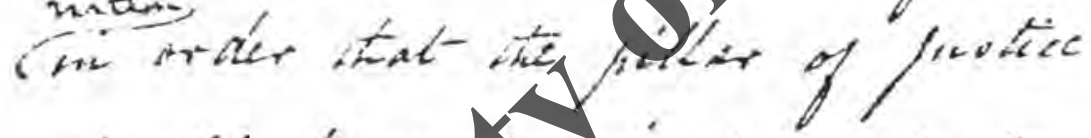

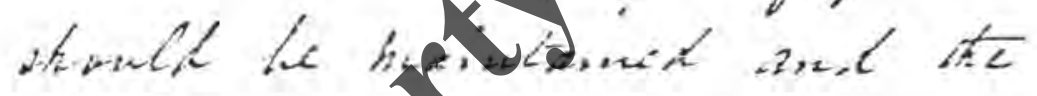

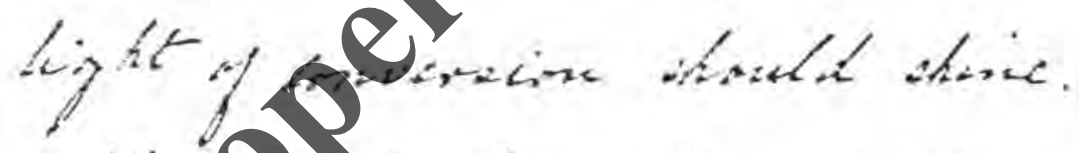

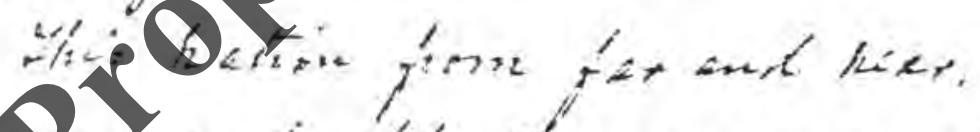

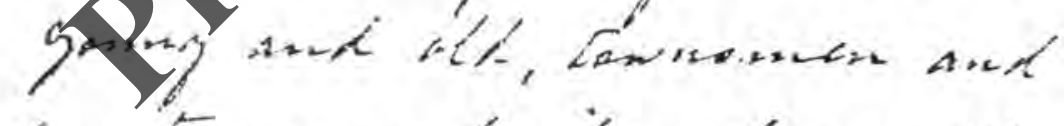

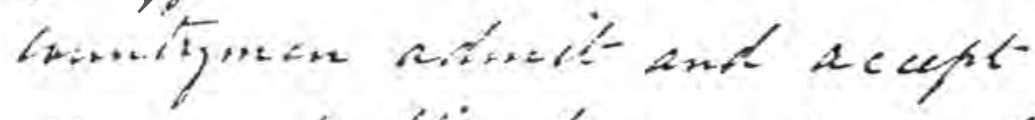

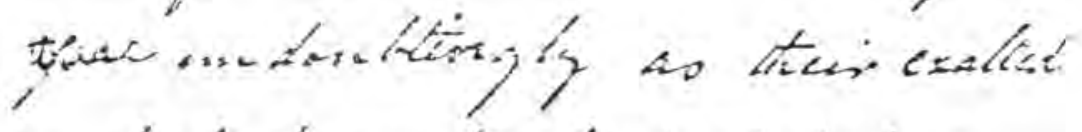
and gad went chici, ctat'ly tho.

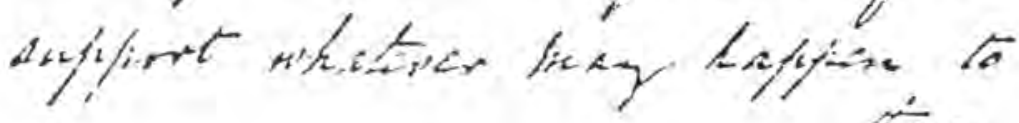
n... 


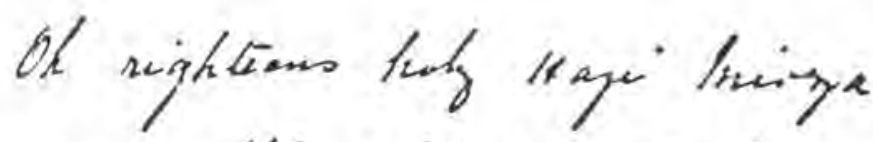
farsan thiragi: sage of the - -

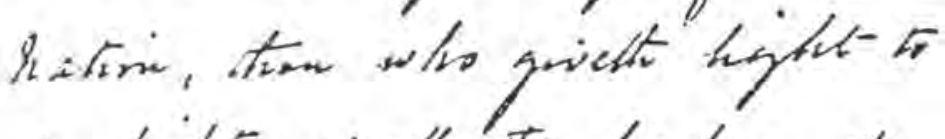
存 ligits of all the leaders, the

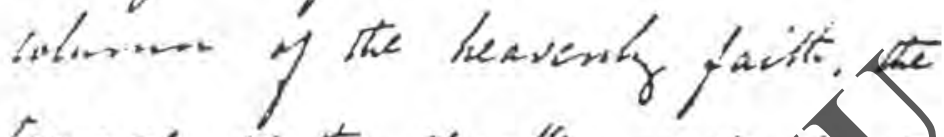
tongue of the chealler of th

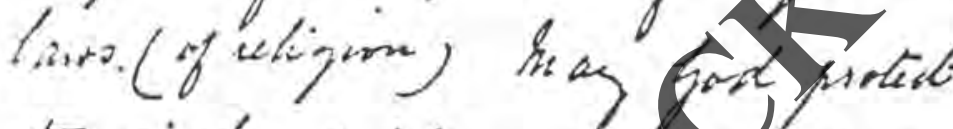

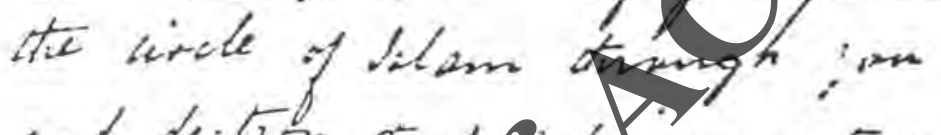

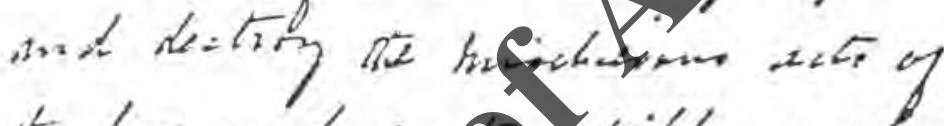

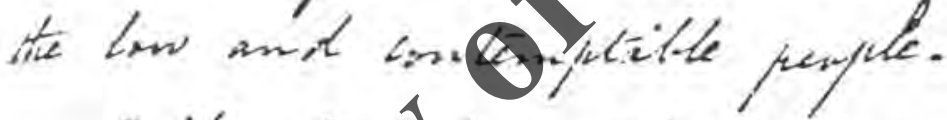

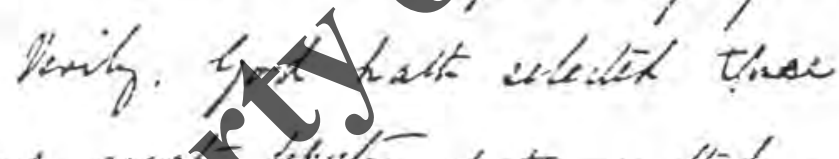

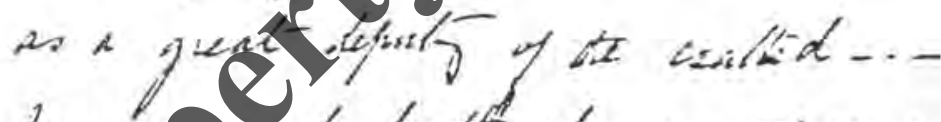

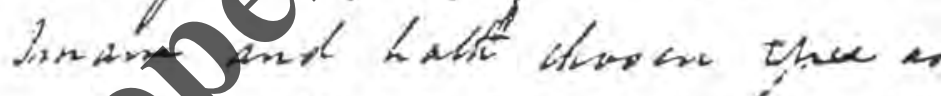
10 yation. An acomatin ato

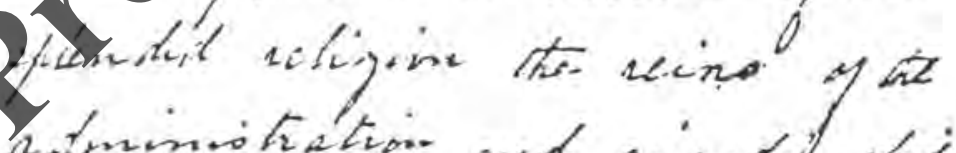

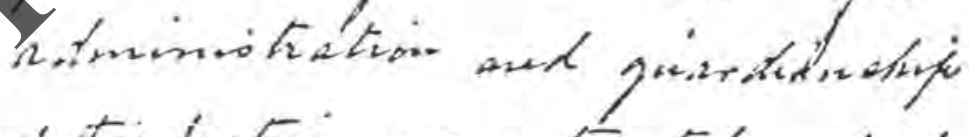

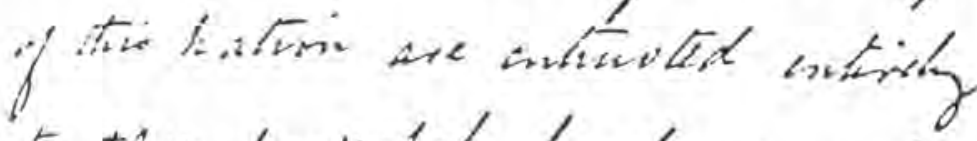
to thry. proverful hand, ai order

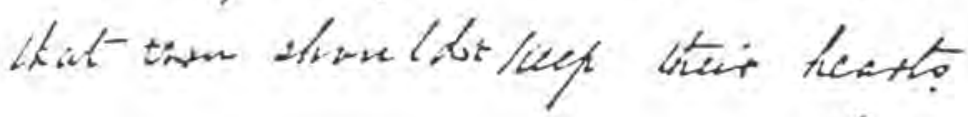
clesen 
loger Yambation of a leler from tyich. pand... Sin, finn Prusareh Io leyi hurga lowesen theisychief

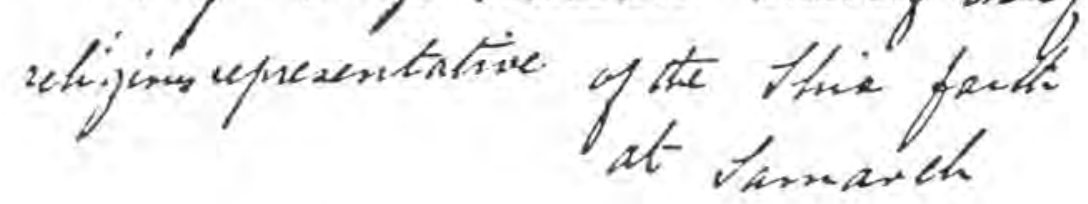

Yhis is a quat and hint tratimant of thrie.

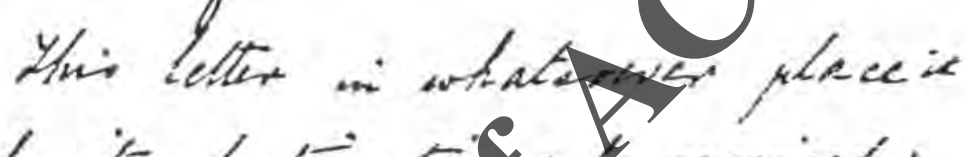
react ito detiniation (te seviex)

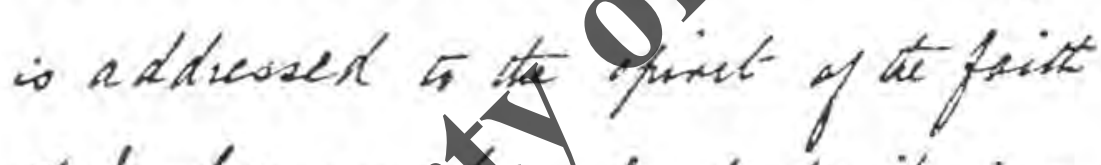
of frota ammex. In fact it 20 an

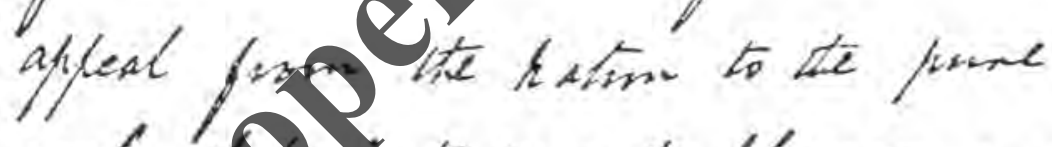

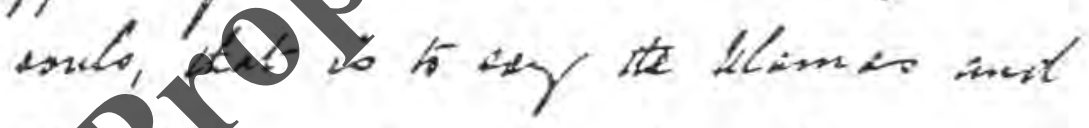

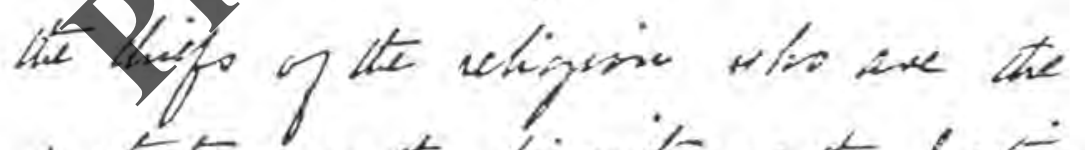

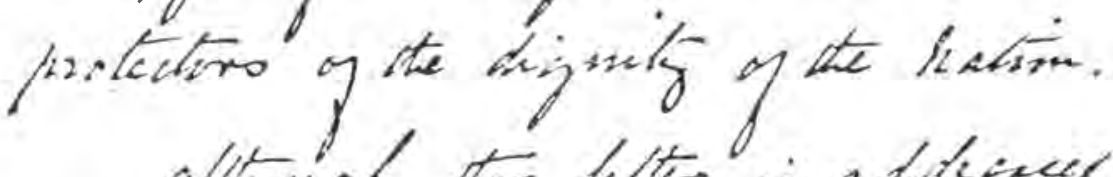

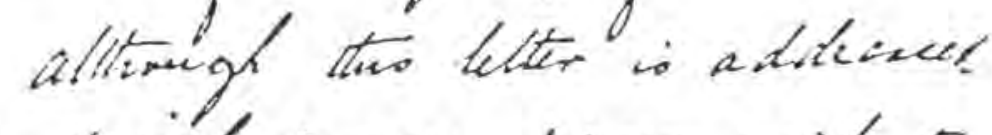

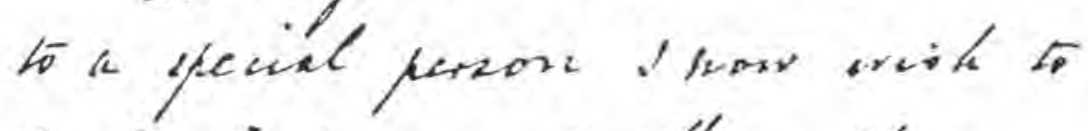

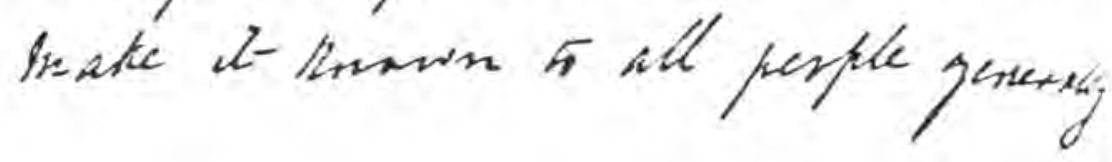




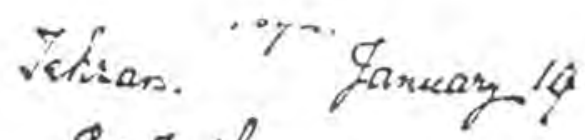

ori $\frac{D}{r}$ aracelío

ใ: $: 14$.

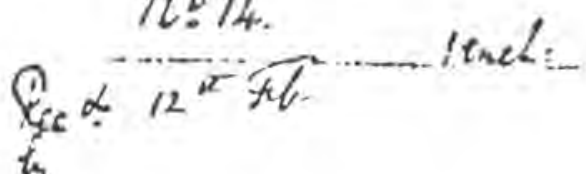

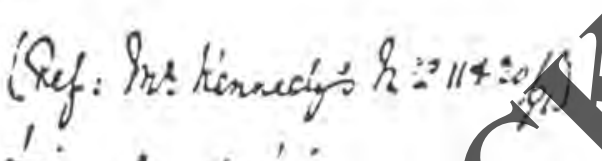

Jimal.ud. in

ranch of ketter ad atroped.

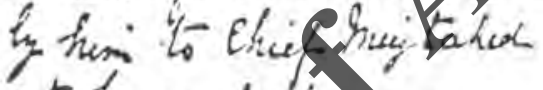

of tamauch aroturey

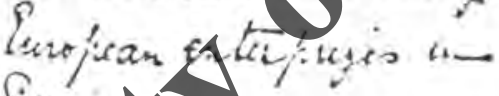

fit: :cen

$x, y$

Nise Luesen $^{\circ}$

-iviria fface for

0 Fines 
126

$r 17$

thorgh dicrounces in his lettes to Haji Giviga tastan thicis:

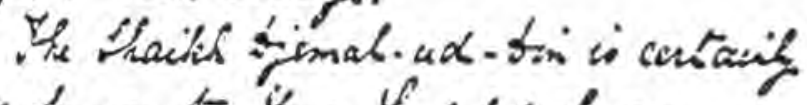
well terown to you dordahi brame.

truing the thise thad the trowe of

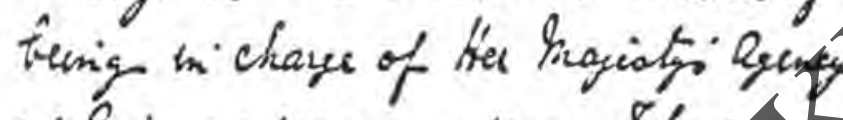
the Eais in is7s and 1879, Shadpoceren

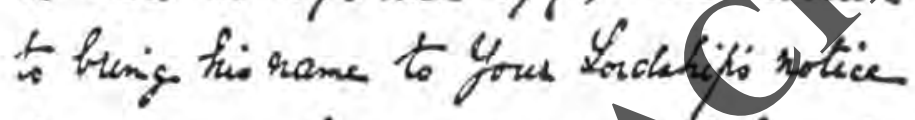
Es a freson who was caffrouses the in

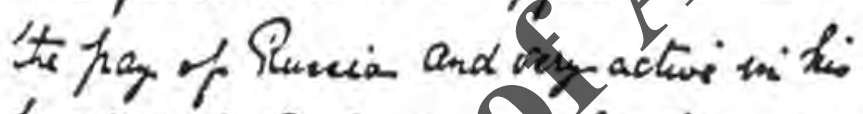
rotiting to licyland, ad las kennedy in

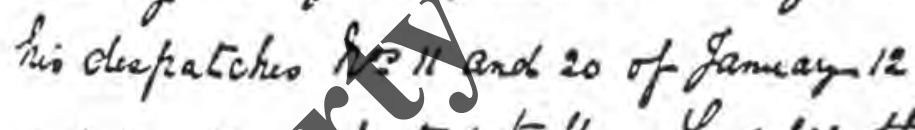

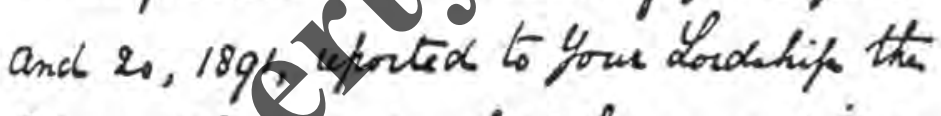
Cicumntas under which he was sxpelled fromplera:

If have the honous to be, with the irghent icopect, Mng Lood,

Your Loodatif:

mot obdeint

humble zerrant

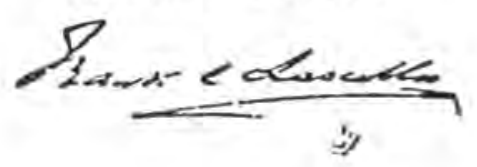


riv

125

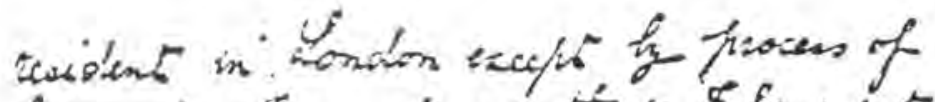
law, $\sin 5$ em afiaia' tiat Yhav hot

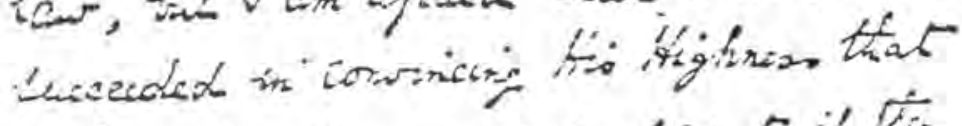

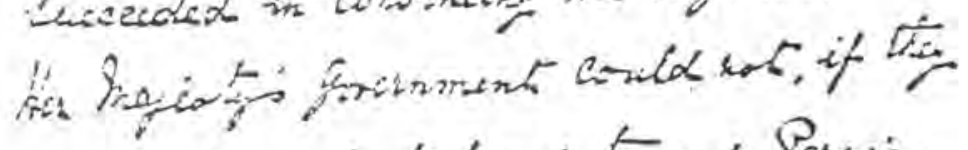

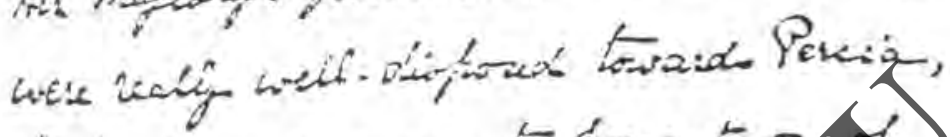

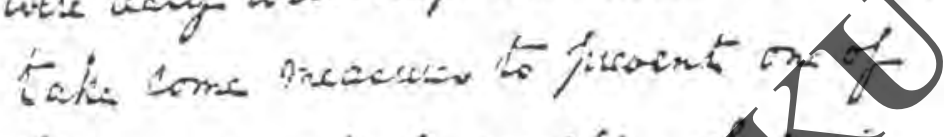
Sis Enimie ofienty acimibing por Wiorden.

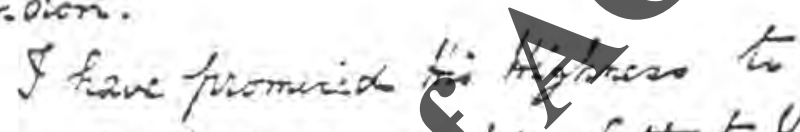

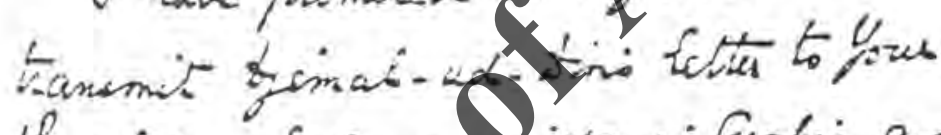

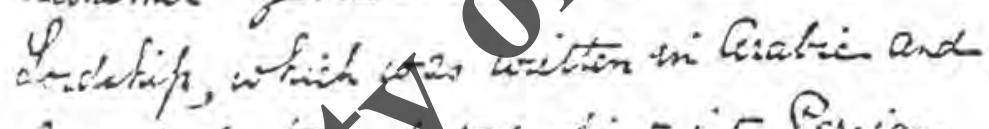

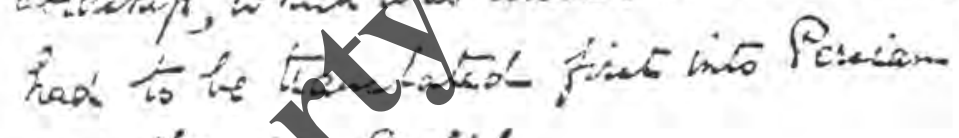

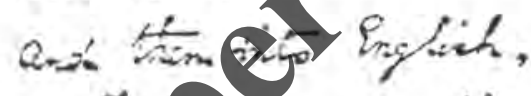

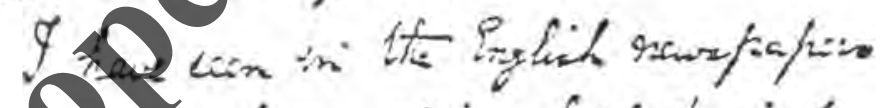

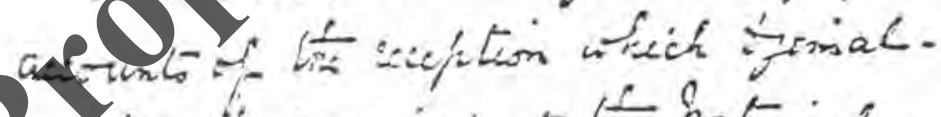

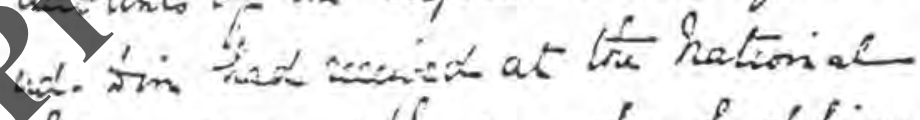

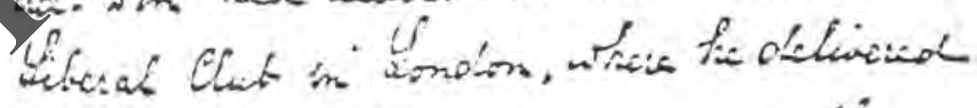

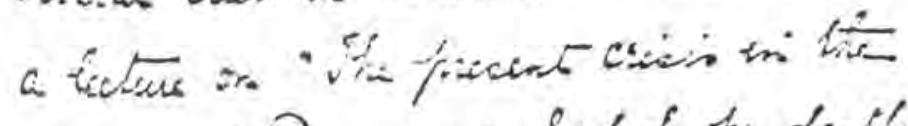

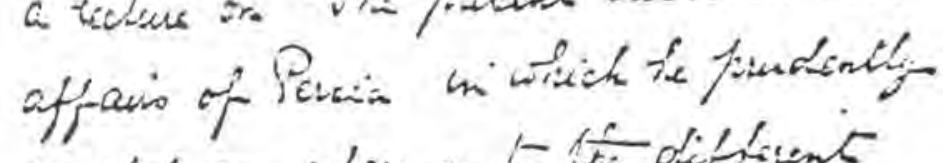

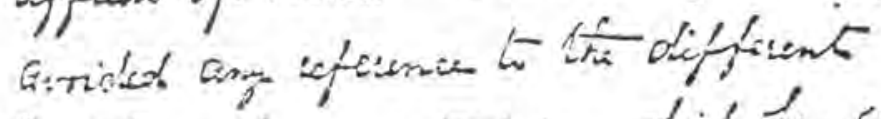

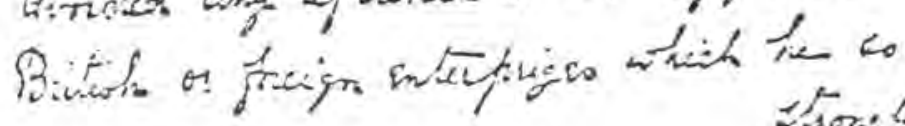
Exongl- 
124

rIA

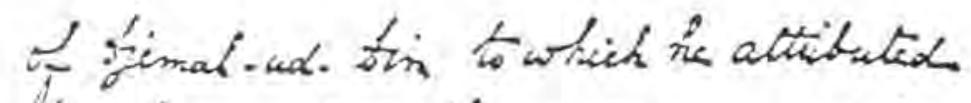
is a laje extent it cecentsorements

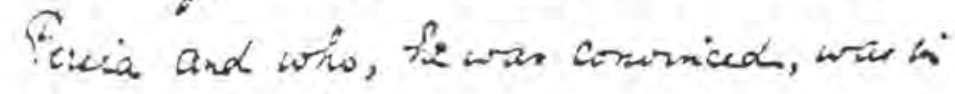

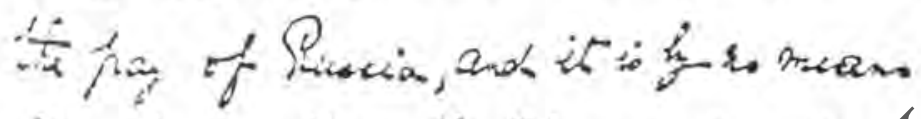

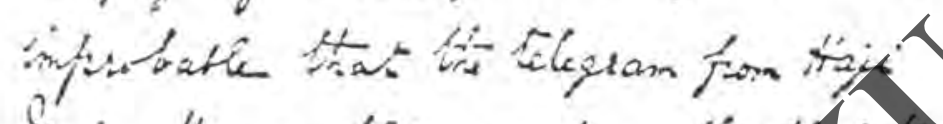

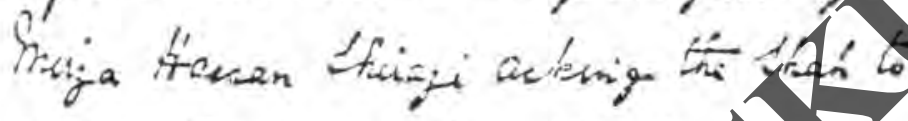

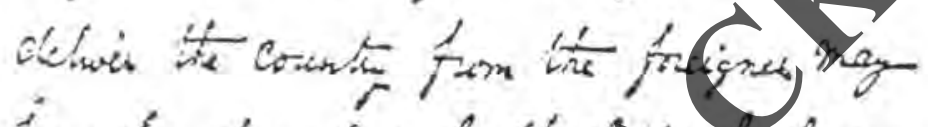

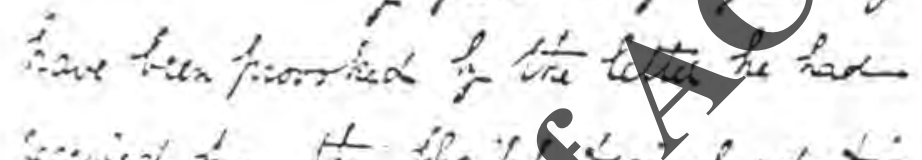

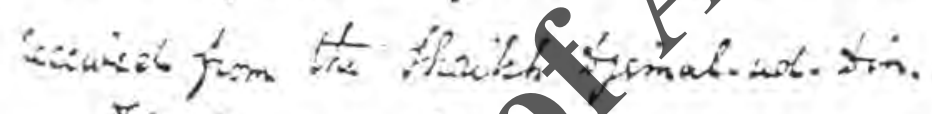

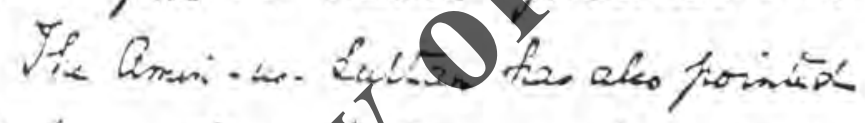

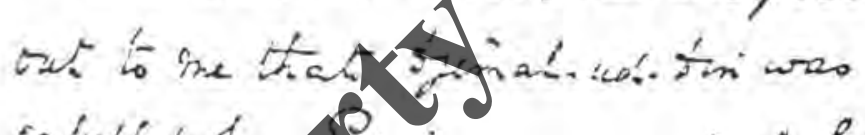

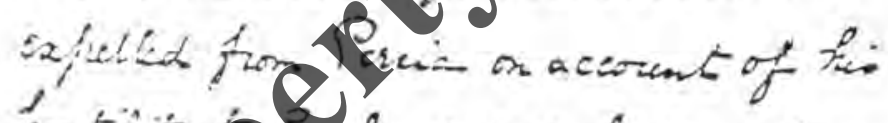

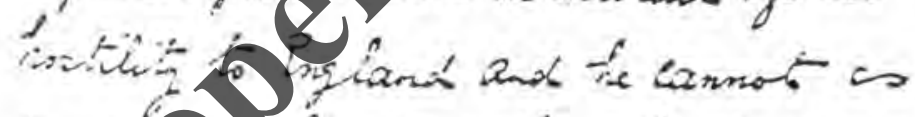

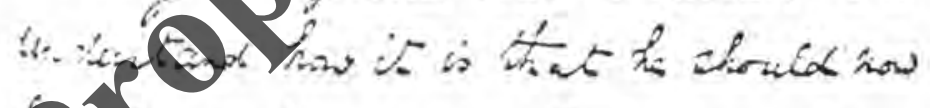

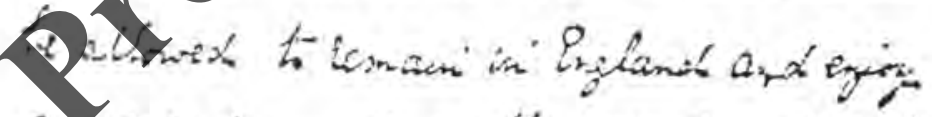

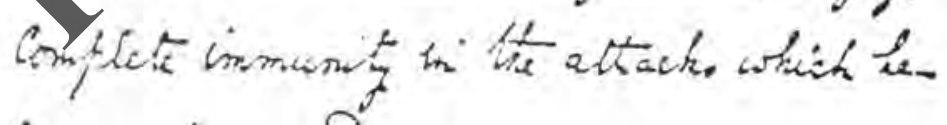
nov inckes on Peicio.

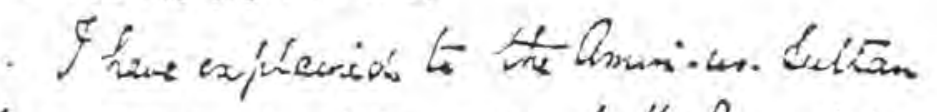

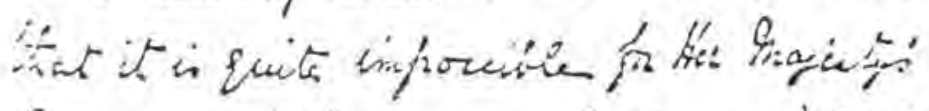

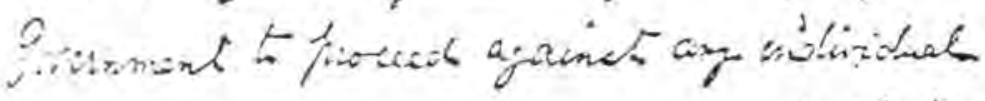
disident 
$\hat{n} \div / 4$.

Iefiar.

Ganuary. 19.1809.

Sison.

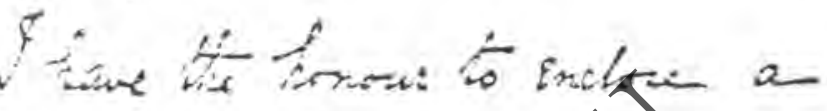

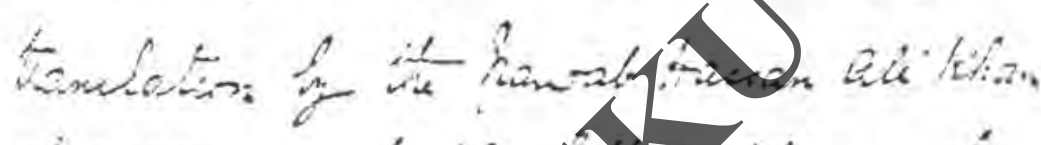

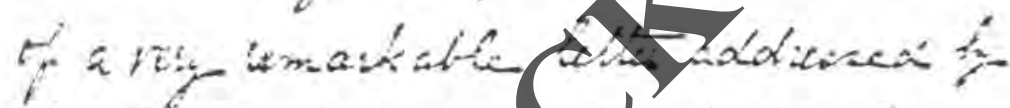

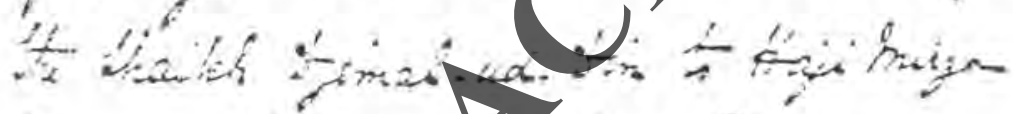

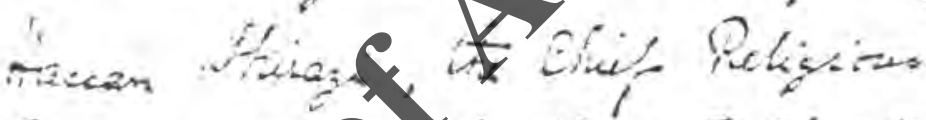

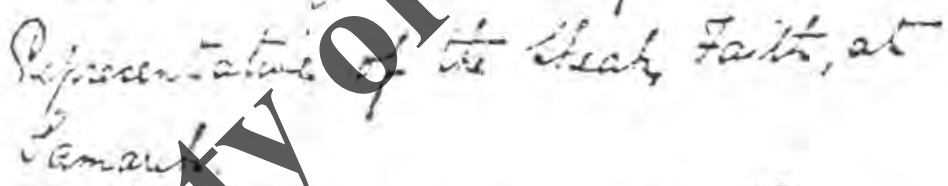

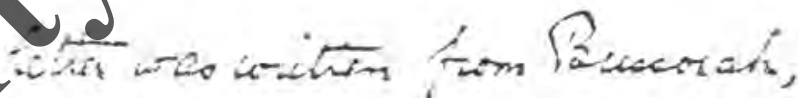

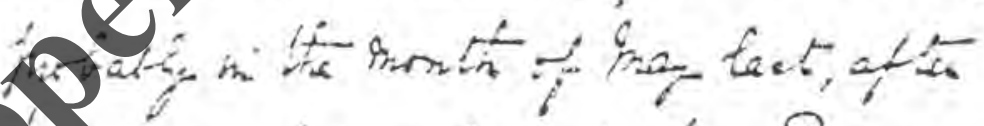

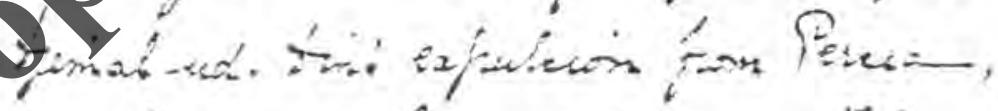

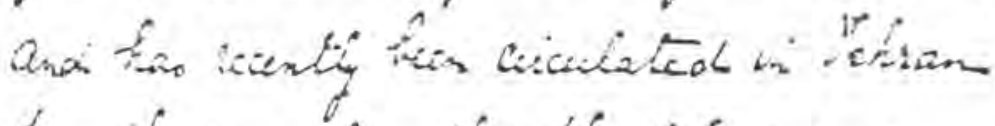

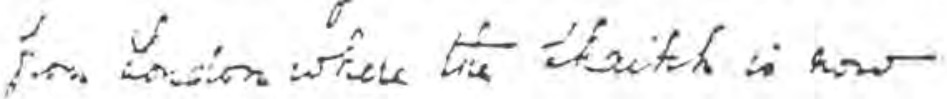
Widing.

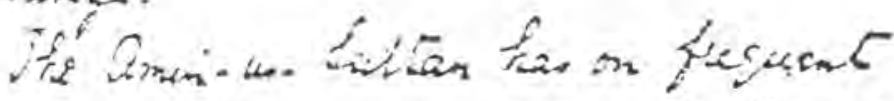

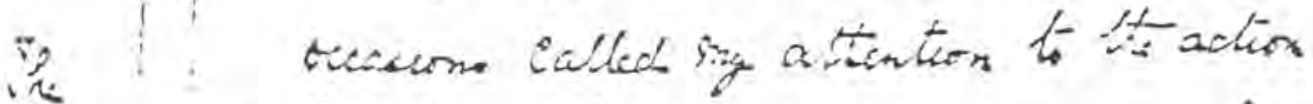

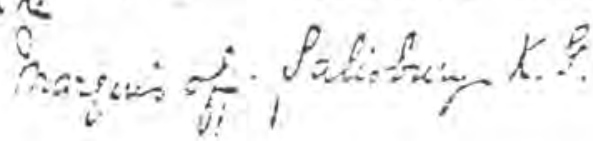




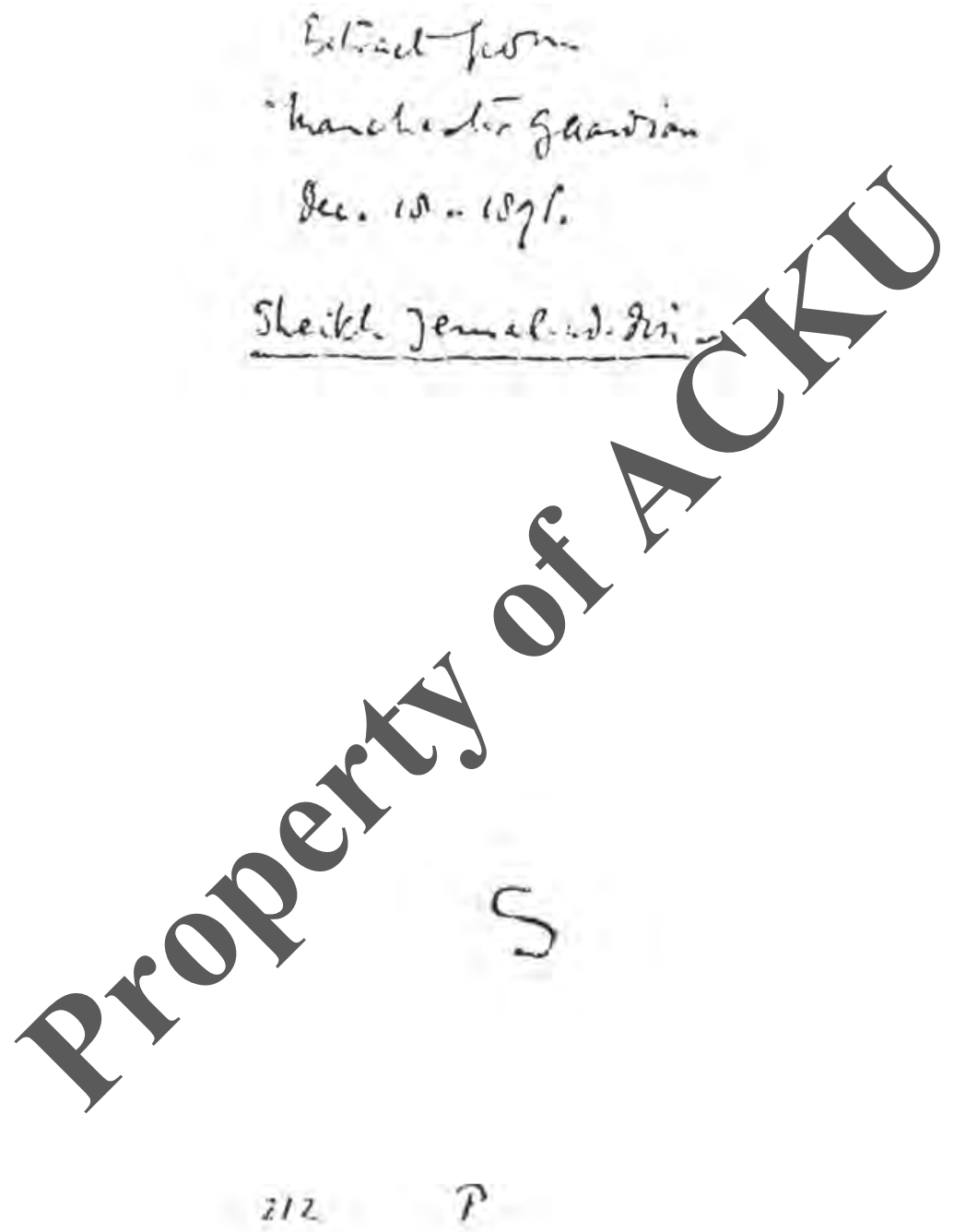




\section{Extract from}

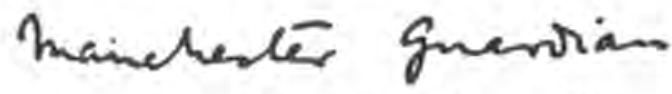

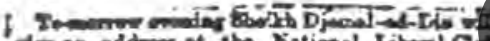

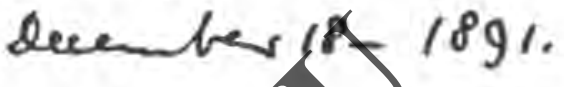

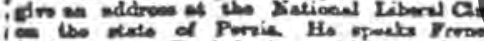

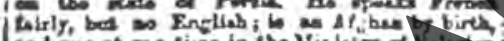

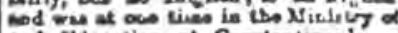

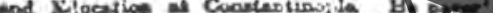

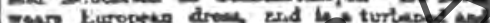

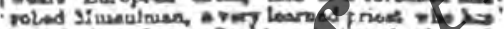

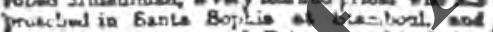

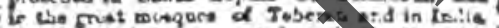

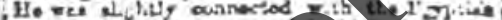

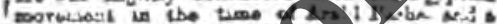

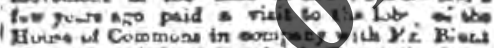

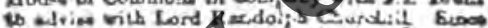

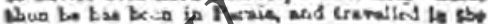

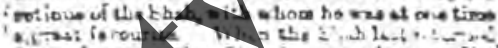

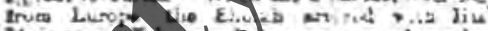

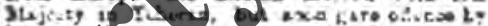

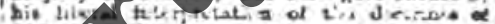

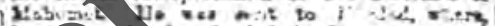

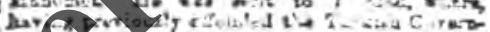

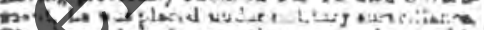

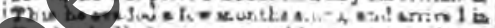

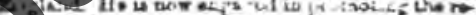

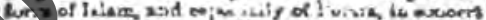

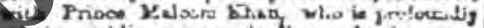

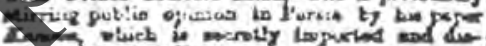

Itribated in etwat reapun

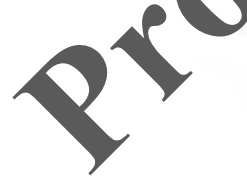




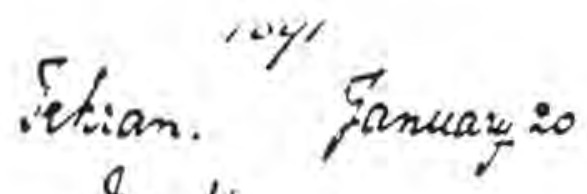

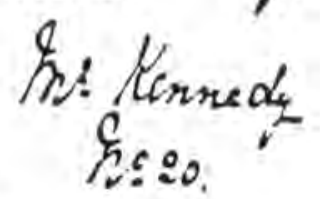

Pes $\alpha x_{\cos 13}$.

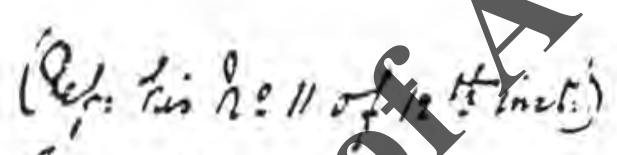

Jamai.kn $=0$

Shan paxtry sut tie 9xay

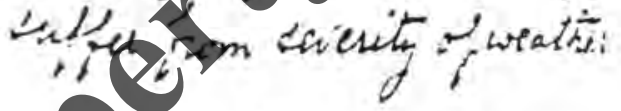

The husenownils

cinf Vinder fifice

sem at lavipule

29 
TTT

119

is is is exjoyment is excellent

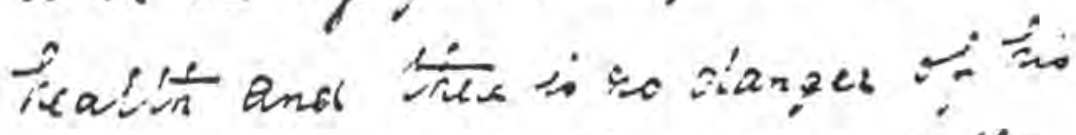

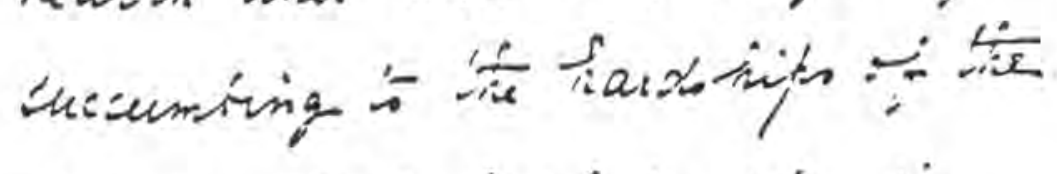
firemey ina' of piring inc Expervence is tis putic scapas

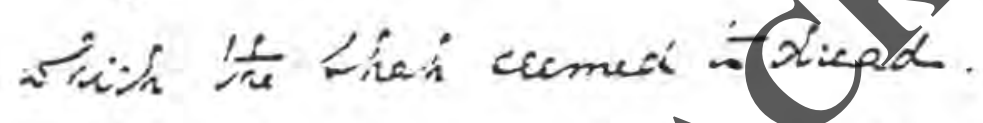

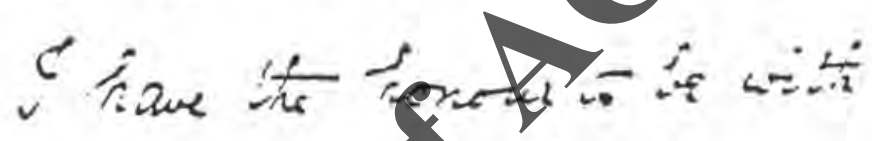

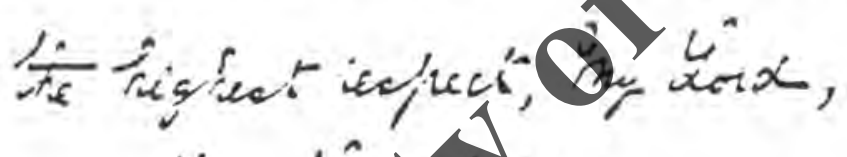
in limesto

Rat retolint

isconite ecisant ins? $11, \ldots \ldots . .$. 
118

rrt

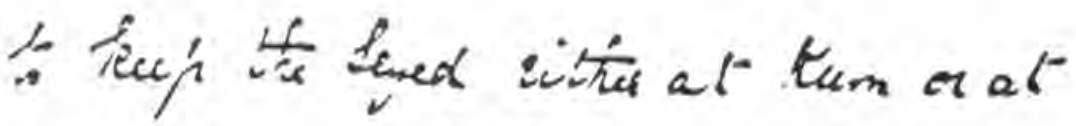

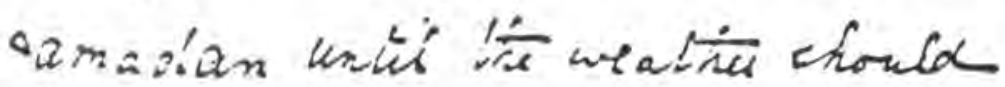

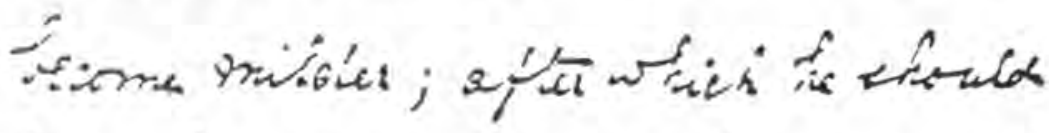

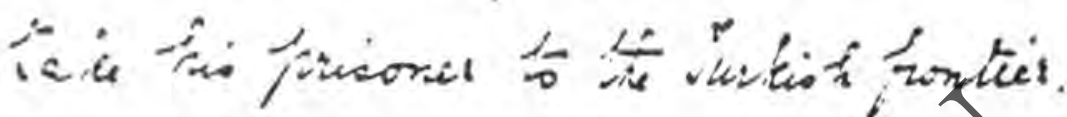

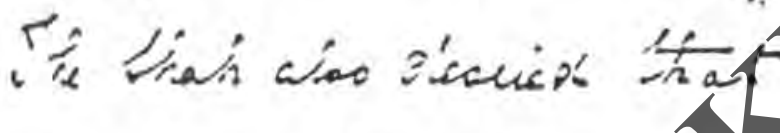

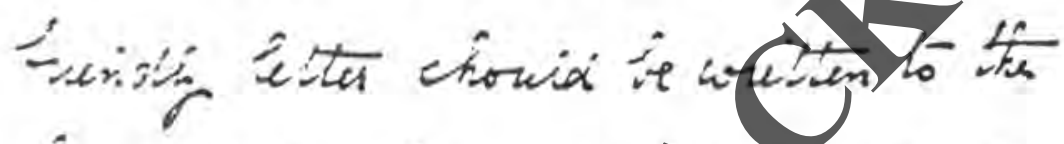

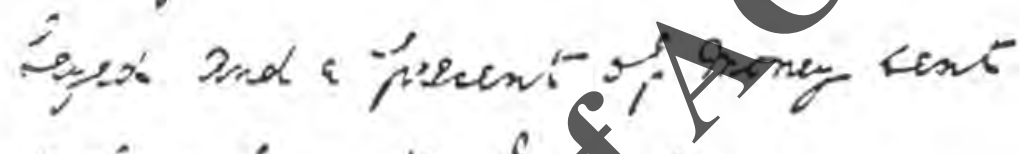
s im fum tir Srogry.

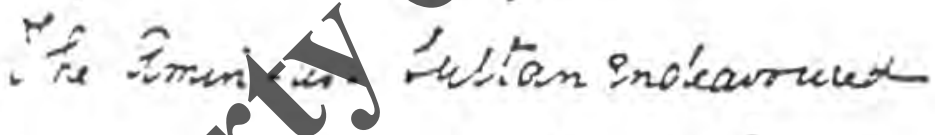

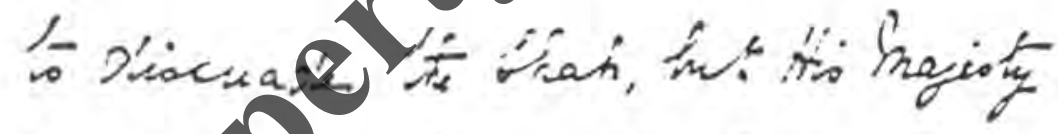

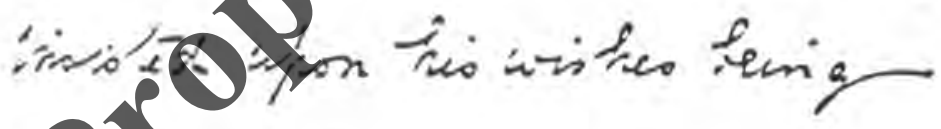

Pover zur.

Tis tistrew informs ane that

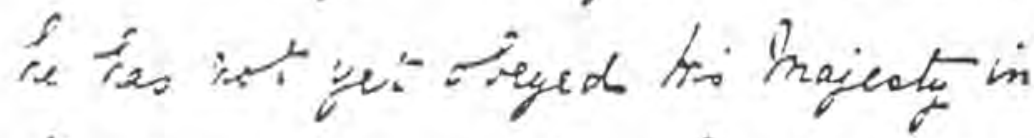

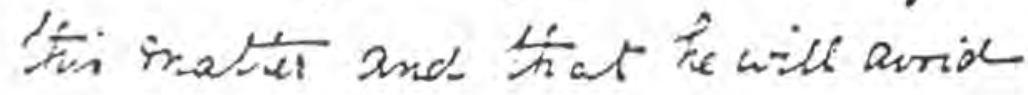

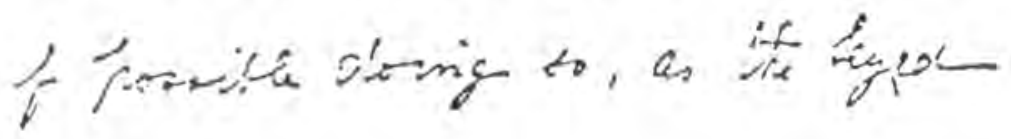


rro

117

refian.

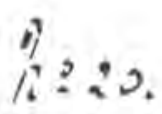

Émuam is. 1501.

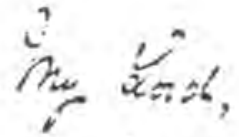

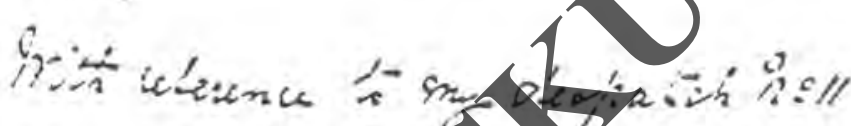

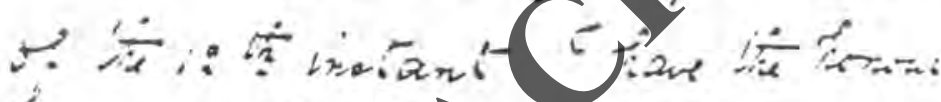

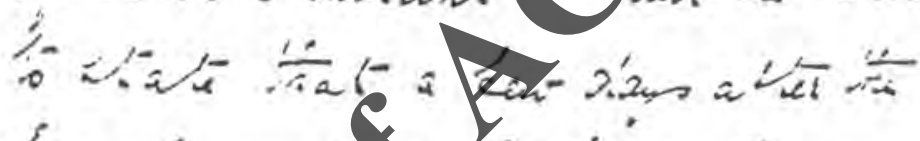

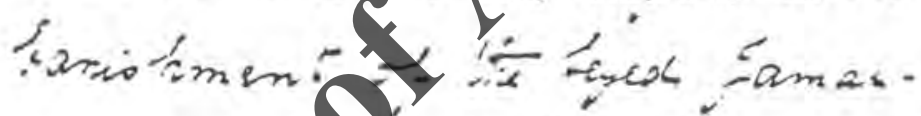

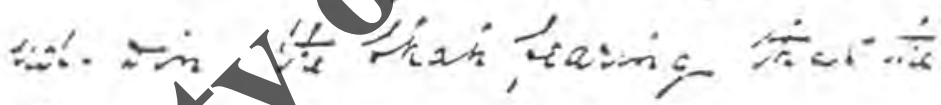

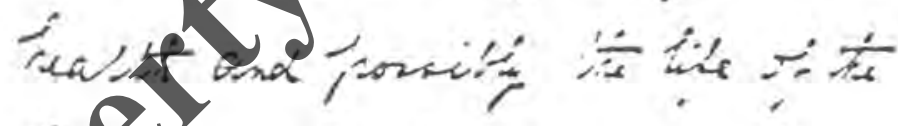

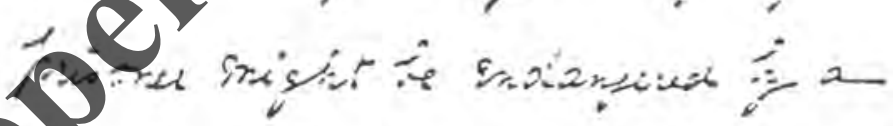

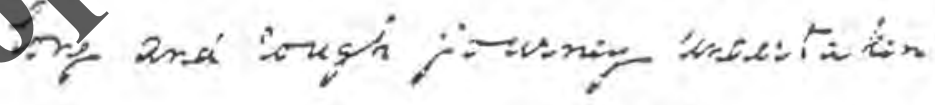

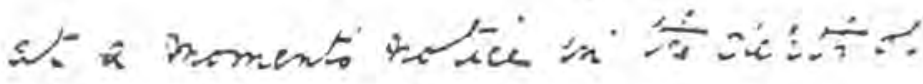

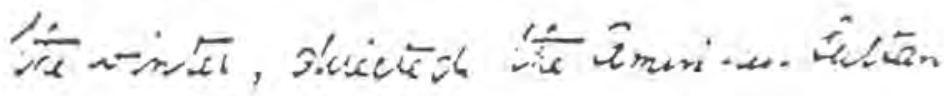

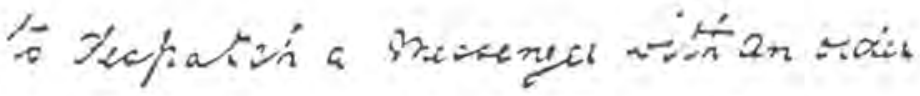

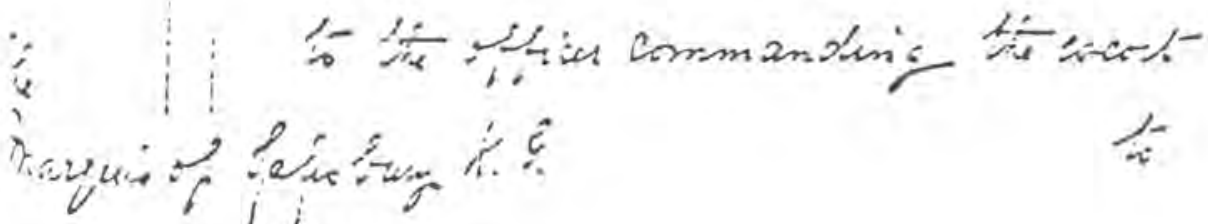


116

rry

$m^{2}$ KStmady, vekrom.

1.25. H. 6 .

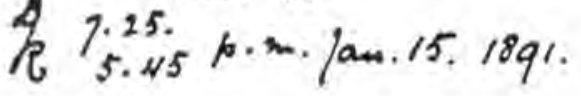

(His so. 5.)

lamal.ed. din

Tussian Recation emplaind

6 Onim that he was lanighes al instigation 1 trim. Lepatio. anim drmies this that panal is Puncers sulged. wh moder Pusper protiction fent Mudie.

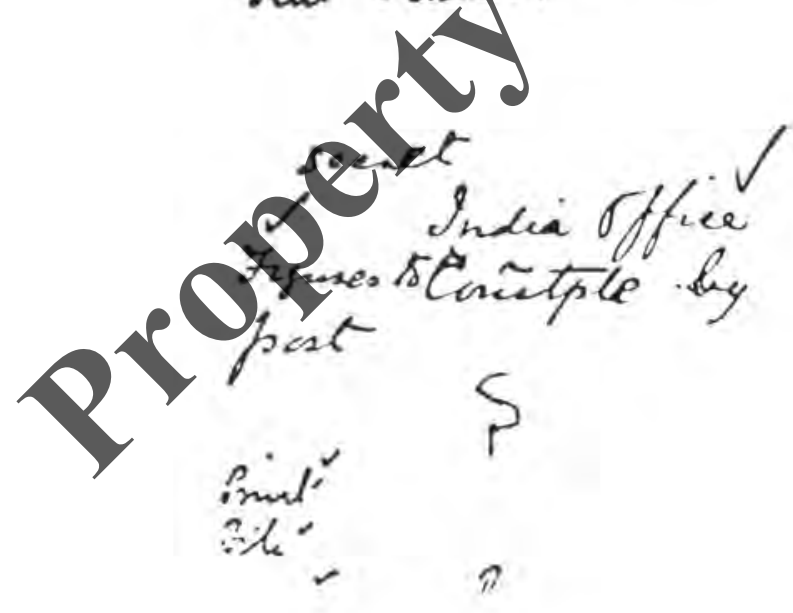


rry

115

Decpipher In: Kromedy Jehram of $7 \dot{5} 25$ p.m. $\tan \cdot 15 \cdot 180 x$. so. 6.

iny Eelegram Sto 5. Pussina legation has implemied to Amin Kagt. Cormal.ed din has

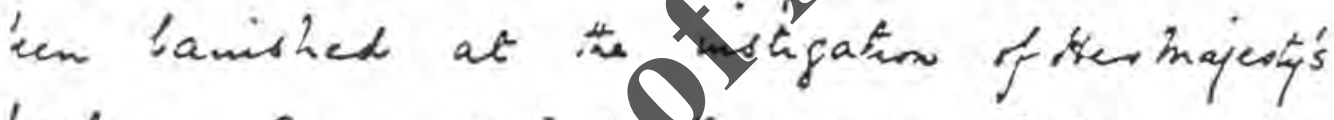
tepation Conmer ásnige thes and pointed ont iat he is a gaten subjed not under Pussian

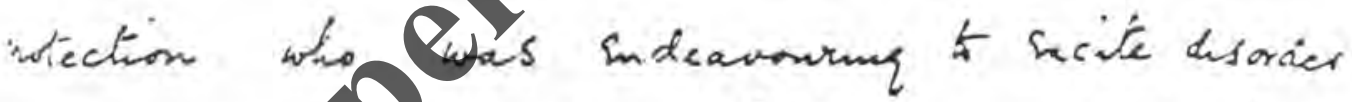
$\rightarrow$ comest. Animess. Sultan addet thatit

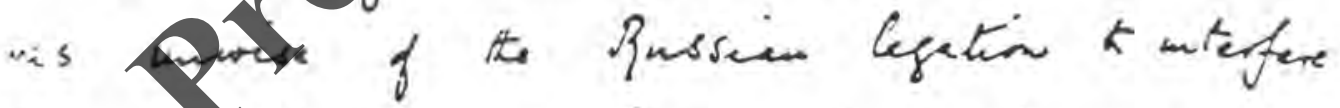
inen inffificially un favour of so dangerousa inshivator. Sent 5 india. 
114

ris

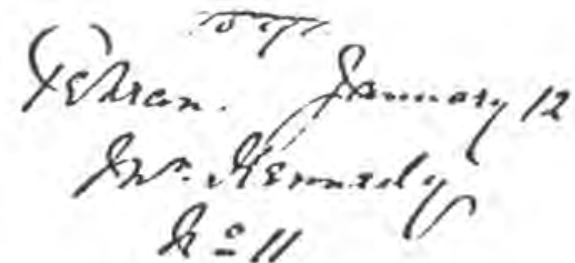

$\% 30 \div 13$

3 promes:

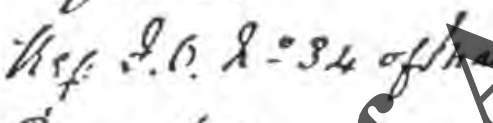

Syed bismat.

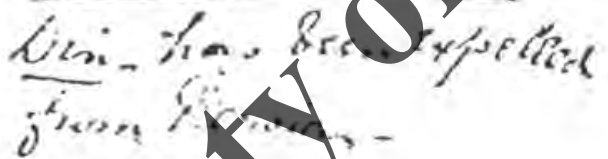

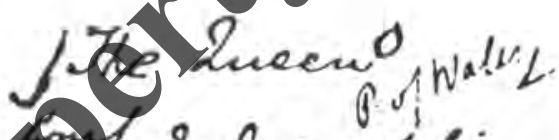

- Indea office seen at fariffite.

S 
rrq

113

to drae no. Ifai frajestin sau foit; \&ut that the fact of

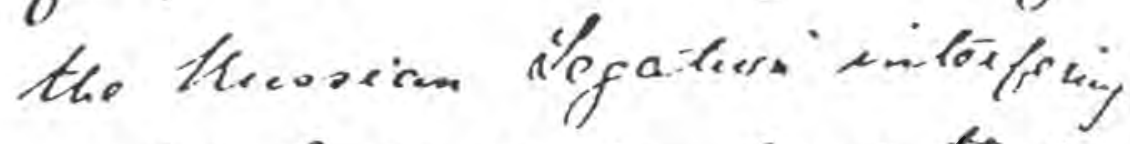
on his behape kerdered fat

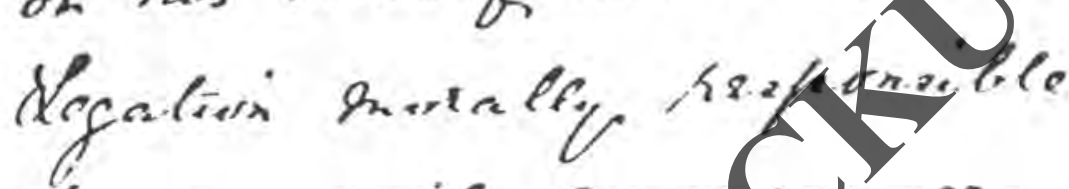

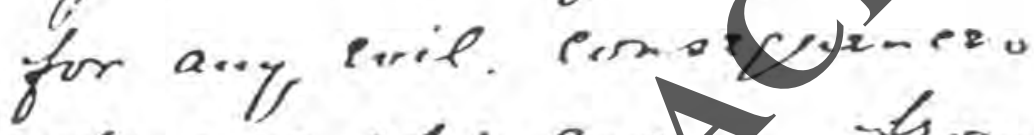

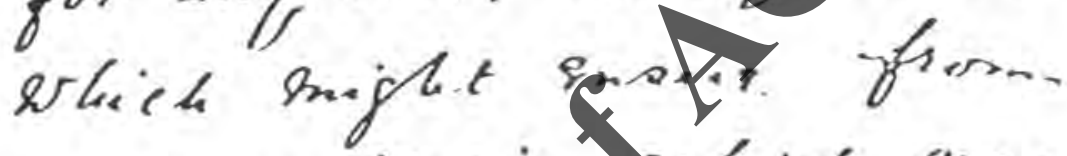
sacrel agitatingerbich aros

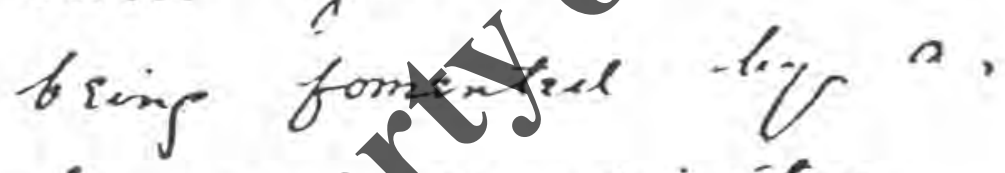
dangeraresernopisator.

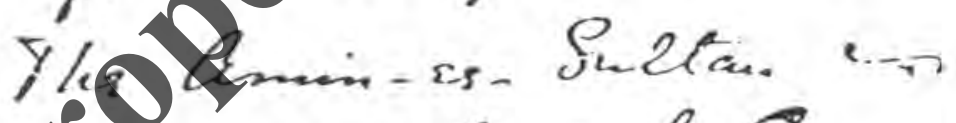

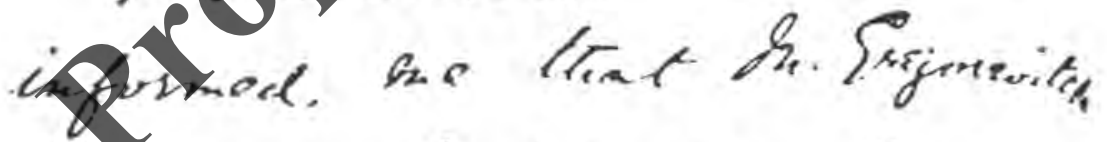
haulesed. to dissernor ane

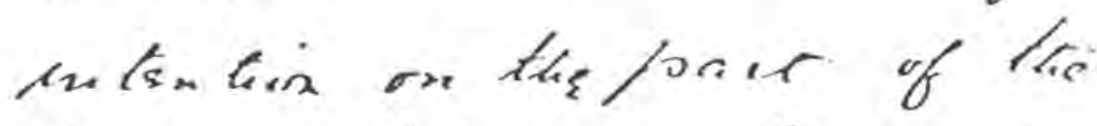

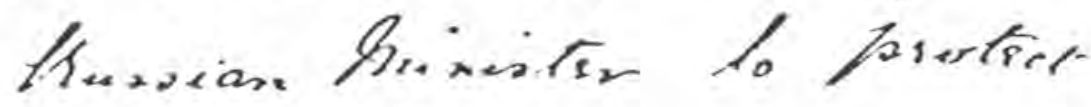
the Sygd.

$R T$ 
112

rr.

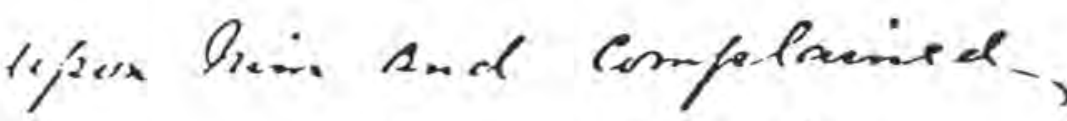
tleat the Sipger had bisan

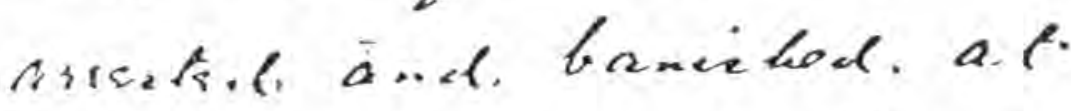

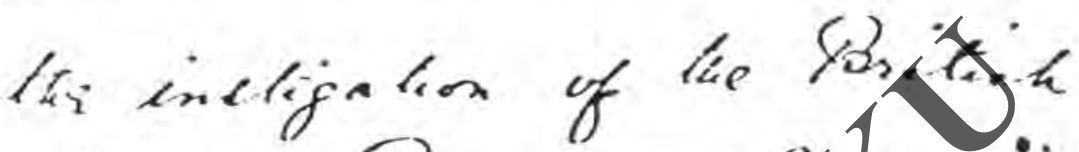

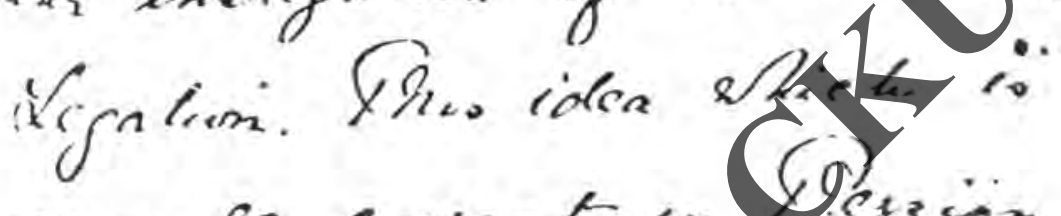
grasuffepessent

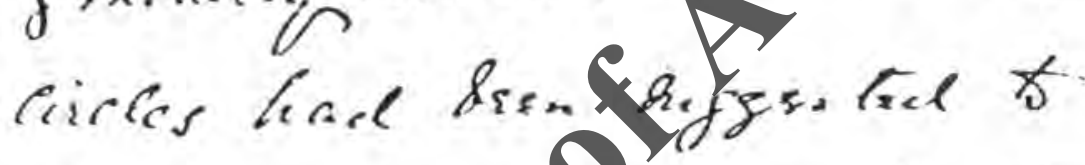

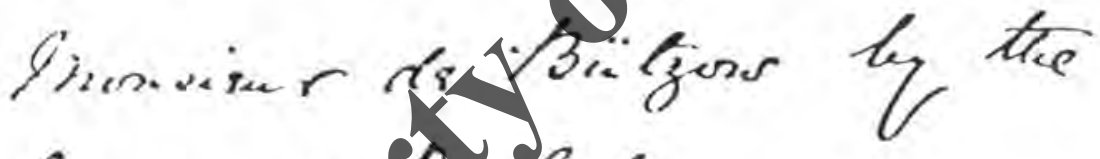
An... sel clowlak.

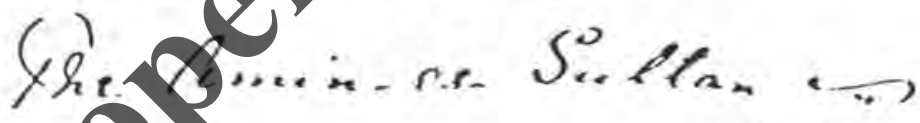
singefertern rey demed the:

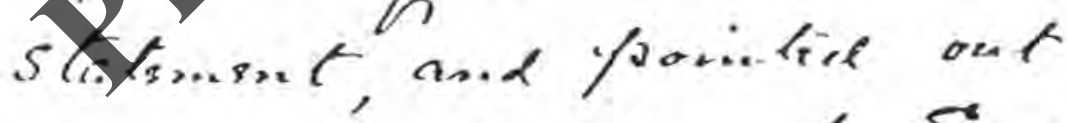

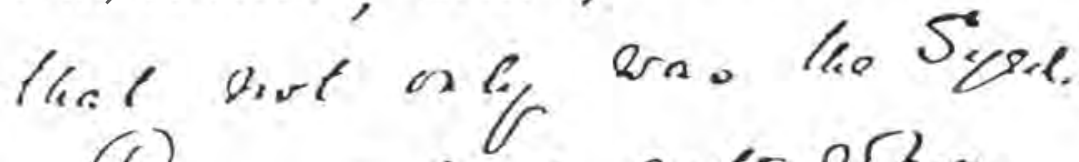
a fervens sulizel, arite arhersu. lac Slask aras at lebientep

1. 
rri

111

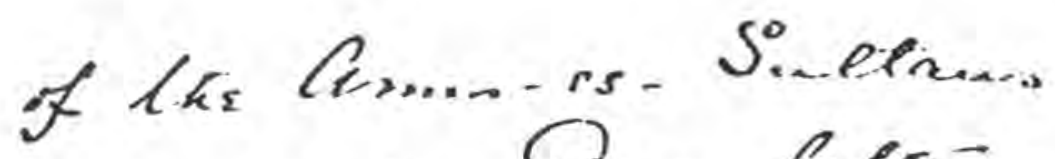

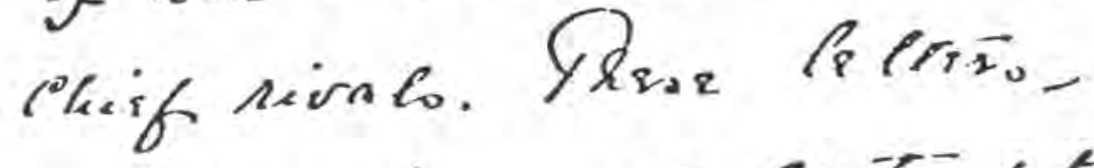
spacal on tarms of corterpot and desiscion of lae shatethe A.min-seolaer ministas.

ollewe the herper to GI, sith the hightor hropect;

luftord,

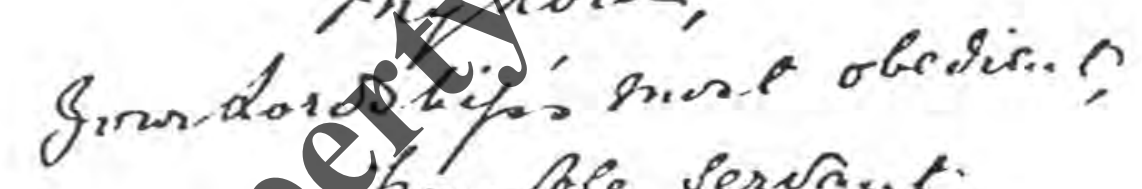

Qhumbles serract; Roovkenmany

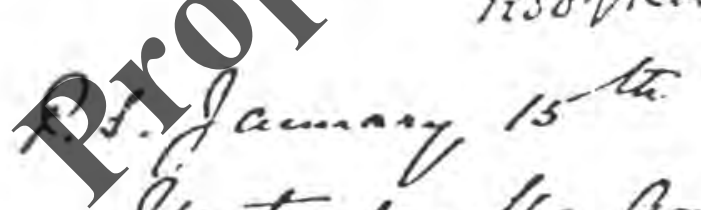

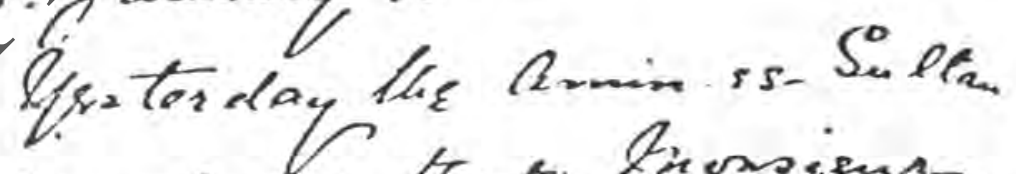
enformed, he that linessiens, Eigurevates, hicgoman of les Hewvion Sfgation, thath calfod iefor. 
110

PSt sidravoresed to riast, bur sosintually the syed way, aftir a phup stinggle, frlaced. on a horst and sint off socosted tey thints soward vermanchak, whence thesule

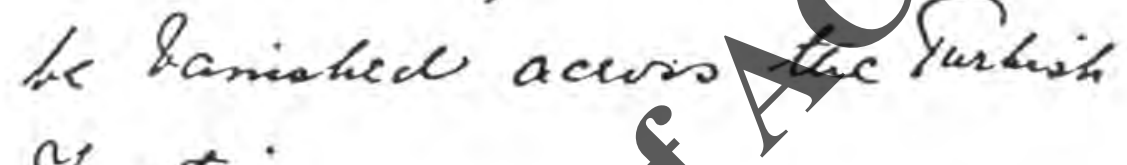
erratis.

Some letedis have bern formd in ter possessuis of the Lefself Bhich compromine

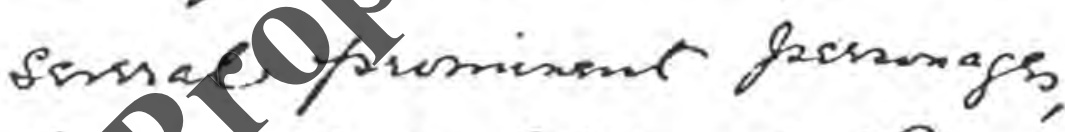
the zyst butable of I Sum no the Rmai-sel- Orwer.h, Priniats of Post and President of the Comail, who is ne of 
rrr

109

lnanary, fo as to avand at possible disturbance, hoss stpricially as the Syid had takm "buee" or Isfugt al the shisis of SAdatadue Azm, clowe. to Pran.

Yhe Sreel gras accendincly arestiel aid fasdem into Shich ugfluad berm snticed and bech, tho' hut actualy szte the phere bondaris extennlly assudend to br a frlace of refager. A slight desturbance was caused by the Sigd and her hepportes, $0<k_{0}$ 
108

TrT

having surssndeced. Persian intreats into Singlish hands.

Fhe Lhat ding cervinge that the rotanim Syals Samal-ud-Din sas fut inztajator, if loote ackine wists of the fereor aidened that he stunted be at once. ixpelecel to conntry anch. Shishoutin kafweed to lis $Q_{1}$ to the Amin-ss-Sultans cosice that thes Expoulair shonled be sffectul hi as quirt and unvelentations Praner 
rro

107

reㅇ‥

Pruan

Sam.ay 12.189.

hry dord,

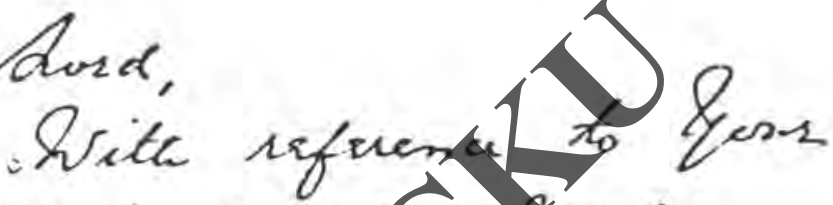

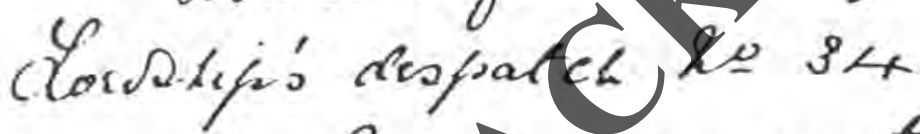
secet of Shard ance to

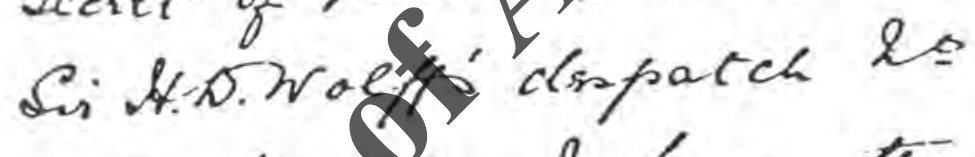

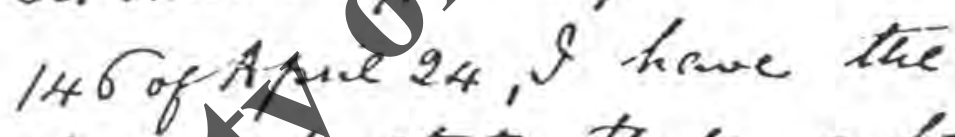
hom dol ago, the Shath Receivid Q. anomprims leter guark abming stes hajisty for the singoremment of Yessia and the smore speciale for harguis of faliabury kaving corp. 
106

rry

hor. Kemnedy

4 gam. $11.18 \mathrm{gl}$

$x_{1} 6$ : 7.0. deot - ho.34/g0

aharive ancompmon wher 10 thal.

the bering convinges

thet Djernal nd

is the nvitene $\mathrm{t}$.

hed him toty

and sent is

olecure in wille hes?

banighest "wos

Juzkth froution

Yadia Chace

"Cimpar

5

sopies 
rry

105

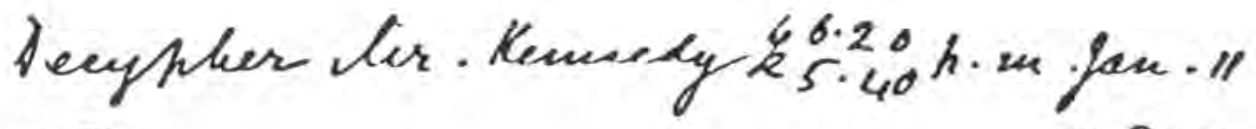
No5

1891

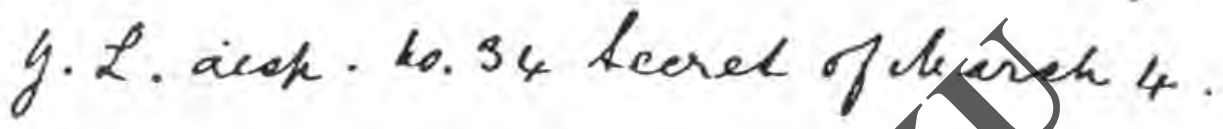

thath received a few dachergo an

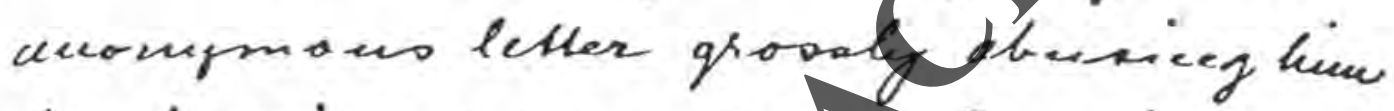
for having eurrender Pet Persein. inserects to Seeplayo. H.m. Beniz convineed that ffe notosions byed Xjinal-ul- J the awthor, cansed him to be reged at the shrime nere Hehran Plere he bad baken refugtail sent us der a- monculed cecost to thersmanshat, whoure he will be havished arovo turbects frantier.

(tent \& hudia). 
104

rra

lehtan deper 24 isa stwoeff.

$$
\text { ho } 146
$$

ve, man 12

$\mathrm{By}$

Limecustin

Opincing of a.m

Sultán re. 1,

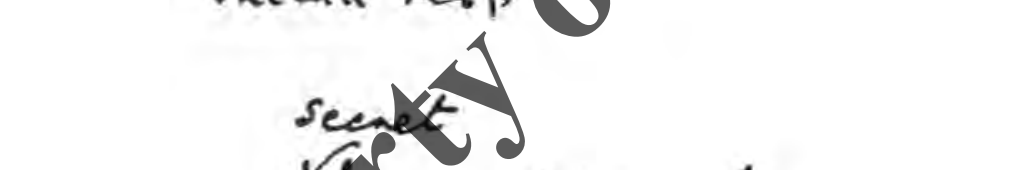

Strii offic with

S

$2 G$ 
rra

103

Enlent a suboratinale of the Sraxe vigin:

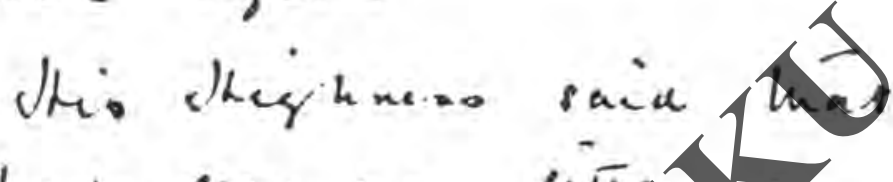
he had reen a. kitag as

porsicee of the Sheiks band. had mi.cracele the orari: to pive -k.... Shint to lence inting an he crued gain wofking by rernaining

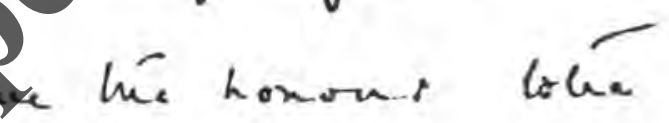

wisi hat hequest reobees. hin Lis a ,

Gouthouthlue inost obebeens Jurnale Servants.

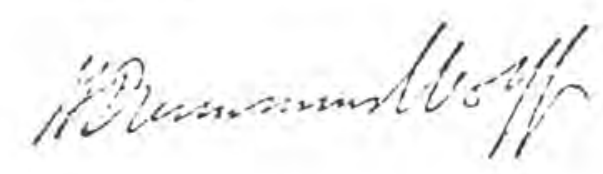


102

rt.

to 146

Ithian

Apree $24 \cdot$ if 90

ky Lang.

bits reference tó yores Lonshipe depacien koj 34 of lnorec 4 in inarbete feeret. Ihace the houfot, Motrace

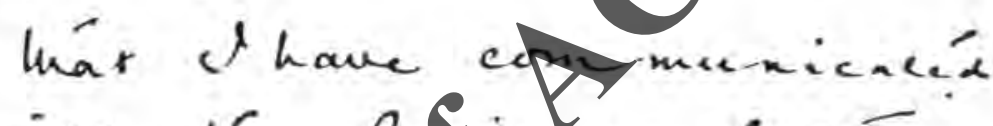
with the Grkin.c. Suctan respecting Qo presince at 9rukad of the sheike farulotution. I he Sheik a inforing at he howse of Areti Insammade Jassan the proprecers of the linae raclutay and. is a cerciain

veght ctorom ravee

cxcens-

harmese frakiburgeres

n.

$r$

ก 
ris

101

ropo

th. Relerabues beb.7. fii R.hories

$15 \div 40$

fecuet

RI hebiz.

byherLaw

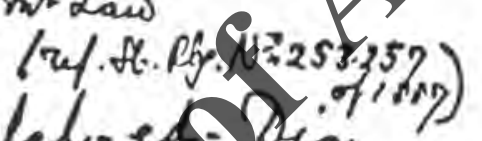

Hehal-exdoin

Persig ondiw. vays he is nov

in Reprorditriguizagets

Inford: Le recomimeats

1icexpulcin therde quitty.

Copy angs

intt. walff

$\sqrt{ }$ 
100

vito Rusian arnw.

Ohave tho hosmu to he

witt the highest reafect.

hyy Lard,

Your Lordshifin mat obe dients

ret 
rT

99

know that he was the author of. the sensational telegram from Tiflis descriptive of the presentation to the Shat of a Persian. Bike by fir Henry rolf ff, the corraguent riot and the flight of Hi j. Finite to Tabrez. It wouptear that the sheikh is a Marian subject, or at least that he was born there, of Affshancterents and ny colleague is tho of opinion that the Perse authorities should expel Q without lo as of time, though suety and circumspectly so thai he should not have a pretext for posing as a victim and rucking into 
98

rit

and witt great anxiety lest it should affear that the information came frow him, that he had reason to know that the their was at prevent in Persia doing munch mischief toter. Se knew for certain that when here the individual had been frequently in communication with hosier zinovieff. He is now foxing at Tot en as under the protection of Russia, and is undoubtedly trying to further Russian interests there. Riga hammond has recon to know 
res

97

St. Retersburgh Zebmary $7 \cdot 1590$

$15 \div 40$ Bry Lords

fecret

It will be in Your Lordikis

recollection that in myydotes

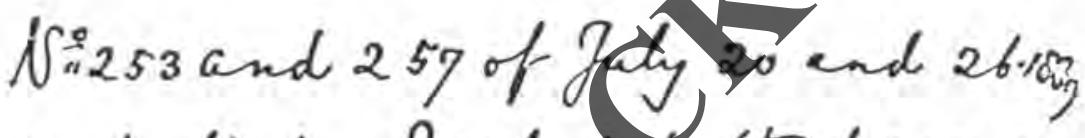
recfectively, 2reporat theresence at huscow of rencterious charatev me theikh Yelal-ed-Din, who subequopty a theared at ft. Reterchurs. andinglas crotfed uf both in Acia. ad Eurofo in varions cafacities w an Offfhan, an Eoyttian and an Arab. Buiza halmond then, ny Rersien cslleague, has now ye informed ne in the stinctert contilase hanquis of Salicbung.?.

axd te. te ite 


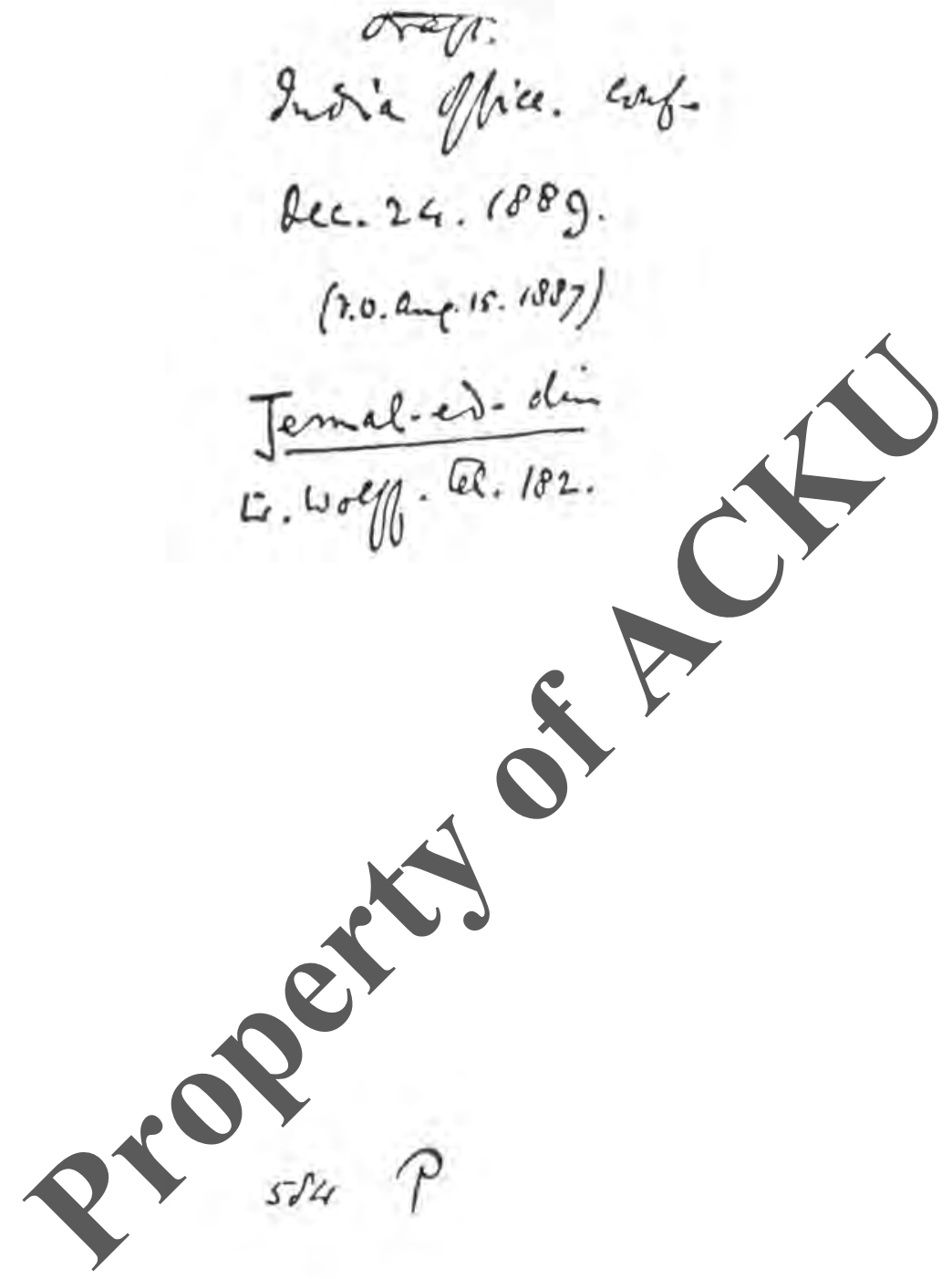




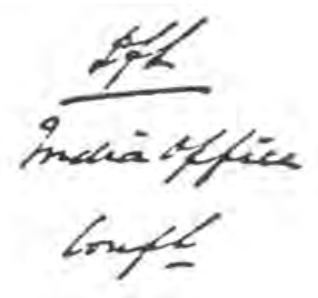

$$
\begin{gathered}
\operatorname{sen} \theta \\
\partial_{\alpha}=4 \cdot \cos g
\end{gathered}
$$

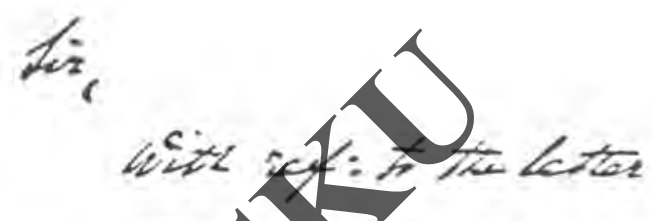

1.H. kolfF

$: 18220.90221$

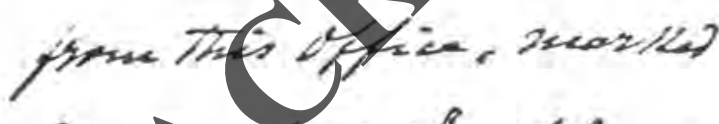
sasectpe ts $15^{2}$ of $a_{p r}$ 18 . 1 wew wiseted ky he

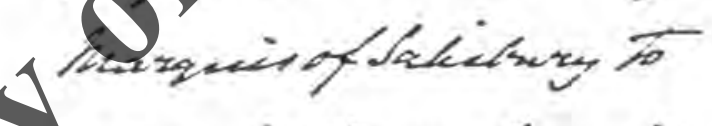

1 dramanit to yow herowsid?

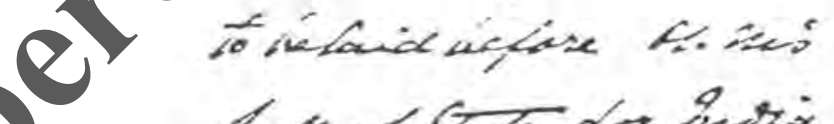
Letsof sate of intix

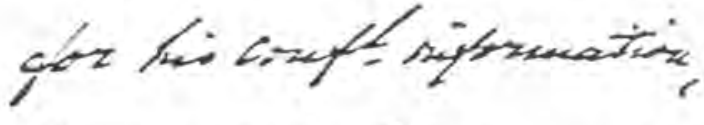
dagipoof the desplike

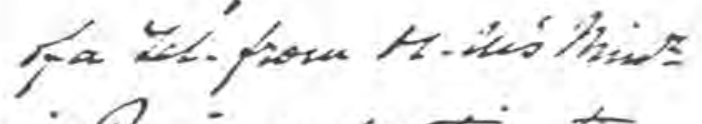
sionsic exporting th pivance of thitom or Shictits Gomat-ed-dir. 
Sii H. Wor
Mo. 152

D.21 Der.usq

Sheite Lomaledem

is hne. Shath unidh ammyed.

(Yhin is the and shoditad

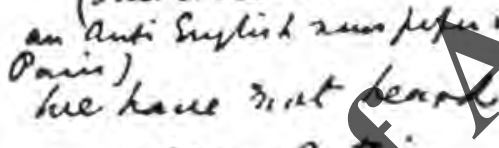

anytaing of tas

persm sane $2 \mathrm{R}$.

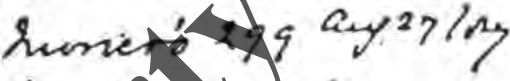

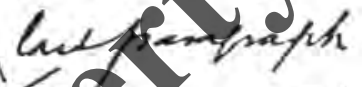

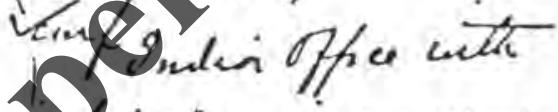

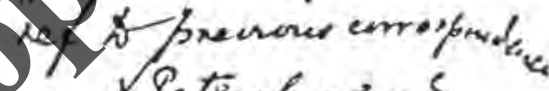

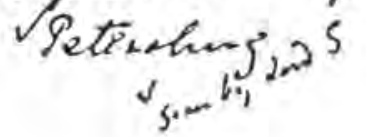




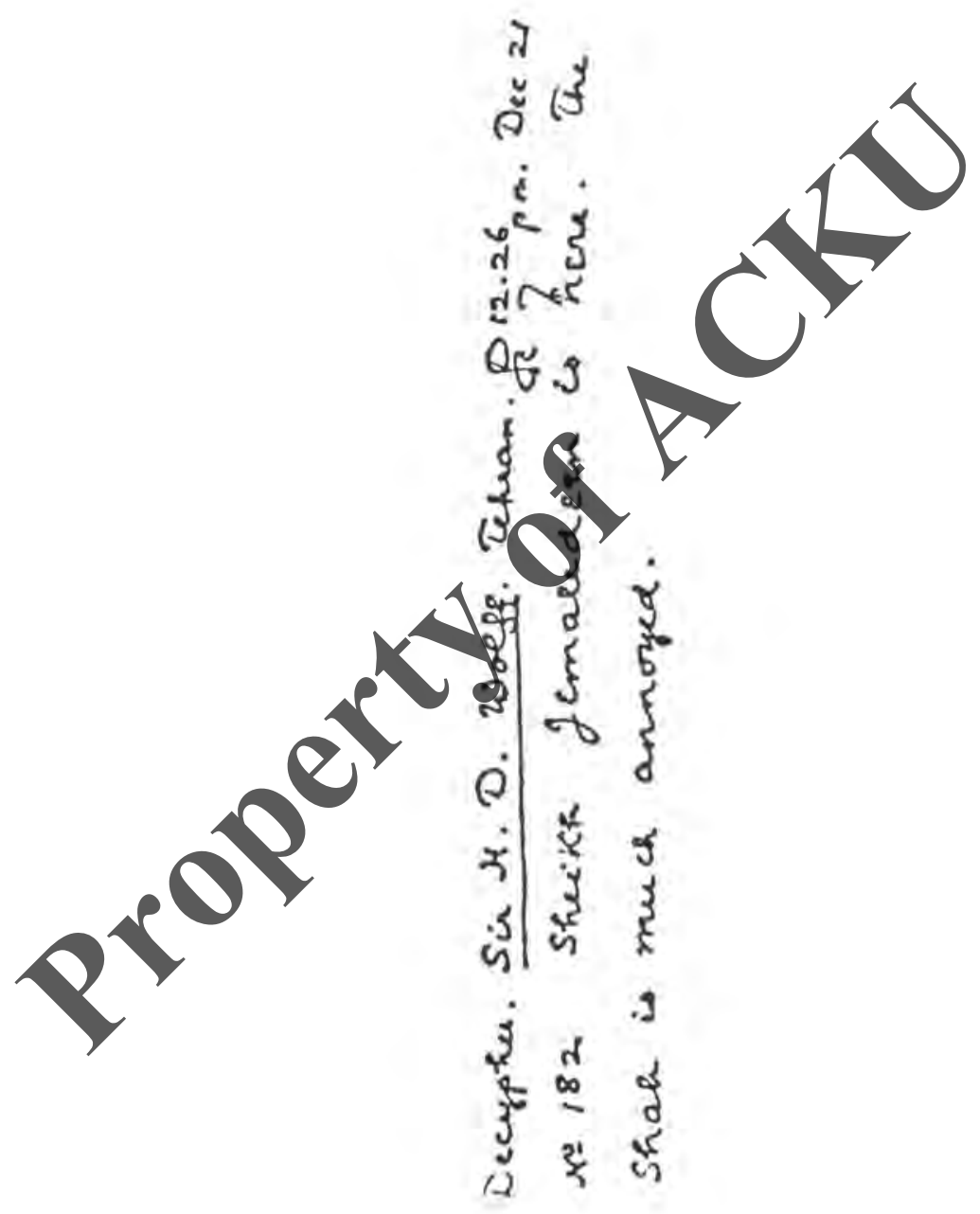


92

ro.

Sheme top hamin to be unte the highajt resfect, ky.Lind, Yom Lisorith' most obectiens,

Ocessuses
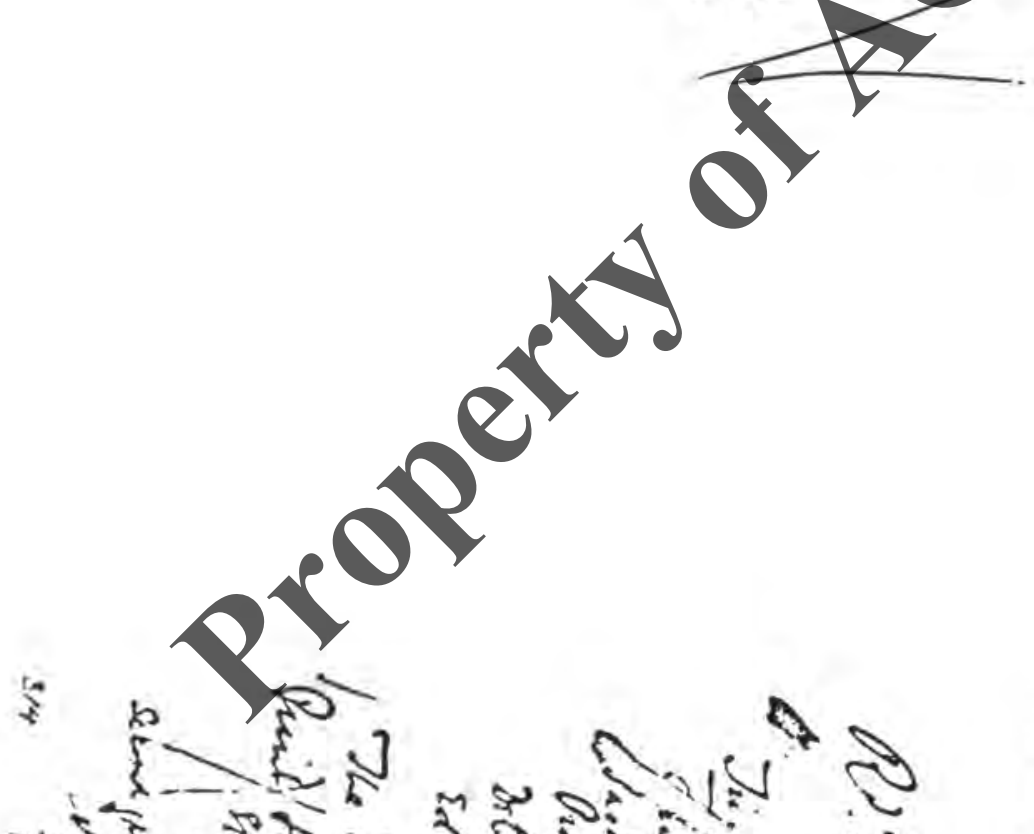

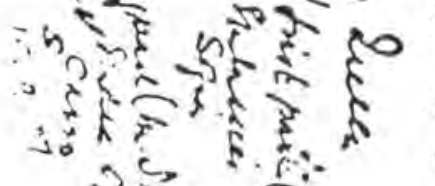

?

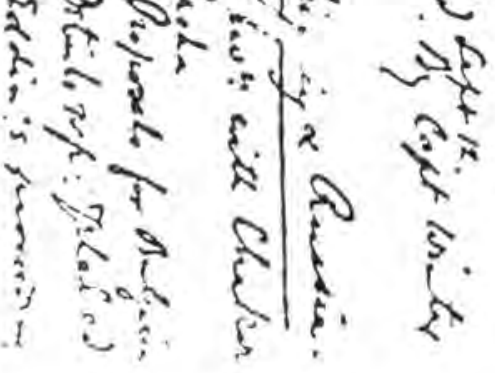
iों के को 
rol

91

he had ione to petarding afte. tha deatte of Katkow, by whims he had been atracted $t$.

lesurw, in the hope of baing well

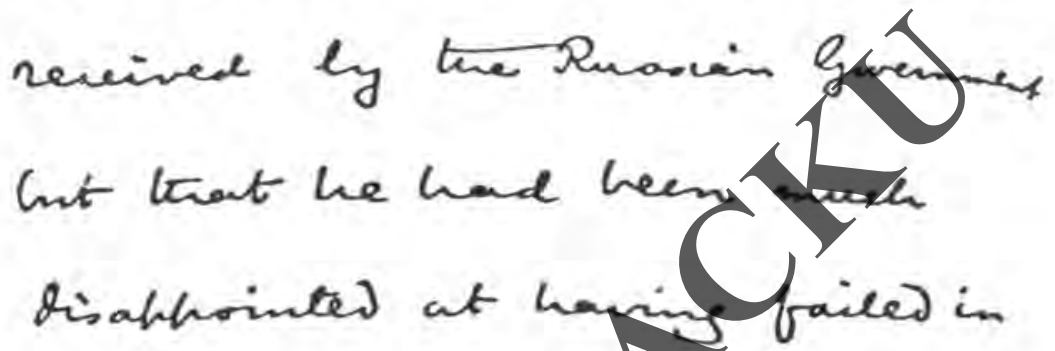
his attempto te set official perarns, who Pre in a manked manmen gros hein the end

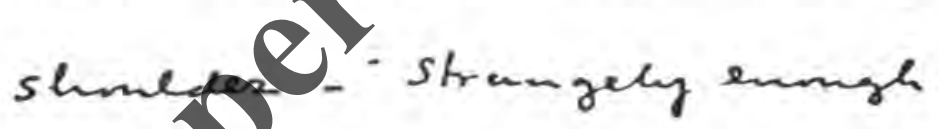
haveles there had been me exceftim, that of M. Poledonosfon, the Proter of the reng Synot, who hade haid heim a visit and interisiendes hein at great lengtro

o 
90

rot

sound him and to song tint as a Mussulman it ames be proper on hes pant to call on the Representative of the caliph of silagato received a civil apposes to the effect the nt frymal-eddin entirely preconize the position of tho sultan as caliph ont that he had his oust political reasons for hst calling on the Timaseh Ambaravalos. Shaker, who hand evidently had him very closely watched, said that he 
ror that ohout firm thes

89

conaiderations, such a furposal would neves be agued to ly tive otites

istieil for Powers.

t

[In the compey of

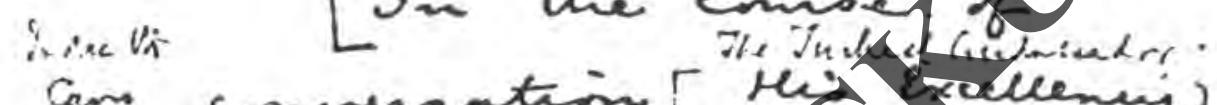
com commentain [ Heig exolleney] mentivisened that (he) had heen infromes of thet thesence at S. Petershing of Jemal-ed-din, with whe antecedents he semex taringhly well acporinted. He had hean pdisus to ascertain what thes literany sheikh was about ande lack sent a confidential peram to sorino

41339 \& 3 
88

rof

shomed be ineligitre as Preine.

Shakein Pasha had of his oun initiative and interent instinction furm the Pate fat lenct so he asesurese fome susgested that a tengr hure and simble ter the Trentyy Benling the the anmalling of the peramal union ef repanative the offue of Bulgmean Regent bont lifat of Emenom Geteral of Eacterm Rommetia mined be the leat and mort logical sintion- But M. de Giens had olienned that 
$r \Delta o$

87

as determines in lacet years protrese; that is to say thent the Temporany Regent shoved he placed in exartly the safe fusition as prince alexpanser of Baterting had ocfutfied

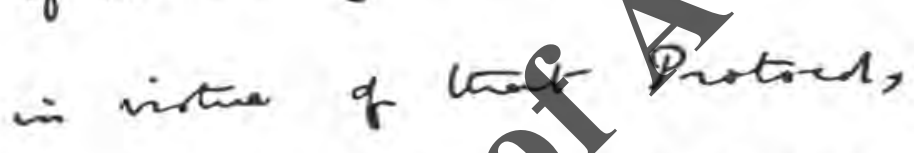

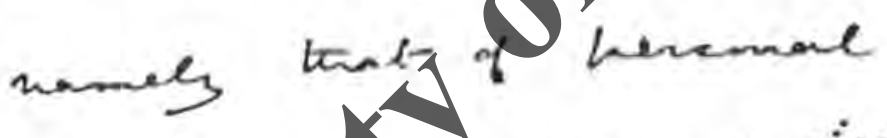
Regent, after difterent tites, of tha hov Princivalities, which

shred rimaien bictinct fem each other withent a comamen solnanje: that the Solgarien conatitution shmed be left intact ande trat the Revent. chomet 
86

rof

form tho Excellenny that the enquing hade been made tiningh him torngh the format anawer, acenting to Ruasian Change' d'at ataices at constantinople - Ghe matter hod. leen afficusared hetween the Minflet ande the Ambuaster wite the frusing poift :- that the purpore Regent shmed he descrited as Prime- Lienterant of Bulganar and Grvernor. General of Ruaterm Rommelia, ens 
roY

85 against the wifful streabing $l y$ the Ruscuin Press, controlle as it was knoum to be ly the Imperial censan, of fulse uphats so denrgatang to the honore of in allied and frientery Grvernment.

I then fatres shakin Pasha what Noty had been given by 10 de Eiens to the enquing stred, understord had afen made ly the Pote resesting the atritutes wita Quici the tempirary Regent. of such a one were to be agreed uptor. was to be invested. I underatords from

$$
3133963
$$


84

$r \Delta A$

Ithen sovide ltiat a mouh more semons change had been binght against M.de Giens who was credited wite having whered the threent that $Y$. Pate ufused to take tore crevente miassures reforyondel by Ruevin againat Ferdiname, the Ryesian Gaverment wonld mate feprisals by the oceupration of Varria on hezerom Shakkin Pacha indignantly

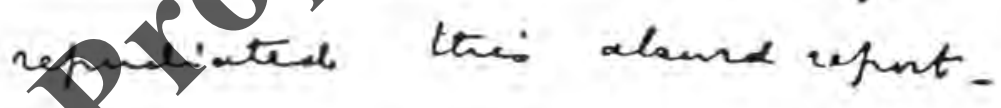
He sand it hade criginates in - Russian. mensfaptes ande thent he have stimgly invteated bo. M. Veangaly against 
$r \Delta q$

83

been undenstord by him as a frophition to be for the present acter upon. As refereme to my previon pesetge will show thin was portealy the light in whid fe. or $_{2}$ Geir himsey desfirives his comersation arot the Tukish Ambacoark 1 excent that shakeif Pacha minimizes evern there than the-Reasian quivister the funtei of the latar language by the statimer respecting the share wrich the other siguating frovers might take in eventinal oceupation. 
82

ri.

of conversation adverted to the necessity of using forte mearemes to hing the Anganions to reason otter means failed of the very disagreeable f presitilities of the pentane, and had seise twat such fiber measures crate only be imenived of the form of joint we cuppoty in which any of mint mich to do so, cackle share The foreign Ministers language hade been entirely academical cane had not been 
ril

81

5. Petwaling -

L: 299 aingust 27. 1857.

my Londe

I have at last been patienate enongh, afters yy unsuccesisful attemprof to find my tinkich whe four, shatein Pashe, at hent aftermorm.

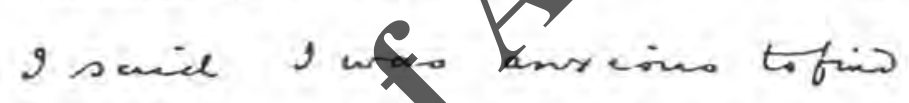
out from 2y' the exact buter of the Appacel of a joint occufftion of Anlgonia, said ty live teen mande to his

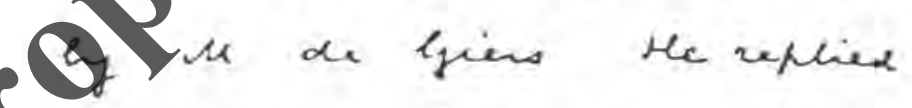
that thene hase neves been masce a formal proposal to tout effect, hat that His 7he Excelleny has in the crme of harquis of Salielny Kly 21337 is 3. 
80

rar

Sheen the Majah blustro a fork deal, be told tho ferman coneul that withim thew usan, thew coneld notbe an Eyhichmen the found in the $\mathrm{c}$ sle of Dutia.

sha.'for to tuleef font

On. Katkoff is hos de Corihatand is nit thely tor thow figlet again.

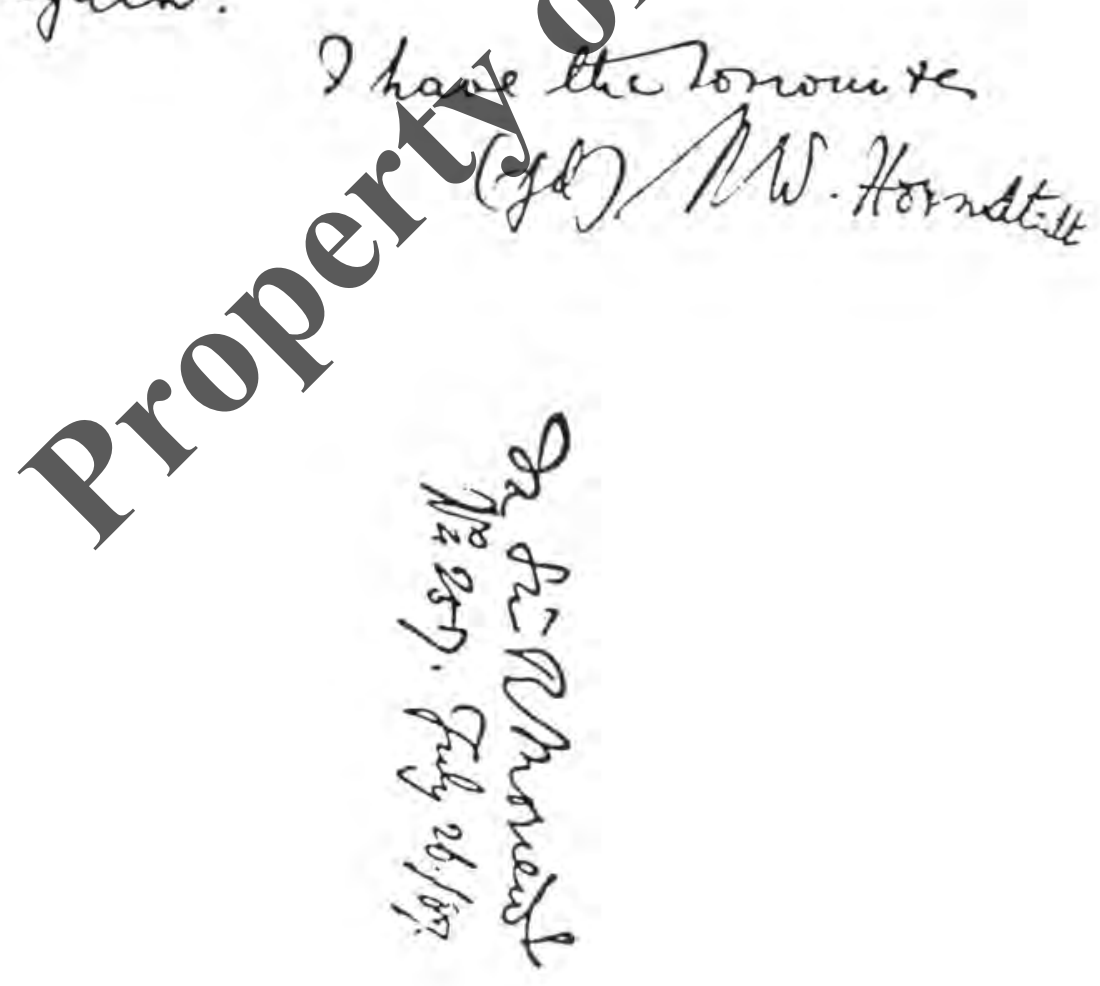


(in rench) and is therem described as Aga Cheych, sfeim al Cdbin. voo or thereycar ago, yein al CAbiu is srid to have sfents math in Inglant staycif with an Bghah tomily, the nance of this. fa nit my informant could ma re erlect, but the body oftes houge is said to be a prosteghas of Lord Bysow, the duntal bard, andreuced fite nimmeniate reipbouthoot of ondon, as rench 20ao ther. "ly this family,

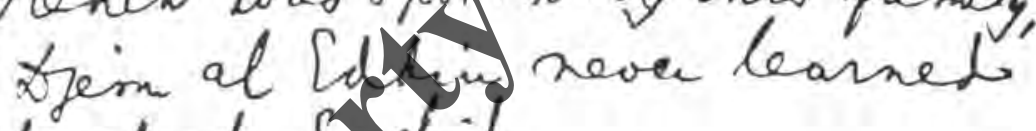
to steak Eyclish.

thaef fingh has taken a coustrine near hoscow, and Q Lave therefre $n$ s-tte rane offortuinty to observe his movements, and feel rome varre in sheaking? of them, but 0 have nor the slyfted indicution of any conneition detioes the Kryah and the Oga Cheych or that the two hoe ever met.? 
of Djem al Cdhin from the dy that he left- Hotel Slaver, until piecta montt sinice when he tooto of his suaters at the Hotel Probl.

fince he has reaided at this Hotel he afpiois to have receing no the visitor but Perrian And hivg in zucow.

Accompantied ly vome feres

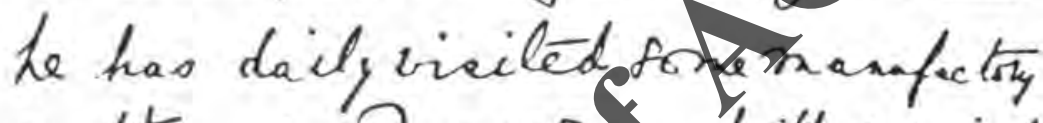
or otter in mocos a y d the vicint and his aly object xeems to he the stidy fersian manfacturs.

His evegring he matty ohend. at his ofel entertainis his fuife on a very modset $x$ cale. He is faid to le well convesant witt mot of the Eactervlanguares, but of the lunsticen he knom hotting, exce It a little. Hench and tet.' inferfectly.

He hordri a Revians lesefont las 
rio

77

Since that 9 have ascertained. that he arrived here about the $10^{\text {th }}$ of (nay, (new so lyle) tofeltew witt the Persian Minister of Finance, Shaven el holt, and som ie so's or five other Fenian, the d. them fut ut at the H tet Hove, but only stayed the on one night, when the $C$ in steven to stay witt the Pesiagonaul fere sal lin, and others went to live among the de Countrymen.

gl on inter and his faff soon for Belgium, Sere * over e bound, for the furfur of studying Trailways, and ales with a view to engage Belgian engines for the construction of railways in Persia.

I can jive account of the docoigs 
Aritie bicice Consulato

Rooseow July 23.189

fire,

$I$ firt became avare of Sjein al Cddin's affearance is this city throuph the "Inocow Gopos.

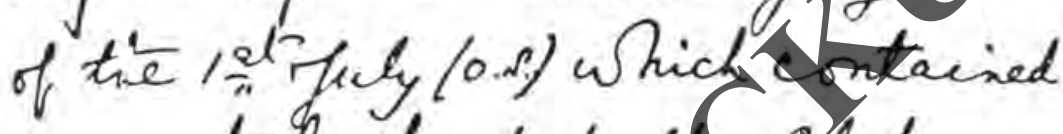
an artecle headed the If phan sheikh in mocar-foring an account of his to a d mentionig that he had garmied here from Persia.

$D$ ingodeately freafected that he has some connection with the Trek Vajah thuleep fingh, and Lonce ma de my inquiries, but could learn sothey about Dfim al Pdbin, int't yecterday, when? diocovered that he was staynif althe Hotel Proyal, a third elas hotel m nuscoss,

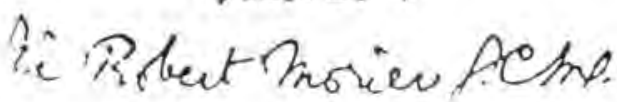

fince 
75

ryy

1807

firptereburg July26

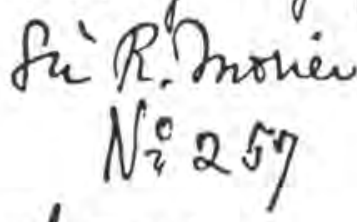

Deloure

dif any'.

hycofit. Lumber

(uf. (15) 59 )

rellas Iddi

Seport by si fornstedt

Qhis novienents at

Qmoceos

$\checkmark$ hreci (tfeci (Seart)

$\checkmark C_{n}$.

5.1 .8

26 
74

$r u$

$N \div 259$

Se. Retersburg

Jul, 26.1807

Mry Lort,

Thave the honows to inclae herewith the Mefor. of Ir Vice conoul Xo Gatedt an the movemento of teflat Ld Lin, sow arrival in 0 cas was refoted in my de fotch No 259 of fuly 20.

2 have the homon to lee

Wth the highectreafuet,

Dny Lord,

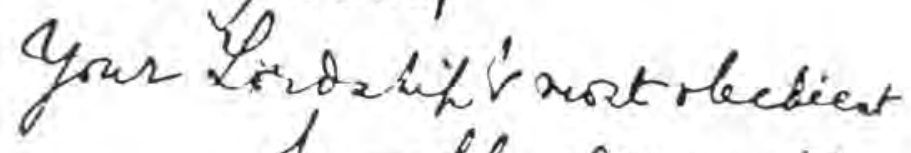
humble servant

haspies of Schiahry kf.

te. te. te.

Drsonina? 


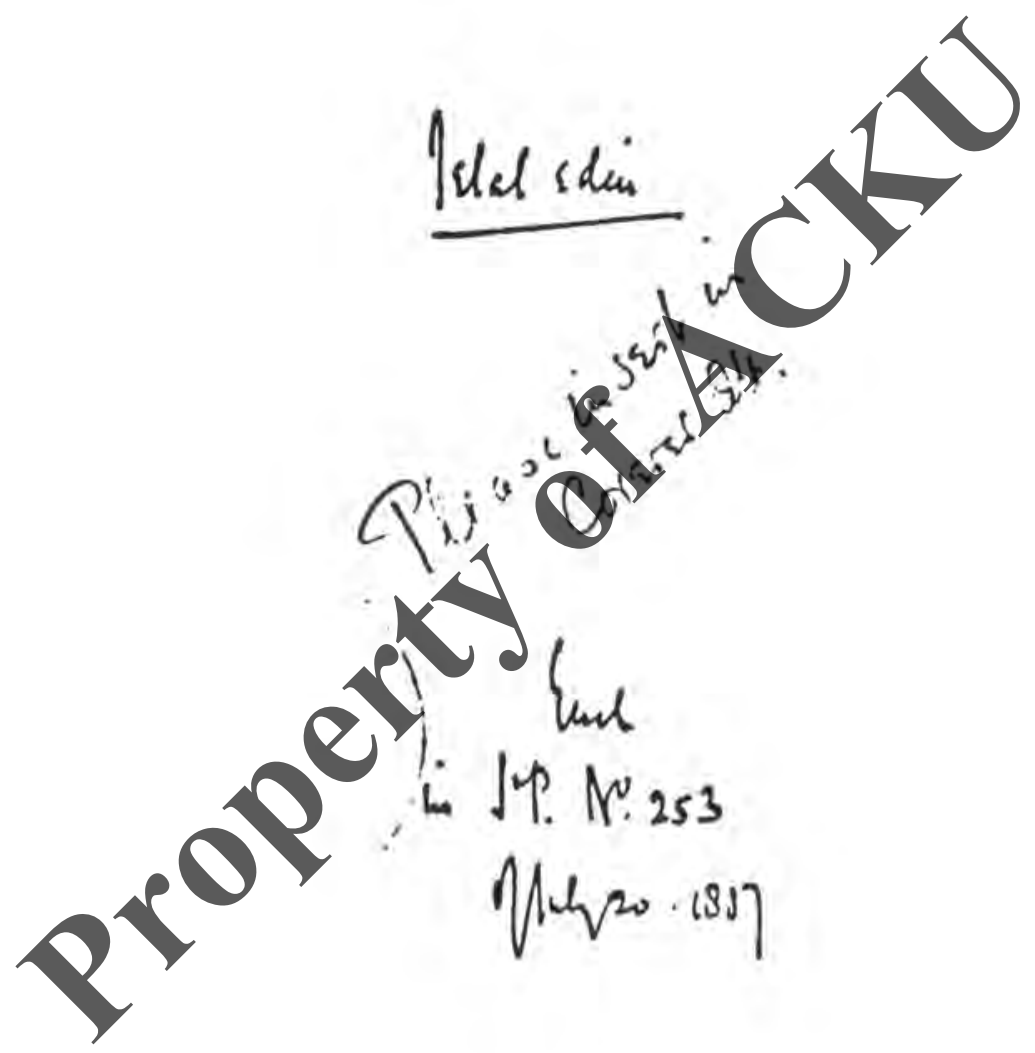


72

ry.

The Noscow queptle of the 1/s fuly roy annowain the fresince in llowear of the Afolan thicith Bimal boldin, a native of Gabut and sho pased some zeas in Trestey, was banithed 18 year and took an ofelizpleart

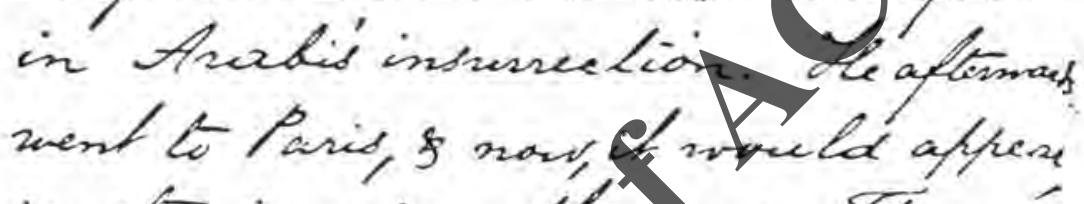

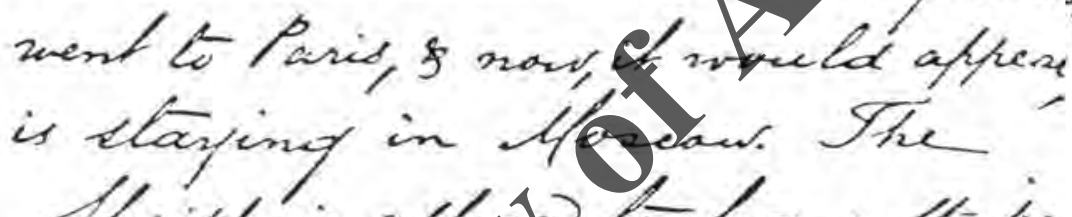
Shich is allyote to have tlatis that his abpes in viciting Ruscia mar lo mates himself, iractivally. acquajer mitt a eandy on

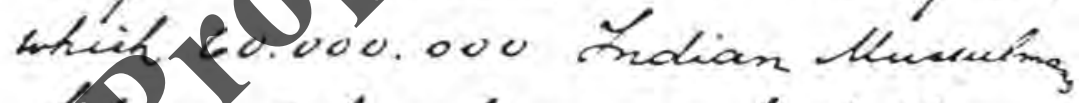
pery tok seliance, $5=$ fivel they rupe mill offant them forotelain and emancifalk them fran the dustid hnglick yoke? -

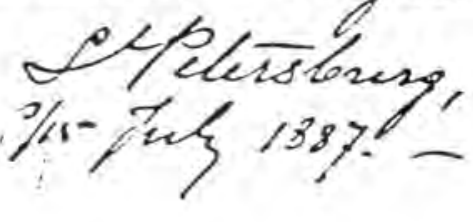


ry

71

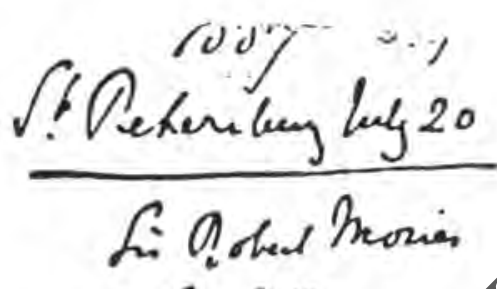
$h: 253$

1 Euchum

Qceigusas

arture

yegaretenied

Yinain Oiflicen

5. 27.7

$265^{\circ}$ 
70

.253 .

'euch

S'Peteranane

20 lug wory

by Sule,

ryt

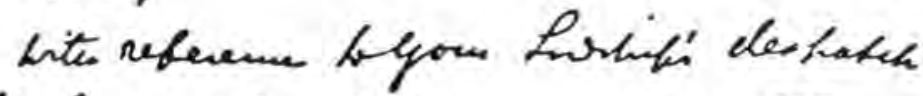

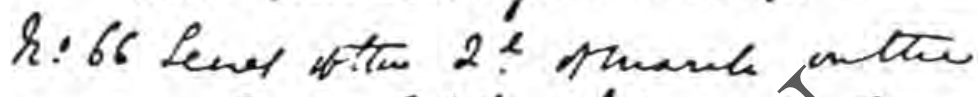

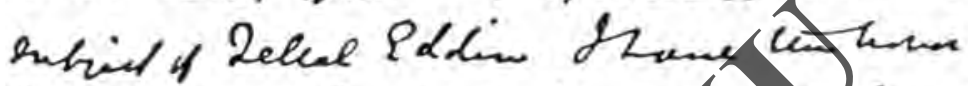

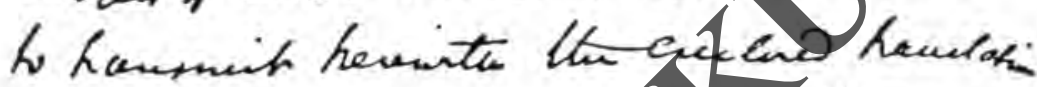

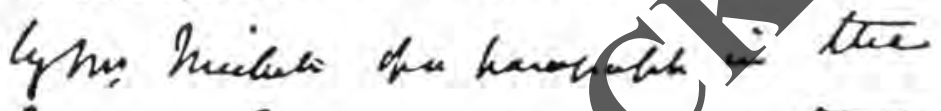
hasers forcke aprosang the.

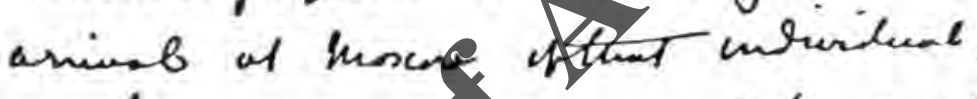
thene cumentaistmation to be send to

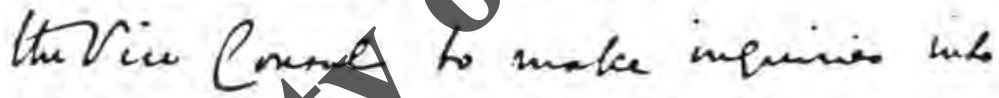
to canses of tar prescee then

1. SJam the han is he vith tio hist narlect.

Ony Lrae.

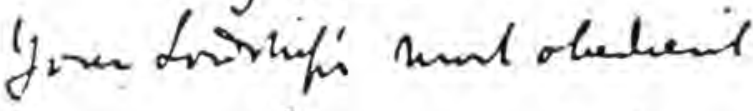
humble serval

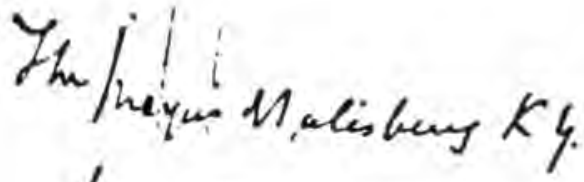

Qisonana 
69

rye

प्यग

S.Potashy Masich 21

Si. R.Mnien $N^{\circ} \cdot 9 \%$

$\operatorname{rec}^{2} 20^{2}$

hy llear Limile

Iref.ro. Nisk)

Jellal-ed Sin:

stef of $2:$ alphrialt

rer.ke,ta.

Ravatursess

Xxdie its rent canio.

100 
68

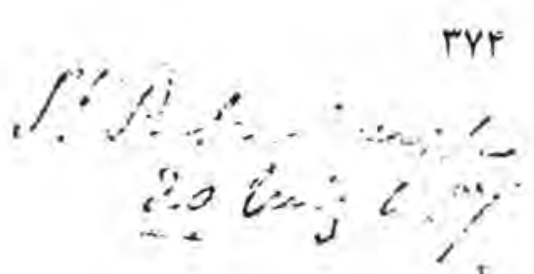

ing Line,

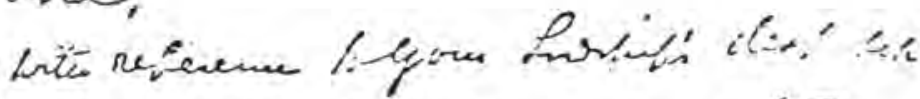

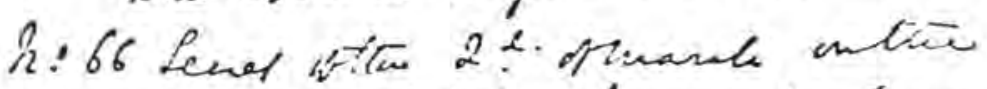

subiet if Deleat Pdilin thane thaner

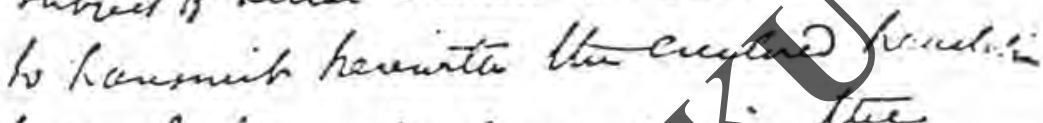

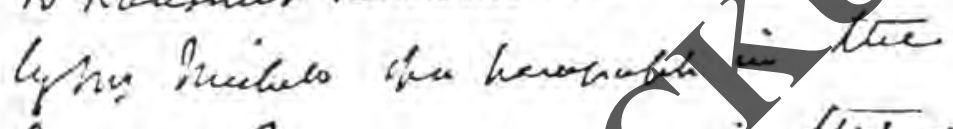

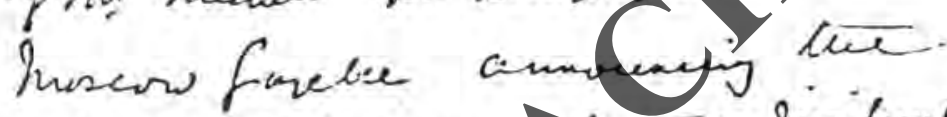

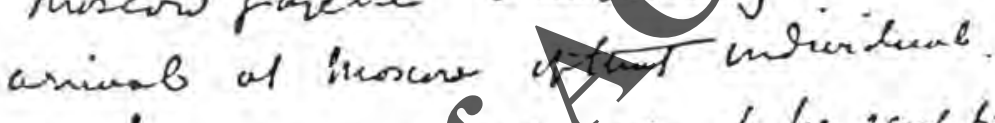

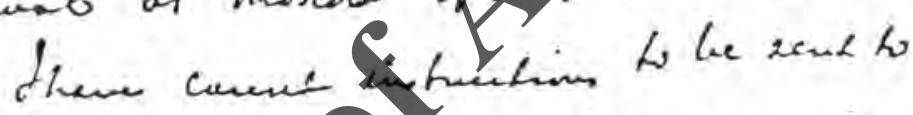

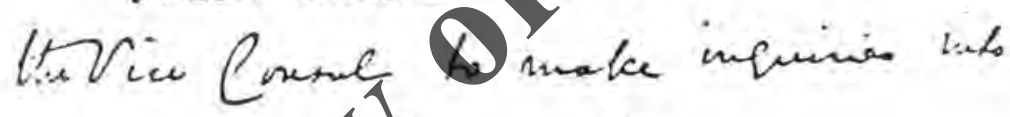

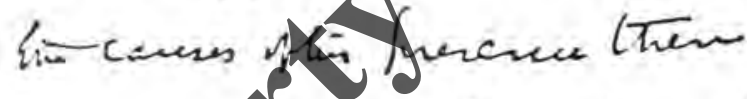

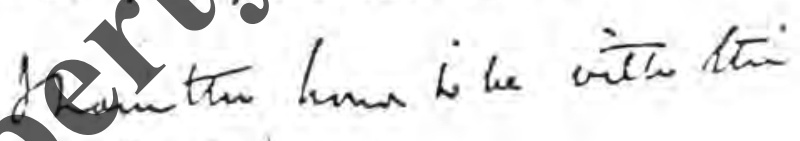
'minsonec. =

Any Line.

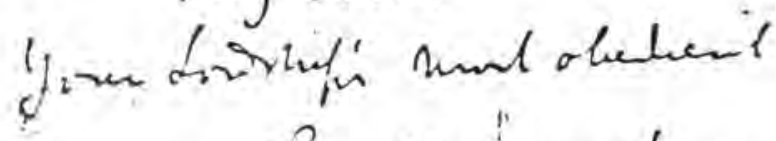
hematle serwart

Y i

(1,0ines)

$+2$ 
ryo

67

Exid.

S.Petersting. Mardin 21.

Si. R. Mrien

$N^{\circ} \cdot 91$.

$\operatorname{rec}^{3} 28^{4}$

ly dher r lumigr

(ref. .0.155\%)

Jellal ex Sin:

2 ter of de: a fhrintt

(2) U...ke,tan

Suctinasos

As Solie oft recut

Cario.

log 5 
66

ryz

to! any Lat is A theisen Tuleretant. D mang ade my oun convictin. that he utary incmits with the offingle tiadition. and worgety Resein -

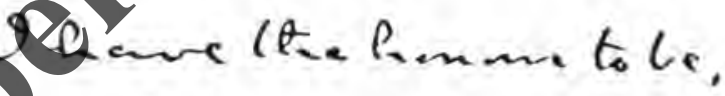

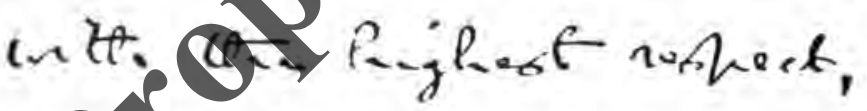
$\operatorname{lng} \operatorname{len}=2$,

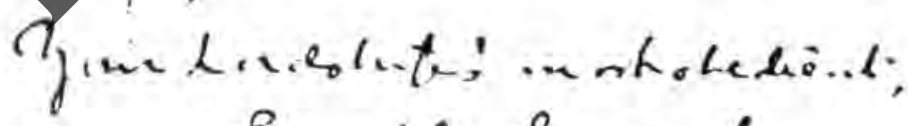
fintrle dewant,

Arsonumin) 
ryY

65

Pani for tiscia, and a rumona hade now gone folto, of while he was presibly himesel the perters. teint he had obtainget a

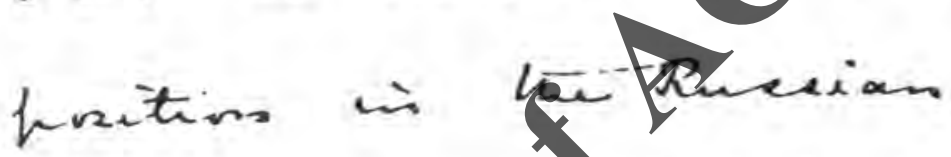

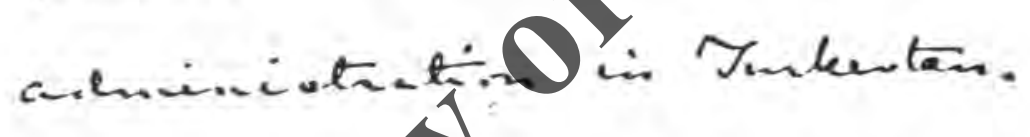

Ine ge Geir oflied lent of comed nat reukest gatolkaning heard the tume of Jellal-ed-bin, hut ltat he conded state wery fositively that no

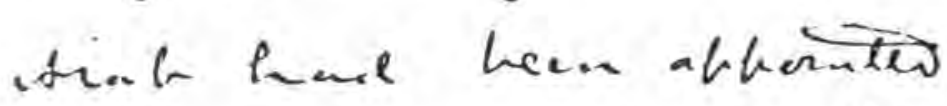
to 
64

rYA

neushafer at Paris, crataining tha most violent altarles on Hes Majestais Evernment who profeease to be intimate relations ftats. Malndi and with the rifferint. religions lexdeg/ 1 stom, ause who vals beliered to have =egeravined, to tia hest of the; a bility, t. kQute disuffechion in Inces: At nohot has reachere as sonere time age that lee haed leff
Prois 
ryq

63

cunel His Excellening was sindertey bleaced at the abbucisti.. of his moth of denence wite len oufleds.

9 thenght terdecasion a gurie one fo Enquice of His Excellenjoltont Jellal ed - bis jrefewere to in

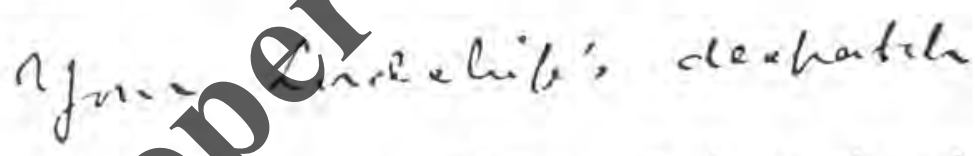

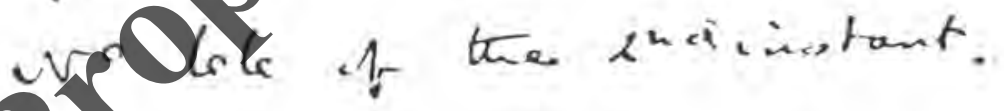

I save teat lais indivicluat wres an Arab adventimen who had edited for some time an Ara's neushater 
S. Pelorsting Uaneh. $21.1 \times 87$.

Nog1. Ny Lerd,

Haveing occasaion to call hor deonsioun de Gions

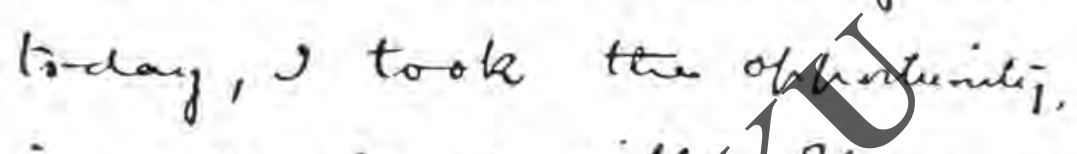
in accendance wilt rym Luclehibú deshate ho. jo of

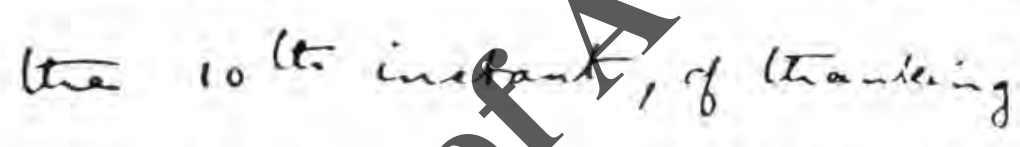
this Excelley of on bihalf of

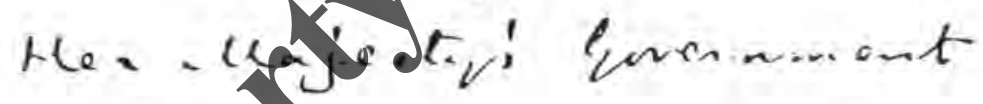
fir log folk and fiendl,

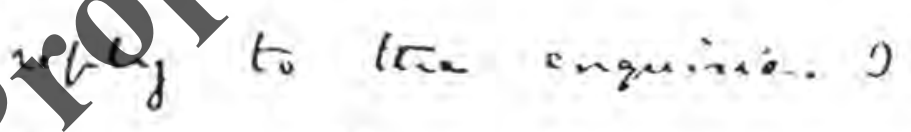

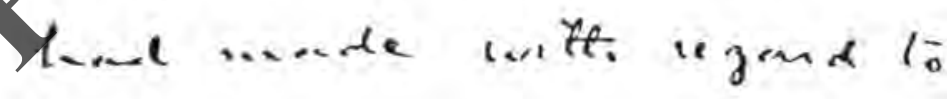
the Kije Aloweok singh

The and Marquie of Sali.eling. K. $P_{1}$. fec et ete 
rAI

61

Dtafe

Pir P Provies

No.66. Secuet.

70.

2 manch 1887.

Ref and Jdderlus desplisert' $x \cdot 2>$

寉 3id Nov

gellel Qdiu

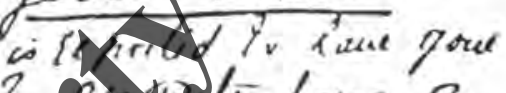

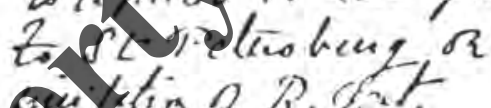

Qajutin ? $P$.

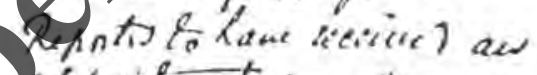

dipmineat a de

Gutle.ton siation.

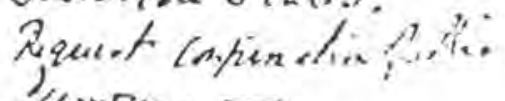

isennas To

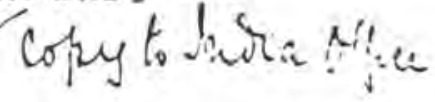


st.

7.0. Aug. $2 y$ usst.

riria afpie.

Senet.

hir.

$I$ am derieid b Eare frandile ta equert tiar ror file cdrues his Prow to the Edre, of

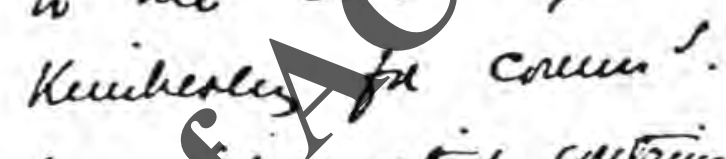
the ixprusation contricus in puesteritur of the 2o. and veature to the afplear fancel $\omega$. ris, and J an t unit at that thic is the rame nirivinat os the are desceithed in the conere nicher in us water of the 
rAt

59

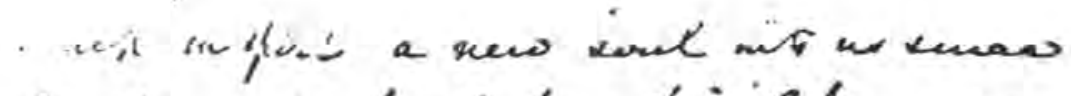

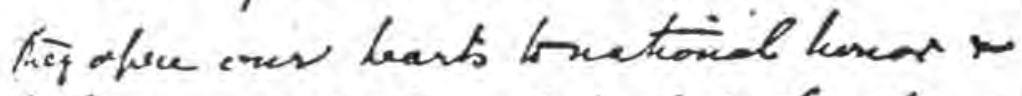

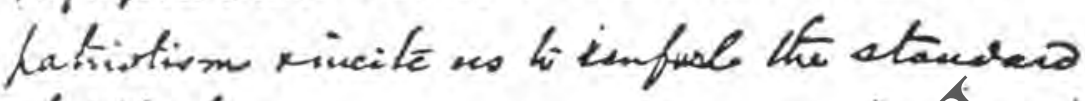
of biterty.

R,t:3.

$9 / 2$ : wie whith the Enchil arferllos a kas?.

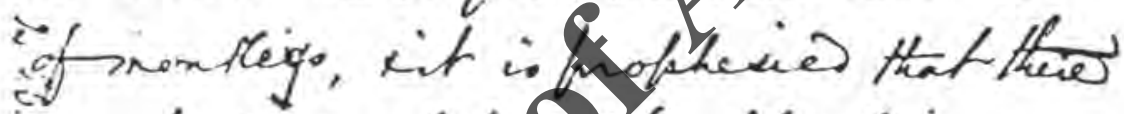

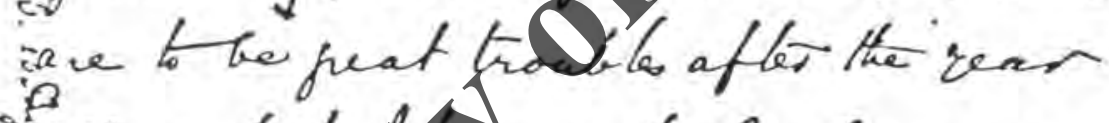

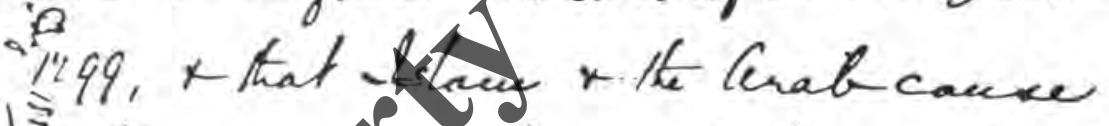

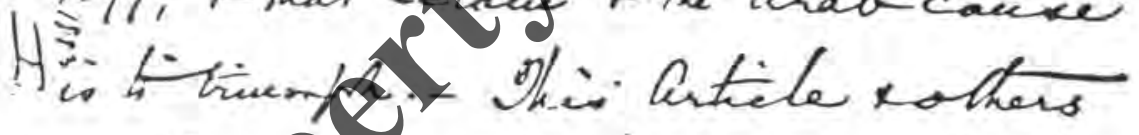
in tho Diter numbers uftetrager capten mang wors mitter in ciphen Which it is sifficall for ma to understana. Peshato they have no giffecently for the initiateo in Erypl. 
58

TAF

Kirics bos bean Goferrad as han.

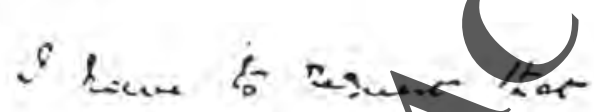

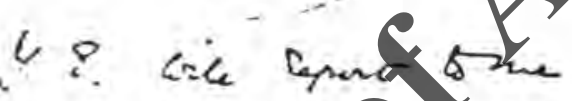

$\therefore$ ans cofformatio of

An: ruplour coun syour

on.<smiles>CCO</smiles> 
TNO

hice, $2-187$.

57

Yrofe

$L_{\text {is }} \mathcal{P}$ hinis

in. 66

fecer.
$\mathcal{P}$.

lite ofference to and

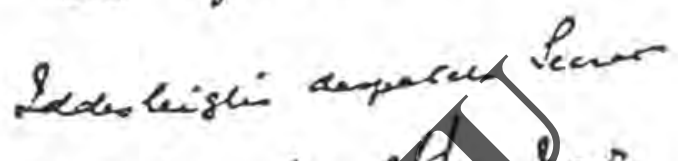
l.277\% tै 3ै? Row?

Cort ispectiog a tpout

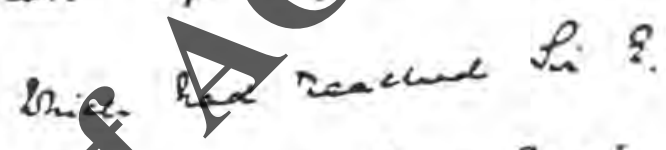

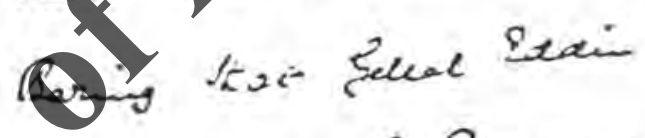
had gow $\&$ Pr Petar burge. on th minitetion it wo

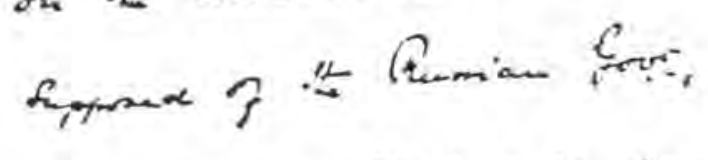

Prane has to stat to tom ter accurating \& information recente fou a bruiac fource, an appinisurers in an of 2 Tintertam

artics 


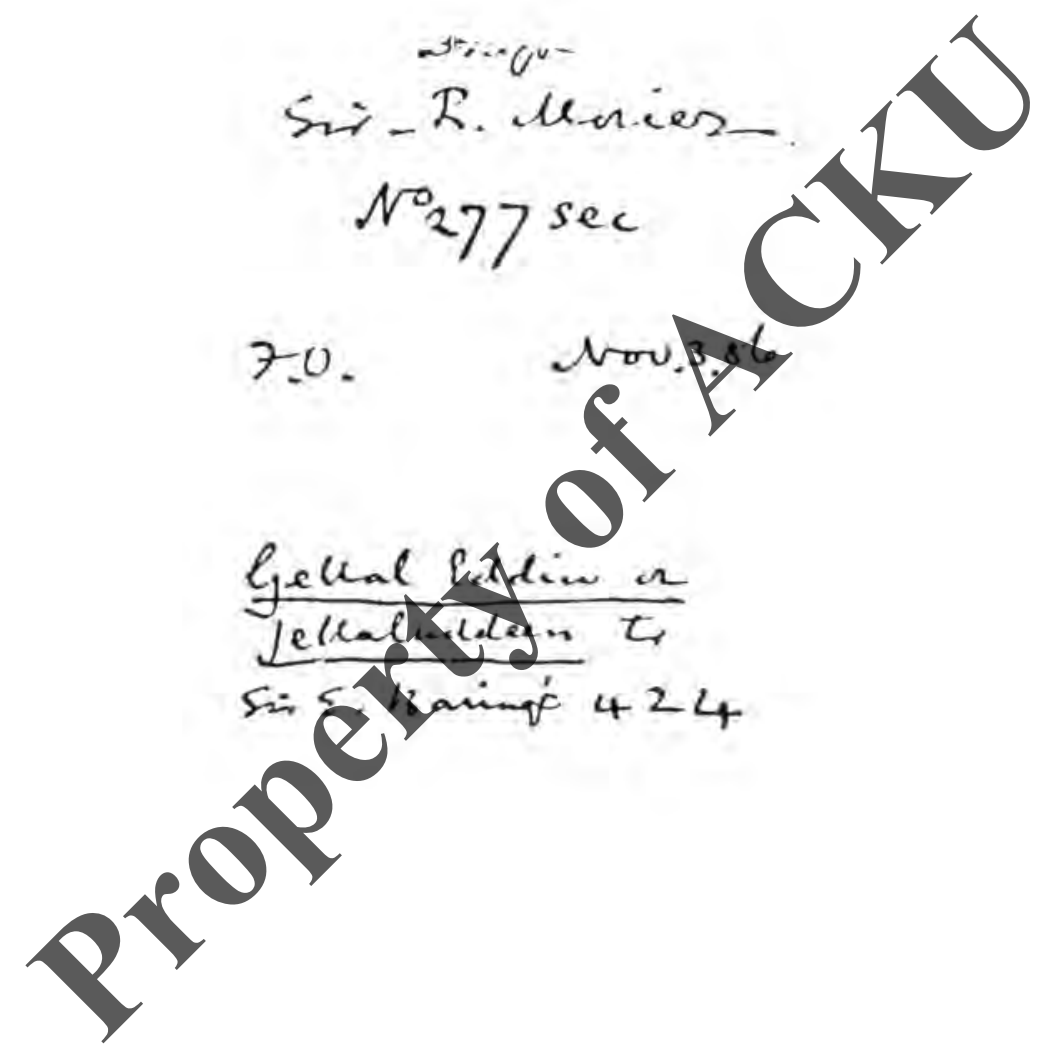


Gue Af: Pelectruy, it

intoryonet at the egrect

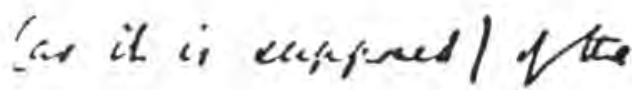

Qucasar fort...

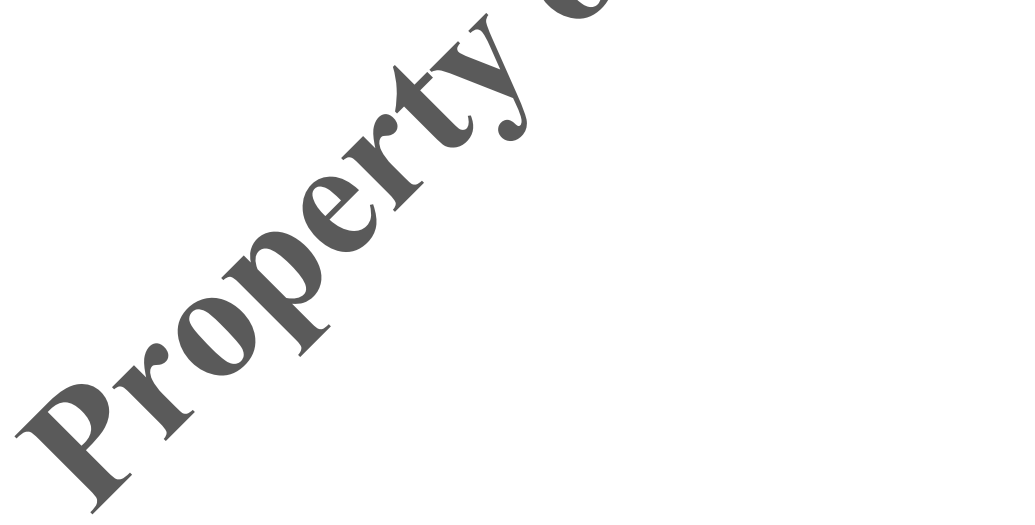


54

rAs

7.5. 083.3. . coor

xraft.

In $\&$ hovie lis,

Itrandinet of dow Le

Lecurts the onte Gupl.

infinactio 6 fins ox

Sevpately for the kgat ad

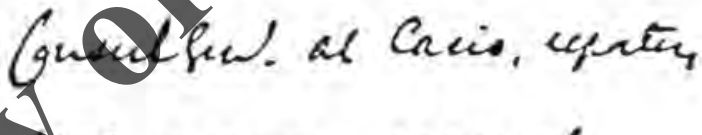

tyal a tuen tramed

sellal Cheis a felcalader.

who has heen po some twe

werent at Vacis, and ha.

then sated Auhe

neusquen intainery

atcreles on the Sraphith and

Sopition biss her wo

fine 
rᄉ9

53

10466

Carir oce 23

Sir E. Baring

he 424 Sengt

Recon Novice

Gelcal Pravi

in there thangen 6

satody ar inta

Q14ascunger.

Filles. S.C.

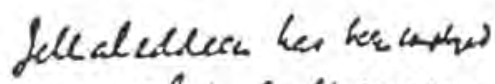

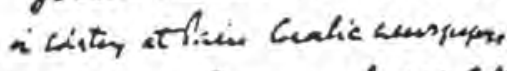

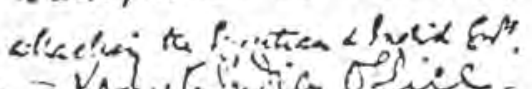

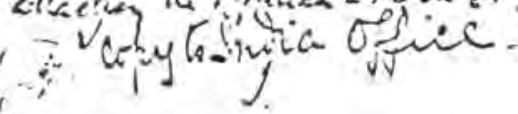


52

rq.

laskfew years; I wrula suggess. cterefre, thas id might be as weel tetas Sis R. hemier sfores

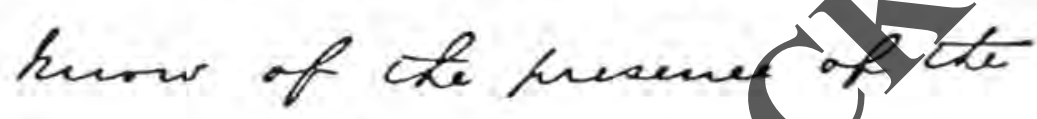
Snan in $S$.

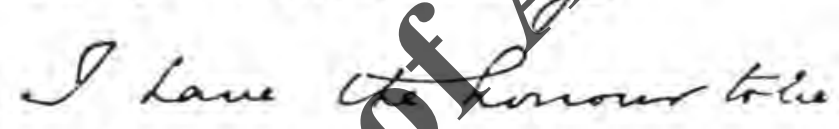

with cRe hiket restect,

Bby trac

undoshifi

hustabedint

tiucuste ecruant

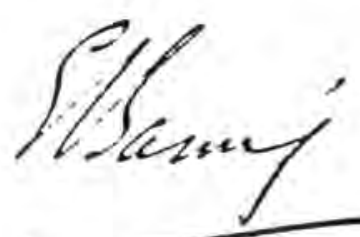


एव

51

duty to infirm Yourtordstix itas I leerns from Hameralla that the weel-hurm gelfortidin. whe waed to reside fflatro ha recenty fone to fatperaburg whitar it as expersed be was

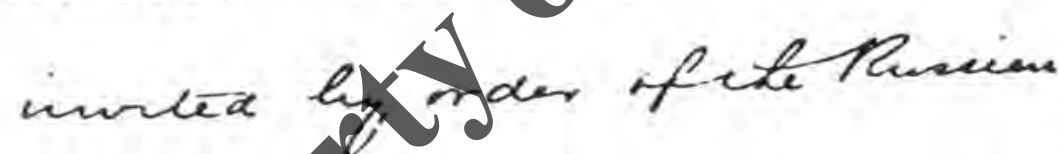
fruerabine. -

Nke hamenty of qeeceldaim twaras hugland is well kurms and hes mone Chan sue

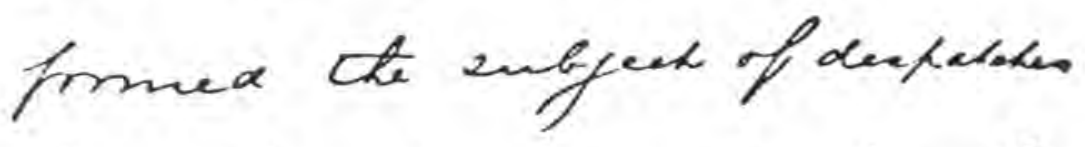
from che comatry dersing cte las 


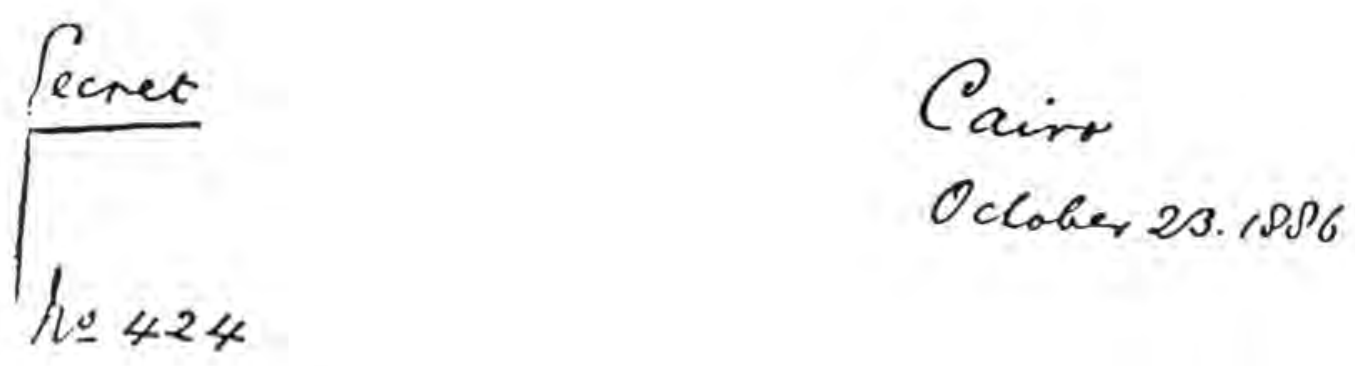

buytord.

'I Lave cteptour a scans chas reafex tule tra

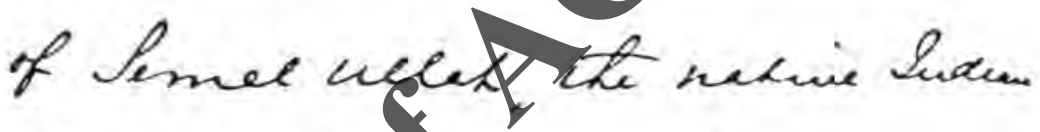
Guage, whoctame olgyet anny

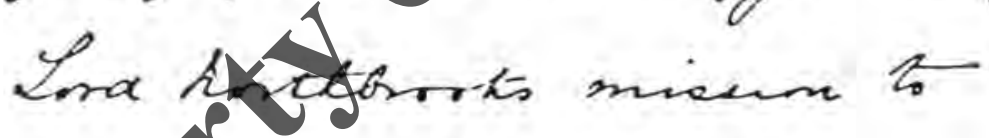
exartyme into the lake of ate Oyptuan legal adminiotration, twas akancunger m boera che shit whith hroght we t 76 Eygt and I chint is my arl of Idocaleigh gers. denty … $x$ : 
Sie refined to.

Shave the kousun to he

with the highot zaspece

hey fond. your fordotifi:

hesst obedion. herento sewant

i

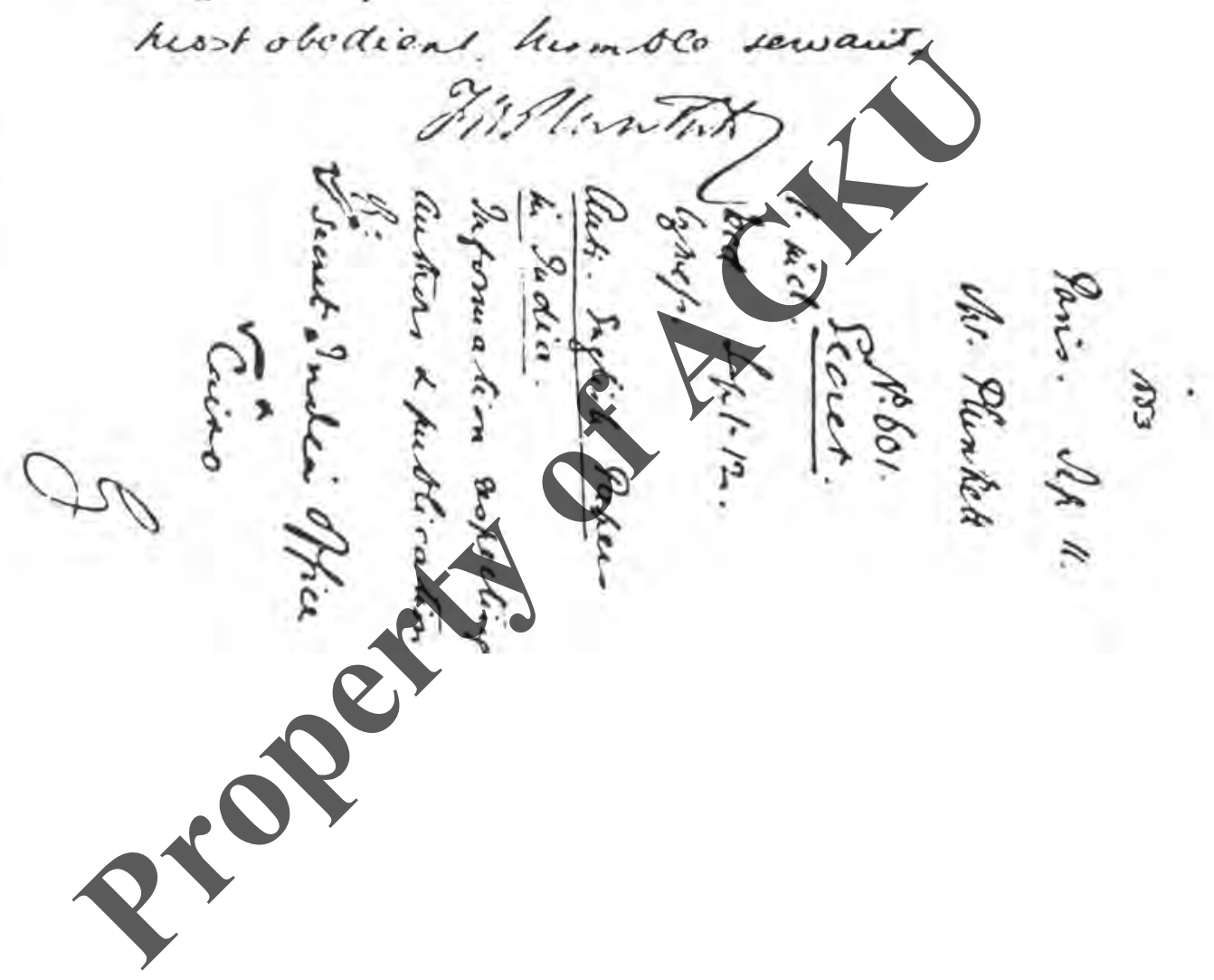


48

rat

hay; but as he her nis

teterancy a squiremeats whateuce and is alnuort a begaar. behive the, are not bikes to keex him kng.

Ini: $\therefore$ the tidisiduar callery hirmely feince Soochoit lingh Nike rase was presed op tote aleintion of the requis of Pakitures Level years apo, $a_{-x} 0$ who is tow hexo in an absoluters, dastitide andition.

I have the-hououn 6 tetum hercioith the nigrial doument,

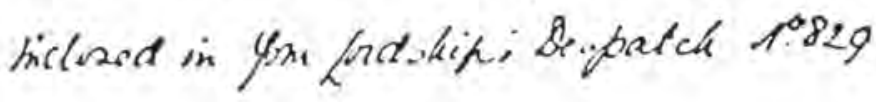


ras

47

hi, firses th is sapected to distribute the Paxen in Judia, and thas those who aie bonkir this gef consider thas the lasiest arabeto hicrease the destere of ingland to loave foyt is to stis ax

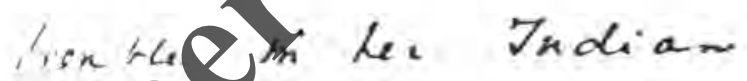
Porestrin.

Anthen Dudian ka. aleb been taken into the farue ancesn. to th are $\alpha$ also if pofocite in the vame, Lan. 
46

pq7

hs. Sawna is thoos

to have frequentes received brcuniasy asistarece frow one of the kombers of tho howse of Camordo Bfopter, and $\therefore$ also tiugum th to te a frequent rigitor to other financiad fistablis acents.

Ien tord thas te tason why fomat al Iin has teen taken inso the affais is that, Riruph

his 
ray

45

ikrueld be hostile to Great Butain?

And an the. suall houes lendero drass ivounces fim the biviper pros. and thes again forntartain Baxkers si Park: clacurkere fate inference $\therefore$ Lot stratinal that he Sacas is supharte $\alpha$. $B$ thint mas be lenrued the inasowable financial initis of artain Espltian lows.

hes. 
Panir.

Lefutember 11. 1805.

rebor thy Lad.

inet. Wilt ceference to your

Indokipis beopalches No $79 y$

Lever of the 2,4 and

atice.

of the $29^{\text {a }}$ altimo. Suave the

howoun to ufope lthar a

Sentleman prom 1 often see,

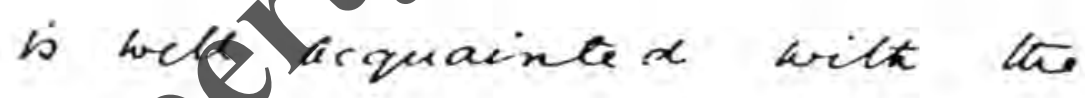

hogranes Samia lith whon

the Sheikh Gamal el deen

(o gamal al 8in) is now

ki

Conductine 


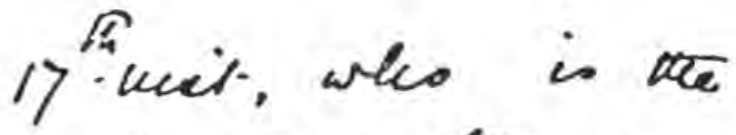
nespecter acelten i sites adrefes $\pi$ the klerive aed his «nsiciac.

S

No 1 . 
42

r..

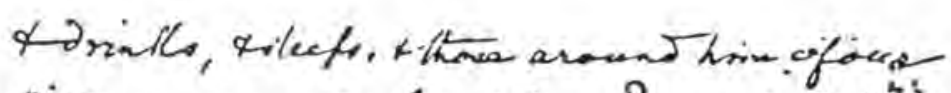
tiva are hean odesficables. Dower is niv the hass of de tarofeens int have furetaxes in

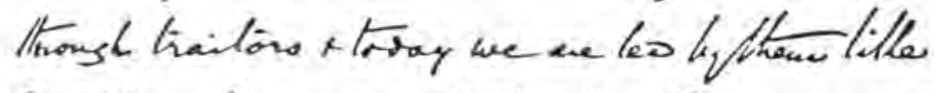

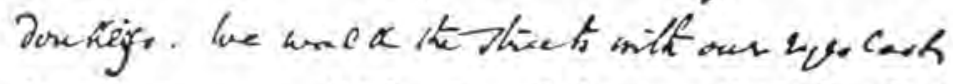

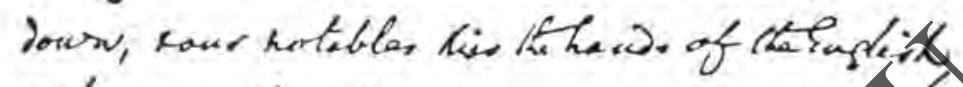

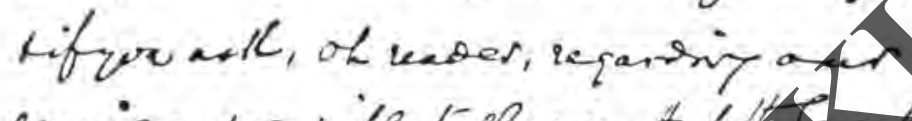

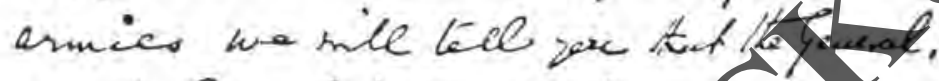

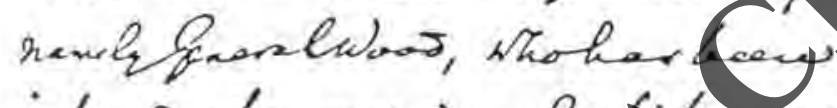

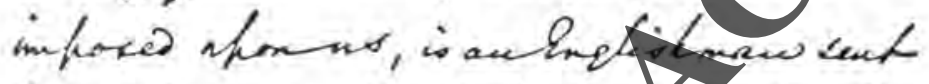
boverive as. He is tiffrit out the bos of fairs t Alecerow frow high

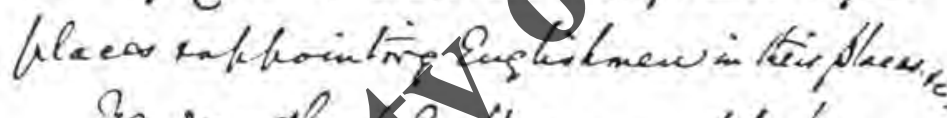

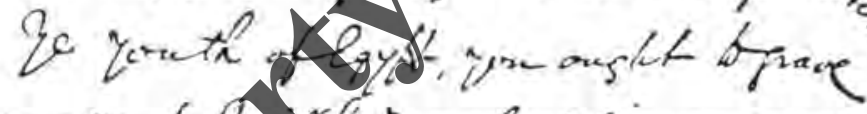

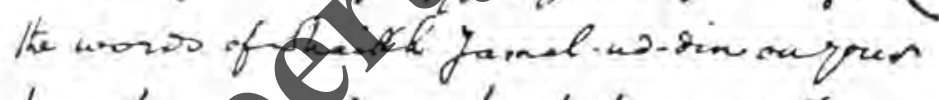
heants, four boets har winttew a bong grosyta kim, a coly, of wheir

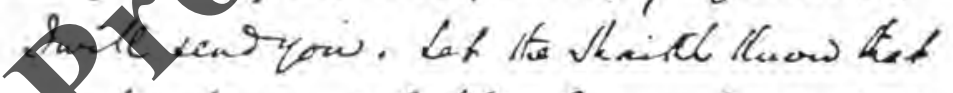

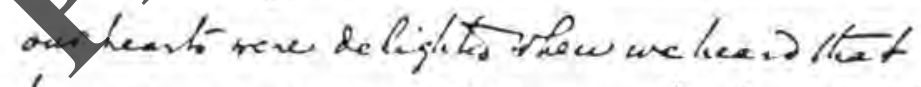

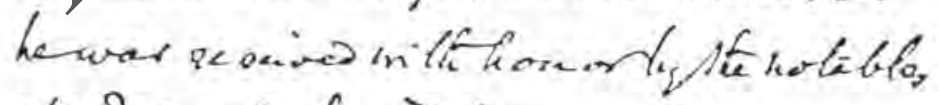

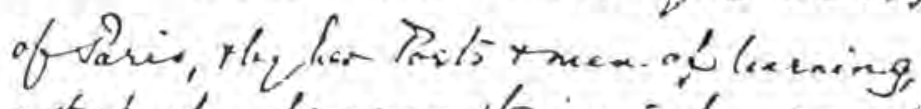

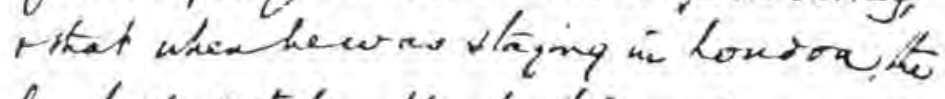

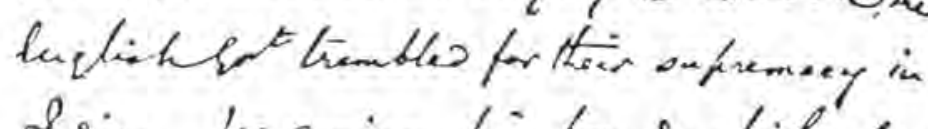

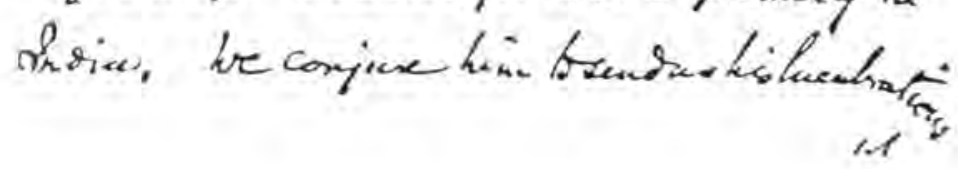


$p=1$

41

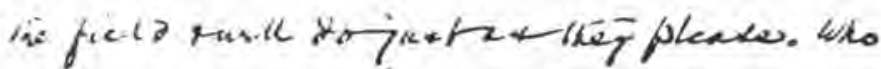

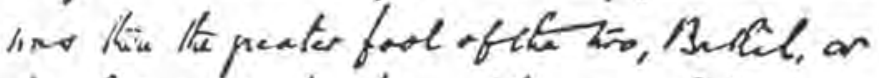

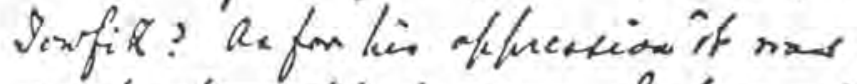

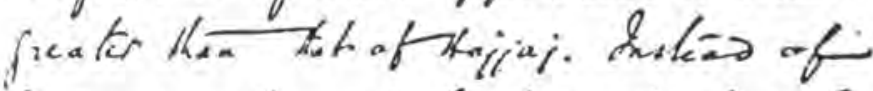

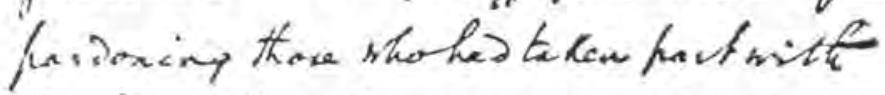

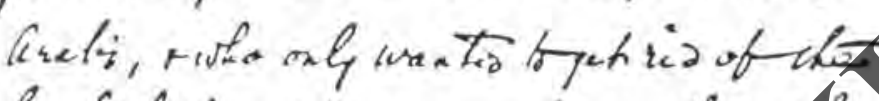

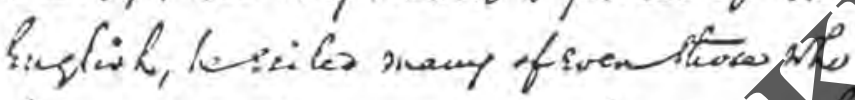

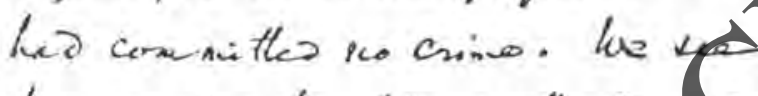

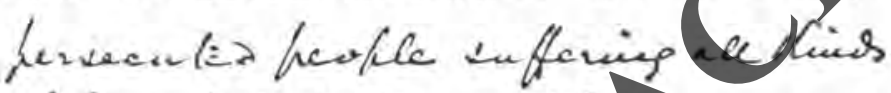

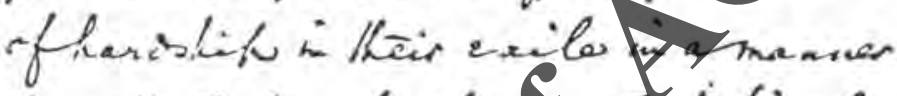

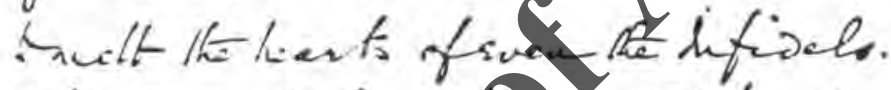

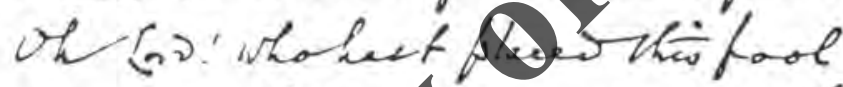
oues ws, what at our vin that how

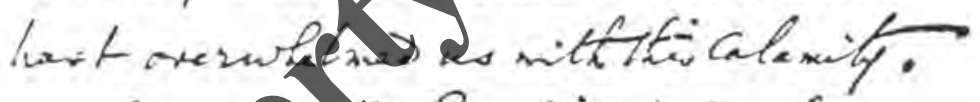

Ine, ter or poi timinth ita

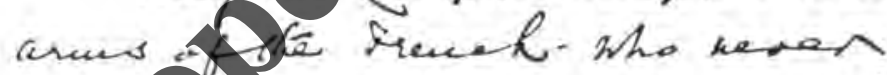
heapose thustumans anoer teas

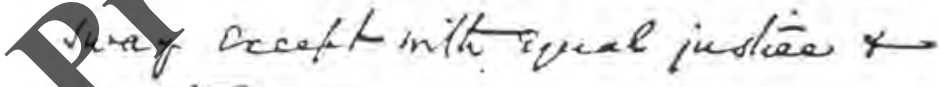
Suity. -

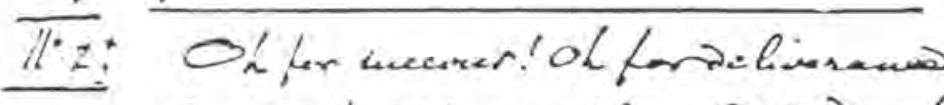
$\times 8.46$ ander.

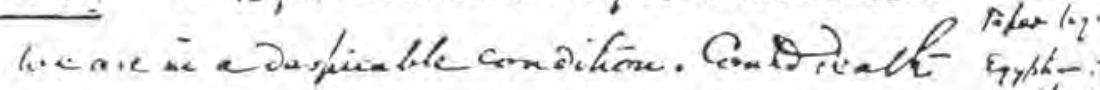
Le lain $h$ of $f$.

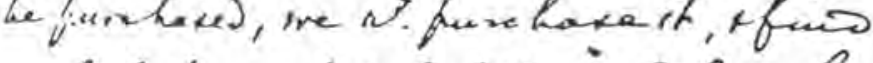

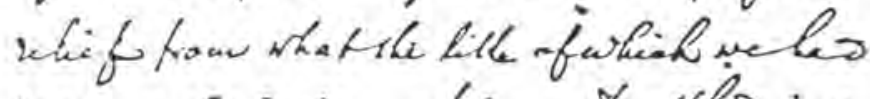

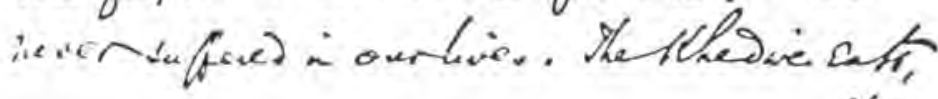


40

rot

Es for the granites be her brought a

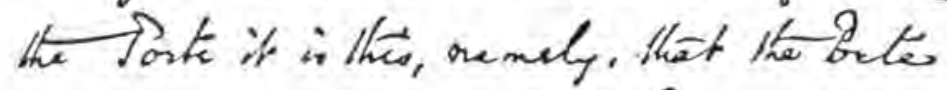

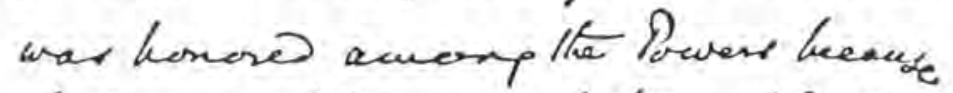

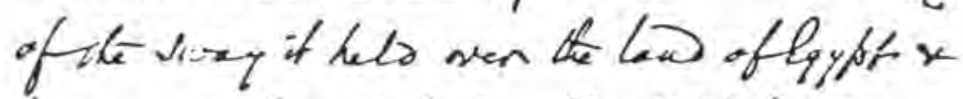
because of yt being is bight hand. Dat now, we account of the forty of is that Dower los his italy o few tox

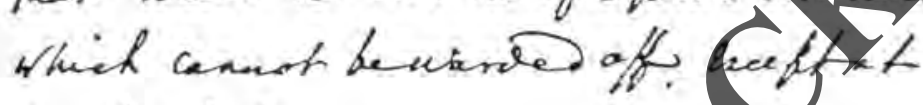
peat sink.

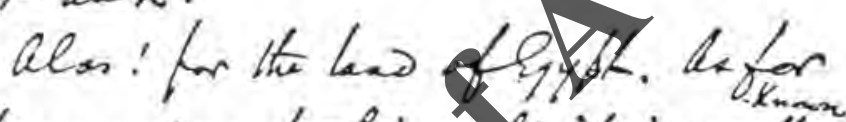
Hat hare dove to hin of it is well ball that the ge th having his

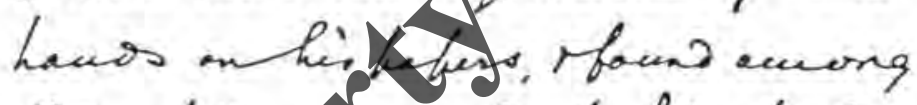

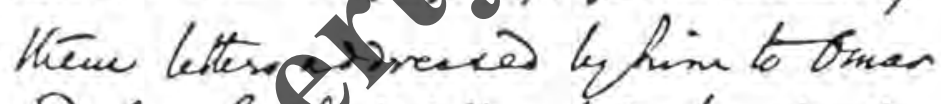
Parka bolo inciting him bat at

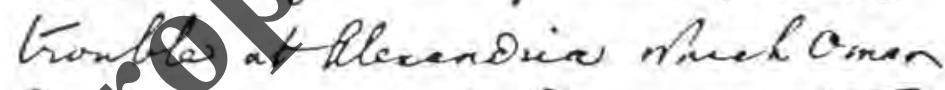
28. aceororidly tia now they

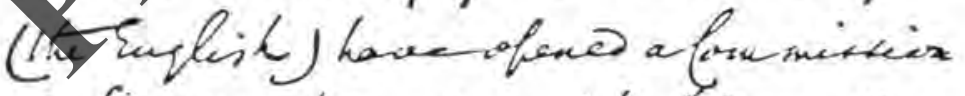

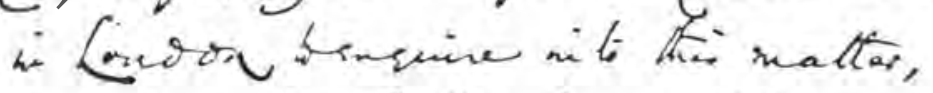

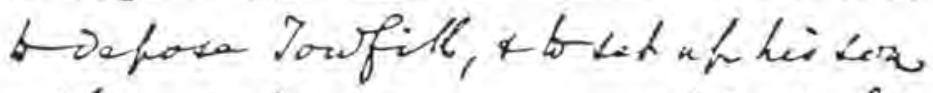

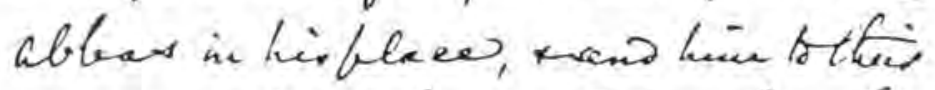
own country the cine ats. In the meantime they will Verein masters of the. 
$r \cdot r$

39

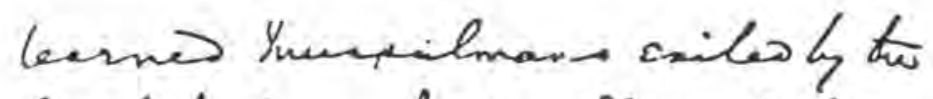

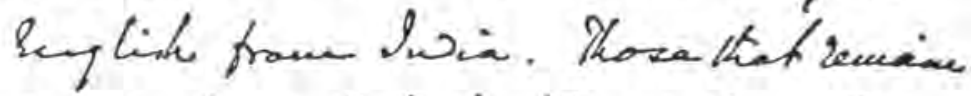
zeciry how thai be thew have him fixates redis certain that they works infer a vioniles fate tees tom thoria

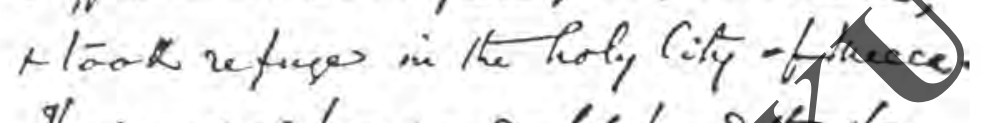

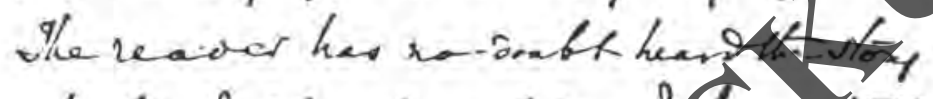

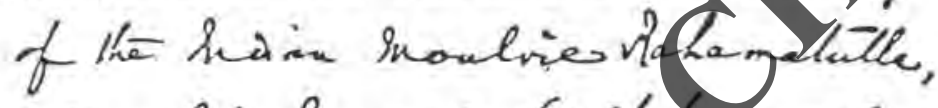

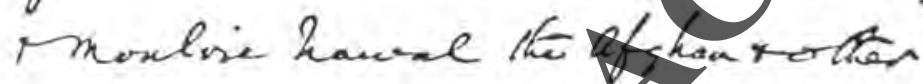

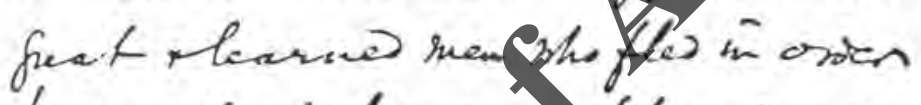

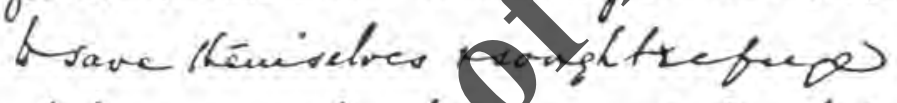

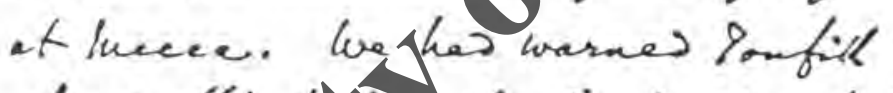
of all this th es inkroglis bath from his 10 at folly he could not

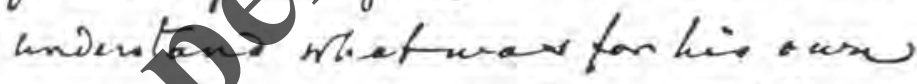

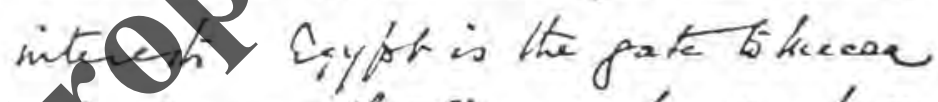
14 has with tic our hans opened * The English who, unless surcoreen corner to us from hor Almighty, with inter the Moly city - become farceuse of the law of the jas. Will religion train thus the ne? This is the pats calamity that Dow fill her rawer averts haw.

As 
38

$r \cdot r$

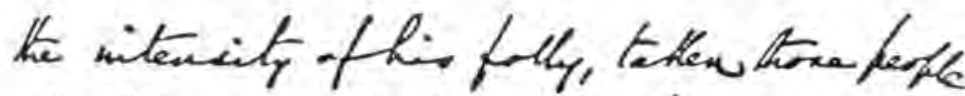

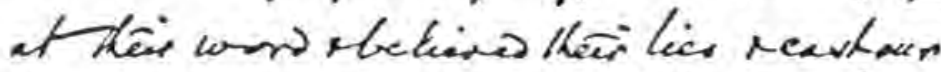

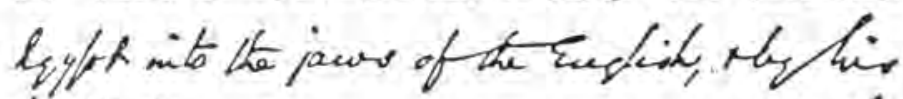
folly hax rawow men a great calemity

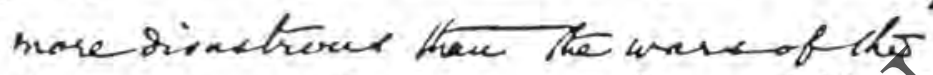
Geacaver. Elypt mecclad

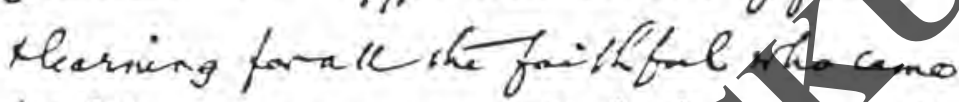

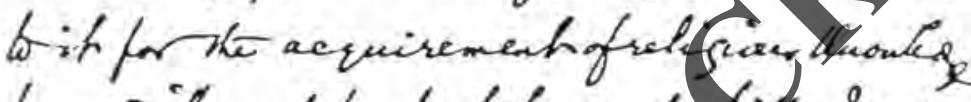
frous riferent farto of the whe the hele.

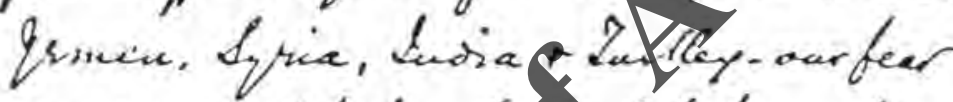
hors is that he for the here beew quat in lari angly the will have.

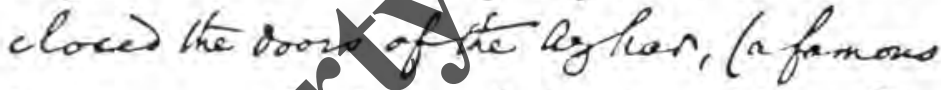

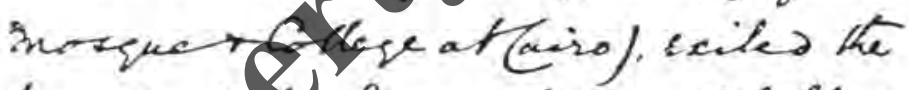

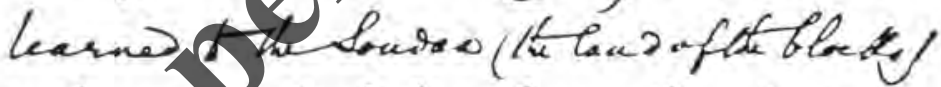
fut Oof theteahing of exhisions

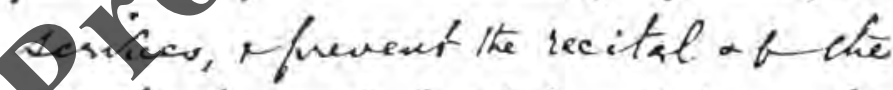

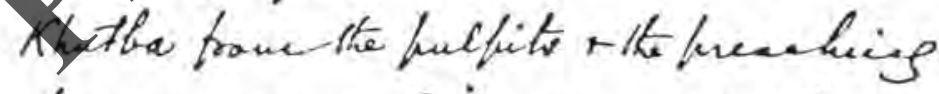
furmones on tridares, as they have

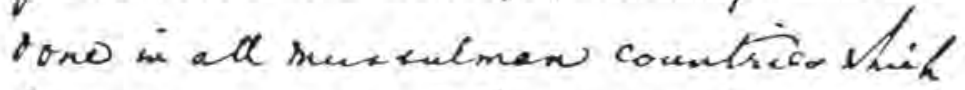
have come unter thein dway. hoodat the Philiffinine o becuod, the Andanens raurthatse which are full of 
$+0$

have sore kics fruphost at vies. thelter heis mi not caudineness by the Rruach fortitis not forme torap

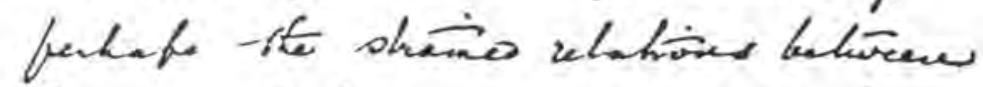

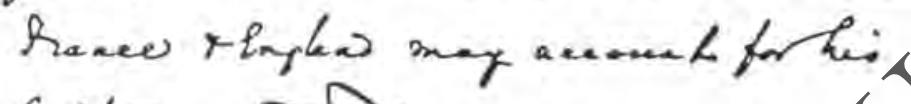

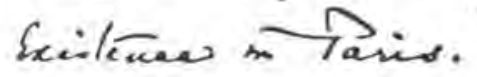

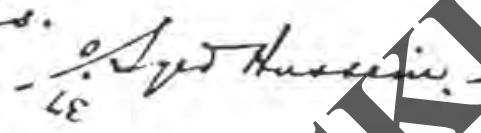

$x: 1-$

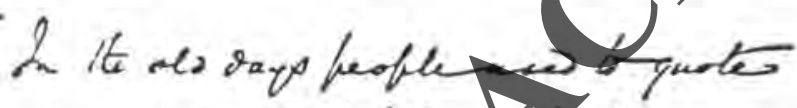

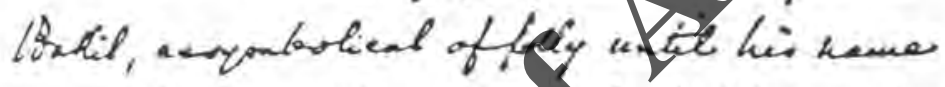

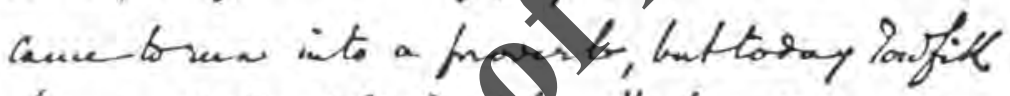

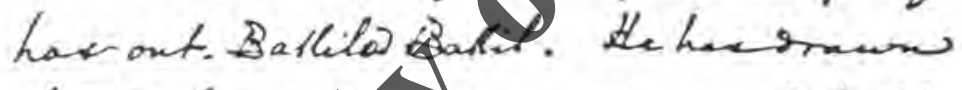

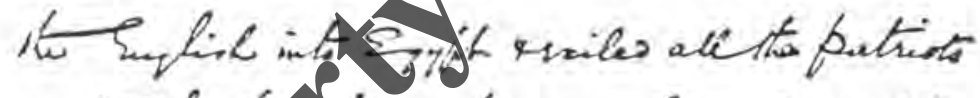

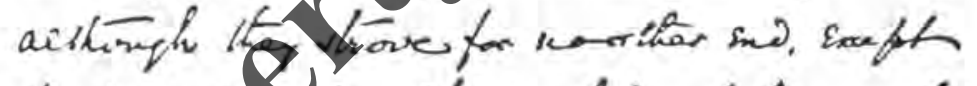
to shang his thesisediff, wh thavent

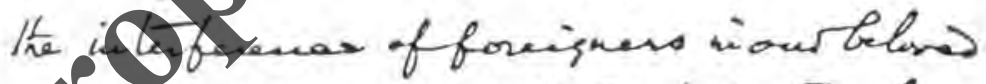

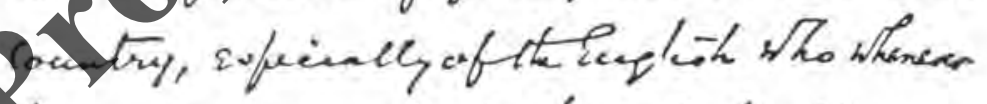

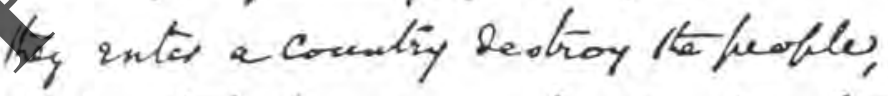
kim theis houses, seterminates the

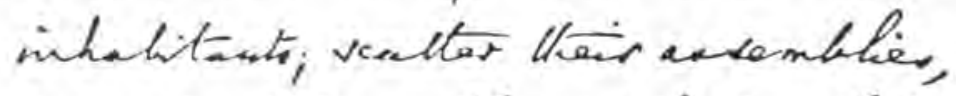
deqrase their nobles, simfricion theis King in duranae vilos as thâs have sones

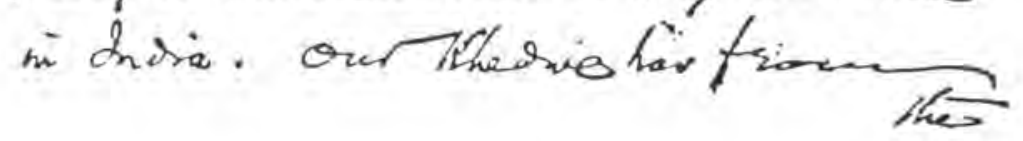


36

$r \cdot 7$

Jfound thatit max eansitaty

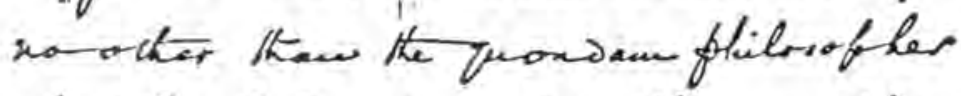

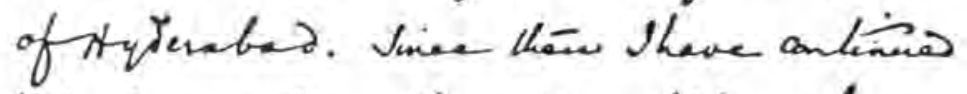
the foorened site copice fit, ar have

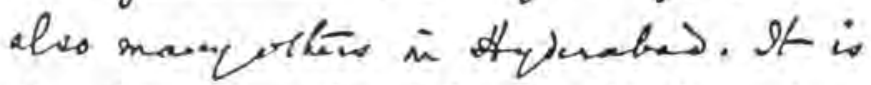

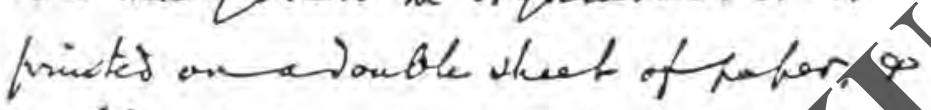
withis the linito of the four be it

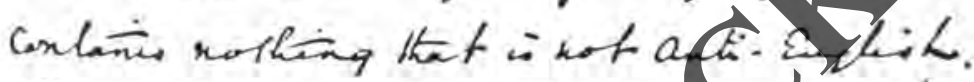
the faper in my humble tin. is not

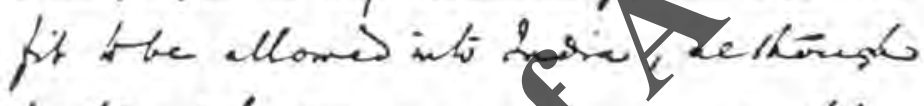
fontimately, there are trang in thes

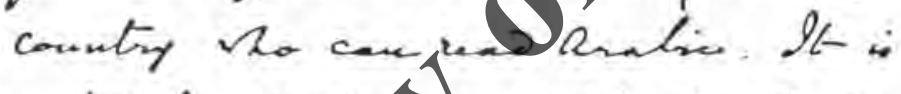
culaing otell guitablo for $\varepsilon_{7 y}$ bl

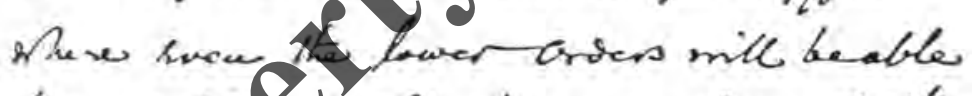
treed if of 1 is hewerver the an hination

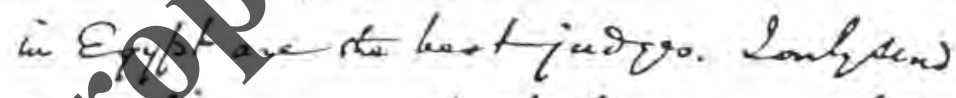
This aceorent of the haw, the

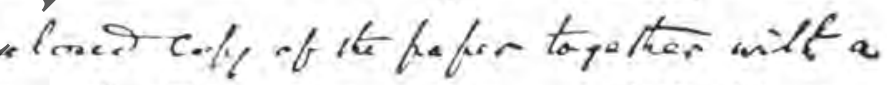
tranclation of fouliom ofit, lfintling

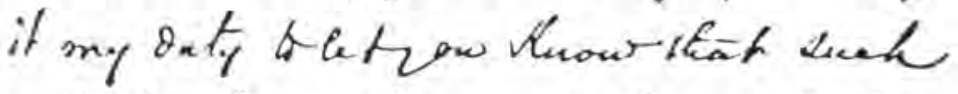
a furkerfins its way theferatiad. Inov ats that torny kuowleser te man is focenilews, anuet thereforo hare 
$r \cdot Y$

35

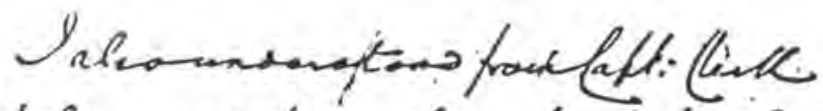

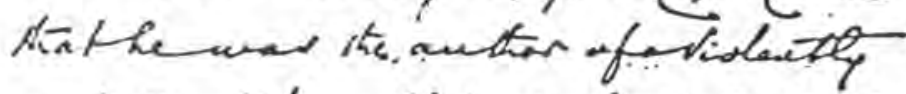

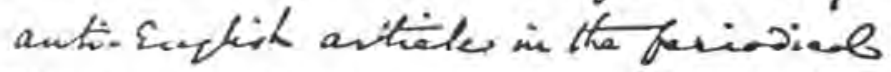

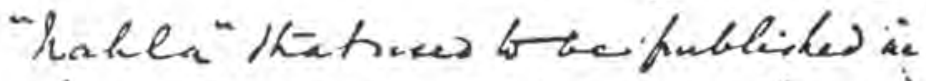
lacetor. Ifex: hat sinistas fare hain a confle of therarand rafke truable him theve the curfto. but ikenes that he dia not terter

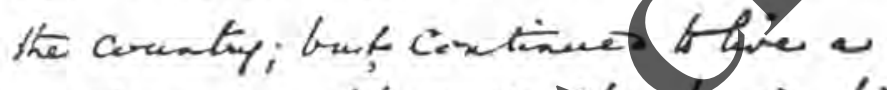
2a her zelives life in the verpending his

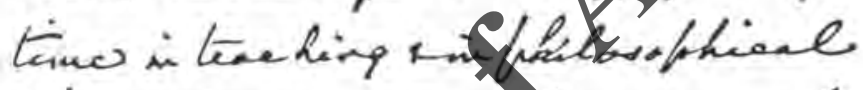
sicuevions - whes hemever the

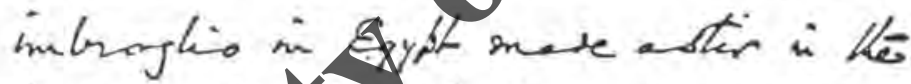

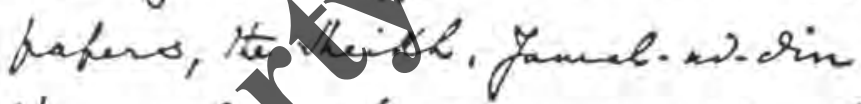

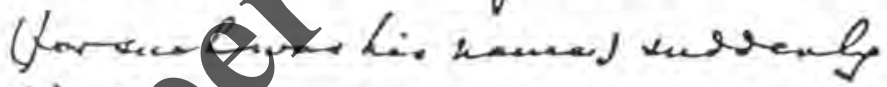

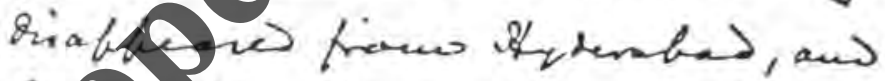
tor fiven $t$ knoustans that he was to Burace. Ifectquite tumes,

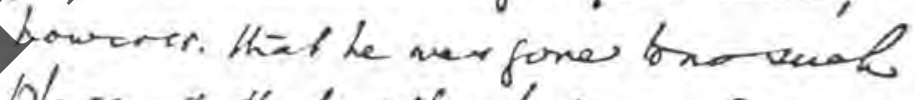
place, t that sitkerlain riasid hawhir redination.

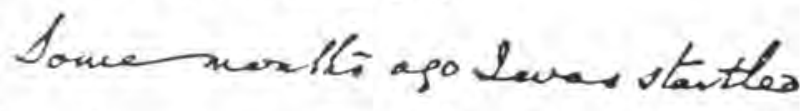
ky havrip an habie ferio sad vent bo me trom Saris, som obening it 
34

$+\lambda$

to: Pane $188 \%$.

lugtarsir.

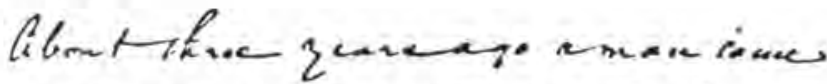

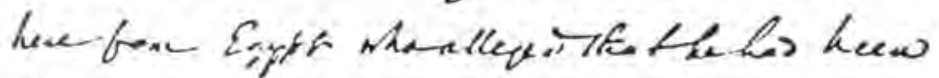

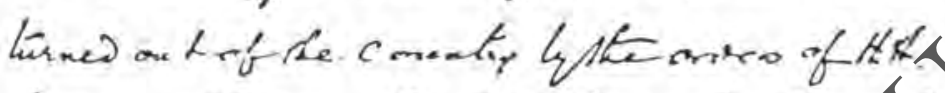

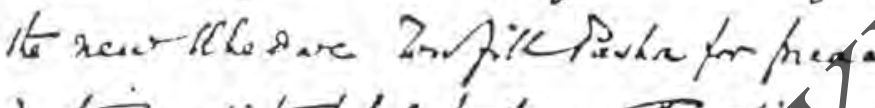

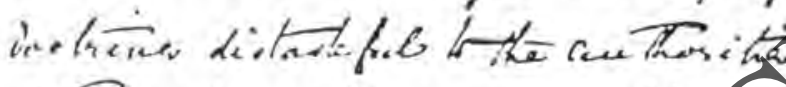

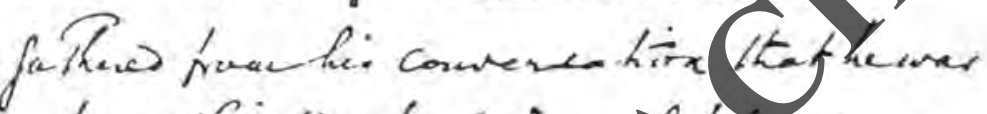

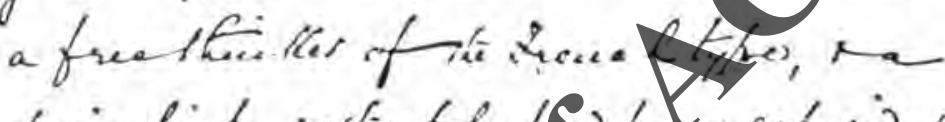

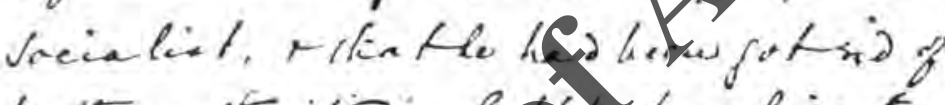

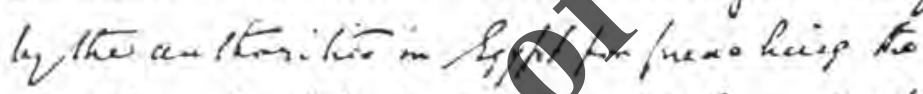

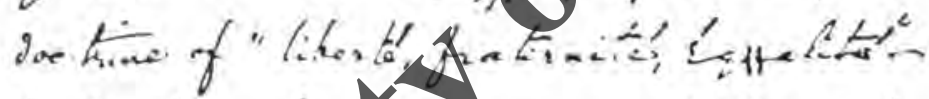

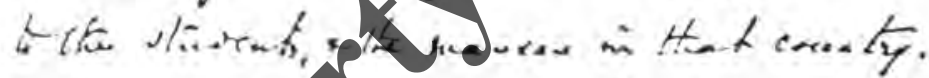

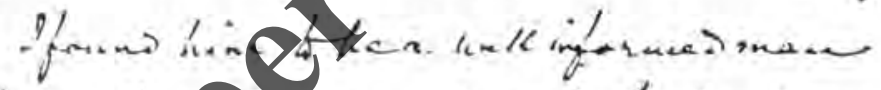

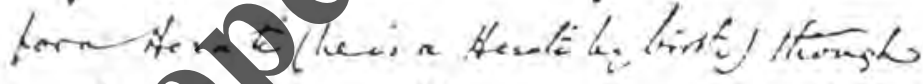

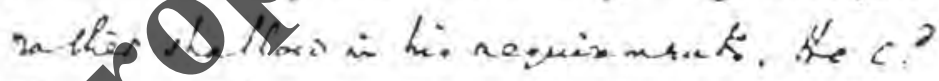

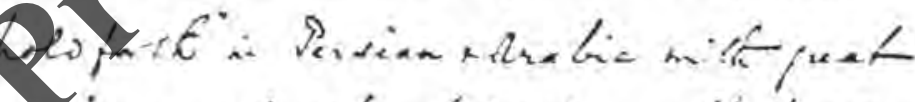

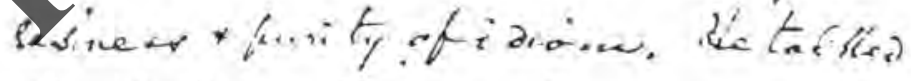

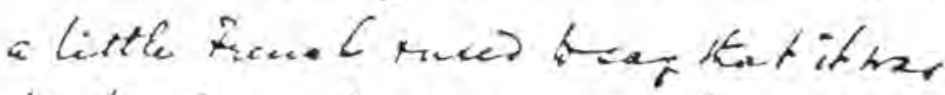

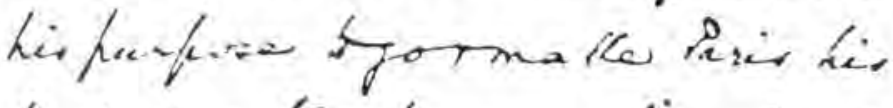
heat ruestirs for vome teries in asen

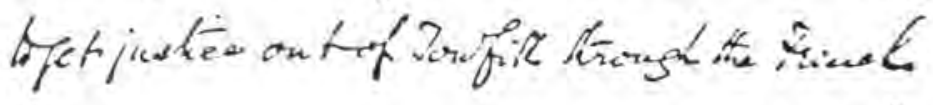

$\vartheta$ 
Cipos

the kexience thierabs

$$
\text { qune } 25^{*} 1 / s
$$

Siviertanat,

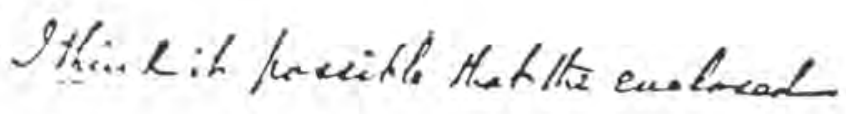

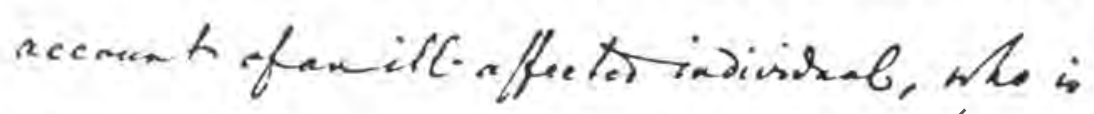

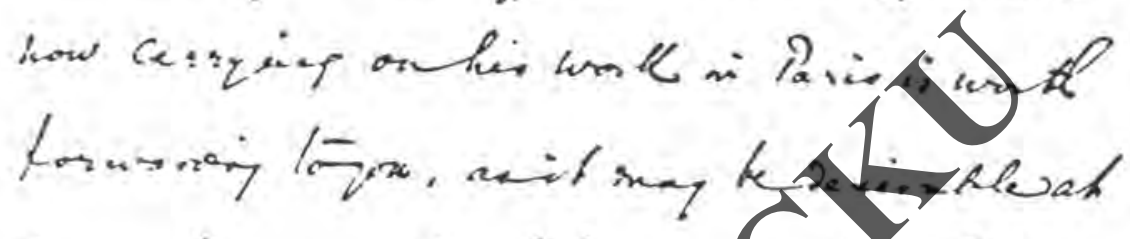
some times or other the ac in sith his ankcesict.. Jaber cuefre tiaurla tir

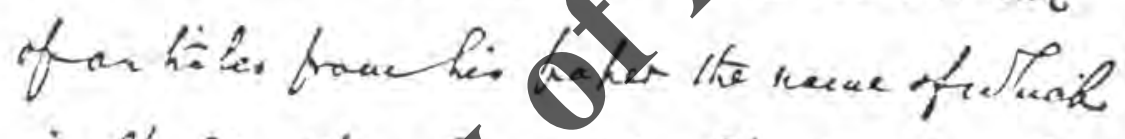
$\therefore$ alo Yarkhar Dent. Oraimlation

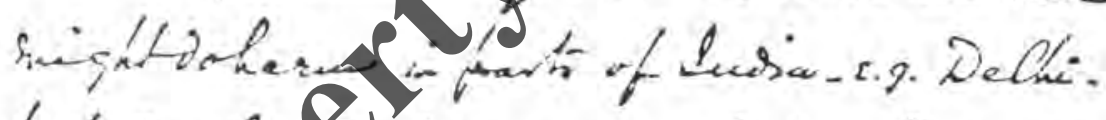

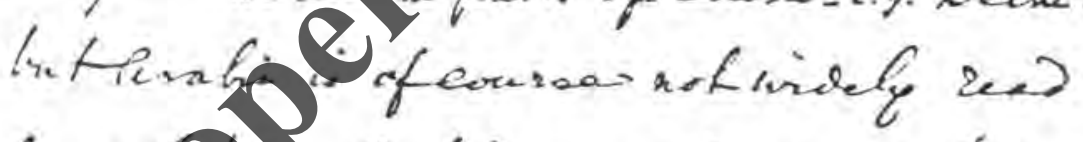

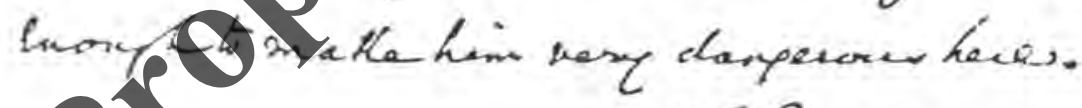
$\because$ Elaveny$\checkmark V$. the rlaumbere of who the 2 men is map pertatos be ase fill

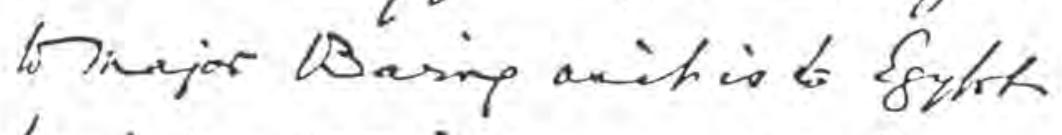
heis now joing. atel 
32

i1.

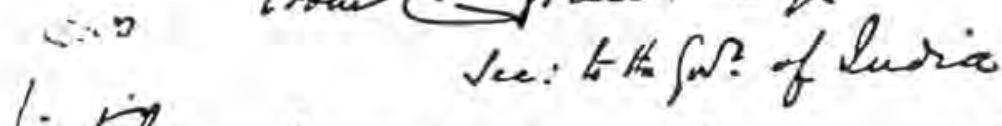

Troul- franters.

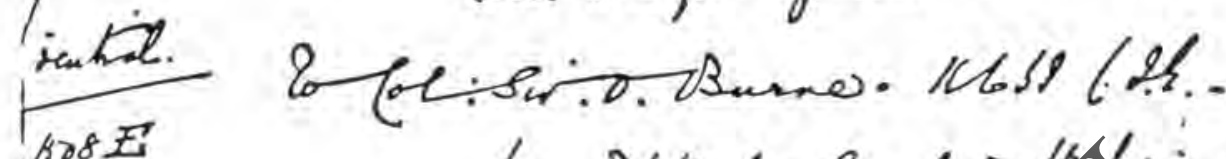

$1308 E$

eiprout:

likical. Si.

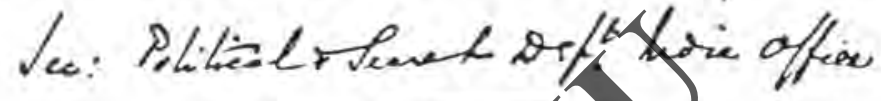

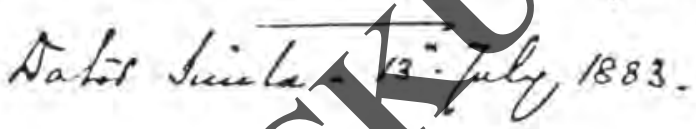

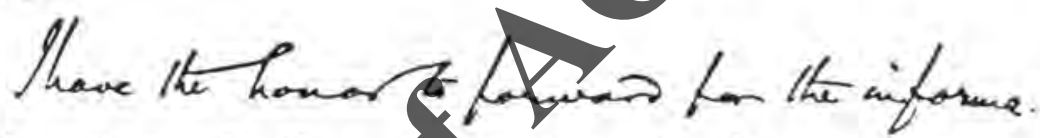

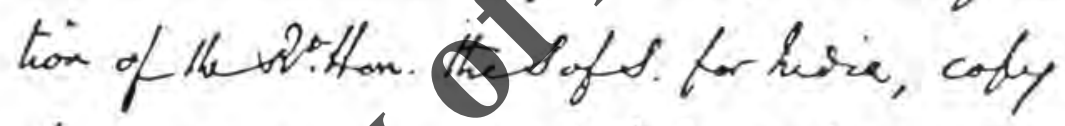

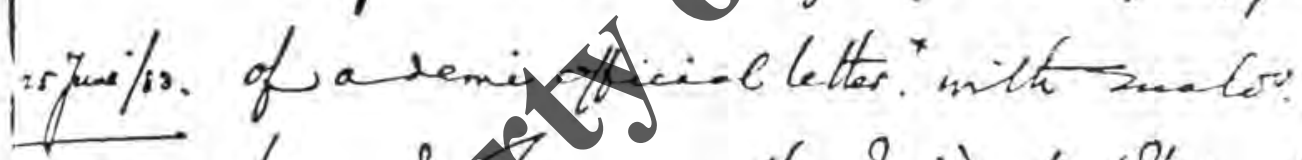

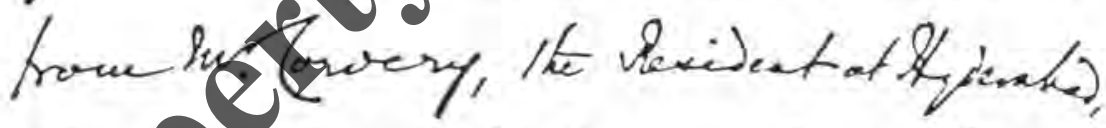

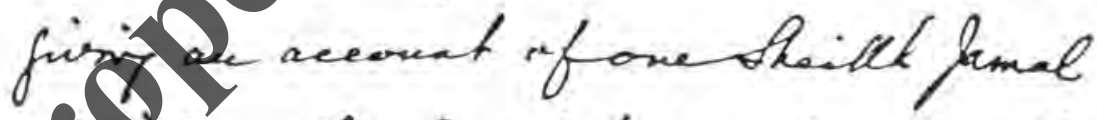

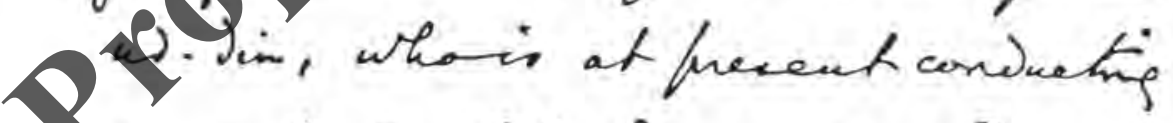

an auti. Eufferk tisionial attaris.

$\because$ Ofnuts.

ter. Alat of hisio

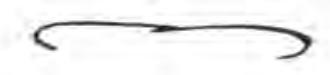




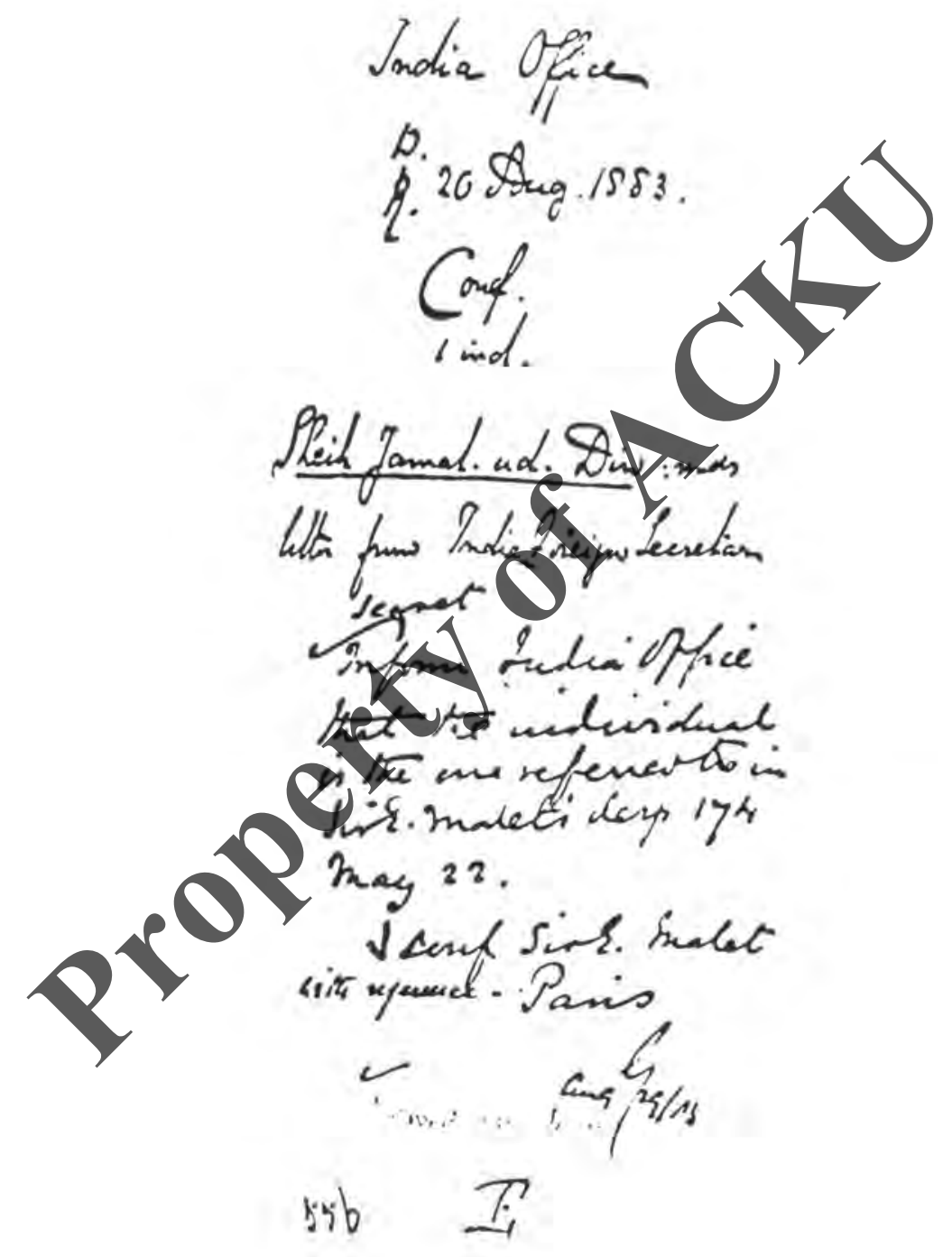


30

PIT

imfintiol.

Limin coffice.

$20^{\text {"Auguat }} 1008$.

Si,

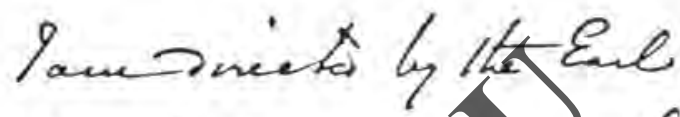

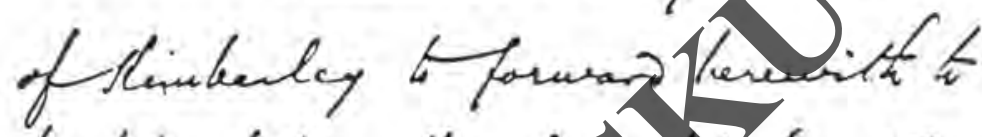

be bis befre the sefesy veculary

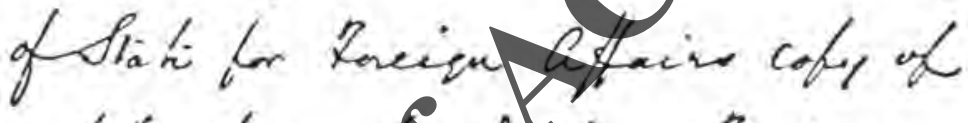

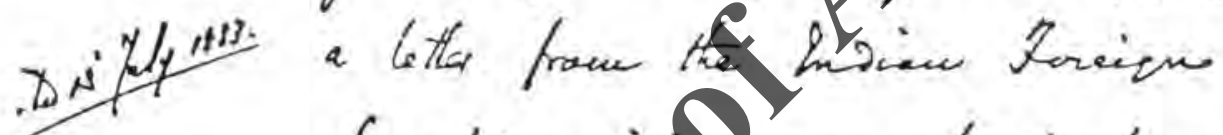

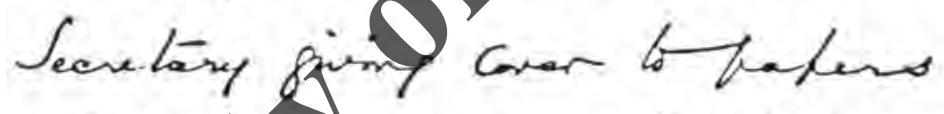

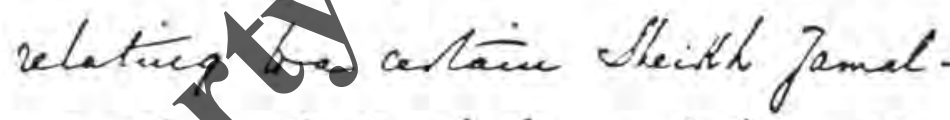

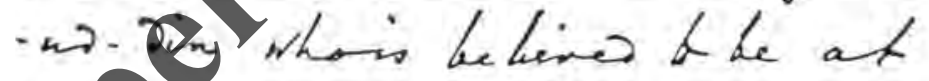

triany i Paris. -

Heree te houm to the.

Si,

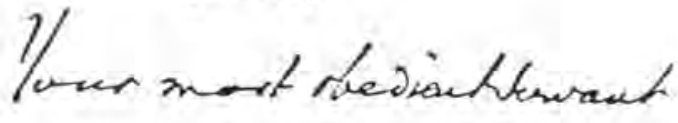

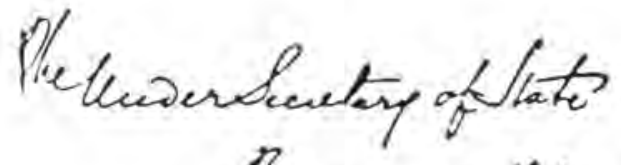

Foreigr Office.

Lrandencied 


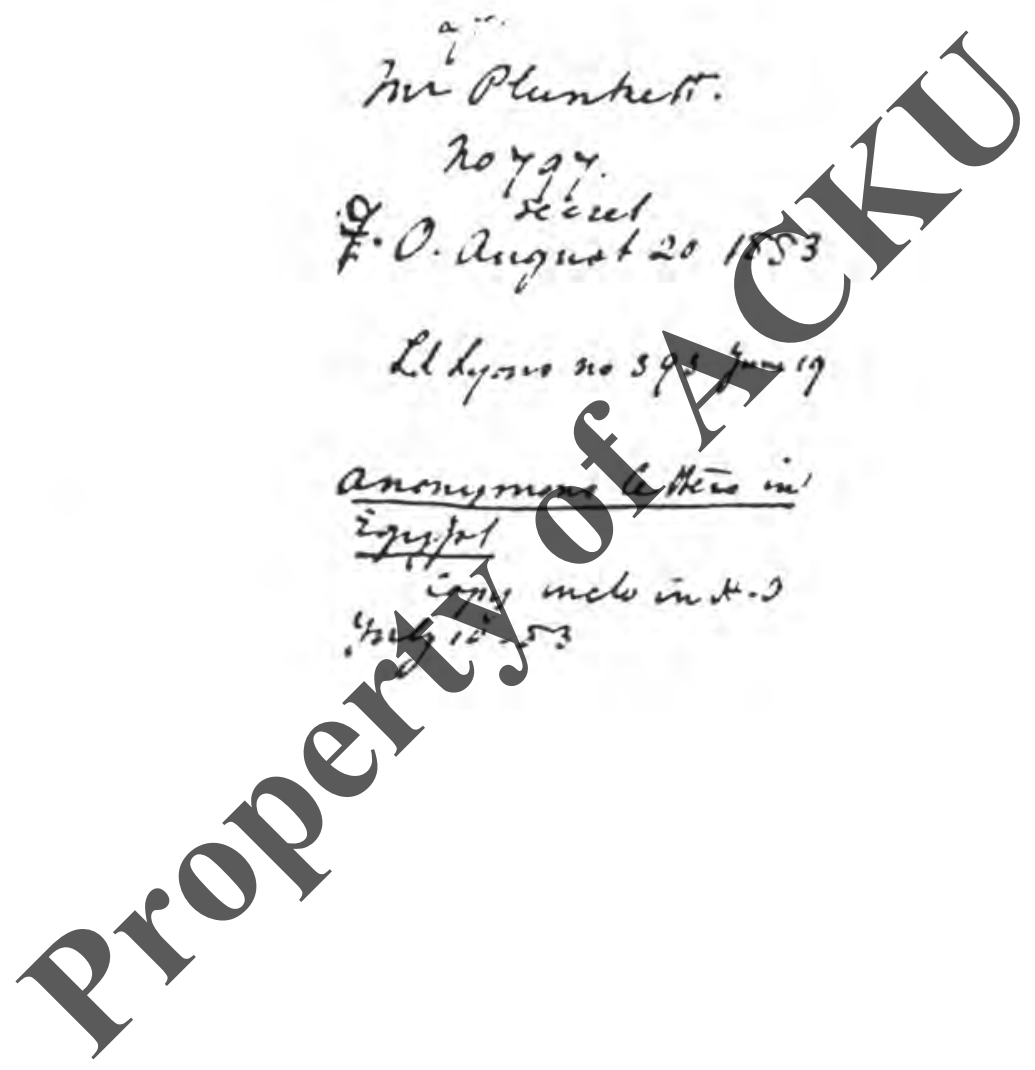


7. $a_{y} \cdot 26$ coss.

Sf.

tw. Plunket. his,

to. 997 Secut mits up. to knes Lqui' desa. Ko.3gs q the 19 " jout

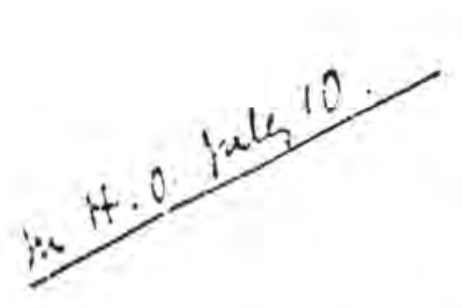

inces niel.

inimitic inelee frit uifrueratis kiss 2 uinte witele bix.

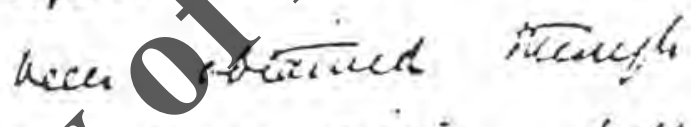
river aifice foren the procie aulit tes is. Oacis in the retpect 7 the afflew formenel.Din, the pesuned autres of certrion

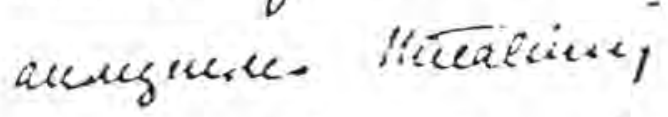
Wtees atrefed to the kicesice ree?

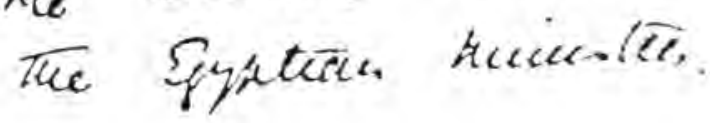


$\therefore$ is cofiacuteri

livel vepetay thes anses siveir has

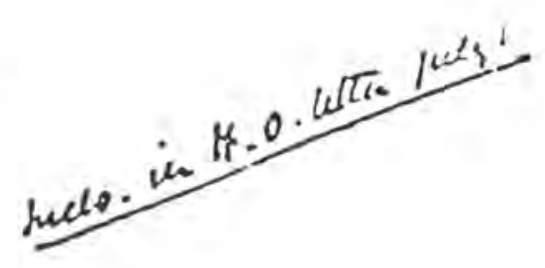

kate frasinger it the

since ivirie \&s the

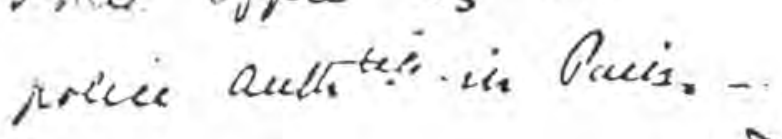
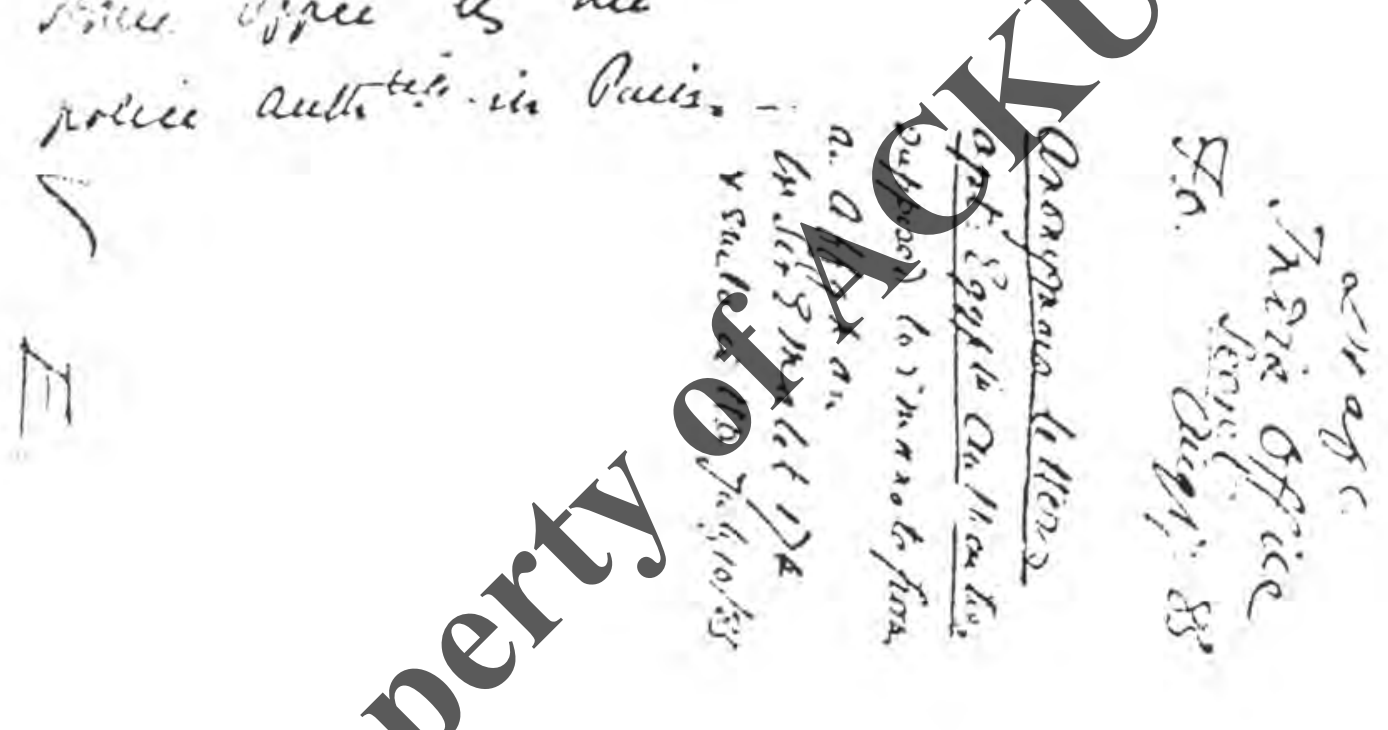
of.

7.0. Acy. 17wos.

nura offic

Seut.

hir,

1 acu Surited bs

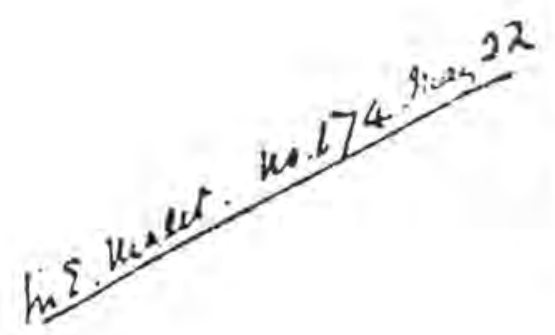

Eave franili $\bar{T}$ enceres of

स्रे infrucation leas is sed tre mia, Pows ? a leoplom inde. quat Eunalf. ar Cain ventere la certacia akruyzum. titures arreped of the Esptean Auth tes which are nepuse? to enaruate. fune an affexin seckest in Gain names fruel -el. deen

Jam ar. it ecelne coss 


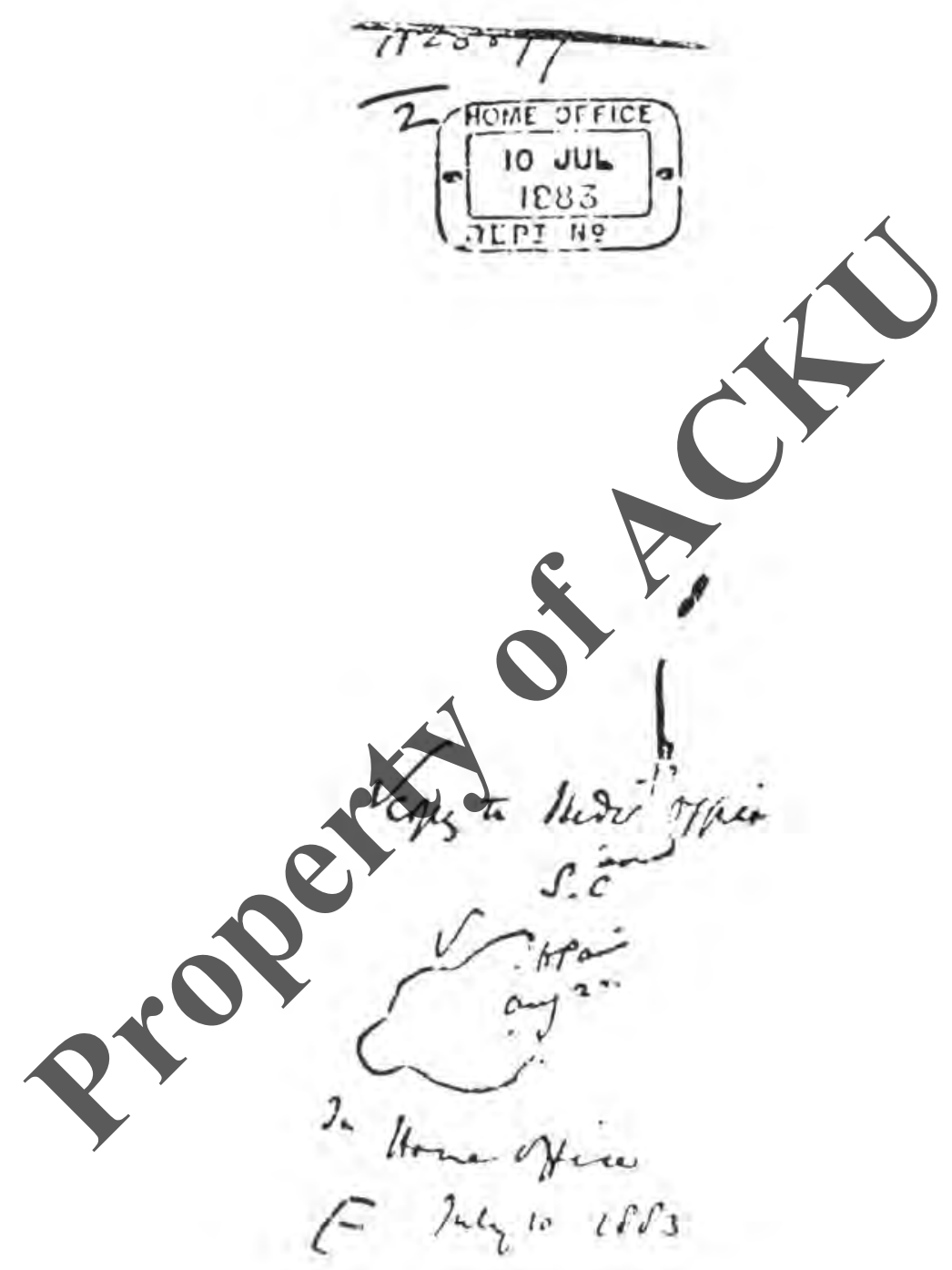


24

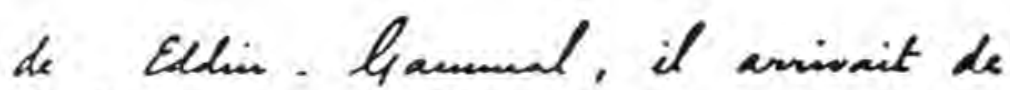
in balcuttar. At it a' etait mene jacmais venue $a^{\circ}$ Parid.

Il fases form itre fort inatrait.

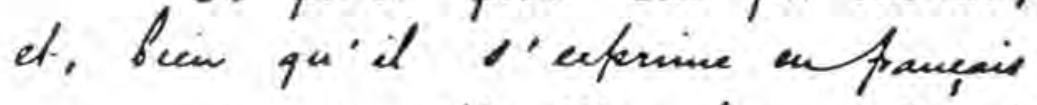
ever aney des diffenties, It coprovitiand tromit langued

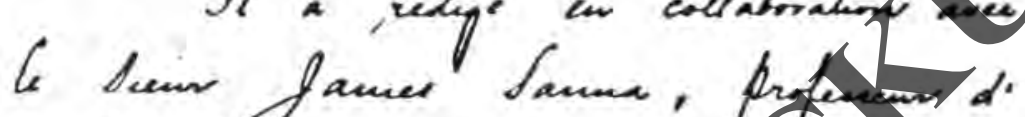
arabe of pedacteur en chat glim jommal ierit en citte bype qui es publie a Paril. "s Pareatue de blich,

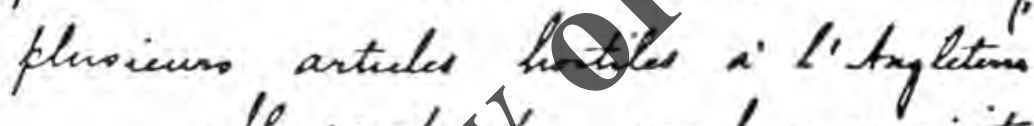
He rent ofes nombremen visits et if paraitip se thomer dous nue Irme siturter de Potme

\$ conduite habitulle et Ae

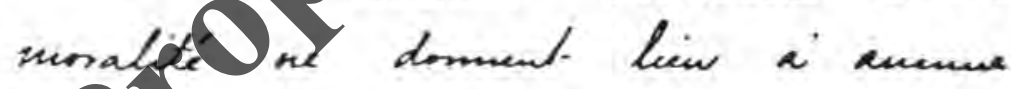
Dengune difarmable

Tenilly agien, Amaienes be

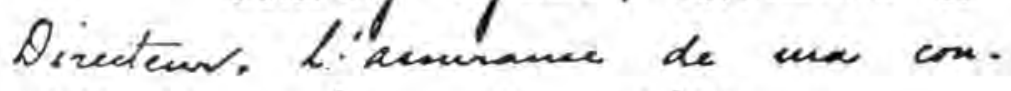
sidination la plas distriygues thy') Le Prefet de Police (uans illyible)

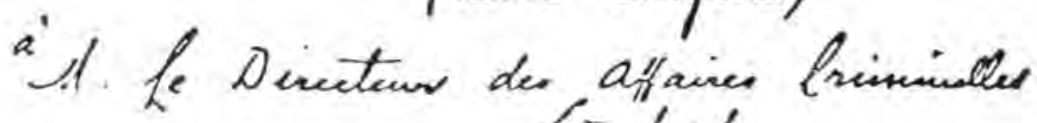


सा

23

Pans le be Givelet 1873

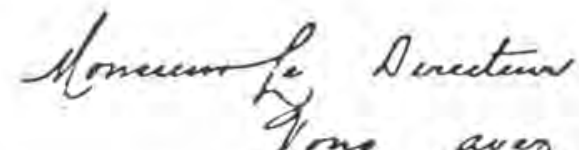

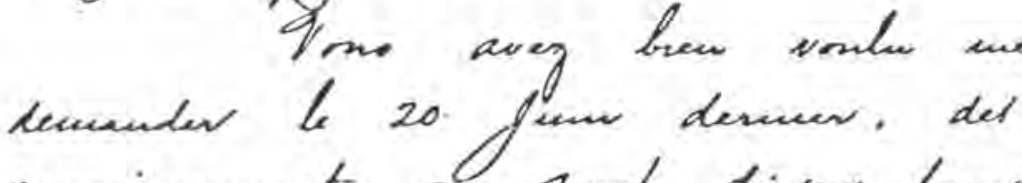
reneciquenerants an supel diun finew

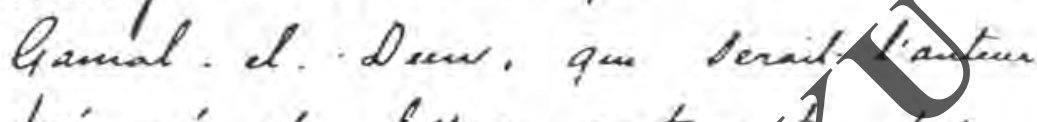
feremune de Letres contenat des menaces de mort adrfiest plowent pereomest Eq

tranomathe a. ghers \&e rémultat de. l'enquite or presente a' celte

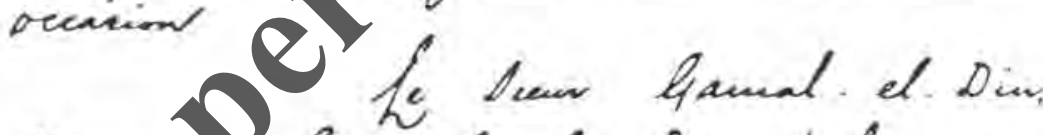
(d) hamal-el. Dewr) homme telted, viginarese d. Afghomitar. de 45 and, edt cétbatave ot loge, defors, le 17 fowner dervier. que de bije.16, an boyer menoual le 50 prande

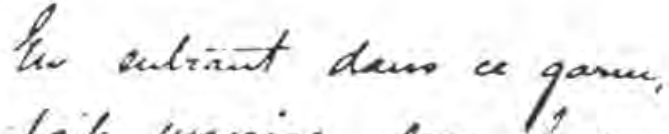

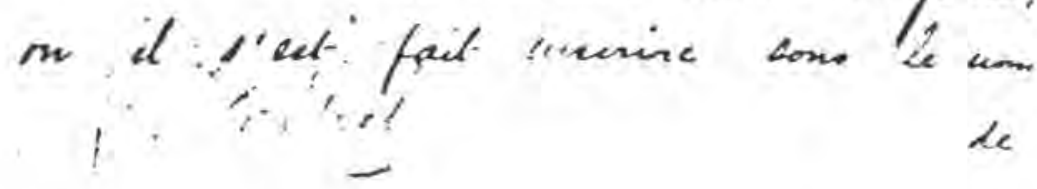


22

ir.

Howe ofine

of 10 I Inly 1883

1 oulon.

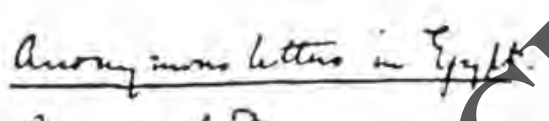

quanal. d. Dan.

Pria ufat nAf Paris.

Rot: $t 015^{2}$ ulte

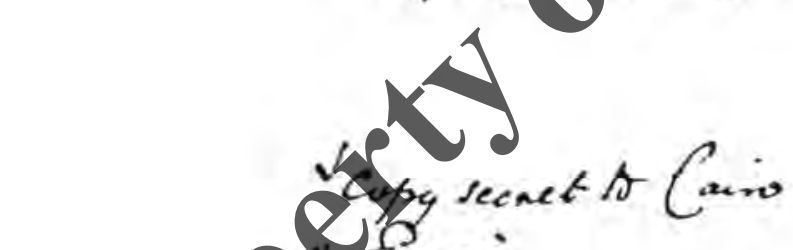

Prone

Tinerme andea

Hice ow reanfol

\& 
Pri

21

Mot inary might he mate

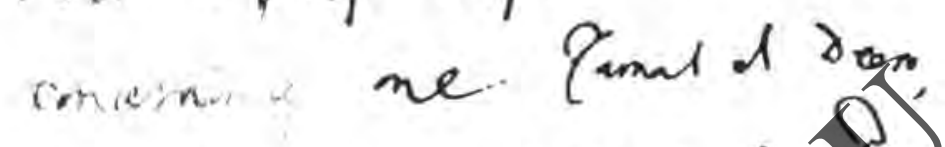
on $A_{f} g$ hen. raident in I.m risected

Jevetary of stere

irenomit to 192 to k laco befoe tho rraluseterter frma. the Plice

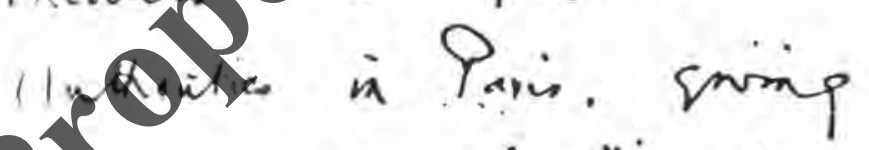
cer newat of $x$. max.

"nsis

your redisis sorvant.

Af Itedell 


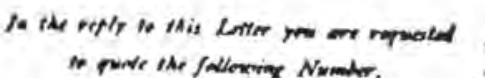

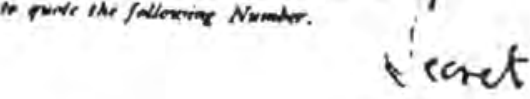

A. 2507 .

Líntelit.

$10^{k g} g_{y}-1583$

$V_{\text {is }}$

Lich eforence

haw of $e^{k}$

formaning of of of 1

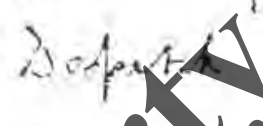

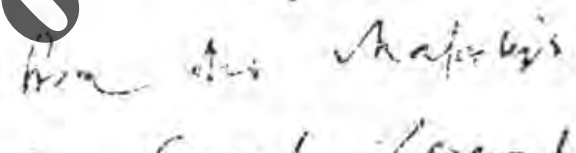

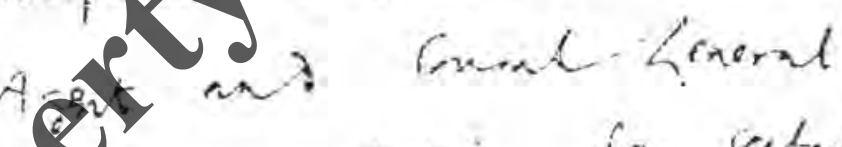

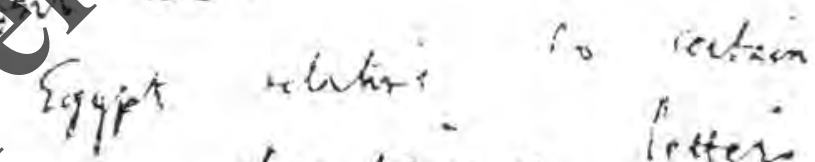

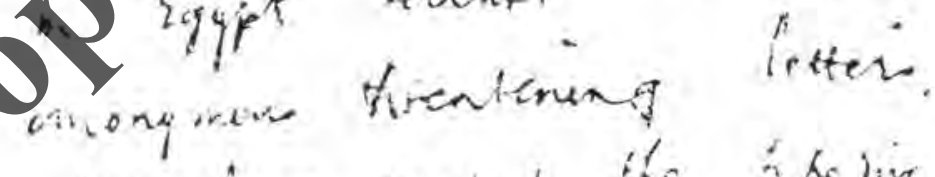

whit hase knowe the shelin and hes Minuters, axis arkeg

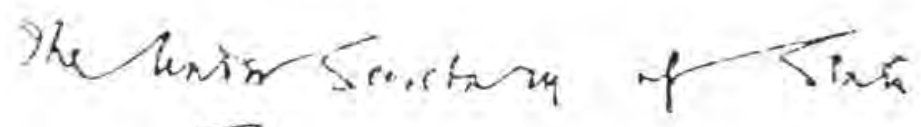

$\nabla$ oreer offino 


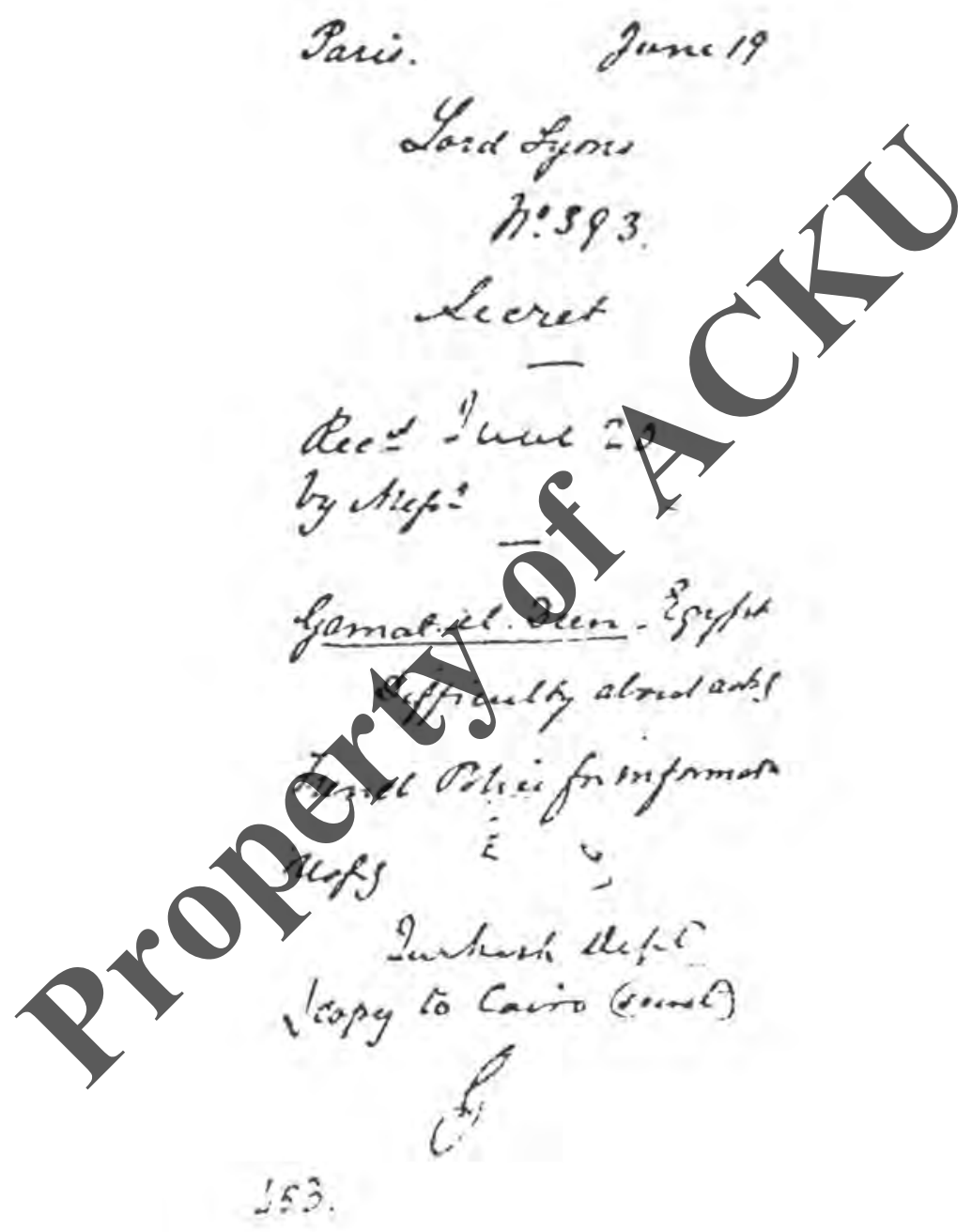


18

irt

any uegfol mformation beang geven by the Hench Goumment, and it wenter mot,

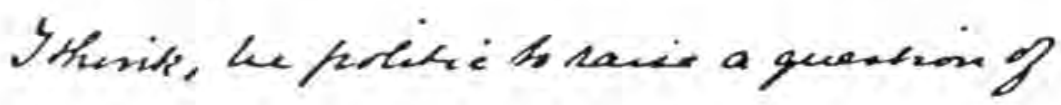
She kind with them at-Bhe; trme.

Inile fowener hearthas

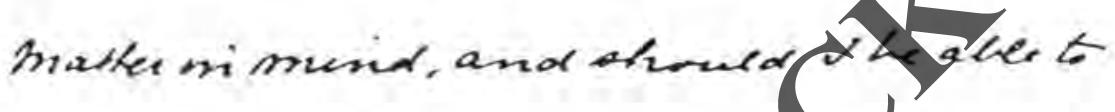

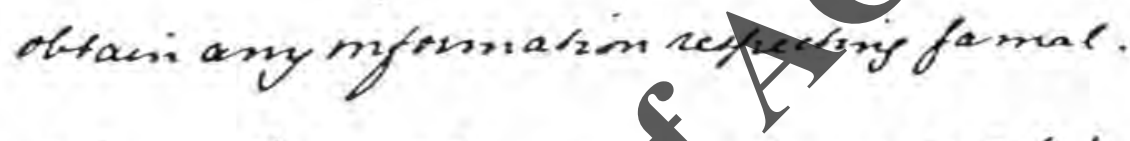

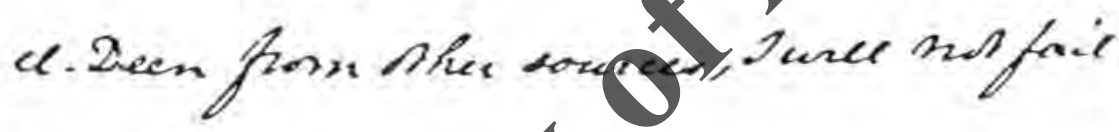

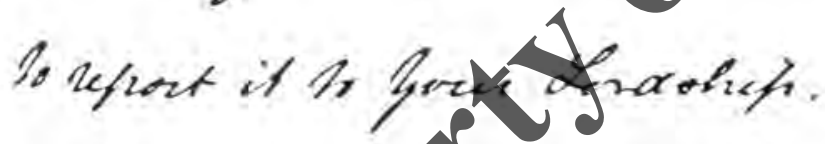

Yhavefis linume, in he

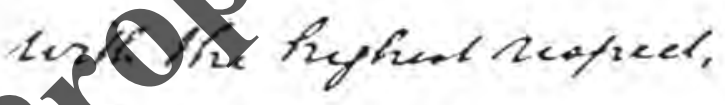

Biyorod,

Zexer indetistio:

hoor dieduit

Kandbe sevant.

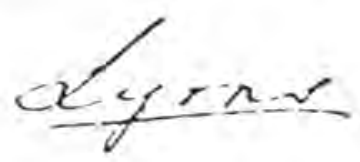


rto

17

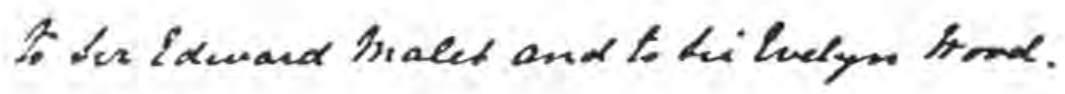

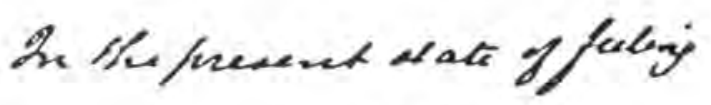

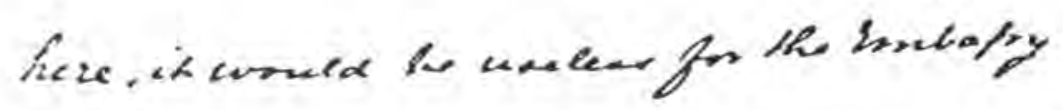
Sapply to the Funch Praci many preteres

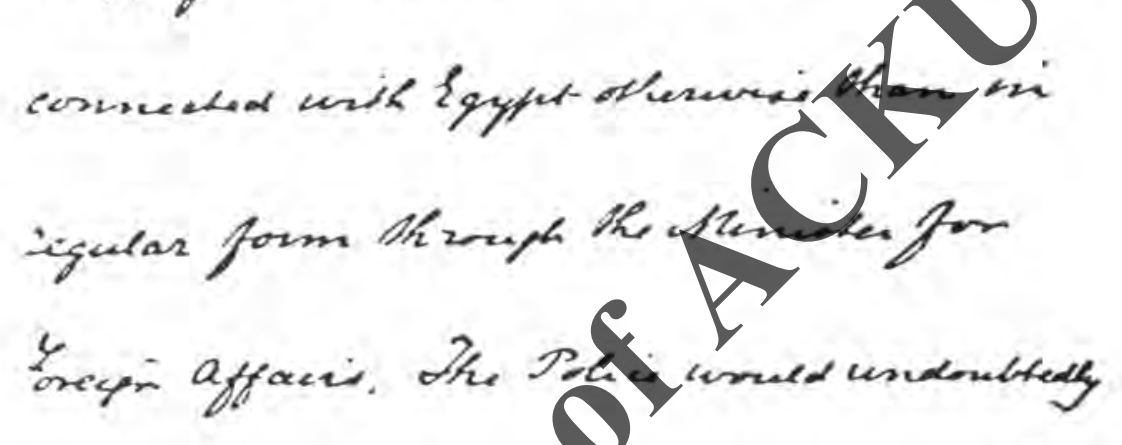

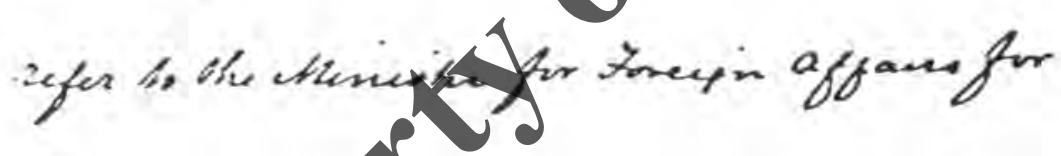

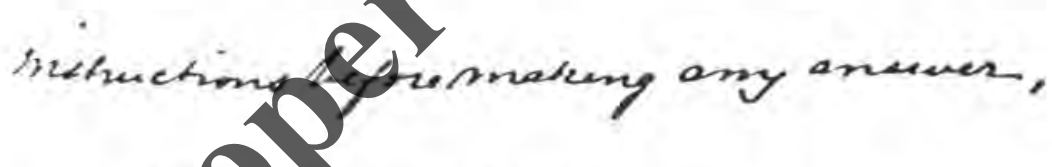

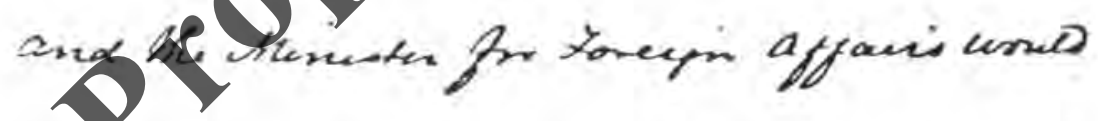

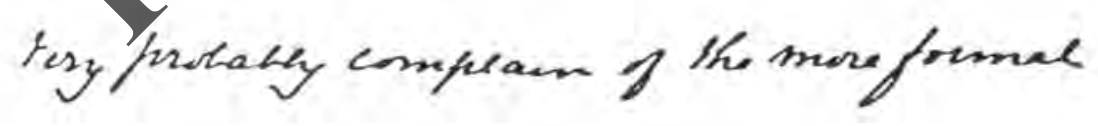
Evine nothaves heen daken.

bn the then hand Idoukt ary 
16

pry

Pari

$k \cdot 393$

Leme19.1883.

decret

Mryoloce,

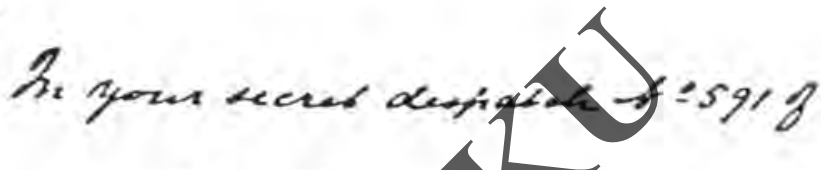

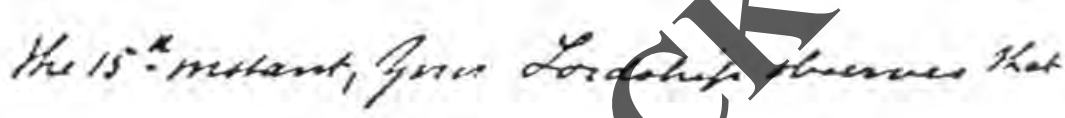

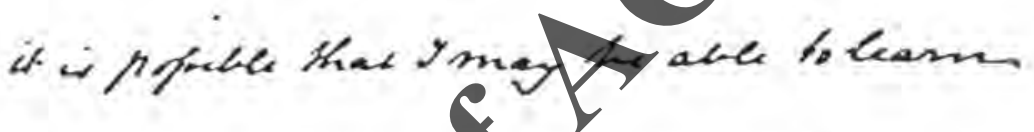

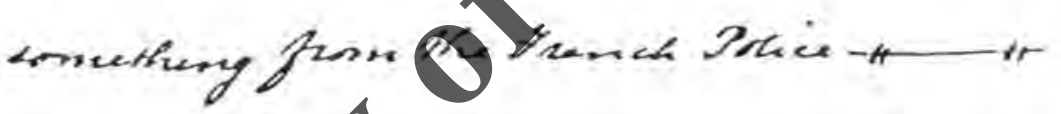

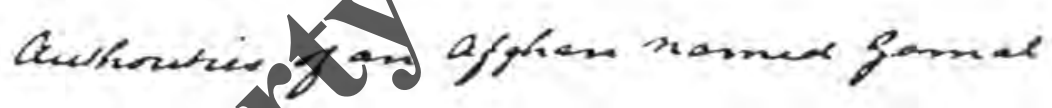

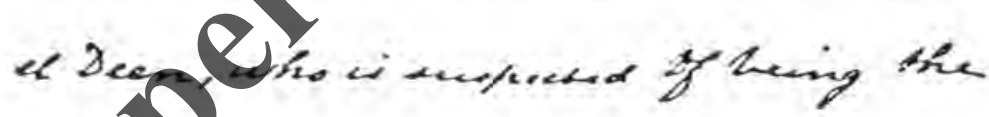

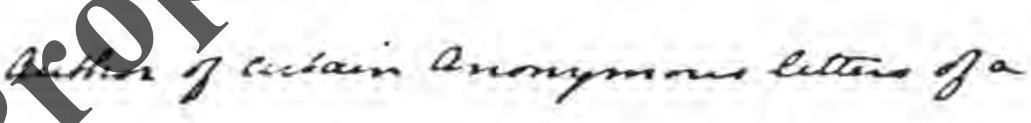

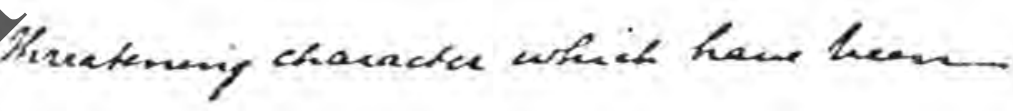

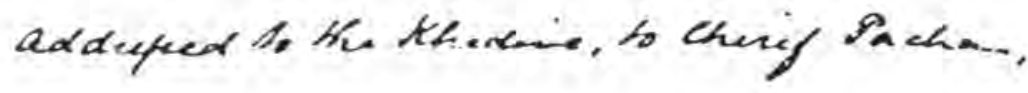

婂 Eare pimance s. g.

* a.

s. $s$. 
FTY

15

in vily, quiney ares recured of tre, Meare. wister hatrig hial ke, wase at rue lises oxplets frue, appesis -

I acer us connus: mitu the sorse uffice ceplentely iki. man, aud it $x$.

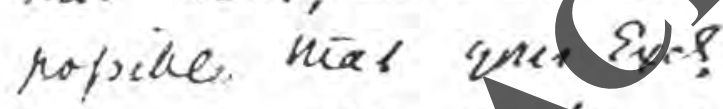

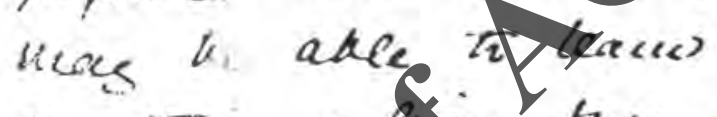

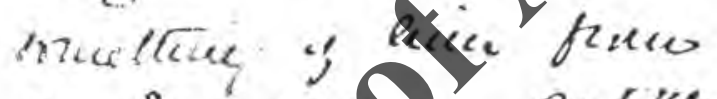

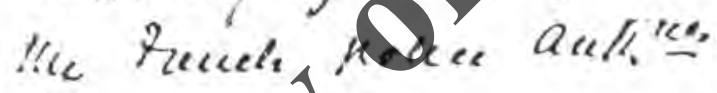


set.

7.0. ruce is $\cos ^{\circ} 3$.

Viscresch keques.

koryotenes. ky knt.

$\checkmark$ hasismat cons

Wo 1040

3 a s.a. , is eneles

pure tone. aruts

circulf is is

ratine to colation

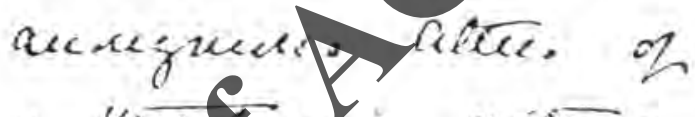

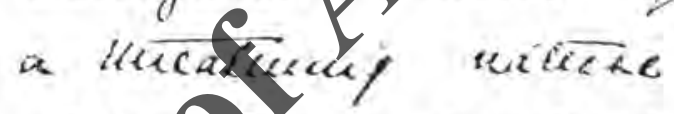
smigh Dame zercelud

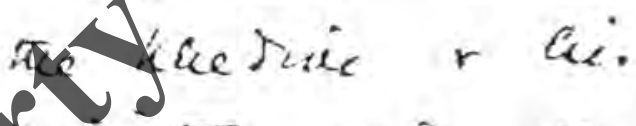

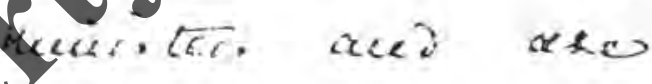
helecued th mucarate fruer an atplares. mened gancal el Rere, wher is is appotiatiod witr

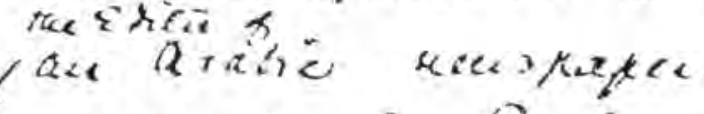
patalinges is Pitizo. (*9. Avenue de carlig,) 'alao euclioe cisy a Ben. Anitua ists 


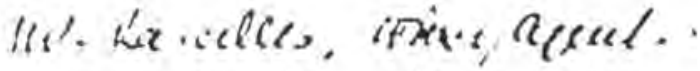

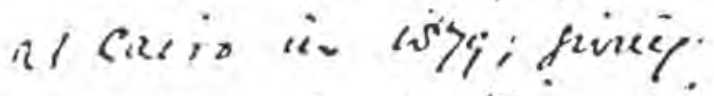

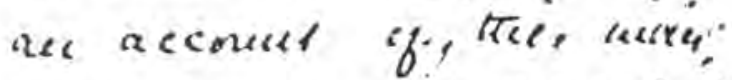
aced I rees ritegelel, "

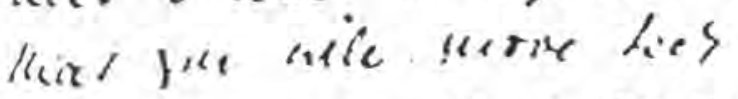

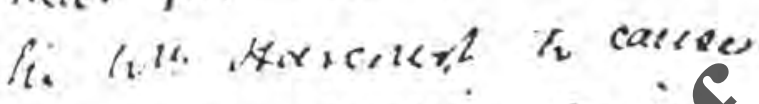

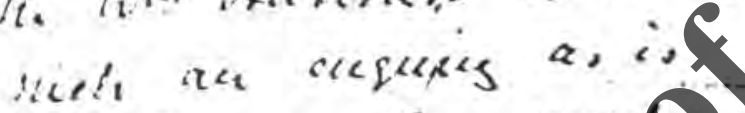

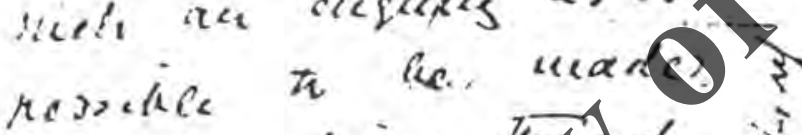

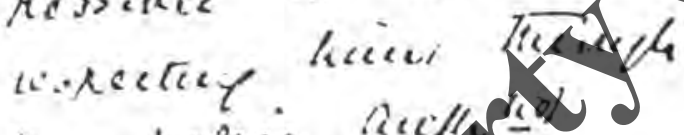
hee troice hathe i)
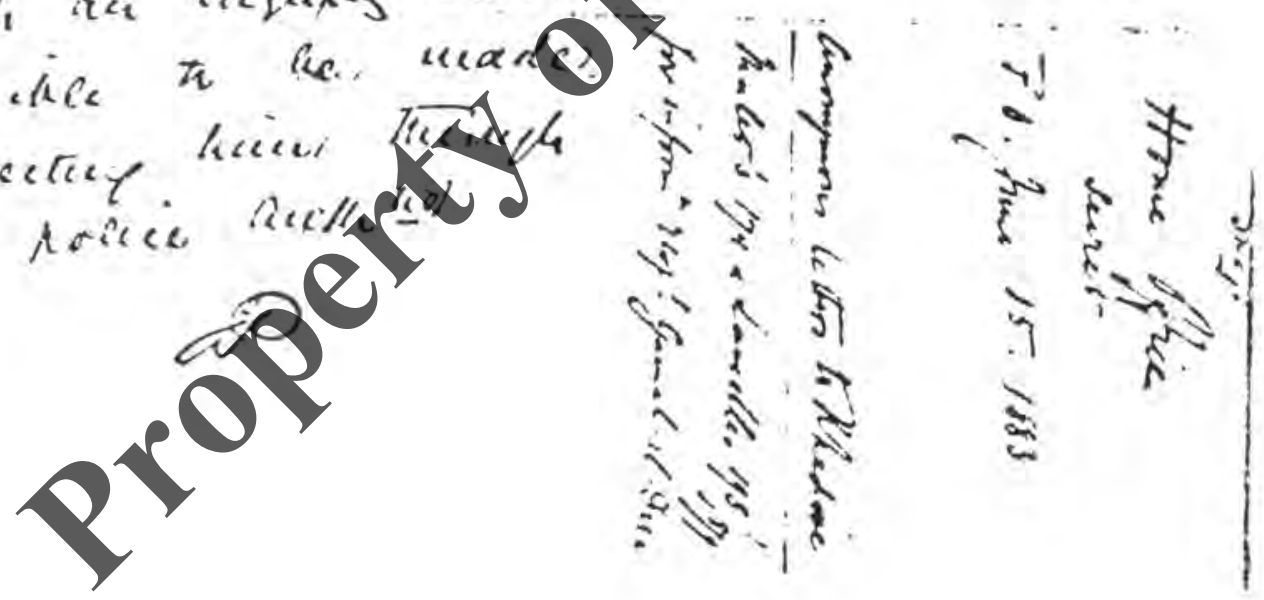
12

rr.

ste.

Zo fruce 15 cops.

Hizees appes:

Secet. Pir,

' sues dreitide of sare fiaunlle

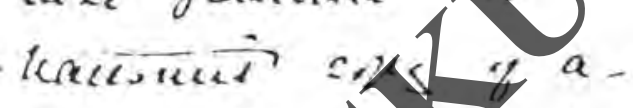

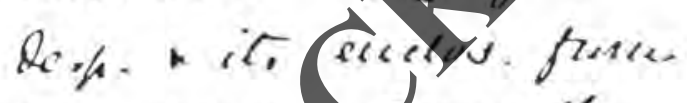

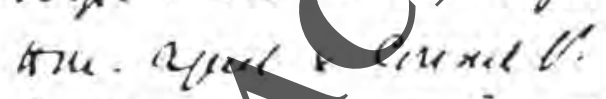

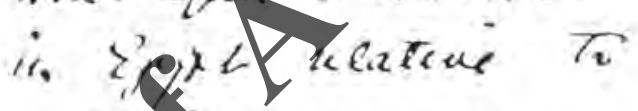

corligery acciseguesed

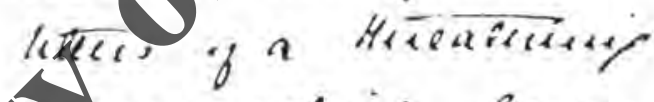

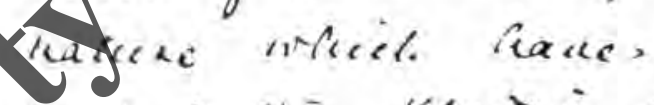

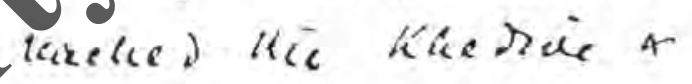
ki. Huicestles aed

are kelieses is sereamet.

fine, are liffleane rearse

Paucel of deces, hitco

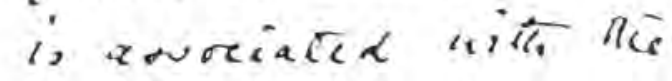

Exict of xer lientia.

resspagie tublibel is Pa....

Inue afos te ececeres, cays y a deoth. fiskis 
pri

11

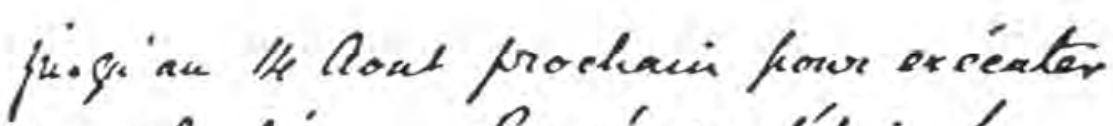
deo rolorte. - Proeé ea délai da ícgice freudica des neareres lex decusacoses forecéc.

royen et e'féfeliexees

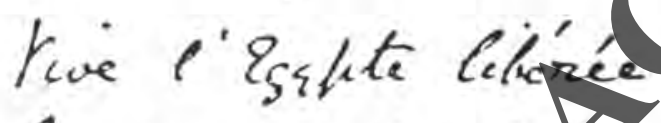
licont aux Ruglact Le lhef dis laligive.

Abropied is (onalie)

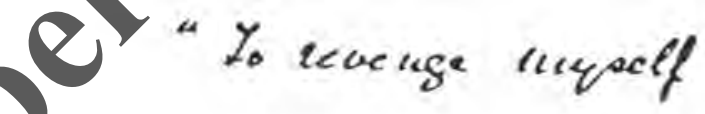


10

pre

Cofy

e Paticoligias

stirsune

diteratiós do cerritore

*) Earleration

Peoperité -

La lagie ardoume an fiefral Hood de demuer ra dévieneiph toun le gredé

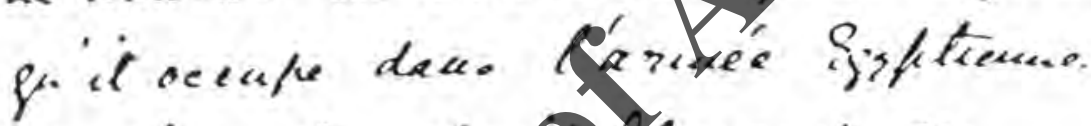

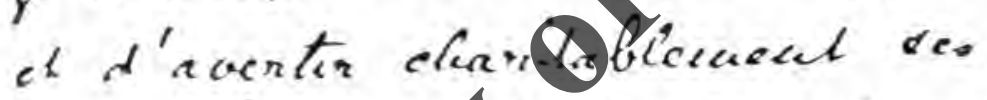
e-kntionausy ágeri de mêues

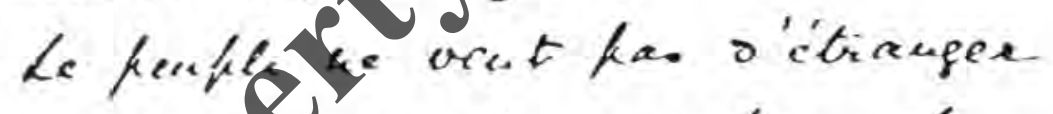
poun leserucueseder ore four le foupener.

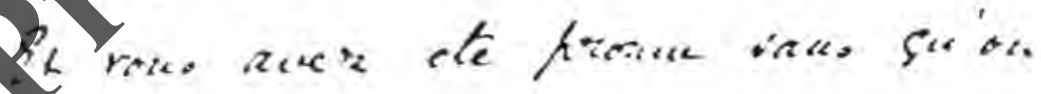
luse dewand on rom:

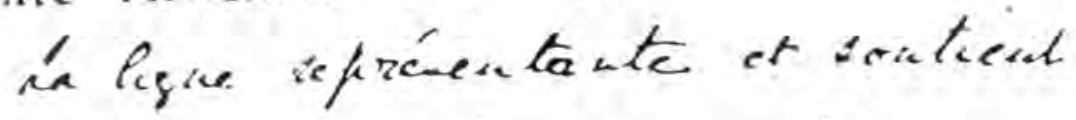

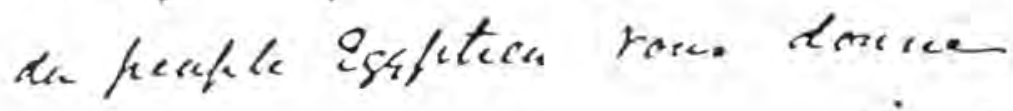

$$
\text { fiopica }
$$




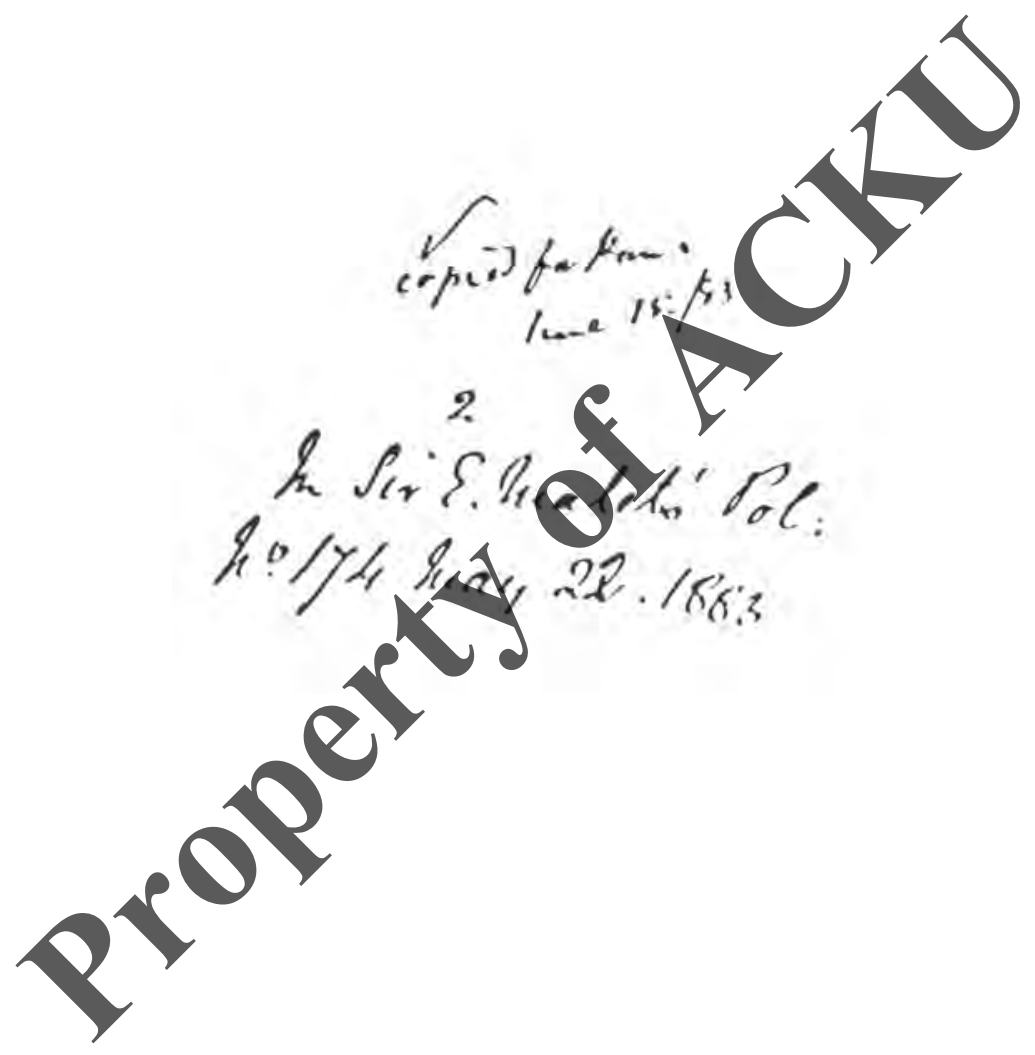


8

prit

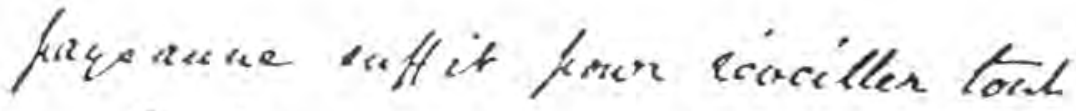

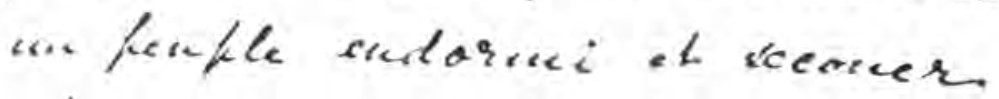

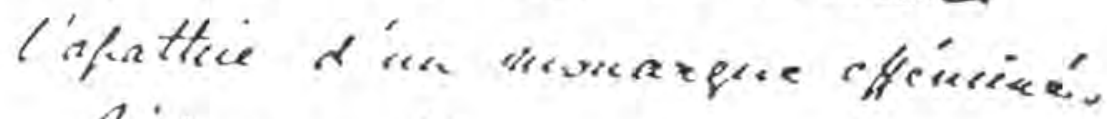

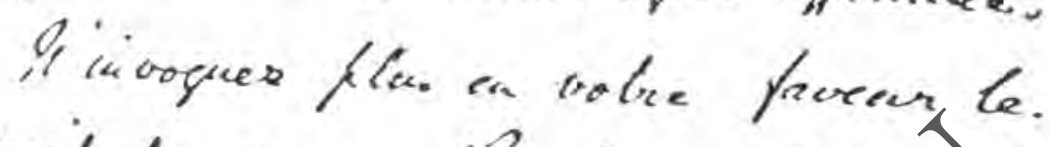

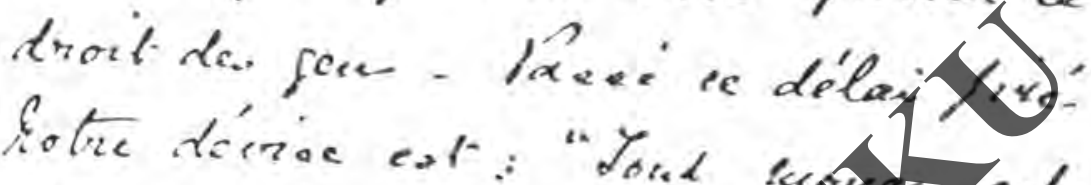

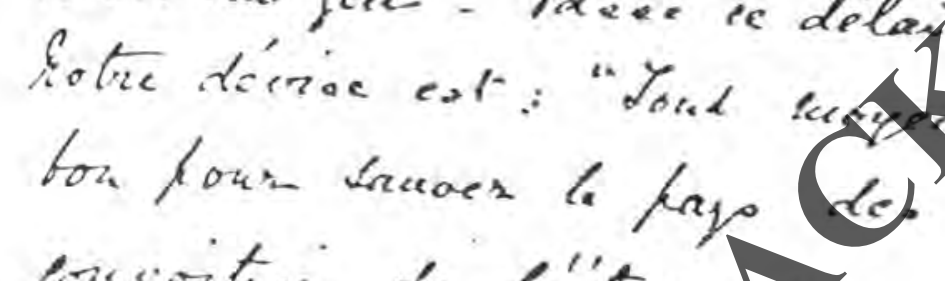

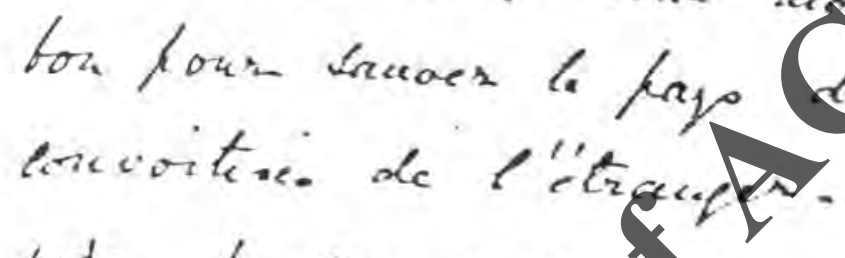
(1.d) de chet de la Sogre

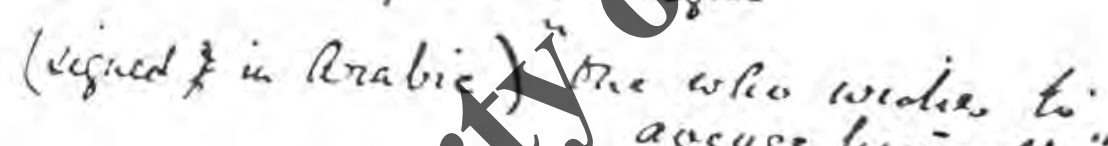


rTo

7

Pafe

- Pratiotigue

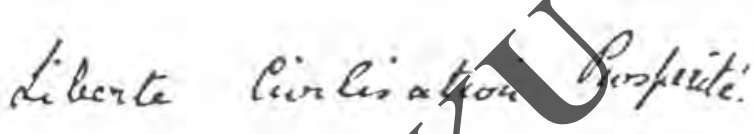

phecues

(*)

Liridound terald

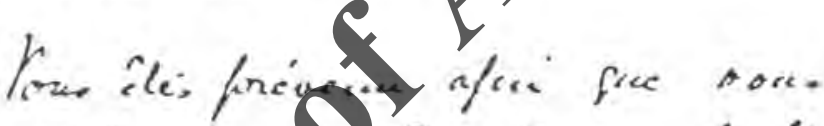

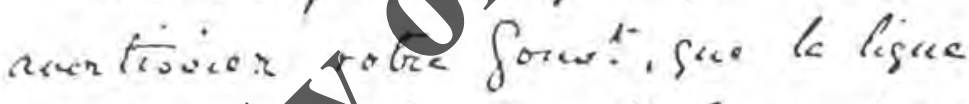

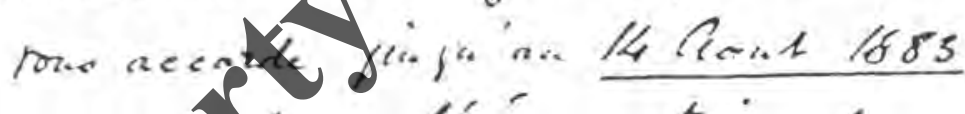

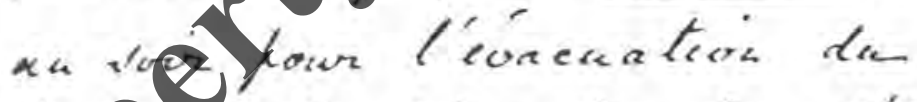

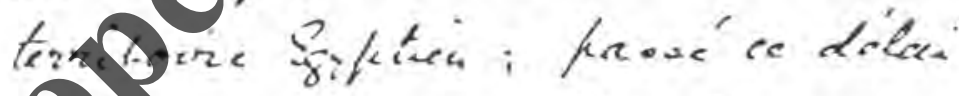

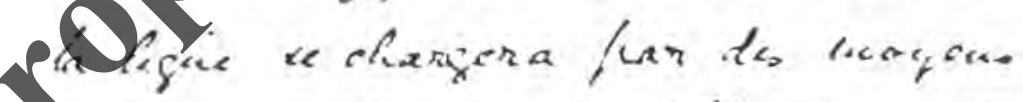

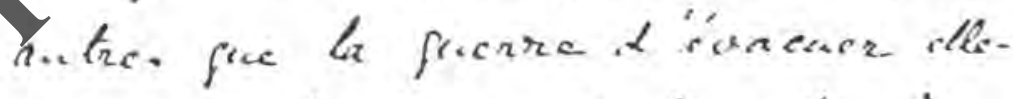

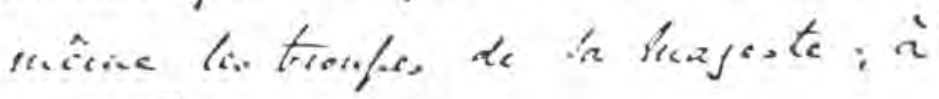

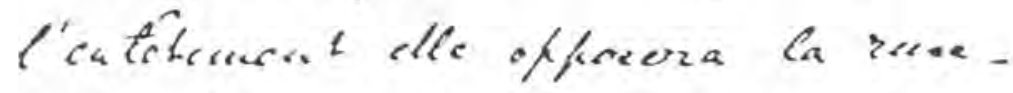

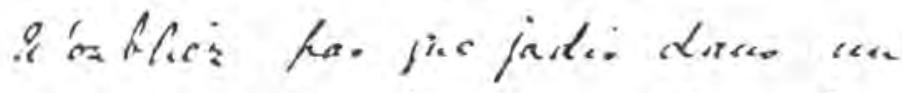

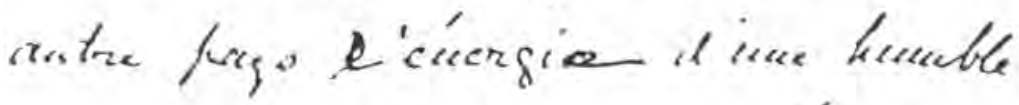
fiasoame 
6

rry

butten hy fusen have high hiviars unerit

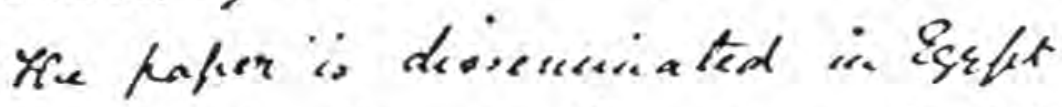

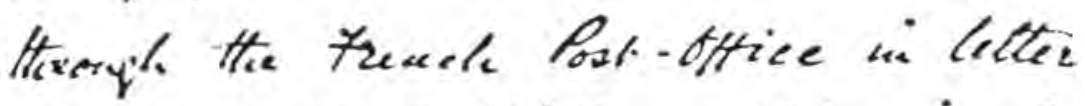
enrers - The fublioliseng of iec io at '́d' Acoume de ficily. Presi

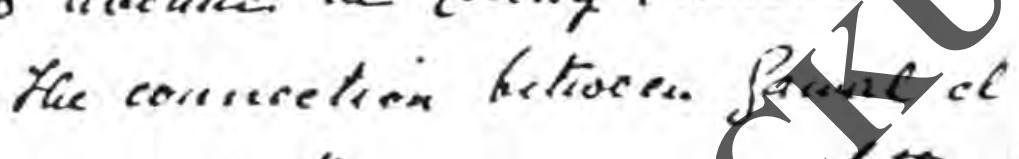

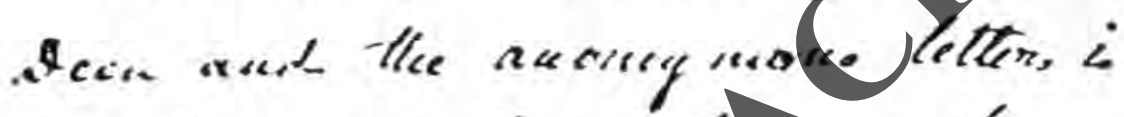

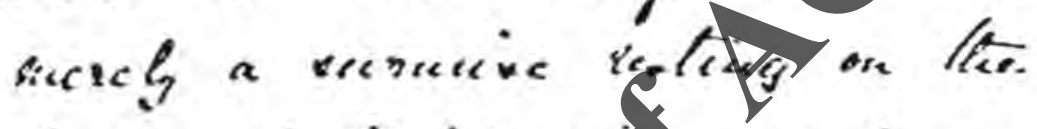

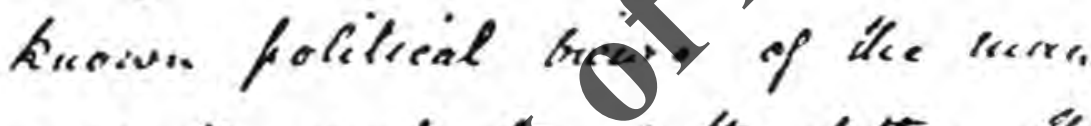
read the emetenty of the letteres. Mlie one to lluerif tizeled italis that the nacilers 80 the d'eague are brottere

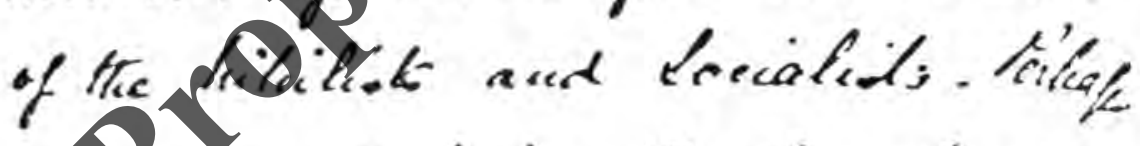

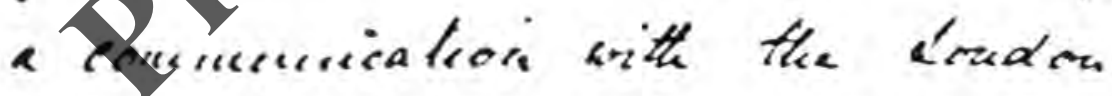
PAice Auttenitisi might-eliect o

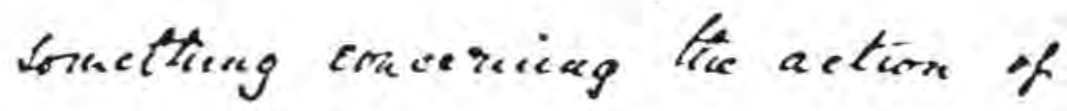
faual ol tocer al Fine; , whome it

locked. 
FTY

5

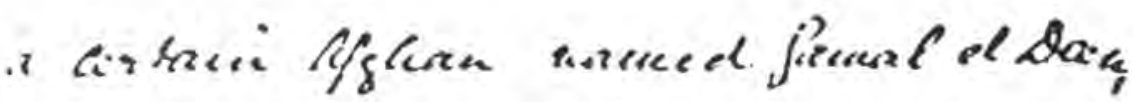

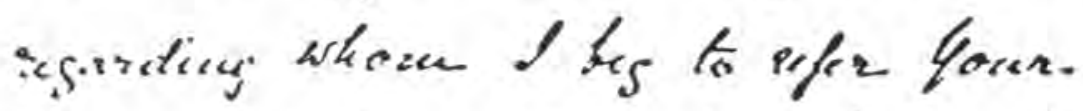
anderiefe to a Dexputelo frowe Hax thecelles to Lard Salcitrury liges if the 2ols of Rucguob lisy 1

If is known that fratuafel bea

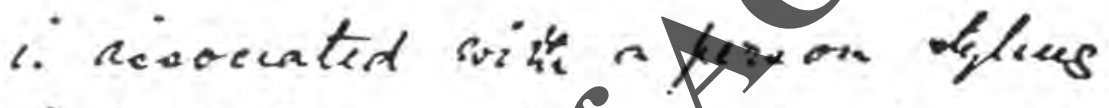

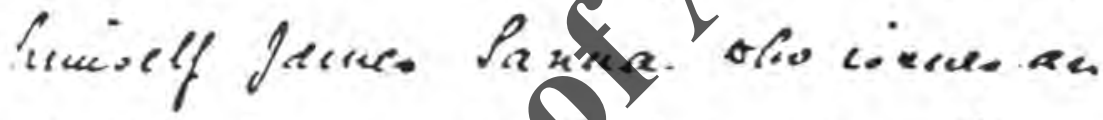

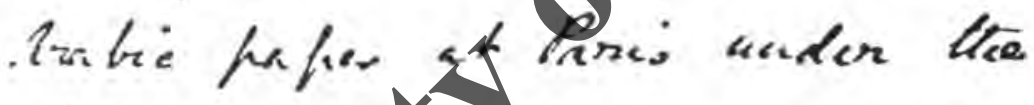
title of "Rporderederen. of whecels

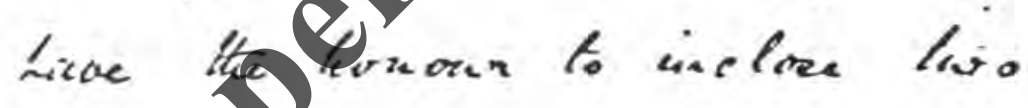

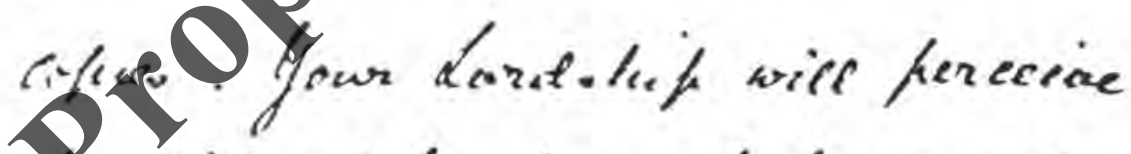
Brifh of the fangur to the caricature.

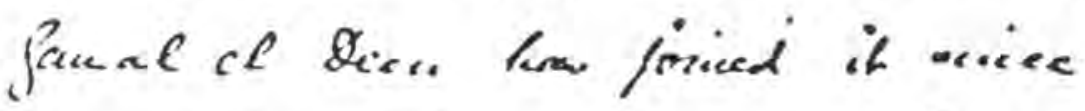
the siclitisy interventeone is isskt, and I am told that lice Artide

ansittea. 
4

FrA

Politieal

$1191 / 4$

fravo

infiterelial

Hiney 28. 1885

Sity land

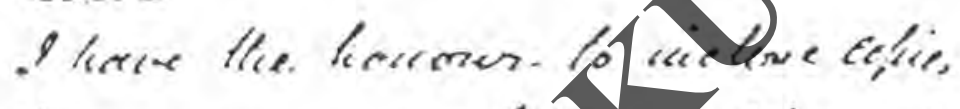

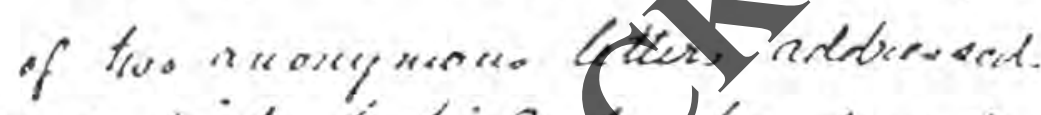

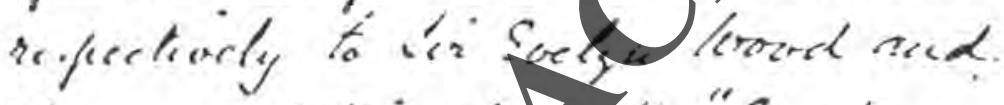

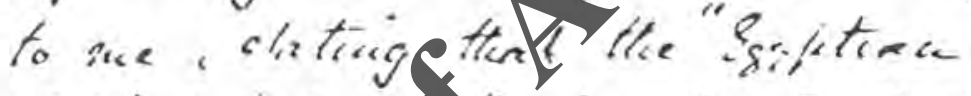

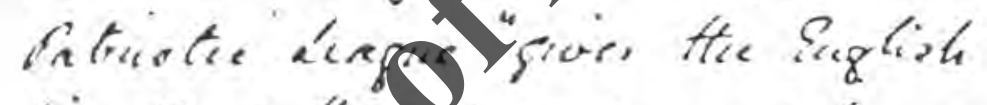

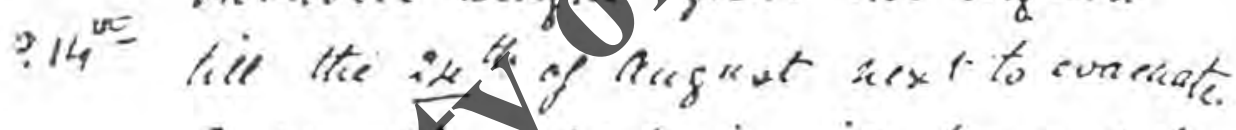

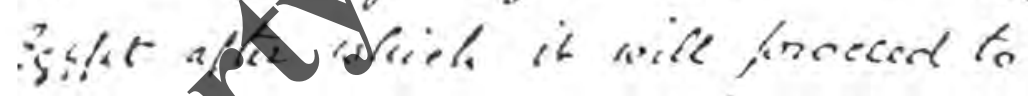

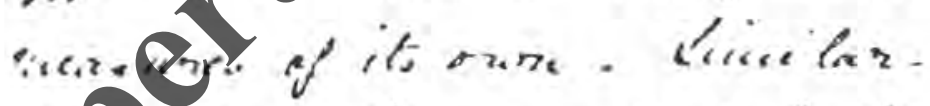

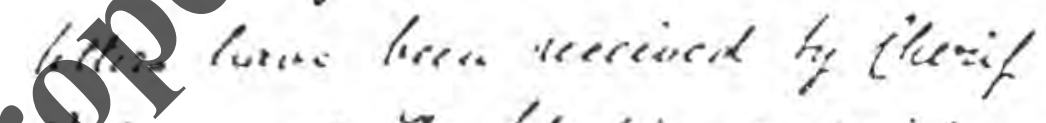

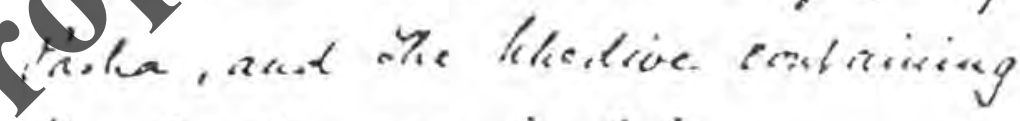
"lacerte of noweverienteris -

It to ke livel tuat these letters

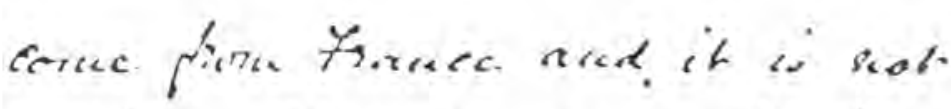

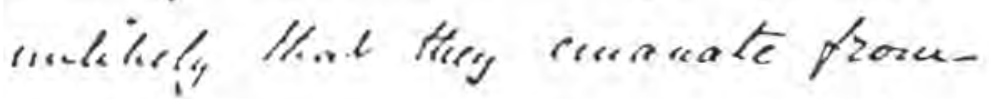
ic Ear fiaroille ky. 
bosons in arabic.

Ihaur not yet heen able to dif coves ardence cormer ke nowes which supports the

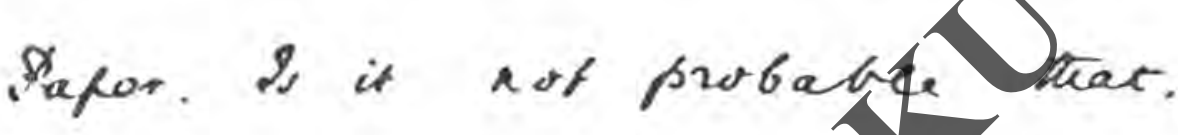
s. me of the frinciate berefits which inclish intwence bill brieg to fintat is to free the Pearant from the nower. baver, ena lo reduce the herent usurious tati changes to the infortimate feliexth, the ncw kones lending kiteñest 
Sriotical in Daxis.

Shave been abee aflis. Some delces to oftain is following pattialars rath heim.

is Pruxa is a dace Sination gew whollives of his sen, which pefis willing to thing topge teot profis. He is lioglent and wiles well; Int it is zeldon his aftain ine proskeross, and he pequenthe honows nones from my Jaformant 
PrI

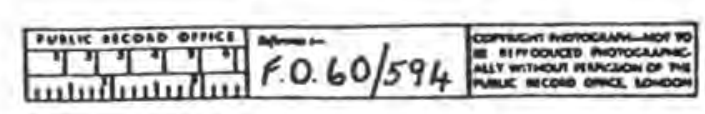

Peisia

Sjemal - ed - Sin (famal-ac-Ain av)

Prosecdinge of. Prespatieste from gent.

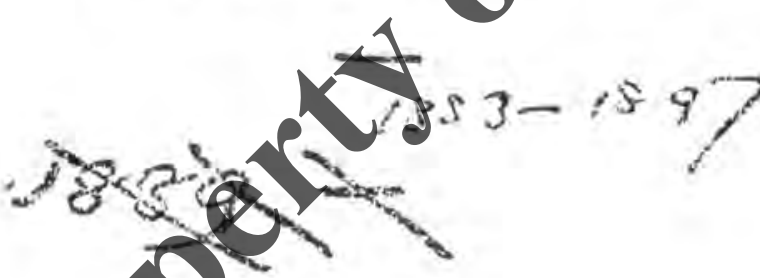

$\stackrel{4}{x \rightarrow m}$

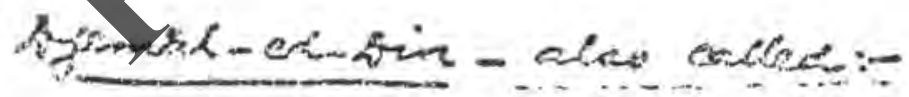

Semaletaen

* Gerral-codalie

Glal-ck-sin

Gervoludain

gemal-uá-dirs

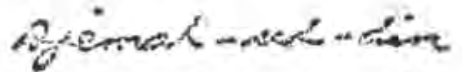

Giomal - ax-and

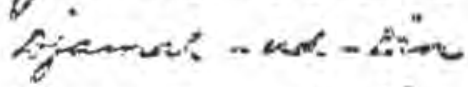

Gamal wne-sinfirmalendir 


\section{فهر ست اعلام}

افشار، ايوج

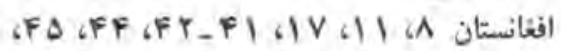

vV

الفغانى - بيدجمال الدين حسينى اسدآبادى

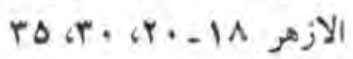

التجاره 19

الجزاير التجاره

rv العنتقمريم

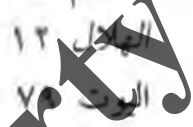

(n)

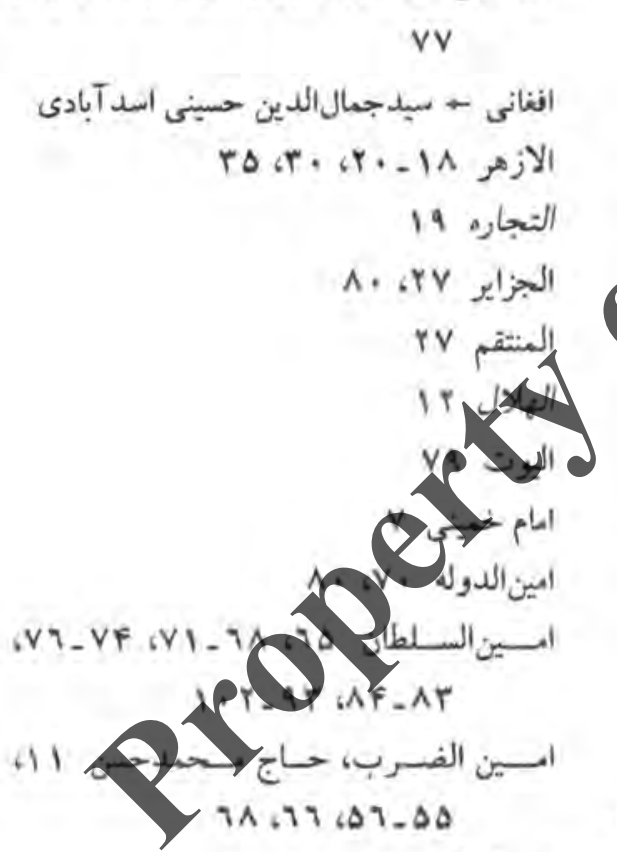

$$
\begin{aligned}
& \text { امين اللكك } 4 \text { انجمن } \\
& \text { انجمن دانش الثي } 11 \\
& \text { iv انجيل }
\end{aligned}
$$

اندوتزى

انقلاب ايران اندون

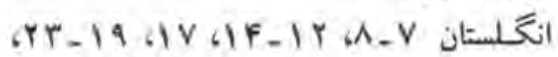
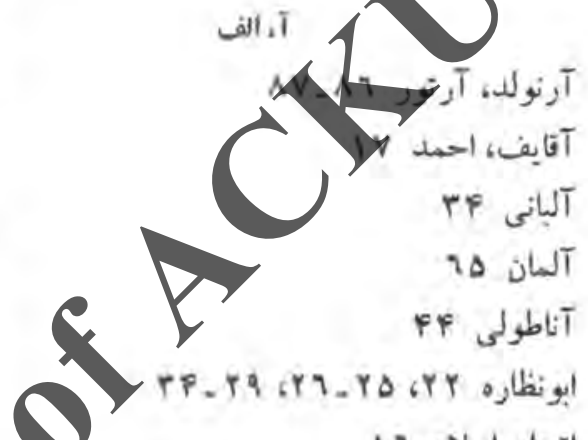

اتحاد اسلام 17

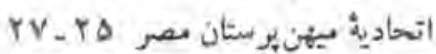

19 اخوانالمسلمبن 19

اديب اسحق أسوان 18

ro أستراليا أستون

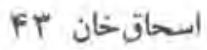

17 اسدآباد كنرون

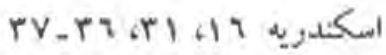
السـلامبول

$\wedge \wedge, \vee q$

PF CrI CY. اسعاعيل باثشا

احفهان OD_OF

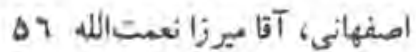

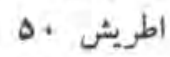

اعتمادالسلطنه، محمدحسن خان هF 


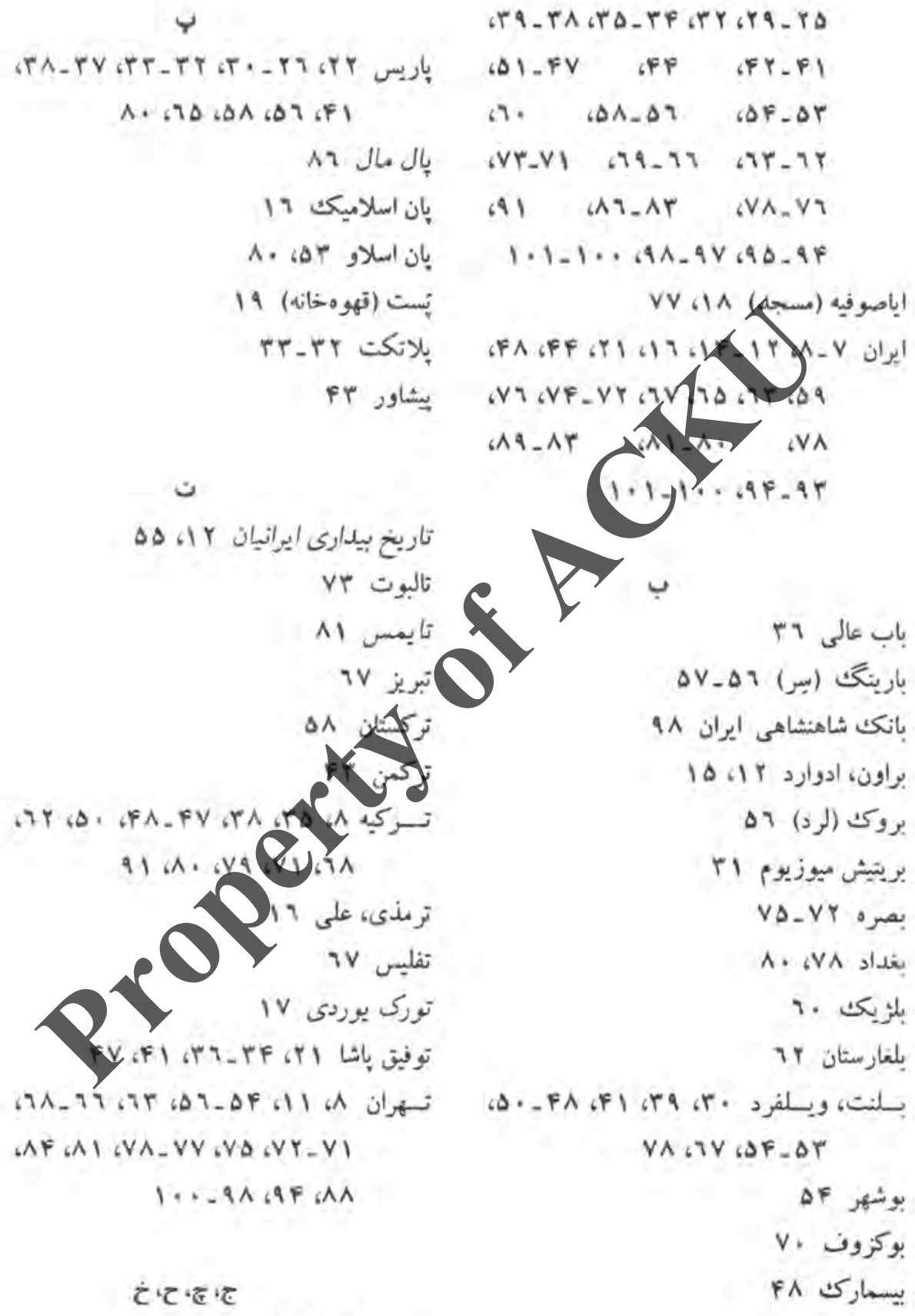

$$
\text { جله ا Y Y Y }
$$


j.j.

$$
\begin{aligned}
& \text { IV دوست محسدخان } \\
& \text { دملى دو }
\end{aligned}
$$

رون.

$$
\begin{aligned}
& \text { رابينو A }
\end{aligned}
$$

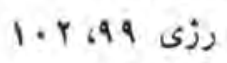

$$
\begin{aligned}
& \text { ركن الدوله } 94 \\
& \text { رنان، ارنست ركن رول }
\end{aligned}
$$

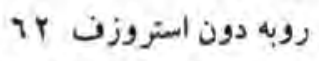

روسيه

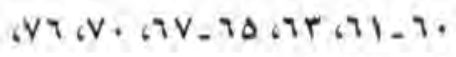

$$
\text { AI-Va เVA }
$$

ه. روم

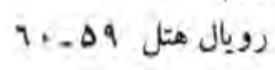
زيدان، جرجى
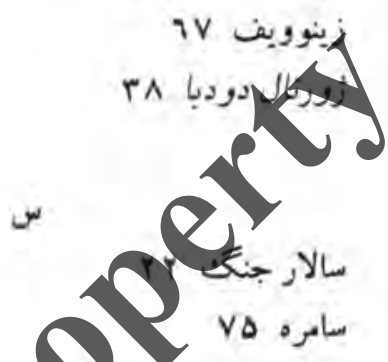

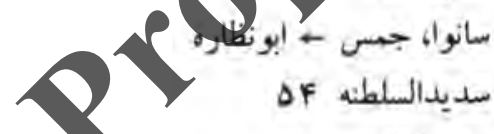
سعد ذغلول 19

سلطان احمد (مسجد) 11 نول

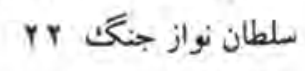$$
\text { سلفي } 17
$$

سنت هترزبورگت

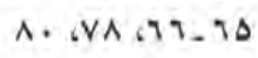

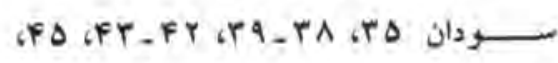

$$
\text { جمال ألدين افغانى ـ هيدجمال الدين حسينى }
$$

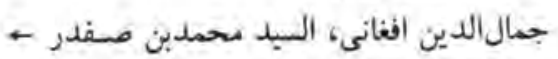$$
\text { سيدجمال الدين حسينى }
$$

Fر جيل، بير وينستن

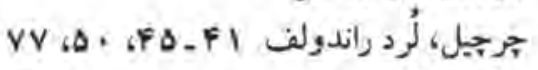

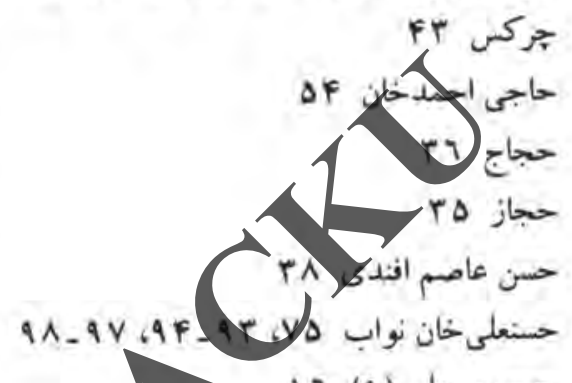

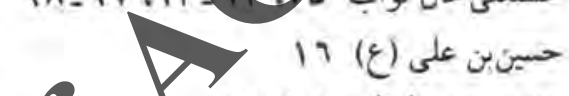

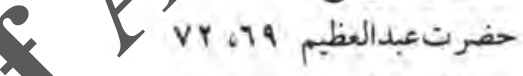
حمدالله حفر

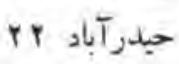
خانقين Vr.VY

خاورميانه خ خانهين FF خزر (بحر) خسرو, خز (بحر) خسروشاهى، سيدمادى 1 خ A-V حواندنيها 2

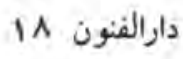

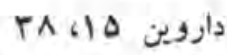

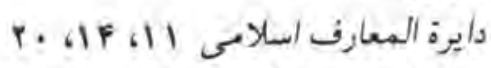

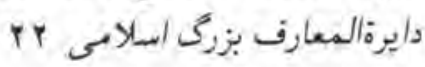

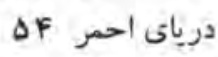

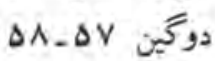




$$
\begin{aligned}
& \text { Fب عبدالرحمان } \\
& \text { or عبدالرسول } \\
& \text { عبده، شيخ محهد } \\
& \text { Fi عنهان دقنان }
\end{aligned}
$$

عثنانى

$$
\wedge \Lambda
$$

Fد عدن

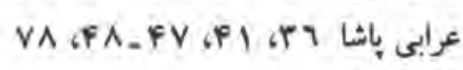
عراق هراب هان هربستان rA عروةالوثقى على Tباد 11

على روقاى بلى باد عمرياشا لطفى جواي

ق.

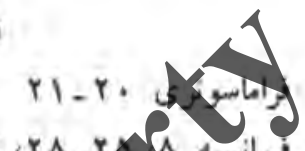

GA ،

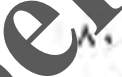

Fی

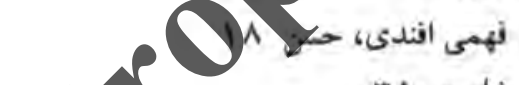

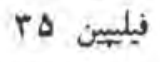$$
\text { قاجاريه }
$$

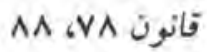

قاهره $V V, \Delta \Lambda, \Delta \backslash, F \Lambda, r V$,

$$
\begin{aligned}
& \text { تبرس } \\
& \text { قفقاز هده، هدر }
\end{aligned}
$$

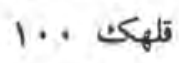

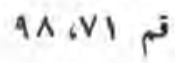

$$
\begin{aligned}
& \Delta V \text { \& } F \text { _ FV } \\
& \text { سوربون rA } \\
& \text { ro سوريه }
\end{aligned}
$$

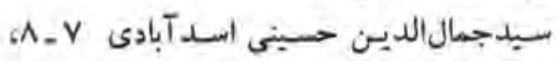
irt-ro 6rT-10 $11 \%-11$

$\Delta I-F V$ GFD-FI 6 TX-TV

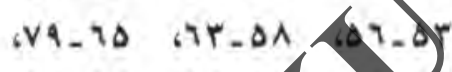

. AN-9T “AN-AV

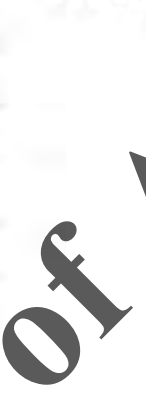

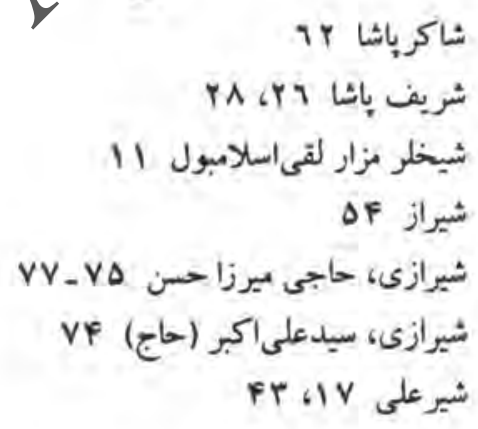

ம VF صياءالخافقين b

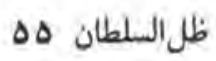
$\varepsilon$ عالى بأشا عالما

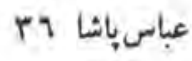


م

عاركيز آن، ساليسبوزى I IFV GT 699 .4D 64 KAF $6 \mathrm{VA} 6 \mathrm{VD}$

$$
1 \cdot T-1,1
$$

مالت، ادوارد

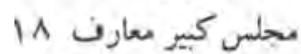
IV محمداعظم VA fF

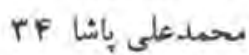
نحملدعلى خان كاشيى Aي مخبرالدوله هديترانه مرآت الثـرت FV مراكث مرات FD_FF مرو مرو

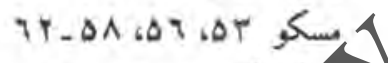

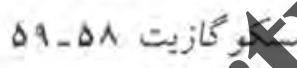
or it

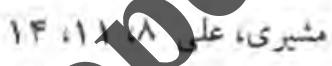
AY-11.17. IF GT-rr Créra irV_ro

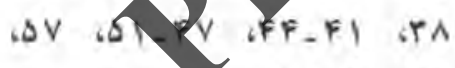

$916 \Lambda+2 \vee 9$
FD

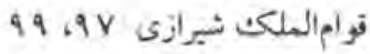
r. $\Delta \wedge,|\nabla| 1)$ كأكون كاموند (ئركت بوادزان كاموند) rF كانالائي

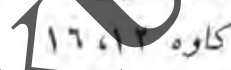
كر مانشاه ) كثمير Fr

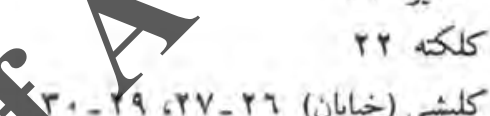
كليثى (خيابان)

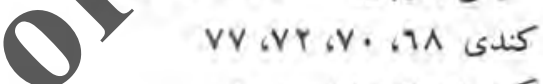
P. كرانويل برانوران

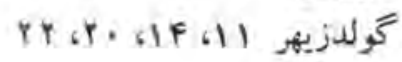

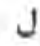

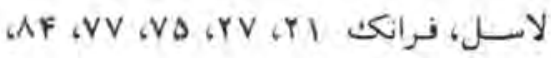
$1+69 \wedge-9 r$

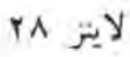
7. لردبايرون

79 ل

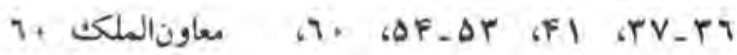

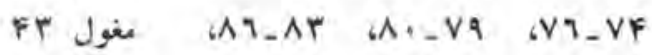

Q1 ITD GT GIV \& 1.. $69 \wedge 697.9 F . \wedge 9-\wedge \Lambda$

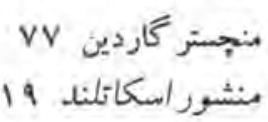
4. لوزاكت 11 


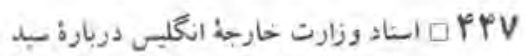

$$
\begin{aligned}
& 1.4-99.97-90
\end{aligned}
$$

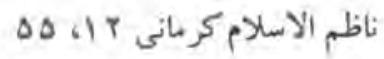

$$
\begin{aligned}
& \text { نجد ناطم الندا } \\
& \text { A. نونى تورورد }
\end{aligned}
$$

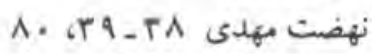

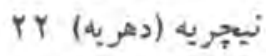

$$
\begin{aligned}
& \text { نيل نيانج }
\end{aligned}
$$

$\sin , 9$

$$
\begin{aligned}
& \text { موريو A }
\end{aligned}
$$

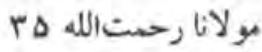

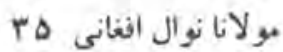

$$
\begin{aligned}
& \text { موئيخ }
\end{aligned}
$$

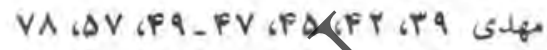
هيرزا جعفرخا هان

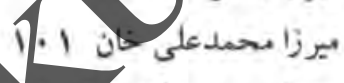
ولادى

ولف، دوروبند

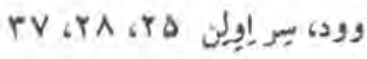

$$
\begin{aligned}
& \text { وهبى بيكت ودو لون } \\
& \text { ويس وهن بله } \\
& \text { 1. } \\
& \text { १ } \Delta, \wedge \Lambda \text { ، } \wedge \Delta_{-} \wedge r
\end{aligned}
$$

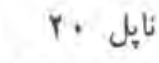

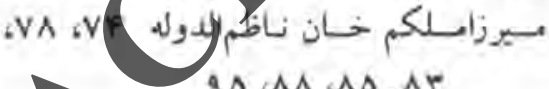
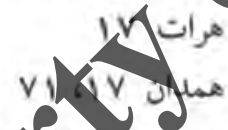

os o.rra

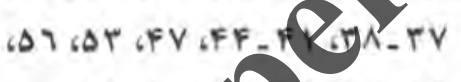

91 , $A$. ARO

نابلئون PF نابل

نادرشاه

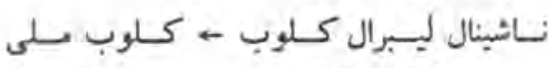
آزاديخوانهان

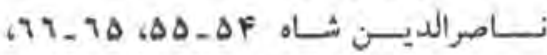

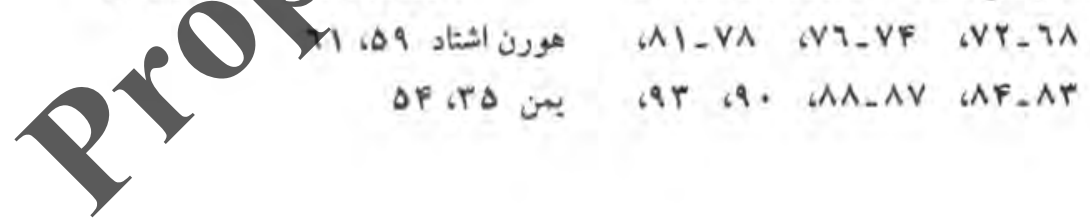




\section{The Original Jext of}

\section{British Foreign M
Ducuments}

\section{Sayyed Djemal-ed din Afghani}

Compiled by:

S. Hadi Khosrowshahi 


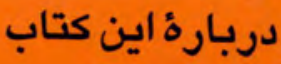

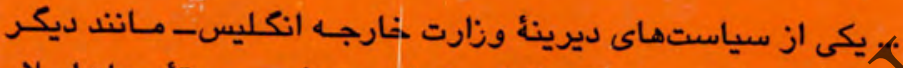

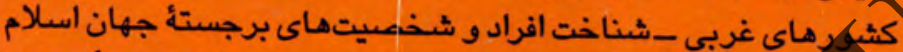

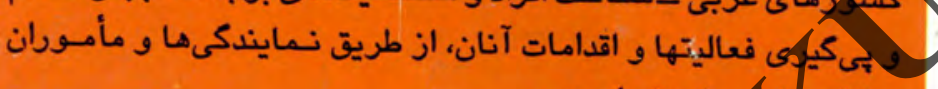

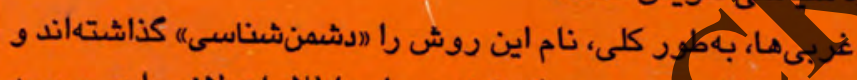

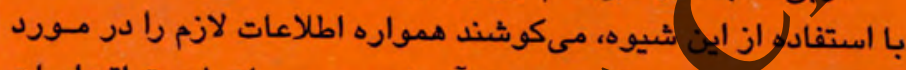

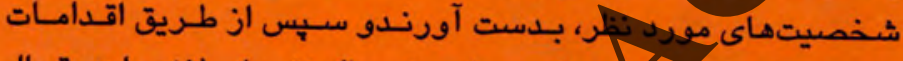

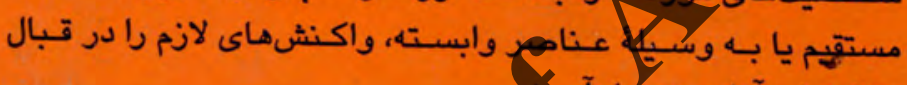

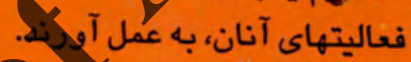

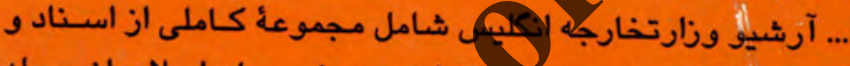

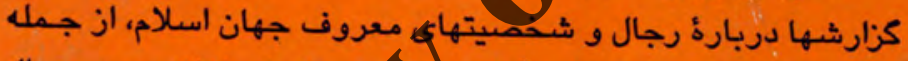

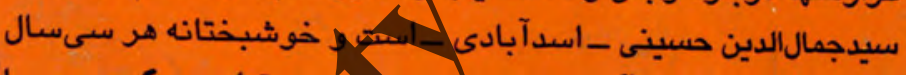

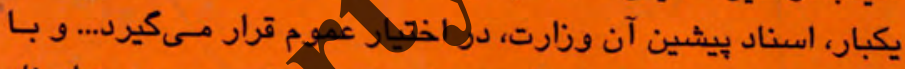

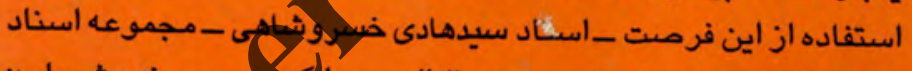

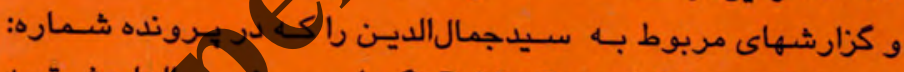

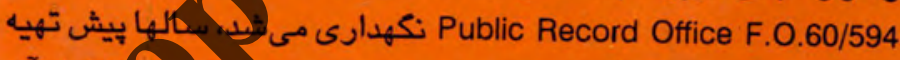

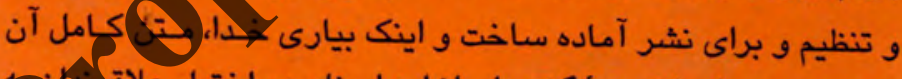

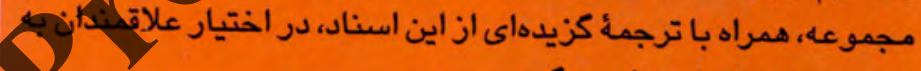

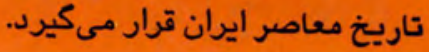

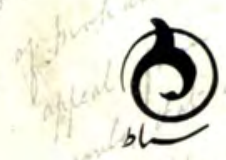

ناشر: سماط و.كلبغ شروق. تهران. خيابان انقلان. مقابل دانشكاه. شمارة IrVA. 